\title{
Exploring the Bidirectional Effects of Personality and Negative Social Interactions across Adulthood
}

Nicole M. S. Belanger

Follow this and additional works at: https://researchrepository.wvu.edu/etd

\section{Recommended Citation}

Belanger, Nicole M. S., "Exploring the Bidirectional Effects of Personality and Negative Social Interactions across Adulthood" (2018). Graduate Theses, Dissertations, and Problem Reports. 5179.

https://researchrepository.wvu.edu/etd/5179

This Dissertation is protected by copyright and/or related rights. It has been brought to you by the The Research Repository @ WVU with permission from the rights-holder(s). You are free to use this Dissertation in any way that is permitted by the copyright and related rights legislation that applies to your use. For other uses you must obtain permission from the rights-holder(s) directly, unless additional rights are indicated by a Creative Commons license in the record and/ or on the work itself. This Dissertation has been accepted for inclusion in WVU Graduate Theses, Dissertations, and Problem Reports collection by an authorized administrator of The Research Repository @ WVU.

For more information, please contact researchrepository@mail.wvu.edu. 
Exploring the Bidirectional Effects of Personality and Negative Social Interactions across Adulthood

Nicole M. S. Belanger, M.S.

Dissertation submitted to the Eberly College of Arts and Sciences at West Virginia University

in partial fulfillment of the requirements for the degree of

Doctor of Philosophy in Psychology

\author{
Nicholas A. Turiano, Ph.D., Chair \\ Aaron Metzger, Ph.D. \\ JoNell Strough, Ph.D. \\ Natalie J. Shook, Ph.D. \\ Megan R. Dillow, Ph.D. \\ Department of Psychology \\ Morgantown, West Virginia \\ 2018
}

Keywords: Big Five personality traits, negative social interactions, reciprocal, transactions Copyright 2018 Nicole Belanger 


\section{ABSTRACT \\ Exploring the Bidirectional Effects of Personality and Negative Social Interactions across Adulthood}

\section{Nicole M. S. Belanger}

Personality and negative social interactions (NSIs; interactions with social network members that are perceived to be a violation of relationship norms; Brooks \& Dunkel Schetter, 2011) are associated with detrimental health outcomes (Hampson \& Friedman, 2008; Hill, Weston, \& Jackson, 2014). Personality is also associated with the occurrence of NSIs (Allemand, Schaffhuser, \& Martin, 2015; Bono, Boles, Judge, \& Lauver, 2002; Silva, Henrie, \& Patrick, 2016). However, both of these constructs change across adulthood (Carstensen, Isaacowitz, \& Charles, 1999; Roberts, Wood, \& Smith, 2005) so it is important to understand how the associations between these two variables may change over time. The few studies that have explored such longitudinal associations by examining the reciprocal associations between them are scarce. Utilizing a sample of 1,530 adults $\left(M_{\text {age }}\right.$ at Time $1=46.03, S D=10.50,49.40 \%$ female) from the Midlife Development in the United States (MIDUS) dataset, the reciprocal associations between the Big Five personality traits and NSIs were examined over 18 years using latent growth curves. Results indicated that (a) personality traits and NSIs change over adulthood; (b) personality traits predict the occurrence of and change in NSIs over time; (c) NSIs predict personality levels and change in personality over time; and (d) age significantly moderated these associations. The findings provide insight into how personality and NSIs exhibit differential associations and patterns of change across adulthood based on one's age.

Keywords: the Big Five personality traits, negative social interactions, reciprocal, transactions 


\section{Acknowledgements}

I would like to thank Dr. Nicholas Turiano, my dissertation chair and advisor, for his guidance throughout this project and my last three years at West Virginia University. I would also like to thank Dr. Julie Patrick for her guidance my first two years at West Virginia University. I am also grateful for the support, recommendations, and time from my committee members Drs. Aaron Metzger, JoNell Strough, Natalie Shook, and Megan Dillow.

I dedicate this dissertation to the following people:

My daughter, Madeline Rose: Always pursue what makes you happy. It might be hard and take longer than what you anticipated, but it will all be worth it in the end. Remember that different roads sometimes lead to the same castle.

To my husband, Brian: Words cannot adequately express my gratitude. Thank you for your sacrifices to ensure that Madeline and I can pursue all of our dreams.

To my friends and family: Thank you for your never ending support. A special thank you to my mother, who took on more than her fair share of child care duties to make all of this work possible. "When the snows fall and the white wind blows, the lone wolf dies but the pack survives."

Finally, to my cousin Derrick and my grandfather Manuel who both passed away unexpectedly during the Fall of 2017 and Spring of 2018, respectively. 


\section{Table of Contents}

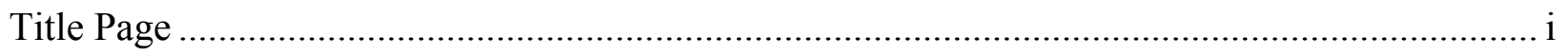

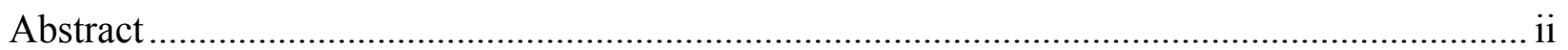

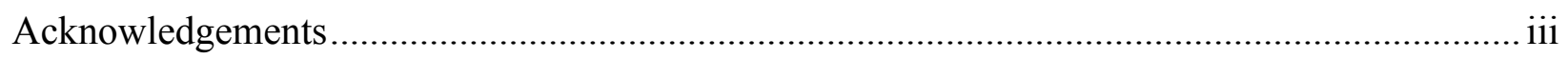

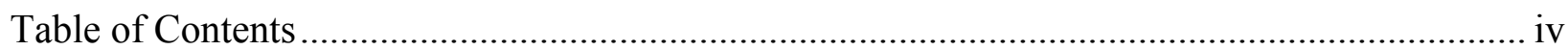

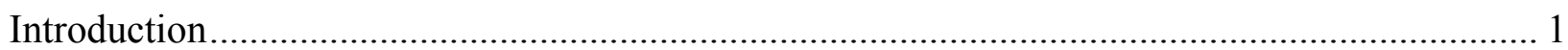

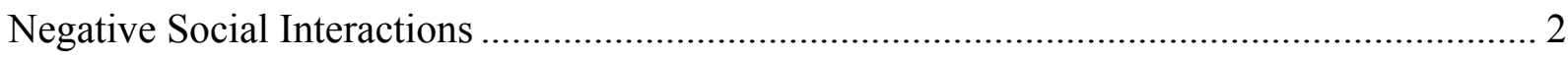

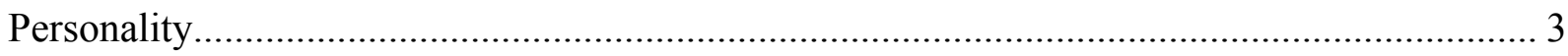

Personality and Negative Social Interactions ........................................................... 4

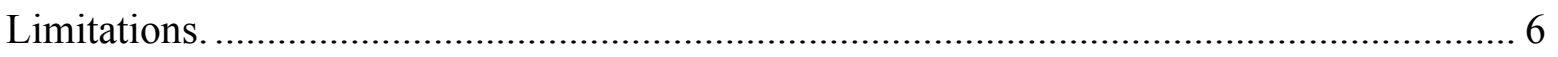

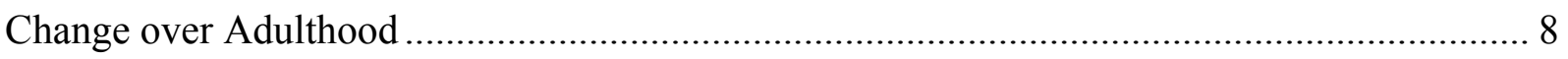

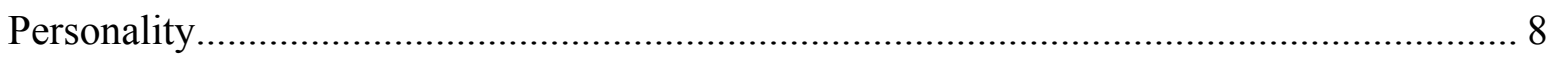

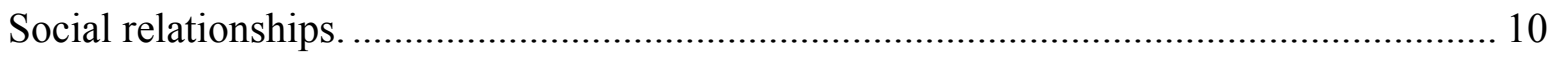

Personality and change in negative social interactions.......................................... 11

Negative social interactions and change in personality.............................................. 12

Change in personality and change in negative social interactions................................... 13

Age as a Potential Moderator............................................................................................ 14

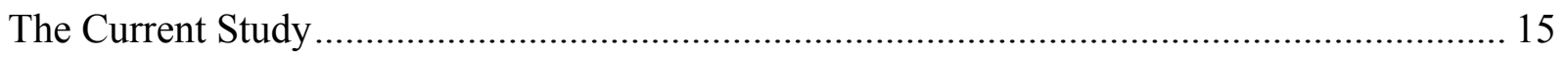

Research Questions and Hypotheses ................................................................ 16

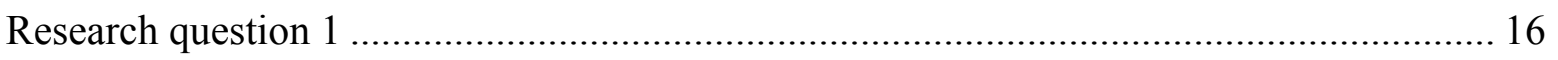

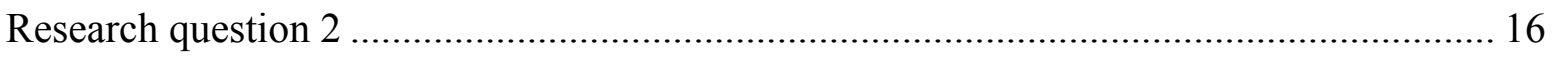

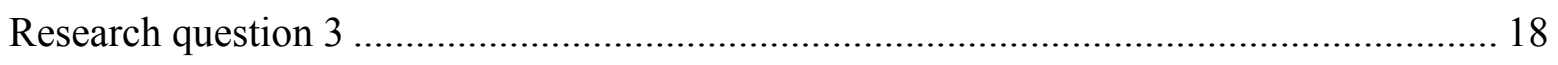

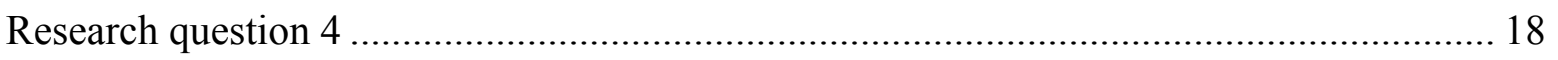

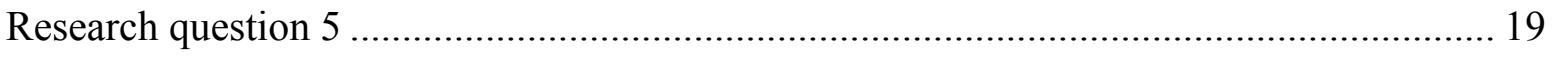

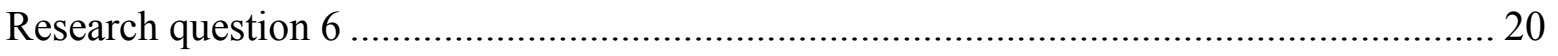

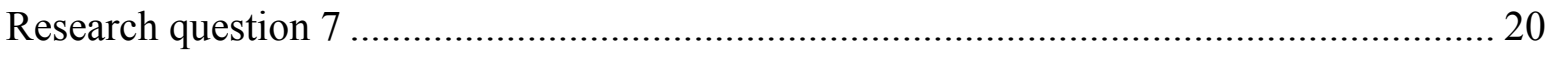

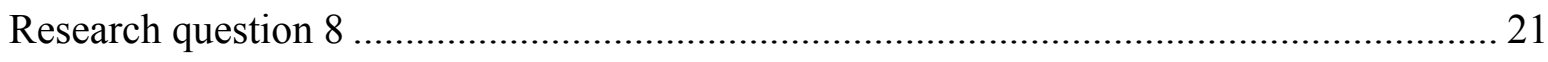

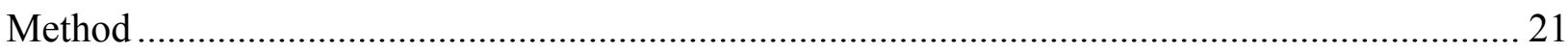

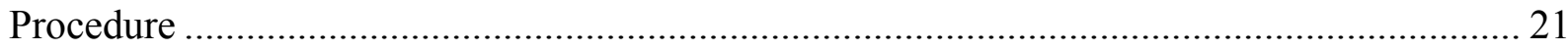

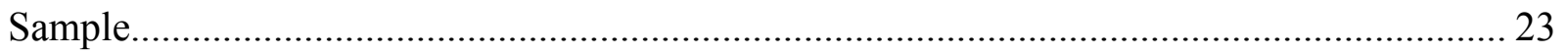

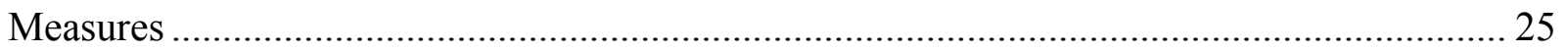

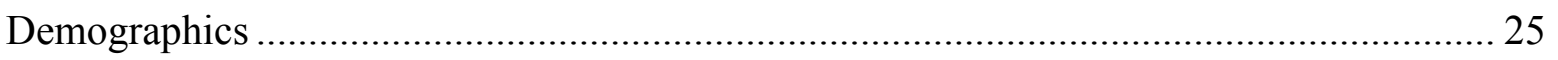

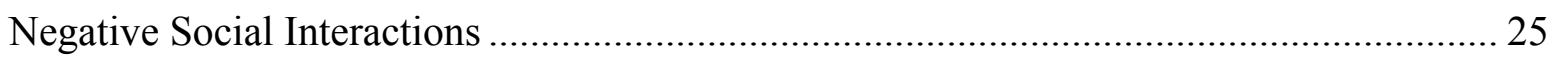




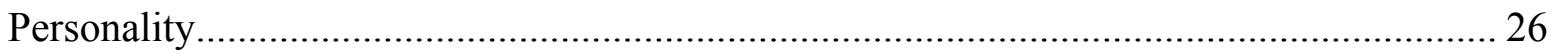

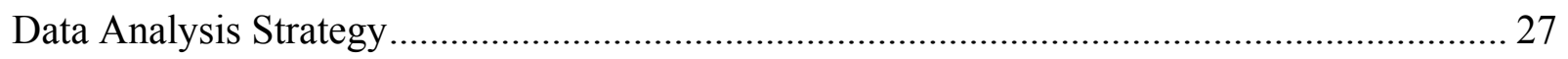

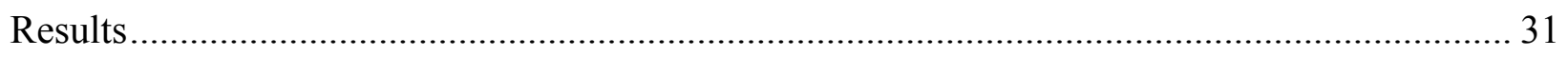

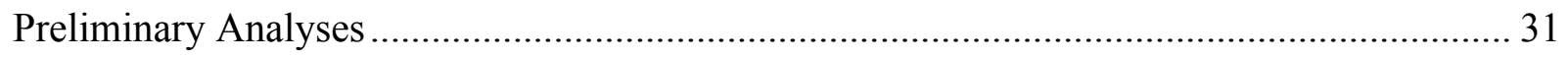

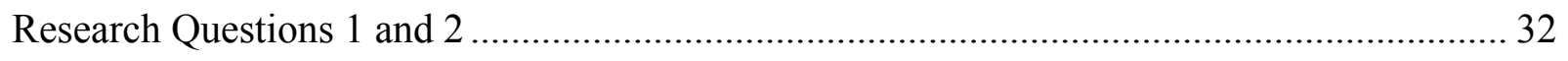

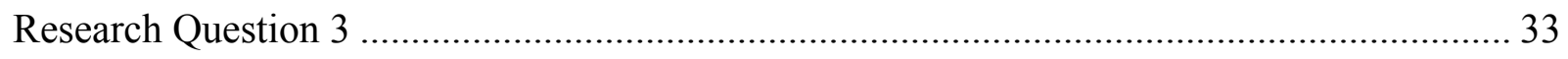

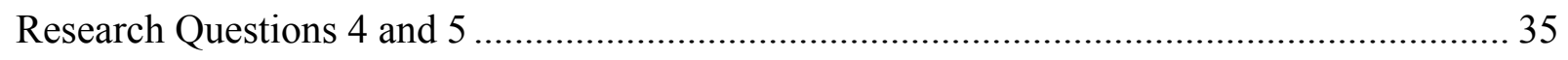

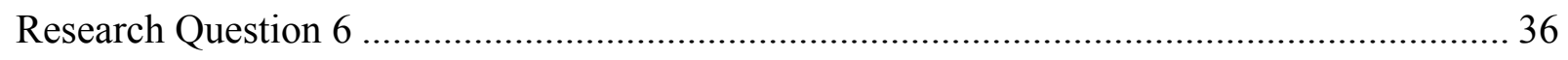

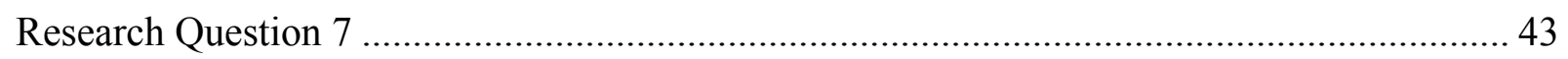

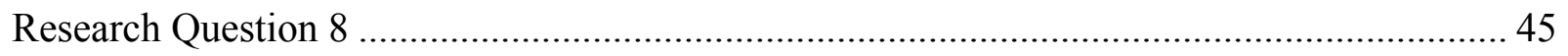

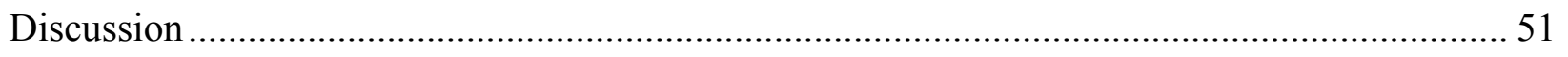

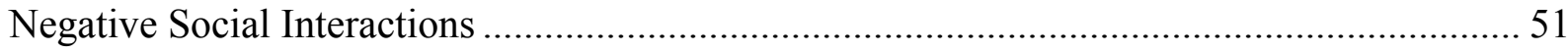

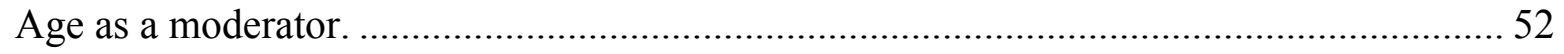

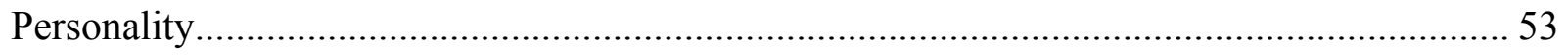

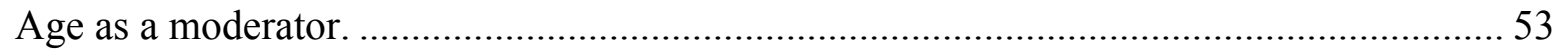

Personality and Negative Social Interactions …………….............................................. 56

Personality predicting negative social interactions....................................................... 56

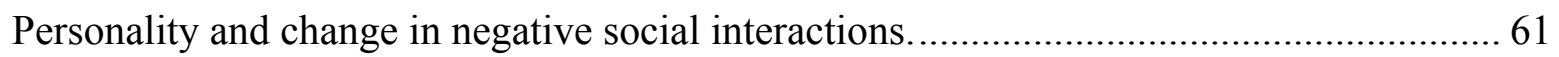

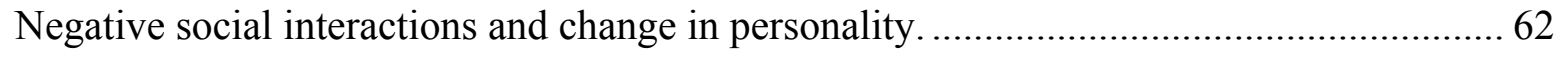

Change in personality and change in negative social interactions........................................ 62

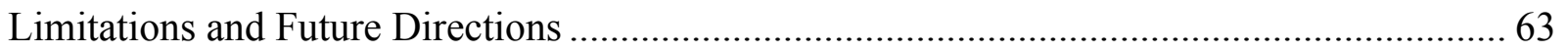

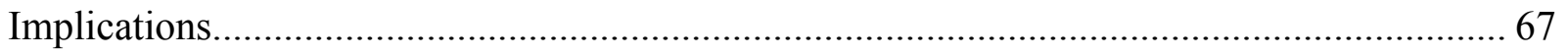

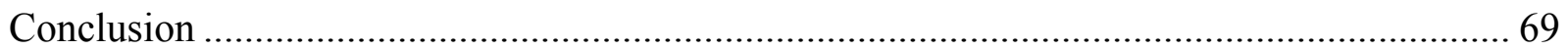

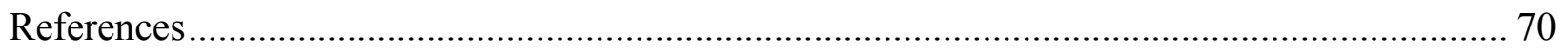

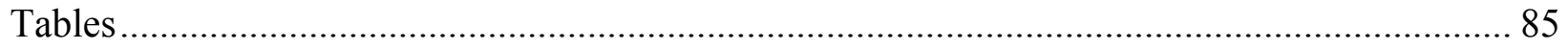

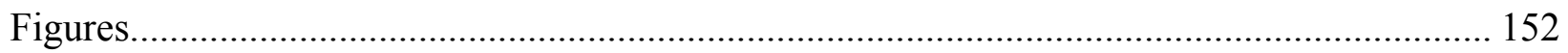

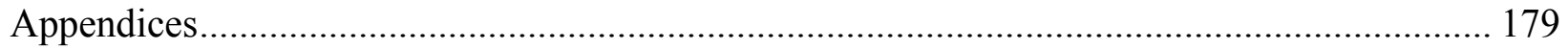


Exploring the Bidirectional Effects of Personality and Negative Social Interactions across

\section{Adulthood}

Personality is associated with health over the life span, and ultimately how long someone lives (Hampson \& Friedman, 2008). With this association now well-established, researchers have focused their attention on understanding why personality is associated with health and longevity. Researchers have examined the role of health behaviors, substance use, and coping patterns as mediators of this association (Kern \& Friedman, 2011; Smith, 2006; Turiano, Chapman, Gruenewald, \& Mroczek, 2015). However, there is only limited support for these pathways (Kern \& Friedman, 2011). The field is now exploring social relationship variables as potential mediators of the personality-health association (Kern \& Friedman, 2011), because personality and social relationships are associated with one another (Asendorpf, 2002; Roberts, Wood, \& Caspi, 2008), and because social relationships are associated with various health outcomes (Cohen, Doyle, Skoner, Rabin, \& Gwaltney, 1997; Holt-Lunstad, Smith, \& Layton, 2010; Uchino, 2004, 2006, 2009). Before the examination of these pathways are explored in further detail, additional research is needed because both personality and social relationships change in adulthood (Carstensen, 1992; Caspi \& Roberts, 2001; Hampson \& Friedman, 2008; Roberts \& Wood, 2006). Thus, understanding how these factors change together will provide insight regarding their differential associations to health across adulthood.

One social relationship construct that is important to examine is negative social interactions (NSIs). NSIs are interactions with social network members that are perceived to be a violation of relationship norms (Brooks \& Dunkel Schetter, 2011). It is important to examine NSIs because they have been associated with a host of negative health outcomes such as diabetes, lung disease, high blood pressure, stroke, and mortality (Hill et al., 2014; Lund, 
Christensen, Nilsson, Kriegbaum, \& Hulvej Rod, 2014). Because of these detrimental health outcomes, it is important to examine factors that predict the occurrence of NSIs. Individual differences in personality can influence who may be at risk of experiencing NSIs. Furthermore, there may be a reciprocal association between personality and NSIs over time (Magnusson, 1990; Magnusson \& Stattin, 1998). Research regarding these associations is scarce and there are considerable limitations within these handful of studies which have not been addressed. Utilizing 18-year longitudinal data from Midlife Development in the United States: A National Longitudinal Study of Health and Well-Being (MIDUS), this study examines whether there is a reciprocal association between the Big Five personality traits and NSIs while addressing several key limitations with prior research.

\section{Negative Social Interactions}

NSIs are interactions with social network members that consist of behaviors perceived to be a violation of relationship norms. NSIs consist of multiple dimensions of aversive interactions such as rejection, neglect, conflict, insensitivity, interference, and unwanted advice (Brooks \& Dunkel Schetter, 2011; Newsom, Rook, Nishishiba, Sorkin, \& Mahan, 2005; Rook, 1998). Although individuals can experience a NSI with anyone, NSIs are more likely to occur within close relationships (Sorkin \& Rook, 2004). This includes, but is not limited to, relationships with parents, children, friends, and romantic partners.

The construct of NSIs has been used interchangeably with terms such as social negativity, social undermining, social conflict, and negative social support (see Brooks \& Dunkel Schetter, 2011). These terms represent specific dimensions of NSIs, whereas the construct of NSIs is more broad and inclusive (Brooks \& Dunkel Schetter, 2011). Although the term negative social support has been used in the literature, NSIs are empirically and conceptually distinct from social 
support (i.e., the actual receipt of, or the perceived availability of, emotional or instrumental support from the social network; Antonucci \& Jackson, 1987; Okun \& Keith, 1998). Individuals can experience NSIs and social support simultaneously (Mavandadi, Sorkin, Rook, \& Newsom, 2007; Newsom et al., 2005; Okun \& Keith, 1998). Although individuals report more positive interactions relative to negative interactions in general (Newsom et al., 2005), NSIs are the most experienced type of daily stressor (Luong \& Charles, 2014).

\section{Personality}

Personality represents individuals' relatively enduring thoughts, behaviors, and emotions. The most prominent trait theory in personality is the Big Five (John \& Srivastava, 1999), which organizes personality traits into a hierarchy. The bottom of the hierarchy consists of the behaviors, thoughts, and emotions individuals engage in or experience in their daily lives. These characteristics combine to create facets, which are correlated traits under each personality trait (e.g., negative affect for neuroticism). The facets combine to create five broad dimensions, which are agreeableness, openness, neuroticism, extraversion, and conscientiousness (Clark \& Watson, 2008; John \& Srivastava, 1999; McCrae \& Costa, 2008).

Agreeableness reflects the motive to maintain social harmony and a willingness to defer to others. Individuals who are higher in agreeableness are generally cooperative, altruistic, and trustworthy. Openness reflects the need for intellectual pursuits and the desire for novelty and variety. Individuals who are higher in openness are inquisitive, insightful, and inventive. Neuroticism reflects the tendency to experience negative affect. Individuals who are higher in neuroticism are tense, anxious, and despondent. Extraversion reflects a preference for social environments and the tendency to experience positive affect. Individuals who are higher in extraversion are gregarious, assertive, and outgoing. Conscientiousness reflects the tendency to 
be goal-directed and achievement-oriented. Individuals who are higher in conscientiousness are responsible, dependable, and practical (John \& Srivastava, 1999; McCrae \& Costa, 2008).

\section{Personality and Negative Social Interactions}

A developmental systems theory utilized to explain the association between personality and the social environment is dynamic interactionism (as demonstrated in Reynolds et al., 2010). Dynamic interactionism posits that individuals are active and purposeful agents in their development and environments, and that there is a continuous and reciprocal interaction between individuals and their environments, which leads to development (Magnusson, 1990; Magnusson \& Stattin, 1998). Individuals as active entities in their environments is demonstrated by personality-by-environment transactions. Specifically, individuals (a) actively pursue social environments that are consistent with their personalities (the attraction/selection principle); (b) manipulate their social environments to be more consistent with their personalities (the manipulation principle), and (c) evoke responses from their social environments that are consistent with their personalities (the evocation principle; Buss, 1987; Roberts et al., 2008).

For example, people who are higher in extraversion prefer jobs that contain a social aspect to them (e.g., teaching), and are more likely to be in social situations when assessed randomly using an experience-sampling method (the attraction/selection principle; Ackerman \& Heggestad, 1997; Wrzus, Wagner, \& Riediger, 2016). Workers actively modify aspects of their job to improve the fit between the demands of their jobs and their preferences (i.e., personality; Tims, Bakker, \& Derks, 2013; Wrzesniewski \& Dutton, 2001). This includes crafting jobs to be more social or creating more autonomy and flexibility within the parameters of the job (the manipulation principle; Wrzesniewski \& Dutton, 2001). Individuals who engage in dominant 
behaviors elicit submissive responses from their interactional partners (the evocation principle; Thorne, 1987).

Personality-by-environment transactions can expand to encompass the association between personality and NSIs. As mentioned previously, those who are higher in agreeableness attempt to maintain social harmony (John \& Srivastava, 1999; McCrae \& Costa, 2008) and are less likely to approach arguments (Blickle, 1997). As such, college students $\left(n=124, M_{\text {age }}\right.$ not provided) higher in agreeableness elicited less conflict from their interactional partners, Dutch workers $\left(n=173, M_{\mathrm{age}}=41.00, S D=11.04\right)$ higher in agreeableness reported less conflict at work, and younger German adults (range of $M_{\text {age }} 17.00-24.40$, range of $S D=0.77-4.60$ ) higher in agreeableness reported less conflict within their social networks (Asendorpf \& Wilpers, 1998; Blickle, 1997; Bono et al., 2002; Dijkstra, van Dierendonck, Evers, \& De Dreu, 2005; Graziano, Jensen-Campbell, \& Hair, 1996; Neyer \& Lehnart, 2007; Parker, Ludtke, Trautwein, \& Roberts, 2012; Sturaro, Denissen, van Aken, \& Asendorpf, 2008).

The association between openness and NSIs has not been explored in great detail relative to the other four personality traits (Bono et al., 2002). The literature that does exist suggests that individuals who are higher in openness are more likely to approach arguments (Bono et al., 2002). This is supported by research indicating that younger German adults $\left(n=2,173, M_{\text {age }}=\right.$ $19.51, S D=0.77$ ) higher in openness reported more conflict with their families (Parker et al., 2012).

Those who are higher in neuroticism are more likely to experience negative affect (John \& Srivastava, 1999; McCrae \& Costa, 2008), and select themselves into unstable and unsatisfying relationships (Jeronimus, 2015). Younger German adults (range of $n=154-2,173$, range of $M_{\text {age }} 17.00-24.40$, range of $S D=0.77-3.70$ ) higher in neuroticism reported more 
conflict with their social networks (Neyer \& Lehnart, 2007; Parker et al., 2012; Sturaro et al., 2008). Similarly, college students $\left(n=203, M_{\text {age }}=18.60, S D=\right.$ not provided $)$ higher in neuroticism reported more conflict with their roommates (Bono et al., 2002).

Findings in the literature regarding extraversion are mixed. This may be a reflection of the different behavioral domains that encompass extraversion that can be conducive to both more NSIs (e.g., the tendency to be forceful) or fewer NSIs (e.g., the tendency to experience positive emotions; John \& Srivastava, 1999). For example, individuals higher in extraversion attempt to avoid arguments, but are simultaneously higher in argumentativeness (Blickle, 1997). Regarding conflict, college students $\left(n=203, M_{\text {age }}=18.60, S D=\right.$ not provided $)$ higher in extraversion reported less conflict with their roommates (Bono et al., 2002), whereas younger German adults $\left(\mathrm{n}=154, M_{\mathrm{age}}=17.00, S D=\right.$ not provided $)$ higher in extraversion reported more conflict with their best friends (Sturaro et al., 2008). Thus, being higher in extraversion could result in experiencing more or fewer NSIs.

Those who are higher in conscientiousness are dependable, reliable, and attempt to follow socially prescribed norms (John \& Srivastava, 1999). Thus, it is not surprising that those who are higher in conscientiousness reported fewer NSIs with their social networks in general, because NSIs are violations of relationship norms (Brooks \& Dunkel Schetter, 2011). More specifically, younger German adults (range of $n=154-2,173$, range of $M_{\text {age }} 17.00-24.40$, range of $S D=$ $0.77-3.70$ ) higher in conscientiousness reported less conflict with their family and friends (Neyer \& Lehnart, 2007; Parker et al., 2012; Sturaro et al., 2008).

Limitations. The studies presented previously are insightful for the examination of personality and NSIs. The majority of the previous studies utilized German samples and although the current study utilizes an American sample, there are no known differences between German 
and American samples regarding personality (McCrae \& Costa, 1997). Thus, it would be important for the current study to replicate findings in a different national sample. The majority of the research has also heavily relied upon (a) models where personality is the predictor of NSIs; (b) younger adult samples; and (c) conflict as the only measure of NSIs. These limitations need to be addressed because (a) it is possible that NSIs predict personality traits; (b) personality and social relationships change over adulthood, which can lead to differential associations in middle and older adulthood; and (c) assessing conflict poses a risk to construct validity because conflict may underrepresent the broader construct of NSIs, which may skew the reported occurrence of, and variability surrounding, NSIs (Hartmann, Pelzel, \& Abbott, 2011).

Compared to studies assessing conflict (i.e., one Likert-type question stating, "How often do you have conflicts with your [spouse, family, friends]" or some variation of that question), studies that have examined the association between personality and a multi-itemed construct of NSIs (i.e., 4 - 12 Likert-type questions assessing multiple dimensions of NSIs) have similar findings for agreeableness, neuroticism, and conscientiousness. Specifically, middle-aged (range of $n=346-783$, range of $M_{\mathrm{age}}=32.55-47.80$, range of $\left.S D=0.90-11.59\right)$ and older adults $(n$ $=1,906, M_{\text {age }}$ not provided, range of age $=62-92$ ) who were higher in agreeableness or conscientiousness reported fewer NSIs with their significant others and their social networks, whereas those who were higher in neuroticism reported more NSIs with their significant others and their social networks (Allemand et al., 2015; Iveniuk, Waite, Laumann, Mcclintock, \& Tiedt, 2014; Silva et al., 2016).

There is some discrepancy regarding openness and NSIs. Openness was negatively associated with conflict (Parker et al., 2012), but there was no association between openness and NSIs (Silva et al., 2016) or a broad measure of social strain (12 Likert-type questions assessing 
the frequency of being rejected, restricted, criticized, and social network members being demanding; Allemand et al., 2015; Fydrich, Sommer, \& Bahler, 2007) Findings between extraversion and conflict were equivocal (Bono et al., 2002; Sturaro et al., 2008), but there was a positive association between extraversion and the broad measure of social strain (Allemand et al., 2015) and no association between extraversion and NSIs (Silva et al., 2016). Thus, one purpose of the current study was to determine if the association between personality and multiitemed construct of NSIs is similar to previous findings regarding personality and conflict.

\section{Change over Adulthood}

As mentioned previously, the studies that investigated the personality and NSIs association are informative, but they heavily rely on cross-sectional studies or studies that have a cross-sectional component to them (e.g., examining the cross-sectional aspects of a longitudinal research design). Although personality and social relationships can be stable for some individuals, they can also change for many individuals across adulthood (Carstensen et al., 1999; Roberts et al., 2008). This stability and change in personality and social relationships can result in changes in the association between personality and NSIs across adulthood.

Personality. Personality exhibits rank-order stability: those who are higher in a specific personality trait stay higher in that personality trait relative to other individuals over time (Bates \& Novosad, 2008). Research indicates that rank-order stability increases linearly until the age of 50 to 59 (Bleidorn, Kandler, Riemann, Spinath, \& Angleitner, 2009; Hopwood \& Donnellan, 2011; Roberts \& DelVecchio, 2000). This stability may have implications on the frequency of NSIs over time. For example, those who are relatively higher in neuroticism remain higher in neuroticism as they age, which may result in more NSIs over time compared to those who are relatively lower in neuroticism. Similarly, those who are higher in conscientiousness will remain 
relatively higher in conscientiousness over time, which may result in fewer NSIs over time compared to those who are relatively lower in conscientiousness.

Personality also exhibits mean-level change across adulthood. During younger adulthood (18 - 39 years old), adults invest in age-graded social roles such as entering a career, marrying, and having a family (Eliason, Mortimer, \& Vuolo, 2015). These new roles lead to new social environments, which is a driving force for personality development (i.e., the social investment principle; Roberts, Wood, \& Smith, 2005). Thus, younger adults (range of $n=173-1,908$, range of $M_{\text {age }}=19.51-30.60$, range of $\left.S D=0.77-9.15\right)$ who invest in age-graded social roles exhibit mean-level increases in agreeableness and conscientiousness, and mean-level decreases in neuroticism across adulthood (Asendorpf \& Wilpers, 1998; Bleidorn et al., 2009; Ludtke, Roberts, Trautwein, \& Nagy, 2011; McCrae et al., 2000; Neyer \& Lehnart, 2007; Roberts et al., 2005). These mean-level changes may have implications on the frequency of NSIs over time. Higher levels of neuroticism have been associated with more NSIs (Bono et al., 2002; Neyer \& Lehnart, 2007; Parker et al., 2012; Sturaro et al., 2008). Thus, the mean-level decreases in neuroticism may result in fewer NSIs over time. Higher levels of agreeableness and conscientiousness have been associated with fewer NSIs (Bono et al., 2002; Graziano et al., 1996; Parker et al., 2012; Sturaro et al., 2008). Thus, the mean-level increases in conscientiousness and agreeableness may result in fewer NSIs over time.

Mean-level changes in openness and extraversion have not been studied as extensively as change in agreeableness, conscientiousness, and neuroticism (Roberts, Walton, \& Viechtbauer, 2006). Furthermore, the pattern of change is not consistent across studies. Openness has been found to exhibit mean-level increases in younger adulthood $\left(n=1,908, M_{\text {age }}=19.51, S D=0.77\right.$; Ludtke et al., 2011), mean-level decreases in younger/middle adulthood $\left(n=374, M_{\text {age }}=30.60\right.$, 
$S D=9.15$; Bleidorn et al., 2009), and mean-level decreases in older adulthood although this decrease was not significant $\left(n=410, M_{\text {age }}=71.32 S D=\right.$ not provided; Kandler, Kornadt, Hagemeyer, \& Neyer, 2015). Extraversion has been found to exhibit mean-level increases and decreases in younger adulthood $\left(n=132\right.$ and $154, M_{\text {age }}=20.20$ and range of age $=18-29$, respectively; Asendorpf \& Wilpers, 1998; McCrae et al., 2000), and mean-level decreases in older adulthood $\left(n=410, M_{\text {age }}=71.32, S D=\right.$ not provided; Kandler et al., 2015). It is not clear whether extraversion and openness would significantly change over the course of the current study. If a significant pattern of change is present, it is also not clear in what direction the change would be in.

Social relationships. Similar to personality, social relationships can remain stable or change across adulthood. Socioemotional selectivity theory (Carstensen et al., 1999) posits that interactions between individuals and their social networks can be classified into two categories: one that is related to the acquisition of knowledge and one that is related to emotional wellbeing. During younger adulthood, adults view their time as unlimited and expansive. This future orientation motivates them to gain knowledge from their social networks to ensure that they have the necessary skills for the future. They are willing to attain this knowledge regardless of the emotional costs of the pursuit (Carstensen, 1992; Carstensen et al., 1999). When these adults age, they begin to view their time as more limited. This limited future orientation motivates them to transfer their pursuit to seek out emotional needs, instead of knowledge, from their social networks. If interactional partners are not contributing to adults' emotional well-being, then those network members are removed (Carstensen, 1992; Carstensen et al., 1999).

The stability and change in the social network may have implications on the frequency of NSIs over time. Younger adults retain negative social network members because they are 
interested in attaining knowledge from these members (Carstensen, 1992; Carstensen et al., 1999). This retention could result in the stability of NSIs in younger adulthood. Moreover, middle-aged and older adults remove negative social network members because those negative members are no longer contributing to their emotional well-being (Carstensen, 1992; Carstensen et al., 1999; Newsom et al., 2008). This change could result in fewer NSIs over time. It is important to note that the removal of negative social network members may not completely diminish the frequency of NSIs. NSIs are more likely to occur within close relationships such as parent-child relationships and spousal/partner relationships (Sorkin \& Rook, 2004). These types of relationships are rarely removed from the network (Laursen \& Hafen, 2010), which can result in some NSIs over time.

Personality and change in negative social interactions. The five longitudinal studies that have examined the association between personality and change in NSIs have primarily relied on samples of younger adults (range of $n=154-2,173$, range of $M_{\text {age }}=17.00-24.40$, range of $S D=0.77-3.70$ ) and time frames that range from two to eight years (with the exception of Mund \& Neyer, 2014). Results from these studies are mixed: some studies found no association between personality and change in NSIs (e.g., Mund \& Neyer, 2014; Neyer \& Asendorpf, 2001; Sturaro et al., 2008), whereas others found an association (e.g., Neyer \& Lehnart, 2007; Parker et al., 2012). Younger German adults $\left(n=339, M_{\text {age }}=24.40, S D=3.70\right)$ who were higher in neuroticism reported a decrease in conflict with family members over time (Neyer \& Lehnart, 2007). Younger German adults $\left(n=2,173, M_{\text {age }}=19.51, S D=0.77\right)$ who were higher in agreeableness or conscientiousness reported a decrease in conflict with their social networks over time (Parker et al., 2012). The studies conducted by Neyer and Asendorpf (2001) or Sturaro and colleagues (2008) may not have found a significant association between personality and change 
in NSIs because these studies sampled from younger adults and the time frames ranged from four to six years. It is possible that these younger adults are seeking knowledge from members of their social network, which would result in the stability of their relationships based on socioemotional selectivity theory (Carstensen et al., 1999) and would lead to the stability of NSIs over time.

To the best of my knowledge, the only study that has examined the association between personality and change in NSIs while utilizing a sample of younger and middle-aged adults was conducted by Mund and Neyer (2014). This study examined the longitudinal and bidirectional associations between personality and conflict over 15 years of adulthood $\left(n=654, M_{\text {age }}\right.$ at Wave $1=24.39, S D=3.69, M_{\text {age }}$ at Wave $2=32.55, S D=4.47, M_{\text {age }}$ at Wave $\left.3=40.20, S D=4.31\right)$. Interestingly, the researchers did not find a significant association between personality and change in conflict (Mund \& Neyer, 2014). It is not clear whether this null finding is the result of not sampling from older adults. Reductions in NSIs are expected to be greater in older adulthood (Carstensen et al., 1999; Wrzus, Hänel, Wagner, \& Neyer, 2012), therefore, it is possible that there was limited power to detect such a change in conflict without the inclusion of older adults. It is also not clear whether this null finding would replicate in the current study because the current sample encompasses a greater range of ages in adulthood and utilizes a multi-itemed construct of NSIs.

Negative social interactions and change in personality. The majority of the research within the field of personality and NSIs has primarily examined whether personality is associated with conflict. One important area of research that is missing from the field is whether NSIs are associated with the trajectories of personality traits over time. Dynamic interactionism posits that there is a continuous and reciprocal interaction between individuals and their environments, 
which leads to development (Magnusson, 1990; Magnusson \& Stattin, 1998). Therefore, it possible that the social environment can influence personality development over time. Only one study has examined whether conflict is associated with the trajectories of personality traits over time (Mund \& Neyer, 2014). German adults $\left(n=654, M_{\text {age }}\right.$ at Wave $\left.1=24.39, S D=3.69\right)$ who reported conflict with their partners and friends reported a decrease in neuroticism over 15 years. It is not clear whether these results would generalize to a multi-itemed construct of NSIs.

Change in personality and change in negative social interactions. Similar to the section presented above, another important area of research that is missing from the field is whether changes in personality are associated with changes in NSIs and vice versa. As mentioned previously, dynamic interactionism posits that there is a continuous and reciprocal interaction between individuals and their environments, which leads to development (Magnusson, 1990; Magnusson \& Stattin, 1998). Therefore, it possible that personality and the social environment are reciprocally associated over time.

To my knowledge, only two studies have examined such associations (Mund \& Neyer, 2014; Parker et al., 2012). Parker and colleagues (2012) examined personality change in a sample of younger German adults $\left(n=2,173, M_{\text {age }}\right.$ at Time $\left.1=19.51, S D=0.77\right)$ and the occurrence of conflict over two years. Mean-level increases in agreeableness and conscientiousness were associated with decreases in conflict with parents and friends. Meanlevel increases in neuroticism was associated with an increase in conflict with parents, siblings, and friends (Parker et al., 2012). Mund and Neyer (2014) also found that mean-level increases in agreeableness were associated with a decrease in conflict over time $\left(n=654, M_{\text {age }}\right.$ at Wave $1=$ 24.39, $S D=3.69$ ). However, differences were present regarding conscientiousness: mean-level increases were associated with an increase in conflict over time (Mund \& Neyer, 2014). It is not 
clear why there are conflicting results regarding mean-level changes in conscientiousness and conflict across the studies. The differences may be the result of examining a sample of youngeryoung adults (Parker et al., 2012) relative to a sample of younger/middle-aged adults (Mund \& Neyer, 2014). The current study explores this conflicting finding and whether it is generalizable to a multi-itemed construct of NSIs or a sample that encompasses all age periods of adulthood.

\section{Age as a Potential Moderator}

Another area of research that is missing is whether age moderates the association between personality and NSIs. As mentioned previously, personality and social relationships change over adulthood. Regarding personality, agreeableness and conscientiousness exhibit mean-level increases in younger adulthood, whereas neuroticism exhibits mean-levels decreases in younger adulthood. These mean-level changes continue across adulthood (Asendorpf \& Wilpers, 1998; Bleidorn et al., 2009; Ludtke et al., 2011; McCrae et al., 2000; Neyer \& Lehnart, 2007; Roberts et al., 2005).

Regarding social relationships, not only do aging adults trim negative social network members from their social networks (Carstensen et al., 1999), but the social network members modify their behaviors, which may have additional implications on the occurrence of NSIs. The social input model (Fingerman \& Charles, 2010; Fingerman, Miller, \& Charles, 2008) posits that interactional partners engage in behaviors that are dependent on the age of the person with whom they are interacting. Specifically, both younger $\left(M_{\mathrm{age}}=25.60, S D=3.64\right.$, range of age $=22.00-$ 35.00) and older adults $\left(M_{\text {age }}=70.36, S D=3.57\right.$, range of age $\left.=65.00-77.00\right)$ engage in fewer confrontational behaviors in response to older adults who are critical or insensitive. In addition, younger and older adults are more likely to send a birthday card to an older adult, rather than to a younger adult, when given the opportunity to do so (Fingerman et al., 2008). As such, people 
would rather maintain a positive relationship with older adults and make their remaining encounters fulfilling and rewarding (Fingerman \& Charles, 2010). This could also lead to fewer NSIs in older adulthood.

To my knowledge, no known studies have examined whether age moderates the association between personality and NSIs. The association between personality and NSIs could be stronger when adults are higher in certain personality traits or when they typically experience more NSIs. For example, it is possible that the negative association among agreeableness, conscientiousness, and NSIs are stronger for middle-aged adults (40 - 59 years) because they have experienced the mean-level increases in agreeableness and conscientiousness that occur in younger adulthood (Roberts et al., 2005). Although older adults (60+ years) also have higher mean-levels of agreeableness and conscientiousness like middle-aged adults, they may have trimmed negative interactional partners from their networks, leaving fewer NSIs to account for. In comparison, the positive association between neuroticism and NSIs could be stronger for younger adults $(18-39$ years old) because younger adults have higher levels of neuroticism relative to middle-aged and older adults (Bleidorn et al., 2009; Roberts et al., 2006, 2005), and because they report more NSIs relative to older adults (Walen \& Lachman, 2000). Thus, the final purpose of this study was to examine whether age moderates the reciprocal association between personality and NSIs.

\section{The Current Study}

Dynamic interactionism suggests that a continuous and reciprocal association between personality and NSIs exists (Magnusson, 1990; Magnusson \& Stattin, 1998), however, the studies that have examined this association are scarce. Additionally, the studies previously conducted utilize a single item to assess NSIs, report conflicting findings, do not examine age as 
a potential moderator, and rely heavily on younger adult samples even though these concepts change across adulthood. This study will add to the existing literature by examining the reciprocal association between personality and NSIs over 18 years in a national sample of American adults, while also addressing the limitations discussed above.

More specifically, this study will (a) utilize a sample that spans all age periods in adulthood; (b) utilize a multi-itemed construct of NSIs; (c) examine changes in NSIs and the Big Five personality traits over 18 years; (d) examine whether interindividual differences are present regarding the baseline and frequency/levels of NSIs and the personality traits and the trajectories of these constructs over time; (e) examine whether demographic variables and the personality traits account for interindividual differences in NSIs; (f) examine whether demographic variables and NSIs account for interindividual differences in the personality traits; $(\mathrm{g})$ examine the reciprocal association between personality and NSIs over 18 years; and (h) examine the moderating effects of age.

\section{Research Questions and Hypotheses}

Research question 1. Is there stability or change in NSIs over 18 years?

Hypothesis. Based on socioemotional selectivity theory, social input theory, and prior research (Carstensen et al., 1999; Fingerman et al., 2008; Walen \& Lachman, 2000), it was hypothesized that NSIs would significantly decrease over time.

Research question 2. Are there interindividual differences regarding the initial frequency of NSIs and the rate of change in NSIs over time? Can demographic variables (i.e., age, gender, and education) and the Big Five personality traits account for these differences?

Hypotheses. Based on prior research, it was hypothesized that significant interindividual differences would be present regarding the initial frequency of NSIs. It was hypothesized that: 
a. Older adults would report fewer NSIs compared to younger adults (Carstensen et al., 1999; Fingerman et al., 2008; Walen \& Lachman, 2000).

b. Females would report more NSIs compared to males based on prior work demonstrating that females are more relationship-oriented, which increases the possibility of experiencing NSIs (Beals \& Rook, 2006; Edwards, Hershberger, Russell, \& Markert, 2001; Walen \& Lachman, 2000).

c. Those who have attained higher levels of education would report fewer NSIs based on prior work that educated individuals experience fewer NSIs (Newsom, Mahan, Rook, \& Krause, 2008).

d. Those higher in agreeableness and conscientiousness would report fewer NSIs (Allemand et al., 2015; Asendorpf \& Wilpers, 1998; Blickle, 1997; Bono et al., 2002; Dijkstra et al., 2005; Graziano et al., 1996; Iveniuk et al., 2014; Neyer \& Lehnart, 2007; Parker et al., 2012; Silva et al., 2016; Sturaro et al., 2008).

e. Those higher in neuroticism would report more NSIs (Allemand et al., 2015; Bono et al., 2002; Iveniuk et al., 2014; Neyer \& Lehnart, 2007; Parker et al., 2012; Silva et al., 2016; Sturaro et al., 2008).

Due to conflicting findings regarding the association among extraversion, openness, and NSIs (Allemand et al., 2015; Bono et al., 2002; Dijkstra et al., 2005; Parker et al., 2012; Sturaro et al., 2008), no hypotheses were made, making these specific analyses exploratory.

It was also hypothesized that interindividual differences would exist regarding the rate of change in NSIs over time. This hypothesis was made in light of research suggesting that older adults remove negative interactional partners from their social networks and additional research 
suggesting that interactional partners treat older adults more favorably (Carstensen et al., 1999;

Fingerman et al., 2008), all of which have implications on change in NSIs over time.

Research question 3. Does age moderate the rate of change in NSIs? Does age also moderate the associations among the demographic variables, initial frequency of NSIs, and rate of change in NSIs over time?

Hypotheses. It was hypothesized that age would moderate the rate of change in NSIs over time because of research suggesting that older adults actively remove negative interactional partners and that interactional partners treat older adults more favorably (Carstensen et al., 1999; Fingerman et al., 2008). Thus, age would have a differential effect on NSIs over time. It was also hypothesized that age would moderate the predictive associations among the Big Five personality traits and rate of change in NSIs. This hypothesis was made in light of research indicating that personality development occurs across adulthood (Asendorpf \& Wilpers, 1998; Bleidorn et al., 2009; Ludtke et al., 2011; McCrae et al., 2000; Neyer \& Lehnart, 2007; Roberts et al., 2005). Thus, personality traits would have differential effects on rate of change in NSIs within different age periods.

Research question 4. Is there stability or change in the Big Five personality traits over 18 years?

Hypotheses. Based on the social investment principle and previous work (Bleidorn et al., 2009; Ludtke et al., 2011; Roberts \& Wood, 2006), it was hypothesized that:

a. Agreeableness and conscientiousness would increase over time.

b. Neuroticism would decrease over time.

Due to conflicting findings regarding the trajectories of openness and extraversion over time, no hypotheses were made, making these specific analyses exploratory. 
Research question 5. Are there interindividual differences regarding the initial levels of the Big Five personality traits and the rate of change in the Big Five personality traits over time? Can demographic variables and NSIs account for these differences?

Hypotheses. Based on prior research, it was hypothesized that significant interindividual differences would be present regarding the initial levels of the Big Five personality traits. It was hypothesized that:

a. Younger adults would have higher levels of neuroticism and lower levels of agreeableness and conscientiousness (Asendorpf \& Wilpers, 1998; Bleidorn et al., 2009; Ludtke et al., 2011; McCrae et al., 2000; Neyer \& Lehnart, 2007; Roberts et al., 2005).

b. Females would have higher levels of agreeableness, neuroticism, extraversion, and conscientiousness (Bleidorn et al., 2009; Costa, Terracciano, \& McCrae, 2001; Goodwin \& Gotlib, 2004; Kandler et al., 2015).

c. Those who report more NSIs would have lower levels of agreeableness and conscientiousness (Allemand et al., 2015; Asendorpf \& Wilpers, 1998; Blickle, 1997; Bono et al., 2002; Dijkstra et al., 2005; Graziano et al., 1996; Iveniuk et al., 2014; Neyer \& Lehnart, 2007; Parker et al., 2012; Silva et al., 2016; Sturaro et al., 2008).

d. Those who report more NSIs would have higher levels of neuroticism (Allemand et al., 2015; Bono et al., 2002; Iveniuk et al., 2014; Neyer \& Lehnart, 2007; Parker et al., 2012; Silva et al., 2016; Sturaro et al., 2008).

Regarding the rate of change in the Big Five personality traits, it was hypothesized that those who report more NSIs would report a decrease in neuroticism over time (Mund \& Neyer, 2014). No hypotheses were made regarding the association among NSIs and the trajectories of 
agreeableness, openness, extraversion, and conscientiousness because of limited available research, making these specific analyses exploratory.

Research question 6. Does age moderate the rate of change in the Big Five personality traits? Does age also moderate the associations among the demographic variables, initial levels of the personality traits, and the rate of change in the personality traits over time?

Hypotheses. It was hypothesized that age would moderate the rate of change in agreeableness, neuroticism, and conscientiousness. These hypotheses were made in light of research indicating that personality development begins when younger adults invest in agegraded social roles (Roberts et al., 2005). Thus, age would have a differential effect on agreeableness, neuroticism, and conscientiousness over time. No hypotheses were made regarding whether age would moderate the rate of change in openness or extraversion, making these specific analyses exploratory.

Research question 7. Is there a bidirectional association between the Big Five personality traits and NSIs over 18 years?

Hypotheses. Based on prior research (Mund \& Neyer, 2014; Parker et al., 2012), it was hypothesized that:

a. Increases in agreeableness would be associated with decreases in NSIs over time.

b. Increases in neuroticism would be associated with increases in NSIs over time.

c. Increases in NSIs would be associated with decreases in neuroticism over time.

d. Increases in NSIs would be associated with increases in conscientiousness over time.

Because increases in conscientiousness was associated with increases (Mund and Neyer, 2014) and decreases in conflict over time (Parker et al., 2012), no hypotheses were created for this association. In addition, no hypotheses were created for changes in openness and 
extraversion as the associated change in NSIs a result of limited research, making these specific analyses exploratory.

Research question 8. Does age moderate the bidirectional association between the Big Five personality traits and NSIs?

Hypothesis. It was hypothesized that age would moderate the bidirectional association between the Big Five personality traits and NSIs. This hypothesis was made because it was previously hypothesized that age would moderate the rate of change in NSIs (Research Question 3) and the rate of change in the Big Five personality traits (Research Question 6) over time. More specifically, it was hypothesized that age would moderate the rate of change in NSIs over time because of research suggesting that older adults actively remove negative interactional partners, and that interactional partners treat older adults more favorably (Carstensen et al., 1999; Fingerman et al., 2008). Thus, age would have a differential effect on NSIs over time. It was also hypothesized that age would moderate the rate of change in agreeableness, neuroticism, and conscientiousness because these personality traits beginning to change in younger adulthood (Roberts et al., 2005). Thus, age would have a differential effect on agreeableness, neuroticism, and conscientiousness over time.

\section{Method}

\section{Procedure}

MIDUS was initiated in 1995 to examine the social, psychological, and behavioral factors that account for variability in age-related processes in a national sample of Americans (University of Wisconsin - Madison, 2011). The first wave of MIDUS (MIDUS 1) was conducted in 1995 and 1996, in which non-institutionalized, English-speaking adults in the continental United States were selected using a random digit dialing method. Specifically, the 
MIDUS research team randomly contacted households and compiled a list of all individuals who were between the ages of 25 and 74 years old. From this list, the researchers randomly selected an individual from the household to participate in the study. In addition to this sampling technique, the MIDUS research team recruited siblings and twin pairs and oversampled from five metropolitan areas (Boston, Atlanta, Chicago, Phoenix, and San Francisco) to increase the diversity of the sample. Once the participants were selected, they completed a telephone interview that lasted 30 minutes. Afterwards, participants were mailed two self-administered questionnaires, which took an hour-and-a-half to complete at home. Participants were compensated $\$ 20$ for their participation. Of the contacted households, $60-70 \%$ of participants completed the telephone interview. Of the participants who completed the telephone interview, $89 \%$ completed the self-administered questionnaires, which resulted in a final sample of 7,108 participants. See Appendix A for the completion rates and participant characteristics broken down by individual samples (e.g., random digit dialing, sibling, twin, and oversampled metropolitan areas) for participants who completed MIDUS 1.

From 2004 to 2006, participants who completed MIDUS 1 were contacted for a followup assessment (MIDUS 2). Some participants were not eligible to complete MIDUS 2 because they (a) were unable to participate for health or other related reasons (3.00\% of MIDUS 1 sample); (b) died (6.00\% of MIDUS 1 sample); (c) did not have a working telephone number and could not be contacted (10.00\% of MIDUS 1 sample); refused to participate $(12.00 \%$ of MIDUS 1 sample). Of the 7,108 participants, $75 \%$ of participants $^{1}(n=4,963)$ agreed to and completed the telephone interview, which lasted 30 minutes. These participants were also mailed two self-

\footnotetext{
${ }^{1}$ This percentage was adjusted to account for mortality rates.
} 
administered questionnaires, which took an hour-and-a-half to complete at home. Eighty-one percent of participants $(n=4,032)$ completed the self-administered questionnaires and were compensated $\$ 60$ for their participation. The time interval between MIDUS 1 and MIDUS 2 ranged from 7.80 to 10.40 years $\left(M_{\text {interval }}=9.00\right.$ years $)$. See Appendix A for the completion rates and participant characteristics broken down by individual samples (e.g., random digit dialing, sibling, twin, and oversampled metropolitan areas) for participants who completed MIDUS 2.

From 2013 to 2014, participants who completed the telephone interview of MIDUS 2 were contacted for an additional follow-up assessment (MIDUS 3). The time interval between MIDUS 2 and MIDUS 3 ranged from 7.90 to 10.30 years $\left(M_{\text {interval }}=9.10\right.$ years $)$. Of the 4,963 contacted participants, $86.25 \%(n=4,281)$ were eligible to participate. Eligibility criteria included whether the participants were alive and resided in the United States and did not have health complications that would prevent them from participating. Of the eligible sample, 76.90\% $(n=3,294)$ completed the telephone interview, which lasted 45 minutes. Twelve participants could not participate in MIDUS after the telephone interviews because they no longer met the eligibility criteria. Participants who completed the telephone interview and were eligible to continue were mailed two self-administered questionnaires, which took two hours to complete at home. Roughly $83 \%$ of participants $(n=2,732)$ completed the self-administered questionnaires and were compensated $\$ 60$ for their participation. See Appendix A for the completion rates and participant characteristics broken down by individual samples (e.g., random digit dialing, sibling, twin, and oversampled metropolitan areas) for participants who completed MIDUS 3.

\section{Sample}

Although MIDUS contains data for 7,108 participants, not all were eligible to be included in the current study. First, the measures used in this study were assessed in the self-administered 
questionnaire portion of data collection. As such, no data are available for participants who did not return these questionnaires. Second, because latent growth curve analyses require that the number of assessments be the same for all participants, and that data must be obtained for at least three measurement occasions for each participant (Byrne, 2010; Duncan \& Duncan, 2004; Little, 2013), participants who were no longer eligible for sequential MIDUS follow-ups were removed from the sample. Lastly, the Indicators of Strain Scale assessed the frequency with which participants experienced NSIs with their partner. However, participants who did not have a partner did not answer the partner portion of the Indicators of Strain Scale and MIDUS did not assess whether participants' partner remained the same over the course of the study. In order to overcome the possibility that a potential change in NSIs over time was the result of not having a partner or having a different partner over time, participants who did not have a partner over all waves of MIDUS were removed from the sample ${ }^{2}$. With these exclusion criteria in place, the sample size decreased from 7,108 to 1,530 participants. Using data from MIDUS 1, participants who were excluded were more likely to be female, not married, and less educated relative to the participants in the final sample (see Appendix A). For a visual representation of sample attrition, please see Figure 1. For attritional analyses organized by the different stages of exclusion, please see Appendix A.

At MIDUS 1, the average age of the utilized sample was 46.03 years $(S D=10.50$, range $=25-74), 49.40 \%$ were female, $95.30 \%$ identified as White/Caucasian, $94.70 \%$ were married, and $46.80 \%$ earned some college credit or more. See Tables $1-3$ for the participant

\footnotetext{
${ }^{2}$ Results were examined separately for participants who did $(n=1,530)$ and $\operatorname{did} \operatorname{not}(n=980)$ consistently have a partner across all waves of MIDUS. Results were not appreciably different across the samples.
} 
characteristics separated by age groups ${ }^{3}$. At MIDUS 2, the average age of the utilized sample was 54.91 years $(S D=10.45$, range $=34-83), 49.40 \%$ were female, $94.40 \%$ identified as White/Caucasian, $96.60 \%$ were married, and $44.80 \%$ earned some college credit or more. See Tables $4-6$ for the participant characteristics separated by age groups. At MIDUS 3, the average age of the utilized sample was 64.02 years $(S D=10.47$, range $=42-92), 44.00 \%$ were male, 91.00\% identified as White/Caucasian, 96.80\% were married, and $43.50 \%$ earned some college credit or more. See Tables $7-9$ for the participant characteristics separated by age groups.

\section{Measures}

Demographics. Participants reported their age, gender $(0=$ Female, $1=$ Male $)$, and highest level of education completed $(1=$ No school/some grade school to $12=$ Graduate or professional degree; Appendix B) at each wave of collection. ${ }^{4}$

Negative Social Interactions. Using the Indicators of Strain Scale (modified measure from Schuster, Kessler, \& Aseltine, 1990; Appendix B), participants reported the frequency with which they experienced NSIs with their family members, significant others, and friends at each wave of collection. Using a 4-point Likert-type scale from 1 (never) to 4 (often), participants indicated how often each group made too many demands, engaged in criticism, let the participants down when being relied on, and got on the participants' nerves. An average score across the 12 items was computed for each wave of MIDUS for the entire sample and the three

${ }^{3}$ Age groups were created for the multigroup analyses, which examine moderation in AMOS. The use of age groups will be discussed in greater detail in the data analysis section.

${ }^{4}$ Participants also reported their marital status $(0=$ Married, $1=$ Separated, divorced, widowed, or never married) and race $(0=$ White/Caucasian, $1=$ Black/African American, Native American/Alaska Native, Asian, Native Hawaiian/Pacific Islander, or other). All analyses were modeled with and without these variables. Results were not appreciably different across the analyses, so marital status and race were removed from the models. 
age groups, with higher scores reflecting a higher reported frequency of experiencing NSIs. The scale had acceptable internal consistency across the entire sample and the three age groups. See Tables $1-12$ for the descriptive statistics and the alpha reliabilities of the entire sample and the three age groups across the three waves of MIDUS ${ }^{5}$.

Personality. Using the Midlife Development Inventory Personality Scale (Lachman \& Weaver, 1997; Appendix B), participants reported how well 25 adjectives (e.g., talkative, careless, warm) described themselves using a 4-point Likert-type scale from 1 (not at all) to 4 (a lot) at each wave of collection. Two items (calm and careless) were reversed coded. An average score across (a) five adjectives was computed for agreeableness; (b) seven adjectives was computed for openness; (c) four adjectives was computed for neuroticism; (d) five adjectives was computed for extraversion; and (e) four adjectives was computed for conscientiousness for the entire sample and the three age groups at each wave of MIDUS. A higher score for a personality trait reflects a greater endorsement of that trait. The scale had acceptable internal consistency across the entire sample and the three age groups, except for the personality trait of conscientiousness. The low reliability has been observed in other studies and is a limitation of the MIDUS dataset (Turiano et al., 2015; Turiano, Mroczek, Moynihan, \& Chapman, 2013; Turiano, Whiteman, Hampson, Roberts, \& Mroczek, 2012). See Tables $1-12$ for the descriptive statistics and the alpha reliabilities of the entire sample and the three age groups across the three waves of MIDUS.

${ }^{5}$ Results were examined separately based on the source of NSIs (i.e., whether NSIs were from family, partner, or friends). To examine the descriptives statistics, reliabilities, and bivariate correlations of these variables, please see Appendix C. To examine the results for the research questions separately by source of NSI, please see Appendices I - K. 


\section{Data Analysis Strategy}

Preliminary analyses were conducted in SPSS which included (a) examining the data for missingness; (b) computing descriptive statistics (c) testing the descriptive statistics against normality assumptions; (d) examining the bivariate associations among all variables for the entire sample and the three age groups; and (e) examining the presence of multivariate outliers.

Once completed, six univariate latent growth curves (NSIs and the Big Five personality traits; see Figure 2 for an example) were modeled independently in AMOS. Univariate latent growth curves allow for the examination of (a) the initial level of the modeled variable (i.e., the intercept; the reported frequency of NSIs or the endorsement of the Big Five personality traits at MIDUS 1); (b) the rate of change of the variable over time (i.e., the slope; whether NSIs or the Big Five personality traits exhibit mean-level increases or decreases over time); (c) the association between the intercept of the modeled variable and its slope (i.e., the covariance; e.g., whether individuals who reported experiencing higher levels of NSIs report steeper declines in NSIs over time relative to other participants); and (d) the variability surrounding the intercept and slope of the modeled variable (i.e., whether there are interindividual differences regarding the intercept and slope of the modeled variable; Duncan \& Duncan, 2004).

After examining the univariate latent growth curves and establishing that significant variability surrounded the intercepts and slopes, predictor variables were used to determine whether they could account for this variance ${ }^{6}$ (see Figure 3 for an example). Age, gender, education, and the Big Five personality traits were used as predictors for the latent growth curve of NSIs. Age, gender, education, and NSIs were used as predictors for the latent growth curves

\footnotetext{
${ }^{6}$ If significant variability was not present around the intercept or slope, the predictors were not regressed onto the respective intercept or slope.
} 
of each individual personality trait. To aid in model estimation and with the interpretation of the estimates, the Big Five personality traits were allowed to covary with one another. Also, the predictor variables of education, NSIs, and the Big Five personality traits were standardized. As such, a unit increase or decrease in these variables represent a standard deviation increase or decrease, respectively. Age was not manipulated so that a unit increase or decrease represents an increase or decrease of a year, respectively.

Once all univariate latent growth curves were examined, preliminary models were analyzed in preparation for the for cross-domain latent growth curves, which examines the reciprocal association between NSIs and the Big Five personality traits. Specifically, the latent growth curves of NSIs and a single personality trait were modeled jointly in which the intercepts and slopes within each latent growth curve, the intercepts across the latent growth curves, and the slopes across the latent growth curves were allowed to covary with one another (see Figure 4 for an example). The covariances across the intercepts and across the slopes were modeled to account for the shared variance between these parameters. Five models were examined to determine whether the intercepts, slopes, and variances were significant after accounting for the shared variance among the variables.

After examining these models, five cross-domain latent growth curves were modeled in AMOS (see Figure 5 for an example). In addition to the intercepts, slopes, and variabilities estimated previously, cross-domain latent growth curves allow for the prediction of (a) slope from intercept (i.e., whether the initial frequency of NSIs predicts rate of change for NSIs or whether the initial level of a personality trait predicts rate of change for that personality trait); (b) the rate of change in the Big Five personality traits from the initial frequency of NSIs; (c) the rate 
of change in NSIs from the initial levels of the Big Five personality traits ${ }^{7}$; and (d) the rate of change in NSIs from the rate of change in the Big Five personality traits and vice versa ${ }^{8}$ (Willet \& Sayer, 1996). Predictors were also used to determine whether they could account for the significant variance surrounding the intercepts and slopes of the cross-domain latent growth curves $^{9}$. Age, gender, and education were used as the predictors for the latent growth curve of a personality trait. Age, gender, education, and the remaining four personality traits were used as the predictors for the latent growth curve of NSIs. To aid in model estimation, the covariance between the intercepts of the two latent growth curves was constrained to be the unstandardized correlation between the variables at MIDUS 1, which does not alter model fit or parameters. To aid with the interpretation of the estimates, the predictor variables of education, NSIs, and the four personality traits were standardized. As such, a unit increase or decrease in these variables represent a standard deviation increase or decrease, respectively. Age was not manipulated so that a unit increase or decrease represents an increase or decrease of a year, respectively.

After, multigroup analyses were conducted in AMOS to explore whether the above associations varied as a function of age. For these analyses, three age groups were specified in AMOS: the younger adults (25 - 39 years old at MIDUS 1), the middle-aged adults $(40-59$

${ }^{7}$ The prediction of change in slope was only analyzed if there was significant change in slope over time.

${ }^{8}$ The models examining the reciprocal association between the rate of change in the Big Five personality traits and the rate of change in NSIs would not converge, so these parameters could not be examined. This issue is discussed in further detail in the results section.

${ }^{9}$ If significant variability was not present around the intercept or slope, the predictor variables were not regressed onto the respective intercept or slope. 
years old at MIDUS 1$)$, and the older adults $(60+\text { years old at MIDUS } 1)^{10}$. Then, two models were simultaneously computed for each univariate latent growth curve. The first model was the unconstrained model, where the parameters were freely estimated across the three groups. The second model was the constrained model, where the parameters were constrained to be equal across the three groups. These models were examined to determine whether the unconstrained or the constrained model fit the data better, which was determined by a CFI change statistic of .01 or greater. The CFI change statistic was used relative to the chi-square difference statistic because the CFI is more robust to larger sample sizes (Little, 2013). If analyses indicated that the unconstrained model fit the data significantly better than the constrained model, then moderation was present (Duncan \& Duncan, 2004). If moderation was present, the univariate and crossdomain latent growth curves were modeled independently for each age group for all analyses ${ }^{11}$.

To account for inflated Type I error as the result of conducting multiple latent growth curve analyses, the false discovery rate technique was utilized in SAS (Benjamini \& Hochberg, 1995). Specifically, the analyses accounted for the possibility that $15 \%$ of the significant findings would be the result of Type I error. This high false discovery rate was determined by recommendations for first-stage exploratory studies (McDonald, 2014). Any corrections that were made are noted throughout the results section.

${ }^{10}$ Ages 18 - 39 typically distinguish younger adulthood, ages 40 - 59 typically distinguish middle adulthood, and ages 60 and older typically distinguish older adulthood (Erikson, 1980). The categorization of these age groups has also been used in prior studies (e.g., Asendorpf \& Wilpers, 1998; Carstensen et al., 1999; Fingerman \& Charles, 2010; Fingerman et al., 2008; Ludtke et al., 2011; Mund \& Neyer, 2014).

${ }^{11}$ Modeling the latent growth curves separately was also supported by the fact that the age groups differed regarding the significance of the variability surrounding the slopes and the significance of change in slope over time. Thus, certain age groups had different parameters that could be predicted, which could only be modeled in separate latent growth curves. 
All latent growth curves described previously were examined to determine how well the models fit the data. Because the commonly used model fit index, the chi-square, is sensitive to large sample size and number of degrees of freedom (Little, 2013), additional model fit parameters were examined such as the CMIN/DF (the chi-square statistic divided by the degrees of freedom), the RMSEA (provides an index of standard errors) and the CFI (provides an index of parsimony). Acceptable model fit as determined by the CMIN/DF is below 3.0, .08 - .01 for the RMSEA, and .90 - .99 for the CFI (Little, 2013). To determine statistical significance, critical ratio scores $>1.96, p<.05$, or CFI change scores $\geq .01$ were used for all analyses.

\section{Results}

\section{Preliminary Analyses}

Missing data were present (see Appendix D), however, the largest percentage of missing data for any variable did not exceed $1.00 \%$. As such, full information maximum likelihood was utilized to account for the missing data in all latent growth curve analyses. Full information maximum likelihood is more desirable relative to other missing data techniques because it results in a less biased estimation of parameters (Little, 2013).

Descriptive statistics and bivariate correlations were conducted for the full sample and separately for the three age groups (see Tables $1-12$ ). The assumption of normality was checked and results indicated that two variables were negatively skewed (conscientiousness at MIDUS 3 for the full sample and for the younger adults), whereas one variable was positively skewed (NSIs at MIDUS 3 for older adults). These variables were transformed with a square root transformation; however, the latent growth curves were not appreciably different with the transformed variables. Thus, the non-transformed variables were reported in the results section. 
Mahalanobis distance scores were computed to examine the presence of multivariate outliers. Results indicated that 127 participants were multivariate outliers, $\chi^{2}(33)=63.87, p<$ .05. Multivariate outliers were more likely to be younger, less educated, not married, not White/Caucasian, higher in openness at MIDUS 1, higher in neuroticism at MIDUS 2 and MIDUS 3, lower in conscientiousness at MIDUS 3, and reported experiencing more NSIs at MIDUS 2 and MIDUS 3 (see Appendix D). All analyses were conducted including and excluding the multivariate outliers and the results were not appreciably different with the inclusion of multivariate outliers. As such, the following results include the multivariate outliers to conserve power.

\section{Research Questions 1 and $2^{12}$}

(1) Is there stability or change in NSIs over 18 years?

(2) Are there interindividual differences regarding the initial frequency of NSIs and the rate of change in NSIs over time? Can demographic variables (i.e., age, gender, and education) and the Big Five personality traits account for these differences?

A univariate latent growth curve was modeled in AMOS to examine the rate of change in NSIs. The model fit the data well (see Appendix E). NSIs significantly decreased over time (see Appendix F). There was no significant association between participants' initial frequency of NSIs and their rate of change over time.

\footnotetext{
${ }^{12}$ NSIs were moderated by age, which is examined in Research Question 3. Because these results were qualified by this moderated effect, a general description of the results were discussed in this section for brevity purposes. For a more detailed description of the results, please see Appendix E.
} 
Age, gender, education, and the Big Five personality traits were added to the model to account for the significant variability surrounding the intercept and slope. The model fit was poor, which indicates that significant individual differences remained unaccounted for in the model (see Appendix E). Being one standard deviation higher in openness or neuroticism was associated with more NSIs at MIDUS 1. Being older, male, or one standard deviation higher in agreeableness or conscientiousness was associated with fewer NSIs at MIDUS 1. Being older or one standard deviation higher in neuroticism was associated with a steeper decrease in NSIs over time.

\section{Research Question 3}

(3) Does age moderate the rate of change in NSIs? Does age also moderate the associations among the demographic variables, initial frequency of NSIs, and rate of change in NSIs over time?

A multigroup latent growth curve was modeled in AMOS to examine whether age moderated the rate of change in NSIs over time. The unconstrained model was compared to the constrained model, and results indicated that the unconstrained model fit the data better, $\Delta \mathrm{CFI}=$ $.032, p<.05$ (see Table 13). Thus, age moderated these associations.

The unconstrained model fit the data well (see Table 14). For the younger adults, the reported frequency of NSIs significantly decreased by 0.004 scale units every year of age. For the middle-aged adults, the reported frequency of NSIs significantly decreased by 0.010 scale units every year of age. For the older adults, reported frequency of NSIs significantly decreased by 0.008 scale units every year of age (see Figure 6 ). There was no significant association between participants' initial frequency of NSIs and their rate of change over time. 
To determine whether the differences among the intercepts and slopes were statistically different from one another, the critical ratio scores were examined. Results indicated that younger and middle-aged adults significantly reported more NSIs compared to older adults (critical ratio comparing younger to older adults $=-4.24, p<.05$; critical ratio comparing middleaged to older adults $=-3.81, p<.05)$. Results also indicated that NSIs for younger adults decreased at a less steep rate compared to the middle-aged and older adults (critical ratio comparing younger to middle-aged adults $=-4.48, p<.05$; critical ratio comparing younger to older adults $=-2.16, p<.05)$.

Younger adults. Age, gender, education, and the Big Five personality traits were added to the model to account for the significant variability surrounding the intercept and slope. The model fit was poor, which indicates that significant individual differences remained unaccounted for in the model (see Table 15). Being one standard deviation higher in neuroticism was associated with more NSIs at MIDUS 1, whereas being male or one standard deviation higher in conscientiousness was associated with fewer NSIs at MIDUS 1. Being older or one standard deviation higher in neuroticism was associated with a steeper decrease in NSIs over time.

Middle-aged adults. Age, gender, education, and the Big Five personality traits were added to the model to account for the significant variability surrounding the intercept and slope. The model fit was poor, which indicates that significant individual differences remained unaccounted for in the model (see Table 15). Being one standard deviation higher in education, openness, or neuroticism was associated with more NSIs at MIDUS 1, whereas being older, male, or one standard deviation higher in agreeableness or conscientiousness was associated with fewer NSIs at MIDUS 1. No variables significantly accounted for the change in NSIs over time. 
Older adults. Age, gender, education, and the Big Five personality traits were added to the model to account for the significant variability surrounding the intercept and slope. The model fit was poor, which indicates that significant individual differences remained unaccounted for in the model (see Table 15). Being male or one standard deviation higher in neuroticism was associated with more NSIs at MIDUS 1 . No variables significantly accounted for the change in NSIs over time.

\section{Research Questions 4 and $5^{13}$}

(4) Is there stability or change in the Big Five personality traits over 18 years?

(5) Are there interindividual differences regarding the initial levels of the Big Five personality traits and the rate of change in the Big Five personality traits over time? Can demographic variables and NSIs account for these differences?

Agreeableness, openness, neuroticism, and conscientiousness. Four univariate latent growth curves were modeled in AMOS to examine the rate of change in these variables. The models fit the data well expect for neuroticism ${ }^{14}$ (see Appendix E). Agreeableness, openness, and neuroticism significantly decreased every year, whereas conscientiousness increased every year, but not significantly (see Appendix F). There were no significant associations between participants' levels of agreeableness, openness, conscientiousness, and their rate of change over

\footnotetext{
${ }^{13}$ Agreeableness, openness, neuroticism, and conscientiousness were moderated by age, which is examined in Research Question 6. Because these results were qualified by this moderated effect, a general description of the results were discussed in this section for brevity purposes. For a more detailed description of these results, please see Appendix E.

14 This poor fitting model may indicate that the rate of change in neuroticism may not fit a linear trend. The literature indicates that neuroticism decreases in younger adulthood and can increase in older adulthood (Bleidorn et al., 2009; Kandler et al., 2015). Testing a quadratic change in neuroticism requires four waves a data, which is not available with the MIDUS dataset.
} 
time. Participants who scored higher in neuroticism experienced a steeper decrease in neuroticism over time.

Age, gender, education, and NSIs were added to each latent growth curve to account for the significant variability surrounding the intercepts and slopes. The models adequately fit the data (see Appendix E). Being one standard deviation higher in NSIs was associated with lower levels of agreeableness and conscientiousness at MIDUS 1, whereas it was associated with higher levels of neuroticism. Being one standard deviation higher in NSIs negated the overall decrease in agreeableness over time. Being one standard deviation higher in NSIs was associated with a steeper decrease in neuroticism over time.

Extraversion. A univariate latent growth curve was modeled in AMOS to examine the rate of change in extraversion. The model fit the data well (see Table 16). Extraversion significantly decreased by 0.006 scale units every year of age (see Figure 7 ). There was no significant association between participants' initial levels of extraversion and their rate of change over time.

Age, gender, education, and NSIs were added to the model to account for the significant variability surrounding the intercept and slope. The model adequately fit the data (see Table 17). Being male or one standard deviation higher in education or NSIs was associated with lower levels of extraversion at MIDUS 1. No variables significantly accounted for the rate of change in extraversion over time.

\section{Research Question 6}

(6) Does age moderate the rate of change in the Big Five personality traits? Does age also moderate the associations among the demographic variables, initial levels of the personality traits, and the rate of change in the personality traits over time? 
Agreeableness. A multigroup latent growth curve was modeled in AMOS to examine whether age moderated the rate of change in agreeableness over time. The unconstrained model was compared to the constrained model, and results indicated that the unconstrained model fit the data better, $\Delta \mathrm{CFI}=.011, p<.05$ (see Table 18). Thus, age moderated these associations.

The unconstrained model fit the data well (see Table 19). For younger adults, agreeableness significantly decreased by 0.003 scale units every year of age. For middle-aged adults, agreeableness decreased by 0.001 scale units every year of age, however, this decrease was not significant. For older adults, agreeableness significantly decreased by 0.005 scale units every year of age (see Figure 8). There was no significant association between participants' levels of agreeableness and their rate of change over time.

To determine whether the differences among the intercepts and slopes were statistically different from one another, the critical ratio scores were examined. Results indicated that the younger adults had significantly lower levels of agreeableness compared to the middle-aged and older adults (critical ratio comparing younger to middle-aged adults $=3.05, p<.05$; critical ratio comparing younger to middle-aged adults $=2.60, p<.05)$. Results also indicated that agreeableness for the middle-aged adults decreased at a less steep rate compared to the older adults (critical ratio comparing middle-aged to older adults $=-2.45, p<.05$ ).

Younger adults. Age, gender, education, and NSIs were added to the model to account for the significant variability surrounding the intercept and slope. The model fit the data well (see Table 20). Being male was associated with lower levels of agreeableness at MIDUS 1. Being older was associated with a less steep decrease in agreeableness over time. Being one standard deviation higher in NSIs negated the overall decrease in agreeableness over time. 
Middle-aged adults. Age, gender, education, and NSIs were added to the model to account for the significant variability surrounding the intercept. The model adequately fit the data (see Table 20). Being male or one standard deviation higher in education or NSIs was associated with lower levels of agreeableness at MIDUS 1.

Older adults. Age, gender, education, and NSIs were added to the model to account for the significant variability surrounding the intercept. The model fit the data well (see Table 20). Being male or one standard deviation higher in education was associated with lower levels of agreeableness at MIDUS 1.

Openness. A multigroup latent growth curve was modeled in AMOS to examine whether age moderated the rate of change in openness over time. The unconstrained model was compared to the constrained model, and results indicated that the unconstrained model fit the data better, $\Delta \mathrm{CFI}=.010, p<.05$ (see Table 18). Thus, age moderated these associations.

The unconstrained model adequately fit the data (see Table 19). For younger adults, openness significantly decreased by 0.007 scale units every year of age. For middle-aged adults, openness significantly decreased by 0.001 scale units every year of age. For older adults, openness significantly decreased by 0.012 scale units every year of age (see Figure 9 ). There was no significant association between participants' levels of openness and their rate of change over time.

To determine whether the differences among the intercepts and slopes were statistically different from one another, the critical ratio scores were examined. Results indicated that the younger adults had significantly lower levels of openness compared to the middle-aged adults (critical ratio comparing younger to middle-aged adults $=2.27, p<.05$ ). Results also indicated that openness for younger and middle-aged adults decreased at a less steep rate compared to the 
older adults (critical ratio comparing younger to older adults $=-2.08, p<.05$; critical ratio comparing middle-aged to older adults $=-3.14, p<.05)$.

Younger adults. Age, gender, education, and NSIs were added to the model to account for the significant variability surrounding the intercept and slope. The model fit the data well (see Table 21). Being male or one standard deviation higher in education was associated with higher levels of openness at MIDUS 1, whereas being older was associated with lower levels. Being older was associated with a less steep decrease in openness over time.

Middle-aged adults. Age, gender, education, and NSIs were added to the model to account for the significant variability surrounding the intercept and slope. The model adequately fit the data (see Table 21). Being one standard deviation higher in education was associated with higher levels of openness at MIDUS 1. No variables significantly accounted for the rate of change in openness over time.

Older adults. Age, gender, education, and NSIs were added to the model to account for the significant variability surrounding the intercept and slope. The model adequately fit the data (see Table 21). No variables significantly accounted for the initial levels of, or rate of change in, openness over time.

Neuroticism. A multigroup latent growth curve was modeled in AMOS to examine whether age moderated the rate of change in neuroticism over time. The unconstrained model was compared to the constrained model, and results indicated that the unconstrained model fit the data better, $\Delta \mathrm{CFI}=.012, p<.05$ (see Table 18). Thus, age moderated these associations.

The unconstrained model adequately fit the data (see Table 19). For younger adults, neuroticism significantly decreased by 0.009 scale units every year of age. For middle-aged adults, neuroticism significantly decreased by 0.009 scale units every year of age. For older 
adults, neuroticism significantly decreased by 0.005 scale units every year of age (see Figure 10). Younger and middle-aged adults who scored higher in neuroticism experienced a steeper decrease in neuroticism over time. There were no significant associations between older adults' initial endorsement of neuroticism and their rate of change over time.

To determine whether the differences among the intercepts and slopes were statistically different from one another, the critical ratio scores were examined. Results indicated that the younger adults had significantly higher levels of neuroticism compared to the middle-aged and older adults (critical ratio comparing younger to middle-aged adults $=-3.83, p<.05$; critical ratio comparing younger to older adults $=-4.31, p<.05)$.

Younger adults. Age, gender, education, and NSIs were added to the model to account for the significant variability surrounding the intercept and slope. The model fit the data well (see Table 22). Being one standard deviation higher in NSIs was associated with higher levels of neuroticism at MIDUS 1, whereas being male or one standard deviation higher in education was associated with lower levels of neuroticism. Being one standard deviation higher in NSIs was associated with a steeper decrease in neuroticism over time.

Middle-aged adults. Age, gender, education, and NSIs were added to the model to account for the significant variability surrounding the intercept and slope. The model fit was poor, which indicates that significant individual differences remained unaccounted for in the model (see Table 22). Being one standard deviation higher in NSIs was associated with higher levels of neuroticism at MIDUS 1, whereas being older, male, or one standard deviation higher in education was associated with lower levels of neuroticism. Being older was associated with a less steep decrease in neuroticism over time. 
Older adults. Age, gender, education, and NSIs were added to the model to account for the significant variability surrounding the intercept. The model fit the data well (see Table 22). Being one standard deviation higher in NSIs was associated with higher levels of neuroticism at MIDUS 1, whereas being male or one standard deviation higher in education was associated with lower levels of neuroticism.

Extraversion. A multigroup latent growth curve was modeled in AMOS to examine whether age moderated the rate of change in extraversion over time. The unconstrained model was compared to the constrained model, and results indicated that there were no significant differences between the models, $\Delta \mathrm{CFI}=.009, p>.05$ (see Table 18). Thus, age did not moderate these associations.

Conscientiousness. A multigroup latent growth curve was modeled in AMOS to examine whether age moderated the rate of change in conscientiousness over time. The unconstrained model was compared to the constrained model, and results indicated that the unconstrained model fit the data better, $\Delta \mathrm{CFI}=.018, p<.05$ (see Table 18). Thus, age moderated these associations.

The unconstrained model fit the data well (Table 19). For younger adults, conscientiousness significantly increased by 0.003 scale units every year of age. For middle-aged adults, conscientiousness increased by 0.001 scale units every year of age, however, this increase was not significant. For older adults, conscientiousness significantly decreased by 0.008 scale units every year of age (see Figure 11). There was no significant association between participants' initial levels of conscientiousness and their rate of change over time.

To determine whether the differences among the intercepts and slopes were statistically different from one another, the critical ratio scores were examined. Results indicated that the 
younger adults had significantly lower levels of conscientiousness compared to the middle-aged adults (critical ratio comparing younger to middle-aged adults $=2.16, p<.05$ ).

Conscientiousness for the older adults significantly decreased over time, whereas conscientiousness for the younger and middle-aged adults increased over time (critical ratio comparing younger to older adults $=-2.66, p<.05$; critical ratio comparing middle-aged to older adults $=-5.04, p<.05)$. Also, conscientiousness for the younger adults increased at a steeper rate compared to the middle-aged adults (critical ratio comparing younger to middle-aged adults $=$ $1.98, p<.05)$

Younger adults. Age, gender, education, and NSIs were added to the model to account for the significant variability surrounding the intercept and slope. The model fit the data well (see Table 23). Being male or one standard deviation higher in NSIs was associated with lower levels of conscientiousness at MIDUS 1. No variables significantly accounted for the rate of change in conscientiousness over time.

Middle-aged adults. Age, gender, education, and NSIs were added to the model to account for the significant variability surrounding the intercept. The model fit the data well (see Table 23). Being one standard deviation higher in education was associated with higher levels of conscientiousness at MIDUS 1, whereas being male or one standard deviation higher in NSIs was associated with lower levels of conscientiousness.

Older adults. Age, gender, education, and NSIs were added to the model to account for the significant variability surrounding the intercept and slope. The model fit was poor, which indicates that significant individual differences remained unaccounted for in the model (see Table 23). No variables significantly accounted for the initial levels of, or rate of change in, conscientiousness over time. 


\section{Research Question $7^{15}$}

(7) Is there a bidirectional association between the Big Five personality traits and NSIs over 18 years?

Before the cross-domain latent growth curves were modeled to examine the reciprocal association between NSIs and the Big Five personality traits, preliminary models were analyzed to determine whether the intercepts, slopes, and variances were significant after accounting for the shared variance among the NSIs and personality variables. Results indicated that significant variability surrounded all of the intercepts and slopes (see Table 24). Thus, after accounting for the shared variance among the NSI and personality variables, interindividual differences were present regarding the initial frequency and rate of change in NSIs, and the initial levels and rate of change in the Big Five personality traits. Results also indicated that conscientiousness was the only variable that did not significantly change over time. As such, the intercepts of NSIs and conscientiousness were not regressed onto the slope of conscientiousness because there was no significant change to predict.

Unfortunately, the originally proposed cross-domain latent growth curves for Research Questions 7 or 8 could not be analyzed. The models would not converge when the parameters of slope predicting slope (i.e., the slope of NSIs predicting the slope of a personality trait or the slope of a personality trait predicting the slope of NSIs) were included in the model. The parameters within the cross-domain latent growth curves were manipulated in many ways to determine whether any variations of the models would result in model convergence. The

\footnotetext{
${ }^{15}$ All of the cross-domain latent growth curves were moderated by age, which is examined in Research Question 8. Because these results were qualified by this moderated effect, a general description of the results were discussed in this section for brevity purposes. For a more detailed description of the results for Research Question 7, please see Appendix E.
} 
following manipulations were attempted: (a) retaining all seven predictors in the model (i.e., age, gender, education, and the remaining four personality traits); (b) removing all predictors from the models so that it was just the two latent growth curves predicting one another; (c) adding a single predictor to the models (e.g., having age as the only predictor) and then alternating each single predictor variable in the model; (d) a combination of the predictor variables (e.g., having only the four personality traits as predictors or having only the demographic variables as predictors); (e) manipulating the age variable so that it only reflected a specific age group to reduce the variance associated with age (the variance associated with age was large because of the wide range of ages in the study); and (f) allowing the covariance between the intercepts to be freely estimated. None of these manipulations worked. As such, the parameter of slope predicting slope was removed from the cross-domain latent growth curves.

This resulted in cross-domain latent growth curves that predicted (a) slope from intercept (i.e., whether the initial frequency of NSIs predicts the rate of change in NSIs or whether the initial level of a personality trait predicts the rate of change for that personality trait); (b) the rate of change in the Big Five personality traits from the initial frequency of NSIs; and (c) the rate of change in NSIs from the initial levels of the Big Five personality traits (see Figure 12). Even with the modifications to Research Questions 7 and 8, these research questions differ from Research Questions 2, 3, 5, and 6 because the cross-domain latent growth curves allow for the simultaneous examination of the predictive pathways between the latent growth curves of NSIs and the Big Five personality traits.

The modified cross-domain latent growth curves were modeled in AMOS to examine the association between NSIs and each individual personality trait. Overall, the models fit the data poorly (see Appendix E). Higher levels of neuroticism were associated with a steeper decrease in 
NSIs over time. There were no significant associations between the latent growth curve of NSIs and the latent growth curves of agreeableness, openness, extraversion, or conscientiousness.

\section{Research Question 8}

(8) Does age moderate the bidirectional association between the Big Five personality traits and NSIs?

Agreeableness. A multigroup latent growth curve was modeled in AMOS to examine whether age moderated the association between NSIs and agreeableness. The unconstrained model was compared to the constrained model, and results indicated that the unconstrained model fit the data better, $\Delta \mathrm{CFI}=.019, p<.05$ (see Table 25). Thus, age moderated these associations.

The unconstrained model fit the data well (see Table 26). For younger adults, NSIs and agreeableness significantly decreased by 0.004 and 0.003 scale units every year of age, respectively. For middle-aged adults, NSIs significantly decreased by 0.0010 scale units every year of age, whereas agreeableness decreased (but not significantly) by 0.001 units every year of age. For older adults, NSIs and agreeableness significantly decreased by 0.008 and 0.005 scale units every year of age, respectively (see Figures $13-15$ ). There were no significant associations among participants' initial frequency of NSIs, initial level of agreeableness, and rate of change over time.

Younger adults. A cross-domain latent growth curve was modeled in AMOS to examine the association between NSIs and agreeableness for younger adults. The model fit was poor, which indicates that significant individual differences remained unaccounted for in the model (see Table 27). There were no significant associations between the latent growth curves of NSIs and agreeableness for younger adults. 
Middle-aged adults. A cross-domain latent growth curve was modeled in AMOS to examine the association between NSIs and agreeableness for middle-aged adults. The model fit was poor, which indicates that significant individual differences remained unaccounted for in the model (see Table 28). There were no significant associations between the latent growth curves of NSIs and agreeableness for middle-aged adults.

Older adults. A cross-domain latent growth curve was modeled in AMOS to examine the reciprocal association between NSIs and agreeableness for older adults. The model fit was poor, which indicates that significant individual differences remained unaccounted for in the model (see Table 29). There were no significant associations between the latent growth curves of NSIs and agreeableness for older adults.

Openness. A multigroup latent growth curve was modeled in AMOS to examine whether age moderated the association between NSIs and openness. The unconstrained model was compared to the constrained model, and results indicated that the unconstrained model fit the data better, $\Delta \mathrm{CFI}=.017, p<.05$ (see Table 25). Thus, age moderated these associations.

The unconstrained model fit the data well (see Table 26). For younger adults, NSIs and openness significantly decreased by 0.004 and 0.007 scale units every year of age, respectively. For middle-aged adults, NSIs and openness significantly decreased by 0.010 and 0.005 scale units every year of age, respectively. For older adults, NSIs and openness significantly decreased by 0.008 and 0.012 scale units every year of age, respectively (see Figures $16-18$ ). There were no significant associations among participants' initial frequency of NSIs, initial level of openness, and rate of change over time.

Younger adults. A cross-domain latent growth curve was modeled in AMOS to examine the reciprocal association between NSIs and openness for younger adults. The model fit was 
poor, which indicates that significant individual differences remained unaccounted for in the model (see Table 30). There were no significant associations between the latent growth curves of NSIs and openness for younger adults.

Middle-aged adults. A cross-domain latent growth curve was modeled in AMOS to examine the association between NSIs and openness for middle-aged adults. The model fit was poor, which indicates that significant individual differences remained unaccounted for in the model (see Table 31). Higher levels of openness at MIDUS 1 was associated with a less steep decrease in NSIs over time. Higher levels of openness at MIDUS 1 was associated with a steeper decrease in openness over time

Older adults. A cross-domain latent growth curve was modeled in AMOS to examine the association between NSIs and openness for older adults. The model fit was poor, which indicates that significant individual differences remained unaccounted for in the model (see Table 32). There were no significant associations between the latent growth curves of NSIs and openness for older adults.

Neuroticism. A multigroup latent growth curve was modeled in AMOS to examine whether age moderated the association between NSIs and neuroticism. The unconstrained model was compared to the constrained model, and results indicated that the unconstrained model fit the data better, $\Delta \mathrm{CFI}=.016, p<.05$ (see Table 25). Thus, age moderated these associations.

The unconstrained model fit the data well (see Table 26). For younger adults, NSIs and neuroticism significantly decreased by 0.004 and 0.009 scale units every year of age, respectively. For middle-aged adults, NSIs and neuroticism significantly decreased by 0.010 and 0.009 scale units every year of age, respectively. For older adults, NSIs and neuroticism significantly decreased by 0.008 and 0.005 scale units every year of age, respectively (see 
Figures 19 -21). Younger and middle-aged adults who were higher in neuroticism experienced a steeper decrease in neuroticism over time. There were no significant associations between older adults' initial endorsement of neuroticism and their rate of change over time.

Younger adults. A cross-domain latent growth curve was modeled in AMOS to examine the association between NSIs and neuroticism for younger adults. The model fit the data well (see Table 33). Higher levels of neuroticism at MIDUS 1 was associated with a steeper decrease in NSIs and neuroticism over time.

Middle-aged adults. A cross-domain latent growth curve was modeled in AMOS to examine the association between NSIs and neuroticism for middle-aged adults. The model adequately fit the data (see Table 34). Higher levels of neuroticism at MIDUS 1 was associated with a steeper decrease in NSIs and neuroticism over time.

Older adults. A cross-domain latent growth curve was modeled in AMOS to examine the reciprocal association between NSIs and neuroticism for older adults. The model fit was poor, which indicates that significant individual differences remained unaccounted for in the model (see Table 35). There were no significant associations between the latent growth curves of NSIs and neuroticism for older adults.

Extraversion. A multigroup latent growth curve was modeled in AMOS to examine whether age moderated the association between NSIs and extraversion. The unconstrained model was compared to the constrained model, and results indicated that the unconstrained model fit the data better, $\Delta \mathrm{CFI}=.017, p<.05$ (see Table 25$)$. Thus, age moderated these associations.

The unconstrained model fit the data well (see Table 26). For younger adults, NSIs and extraversion significantly decreased by 0.004 and 0.008 scale units every year of age, respectively. For middle-aged adults, NSIs and extraversion significantly decreased by 0.010 and 
0.004 scale units every year of age, respectively. For older adults, NSIs and extraversion significantly decreased by 0.008 and 0.014 scale units every year of age, respectively (see Figures 22 -24). There were no significant associations among participants' initial frequency of NSIs, initial endorsement of extraversion, and rate of change over time.

Younger adults. A cross-domain latent growth curve was modeled in AMOS to examine the association between NSIs and extraversion for younger adults. The model fit was poor, which indicates that significant individual differences remained unaccounted for in the model (see Table 36). There were no significant associations between the latent growth curves of NSIs and extraversion for younger adults.

Middle-aged adults. A cross-domain latent growth curve was modeled in AMOS to examine the association between NSIs and extraversion for middle-aged adults. The model fit was poor, which indicates that significant individual differences remained unaccounted for in the model (see Table 37). There were no significant associations between the latent growth curves of NSIs and extraversion for middle-aged adults.

Older adults. A cross-domain latent growth curve was modeled in AMOS to examine the reciprocal between NSIs and extraversion for older adults. The model fit was poor, which indicates that significant individual differences remained unaccounted for in the model (see Table 38). There were no significant associations between the latent growth curves of NSIs and extraversion for older adults.

Conscientiousness. A multigroup latent growth curve was modeled in AMOS to examine whether age moderated the association between NSIs and conscientiousness. The unconstrained model was compared to the constrained model, and results indicated that the unconstrained 
model fit the data better, $\Delta \mathrm{CFI}=.025, p<.05$ (see Table 25). Thus, age moderated these associations.

The unconstrained model fit the data well (see Table 26). For younger adults, NSIs significantly decreased by 0.004 scale units every year of age, whereas conscientiousness significantly increased by 0.003 scale units every year of age. For middle-aged adults, NSIs significantly decreased by 0.010 scale units every year of age, whereas conscientiousness increased by 0.001 scale units every year of age. For older adults, NSIs and conscientiousness significantly decreased by 0.008 scale units every year of age (see Figures $25-27$ ). Older adults who scored higher in conscientiousness experienced a steeper decrease in conscientiousness over time.

Younger adults. A cross-domain latent growth curve was modeled in AMOS to examine the association between NSIs and conscientiousness for younger adults. The model fit the data well (see Table 39). There were no significant associations between the latent growth curves of NSIs and conscientiousness for younger adults.

Middle-aged adults. A cross-domain latent growth curve was modeled in AMOS to examine the association between NSIs and conscientiousness for middle-aged adults. The model fit was poor, which indicates that significant individual differences remained unaccounted for in the model (see Table 40). There were no significant associations between the latent growth curves of NSIs and conscientiousness for middle-aged adults.

Older adults. A cross-domain latent growth curve was modeled in AMOS to examine the association between NSIs and conscientiousness for older adults. The model fit was poor, which indicates that significant individual differences remained unaccounted for in the model (see 
Table 41). There were no significant associations between the latent growth curves of NSIs and conscientiousness for older adults. For a brief summary of the results, please see Appendix G.

\section{Discussion}

The Big Five personality traits and social relationships, more specifically NSIs, are associated with one another (Asendorpf, 2002; Roberts et al., 2008). However, these concepts change over adulthood and the longitudinal associations between personality and NSIs have only been examined in a handful of studies (Mund \& Neyer, 2014; Neyer \& Lehnart, 2007; Parker et al., 2012; Sturaro et al., 2008). Furthermore, the conducted studies utilize a single item to assess NSIs, report conflicting findings, do not examine age as a potential moderator, rely heavily on younger adult samples, and have not examined the reciprocal association between personality and NSIs (with the exception of Mund and Neyer, 2014 and Parker et al., 2012). The purpose of the current study was to examine the longitudinal and reciprocal associations between the Big Five personality traits and NSIs in a national sample of American adults using 18-year longitudinal data. Results indicated that personality traits and NSIs change over the course of adulthood, personality traits predict the occurrence of and change in NSIs over time, NSIs predict personality levels and change in personality over time, and that age significantly moderated these associations with stronger effects often occurring in middle adulthood.

\section{Negative Social Interactions}

Socioemotional selectivity theory (Carstensen et al., 1999) posits that older adults trim negative social network members who are not contributing to older adults' well-being. In addition, the social input model (Fingerman \& Charles, 2010; Fingerman et al., 2008) posits that interactional partners treat older adults more favorably and attempt to maintain a positive relationship with older adults. As such, interactions with social network members should 
improve with age, a claim which the results of the current study support. Not only did NSIs significantly decrease over the course of 18 years, which supports the first hypothesis, but older adults $(60+$ years old) reported significantly fewer NSIs relative to younger adults $(18-39$ years old) at the beginning of the study (MIDUS 1).

Age as a moderator. Age moderated the rate of change in NSIs over time, which supported my third hypothesis. Middle-aged adults (40 - 59 years old) exhibited the steepest declines in NSIs, followed by older adults and then younger adults. This parallels socioemotional selectivity theory, which suggests that younger adults are not trimming negative social network members from their social networks, perhaps because they are receiving knowledge from these members. As a result, they keep these negative members regardless of the emotional costs of doing so (Carstensen, 1992; Carstensen et al., 1999). Middle-aged adults experienced the steepest declines in NSIs over time because they are starting to trim negative social network members during this age period (Carstensen et al., 1999). Also, their available time and resources could be more restricted compared to younger adults because they are a sandwich generation (i.e., having a parent that is $65+$ years old and raising minor children/supporting grown children; Pew Research Center, 2013). This may reduce the amount of time middle-aged adults can spend with their friends (Wrzus et al., 2016), which may further reduce the frequency of NSIs.

Although the older adults' declines in NSIs were not as steep as the middle-aged adults' declines, there were no significant differences between the two slopes. However, older adults did report significantly fewer NSIs at MIDUS 1 compared to middle-aged adults. Older adults could have trimmed some of their negative social network members before the beginning of the study (Carstensen et al., 1999), which would have resulted in the fewer NSIs at MIDUS 1. Furthermore, the interactional partners could be actively modifying their interactions with older 
adults to avoid NSIs, which is consistent with the social input model (Fingerman \& Charles, 2010; Fingerman et al., 2008). As such, the continued decrease in NSIs over time could be explained by the socioemotional selectivity theory, the social input model, or both.

\section{Personality}

The social investment principle postulates that investing in age-graded social roles, which typically occurs in younger adulthood (Eliason et al., 2015), results in significant mean-level decreases in neuroticism and mean-level increases in agreeableness and conscientiousness (Roberts et al., 2005). This type of hypothesized change was only partially supported in the current study. Neuroticism did significantly decrease over the 18 years, but so did agreeableness, which was counter to the fourth hypothesis. Furthermore, conscientiousness increased over time, but this increase was not significant. These results were qualified by age, which supports the sixth hypothesis and gives insight into the differential patterns of personality development.

Age as a moderator. Agreeableness decreased over time for all age groups, however, this decline was not significant for middle-aged adults. Although the decrease in agreeableness could reflect true change in this cohort of adults, the decrease in agreeableness may be the result of a regression to the mean (Hartmann et al., 2011). Specifically, the participants who consented into the study may have been higher in agreeableness at MIDUS 1 relative to the individuals who declined to participate. Although this has not been specifically studied, the covariance for middle-aged adults was negative which suggests regression to the mean. Furthermore, those who are higher in agreeableness are more interested in research studies and believe that research studies are a good use of their time (Meade \& Craig, 2012; Meade \& Pappalardo, 2013), which makes this rationale plausible. As such, these participants could have regressed in agreeableness 
over time because regression occurs toward the direction of the population mean of a group (Cook \& Campbell, 1979).

It is important to note that the younger adults had significantly lower levels of agreeableness at MIDUS 1 relative to the middle-aged and older adults. This finding does lend some support to the social investment principle. Social investment principle posits that meanlevel increases in agreeableness occurs when adults engage in age-graded social roles, which typically occurs in younger adulthood (Eliason et al., 2015; Roberts et al., 2005). But younger adults are delaying their investment in age-graded social roles (Arnett, 2004), which may postpone the mean-level increases in agreeableness. Thus, the younger adults' lower levels of agreeableness compared to the middle-aged and older adults may be the result of this cohort of younger adults delaying their engagement in age-graded social roles.

Neuroticism did significantly decrease for all age groups, which supports the social investment principle (Roberts et al., 2005). However, there were no significant differences among the slopes for each age group. There were significant differences regarding levels of neuroticism at MIDUS 1, such that younger adults had higher levels of neuroticism relative to middle-aged and older adults. Similar to agreeableness, this finding does lend some support to the social investment principle. Younger adults are postponing their engagements in age-graded social roles (Arnett, 2004), which may postpone the mean-level decreases in neuroticism. Thus, the younger adults' higher levels of neuroticism relative to middle-aged and older adults may be the result of the younger adults not engaging in age-graded social roles.

Conscientiousness significantly increased for younger adults, increased for middle-aged adults (but not significantly), and significantly decreased for older adults. The non-significant increase in conscientiousness for middle-aged adults may be the result of a ceiling effect (Cook 
\& Campbell, 1979; Howell, 2010). A ceiling effect is when a large portion of participants select the highest anchor available within a scale (Cook \& Campbell, 1979; Hartmann et al., 2011). Because the highest anchor within the Midlife Development Inventory Personality Scale was 4, and the average of conscientiousness for middle-aged adults was $3.51(S D=0.41)$ at MIDUS 1, participants did not have the opportunity to select anything higher than a 4 at sequential time points. Thus, there was little potential for participants to significantly increase in conscientiousness using this scale. For older adults, research suggests that conscientiousness can exhibit mean-level decreases in late life (Kandler et al., 2015; Mottus, Johnson, \& Deary, 2012; Wortman, Lucas, \& Donnellan, 2012), which parallels the findings in the current study.

It is important to note, however, that the Cronbach's alpha for conscientiousness was low (range of $\alpha=.51-.61$ ), which is a limitation of the MIDUS dataset. Measures that have low reliabilities have inflated standard errors of estimates and should not be heavily relied upon to assess true change (Cook \& Campbell, 1979). Therefore, there might be some error regarding the slope parameters of conscientiousness. Future research should utilize measures that have higher reliability estimates compared to the Midlife Development Inventory Personality Scale to assess change over time.

Mean-level changes in openness and extraversion have not been studied as extensively as change in agreeableness, neuroticism, and conscientiousness (Roberts et al., 2006). The studies that have examined changes in openness and extraversion report conflicting findings (Asendorpf \& Wilpers, 1998; Bleidorn et al., 2009; Kandler et al., 2015; Ludtke et al., 2011; McCrae et al., 2000). Similar to the study conducted by Kandler and colleagues (2015), extraversion significantly decreased over the course of the study and age did not moderate this association. The mean-level decreases in extraversion could be the result of adults becoming more selective 
in the activities that they pursue because time becomes more restricted when engaging in agegraded social roles, especially for younger and middle-aged adults (Eliason et al., 2015; Kandler et al., 2015; Wrzus et al., 2016). Furthermore, the mean-level decreases in extraversion could be the result of adults becoming more selective in the social relationships that they pursue (Kandler et al., 2015), which is especially relevant for middle-aged and older adults (Carstensen et al., 1999).

Similar to the study conducted by Bleidorn and colleagues (2009), openness significantly decreased over the course of the study. This result was moderated by age in which older adults reported the steepest declines in openness compared to younger and middle-aged adults. Older adults could be reporting the steepest declines in openness because they are becoming increasingly aware of the physical challenges (e.g., less efficient immune system functioning; De la Fuente \& Miquel, 2009), cognitive challenges (e.g., declines in processing speed; Finkel, Reynolds, McArdle, \& Pedersen, 2007), and socioemotional losses (e.g., death of loved ones; Stroebe, Hansson, Stroebe, \& Schut, 2001) that they face (Baltes, Lindenberger, \& Staudinger, 2006; Freund \& Baltes, 1998). As such, older adults could be allocating their energy and resources into maintaining, regulating, and compensating for their losses (i.e., selection, optimization, and compensation; Baltes et al., 2006; Freund \& Baltes, 1998) instead of pursuing new experiences and adventures.

\section{Personality and Negative Social Interactions}

Personality predicting negative social interactions. Dynamic interactionism posits that individuals are active and purposeful agents in their development and environments (Magnusson, 1990; Magnusson \& Stattin, 1998). This has been demonstrated in the literature with personalityby-environment transactions (Buss, 1987; Roberts et al., 2008), which state that individuals 
actively pursue and manipulate their social environments, and that individuals evoke responses from their social environments that are consistent with their personalities. These transactions have been expanded to include the association among personality, conflict, and NSIs (e.g., Allemand et al., 2015; Blickle, 1997; Bono et al., 2002; Dijkstra et al., 2005; Graziano et al., 1996; Mund \& Neyer, 2014; Neyer \& Lehnart, 2007; Parker et al., 2012; Silva et al., 2016; Sturaro et al., 2008).

Similar to previous research, participants who were higher in agreeableness and conscientiousness reported fewer NSIs at MIDUS 1 (Allemand et al., 2015; Asendorpf \& Wilpers, 1998; Blickle, 1997; Bono et al., 2002; Dijkstra et al., 2005; Graziano et al., 1996; Iveniuk et al., 2014; Neyer \& Lehnart, 2007; Parker et al., 2012; Silva et al., 2016; Sturaro et al., 2008). This finding is consistent with previous research because individuals who are higher in agreeableness attempt to maintain social harmony, whereas those who are higher in conscientiousness attempt to follow socially prescribed norms (John \& Srivastava, 1999; McCrae \& Costa, 2008). Thus, this reduces the opportunity to experience NSIs from social network members, which supports the second hypothesis. Furthermore, participants who were higher in neuroticism reported more NSIs at MIDUS 1, which also supports the second hypothesis. Individuals who are higher in neuroticism tend to select themselves into unstable relationships that frequently experience conflict (Jeronimus, 2015). As such, those who are higher in neuroticism are prone to experience more NSIs from social network members.

The association between openness and NSIs has not been explored in great detail relative to the other personality traits (Bono et al., 2002). Despite this, research does suggest that those who are higher in openness report more NSIs (Parker et al., 2012), which the results of this study support. Individuals who are higher in openness have a desire for novelty and variety (John \& 
Srivastava, 1999), which could compel these individuals into new environments that may lead to NSIs. For example, individuals who are higher in openness are more likely to use marijuana, whereas those who are higher in openness' facets of values (e.g., being unconventional) and actions (e.g., having wide interests) are more likely to use cocaine or heroin (Terracciano, Löckenhoff, Crum, Bienvenu, \& Costa, 2008). This could lead to criticism, excess demands to stop the drug use, or annoyance from social network members, which are NSIs. Furthermore, those who are higher in openness seek out new activities and social environments (John \& Srivastava, 1999), which can lead to new acquaintaces and friends. Thus, those who are higher in openess could experience more NSIs relative to those who are lower in openess because having exposure to a larger social network is a risk factor of experiencing more NSIs (Beals \& Rook, 2006).

Regarding extraversion and NSIs, findings in the literature are mixed with some studies finding a positive association (Allemand et al., 2015; Sturaro et al., 2008), a negative association (Bono et al., 2002), or no association (Silva et al., 2016). Extraversion was not associated with NSIs in this study. This may be the result of how broad the domain of extraversion is (John \& Srivastava, 1999; McCrae \& Costa, 2008). An association between extraversion and NSIs may exist when measuring the facets of extraversion because facets are more strongly related to social relationship variables relative to the broad dimensions of the Big Five personality traits (Mund \& Neyer, 2014). For example, the tendency to experience positive emotions could be negatively associated with NSIs, whereas the tendency to be forceful could be positively associated with NSIs. Examining the association between the personality facets and NSIs is not possible in the MIDUS dataset because the measure is too brief. 
Age as a moderator. In support of the third hypothesis, these previous findings were moderated by age. Specifically, middle-aged adults who were higher in agreeableness reported fewer NSIs at MIDUS 1 . The middle-aged participants may have engaged in age-graded social roles when they were in younger adulthood, where investment in these roles typically occur (Eliason et al., 2015). This would lead to higher levels of agreeableness in middle adulthood, which is indicative of the social investment principle (Roberts et al., 2005). This rationale is also supported by the examination of the critical ratio scores of agreeableness at MIDUS 1, in which middle-aged adults endorsed significantly higher levels of agreeableness compared to the younger adults. Furthermore, fewer NSIs in middle adulthood can be explained by the socioemotional selectivity theory (Carstensen et al., 1999), which posits that adults trim negative social network members from their networks as they age to maintain and enhance emotional well-being. Examination of the critical ratio scores of NSIs at MIDUS 1 indicate that middleaged adults report significantly more NSIs relative to older adults. Thus, the higher levels of agreeableness and relatively more NSIs compound to create this unique association for middleaged adults.

Age also moderated the association between neuroticism and NSIs. All adults who were higher in neuroticism reported more NSIs at MIDUS 1. However, younger adults who were higher in neuroticism at MIDUS 1 reported a steeper decrease in NSIs over time relative to middle-aged and older adults. The steeper decrease in NSIs over time for younger adults may be the result of the (counterintuitive at first glance) benefits associated with NSIs (Mund \& Neyer, 2014). Of course, NSIs can have detrimental consequences on social relationships. Specifically, NSIs and conflict can threaten the stability of voluntary relationships (i.e., friendships) and can deteriorate the perceived relationship quality of obligatory relationships (i.e., family; Laursen $\&$ 
Hafen, 2010). However, NSIs and conflict can have beneficial consequences on social relationships (Mund \& Neyer, 2014). Being criticized can lead to challenging and constructive conversations, which can result in the improvement of self-expression and interpersonal skills (Laursen \& Hafen, 2010).

Because younger adults endorsed higher levels of neuroticism relative to middle-aged and older adults, the criticism or unwanted advice that they received from their social network members could have been constructive and insightful about their neurotic behaviors (e.g., being moody or worrying a lot; Mund \& Neyer, 2014). This type of NSI could lead to a decrease in neurotic behaviors at a faster rate, which was also supported in the current study (Research Question 3). The decrease in neuroticism may have led to a steeper decrease in NSIs, because those younger adults are no longer endorsing the neurotic behaviors as much, which would remove the constructive criticism between social network members.

Lastly, age also moderated the association between conscientiousness and NSIs, such that younger and middle-aged adults who were higher in conscientiousness reported fewer NSIs. Those who are higher in conscientiousness are dependable, reliable, and attempt to follow socially prescribed norms (John \& Srivastava, 1999). Thus, it is not surprising that younger and middle-aged adults who are higher in conscientiousness reported fewer NSIs because NSIs are violations of relationship norms (Brooks \& Dunkel Schetter, 2011). It is surprising, however, that this association was not significant for the older adults. While comparing the latent growth curves of conscientiousness across the age groups, the older adults' latent growth curve fit the data significantly worse than the younger and middle-aged adults' $(\mathrm{CFI}>.01, p<.05)$. This indicates that there is significant variability within the initial levels of and trajectories of conscientiousness for older adults that was not accounted for. Thus, it is possible that 
conscientiousness was not associated with NSIs for older adults because the variance of conscientiousness was much larger and unaccounted for the older adults.

Personality and change in negative social interactions. The longitudinal studies that have examined the association between personality and change in NSIs have primarily relied on samples of younger adults and time frames that range from two to eight years (with the exception of Mund and Neyer, 2014). Results from these studies are mixed: some studies found no association between personality and change in NSIs (e.g., Neyer \& Asendorpf, 2001; Sturaro et al., 2008), whereas others found an association (e.g., Neyer \& Lehnart, 2007; Parker et al., 2012). Similar to results from Neyer and Lehnart, adults who were higher in neuroticism reported a steeper decrease in NSIs over 18 years. However, this result was qualified by age, such that this trend was only applicable for younger and middle-aged adults. The explanation for this result was discussed in some detail in the previous section. Specifically, NSIs are not entirely harmful to social relationships (Mund \& Neyer, 2014), NSIs can lead to emotionallycloser relationships (Fung, Yeung, Li, \& Lang, 2009) and can lead to difficult but constructive conversations that involve self-reflection (Laursen \& Hafen, 2010). The criticism or unwanted advice that younger and middle-aged adults received from their social network members could be constructive and insightful about their neurotic behaviors (e.g., being moody or worrying a lot; Mund \& Neyer, 2014). This type of NSI could lead to a decrease in neurotic behaviors at a faster rate. The MIDUS dataset does not assess the context of the NSI (i.e., whether the NSI was constructive or not), however, making it difficult to tease apart these effects.

The significant and steeper decrease in neuroticism was only significant for younger adults for Research Question 3, but significant for both younger and middle-aged adults for Research Question 8. The significant parameter for the middle-aged adults in Research Question 
8 may have been the result of utilizing a cross-domain latent growth curve instead of a univariate growth curve. The cross-domain latent growth curve does not only simultaneously predict the latent growth curves of NSIs and neuroticism, but it also includes the neuroticism variables from MIDUS 2 and MIDUS 3 in the model. The inclusion of these additional variables in the model accounted for additional variance $\left(R^{2}\right.$ of Research Question 3 accounted for $=.054, R^{2}$ of Research Question 8 accounted for $\left.=.113, \Delta R^{2}=.079\right)$, which may have resulted in the significant result for middle-aged adults.

Negative social interactions and change in personality. There were no significant associations among NSIs and change in the Big Five personality traits. This null finding may be the result of using the broad dimensions of the Big Five personality traits, rather than the narrower facets that make up the broad personality dimensions. Social relationship variables are not constant over time; they have the capacity to change from day to day (Mund, Finn, Hagemeyer, \& Neyer, 2016; Mund \& Neyer, 2014). On the other hand, the Big Five personality traits are relatively constant (John \& Srivastava, 1999). As such, the use of the Big Five personality traits may have led to a reduction of significant findings because facets exhibit stronger effects with social relationship variables (Mund et al., 2016; Mund \& Neyer, 2014). Researchers should utilize questionnaires in their study that assess personality facets, such as the NEO-PI-R (Costa \& McCrae, 1992) to tease out these effects.

Change in personality and change in negative social interactions. Unfortunately, the predictive associations among change in personality predicting change in NSIs and vice versa could not be examined in the current study. The models would not converge in AMOS even after manipulating the models in six different ways, leaving the question of why? It is possible that these models would not converge because there was not enough meaningful change in the 
variables over time. For example, the unstandardized betas for change in NSIs and the Big Five personality traits ranged from 0.001 to 0.014 . This small range in change may not be powerful enough to predict further change. Also, the variability surrounding these unstandardized estimates were relatively non-existent, even though it was statistically significant. For example, the majority of the unstandardized betas for the variance parameters of slope were $0.000(S . E .=$ 0.000). It is possible that the findings of significant change in the variables and significant variability surrounding the change may be the result of utilizing a large dataset. It is important to examine the reciprocal associations between personality and NSIs in future research because the reciprocal changes in personality and NSIs could have positive (e.g., fewer NSIs, higher levels of conscientiousness) or negative (e.g., more NSIs) consequences on personality development and the occurrence of NSIs.

\section{Limitations and Future Directions}

The following limitations need to be taken into consideration when interpreting the results of the current study. The generalizability of these results may be limited for the following reasons. First, the MIDUS sample mainly consisted of highly educated Caucasian individuals. These results cannot be generalized to other racial or ethnic groups, however, I am unaware of any research that has documented racial or ethnic differences between personality and NSIs. Furthermore, the sample utilized for this study $(n=1,530)$ is selective due to the missing data techniques that were implemented for this study (e.g., the requirement of having data and a partner at all three waves of assessment). Researchers should not only examine these associations in a more diverse sample to determine the generalizability of these results, but should also use missing data techniques such as full information maximum likelihood to account for missing 
data. Such techniques would allow for a larger and more representative sample of the adult population.

Although the creation of age groups has been done in previous literature (e.g., Asendorpf \& Wilpers, 1998; Carstensen et al., 1999; Fingerman \& Charles, 2010; Fingerman et al., 2008; Ludtke et al., 2011; Mund \& Neyer, 2014) and represents developmentally important age periods within adulthood (Erikson, 1980), there are methodological concerns associated with this categorization. Creating a categorical variable from an interval variable restricts the variance associated with the variable, which can lead to reduced power and an increased probability of Type II error (Streiner, 2002). Although the categorization of age was used to be consistent with prior research and theory, future research should examine the moderating effects of age as an interval variable so the true variability in age differences can be examined.

In addition, the sample sizes across the age groups were vastly different from one another. The younger adult group consisted of 448 participants, the middle-aged adult group consisted of 909 participants, and the older adult group consisted of 173 participants. This could have increased the probability of Type I and Type II errors simultaneously. The results associated with older adults could have inflated Type II error because the power associated with this age group is lower compared to the other age groups. Furthermore, the results associated with the middle-aged adults could have increased the probability of Type I error because these analyses may have been overpowered as the result of such a large sample. The false discovery rate technique was utilized (Benjamini \& Hochberg, 1995) and a high false discovery rate was set at $15 \%$ (McDonald, 2014) to account for not only this issue, but an additionally inflated Type I error as a result of running multiple latent growth curve models. 
The Indicators of Strain Scale (modified measure from Schuster et al., 1990) relies on individuals' subjective assessments of their social network's behaviors. It is possible that these self-report measures do not fully capture the occurrence of NSIs individuals experience because (a) they may not know what constitutes a NSI; (b) they may perceive behaviors that are NSIs (e.g., criticism) as not aversive; and (c) they may not be willing to report that they experience NSIs due to social desirability. Furthermore, age may influence the interpretation of the Indicators of Strain Scale because older adults do not focus on negative events (Strough et al., 2016). As such, it is possible that the reported frequency of NSIs may be negatively skewed.

The Indicators of Strain Scale (modified measure from Schuster et al., 1990) also assessed the frequency of NSIs participants experienced with their family members, spouse/partner, and friends. However, MIDUS did not assess whether participants' partners remained the same over the 18 -year time frame. In order to account for the possibility that changes in NSIs were the result of a different partner, participants who did not have a partner at any of the three waves were removed from the sample (980 participants). Although this procedure was the best possible way to handle this issue within the MIDUS dataset, it is not without its limitations because a participant could have changed their partner in between the waves of assessment. This participant would have been kept in the current sample because they technically had a partner across the waves, even though it was a different partner. Despite the fact that an average score of NSIs was created, which included the reported frequency of NSIs from family members, partner, and friends, there is a possibility that this change estimate might be slightly skewed as a result of participants potentially having different partners over the study. Future research should take into account whether participants' partners are the same over time to ensure the validity of the change estimates. 
The majority of the latent growth curve models did not fit the data well. This indicates that significant interindividual differences remained unaccounted for in the model (Grady, Karraker, \& Metzger, 2012). Furthermore, the majority of the significant effects were small (Howell, 2010). Despite the poor fitting models and small effects, the models did well at accounting for the variance in NSIs and the Big Five personality traits. Across all research questions, the models accounted for up to $21.60 \%$ of the variance in the intercepts of the latent growth curves and up to $58.10 \%$ of the variance in the slopes of the latent growth curves (see Table 42 for a breakdown of the variance accounted for by variable). As such, the demographic variables, NSIs, and the Big Five personality traits accounted for a good portion of the frequency/levels of NSIs and the personality traits at MIDUS 1 and the trajectories of these concepts over time.

Future research should also take into consideration interactional partners' personalities when examining the association between personality and NSIs. Interactional partners' personalities can also influence the occurrence of NSIs (Bono et al., 2002). For example, participants who have an interactional partner who is higher in extraversion report experiencing more conflict with their interactional partner (Bono et al., 2002). As such, interactional partners can either increase or decrease the occurrence of NSIs within a relationship. Future research should use dyadic data to disentangle these effects by using actor partner models.

Furthermore, dyadic data could be utilized to determine whether individuals who are higher in certain personality traits are biased in their reporting of NSIs. Although this specifically has not been examined, certain individuals may over-report or under-report the frequency of NSIs that they experience because research suggests that those who are higher in certain personality traits over-report or under-report the number of somatic illnesses that they have 
(Friedman \& Kern, 2014). Dyadic data could disentangle this by examining whether there are discrepancies between interactional partners regarding how often they engage in NSIs and how often they receive NSIs. If there are discrepancies between the partners, their personality traits could be used to predict the occurrence of discrepancies.

The results in this study were not only similar to previous studies that utilize German samples and a single Likert-type question to assess conflict, but these results were also similar when examining the separate sources of NSIs (i.e., NSIs from family members, partners, and friends). This similarity suggests that the associations between personality and NSIs are similar across different individualistic countries and that a single indicator may be an efficient way to measure NSIs. Single-itemed measures reduce assessment time and the burden on participants (Burisch, 1984), and have been found to have similar predictive validity to larger measures in other fields (Bergkvist \& Rossiter, 2007; Fisher, Matthews, \& Gibbons, 2016; Wanous, Reichers, \& Hudy, 1997). However, studies should empirically examine the validity of a multi-itemed construct of NSIs compared to a single-itemed measure of conflict in more detail before relying on a single-itemed measure, because relationship dynamics are complex and it may be unlikely that a single item will capture this complexity.

\section{Implications}

The current study does have implications for research within the personality-social environment field. Forming close social relationships is a normative process and these relationships are established early in the life span. Specifically, parent-child relationships typically begin at birth and are present throughout the majority of the life span, friendships begin to bud once individuals enter formal schooling, and romantic relationships begin to emerge as early as middle childhood (Levitt \& Cici-Gokaltun, 2011). The quality of these relationships has 
been associated with school involvement and achievement, job attainment and success, psychological well-being, health, and mortality (Hartup, 1996; Hartup \& Stevens, 1997; Lund et al., 2014; Newsom et al., 2005; Uchino, 2004, 2006, 2009). Results from this study indicate that individuals' personalities can influence the types of interactions that they have with social network members, which may influence the quality of the relationship over time and have implications on many important developmental outcomes across the life span. Knowing this, researchers can use behavioral activation (Magidson, Roberts, Collado-Rodrigues, \& Lejuez, 2014) to alter personality traits (e.g., to decrease neuroticism or to increase agreeableness or conscientiousness) to reduce the occurrence of NSIs and potentially increase the quality of the social relationships, which may have positive effects on psychological well-being and health over time.

Furthermore, the results suggest that NSIs could be a plausible mediator for the personality-health association because personality is associated with the occurrence of NSIs. Researchers may be able to target individuals who endorse specific personality traits (e.g., those who are higher in neuroticism or openness) so that any increases or decreases in specific personality traits would have a downstream effect on health via more optimal social functioning. Specifically, researchers can use behavioral activation (Magidson et al., 2014) to reduce levels of neuroticism or openness to potentially reduce the frequency of NSIs. In turn, there may be reductions in cardiovascular reactivity and the possible protective effect on health from having more supportive social networks (Reblin \& Uchino, 2008). Although the feasibility of modifying personality characteristics to improve health is still in its early stages, this study suggests that personality and the social environment are associated across adulthood and should be further examined. Second, the results suggest that there are differential associations between personality 
and NSIs across adulthood, which could lead to differential effects on health. An additional examination of the reciprocal association between the Big Five personality traits and NSIs is needed to confirm the replicability of these results.

Regarding the effects of the social environment on personality development, researchers typically examine whether entrance into new social environments promote personality development (e.g., Lang, Reschke, \& Neyer, 2006; Neyer \& Asendorpf, 2001; Roberts et al., 2005). The current study expands on this and suggests that not only do social environments have the capacity to influence the development of personality traits over time, but so does the everyday interactions that individuals have with social network members. More specifically, that younger and middle-aged adults who experience more NSIs in their networks decrease in neuroticism at a steeper rate relative to those who do not experience more NSIs. This finding highlights the importance of interactions between social network members and should be examined in greater detail in the future.

\section{Conclusion}

This is the first study to examine the associations between the Big Five personality traits and a multi-itemed construct of NSIs in a large national sample. Furthermore, this was the first study to examine whether age moderated the change in personality traits and NSIs over 18 years, and whether age moderated the association between the two constructs. Results indicated that personality traits and NSIs change across adulthood, personality traits predict the occurrence of and change in NSIs over time, and that NSIs predict personality levels and change in personality over time. The results also highlight the need for future research to examine age as a moderator because the overwhelming majority of the results were moderated by age (with the exception of the univariate latent growth curve of extraversion). 


\section{References}

Ackerman, P. L., \& Heggestad, E. D. (1997). Intelligence, personality, and interests: Evidence for overlapping traits. Psychological Bulletin, 121, 219-245.

Allemand, M., Schaffhuser, K., \& Martin, M. (2015). Long-term correlated change between personality traits and perceived social support in middle adulthood. Personality and Social Psychology Bulletin, 41, 420-432. doi.org/10.1177/0146167215569492

Antonucci, T. C., \& Jackson, J. S. (1987). Social support, interpersonal efficacy, and health: A life course perspective. In L. L. Carstensen \& B. A. Edelstein (Eds.), Handbook of clinical gerontology (pp. 291-311). Elmsford, NY: Pergamon Press.

Arnett, J. J. (2004). Emerging adulthood: The winding road from the late teens through the twenties. New York, NY: Oxford University Press.

Asendorpf, J. B. (2002). Personality effects on personal relations over the life span. In A. L. Vangelisti, H. T. Reis, \& M. A. Fitzpatrick (Eds.), Stability and change in relationships (pp. 35-56). Cambridge, UK: Cambridge University Press.

Asendorpf, J. B., \& Wilpers, S. (1998). Personality effects on social relationships. Journal of Personality and Social Psychology, 74, 1531-1544. doi.org/10.1037/0022-3514.74.6.1531

Baltes, P. B., Lindenberger, U., \& Staudinger, U. M. (2006). Life span theory in developmental psychology. In R. M. Lerner (Ed.), Handbook of child psychology: Theoretical models of human development (pp. 569-664). John Wiley \& Sons, Inc.

Bates, J. E., \& Novosad, C. (2008). Measurement of individual difference constructs in child development, or taking aim at moving targets. In D. M. Teti (Ed.), Handbook of research methods in developmental science (pp. 103-122). Oxford, UK: Blackwell Publishing Ltd.

Beals, K. P., \& Rook, K. S. (2006). Gender differences in negative social exchanges: Frequency, 
reactions, and impact. In V. H. Bedford \& B. F. Turner (Eds.), Men in relationships: A new look from a life course perspective (pp. 197-217). New York, NY: Springer Publishing Company.

Benjamini, Y., \& Hochberg, Y. (1995). Controlling the false discovery rate: A practical and powerful approach to multiple testing. Journal of Royal Statistical Society, 57, 289-300.

Bergkvist, L., \& Rossiter, J. R. (2007). The predictive validity of multiple-item versus singleitem measures of the same constructs. Journal of Marketing Research, 44, 175-184. doi.org/10.1509/jmkr.44.2.175

Bleidorn, W., Kandler, C., Riemann, R., Spinath, F. M., \& Angleitner, A. (2009). Patterns and sources of adult personality development: Growth curve analyses of the NEO PI-R scales in a longitudinal twin study. Journal of Personality and Social Psychology, 97, 142-155. doi.org/10.1037/a0015434

Blickle, G. (1997). Argumentativeness and the facets of the big five. Psychological Reports, 81, $1379-1385$.

Bono, J. E., Boles, T. L., Judge, T. A., \& Lauver, K. J. (2002). The role of personality in task and relationship conflict. Journal of Personality, 70, 311-344. doi.org/10.1111/14676494.05007

Brooks, K. P., \& Dunkel Schetter, C. (2011). Social negativity and health: Conceptual and measurement issues. Social and Personality Psychology Compass, 5, 904-918. doi.org/10.1111/j.1751-9004.2011.00395.x

Burisch, M. (1984). Approaches to personality inventory construction: A comparison of merits. American Psychologist, 39, 214-227. doi.org/10.1037/0003-066X.39.3.214

Buss, D. M. (1987). Selection, evocation, and manipulation. Journal of Personality and Social 
Psychology, 53, 1214-1221. doi.org/10.1037/0022-3514.53.6.1214

Byrne, B. M. (2010). Structural equation modeling with AMOS: Basic concepts, applications, and programming. (2nd ed.). New York, NY: Routledge: Taylor \& Francis Group.

Carstensen, L. L. (1992). Social and emotional patterns in adulthood: Support for socioemotional selectivity theory. Psychology and Aging, 7, 331-338. doi.org/10.1037/0882-7974.7.3.331

Carstensen, L. L., Isaacowitz, D. M., \& Charles, S. T. (1999). Taking time seriously: A theory of socioemotional selectivity. American Psychologist, 54, 165-181. doi.org/10.1037/0003066X.54.3.165

Caspi, A., \& Roberts, B. W. (2001). Personality development across the life course: The argument for change and continuity. Psychological Inquiry, 12, 49-66. doi.org/10.1207/S15327965PLI1202_01

Clark, L. A., \& Watson, D. (2008). Temperament: An organizing paradigm for trait psychology. In O. P. John, R. W. Robins, \& L. A. Pervin (Eds.), Handbook of personality: Theory and research (3rd ed., pp. 265-286). New York, NY: The Guilford Press.

Cohen, S., Doyle, W. J., Skoner, D. P., Rabin, B. S., \& Gwaltney, J. M. (1997). Social ties and susceptibility to the common cold. Journal of the American Medical Association, 277, 1940-1944. doi.org/10.1001/jama.1997.03540480040036

Cook, T. D., \& Campbell, D. T. (1979). Validity. In Quasi-experimentation: Design and data analysis issues for field settings. (pp. 37-94). Chicago, IL.: Rand McNally College Publishing Company.

Costa, P. T., Jr., \& McCrae, R. R. (1992). Revised NEO Personality Inventory (NEO-PI-R) and NEO Five-Factor Inventory (NEO-FFI) professional manual. Odessa, FL: Psychological Assessment Resources. 
Costa, P. T., Terracciano, A., \& McCrae, R. R. (2001). Gender differences in personality traits across cultures: Robust and surprising findings. Journal of Personality and Social Psychology, 81, 322-331. doi.org/10.1037/0022-3514.81.2.322

De la Fuente, M., \& Miquel, J. (2009). An update of the oxidation-inflammation theory of aging: The involvement of the immune system in oxi-inflamma-aging. Current Pharmaceutical Design, 15, 3003-3026. doi.org/ 10.2174/138161209789058110

Dijkstra, M. T. M., van Dierendonck, D., Evers, A., \& De Dreu, C. K. W. (2005). Conflict and well-being at work: The moderating role of personality. Journal of Managerial Psychology, 20, 87-104. doi.org/10.1108/02683940510579740

Duncan, T. E., \& Duncan, S. C. (2004). An introduction to latent growth curve modeling. Behavior Therapy, 35, 333-363. doi.org/10.1016/S0005-7894(04)80042-X

Edwards, K. J., Hershberger, P. J., Russell, R. K., \& Markert, R. J. (2001). Stress, negative social exchange, and health symptoms in university student. The Journal of American College Health, 50, 75-79. doi.org/10.1080/07448480109596010

Eliason, S. R., Mortimer, J. T., \& Vuolo, M. (2015). The transition to adulthood: Life course structures and subjective perceptions. Social Psychology Quarterly, 78, 205-227. doi.org/10.1177/0190272515582002

Erikson, E. H. (1980). Identity and the life cycle. New York, NY: W. W. Norton.

Fingerman, K. L., \& Charles, S. T. (2010). It takes two to tango: Why older people have the best relationships. Current Directions in Psychological Science, 19, 172-176. doi.org/10.1177/0963721410370297

Fingerman, K. L., Miller, L., \& Charles, S. (2008). Saving the best for last: How adults treat social partners of different ages. Psychology and Aging, 23, 399-409. 
doi.org/10.1037/0882-7974.23.2.399

Finkel, D., Reynolds, C. A., McArdle, J. J., \& Pedersen, N. L. (2007). Age changes in processing speed as a leading indicator of cognitive aging. Psychology and Aging, 22, 558-568. doi.org/ 10.1037/0882-7974.22.3.558

Fisher, G. G., Matthews, R. A., \& Gibbons, A. M. (2016). Developing and investigating the use of single-item measures in organizational research. Journal of Occupational Health Psychology, 21, 3-23. doi.org/10.1037/a0039139

Freund, A. M., \& Baltes, P. B. (1998). Selection, optimization, and compensation as strategies of life management: Correlations with subjective indicators of successful aging. Psychology and Aging, 13, 531-543. doi.org/10.1037/0882-7974.13.4.531

Friedman, H. S., \& Kern, M. L. (2014). Personality, well-being, and health. Annual Review of Psychology, 65, 719-742. doi.org/10.1146/annurev-psych-010213-115123

Fung, H. H., Yeung, D. Y., Li, K. K., \& Lang, F. R. (2009). Benefits of negative social exchanges for emotional closeness. Journal of Gerontology: Psychological Sciences, 64B, 612-621. doi.org/10.1093/geronb/gbp065

Fydrich, T., Sommer, G., \& Bahler, E. (2007). F-SozU: Fragebogen zur sozialen Unterstützung. Göttingen, Germany: Hogrefe.

Goodwin, R. D., \& Gotlib, I. H. (2004). Gender differences in depression: The role of personality factors. Psychiatry Research, 126(2), 135-142. doi.org/10.1016/j.psychres.2003.12.024

Grady, J. S., Karraker, K., \& Metzger, A. (2012). Shyness trajectories in slow-to-warm-up infants: Relations with child sex and maternal parenting. Journal of Applied Developmental Psychology, 33, 91-101. doi.org/10.1016/j.appdev.2011.11.002 
Graziano, W. G., Jensen-Campbell, L. A., \& Hair, E. C. (1996). Perceiving interpersonal conflict and reacting to it: The case for agreeableness. Journal of Personality and Social Psychology, 70, 820-835. doi.org/10.1037/0022-3514.70.4.820

Hampson, S. E., \& Friedman, H. S. (2008). Personality and health. In O. P. John, R. W. Robins, \& L. A. Pervin (Eds.), Handbook of personality: Theory and research (3rd ed., pp. 770794). New York, NY: The Guilford Press.

Hartmann, D. P., Pelzel, K. E., \& Abbott, C. B. (2011). Design, measurement, and analysis in developmental research. In M. H. Bornstein \& M. E. Lamb (Eds.), Developmental science: An advanced textbook (6th ed., pp. 109-197). New York, NY.: Psychology Press.

Hartup, W. W. (1996). The company they keep: Friendships and their developmental significance. Child Development, 67, 1-13. doi.org/10.1111/1467-8624.ep9602271141

Hartup, W. W., \& Stevens, N. (1997). Friendship and adaptation in the life course. Psychological Bulletin, 121, 335-370.

Hill, P. L., Weston, S. J., \& Jackson, J. J. (2014). Connecting social environment variables to the onset of major specific health outcomes. Psychology \& Health, 29, 753-767. doi.org/10.1080/08870446.2014.884221

Holt-Lunstad, J., Smith, T. B., \& Layton, J. B. (2010). Social relationships and mortality risk: A meta-analytic review. PLoS Medicine, 7, 1-20. doi.org/10.1371/journal.pmed.1000316

Hopwood, C. J., \& Donnellan, M. B. (2011). Genetic and environmental influences on personality trait stability and growth during the transition to adulthood: A three-wave longitudinal study. Journal of Personality and Social Psychology, 100, 545-556. doi.org/10.1037/a0022409

Howell, D. C. (2010). Statistical methods for psychology. Belmont, CA: Duxbury Press. 
Iveniuk, J., Waite, L. J., Laumann, E., Mcclintock, M. K., \& Tiedt, A. D. (2014). Marital conflict in older couples: Positivity, personality, and health. Journal of Marriage and Family, 76, 130-144. doi.org/10.1111/jomf.12085

Jeronimus, B. F. (2015). Environmental influences on neuroticism: A story about emotional (in) stability. University of Groningen. doi.org/10.13140/2.1.3452.2407

John, O. P., \& Srivastava, S. (1999). The big five trait taxonomy: History, measurement, and theoretical perspectives. In L. A. Pervin \& O. P. John (Eds.), Handbook of personality: Theory and research (2nd ed., pp. 102-138). New York, NY: The Guilford Press.

Kandler, C., Kornadt, A. E., Hagemeyer, B., \& Neyer, F. J. (2015). Patterns and sources of personality development in old age. Journal of Personality and Social Psychology, 109, 175-191. doi.org/10.1037/pspp0000028

Kern, M. L., \& Friedman, H. S. (2011). Personality and pathways of influence on physical health. Social and Personality Psychology Compass, 5, 76-87. doi.org/10.1111/j.17519004.2010.00331.x

Lachman, M. E., \& Weaver, S. L. (1997). The midlife development inventory (MIDI) personality scales: Scale construction and scoring. Waltham, MA: Brandeis University.

Lang, F. R., Reschke, F. R., \& Neyer, F. J. (2006). Social relationships, transitions, and personality development across the life span. In D. K. Mroczek \& T. D. Little (Eds.), Handbook of personality development (pp. 445-466). Mahwah, NJ: Lawrence Erlbaum Associates.

Laursen, B., \& Hafen, C. (2010). Future directions in the study of close relationships: Conflict is bad (except when it's not). Social Development, 19, 858-872. doi.org/10.1111/j.14679507.2009.00546.x. 
Levitt, M. J., \& Cici-Gokaltun, A. (2011). Close relationships across the life span. In K. L. Fingerman, C. A. Berg, J. Smith, \& T. C. Antonucci (Eds.), Hanbook of life-span development (pp. 457-486). New York, NY: Springer Publishing Company.

Little, T. D. (2013). Longitudinal structural equation modeling. New York, NY: The Guilford Press.

Ludtke, O., Roberts, B. W., Trautwein, U., \& Nagy, G. (2011). A random walk down university avenue: Life paths, life events, and personality trait change at the transition to university life. Journal of Personality and Social Psychology, 101, 620-637. doi.org/10.1037/a0023743

Lund, R., Christensen, U., Nilsson, C. J., Kriegbaum, M., \& Hulvej Rod, N. (2014). Stressful social relations and mortality: A prospective cohort study. Journal of Epidemiology and Community Health, 68, 720-727. doi.org/10.1136/jech-2013-203675

Luong, G., \& Charles, S. T. (2014). Age differences in affective and cardiovascular responses to a negative social interaction: The role of goals, appraisals, and emotion regulation. Developmental Psychology, 50, 1919-1930. doi.org/10.1037/a0036621

Magidson, J. F., Roberts, B. W., Collado-Rodrigues, A., \& Lejuez, C. W. (2014). Theory-driven intervention for changing personality: Expectancy value theory, behavioral activation, and conscientiousness. Developmental Psychology, 50, 1442-1450. doi.org/10.1037/a0030583

Magnusson, D. (1990). Personality development from an interactional perspective. In L. A. Pervin (Ed.), Handbook of Personality: Theory and research (pp. 193-222). New York, NY: Guilford Press.

Magnusson, D., \& Stattin, H. (1998). Person-context interaction theories. In W. Damon \& R. M. Lerner (Eds.), Handbook of child psychology: Theoretical models of human development 
(pp. 685-759). Hoboken, NJ: John Wiley \& Sons, Inc.

Mavandadi, S., Sorkin, D. H., Rook, K. S., \& Newsom, J. T. (2007). Pain, positive and negative social exchanges, and depressive symptomatology in later life. Journal of Aging and Health, 19, 813-830. doi.org/10.1177/0898264307305179

McCrae, R. R., \& Costa, P. T. (1997). Personality trait structure as a human universal. The American Psychologist, 52, 509-516. doi.org/10.1037/0003-066X.52.5.509

McCrae, R. R., \& Costa, P. T. (2008). The five-factor theory of personality. In O. P. John, R. W. Robins, \& L. A. Pervin (Eds.), Handbook of personality: Theory and research. (pp. 159181). New York, NY: The Guilford Press.

McCrae, R. R., Costa, P. T., Ostendorf, F., Angleitner, A., Hrebícková, M., Avia, M. D., ... Smith, P. B. (2000). Nature over nurture: Temperament, personality, and life span development. Journal of Personality and Social Psychology, 78, 173-186. doi.org/10.1037/0022-3514.78.1.173

McDonald, J. H. (2014). Multiple tests. In Handbook of biological statistics (3rd ed., pp. 254260). Baltimore, MD: Sparky House Publishing.

Meade, A. W., \& Craig, S. B. (2012). Identifying careless responses in survey data. Psychological Methods, 17, 437-455. doi.org/10.1037/a0028085

Meade, A. W., \& Pappalardo, G. (2013). Predicting careless responses and attrition in survey data with personality. 28th Annual Meeting of the Society for Industrial and Organizational Psychology.

Mottus, R., Johnson, W., \& Deary, I. J. (2012). Personality traits in old age: Measurement and rank-order stability and some mean-level change. Psychology and Aging, 27, 243-249. doi.org/10.1037/a0023690 
Mund, M., Finn, C., Hagemeyer, B., \& Neyer, F. J. (2016). Understanding dynamic transactions between personality traits and partner relationships. Current Directions in Psychological Science, 25, 411-416. doi.org/10.1177/0963721416659458

Mund, M., \& Neyer, F. J. (2014). Treating personality-relationship transactions with respect: Narrow facets, advanced models, and extended time frames. Journal of Personality and Social Psychology, 107, 352-68. doi.org/10.1037/a0036719

Newsom, J. T., Mahan, T. L., Rook, K. S., \& Krause, N. M. (2008). Stable negative social exchanges and health. Health Psychology, 27, 78-86. doi.org/10.1037/0278-6133.27.1.78

Newsom, J. T., Rook, K. S., Nishishiba, M., Sorkin, D. H., \& Mahan, T. L. (2005).

Understanding the relative importance of positive and negative social exchanges:

Examining specific domains and appraisals. Journal of Gerontology: Psychological Sciences, 60B, P304-P312. doi.org/10.1093/geronb/60.6.P304

Neyer, F. J., \& Asendorpf, J. B. (2001). Personality-relationship transaction in young adulthood. Journal of Personality and Social Psychology, 81, 1190-1204. doi.org/10.1037/00223514.81.6.1190

Neyer, F. J., \& Lehnart, J. (2007). Relationships matter in personality development: Evidence from an 8-year longitudinal study across young adulthood. Journal of Personality, 75, 535568. doi.org/10.1111/j.1467-6494.2007.00448.x

Okun, M. A., \& Keith, V. M. (1998). Effects of positive and negative social exchanges with various sources on depressive symptoms in younger and older adults. Journal of Gerontology: Psychological Sciences, 53B, 4-20. doi.org/ 10.1093/geronb/53B.1.P4

Parker, P. D., Ludtke, O., Trautwein, U., \& Roberts, B. W. (2012). Personality and relationship quality during the transition from high school to early adulthood. Journal of Personality, 80, 
1061-1089. doi.org/10.1111/j.1467-6494.2012.00766.x

Pew Research Center (2013). The sandwich generation: Rising financial burdens for middle-aged americans. Retrieved from http://www.pewsocialtrends.org/2013/01/30/the-sandwichgeneration/

Reblin, M., \& Uchino, B. N. (2008). Social and emotional support and its implication for health. Current Opinion in Psychiatry, 21, 201-205. doi.org/10.1097/YCO.0b013e3282f3ad89.Social

Reynolds, K. J., Turner, J. C., Branscombe, N. R., Mavor, K. I., Bizumic, B., \& Subasic, E. (2010). Interactionism in personality and social psychology: An integrated approach to understanding the mind and behavior. European Journal of Personality, 24, 458-482. doi.org/10.1002/per. 782

Roberts, B. W., \& DelVecchio, W. F. (2000). The rank-order consistency of personality traits from childhood to old age: A quantitative review of longitudinal studies. Psychological Bulletin, 126, 3-25. doi.org/10.1037/0033-2909.126.1.3

Roberts, B. W., Walton, K. E., \& Viechtbauer, W. (2006). Patterns of mean-level change in personality traits across the life course: A meta-analysis of longitudinal studies. Psychological Bulletin, 132, 1-25. doi.org/10.1037/0033-2909.132.1.1

Roberts, B. W., \& Wood, D. (2006). Personality development in the context of the NeoSocioanalytic Model of personality. (D. K. Mroczek \& T. D. Little, Eds.). Mahwah, NJ: Erlbaum.

Roberts, B. W., Wood, D., \& Caspi, A. (2008). The development of personality traits in adulthood. In O. P. John, R. W. Robins, \& L. A. Pervin (Eds.), Handbook of personality: Theory and research. (3rd ed., pp. 375-398). New York, NY: The Guilford Press. 
Roberts, B. W., Wood, D., \& Smith, J. L. (2005). Evaluating five factor theory and social investment perspectives on personality trait development. Journal of Research in Personality, 39, 166-184. doi.org/10.1016/j.jrp.2004.08.002

Rook, K. S. (1998). Investigating the positive and negative sides of personal relationships: Through a glass darkly. In B. H. Spitzberg \& W. R. Cupach (Eds.), The dark side of close relaitonships. Mahwah, NJ: Laerence Erlbaum Associates.

Schuster, T. L., Kessler, R. C., \& Aseltine, R. H. (1990). Supportive interactions, negative interactions, and depressive mood. American Journal of Community Psychology, 18, 423438.

Silva, N. M. M., Henrie, J. A., \& Patrick, J. H. H. (2016). Personality, negative social exchanges, and physical health among bereaved adults. Health Psychology Open, 3, 1-14. doi.org/10.1177/2055102916637877

Smith, T. W. (2006). Personality as risk and resilience in physical health. Current Directions in Psychological Science, 15, 227-231. doi.org/10.1111/j.1467-8721.2006.00441.x

Sorkin, D. H., \& Rook, K. S. (2004). Interpersonal control strivings and vulnerability to negative social exchanges in later life. Psychology and Aging, 19, 555-564. doi.org/10.1037/08827974.19.4.555

Streiner, D. L. (2002). Breaking up is hard to do: The heartbreaker of dichotomizing continuos data. The Canadian Journal of Psychiatry, 47, 262-266. doi.org/10.1177/070674370204700307

Stroebe, M., Hansson, R. O., Stroebe, W., \& Schut, H. (2001). Handbook of bereavement research: Consequences, coping and care. Washington, D.C.: American Psychological Association. 
Sturaro, C., Denissen, J. J. A., van Aken, M. A. G., \& Asendorpf, J. B. (2008). Personenvironment transactions during emerging adulthood: The interplay between personality characteristics and social relationships. European Psychologist, 13, 1-11. doi.org/10.1027/1016-9040.13.1.1

Terracciano, A., Löckenhoff, C. E., Crum, R. M., Bienvenu, O. J., \& Costa, P. T. (2008). Fivefactor model personality profiles of drug users. BMC Psychiatry, 8, 1-10. doi.org/10.1186/1471-244X-8-22

Thorne, A. (1987). The press of personality: A study of conversations between introverts and extraverts. Journal of Personality and Social Psychology, 53, 718-726.

Tims, M., Bakker, A. B., \& Derks, D. (2013). The impact of job crafting on job demands, job resources, and well-being. Journal of Occupational Health Psychology, 18, 230-240. doi.org/10.1037/a0032141

Turiano, N. A., Chapman, B. P., Gruenewald, T. L., \& Mroczek, D. K. (2015). Personality and the leading behavioral contributors of mortality. Health Psychology, 34, 51-60. doi.org/10.1037/hea0000038

Turiano, N. A., Mroczek, D. K., Moynihan, J., \& Chapman, B. P. (2013). Big 5 personality traits and interleukin-6: Evidence for "healthy neuroticism" in a U.S. population sample. Brain, Behavior, and Immunity, 28, 83-89. doi.org/10.1016/j.bbi.2012.10.020

Turiano, N. A., Whiteman, S. D., Hampson, S. E., Roberts, B. W., \& Mroczek, D. K. (2012). Personality and substance use in midlife: Conscientiousness as a moderator and the effects of trait change. Journal of Research in Personality, 29, 997-1003. doi.org/10.1016/j.jrp.2012.02.009 Uchino, B. N. (2004). Social support and physical health: Understanding the health 
consequences of relationships. New Haven, CT: Yale University Press.

Uchino, B. N. (2006). Social support and health: A review of physiological processes potentially underlying links to disease outcomes. Journal of Behavioral Medicine, 29, 377-387. doi.org/10.1007/s10865-006-9056-5

Uchino, B. N. (2009). Understanding the links between social support and physical health: A life-span perspective with emphasis on the separability of perceived and received support. Perspectives on Psychological Science, 4(3), 236-255.

University of Wisconsin - Madison (2011). History and overview of MIDUS. Retrieved from http://midus.wisc.edu/scopeofstudy.php

Walen, H. R., \& Lachman, M. E. (2000). Social support and strain from partner, family, and friends: Costs and benefits for men and women in adulthood. Journal of Social and Personal Relationships, 17, 5-30. doi.org/10.1177/0265407500171001

Wanous, J. P., Reichers, A. E., \& Hudy, M. J. (1997). Overall job satisfaction: How good are single-item measures? Journal of Applied Psychology, 82, 247-252. doi.org/10.1037/00219010.82 .2 .247

Willet, J. B., \& Sayer, A. G. (1996). Cross-domain analyses of change over time: Combining growth modeling and covariance structure analysis. In G. A. Marcoulides \& R. E. Schumacker (Eds.), Advanced structural equation modeling: Issues and techniques (pp. 125-157). Mahwah, NJ: Erlbaum Associates.

Wortman, J., Lucas, R. E., \& Donnellan, M. B. (2012). Stability and change in the Big Five personality domains: Evidence from a longitudinal study of Australians. Psychology and Aging, 27, 867-874. doi.org/10.1037/a0029322

Wrzesniewski, A., \& Dutton, J. E. (2001). Crafting a job: Revisioning employees as active 
crafters of their work. The Academy of Management Review, 26, 179-201.

Wrzus, C., Hänel, M., Wagner, J., \& Neyer, F. J. (2012). Social network changes and life events across the life span: A meta-analysis. Psychological Bulletin, 139, 53-80. doi.org/10.1037/a0028601

Wrzus, C., Wagner, G. G., \& Riediger, M. (2016). Personality-situation transactions from adolescence to old age. Journal of Personality and Social Psychology, 110, 782-799. doi.org/10.1037/pspp0000054 
Table 1

Descriptive Statistics and Bivariate Correlations for MIDUS 1 - Younger Adults

\begin{tabular}{|c|c|c|c|c|c|c|c|c|c|c|}
\hline & $M, S D$, or $\%$ & $\alpha$ & 1 & 2 & 3 & 4 & 5 & 6 & 7 & 8 \\
\hline 1. Age & $33.67,3.90$ & - & - & & & & & & & \\
\hline 2. Gender & $55.40 \%$ Female & - & .00 & - & & & & & & \\
\hline \multicolumn{11}{|l|}{$(0=$ Female $)$} \\
\hline 3. Education & $7.54,2.29$ & - & -.04 & .02 & - & & & & & \\
\hline 4. NSIs & $2.05,0.39$ & .78 & .01 & -.10 & -.07 & - & & & & \\
\hline 5. Agreeableness & $3.40,0.49$ & .79 & .03 & -.32 & -.01 & -.03 & - & & & \\
\hline 6. Openness & $2.95,0.49$ & .75 & -.09 & .13 & .13 & -.02 & .32 & - & & \\
\hline 7. Neuroticism & $2.29,0.67$ & .74 & -.06 & -.12 & -.18 & .32 & -.07 & -.11 & - & \\
\hline 8. Extraversion & $3.18,0.55$ & .77 & -.07 & -.07 & .00 & -.08 & .43 & .44 & -.12 & - \\
\hline 9. Conscientiousness & $3.45,0.42$ & .52 & -.02 & -.26 & .03 & -.12 & .24 & .07 & -.10 & .10 \\
\hline
\end{tabular}

Note. Significant statistics $(p<.05)$ are bolded. Younger adults' $(n=448)$ ages ranged from $25-39,91.10 \%$ were married, and 95.50\% identified as White/Caucasian. 
Table 2

Descriptive Statistics and Bivariate Correlations for MIDUS 1 -Middle-Aged Adults

\begin{tabular}{|c|c|c|c|c|c|c|c|c|c|c|}
\hline & $M, S D$, or $\%$ & $\alpha$ & 1 & 2 & 3 & 4 & 5 & 6 & 7 & 8 \\
\hline 1. Age & $48.65,5.47$ & - & - & & & & & & & \\
\hline 2. Gender & $48.20 \%$ Female & - & .08 & - & & & & & & \\
\hline \multicolumn{11}{|l|}{$(0=$ Female $)$} \\
\hline 3. Education & $7.42,2.41$ & - & -.04 & .19 & - & & & & & \\
\hline 4. NSIs & $2.03,0.40$ & .82 & -.16 & -.09 & .03 & - & & & & \\
\hline 5. Agreeableness & $3.47,0.47$ & .80 & .06 & -.25 & -.11 & -.11 & - & & & \\
\hline 6. Openness & $3.00,0.50$ & .76 & .00 & .08 & .17 & -.04 & .30 & - & & \\
\hline 7. Neuroticism & $2.16,0.64$ & .75 & -.15 & -.14 & -.13 & .30 & -.04 & -.21 & - & \\
\hline 8. Extraversion & $3.18,0.54$ & .76 & .07 & -.07 & -.11 & -.10 & .51 & .46 & -.14 & - \\
\hline 9. Conscientiousness & $3.49,0.40$ & .55 & .02 & -.11 & .10 & -.14 & .29 & .35 & -.19 & .28 \\
\hline
\end{tabular}

Note. Significant statistics $(p<.05)$ are bolded. Middle-aged adults' $(n=909)$ ages ranged from $40-59,95.70 \%$ were married, and 95.60\% identified as White/Caucasian. 
Table 3

Descriptive Statistics and Bivariate Correlations for MIDUS 1 - Older Adults

\begin{tabular}{|c|c|c|c|c|c|c|c|c|c|c|}
\hline & $M, S D$, or $\%$ & $\alpha$ & 1 & 2 & 3 & 4 & 5 & 6 & 7 & 8 \\
\hline 1. Age & $64.28,3.71$ & - & - & & & & & & & \\
\hline 2. Gender & $40.50 \%$ Female & - & .16 & - & & & & & & \\
\hline \multicolumn{11}{|l|}{$(0=$ Female $)$} \\
\hline 3. Education & $7.15,2.49$ & - & .02 & .19 & - & & & & & \\
\hline 4. NSIs & $1.91,0.36$ & .80 & -.13 & .04 & .04 & - & & & & \\
\hline 5. Agreeableness & $3.50,0.54$ & .85 & -.02 & -.23 & -.16 & -.06 & - & & & \\
\hline 6. Openness & $2.96,0.52$ & .77 & .07 & .07 & .16 & .04 & .39 & - & & \\
\hline 7. Neuroticism & $2.07,0.61$ & .70 & .00 & -.15 & -.15 & .24 & -.02 & -.18 & - & \\
\hline 8. Extraversion & $3.23,0.58$ & .79 & .12 & .03 & -.15 & -.06 & .63 & .59 & -.18 & - \\
\hline 9. Conscientiousness & $3.47,0.44$ & .53 & -.02 & -.04 & -.01 & -.13 & .13 & .22 & -.24 & .16 \\
\hline
\end{tabular}

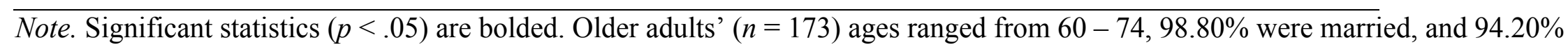
identified as White/Caucasian. 
Table 4

Descriptive Statistics and Bivariate Correlations for MIDUS 2 - Younger Adults

\begin{tabular}{|c|c|c|c|c|c|c|c|c|c|c|}
\hline & $M, S D$, or $\%$ & $\alpha$ & 1 & 2 & 3 & 4 & 5 & 6 & 7 & 8 \\
\hline 1. Age & $42.63,3.89$ & - & - & & & & & & & \\
\hline 2. Gender & $55.40 \%$ Female & - & .01 & - & & & & & & \\
\hline \multicolumn{11}{|l|}{$(0=$ Female $)$} \\
\hline 3. Education & $7.87,2.41$ & - & -.08 & -.02 & - & & & & & \\
\hline 4. NSIs & $2.02,0.42$ & .83 & -.06 & -.06 & -.06 & - & & & & \\
\hline 5. Agreeableness & $3.33,0.54$ & .83 & .08 & -.33 & -.01 & -.04 & - & & & \\
\hline 6. Openness & $2.83,0.56$ & .80 & -.04 & .12 & .15 & -.08 & .27 & - & & \\
\hline 7. Neuroticism & $2.15,0.64$ & .75 & -.04 & -.12 & -.14 & .28 & -.15 & -.24 & - & \\
\hline 8. Extraversion & $3.05,0.58$ & .76 & -.01 & -.09 & .01 & -.02 & .44 & .48 & -.19 & - \\
\hline 9. Conscientiousness & $3.50,0.42$ & .54 & .01 & -.17 & .01 & -.20 & .20 & .09 & -.12 & .12 \\
\hline
\end{tabular}

Note. Significant statistics $(p<.05)$ are bolded. Younger adults' $(n=448)$ ages ranged from $34-49,94.40 \%$ were married, and 94.00\% identified as White/Caucasian. 
Table 5

Descriptive Statistics and Bivariate Correlations for MIDUS 2 - Middle-Aged Adults

\begin{tabular}{|c|c|c|c|c|c|c|c|c|c|c|}
\hline & $M, S D$, or $\%$ & $\alpha$ & 1 & 2 & 3 & 4 & 5 & 6 & 7 & 8 \\
\hline 1. Age & $57.50,5.48$ & - & - & & & & & & & \\
\hline 2. Gender & $48.20 \%$ Female & - & .08 & - & & & & & & \\
\hline \multicolumn{11}{|l|}{$(0=$ Female $)$} \\
\hline 3. Education & $7.51,2.51$ & - & -.08 & .19 & - & & & & & \\
\hline 4. NSIs & $1.95,0.41$ & .84 & -.20 & -.10 & .01 & - & & & & \\
\hline 5. Agreeableness & $3.47,0.45$ & .76 & .06 & -.27 & -.18 & -.14 & - & & & \\
\hline 6. Openness & $2.94,0.49$ & .74 & .02 & .06 & .16 & -.07 & .27 & - & & \\
\hline 7. Neuroticism & $1.98,0.59$ & .73 & -.13 & -.13 & -.13 & .28 & -.14 & -.23 & - & \\
\hline 8. Extraversion & $3.13,0.54$ & .74 & .07 & -.05 & -.10 & -.11 & .48 & .48 & -.22 & - \\
\hline 9. Conscientiousness & $3.54,0.39$ & .55 & .02 & -.07 & .07 & -.14 & .24 & .33 & -.24 & .27 \\
\hline
\end{tabular}

Note. Significant statistics $(p<.05)$ are bolded. Middle-aged adults' $(n=909)$ ages ranged from $48-69,97.60 \%$ were married, and $94.60 \%$ identified as White/Caucasian. 
Table 6

Descriptive Statistics and Bivariate Correlations for MIDUS 2 - Older Adults

\begin{tabular}{|c|c|c|c|c|c|c|c|c|c|c|}
\hline & $M, S D$, or $\%$ & $\alpha$ & 1 & 2 & 3 & 4 & 5 & 6 & 7 & 8 \\
\hline 1. Age & $73.08,3.76$ & - & - & & & & & & & \\
\hline 2. Gender & $40.50 \%$ Female & - & .16 & - & & & & & & \\
\hline \multicolumn{11}{|l|}{$(0=$ Female $)$} \\
\hline 3. Education & $7.19,2.56$ & - & .01 & .17 & - & & & & & \\
\hline 4. NSIs & $1.84,0.38$ & .80 & -.13 & .05 & -.01 & - & & & & \\
\hline 5. Agreeableness & $3.49,0.50$ & .83 & -.07 & -.33 & -.19 & -.21 & - & & & \\
\hline 6. Openness & $2.81,0.51$ & .75 & .03 & .00 & .16 & -.09 & .37 & - & & \\
\hline 7. Neuroticism & $1.90,0.57$ & .72 & -.11 & -.21 & -.23 & .29 & .03 & -.14 & - & \\
\hline 8. Extraversion & $3.13,0.57$ & .76 & -.08 & .01 & -.09 & -.02 & .48 & .56 & -.14 & - \\
\hline 9. Conscientiousness & $3.46,0.44$ & .59 & -.10 & -.12 & -.07 & -.25 & .36 & .21 & -.08 & .24 \\
\hline
\end{tabular}

Note. Significant statistics $(p<.05)$ are bolded. Older adults' $(n=173)$ ages ranged from $68-83,97.10 \%$ were married, and $94.80 \%$ identified as White/Caucasian. 
Table 7

Descriptive Statistics and Bivariate Correlations for MIDUS 3 - Younger Adults

\begin{tabular}{|c|c|c|c|c|c|c|c|c|c|c|}
\hline & $M, S D$, or $\%$ & $\alpha$ & 1 & 2 & 3 & 4 & 5 & 6 & 7 & 8 \\
\hline 1. Age & $51.72,3.89$ & - & - & & & & & & & \\
\hline 2. Gender & $55.40 \%$ Female & - & .02 & - & & & & & & \\
\hline \multicolumn{11}{|l|}{$(0=$ Female $)$} \\
\hline 3. Education & $7.93,2.37$ & - & -.07 & .00 & - & & & & & \\
\hline 4. NSIs & $1.98,0.43$ & .82 & -.15 & -.01 & -.01 & - & & & & \\
\hline 5. Agreeableness & $3.35,0.52$ & .79 & .11 & -.27 & -.03 & -.03 & - & & & \\
\hline 6. Openness & $2.83,0.56$ & .80 & .04 & .11 & .16 & -.01 & .38 & - & & \\
\hline 7. Neuroticism & $2.14,0.64$ & .72 & -.12 & -.12 & -.15 & .26 & -.13 & -.18 & - & \\
\hline 8. Extraversion & $3.04,0.56$ & .73 & .00 & -.04 & -.03 & -.06 & .42 & .55 & -.13 & - \\
\hline 9. Conscientiousness & $3.51,0.46$ & .61 & .04 & -.16 & .03 & -.21 & .24 & .16 & -.19 & .15 \\
\hline
\end{tabular}

Note. Significant statistics $(p<.05)$ are bolded. Younger adults' $(n=448)$ ages ranged from $42-58,94.40 \%$ were married, and 91.30\% identified as White/Caucasian. 
Table 8

Descriptive Statistics and Bivariate Correlations for MIDUS 3 - Middle-Aged Adults

\begin{tabular}{|c|c|c|c|c|c|c|c|c|c|c|}
\hline & $M, S D$, or $\%$ & $\alpha$ & 1 & 2 & 3 & 4 & 5 & 6 & 7 & 8 \\
\hline 1. Age & $66.63,5.50$ & - & - & & & & & & & \\
\hline 2. Gender & $48.20 \%$ Female & - & .08 & - & & & & & & \\
\hline \multicolumn{11}{|l|}{$(0=$ Female $)$} \\
\hline 3. Education & $7.54,2.48$ & - & -.07 & .15 & - & & & & & \\
\hline 4. NSIs & $1.85,0.44$ & .85 & -.10 & -.08 & -.04 & - & & & & \\
\hline 5. Agreeableness & $3.46,0.47$ & .76 & -.02 & -.33 & -.10 & -.11 & - & & & \\
\hline 6. Openness & $2.91,0.51$ & .76 & -.03 & .03 & .18 & -.10 & .33 & - & & \\
\hline 7. Neuroticism & $2.00,0.60$ & .71 & -.08 & -.14 & -.14 & .32 & -.09 & -.20 & - & \\
\hline 8. Extraversion & $3.12,0.55$ & .73 & .04 & -.04 & -.05 & -.12 & .48 & .48 & -.18 & - \\
\hline 9. Conscientiousness & $3.51,0.41$ & .51 & -.07 & -.08 & .10 & -.16 & .26 & .32 & -.21 & .25 \\
\hline
\end{tabular}

Note. Significant statistics $(p<.05)$ are bolded. Middle-aged adults' ages $(n=909)$ ranged from $57-78,98.00 \%$ were married, and 91.70\% identified as White/Caucasian. 
Table 9

Descriptive Statistics and Bivariate Correlations for MIDUS 3 - Older Adults

\begin{tabular}{|c|c|c|c|c|c|c|c|c|c|c|}
\hline & $M, S D$, or $\%$ & $\alpha$ & 1 & 2 & 3 & 4 & 5 & 6 & 7 & 8 \\
\hline 1. Age & $82.16,3.79$ & - & - & & & & & & & \\
\hline 2. Gender & $40.50 \%$ Female & - & .16 & - & & & & & & \\
\hline \multicolumn{11}{|l|}{$(0=$ Female $)$} \\
\hline 3. Education & $7.12,2.62$ & - & .00 & .18 & - & & & & & \\
\hline 4. NSIs & $1.76,0.41$ & .82 & -.10 & .07 & .07 & - & & & & \\
\hline 5. Agreeableness & $3.40,0.48$ & .73 & -.04 & -.32 & -.19 & -.14 & - & & & \\
\hline 6. Openness & $2.76,0.53$ & .77 & .00 & -.07 & .14 & .16 & .39 & - & & \\
\hline 7. Neuroticism & $1.98,0.57$ & .67 & -.04 & -.17 & -.21 & .25 & .07 & -.07 & - & \\
\hline 8. Extraversion & $2.99,0.63$ & .80 & -.03 & -.09 & -.14 & .07 & .57 & .62 & -.03 & - \\
\hline 9. Conscientiousness & $3.34,0.49$ & .51 & -.04 & -.01 & .01 & -.09 & .26 & .40 & -.17 & .34 \\
\hline
\end{tabular}

Note. Significant statistics $(p<.05)$ are bolded. Older adults' $(n=173)$ ages ranged from $73-92,96.50 \%$ were married, and 91.20\% identified as White/Caucasian. 
Table 10

Descriptive Statistics and Bivariate Correlations for MIDUS 1

\begin{tabular}{|c|c|c|c|c|c|c|c|c|c|c|}
\hline & $M, S D$, or $\%$ & $\alpha$ & 1 & 2 & 3 & 4 & 5 & 6 & 7 & 8 \\
\hline 1. Age & $46.03,10.50$ & - & - & & & & & & & \\
\hline 2. Gender & $49.40 \%$ Female & - & .11 & - & & & & & & \\
\hline \multicolumn{11}{|l|}{$(0=$ Female $)$} \\
\hline 3. Education & $7.43,2.40$ & - & -.05 & .13 & - & & & & & \\
\hline 4. NSIs & $2.02,0.39$ & .81 & -.13 & -.08 & .01 & - & & & & \\
\hline 5. Agreeableness & $3.45,0.49$ & .80 & .08 & -.26 & -.09 & -.08 & - & & & \\
\hline 6. Openness & $2.98,0.50$ & .76 & .00 & .09 & .15 & -.02 & .32 & - & & \\
\hline 7. Neuroticism & $2.19,0.65$ & .74 & -.14 & -.14 & -.14 & .30 & -.05 & -.17 & - & \\
\hline 8. Extraversion & $3.19,0.55$ & .77 & .03 & -.53 & -.08 & -.09 & .50 & .47 & -.13 & - \\
\hline 9. Conscientiousness & $3.48,0.41$ & .54 & .03 & -.14 & .06 & -.13 & .25 & .24 & -.17 & .20 \\
\hline
\end{tabular}

Note. Significant statistics $(p<.05)$ are bolded. Participants' $(n=1,530)$ ages ranged from $25-74,94.70 \%$ were married, and $95.30 \%$ identified as White/Caucasian. 
Table 11

Descriptive Statistics and Bivariate Correlations for MIDUS 2

\begin{tabular}{|c|c|c|c|c|c|c|c|c|c|c|}
\hline & $M, S D$, or $\%$ & $\alpha$ & 1 & 2 & 3 & 4 & 5 & 6 & 7 & 8 \\
\hline 1. Age & $54.91,10.45$ & - & - & & & & & & & \\
\hline 2. Gender & $49.40 \%$ Female & - & .11 & - & & & & & & \\
\hline \multicolumn{11}{|l|}{$(0=$ Female $)$} \\
\hline 3. Education & $7.58,2.49$ & - & -.11 & .12 & - & & & & & \\
\hline 4. NSIs & $1.95,0.41$ & .83 & -.18 & -.08 & .00 & - & & & & \\
\hline 5. Agreeableness & $3.43,0.49$ & .80 & .13 & -.28 & -.14 & -.12 & - & & & \\
\hline 6. Openness & $2.89,0.52$ & .76 & .03 & .08 & .16 & -.08 & .29 & - & & \\
\hline 7. Neuroticism & $2.02,0.61$ & .74 & -.17 & -.15 & -.13 & .29 & -.14 & -.23 & - & \\
\hline 8. Extraversion & $3.10,0.56$ & .75 & .07 & -.05 & -.07 & -.08 & .47 & .49 & -.21 & - \\
\hline 9. Conscientiousness & $3.52,0.41$ & .55 & .00 & -.11 & .03 & -.17 & .24 & .24 & -.18 & .22 \\
\hline
\end{tabular}

Note. Significant statistics $(p<.05)$ are bolded. Participants' $(n=1,530)$ ages ranged from $34-83,96.60 \%$ were married, and $94.40 \%$ identified as White/Caucasian. 
Table 12

Descriptive Statistics and Bivariate Correlations for MIDUS 3

\begin{tabular}{|c|c|c|c|c|c|c|c|c|c|c|}
\hline & $M, S D$, or $\%$ & $\alpha$ & 1 & 2 & 3 & 4 & 5 & 6 & 7 & 8 \\
\hline 1. Age & $64.02,10.47$ & - & - & & & & & & & \\
\hline 2. Gender & $49.40 \%$ Female & - & .11 & - & & & & & & \\
\hline \multicolumn{11}{|l|}{$(0=$ Female $)$} \\
\hline 3. Education & $7.61,2.48$ & - & -.12 & .10 & - & & & & & \\
\hline 4. NSIs & $1.88,0.44$ & .84 & -.19 & -.06 & .00 & - & & & & \\
\hline 5. Agreeableness & $3.42,0.49$ & .77 & .06 & -.30 & -.10 & -.10 & - & & & \\
\hline 6. Openness & $2.87,0.53$ & .77 & -.01 & .04 & .17 & -.04 & .36 & - & & \\
\hline 7. Neuroticism & $2.04,0.61$ & .71 & -.12 & -.15 & -.14 & .31 & -.09 & -.19 & - & \\
\hline 8. Extraversion & $3.08,0.57$ & .74 & .01 & -.05 & -.06 & -.08 & .47 & .52 & -.15 & - \\
\hline 9. Conscientiousness & $3.49,0.44$ & .55 & -.09 & -.10 & .08 & -.15 & .25 & .28 & -.19 & .24 \\
\hline
\end{tabular}

Note. Significant statistics $(p<.05)$ are bolded. Participants' $(n=1,530)$ ages ranged from $42-92,96.80 \%$ were married, and $91.00 \%$ identified as White/Caucasian. 
Table 13

The Unconstrained Model Compared to the Constrained Model for the Multigroup Latent Growth Curve of NSIS

$\chi^{2} \quad$ CFI $\quad \Delta \chi^{2} \quad \Delta$ CFI

NSIs

Unconstrained Model $\quad \chi^{2}(\mathbf{1 1})=\mathbf{1 7 . 2 6} \quad .996$

$\begin{array}{lllll}\text { Constrained Model } & \chi^{2}(\mathbf{1 7})=\mathbf{7 1 . 3 3} & .964 & \chi^{2}(\mathbf{6})=\mathbf{5 4 . 0 7} & \mathbf{. 0 3 2}\end{array}$

Note. Significant statistics $(p<.05)$ are bolded. The unconstrained model is where the parameters were freely estimated across the three age groups. The constrained model is where the parameters were constrained to be equal across the three age groups. A change of .01 or greater for the CFI difference statistic was used to determine significance (Little, 2013). Both the chi-square and the CFI difference statistics were computed because the chi-square statistic is commonly used, however, it is sensitive to large sample sizes. The CFI is more robust to larger sample sizes (Little, 2013). 
Table 14

Research Question 3 - Multigroup Analyses for the Latent Growth Curve of NSIS

\begin{tabular}{|c|c|c|c|c|c|c|c|c|}
\hline & \multirow[t]{2}{*}{ Model Fit } & & \multicolumn{2}{|c|}{ Intercept } & \multicolumn{2}{|c|}{ Slope } & \multicolumn{2}{|c|}{ Covariance } \\
\hline & & & $b$ & S.E. & $b$ & S.E. & $b$ & S.E. \\
\hline \multirow[t]{4}{*}{ NSIs } & $\chi^{2}(11)=17.26$ & Younger Adults & $2.057 *^{b}$ & 0.018 & $-0.004 * a, b$ & 0.001 & 0.000 & 0.000 \\
\hline & $\mathrm{CMIN} / \mathrm{DF}=1.57$ & Middle-Aged Adults & $2.033 *^{\mathrm{c}}$ & 0.013 & $-0.010 *$ & 0.001 & 0.000 & 0.000 \\
\hline & $\mathrm{CFI}=.996$ & Older Adults & $1.915^{*}$ & 0.028 & $-0.008 *$ & 0.002 & -0.001 & 0.001 \\
\hline & $\mathrm{RMSEA}=.01$ & & & & & & & \\
\hline
\end{tabular}

Note. Significant statistics $(p<.05)$ are bolded. An asterisk indicates that there is significant variability surrounding the estimate. A blue value indicates that the estimate is significantly different than zero. ${ }^{a}$ indicates that the estimate is significantly different between younger and middle-aged adults. ${ }^{\mathrm{b}}$ indicates that the estimate is significantly different between younger and older adults. ${ }^{\mathrm{c}}$ indicates that the estimate is significantly different between middle-aged and older adults. 
Table 15

Research Question 3-Multigroup Analyses for the Latent Growth Curve of NSIS

\begin{tabular}{|c|c|c|c|c|c|c|c|c|c|c|c|c|}
\hline & \multicolumn{4}{|c|}{ Younger Adults } & \multicolumn{4}{|c|}{ Middle-Aged Adults } & \multicolumn{4}{|c|}{ Older Adults } \\
\hline & \multicolumn{2}{|c|}{ Intercept } & \multicolumn{2}{|c|}{ Slope } & \multicolumn{2}{|c|}{ Intercept } & \multicolumn{2}{|c|}{ Slope } & \multicolumn{2}{|c|}{ Intercept } & \multicolumn{2}{|c|}{ Slope } \\
\hline & $b$ & S.E. & $b$ & S.E. & $b$ & S.E. & $b$ & S.E. & $b$ & S.E. & $b$ & S.E. \\
\hline Estimate & $1.972 *$ & 0.148 & 0.030 & 0.009 & $2.535 *$ & 0.108 & $-0.016^{*}$ & 0.007 & $2.880 *$ & 0.464 & $-0.002 *$ & 0.031 \\
\hline Age & 0.003 & 0.004 & -0.001 & 0.000 & -0.009 & 0.002 & 0.000 & 0.000 & -0.015 & 0.007 & 0.000 & 0.000 \\
\hline Gender & -0.087 & 0.034 & 0.002 & 0.002 & -0.086 & 0.024 & 0.000 & 0.001 & 0.052 & 0.054 & 0.001 & 0.004 \\
\hline Education & -0.013 & 0.018 & 0.001 & 0.001 & 0.028 & 0.012 & -0.001 & 0.001 & 0.007 & 0.026 & 0.001 & 0.002 \\
\hline Agreeableness & -0.002 & 0.019 & 0.000 & 0.001 & -0.041 & 0.015 & 0.000 & 0.001 & -0.024 & 0.031 & 0.000 & 0.002 \\
\hline Openness & 0.023 & 0.019 & -0.001 & 0.001 & 0.037 & 0.014 & 0.000 & 0.001 & 0.042 & 0.032 & -0.001 & 0.002 \\
\hline Neuroticism & 0.102 & 0.017 & -0.004 & 0.001 & 0.108 & 0.013 & -0.001 & 0.001 & 0.099 & 0.030 & -0.001 & 0.002 \\
\hline Extraversion & -0.017 & 0.020 & 0.000 & 0.001 & -0.004 & 0.015 & 0.000 & 0.001 & -0.010 & 0.038 & 0.002 & 0.003 \\
\hline Conscientiousness & -0.044 & 0.017 & -0.002 & 0.001 & -0.042 & 0.014 & 0.000 & 0.001 & -0.036 & 0.026 & 0.000 & 0.002 \\
\hline Covariance & -0.001 & 0.000 & & & 0.000 & 0.000 & & & -0.001 & 0.001 & & \\
\hline$R^{2}$ & .189 & & .416 & & .216 & & .054 & & .170 & & .026 & \\
\hline \multicolumn{5}{|c|}{$\chi^{2}(29)=157.00$} & \multicolumn{4}{|c|}{$\chi^{2}(29)=272.83$} & \multicolumn{4}{|c|}{$\chi^{2}(29)=73.91$} \\
\hline Model Fit & \multicolumn{4}{|c|}{$\mathrm{CMIN} / \mathrm{DF}=5.41$} & \multicolumn{4}{|c|}{$\mathrm{CMIN} / \mathrm{DF}=9.40$} & \multicolumn{4}{|c|}{$\mathrm{CMIN} / \mathrm{DF}=2.54$} \\
\hline
\end{tabular}




$$
\begin{array}{llr}
\mathrm{CFI}=.848 & \mathrm{CFI}=.879 & \mathrm{CFI}=.877 \\
\mathrm{RMSEA}=.09 & \mathrm{RMSEA}=.09 & \mathrm{RMSEA}=.09
\end{array}
$$

Note. Significant statistics $(p<.05)$ are bolded. $R^{2}=$ variance accounted for. An asterisk indicates that there is significant variability surrounding the estimate. A blue value indicates that the estimate is significantly different than zero. Type I errors were accounted for utilizing the false discovery rate adjustment. A green value indicates that the estimate was no longer statistically significant after the false discovery rate adjustments. To aid in the interpretation of the estimates, education, agreeableness, openness, neuroticism, extraversion, and conscientiousness were standardized. As such, a unit increase or decrease in these variables represent a standard deviation increase or decrease, respectively. Age was not manipulated so that a unit increase or decrease represents an increase or decrease of a year, respectively. 
Table 16

Research Question 4 - The Latent Growth Curves of Extraversion

\begin{tabular}{|c|c|c|c|c|c|c|c|}
\hline & \multirow[t]{2}{*}{ Model Fit } & \multicolumn{2}{|c|}{ Intercept } & \multicolumn{2}{|c|}{ Slope } & \multicolumn{2}{|c|}{ Covariance } \\
\hline & & $b$ & S.E. & $b$ & S.E. & $b$ & S.E. \\
\hline & $\chi^{2}(3)=11.00$ & & & & & & \\
\hline \multirow[t]{3}{*}{ Extraversion } & $\mathrm{CMIN} / \mathrm{DF}=3.66$ & $3.183 *$ & 0.014 & $-0.006 *$ & 0.001 & 0.000 & 0.000 \\
\hline & $\mathrm{CFI}=.996$ & & & & & & \\
\hline & RMSEA $=.04$ & & & & & & \\
\hline
\end{tabular}

Note. Significant statistics $(p<.05)$ are bolded. An asterisk indicates that there is significant variability surrounding the estimate. A blue value indicates that the estimate is significantly different than zero. 
Table 17

Research Question 5 - Demographic Variables and NSIs Predicting the Latent Growth Curve of Extraversion

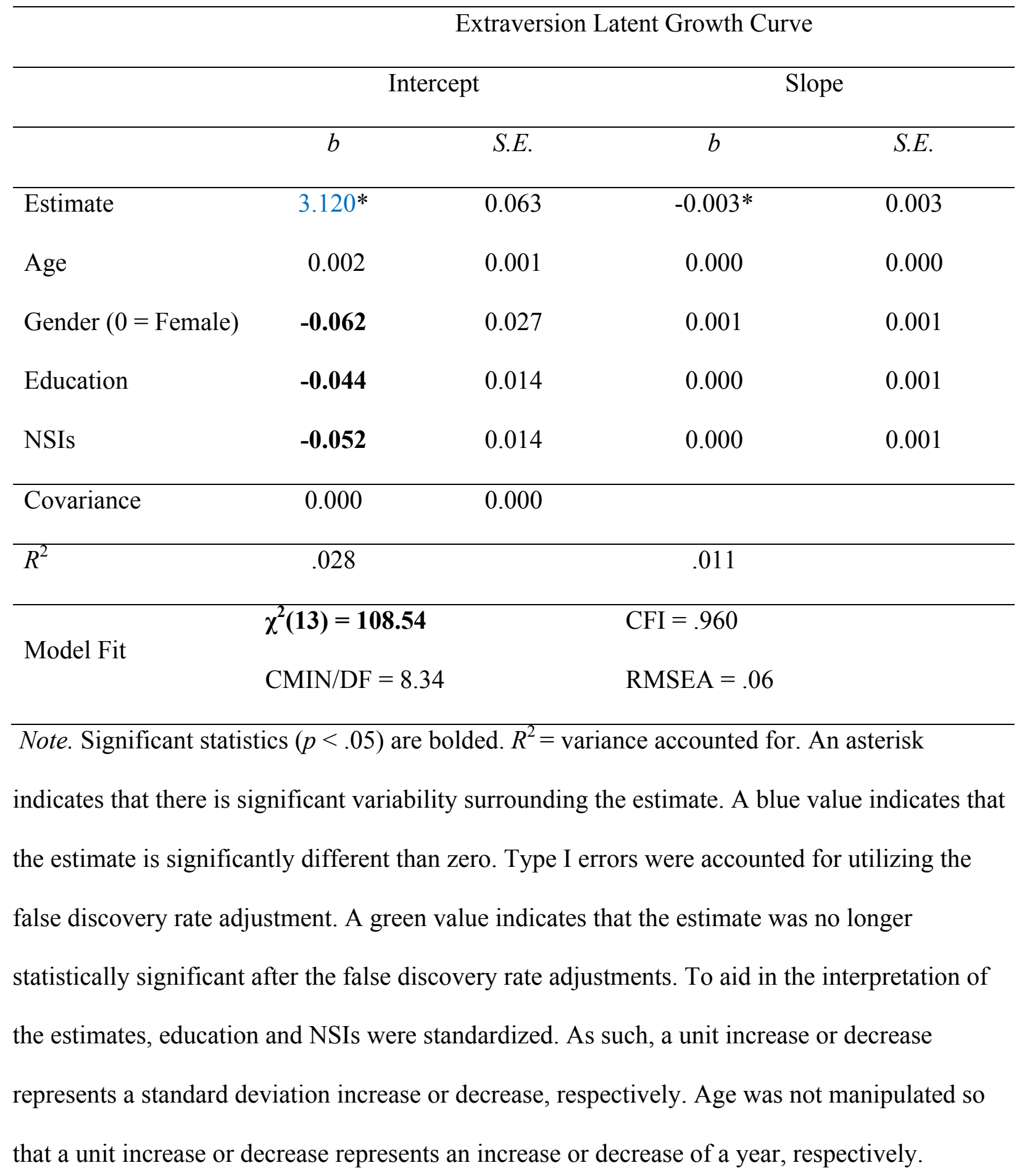


Table 18

The Unconstrained Compared to the Constrained Model for the Multigroup Latent Growth Curve of the Big Five Personality Traits

\begin{tabular}{|c|c|c|c|c|}
\hline & $\chi^{2}$ & CFI & $\Delta \chi^{2}$ & $\Delta \mathrm{CFI}$ \\
\hline \multicolumn{5}{|l|}{ Agreeableness } \\
\hline Unconstrained Model & $\chi^{2}(11)=30.41$ & .990 & & \\
\hline Constrained Model & $\chi^{2}(17)=56.84$ & .979 & $\chi^{2}(6)=26.43$ & .011 \\
\hline \multicolumn{5}{|l|}{ Openness } \\
\hline Unconstrained Model & $\chi^{2}(11)=37.82$ & .988 & & \\
\hline Constrained Model & $\chi^{2}(17)=64.88$ & .978 & $\chi^{2}(6)=27.06$ & .010 \\
\hline \multicolumn{5}{|l|}{ Neuroticism } \\
\hline Unconstrained Model & $\chi^{2}(11)=89.25$ & .959 & & \\
\hline Constrained Model & $\chi^{2}(17)=117.99$ & .947 & $\chi^{2}(6)=28.74$ & .012 \\
\hline \multicolumn{5}{|l|}{ Extraversion } \\
\hline Unconstrained Model & $\chi^{2}(11)=23.79$ & .994 & & \\
\hline Constrained Model & $\chi^{2}(17)=51.79$ & .985 & $\chi^{2}(6)=28.00$ & .009 \\
\hline \multicolumn{5}{|l|}{ Conscientiousness } \\
\hline Unconstrained Model & $\chi^{2}(11)=52.34$ & .974 & & \\
\hline Constrained Model & $\chi^{2}(17)=87.74$ & .956 & $\chi^{2}(6)=35.40$ & .018 \\
\hline
\end{tabular}


Note. Significant statistics $(p<.05)$ are bolded. The unconstrained model is where the parameters were freely estimated across the three age groups. The constrained model is where the parameters were constrained to be equal across the three age groups. A change of .01 or greater for the CFI difference statistic was used to determine significance (Little, 2013). Both the chi-square and the CFI difference statistics were computed because the chi-square statistic is commonly used, however, it is sensitive to large sample sizes. The CFI is more robust to larger sample sizes (Little, 2013). 
Table 19

Research Question 6-Multigroup Analyses for the Latent Growth Curves of the Personality Traits

\begin{tabular}{|c|c|c|c|c|c|c|c|c|}
\hline & \multirow[t]{2}{*}{ Model Fit } & & \multicolumn{2}{|c|}{ Intercept } & \multicolumn{2}{|c|}{ Slope } & \multicolumn{2}{|c|}{ Covariance } \\
\hline & & & $b$ & S.E. & $b$ & S.E. & $b$ & S.E. \\
\hline \multirow[t]{4}{*}{ Agreeableness } & $\chi^{2}(11)=30.41$ & Younger Adults & $3.392 * a, b$ & 0.023 & $-0.003 *$ & 0.001 & 0.000 & 0.000 \\
\hline & $\mathrm{CMIN} / \mathrm{DF}=2.76$ & Middle-Aged Adults & $3.477^{*}$ & 0.015 & $-0.001^{c}$ & 0.001 & -0.001 & 0.000 \\
\hline & $\mathrm{CFI}=.990$ & Older Adults & $3.513 *$ & 0.040 & -0.005 & 0.002 & -0.002 & 0.001 \\
\hline & $\mathrm{RMSEA}=.03$ & & & & & & & \\
\hline \multirow[t]{4}{*}{ Openness } & $\chi^{2}(11)=37.82$ & Younger Adults & $2.937 *^{\mathrm{a}}$ & 0.024 & $-0.007 * b$ & 0.001 & 0.000 & 0.001 \\
\hline & $\mathrm{CMIN} / \mathrm{DF}=3.43$ & Middle-Aged Adults & $3.002 *$ & 0.016 & $-0.005^{\mathrm{c}}$ & 0.001 & -0.001 & 0.000 \\
\hline & $\mathrm{CFI}=.988$ & Older Adults & $2.951 *$ & 0.039 & $-0.012 *$ & 0.002 & -0.002 & 0.001 \\
\hline & $\mathrm{RMSEA}=.04$ & & & & & & & \\
\hline \multirow[t]{3}{*}{ Neuroticism } & $\chi^{2}(11)=89.25$ & Younger Adults & $2.274^{* a, b}$ & 0.031 & $-0.009 *$ & 0.002 & -0.004 & 0.001 \\
\hline & $\mathrm{CMIN} / \mathrm{DF}=8.11$ & Middle-Aged Adults & $2.131 *$ & 0.021 & $-0.009 *$ & 0.001 & -0.004 & 0.001 \\
\hline & $\mathrm{CFI}=.959$ & Older Adults & $2.037^{*}$ & 0.046 & -0.005 & 0.002 & -0.003 & 0.002 \\
\hline
\end{tabular}




\begin{tabular}{|c|c|c|c|c|c|c|c|c|}
\hline \multirow[t]{3}{*}{ Conscientiousness } & $\chi^{2}(11)=52.34$ & Younger Adults & $3.459 * \mathrm{a}$ & 0.020 & $0.003^{* a, b}$ & 0.001 & 0.000 & 0.000 \\
\hline & $\mathrm{CMIN} / \mathrm{DF}=4.75$ & Middle-Aged Adults & $3.510^{*}$ & 0.013 & $0.001^{\mathrm{c}}$ & 0.001 & 0.000 & 0.000 \\
\hline & $\mathrm{CFI}=.974$ & Older Adults & $3.495 *$ & 0.034 & $-0.008 *$ & 0.002 & -0.002 & 0.001 \\
\hline
\end{tabular}

RMSEA $=.05$

Note. Significant statistics $(p<.05)$ are bolded. An asterisk indicates that there is significant variability surrounding the estimate. A blue value indicates that the estimate is significantly different than zero. ${ }^{a}$ indicates that the estimate is significantly different between younger and middle-aged adults. ${ }^{b}$ indicates that the estimate is significantly different between younger and older adults. ${ }^{c}$ indicates that the estimate is significantly different between middle-aged and older adults. 
Table 20

Research Question 6-Multigroup Analyses for the Latent Growth Curve of Agreeableness

\begin{tabular}{|c|c|c|c|c|c|c|c|c|c|c|c|c|}
\hline & \multicolumn{4}{|c|}{ Younger Adults } & \multicolumn{4}{|c|}{ Middle-Aged Adults } & \multicolumn{4}{|c|}{ Older Adults } \\
\hline & \multicolumn{2}{|c|}{ Intercept } & \multicolumn{2}{|c|}{ Slope } & \multicolumn{2}{|c|}{ Intercept } & \multicolumn{2}{|c|}{ Slope } & \multicolumn{2}{|c|}{ Intercept } & \multicolumn{2}{|c|}{ Slope } \\
\hline & $b$ & S.E. & $b$ & S.E. & $b$ & S.E. & $b$ & S.E. & $b$ & S.E. & $b$ & S.E. \\
\hline Estimate & $3.417 *$ & 0.191 & -0.025 & 0.010 & $3.482 *$ & 0.113 & -0.001 & 0.001 & $3.660 *$ & 0.561 & -0.005 & 0.002 \\
\hline Age & 0.004 & 0.006 & 0.001 & 0.000 & 0.003 & 0.002 & - & - & 0.000 & 0.009 & - & - \\
\hline Gender $(0=$ Female $)$ & -0.344 & 0.044 & 0.003 & 0.002 & -0.268 & 0.025 & - & - & -0.285 & 0.065 & - & - \\
\hline Education & -0.013 & 0.023 & 0.000 & 0.001 & -0.036 & 0.012 & - & - & -0.068 & 0.031 & - & - \\
\hline NSIs & -0.030 & 0.022 & 0.003 & 0.001 & -0.053 & 0.012 & - & - & -0.034 & 0.035 & - & - \\
\hline Covariance & 0.001 & 0.001 & & & -0.001 & 0.000 & & & -0.002 & 0.001 & & \\
\hline \multirow[t]{3}{*}{$R^{2}$} & .188 & & .080 & & .145 & & .000 & & .114 & & .000 & \\
\hline & \multicolumn{4}{|c|}{$\chi^{2}(13)=20.64$} & \multicolumn{4}{|c|}{$\chi^{2}(17)=105.62$} & \multicolumn{4}{|c|}{$\chi^{2}(17)=20.72$} \\
\hline & \multicolumn{4}{|c|}{$\mathrm{CMIN} / \mathrm{DF}=1.58$} & \multicolumn{4}{|c|}{$\mathrm{CMIN} / \mathrm{DF}=6.21$} & \multicolumn{4}{|c|}{$\mathrm{CMIN} / \mathrm{DF}=1.21$} \\
\hline \multirow[t]{2}{*}{ Model Fit } & \multicolumn{4}{|c|}{$\mathrm{CFI}=.987$} & \multicolumn{4}{|c|}{$\mathrm{CFI}=.931$} & \multicolumn{4}{|c|}{$\mathrm{CFI}=.987$} \\
\hline & \multicolumn{4}{|c|}{$\mathrm{RMSEA}=.03$} & \multicolumn{4}{|c|}{ RMSEA $=.07$} & \multicolumn{4}{|c|}{$\mathrm{RMSEA}=.03$} \\
\hline
\end{tabular}

Note. Significant statistics $(p<.05)$ are bolded. $R^{2}=$ variance accounted for. An asterisk indicates that there is significant variability surrounding the estimate. A blue value indicates that the estimate is significantly different than zero. Type I errors were accounted for 
utilizing the false discovery rate adjustment. A green value indicates that the estimate was no longer statistically significant after the false discovery rate adjustments. To aid in the interpretation of the estimates, education and NSIs were standardized. As such, a unit increase or decrease in these variables represent a standard deviation increase or decrease, respectively. Age was not manipulated so that a unit increase or decrease represents an increase or decrease of a year, respectively. 
Table 21

Research Question 6-Multigroup Analyses for the Latent Growth Curve of Openness

\begin{tabular}{|c|c|c|c|c|c|c|c|c|c|c|c|c|}
\hline & \multicolumn{4}{|c|}{ Younger Adults } & \multicolumn{4}{|c|}{ Middle-Aged Adults } & \multicolumn{4}{|c|}{ Older Adults } \\
\hline & \multicolumn{2}{|c|}{ Intercept } & \multicolumn{2}{|c|}{ Slope } & \multicolumn{2}{|c|}{ Intercept } & \multicolumn{2}{|c|}{ Slope } & \multicolumn{2}{|c|}{ Intercept } & \multicolumn{2}{|c|}{ Slope } \\
\hline & $b$ & S.E. & $b$ & S.E. & $b$ & S.E. & $b$ & S.E. & $b$ & S.E. & $b$ & S.E. \\
\hline Estimate & $3.273 *$ & 0.201 & $-0.038 *$ & 0.010 & $2.981 *$ & 0.145 & $0.005^{*}$ & 0.007 & $2.383 *$ & 0.678 & 0.024 & 0.033 \\
\hline Age & -0.012 & 0.006 & 0.001 & 0.000 & 0.000 & 0.003 & 0.000 & 0.000 & 0.009 & 0.010 & -0.001 & 0.001 \\
\hline Gender $(0=$ Female $)$ & 0.127 & 0.046 & 0.000 & 0.002 & 0.047 & 0.032 & -0.003 & 0.002 & 0.030 & 0.079 & -0.006 & 0.004 \\
\hline Education & 0.072 & 0.024 & 0.001 & 0.001 & 0.075 & 0.016 & 0.001 & 0.001 & 0.072 & 0.038 & -0.001 & 0.002 \\
\hline NSIs & -0.004 & 0.024 & 0.000 & 0.001 & -0.028 & 0.016 & -0.001 & 0.001 & 0.017 & 0.042 & -0.003 & 0.002 \\
\hline Covariance & 0.001 & 0.001 & & & -0.001 & 0.000 & & & -0.001 & 0.001 & & \\
\hline \multirow[t]{3}{*}{$R^{2}$} & .066 & & .115 & & .039 & & .022 & & .034 & & .124 & \\
\hline & \multicolumn{4}{|c|}{$\chi^{2}(13)=35.34$} & \multicolumn{4}{|c|}{$\chi^{2}(13)=81.88$} & \multicolumn{4}{|c|}{$\chi^{2}(13)=23.20$} \\
\hline & \multicolumn{4}{|c|}{$\mathrm{CMIN} / \mathrm{DF}=2.71$} & \multicolumn{4}{|c|}{$\mathrm{CMIN} / \mathrm{DF}=6.29$} & \multicolumn{4}{|c|}{$\mathrm{CMIN} / \mathrm{DF}=1.78$} \\
\hline \multirow[t]{2}{*}{ Model Fit } & \multicolumn{4}{|c|}{$\mathrm{CFI}=.968$} & \multicolumn{4}{|c|}{$\mathrm{CFI}=.950$} & \multicolumn{4}{|c|}{$\mathrm{CFI}=.959$} \\
\hline & \multicolumn{4}{|c|}{$\mathrm{RMSEA}=.06$} & \multicolumn{4}{|c|}{$\mathrm{RMSEA}=.07$} & \multicolumn{4}{|c|}{$\mathrm{RMSEA}=.06$} \\
\hline
\end{tabular}

Note. Significant statistics $(p<.05)$ are bolded. $R^{2}=$ variance accounted for. An asterisk indicates that there is significant variability surrounding the estimate. A blue value indicates that the estimate is significantly different than zero. Type I errors were accounted for 
utilizing the false discovery rate adjustment. A green value indicates that the estimate was no longer statistically significant after the false discovery rate adjustments. To aid in the interpretation of the estimates, education and NSIs were standardized. As such, a unit increase or decrease in these variables represent a standard deviation increase or decrease, respectively. Age was not manipulated so that a unit increase or decrease represents an increase or decrease of a year, respectively. 
Table 22

Research Question 6-Multigroup Analyses for the Latent Growth Curve of Neuroticism

\begin{tabular}{|c|c|c|c|c|c|c|c|c|c|c|c|c|}
\hline & \multicolumn{4}{|c|}{ Younger Adults } & \multicolumn{4}{|c|}{ Middle-Aged Adults } & \multicolumn{4}{|c|}{ Older Adults } \\
\hline & \multicolumn{2}{|c|}{ Intercept } & \multicolumn{2}{|c|}{ Slope } & \multicolumn{2}{|c|}{ Intercept } & \multicolumn{2}{|c|}{ Slope } & \multicolumn{2}{|c|}{ Intercept } & \multicolumn{2}{|c|}{ Slope } \\
\hline & $b$ & S.E. & $b$ & S.E. & $b$ & S.E. & $b$ & S.E. & $b$ & S.E. & $b$ & S.E. \\
\hline Estimate & $2.660 *$ & 0.250 & $0.007^{*}$ & 0.013 & $2.809 *$ & 0.176 & $-0.031 *$ & 0.009 & $2.118 *$ & 0.626 & -0.005 & 0.002 \\
\hline Age & -0.010 & 0.007 & 0.000 & 0.000 & -0.013 & 0.004 & 0.000 & 0.000 & 0.001 & 0.010 & - & - \\
\hline Gender $(0=$ Female $)$ & -0.125 & 0.058 & 0.000 & 0.003 & -0.098 & 0.039 & 0.000 & 0.002 & -0.181 & 0.073 & - & - \\
\hline Education & -0.107 & 0.030 & 0.002 & 0.002 & -0.076 & 0.019 & 0.000 & 0.001 & -0.094 & 0.035 & - & - \\
\hline NSIs & 0.196 & 0.029 & -0.004 & 0.002 & 0.178 & 0.019 & -0.002 & 0.001 & 0.122 & 0.039 & - & - \\
\hline Covariance & -0.002 & 0.001 & & & -0.003 & 0.001 & & & -0.002 & 0.002 & & \\
\hline \multirow[t]{3}{*}{$R^{2}$} & .170 & & .073 & & .155 & & .034 & & .122 & & .000 & \\
\hline & \multicolumn{4}{|c|}{$\chi^{2}(13)=28.63$} & \multicolumn{4}{|c|}{$\chi^{2}(13)=130.06$} & \multicolumn{4}{|c|}{$\chi^{2}(17)=36.64$} \\
\hline & \multicolumn{4}{|c|}{$\mathrm{CMIN} / \mathrm{DF}=2.20$} & \multicolumn{4}{|c|}{$\mathrm{CMIN} / \mathrm{DF}=10.00$} & \multicolumn{4}{|c|}{$\mathrm{CMIN} / \mathrm{DF}=2.15$} \\
\hline \multirow[t]{2}{*}{ Model Fit } & \multicolumn{4}{|c|}{$\mathrm{CFI}=.975$} & \multicolumn{4}{|c|}{$\mathrm{CFI}=.914$} & \multicolumn{4}{|c|}{$\mathrm{CFI}=.906$} \\
\hline & \multicolumn{4}{|c|}{$\mathrm{RMSEA}=.05$} & \multicolumn{4}{|c|}{$\mathrm{RMSEA}=.10$} & \multicolumn{4}{|c|}{ RMSEA $=.08$} \\
\hline
\end{tabular}

Note. Significant statistics $(p<.05)$ are bolded. $R^{2}=$ variance accounted for. An asterisk indicates that there is significant variability surrounding the estimate. A blue value indicates that the estimate is significantly different than zero. Type I errors were accounted for 
utilizing the false discovery rate adjustment. A green value indicates that the estimate was no longer statistically significant after the false discovery rate adjustments. To aid in the interpretation of the estimates, education and NSIs were standardized. As such, a unit increase or decrease in these variables represent a standard deviation increase or decrease, respectively. Age was not manipulated so that a unit increase or decrease represents an increase or decrease of a year, respectively. 
Table 23

Research Question 6-Multigroup Analyses for the Latent Growth Curve of Conscientiousness

\begin{tabular}{|c|c|c|c|c|c|c|c|c|c|c|c|c|}
\hline & \multicolumn{4}{|c|}{ Younger Adults } & \multicolumn{4}{|c|}{ Middle-Aged Adults } & \multicolumn{4}{|c|}{ Older Adults } \\
\hline & \multicolumn{2}{|c|}{ Intercept } & \multicolumn{2}{|c|}{ Slope } & \multicolumn{2}{|c|}{ Intercept } & \multicolumn{2}{|c|}{ Slope } & \multicolumn{2}{|c|}{ Intercept } & \multicolumn{2}{|c|}{ Slope } \\
\hline & $b$ & S.E. & $b$ & S.E. & $b$ & S.E. & $b$ & S.E. & $b$ & S.E. & $b$ & S.E. \\
\hline Estimate & $3.634^{*}$ & 0.165 & $-0.010 *$ & 0.009 & $3.643 *$ & 0.101 & $0.001 *$ & 0.001 & $3.796 *$ & 0.584 & $0.005 *$ & 0.034 \\
\hline Age & -0.002 & 0.005 & 0.000 & 0.000 & -0.002 & 0.002 & - & - & -0.004 & 0.009 & 0.000 & 0.001 \\
\hline Gender $(0=$ Female $)$ & -0.223 & 0.038 & 0.004 & 0.002 & -0.100 & 0.022 & - & - & -0.059 & 0.068 & 0.002 & 0.004 \\
\hline Education & 0.010 & 0.020 & 0.000 & 0.001 & 0.046 & 0.011 & - & - & -0.001 & 0.033 & 0.000 & 0.002 \\
\hline NSIs & -0.062 & 0.019 & -0.001 & 0.001 & -0.061 & 0.011 & - & - & -0.051 & 0.036 & 0.000 & 0.002 \\
\hline Covariance & 0.000 & 0.001 & & & 0.000 & 0.000 & & & -0.001 & 0.001 & & \\
\hline \multirow[t]{3}{*}{$R^{2}$} & .133 & & .069 & & .085 & & .000 & & .026 & & .015 & \\
\hline & \multicolumn{4}{|c|}{$\chi^{2}(13)=16.11$} & \multicolumn{4}{|c|}{$\chi^{2}(17)=102.00$} & \multicolumn{4}{|c|}{$\chi^{2}(13)=44.77$} \\
\hline & \multicolumn{4}{|c|}{$\mathrm{CMIN} / \mathrm{DF}=1.24$} & \multicolumn{4}{|c|}{$\mathrm{CMIN} / \mathrm{DF}=6.00$} & \multicolumn{4}{|c|}{$\mathrm{CMIN} / \mathrm{DF}=3.44$} \\
\hline \multirow[t]{2}{*}{ Model Fit } & \multicolumn{4}{|c|}{$\mathrm{CFI}=.994$} & \multicolumn{4}{|c|}{$\mathrm{CFI}=.919$} & \multicolumn{4}{|c|}{$\mathrm{CFI}=.814$} \\
\hline & \multicolumn{4}{|c|}{$\mathrm{RMSEA}=.02$} & \multicolumn{4}{|c|}{$\mathrm{RMSEA}=.07$} & \multicolumn{4}{|c|}{ RMSEA $=.11$} \\
\hline
\end{tabular}

Note. Significant statistics $(p<.05)$ are bolded. $R^{2}=$ variance accounted for. An asterisk indicates that there is significant variability surrounding the estimate. A blue value indicates that the estimate is significantly different than zero. Type I errors were accounted for 
utilizing the false discovery rate adjustment. A green value indicates that the estimate was no longer statistically significant after the false discovery rate adjustments. To aid in the interpretation of the estimates, education and NSIs were standardized. As such, a unit increase or decrease in these variables represent a standard deviation increase or decrease, respectively. Age was not manipulated so that a unit increase or decrease represents an increase or decrease of a year, respectively. 
Table 24

Preliminary Models that Examine the Significant Parameters that can be Accounted for with the Cross-Domain Latent Growth Curves

\begin{tabular}{|c|c|c|c|c|c|c|c|}
\hline & \multirow[t]{2}{*}{ Model Fit } & \multicolumn{2}{|c|}{ Intercept } & \multicolumn{2}{|c|}{ Slope } & \multicolumn{2}{|c|}{ Covariance } \\
\hline & & $b$ & S.E. & $b$ & S.E. & $b$ & S.E. \\
\hline NSIs and & $\chi^{2}(14)=28.09$ & $2.027 *$ & 0.010 & $-0.008 *$ & 0.001 & 0.000 & 0.000 \\
\hline \multirow[t]{3}{*}{ Agreeableness } & $\mathrm{CMIN} / \mathrm{DF}=2.00$ & $3.456 *$ & 0.012 & $-0.002 *$ & 0.001 & -0.001 & 0.000 \\
\hline & $\mathrm{CFI}=.996$ & & & & & & \\
\hline & $\mathrm{RMSEA}=.02$ & & & & & & \\
\hline NSIs and & $\chi^{2}(14)=33.98$ & $2.027 *$ & 0.010 & $-0.008 *$ & 0.001 & 0.000 & 0.000 \\
\hline \multirow[t]{3}{*}{ Openness } & $\mathrm{CMIN} / \mathrm{DF}=2.42$ & $2.977^{*}$ & 0.013 & $-0.006 *$ & 0.001 & 0.000 & 0.000 \\
\hline & $\mathrm{CFI}=.995$ & & & & & & \\
\hline & $\mathrm{RMSEA}=.03$ & & & & & & \\
\hline NSIs and & $\chi^{2}(14)=124.78$ & $2.027^{*}$ & 0.010 & $-0.008 *$ & 0.001 & 0.000 & 0.000 \\
\hline \multirow[t]{3}{*}{ Neuroticism } & $\mathrm{CMIN} / \mathrm{DF}=8.91$ & $2.163 *$ & 0.016 & $-0.008 *$ & 0.001 & -0.003 & 0.001 \\
\hline & $\mathrm{CFI}=.970$ & & & & & & \\
\hline & $\mathrm{RMSEA}=.07$ & & & & & & \\
\hline
\end{tabular}




\begin{tabular}{|c|c|c|c|c|c|c|c|}
\hline NSIs and & $\chi^{2}(14)=19.89$ & $2.027 *$ & 0.010 & $-0.008 *$ & 0.001 & 0.000 & 0.000 \\
\hline \multirow[t]{3}{*}{ Extraversion } & $\mathrm{CMIN} / \mathrm{DF}=1.42$ & $3.183 *$ & 0.014 & $-0.006^{*}$ & 0.001 & 0.000 & 0.000 \\
\hline & $\mathrm{CFI}=.998$ & & & & & & \\
\hline & $\mathrm{RMSEA}=.01$ & & & & & & \\
\hline NSIs and & $\chi^{2}(14)=45.17$ & $2.027 *$ & 0.010 & $-0.008 *$ & 0.001 & 0.000 & 0.000 \\
\hline \multirow[t]{3}{*}{ Conscientiousness } & $\mathrm{CMIN} / \mathrm{DF}=3.22$ & $3.493 *$ & 0.010 & $0.001 *$ & 0.001 & 0.000 & 0.000 \\
\hline & $\mathrm{CFI}=.990$ & & & & & & \\
\hline & $\mathrm{RMSEA}=.03$ & & & & & & \\
\hline
\end{tabular}

Note. Significant statistics $(p<.05)$ are bolded. An asterisk indicates that there is significant variability surrounding the estimate. A blue value indicates that the estimate is significantly different than zero. 
Table 25

The Unconstrained Models Compared to the Constrained Models for the Multigroup Cross-Domain Latent Growth Curves

\begin{tabular}{|c|c|c|c|c|}
\hline & $\chi^{2}$ & CFI & $\Delta \chi^{2}$ & $\Delta \mathrm{CFI}$ \\
\hline \multicolumn{5}{|l|}{ NSIs and Agreeableness } \\
\hline Unconstrained Model & $\chi^{2}(43)=92.41$ & .985 & & \\
\hline Constrained Model & $\chi^{2}(55)=171.46$ & .966 & $\chi^{2}(12)=79.05$ & .019 \\
\hline \multicolumn{5}{|l|}{ NSIs and Openness } \\
\hline Unconstrained Model & $\chi^{2}(43)=95.05$ & .986 & & \\
\hline Constrained Model & $\chi^{2}(55)=171.62$ & .969 & $\chi^{2}(12)=76.57$ & .017 \\
\hline \multicolumn{5}{|l|}{ NSIs and Neuroticism } \\
\hline Unconstrained Model & $\chi^{2}(43)=171.66$ & .965 & & \\
\hline Constrained Model & $\chi^{2}(55)=241.43$ & .949 & $\chi^{2}(12)=69.77$ & .016 \\
\hline \multicolumn{5}{|l|}{ NSIs and Extraversion } \\
\hline Unconstrained Model & $\chi^{2}(43)=60.37$ & .995 & & \\
\hline Constrained Model & $\chi^{2}(55)=138.32$ & .978 & $\chi^{2}(12)=77.95$ & .017 \\
\hline \multicolumn{5}{|c|}{ NSIs and Conscientiousness } \\
\hline Unconstrained Model & $\chi^{2}(43)=98.79$ & .982 & & \\
\hline Constrained Model & $\chi^{2}(55)=191.76$ & .957 & $\chi^{2}(12)=92.97$ & .025 \\
\hline
\end{tabular}


Note. Significant statistics $(p<.05)$ are bolded. The unconstrained model is where the parameters were freely estimated across the three age groups. The constrained model is where the parameters were constrained to be equal across the three age groups. A change of .01 or greater for the CFI difference statistic was used to determine significance (Little, 2013). Both the chi-square and the CFI difference statistics were computed because the chi-square statistic is commonly used, however, it is sensitive to large sample sizes. The CFI is more robust to larger sample sizes (Little, 2013). 
Table 26

Research Question 8 - Multigroup Cross-Domain Latent Growth Curves

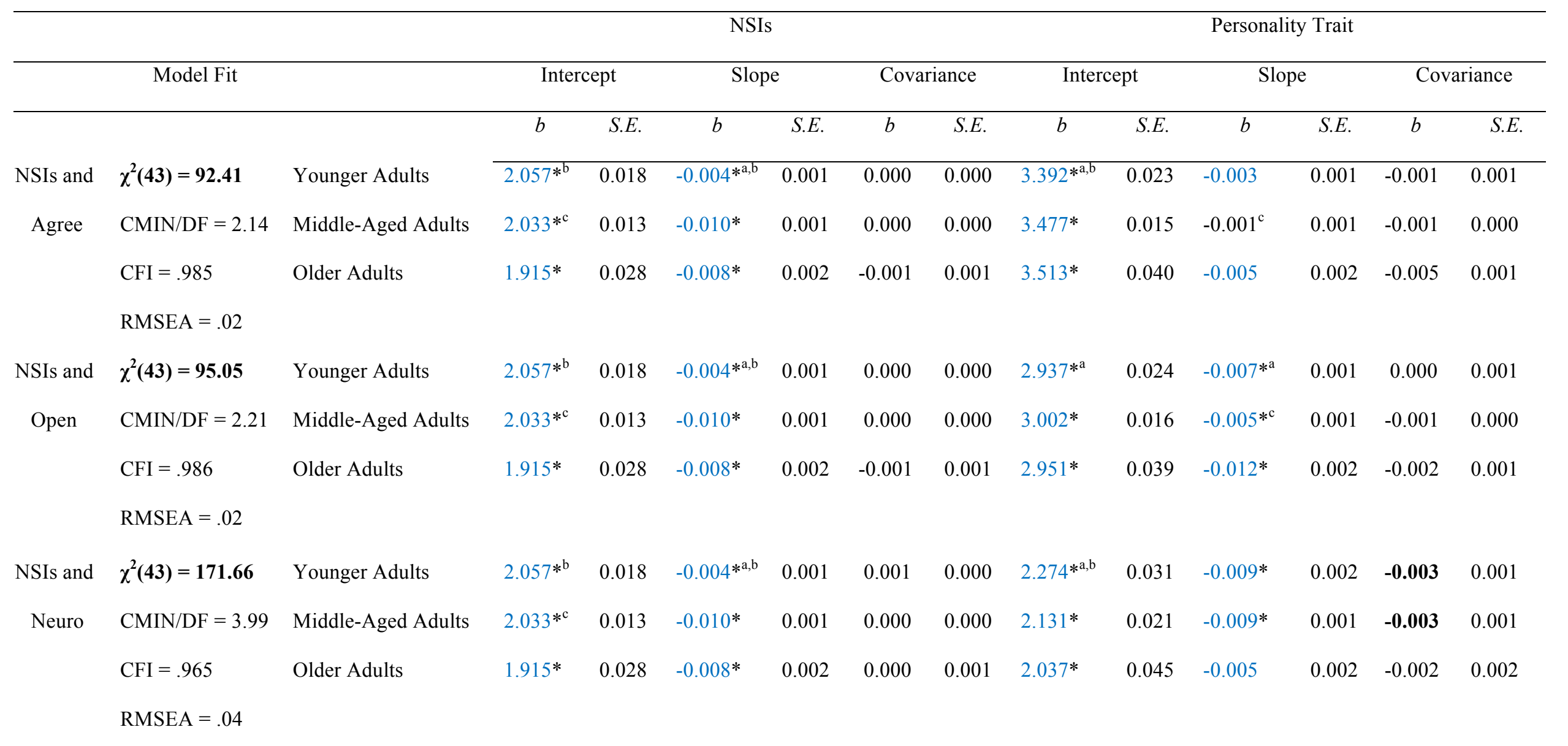


PERSONALITY AND NEGATIVE SOCIAL INTERACTIONS

\begin{tabular}{|c|c|c|c|c|c|c|c|c|c|c|c|c|c|c|}
\hline NSIs and & $\chi^{2}(43)=60.37$ & Younger Adults & $2.057 * \mathrm{~b}$ & 0.018 & $-0.004^{* a, b}$ & 0.001 & 0.000 & 0.000 & $3.167 *$ & 0.026 & $-0.008^{* a, b}$ & 0.001 & -0.001 & 0.001 \\
\hline \multirow[t]{3}{*}{ Extra } & $\mathrm{CMIN} / \mathrm{DF}=1.40$ & Middle-Aged Adults & $2.033 *^{\mathrm{c}}$ & 0.013 & $-0.010 *$ & 0.001 & 0.000 & 0.000 & $3.180 *$ & 0.018 & $-0.004 * \mathrm{c}$ & 0.001 & 0.000 & 0.001 \\
\hline & $\mathrm{CFI}=.995$ & Older Adults & $1.915^{*}$ & 0.028 & $-0.008 *$ & 0.002 & -0.001 & 0.001 & $3.243 *$ & 0.043 & -0.014 & 0.002 & 0.001 & 0.001 \\
\hline & $\mathrm{RMSEA}=.01$ & & & & & & & & & & & & & \\
\hline NSIs and & $\chi^{2}(43)=98.79$ & Younger Adults & $2.057^{\mathrm{b}}$ & 0.018 & $-0.004^{* a, b}$ & 0.001 & 0.000 & 0.000 & $3.459 *^{\mathrm{a}}$ & 0.020 & $0.003 * a, b$ & 0.001 & 0.000 & 0.000 \\
\hline \multirow[t]{2}{*}{ Consc } & $\mathrm{CMIN} / \mathrm{DF}=2.29$ & Middle-Aged Adults & $2.033^{\mathrm{c}}$ & 0.013 & $-0.010 *$ & 0.001 & 0.000 & 0.000 & $3.510 *$ & 0.013 & $0.001^{\mathrm{c}}$ & 0.001 & 0.000 & 0.000 \\
\hline & $\mathrm{CFI}=.982$ & Older Adults & $1.915^{*}$ & 0.028 & $-0.008 *$ & 0.002 & -0.001 & 0.001 & $3.495 *$ & 0.034 & $-0.008 *$ & 0.002 & -0.002 & 0.001 \\
\hline
\end{tabular}

Note. Significant statistics $(p<.05)$ are bolded. Agree $=$ agreeableness. Open $=$ openness. Neuro $=$ neuroticism. Extra $=$ extraversion.

Consc $=$ conscientiousness. An asterisk indicates that there is significant variability surrounding the estimate. A blue value indicates that the estimate is significantly different than zero. ${ }^{a}$ indicates that the estimate is significantly different between younger and middleaged adults. ${ }^{\mathrm{b}}$ indicates that the estimate is significantly different between younger and older adults. ${ }^{\mathrm{c}}$ indicates that the estimate is significantly different between middle-aged and older adults. 
Table 27

Research Question 8 - Examination of the Bidirectional Association between the Latent Growth Curves of NSIs and Agreeableness After Accounting for the Demographic Variables and Remaining Personality Traits for Younger Adults

\begin{tabular}{|c|c|c|c|c|c|c|c|c|}
\hline & \multicolumn{4}{|c|}{ NSIs Latent Growth Curve } & \multicolumn{4}{|c|}{ Agreeableness Latent Growth Curve } \\
\hline & \multicolumn{2}{|c|}{ Intercept } & \multicolumn{2}{|c|}{ Slope } & \multicolumn{2}{|c|}{ Intercept } & \multicolumn{2}{|c|}{ Slope } \\
\hline & $b$ & S.E. & $b$ & S.E. & $b$ & S.E. & $b$ & S.E. \\
\hline Estimate & $1.956 *$ & 0.148 & 0.015 & 0.020 & $3.229 *$ & 0.173 & -0.025 & 0.017 \\
\hline Age & 0.004 & 0.004 & -0.001 & 0.000 & 0.009 & 0.005 & - & - \\
\hline Gender & -0.085 & 0.034 & 0.002 & 0.003 & -0.309 & 0.041 & - & - \\
\hline \multicolumn{9}{|l|}{$(0=$ Female $)$} \\
\hline Education & -0.014 & 0.018 & 0.001 & 0.001 & -0.013 & 0.021 & - & - \\
\hline Openness & -0.028 & 0.019 & -0.001 & 0.001 & - & - & - & - \\
\hline Neuroticism & 0.099 & 0.017 & -0.005 & 0.001 & - & - & - & - \\
\hline Extraversion & -0.017 & 0.019 & 0.000 & 0.001 & - & - & - & - \\
\hline Conscientiousness & -0.043 & 0.017 & -0.001 & 0.001 & - & - & - & - \\
\hline NSIs Intercept & - & - & 0.010 & 0.007 & - & - & 0.008 & 0.005 \\
\hline Agreeableness Intercept & - & - & -0.001 & 0.003 & - & - & 0.002 & 0.004 \\
\hline$R^{2}$ & .179 & & .483 & & .151 & & .179 & \\
\hline
\end{tabular}




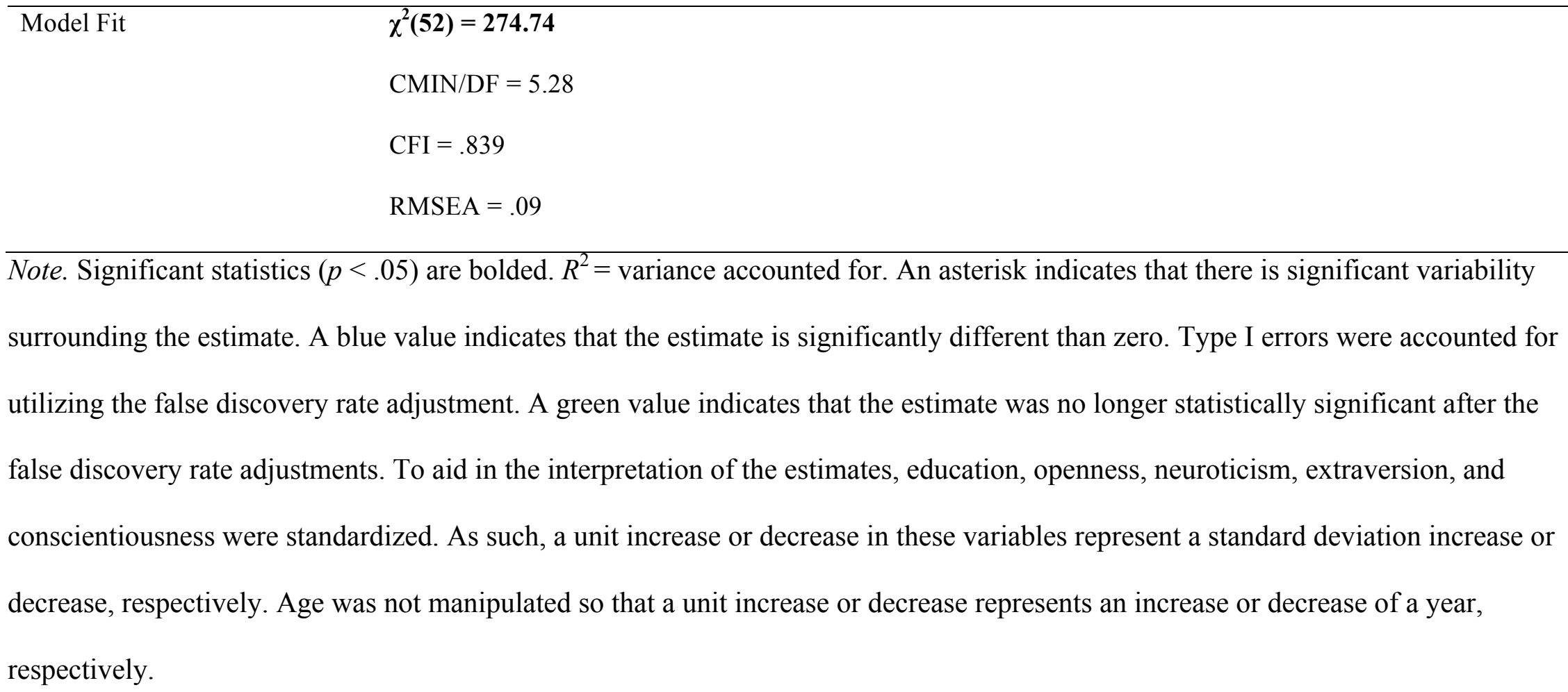


Table 28

Research Question 8 - Examination of the Bidirectional Association between the Latent Growth Curves of NSIs and Agreeableness After Accounting for the Demographic Variables and Remaining Personality Traits for Middle-Aged Adults

\begin{tabular}{|c|c|c|c|c|c|c|c|c|}
\hline & \multicolumn{4}{|c|}{ NSIs Latent Growth Curve } & \multicolumn{4}{|c|}{ Agreeableness Latent Growth Curve } \\
\hline & \multicolumn{2}{|c|}{ Intercept } & \multicolumn{2}{|c|}{ Slope } & \multicolumn{2}{|c|}{ Intercept } & \multicolumn{2}{|c|}{ Slope } \\
\hline & $b$ & S.E. & $b$ & S.E. & $b$ & S.E. & $b$ & S.E. \\
\hline Estimate & $2.555^{*}$ & 0.109 & -0.034 & 0.018 & $3.401 *$ & 0.114 & $-0.001 *$ & 0.001 \\
\hline Age & -0.010 & 0.002 & 0.000 & 0.000 & 0.004 & 0.002 & - & - \\
\hline $\begin{array}{l}\text { Gender } \\
\qquad(0=\text { Female })\end{array}$ & -0.066 & 0.024 & 0.001 & 0.002 & -0.259 & 0.025 & - & - \\
\hline Education & 0.031 & 0.012 & -0.001 & 0.001 & -0.038 & 0.013 & - & - \\
\hline Openness & 0.036 & 0.014 & 0.000 & 0.001 & - & - & - & - \\
\hline Neuroticism & 0.107 & 0.013 & -0.002 & 0.001 & - & - & - & - \\
\hline Extraversion & -0.001 & 0.014 & 0.000 & 0.001 & - & - & - & - \\
\hline Conscientiousness & -0.040 & 0.013 & 0.001 & 0.001 & - & - & - & - \\
\hline NSIs Intercept & - & - & 0.006 & 0.005 & - & - & - & - \\
\hline Agreeableness Intercept & - & - & 0.001 & 0.002 & - & - & - & - \\
\hline$R^{2}$ & .187 & & .095 & & .122 & & .000 & \\
\hline
\end{tabular}




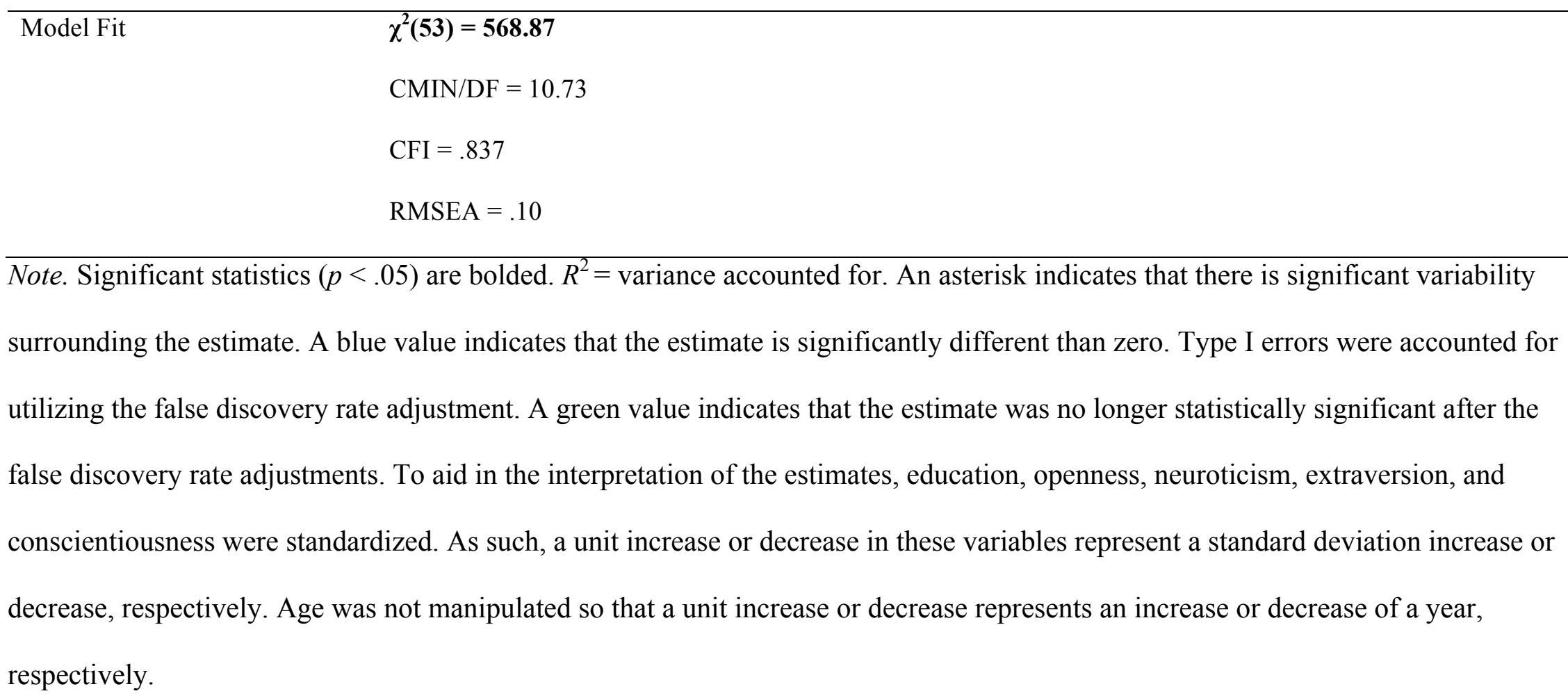


Table 29

Research Question 8 - Examination of the Bidirectional Association between the Latent Growth Curves of NSIs and Agreeableness After Accounting for the Demographic Variables and Remaining Personality Traits for Older Adults

\begin{tabular}{|c|c|c|c|c|c|c|c|c|}
\hline & \multicolumn{4}{|c|}{ NSIs Latent Growth Curve } & \multicolumn{4}{|c|}{ Agreeableness Latent Growth Curve } \\
\hline & \multicolumn{2}{|c|}{ Intercept } & \multicolumn{2}{|c|}{ Slope } & \multicolumn{2}{|c|}{ Intercept } & \multicolumn{2}{|c|}{ Slope } \\
\hline & $b$ & S.E. & $b$ & S.E. & $b$ & S.E. & $b$ & S.E. \\
\hline Estimate & $2.898 *$ & 0.467 & $0.057 *$ & 0.042 & $3.616^{*}$ & 0.616 & 0.022 & 0.021 \\
\hline Age & -0.016 & 0.007 & 0.000 & 0.000 & 0.001 & 0.010 & - & - \\
\hline Gender & 0.064 & 0.055 & 0.000 & 0.003 & -0.294 & 0.073 & - & - \\
\hline \multicolumn{9}{|l|}{$(0=$ Female $)$} \\
\hline Education & 0.011 & 0.026 & 0.001 & 0.002 & -0.073 & 0.034 & - & - \\
\hline Openness & 0.044 & 0.032 & 0.000 & 0.002 & - & - & - & - \\
\hline Neuroticism & 0.101 & 0.030 & 0.001 & 0.002 & - & - & - & - \\
\hline Extraversion & -0.003 & 0.032 & 0.002 & 0.002 & - & - & - & - \\
\hline Conscientiousness & -0.034 & 0.026 & 0.000 & 0.002 & - & - & - & - \\
\hline NSIs Intercept & - & - & -0.016 & 0.008 & - & - & -0.001 & 0.006 \\
\hline Agreeableness Intercept & - & - & -0.003 & 0.004 & - & - & -0.007 & 0.005 \\
\hline$R^{2}$ & .169 & & .114 & & .126 & & .419 & \\
\hline
\end{tabular}




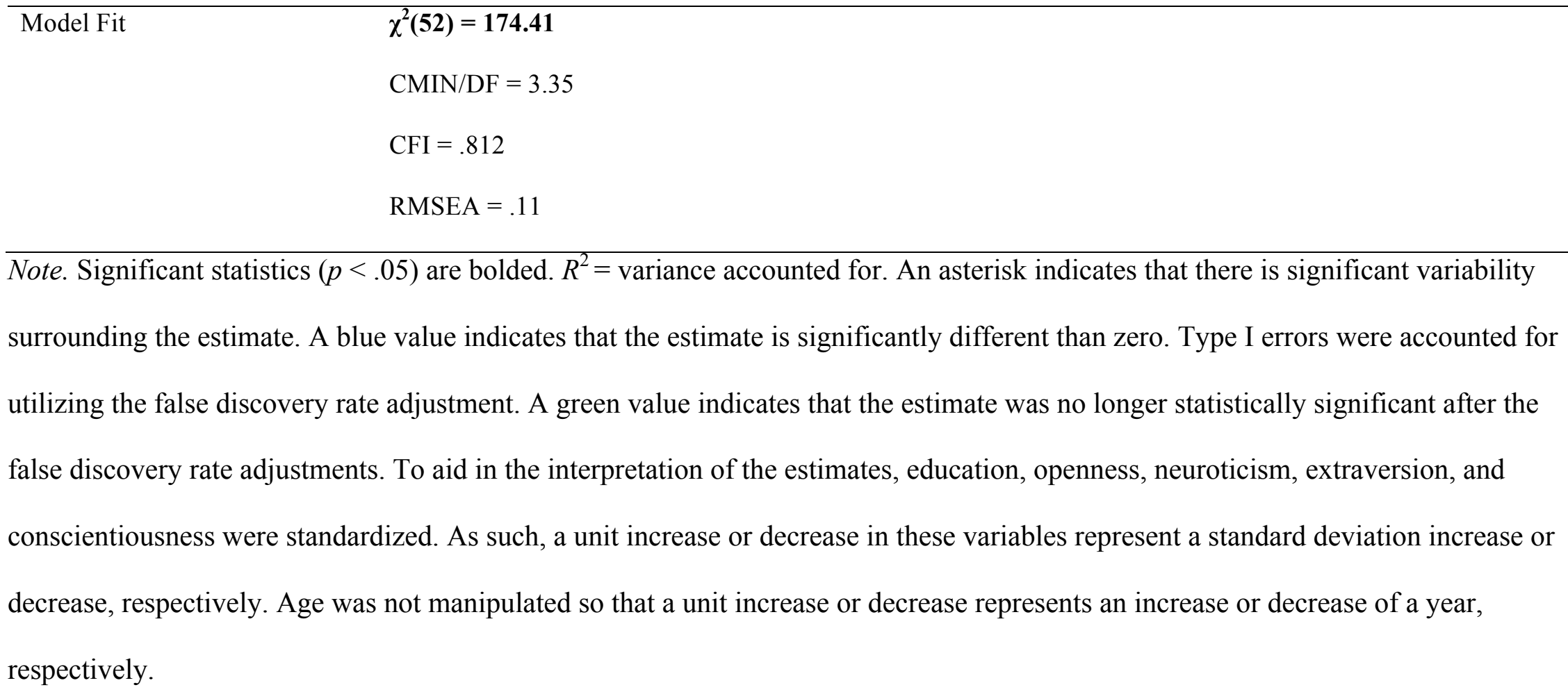


Table 30

Research Question 8 - Examination of the Bidirectional Association between the Latent Growth Curves of NSIs and Openness After Accounting for the Demographic Variables and Remaining Personality Traits for Younger Adults

\begin{tabular}{|c|c|c|c|c|c|c|c|c|}
\hline & \multicolumn{4}{|c|}{ NSIs Latent Growth Curve } & \multicolumn{4}{|c|}{ Openness Latent Growth Curve } \\
\hline & \multicolumn{2}{|c|}{ Intercept } & \multicolumn{2}{|c|}{ Slope } & \multicolumn{2}{|c|}{ Intercept } & \multicolumn{2}{|c|}{ Slope } \\
\hline & $b$ & S.E. & $b$ & S.E. & $b$ & S.E. & $b$ & S.E. \\
\hline Estimate & $1.977 *$ & 0.149 & 0.011 & 0.020 & $3.272 *$ & 0.202 & -0.053 & 0.020 \\
\hline Age & 0.003 & 0.004 & -0.001 & 0.000 & -0.012 & 0.006 & 0.001 & 0.000 \\
\hline Gender & -0.072 & 0.034 & 0.002 & 0.002 & 0.128 & 0.046 & -0.001 & 0.003 \\
\hline \multicolumn{9}{|l|}{$(0=$ Female $)$} \\
\hline Education & -0.010 & 0.018 & 0.001 & 0.001 & 0.072 & 0.024 & 0.001 & 0.001 \\
\hline Agreeableness & 0.007 & 0.019 & 0.000 & 0.001 & - & - & - & - \\
\hline Neuroticism & 0.102 & 0.017 & -0.005 & 0.001 & - & - & - & - \\
\hline Extraversion & -0.003 & 0.019 & -0.001 & 0.001 & - & - & - & - \\
\hline Conscientiousness & -0.043 & 0.017 & -0.002 & 0.001 & - & - & - & - \\
\hline NSIs Intercept & - & - & 0.009 & 0.007 & - & - & -0.002 & 0.005 \\
\hline Openness Intercept & - & - & 0.000 & 0.003 & - & - & 0.006 & 0.004 \\
\hline$R^{2}$ & .172 & & .480 & & .060 & & .161 & \\
\hline
\end{tabular}




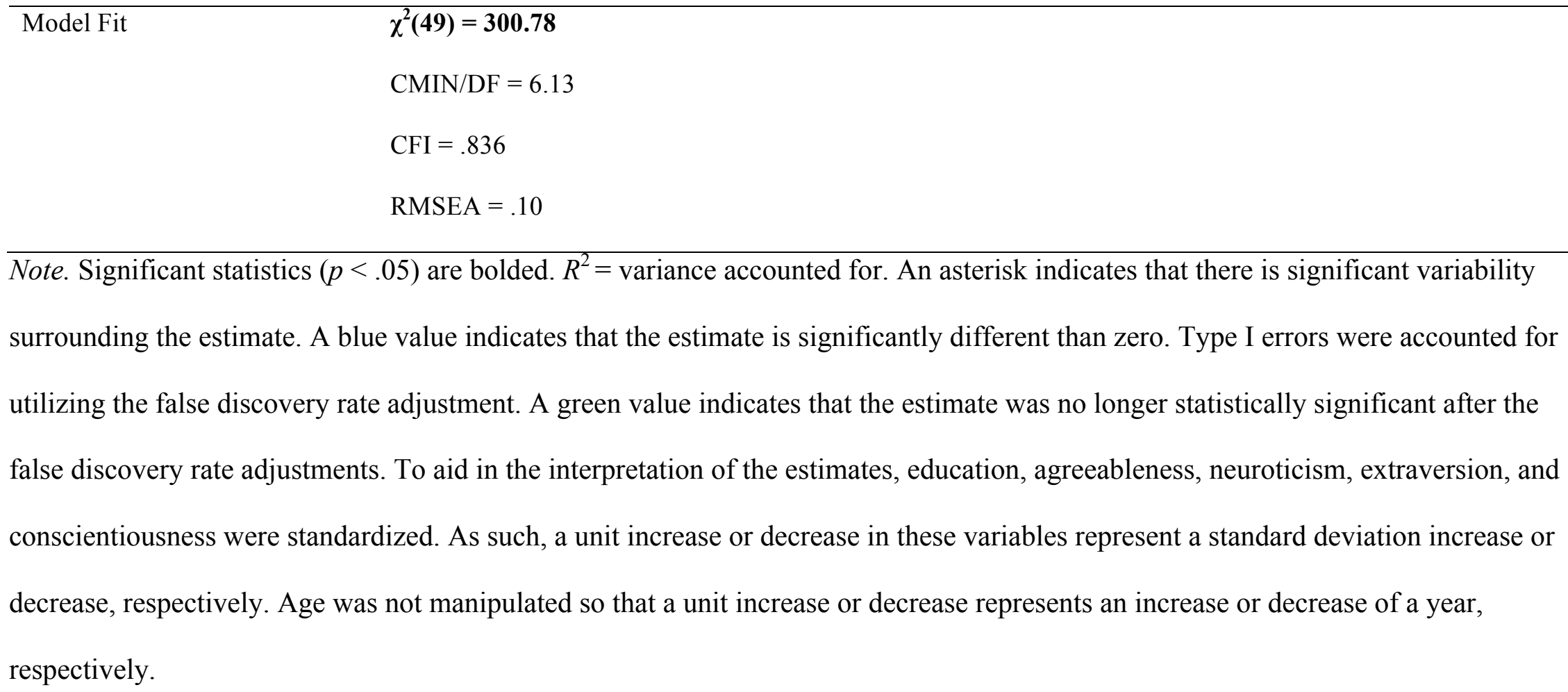


Table 31

Research Question 8 - Examination of the Bidirectional Association between the Latent Growth Curves of NSIs and Openness After Accounting for the Demographic Variables and Remaining Personality Traits for Middle-Aged Adults

\begin{tabular}{|c|c|c|c|c|c|c|c|c|}
\hline & \multicolumn{4}{|c|}{ NSIs Latent Growth Curve } & \multicolumn{4}{|c|}{ Openness Latent Growth Curve } \\
\hline & \multicolumn{2}{|c|}{ Intercept } & \multicolumn{2}{|c|}{ Slope } & \multicolumn{2}{|c|}{ Intercept } & \multicolumn{2}{|c|}{ Slope } \\
\hline & $b$ & S.E. & $b$ & S.E. & $b$ & S.E. & $b$ & S.E. \\
\hline Estimate & $2.557 *$ & 0.109 & $-0.043 *$ & 0.015 & $2.939 *$ & 0.145 & $0.032 *$ & 0.013 \\
\hline Age & -0.010 & 0.002 & 0.000 & 0.000 & 0.001 & 0.003 & 0.000 & 0.000 \\
\hline Gender & -0.075 & 0.024 & 0.000 & 0.002 & 0.052 & 0.032 & -0.003 & 0.002 \\
\hline \multicolumn{9}{|l|}{$(0=$ Female $)$} \\
\hline Education & 0.034 & 0.012 & -0.002 & 0.001 & 0.074 & 0.016 & 0.001 & 0.001 \\
\hline Agreeableness & -0.036 & 0.015 & 0.000 & 0.001 & - & - & - & - \\
\hline Neuroticism & 0.103 & 0.013 & -0.002 & 0.001 & - & - & - & - \\
\hline Extraversion & 0.017 & 0.015 & 0.000 & 0.001 & - & - & - & - \\
\hline Conscientiousness & -0.031 & 0.013 & 0.000 & 0.001 & - & - & - & - \\
\hline NSIs Intercept & - & - & 0.006 & 0.005 & - & - & -0.004 & 0.003 \\
\hline Openness Intercept & - & - & 0.004 & 0.002 & - & - & -0.006 & 0.002 \\
\hline$R^{2}$ & .196 & & .119 & & .034 & & .062 & \\
\hline
\end{tabular}




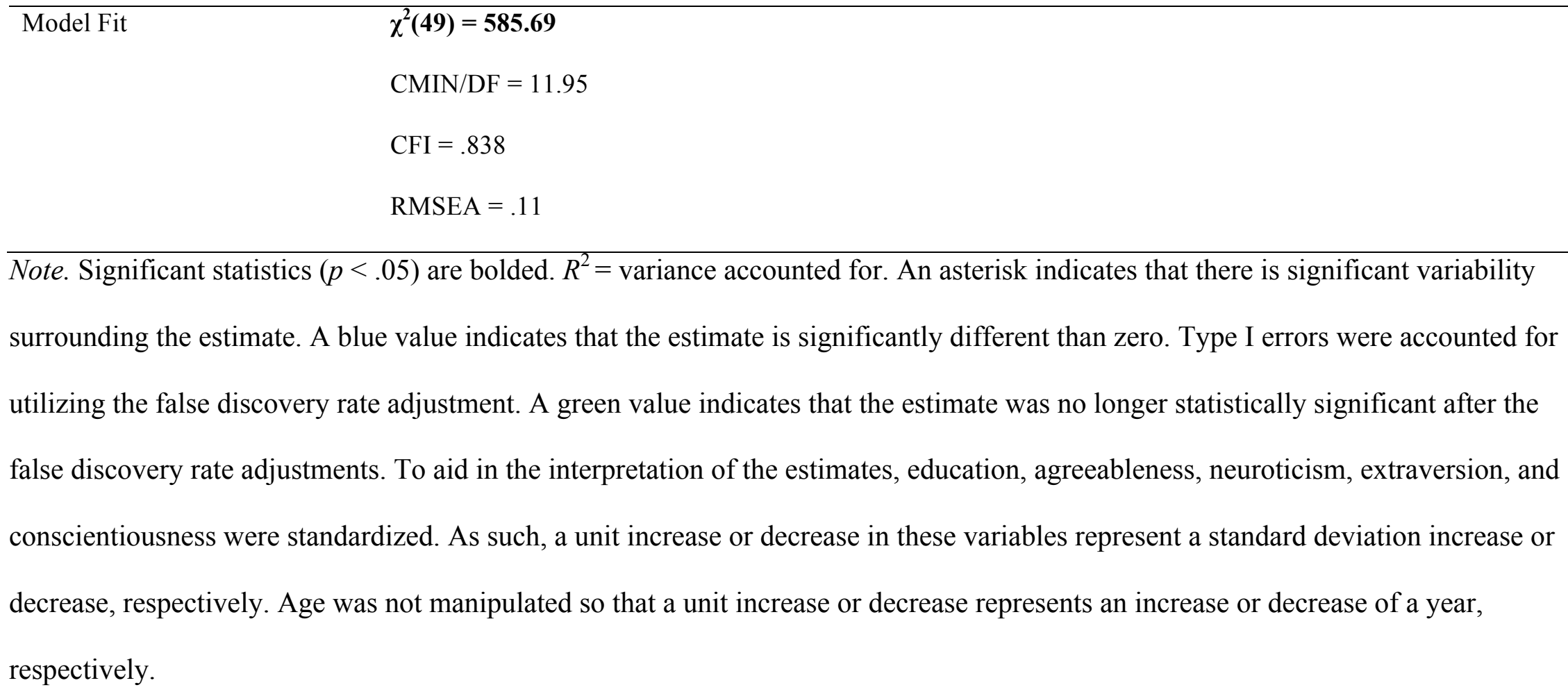


Table 32

Research Question 8 - Examination of the Bidirectional Association between the Latent Growth Curves of NSIs and Openness After Accounting for the Demographic Variables and Remaining Personality Traits for Older Adults

\begin{tabular}{|c|c|c|c|c|c|c|c|c|}
\hline & \multicolumn{4}{|c|}{ NSIs Latent Growth Curve } & \multicolumn{4}{|c|}{ Openness Latent Growth Curve } \\
\hline & \multicolumn{2}{|c|}{ Intercept } & \multicolumn{2}{|c|}{ Slope } & \multicolumn{2}{|c|}{ Intercept } & \multicolumn{2}{|c|}{ Slope } \\
\hline & $b$ & S.E. & $b$ & S.E. & $b$ & S.E. & $b$ & S.E. \\
\hline Estimate & $2.894^{*}$ & 0.468 & $0.049 *$ & 0.039 & $2.411 *$ & 0.681 & $0.062 *$ & 0.040 \\
\hline Age & -0.015 & 0.007 & 0.000 & 0.000 & 0.008 & 0.011 & 0.000 & 0.001 \\
\hline Gender & 0.053 & 0.055 & 0.002 & 0.003 & 0.032 & 0.079 & -0.006 & 0.004 \\
\hline \multicolumn{9}{|l|}{$(0=$ Female $)$} \\
\hline Education & 0.019 & 0.026 & 0.001 & 0.002 & 0.072 & 0.038 & 0.000 & 0.002 \\
\hline Agreeableness & -0.021 & 0.032 & 0.000 & 0.002 & - & - & - & - \\
\hline Neuroticism & 0.098 & 0.030 & 0.000 & 0.002 & - & - & - & - \\
\hline Extraversion & 0.021 & 0.034 & 0.002 & 0.002 & - & - & - & - \\
\hline Conscientiousness & -0.030 & 0.026 & -0.001 & 0.002 & - & - & - & - \\
\hline NSIs Intercept & - & - & -0.015 & 0.008 & - & - & -0.009 & 0.007 \\
\hline Openness Intercept & - & - & -0.002 & 0.004 & - & - & -0.007 & 0.005 \\
\hline$R^{2}$ & .159 & & .112 & & .033 & & .112 & \\
\hline
\end{tabular}




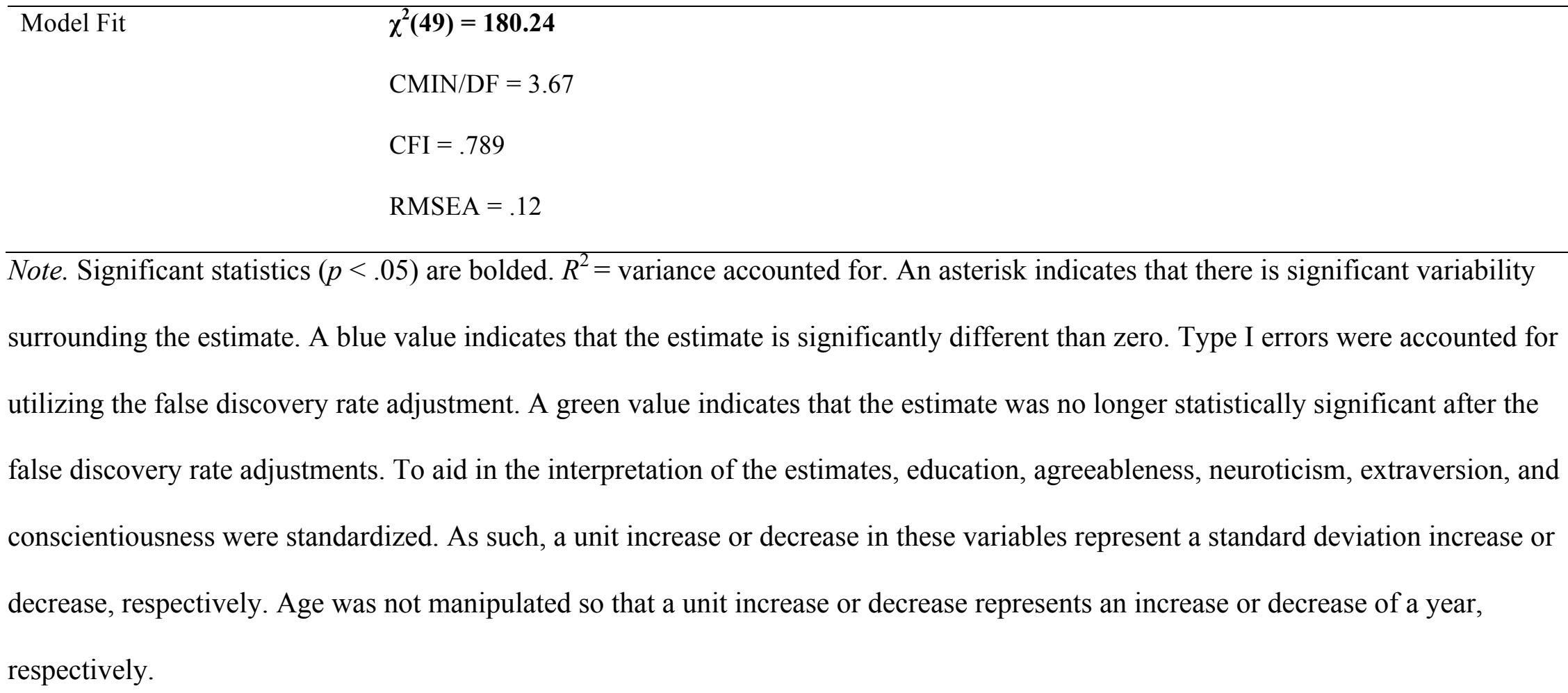


Table 33

Research Question 8 - Examination of the Bidirectional Association between the Latent Growth Curves of NSIs and Neuroticism After Accounting for the Demographic Variables and Remaining Personality Traits for Younger Adults

\begin{tabular}{|c|c|c|c|c|c|c|c|c|}
\hline & \multicolumn{4}{|c|}{ NSIs Latent Growth Curve } & \multicolumn{4}{|c|}{ Neuroticism Latent Growth Curve } \\
\hline & \multicolumn{2}{|c|}{ Intercept } & \multicolumn{2}{|c|}{ Slope } & \multicolumn{2}{|c|}{ Intercept } & \multicolumn{2}{|c|}{ Slope } \\
\hline & $b$ & S.E. & $b$ & S.E. & $b$ & S.E. & $b$ & S.E. \\
\hline Estimate & $2.061 *$ & 0.153 & 0.029 & 0.016 & $2.674^{*}$ & 0.261 & $0.035^{*}$ & 0.018 \\
\hline Age & 0.001 & 0.004 & -0.001 & 0.000 & -0.010 & 0.008 & -0.001 & 0.000 \\
\hline Gender & -0.112 & 0.035 & 0.003 & 0.002 & -0.162 & 0.060 & -0.001 & 0.003 \\
\hline \multicolumn{9}{|l|}{$(0=$ Female $)$} \\
\hline Education & -0.033 & 0.018 & 0.001 & 0.001 & -0.120 & 0.031 & 0.001 & 0.002 \\
\hline Agreeableness & -0.001 & 0.019 & 0.000 & 0.001 & - & - & - & - \\
\hline Openness & 0.024 & 0.019 & -0.001 & 0.001 & - & - & - & - \\
\hline Extraversion & -0.017 & 0.020 & 0.000 & 0.001 & - & - & - & - \\
\hline Conscientiousness & -0.045 & 0.017 & -0.001 & 0.001 & - & - & - & - \\
\hline NSIs Intercept & - & - & 0.012 & 0.008 & - & - & -0.002 & 0.007 \\
\hline Neuroticism Intercept & - & - & -0.011 & 0.003 & - & - & -0.009 & 0.004 \\
\hline$R^{2}$ & .077 & & .581 & & .068 & & .126 & \\
\hline
\end{tabular}




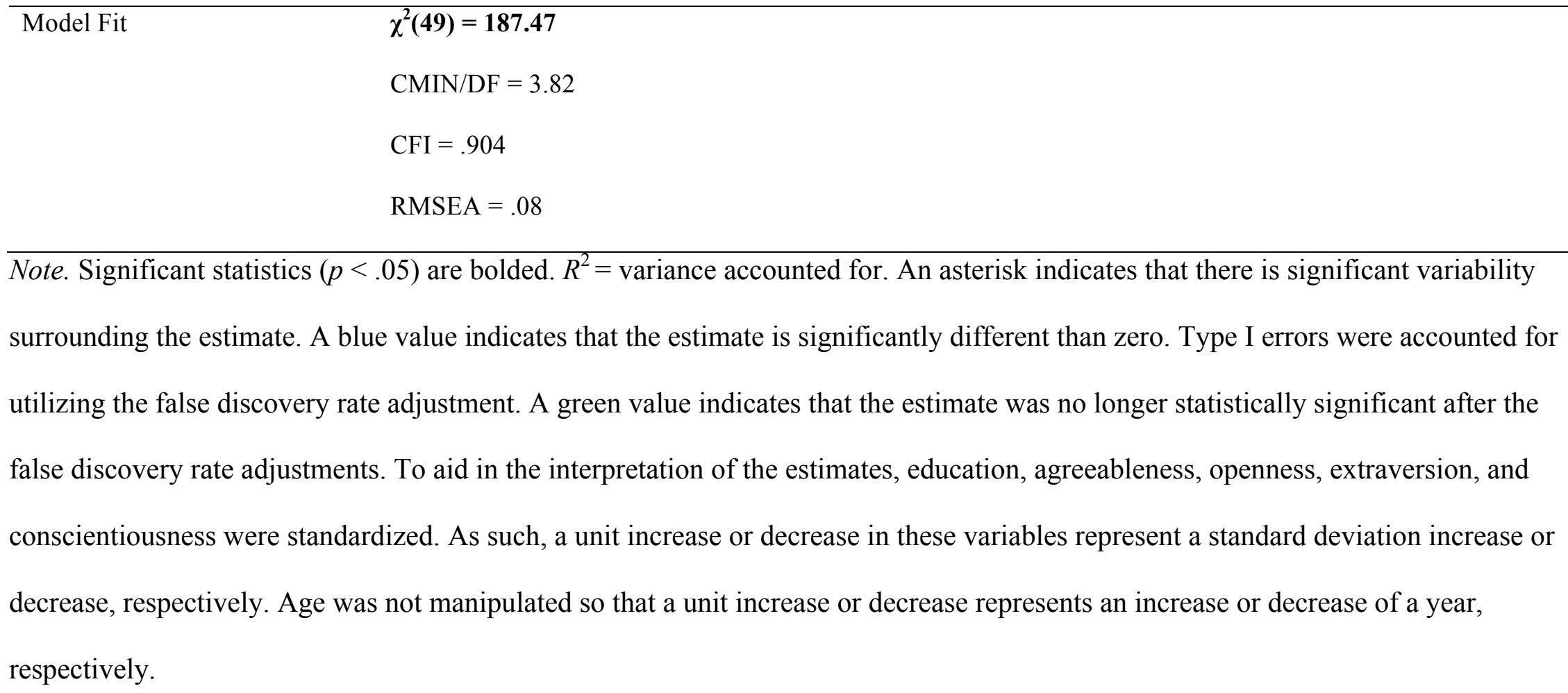


Table 34

Research Question 8 - Examination of the Bidirectional Association between the Latent Growth Curves of NSIs and Neuroticism After Accounting for the Demographic Variables and Remaining Personality Traits for Middle-Aged Adults

\begin{tabular}{|c|c|c|c|c|c|c|c|c|}
\hline & \multicolumn{4}{|c|}{ NSIs Latent Growth Curve } & \multicolumn{4}{|c|}{ Neuroticism Latent Growth Curve } \\
\hline & \multicolumn{2}{|c|}{ Intercept } & \multicolumn{2}{|c|}{ Slope } & \multicolumn{2}{|c|}{ Intercept } & \multicolumn{2}{|c|}{ Slope } \\
\hline & $b$ & S.E. & $b$ & S.E. & $b$ & S.E. & $b$ & S.E. \\
\hline Estimate & $2.683 *$ & 0.114 & -0.022 & 0.013 & $3.077 *$ & 0.185 & $0.003 *$ & 0.013 \\
\hline Age & -0.012 & 0.002 & 0.000 & 0.000 & -0.018 & 0.004 & 0.000 & 0.000 \\
\hline Gender & 0.107 & 0.025 & 0.000 & 0.002 & -0.130 & 0.041 & -0.002 & 0.002 \\
\hline \multicolumn{9}{|l|}{$(0=$ Female $)$} \\
\hline Education & 0.016 & 0.012 & -0.002 & 0.001 & -0.069 & 0.020 & -0.001 & 0.001 \\
\hline Agreeableness & -0.041 & 0.015 & 0.000 & 0.001 & - & - & - & - \\
\hline Openness & 0.037 & 0.014 & 0.000 & 0.001 & - & - & - & - \\
\hline Extraversion & -0.002 & 0.015 & 0.000 & 0.001 & - & - & - & - \\
\hline Conscientiousness & -0.039 & 0.014 & 0.001 & 0.001 & - & - & - & - \\
\hline NSIs Intercept & - & - & 0.008 & 0.005 & - & - & 0.003 & 0.004 \\
\hline Neuroticism Intercept & - & - & -0.005 & 0.002 & - & - & -0.014 & 0.003 \\
\hline$R^{2}$ & .110 & & .133 & & .061 & & .221 & \\
\hline
\end{tabular}




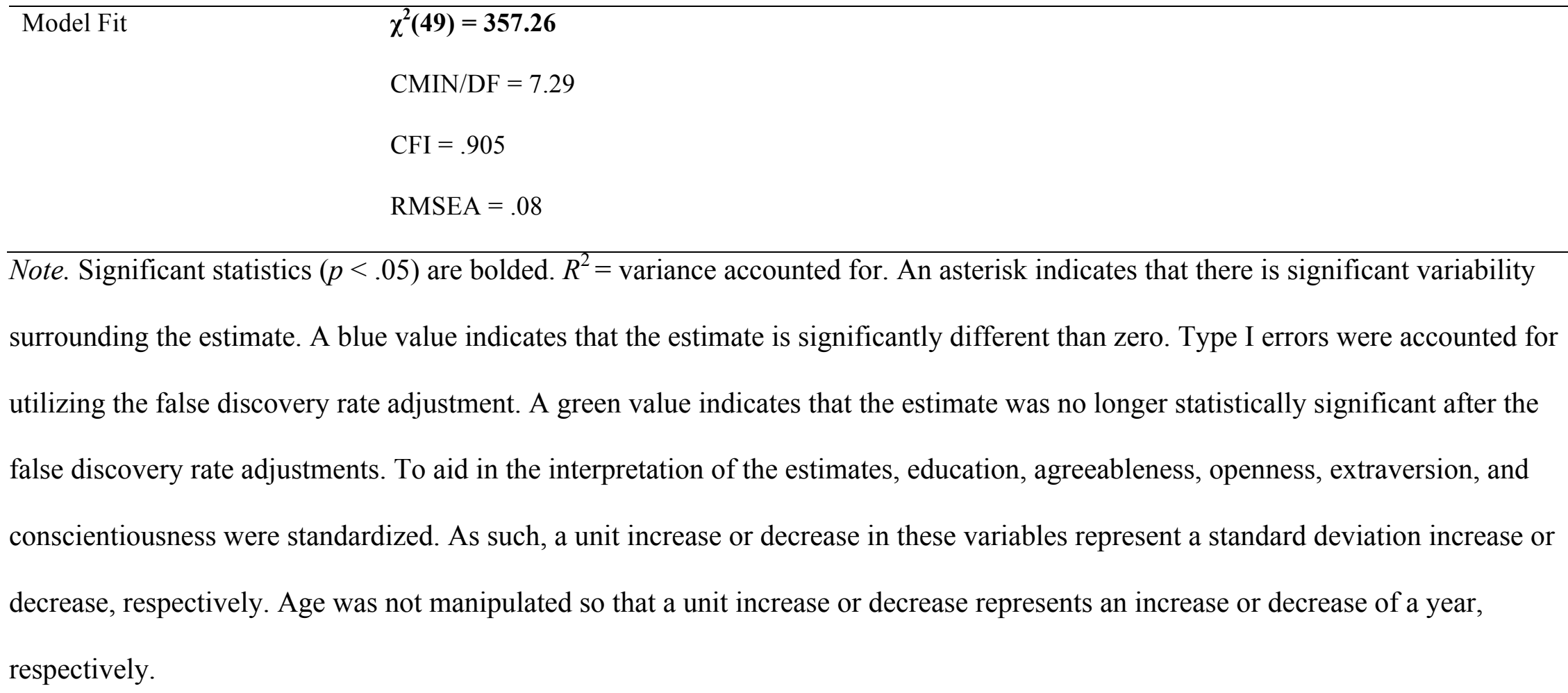


Table 35

Research Question 8 - Examination of the Bidirectional Association between the Latent Growth Curves of NSIs and Neuroticism After Accounting for the Demographic Variables and Remaining Personality Traits for Older Adults

\begin{tabular}{|c|c|c|c|c|c|c|c|c|}
\hline & \multicolumn{4}{|c|}{ NSIs Latent Growth Curve } & \multicolumn{4}{|c|}{ Neuroticism Latent Growth Curve } \\
\hline & \multicolumn{2}{|c|}{ Intercept } & \multicolumn{2}{|c|}{ Slope } & \multicolumn{2}{|c|}{ Intercept } & \multicolumn{2}{|c|}{ Slope } \\
\hline & $b$ & S.E. & $b$ & S.E. & $b$ & S.E. & $b$ & S.E. \\
\hline Estimate & $2.818 *$ & 0.474 & $0.038 *$ & 0.035 & $2.441 *$ & 0.701 & 0.035 & 0.016 \\
\hline Age & -0.014 & 0.007 & 0.000 & 0.000 & -0.005 & 0.011 & - & - \\
\hline Gender & 0.027 & 0.055 & 0.002 & 0.003 & -0.171 & 0.082 & - & - \\
\hline \multicolumn{9}{|l|}{$(0=$ Female $)$} \\
\hline Education & -0.003 & 0.026 & 0.001 & 0.002 & -0.092 & 0.039 & - & - \\
\hline Agreeableness & -0.026 & 0.031 & 0.000 & 0.002 & - & - & - & - \\
\hline Openness & 0.038 & 0.032 & 0.000 & 0.002 & - & - & - & - \\
\hline Extraversion & -0.008 & 0.037 & 0.002 & 0.002 & - & - & - & - \\
\hline Conscientiousness & -0.042 & 0.025 & -0.001 & 0.002 & - & - & - & - \\
\hline NSIs Intercept & - & - & -0.016 & 0.009 & - & - & -0.015 & 0.009 \\
\hline Neuroticism Intercept & - & - & 0.001 & 0.005 & - & - & -0.006 & 0.007 \\
\hline$R^{2}$ & .069 & & .107 & & .069 & & .482 & \\
\hline
\end{tabular}




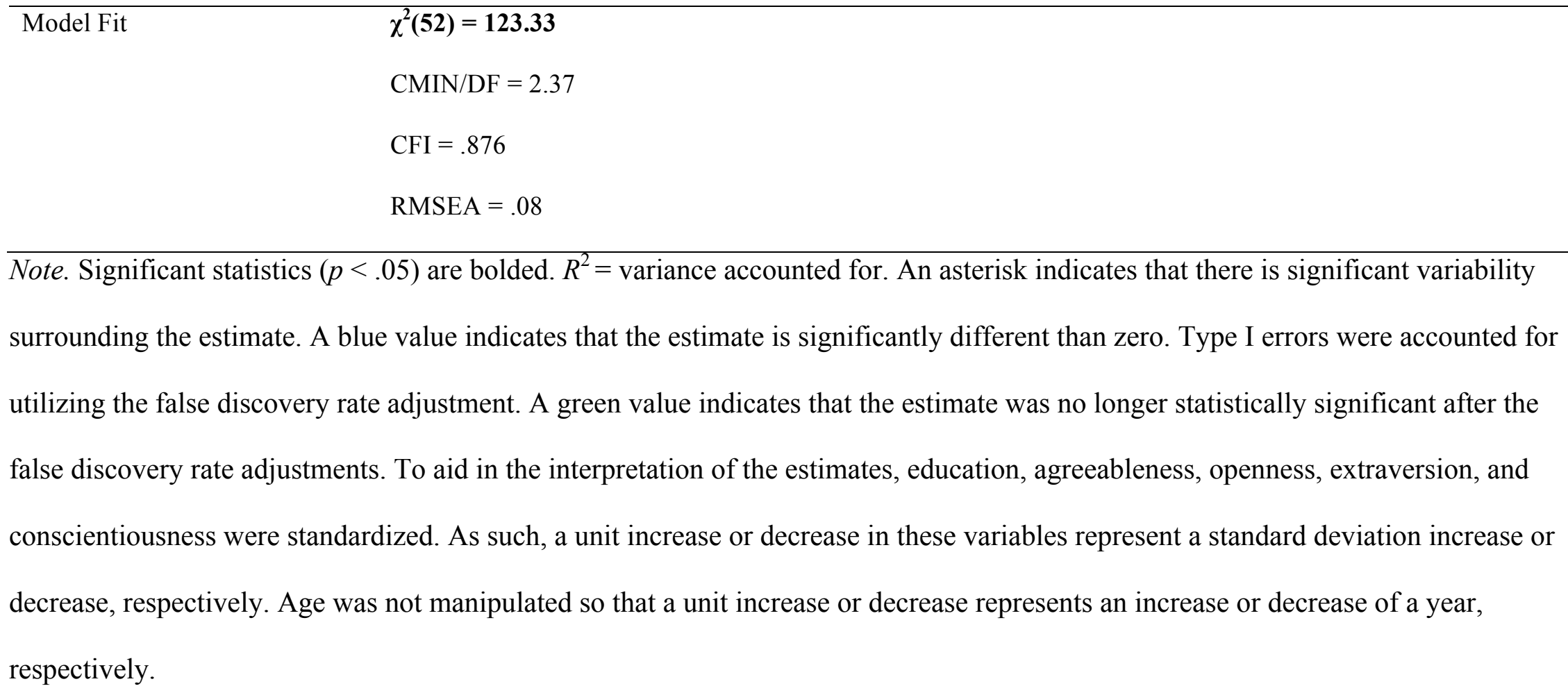


Table 36

Research Question 8 - Examination of the Bidirectional Association between the Latent Growth Curves of NSIs and Extraversion After Accounting for the Demographic Variables and Remaining Personality Traits for Younger Adults

\begin{tabular}{|c|c|c|c|c|c|c|c|c|}
\hline & \multicolumn{4}{|c|}{ NSIs Latent Growth Curve } & \multicolumn{4}{|c|}{ Extraversion Latent Growth Curve } \\
\hline & \multicolumn{2}{|c|}{ Intercept } & \multicolumn{2}{|c|}{ Slope } & \multicolumn{2}{|c|}{ Intercept } & \multicolumn{2}{|c|}{ Slope } \\
\hline & $b$ & S.E. & $b$ & S.E. & $b$ & S.E. & $b$ & S.E. \\
\hline Estimate & $1.959 *$ & 0.148 & 0.012 & 0.020 & $3.483 *$ & 0.227 & -0.024 & 0.020 \\
\hline Age & 0.004 & 0.004 & -0.001 & 0.000 & -0.008 & 0.007 & 0.000 & 0.000 \\
\hline Gender & -0.086 & 0.034 & 0.003 & 0.002 & -0.094 & 0.052 & 0.002 & 0.003 \\
\hline \multicolumn{9}{|l|}{$(0=$ Female $)$} \\
\hline Education & -0.014 & 0.018 & 0.001 & 0.001 & 0.002 & 0.027 & -0.001 & 0.001 \\
\hline Agreeableness & -0.002 & 0.018 & 0.000 & 0.001 & - & - & - & - \\
\hline Openness & 0.027 & 0.018 & -0.001 & 0.001 & - & - & - & - \\
\hline Neuroticism & 0.101 & 0.017 & -0.005 & 0.001 & - & - & - & - \\
\hline Conscientiousness & -0.044 & 0.017 & -0.001 & 0.001 & - & - & - & - \\
\hline NSIs Intercept & - & - & 0.010 & 0.007 & - & - & 0.002 & 0.005 \\
\hline Extraversion Intercept & - & - & -0.001 & 0.002 & - & - & -0.001 & 0.004 \\
\hline$R^{2}$ & .182 & & .495 & & .014 & & .055 & \\
\hline
\end{tabular}




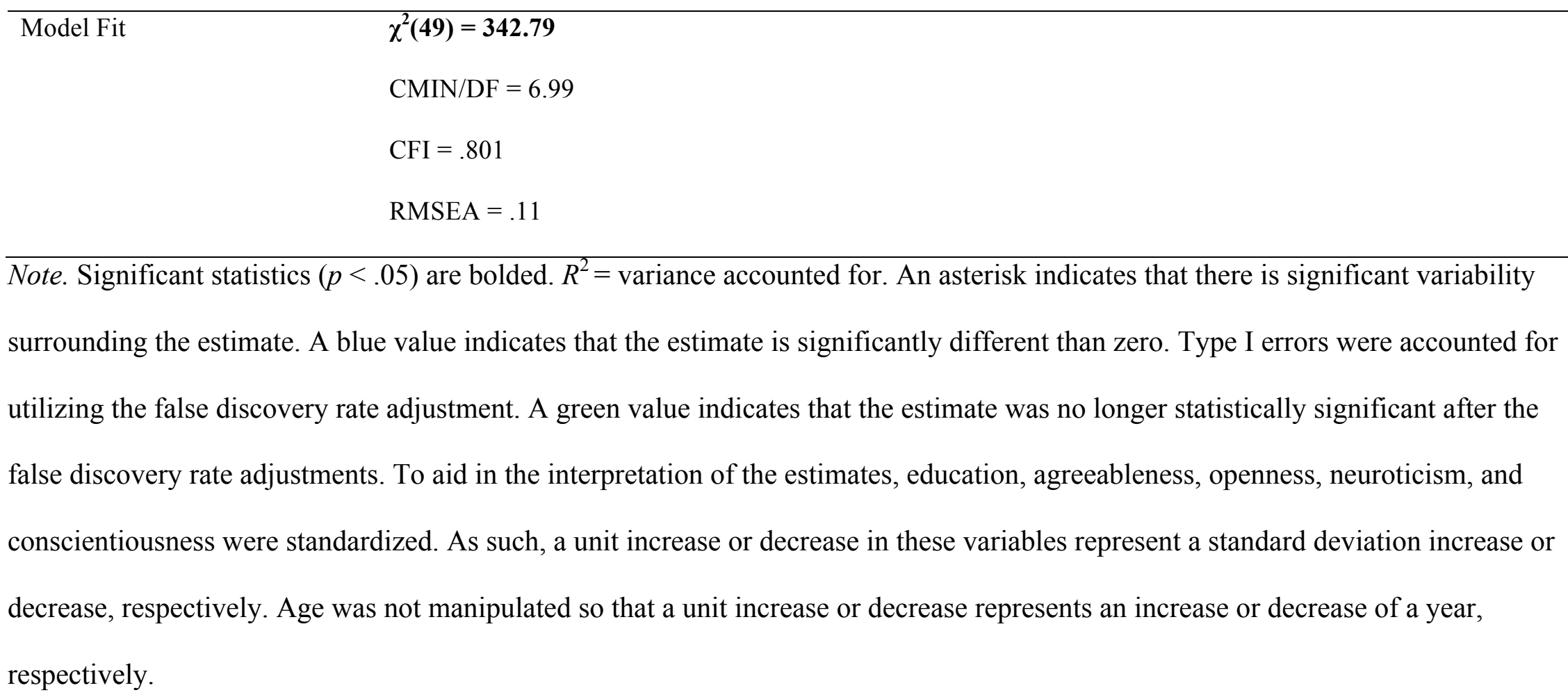


Table 37

Research Question 8 - Examination of the Bidirectional Association between the Latent Growth Curves of NSIs and Extraversion After Accounting for the Demographic Variables and Remaining Personality Traits for Middle-Aged Adults

\begin{tabular}{|c|c|c|c|c|c|c|c|c|}
\hline & \multicolumn{4}{|c|}{ NSIs Latent Growth Curve } & \multicolumn{4}{|c|}{ Extraversion Latent Growth Curve } \\
\hline & \multicolumn{2}{|c|}{ Intercept } & \multicolumn{2}{|c|}{ Slope } & \multicolumn{2}{|c|}{ Intercept } & \multicolumn{2}{|c|}{ Slope } \\
\hline & $b$ & S.E. & $b$ & S.E. & $b$ & S.E. & $b$ & S.E. \\
\hline Estimate & $2.546^{*}$ & 0.109 & $-0.037^{*}$ & 0.016 & $2.853 *$ & 0.159 & $0.015 *$ & 0.014 \\
\hline Age & -0.010 & 0.002 & 0.000 & 0.000 & 0.007 & 0.003 & 0.000 & 0.000 \\
\hline Gender & -0.080 & 0.024 & 0.000 & 0.002 & -0.056 & 0.035 & 0.001 & 0.002 \\
\hline \multicolumn{9}{|l|}{$(0=$ Female $)$} \\
\hline Education & 0.027 & 0.012 & -0.001 & 0.001 & -0.059 & 0.017 & 0.001 & 0.001 \\
\hline Agreeableness & -0.028 & 0.013 & 0.000 & 0.001 & - & - & - & - \\
\hline Openness & 0.049 & 0.013 & 0.000 & 0.000 & - & - & - & - \\
\hline Neuroticism & 0.105 & 0.013 & -0.002 & 0.001 & - & - & - & - \\
\hline Conscientiousness & -0.040 & 0.014 & 0.001 & 0.001 & - & - & - & - \\
\hline NSIs Intercept & - & - & 0.006 & 0.005 & - & - & -0.002 & 0.003 \\
\hline Extraversion Intercept & - & - & 0.002 & 0.002 & - & - & -0.003 & 0.002 \\
\hline$R^{2}$ & .194 & & .040 & & .027 & & .040 & \\
\hline
\end{tabular}




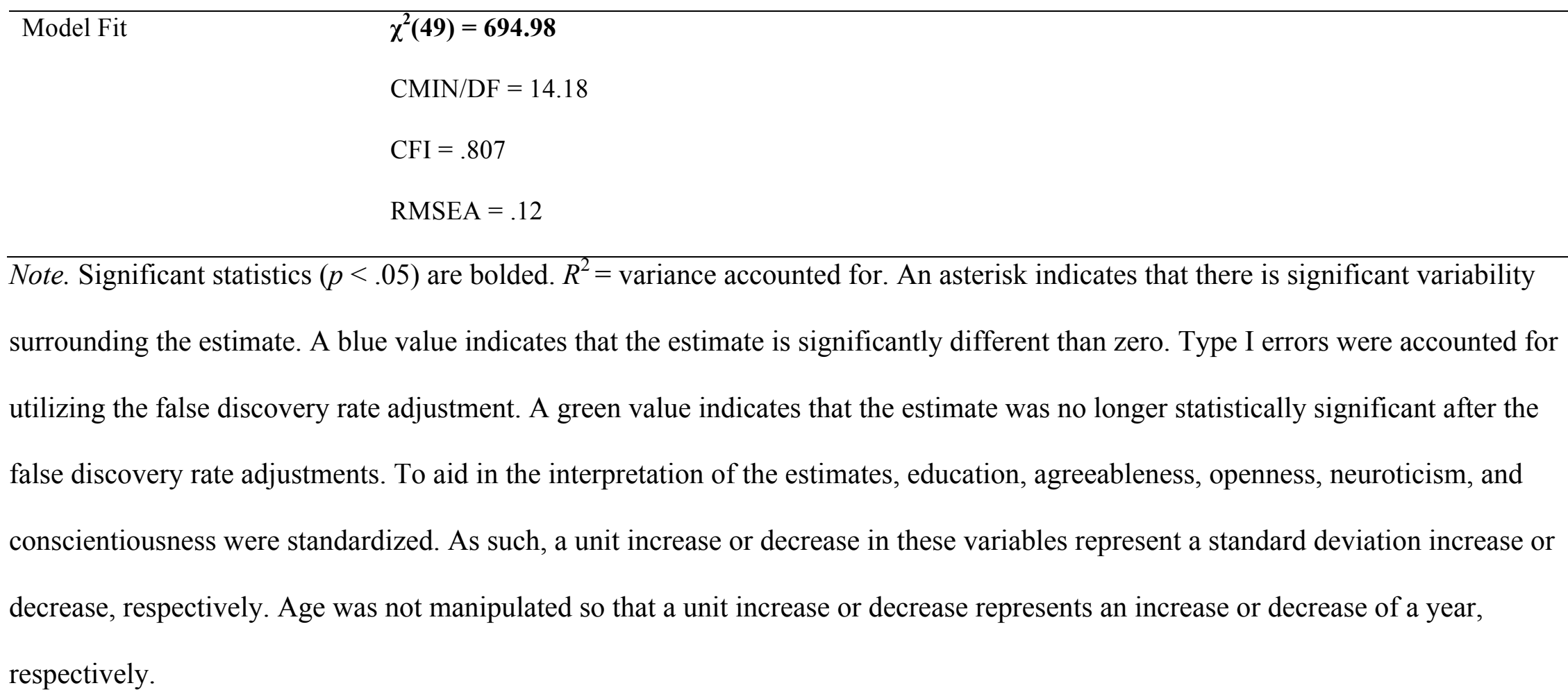


Table 38

Research Question 8 - Examination of the Bidirectional Association between the Latent Growth Curves of NSIs and Extraversion After Accounting for the Demographic Variables and Remaining Personality Traits for Older Adults

\begin{tabular}{|c|c|c|c|c|c|c|c|c|}
\hline & \multicolumn{4}{|c|}{ NSIs Latent Growth Curve } & \multicolumn{4}{|c|}{ Extraversion Latent Growth Curve } \\
\hline & \multicolumn{2}{|c|}{ Intercept } & \multicolumn{2}{|c|}{ Slope } & \multicolumn{2}{|c|}{ Intercept } & \multicolumn{2}{|c|}{ Slope } \\
\hline & $b$ & S.E. & $b$ & S.E. & $b$ & S.E. & $b$ & S.E. \\
\hline Estimate & $2.911 *$ & 0.466 & $0.027^{*}$ & 0.039 & $3.106 *$ & 0.700 & -0.019 & 0.024 \\
\hline Age & -0.016 & 0.007 & 0.000 & 0.000 & 0.002 & 0.011 & - & - \\
\hline Gender & 0.053 & 0.054 & 0.003 & 0.003 & 0.032 & 0.082 & - & - \\
\hline \multicolumn{9}{|l|}{$(0=$ Female $)$} \\
\hline Education & 0.008 & 0.026 & 0.001 & 0.002 & -0.091 & 0.039 & - & - \\
\hline Agreeableness & -0.018 & 0.026 & 0.001 & 0.002 & - & - & - & - \\
\hline Openness & 0.049 & 0.029 & 0.000 & 0.002 & - & - & - & - \\
\hline Neuroticism & 0.098 & 0.030 & 0.000 & 0.002 & - & - & - & - \\
\hline Conscientiousness & -0.035 & 0.026 & -0.001 & 0.002 & - & - & - & - \\
\hline NSIs Intercept & - & - & -0.015 & 0.008 & - & - & 0.000 & 0.007 \\
\hline Extraversion Intercept & - & - & 0.000 & 0.004 & - & - & 0.001 & 0.005 \\
\hline$R^{2}$ & .165 & & .097 & & .037 & & .003 & \\
\hline
\end{tabular}




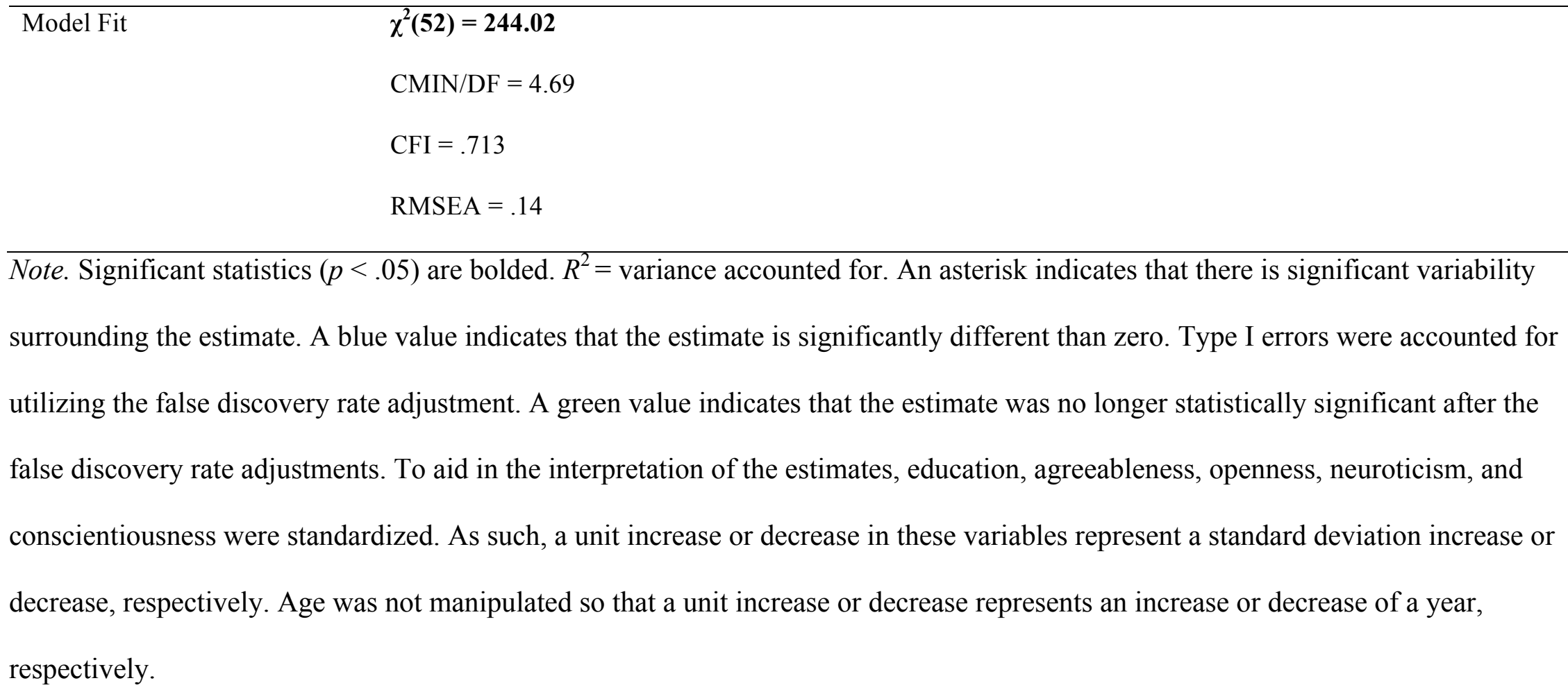


Table 39

Research Question 8 - Examination of the Bidirectional Association between the Latent Growth Curves of NSIs and

Conscientiousness After Accounting for the Demographic Variables and Remaining Personality Traits for Younger Adults

\begin{tabular}{|c|c|c|c|c|c|c|c|c|}
\hline & \multicolumn{4}{|c|}{ NSIs Latent Growth Curve } & \multicolumn{4}{|c|}{ Conscientiousness Latent Growth Curve } \\
\hline & \multicolumn{2}{|c|}{ Intercept } & \multicolumn{2}{|c|}{ Slope } & \multicolumn{2}{|c|}{ Intercept } & \multicolumn{2}{|c|}{ Slope } \\
\hline & $b$ & S.E. & $b$ & S.E. & $b$ & S.E. & $b$ & S.E. \\
\hline Estimate & $1.959 *$ & 0.150 & 0.016 & 0.027 & $3.630 *$ & 0.168 & 0.009 & 0.024 \\
\hline Age & 0.003 & 0.004 & -0.001 & 0.000 & -0.002 & 0.005 & 0.000 & 0.000 \\
\hline Gender & -0.062 & 0.035 & 0.003 & 0.002 & -0.212 & 0.039 & 0.003 & 0.002 \\
\hline \multicolumn{9}{|l|}{$(0=$ Female $)$} \\
\hline Education & -0.015 & 0.018 & 0.001 & 0.001 & 0.015 & 0.020 & 0.000 & 0.001 \\
\hline Agreeableness & 0.001 & 0.019 & 0.000 & 0.001 & - & - & - & - \\
\hline Openness & 0.023 & 0.019 & -0.001 & 0.001 & - & - & - & - \\
\hline Neuroticism & 0.100 & 0.017 & -0.005 & 0.001 & - & - & - & - \\
\hline Extraversion & -0.018 & 0.020 & 0.000 & 0.001 & - & - & - & - \\
\hline NSIs Intercept & - & - & 0.010 & 0.008 & - & - & -0.005 & 0.004 \\
\hline Conscientiousness Intercept & - & - & -0.002 & 0.004 & - & - & -0.002 & 0.005 \\
\hline$R^{2}$ & .139 & & .492 & & .092 & & .080 & \\
\hline
\end{tabular}




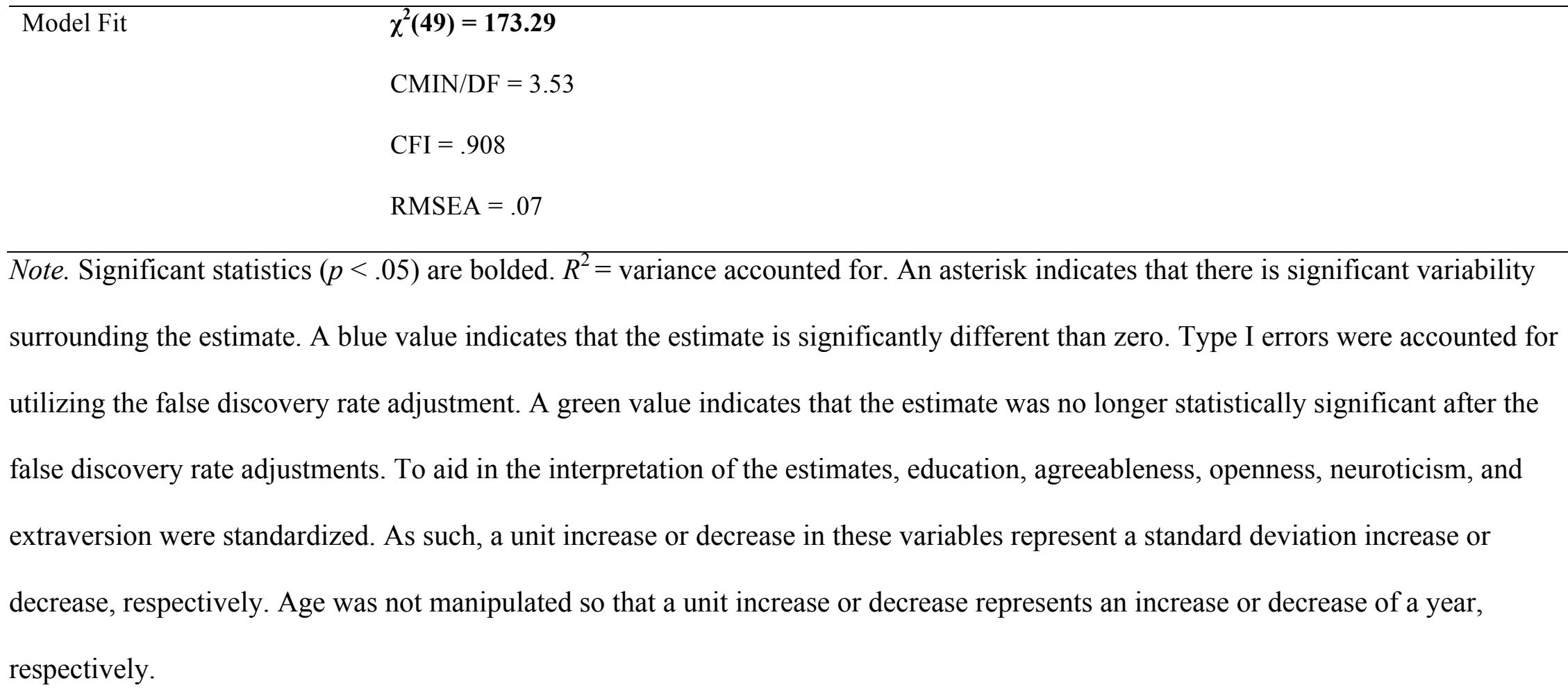


Table 40

Research Question 8 - Examination of the Bidirectional Association between the Latent Growth Curves of NSIs and

Conscientiousness After Accounting for the Demographic Variables and Remaining Personality Traits for Middle-Aged Adults

\begin{tabular}{|c|c|c|c|c|c|c|c|c|}
\hline & \multicolumn{4}{|c|}{ NSIs Latent Growth Curve } & \multicolumn{4}{|c|}{ Conscientiousness Latent Growth Curve } \\
\hline & \multicolumn{2}{|c|}{ Intercept } & \multicolumn{2}{|c|}{ Slope } & \multicolumn{2}{|c|}{ Intercept } & \multicolumn{2}{|c|}{ Slope } \\
\hline & $b$ & $S . E$. & $b$ & S.E. & $b$ & S.E. & $b$ & S.E. \\
\hline Estimate & $2.547^{*}$ & 0.110 & $-0.043 *$ & 0.020 & $3.550 *$ & 0.103 & $0.001 *$ & 0.001 \\
\hline Age & -0.010 & 0.002 & 0.000 & 0.000 & 0.000 & 0.002 & - & - \\
\hline Gender & -0.076 & 0.024 & 0.001 & 0.002 & $-\mathbf{0 . 0 8 9}$ & 0.023 & - & - \\
\hline \multicolumn{9}{|l|}{$(0=$ Female $)$} \\
\hline Education & 0.023 & 0.012 & $-\mathbf{0 . 0 0 2}$ & 0.001 & 0.043 & 0.011 & - & - \\
\hline Agreeableness & -0.043 & 0.015 & 0.000 & 0.001 & - & - & - & - \\
\hline Openness & 0.040 & 0.014 & 0.000 & 0.001 & - & - & - & - \\
\hline Neuroticism & 0.104 & 0.012 & -0.002 & 0.001 & - & - & - & - \\
\hline Extraversion & -0.001 & 0.015 & 0.000 & 0.001 & - & - & - & - \\
\hline NSIs Intercept & - & - & 0.006 & 0.005 & - & - & - & - \\
\hline Conscientiousness Intercept & - & - & 0.004 & 0.003 & - & - & - & - \\
\hline$R^{2}$ & .171 & & .098 & & .038 & & .000 & \\
\hline
\end{tabular}




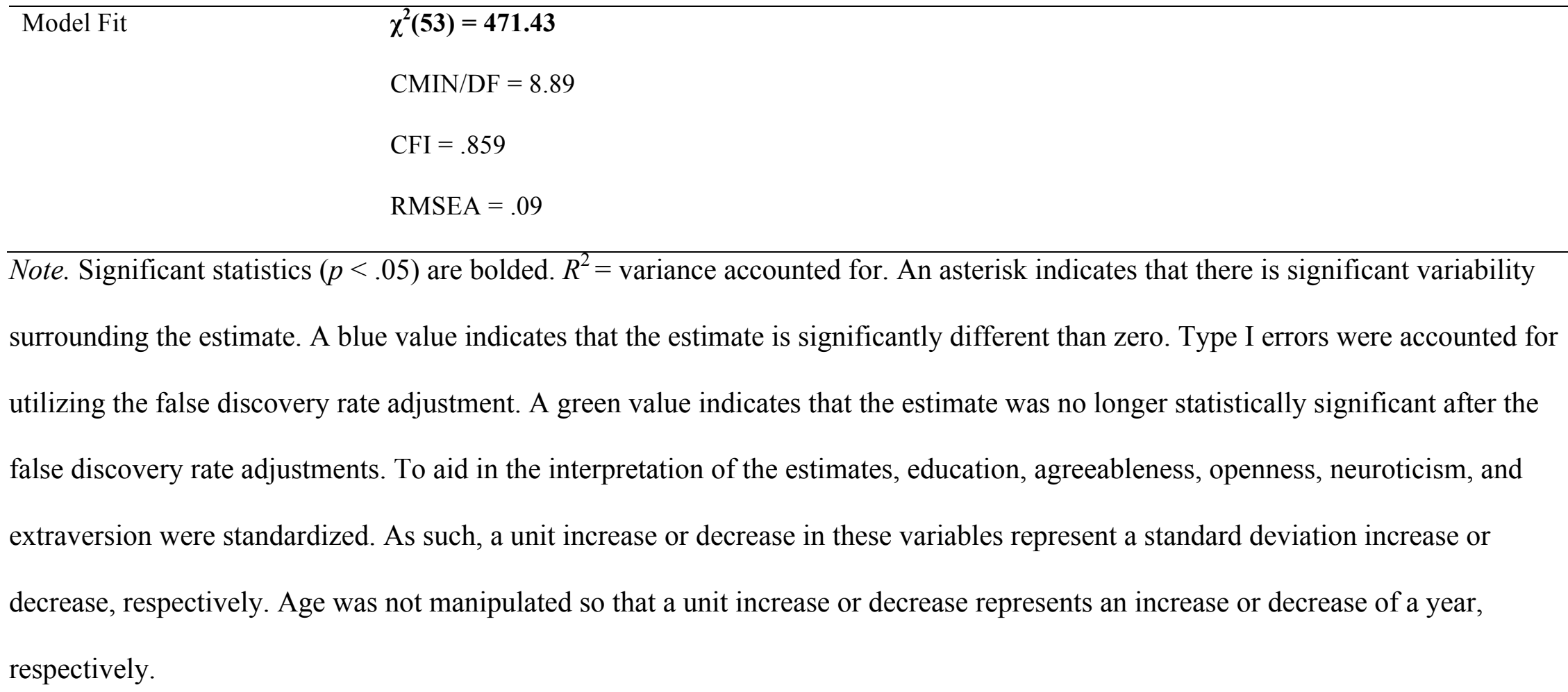


Table 41

Research Question 8 - Examination of the Bidirectional Association between the Latent Growth Curves of NSIs and Conscientiousness After Accounting for the Demographic Variables and Remaining Personality Traits for Older Adults

\begin{tabular}{|c|c|c|c|c|c|c|c|c|}
\hline & \multicolumn{4}{|c|}{ NSIs Latent Growth Curve } & \multicolumn{4}{|c|}{ Conscientiousness Latent Growth Curve } \\
\hline & \multicolumn{2}{|c|}{ Intercept } & \multicolumn{2}{|c|}{ Slope } & \multicolumn{2}{|c|}{ Intercept } & \multicolumn{2}{|c|}{ Slope } \\
\hline & $b$ & S.E. & $b$ & S.E. & $b$ & S.E. & $b$ & S.E. \\
\hline Estimate & $2.874^{*}$ & 0.472 & $0.060^{*}$ & 0.046 & $3.703 *$ & 0.593 & 0.039 & 0.052 \\
\hline Age & -0.015 & 0.007 & 0.000 & 0.000 & -0.003 & 0.009 & 0.000 & 0.001 \\
\hline Gender & 0.056 & 0.055 & 0.002 & 0.003 & -0.064 & 0.069 & 0.002 & 0.004 \\
\hline \multicolumn{9}{|l|}{$(0=$ Female $)$} \\
\hline Education & 0.007 & 0.026 & 0.001 & 0.002 & -0.003 & 0.033 & 0.000 & 0.002 \\
\hline Agreeableness & -0.021 & 0.031 & 0.000 & 0.002 & - & - & - & - \\
\hline Openness & 0.045 & 0.032 & 0.000 & 0.002 & - & - & - & - \\
\hline Neuroticism & 0.097 & 0.029 & 0.000 & 0.002 & - & - & - & - \\
\hline Extraversion & -0.010 & 0.038 & 0.002 & 0.002 & - & - & - & - \\
\hline NSIs Intercept & - & - & -0.016 & 0.008 & - & - & -0.002 & 0.007 \\
\hline Conscientiousness Intercept & - & - & -0.004 & 0.006 & - & - & -0.008 & 0.008 \\
\hline$R^{2}$ & .137 & & .118 & & .008 & & .063 & \\
\hline
\end{tabular}




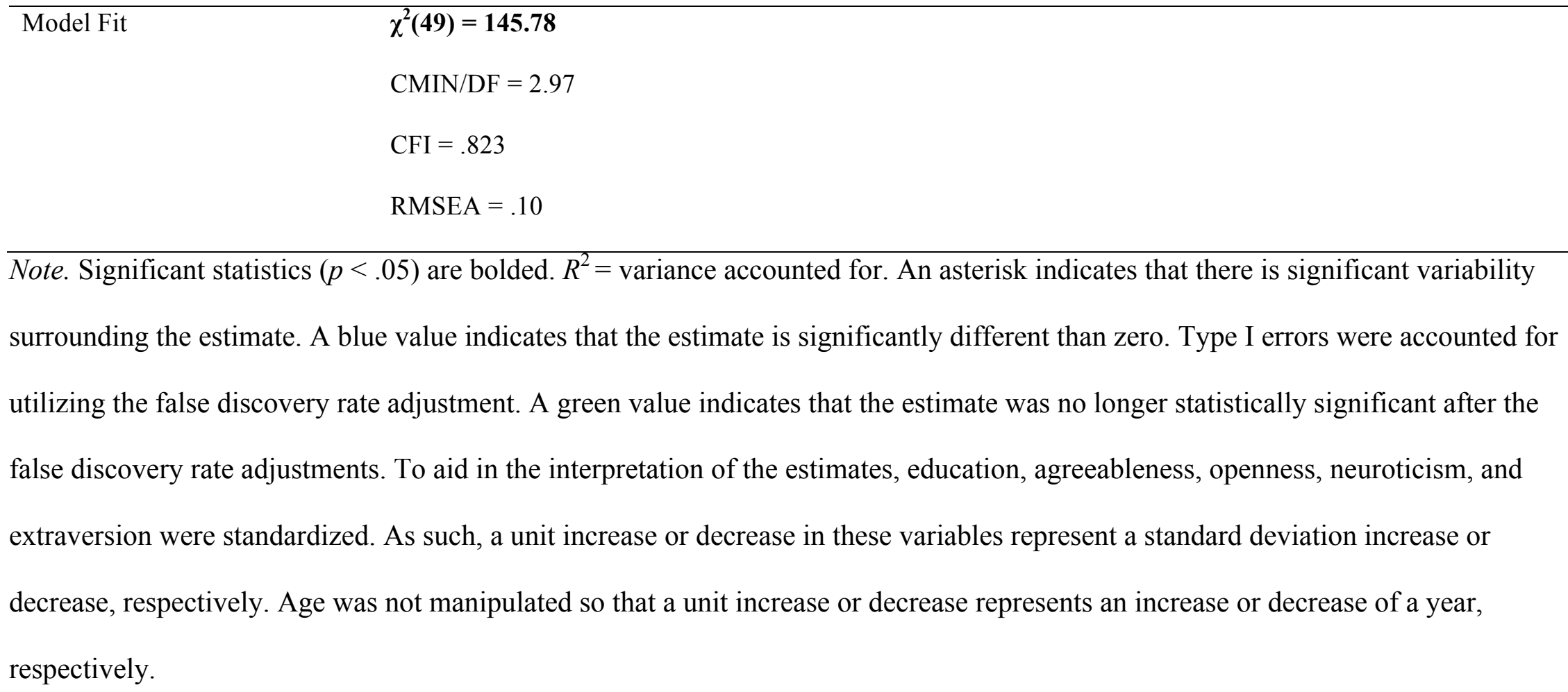


Table 42

The Percentage of Variance Accounted for the Intercepts and Slopes of the Latent Growth Curves across all Research Question

\begin{tabular}{lcc}
\hline & $\begin{array}{c}\text { Highest percentage of variance } \\
\text { accounted for in the intercept of: }\end{array}$ & $\begin{array}{c}\text { Highest percentage of variance } \\
\text { accounted for in the slope of: }\end{array}$ \\
\hline NSIs & $21.60 \%$ & $58.10 \%$ \\
Agreeableness & $18.80 \%$ & $41.90 \%$ \\
Openness & $6.60 \%$ & $16.10 \%$ \\
Neuroticism & $17.00 \%$ & $48.20 \%$ \\
Extraversion & $2.80 \%$ & $5.50 \%$ \\
Conscientiousness & $13.30 \%$ & $12.20 \%$ \\
\hline
\end{tabular}




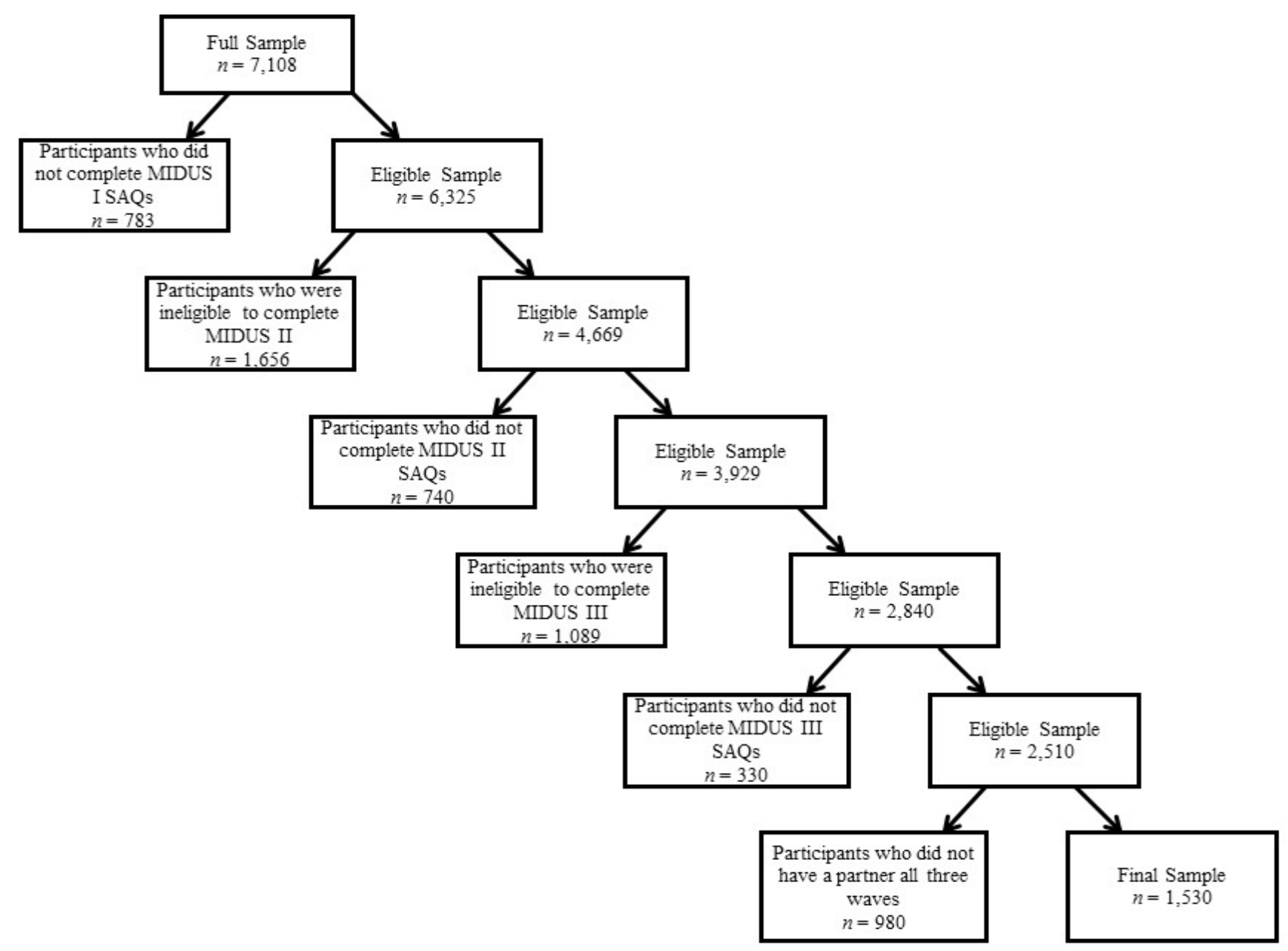

Figure 1. Sample attrition flow chart. "Ineligible participants" was defined as participants who died, were unable to participate for health or other related reasons, and participants who no longer resided in the United States. 


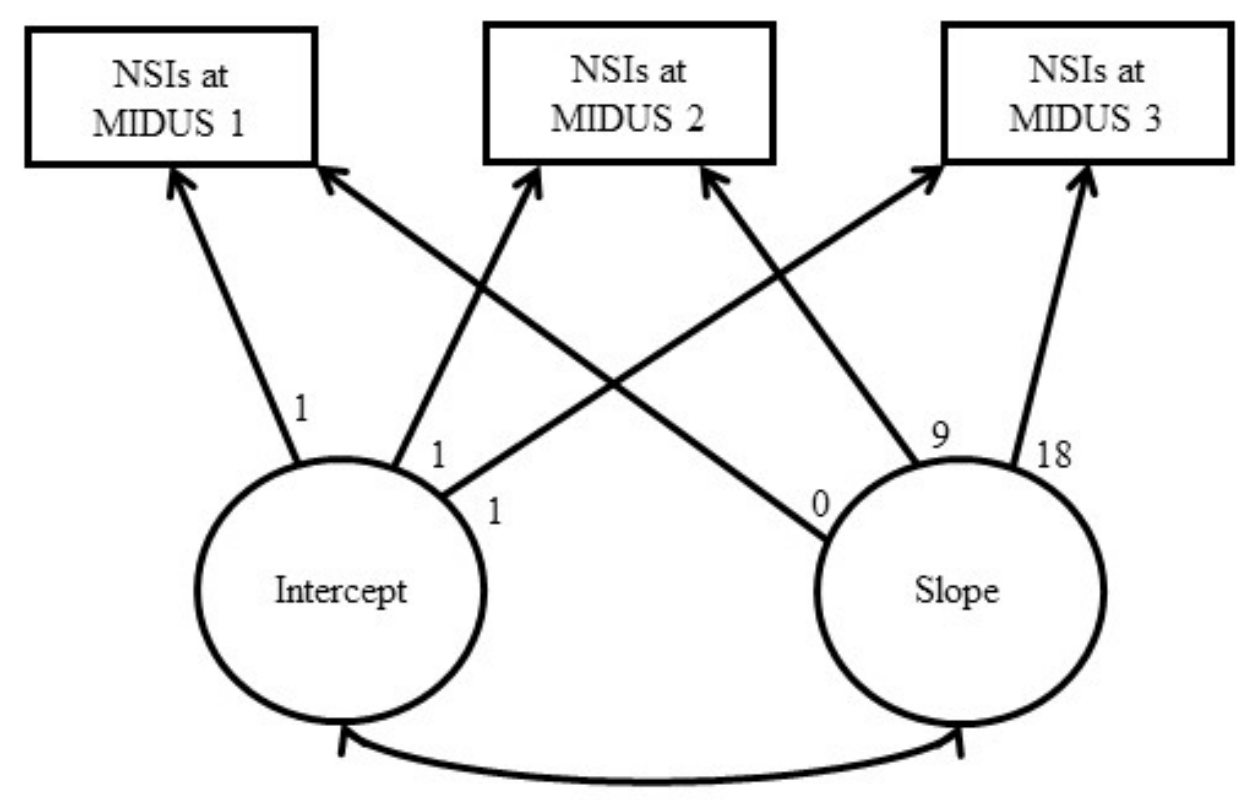

Figure 2. The univariate growth curve of NSIs. This univariate latent growth curve allows for the examination of the reported frequency of NSIs at MIDUS 1 (i.e., the intercept), whether NSIs exhibit mean-level increases or decreases over time (i.e., the slope), the association between the intercept and the slope (i.e., the covariance; e.g., whether individuals who reported experiencing higher levels of NSIs report steeper declines relative to other participants) and whether there are interindividual differences regarding the intercept and slope (Duncan \& Duncan, 2004). Five additional latent growth curves were modeled for the individual personality traits. 


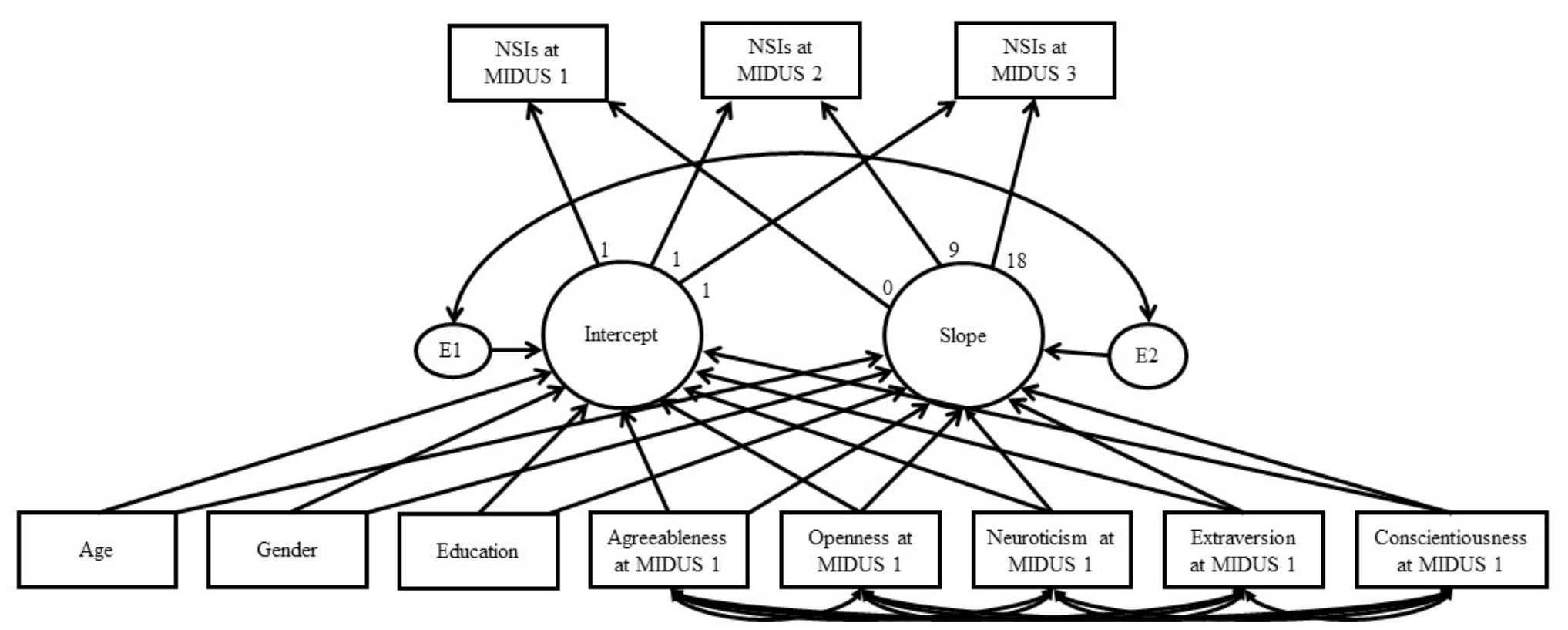

Figure 3. Demographic variables and the Big Five personality traits predicting the latent growth curve of NSIs. To aid in model estimation and with the interpretation of the estimates, the predictor variables of education and the Big Five personality traits were standardized. As such, a unit increase or decrease in these variables represent a standard deviation increase or decrease, respectively. Age was not manipulated so that a unit increase or decrease represents an increase or decrease of a year, respectively. Five additional latent growth curves were modeled for the individual personality traits, where the demographic variables and NSIs were the predictors. 


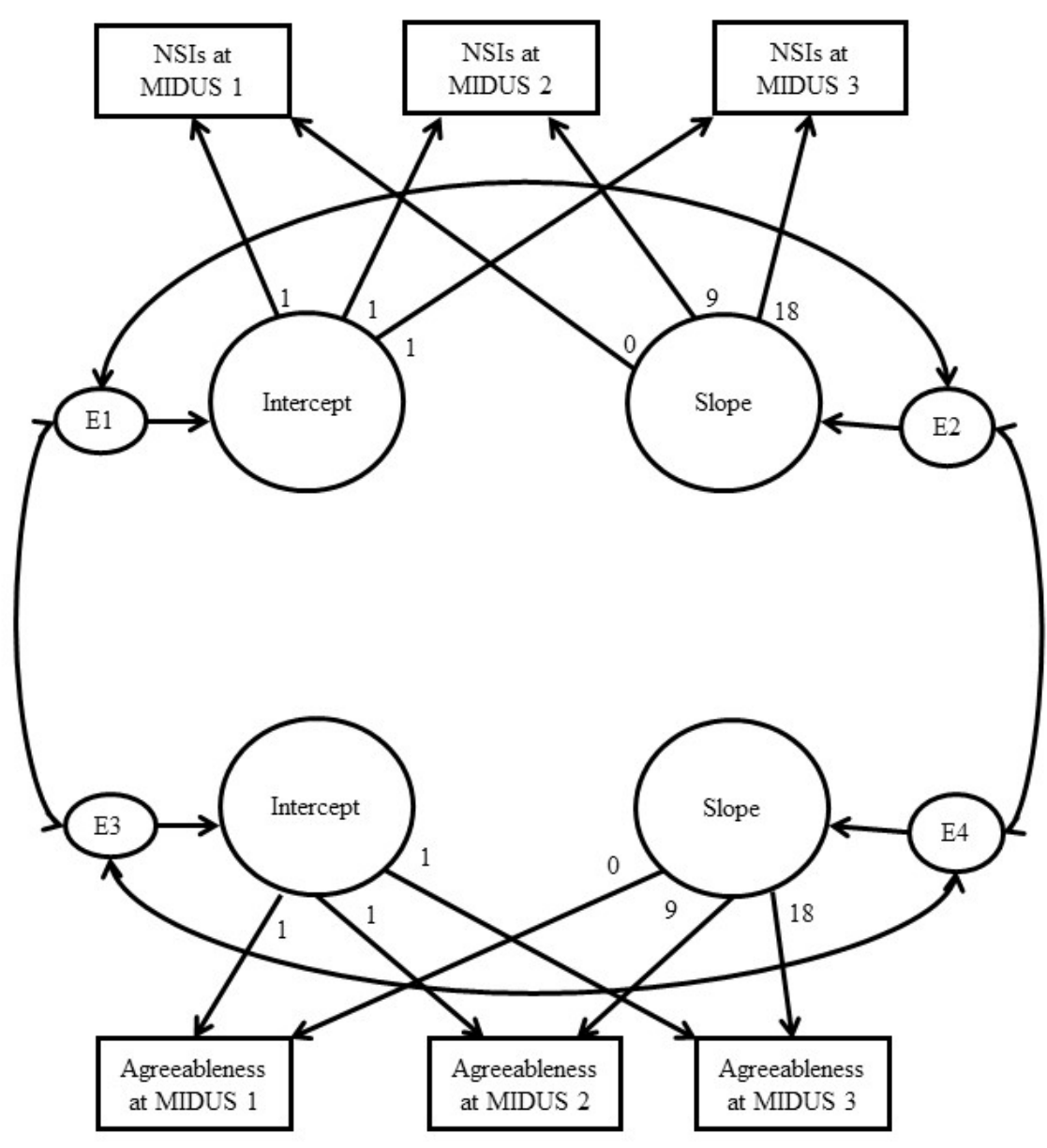

Figure 4. A preliminary model to examine whether the intercepts, slopes, and variances of NSIs and agreeableness were significant after accounting for the shared variance among the variables. Four additional preliminary models were analyzed to account for the shared variance among the remaining four personality traits and NSIs. 


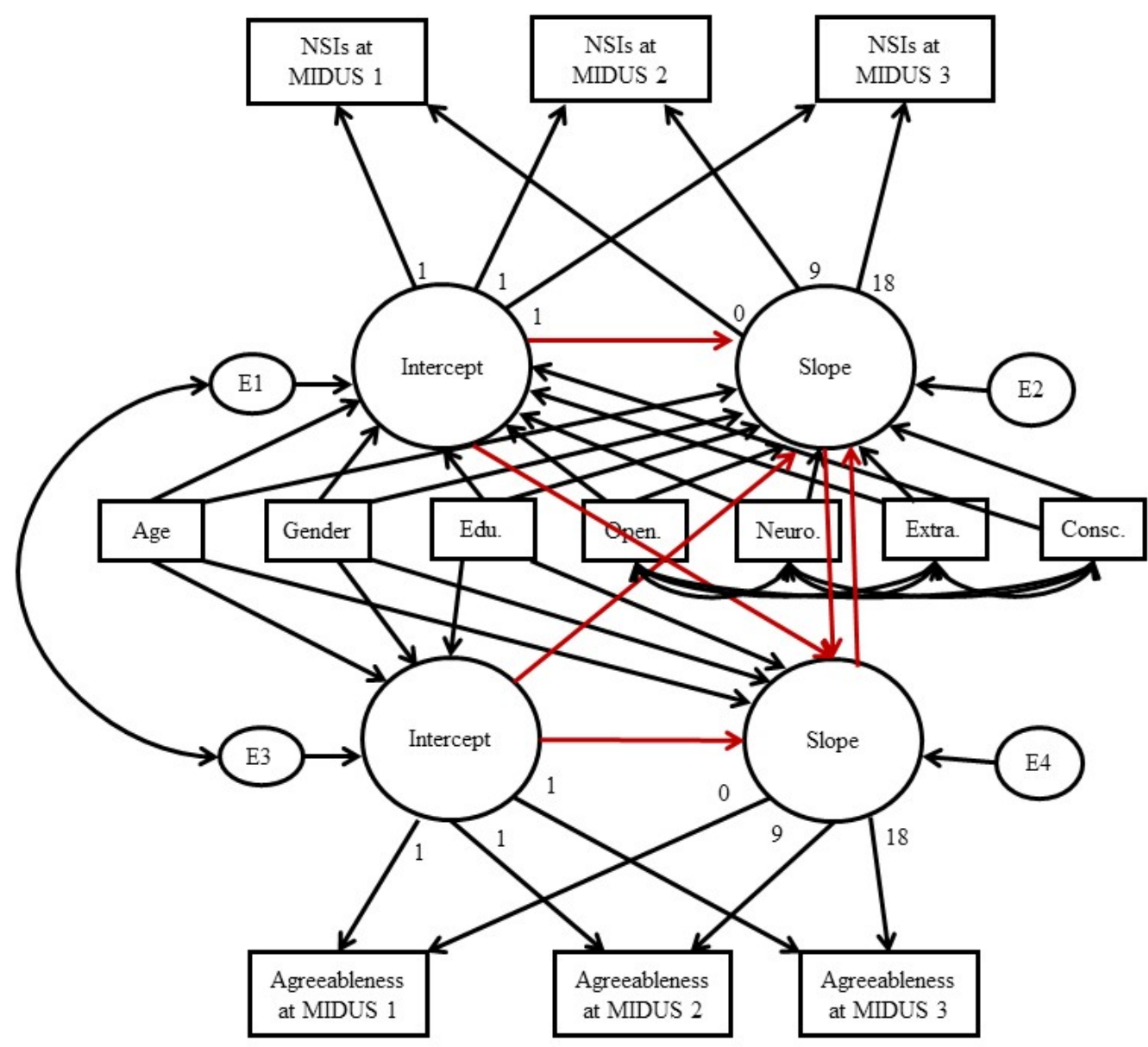

Figure 5. A cross-domain latent growth curve. In addition to the parameters examined previously, cross-domain latent growth curves allow for the prediction of (a) slope from intercept (i.e., whether the initial frequency of NSIs predicts rate of change for NSIs or whether the initial level of a personality trait predicts rate of change for that personality trait); (b) the rate of change in agreeableness from the initial frequency of NSIs; (c) the rate of change in NSIs from the initial levels of agreeableness; and (d) the rate of change in NSIs from the rate of change in the agreeableness and vice versa (highlighted in red; Willet \& Sayer, 1996). Four additional crossdomain latent growth curves were analyzed to examine the associations among NSIs and the remaining four personality traits. 


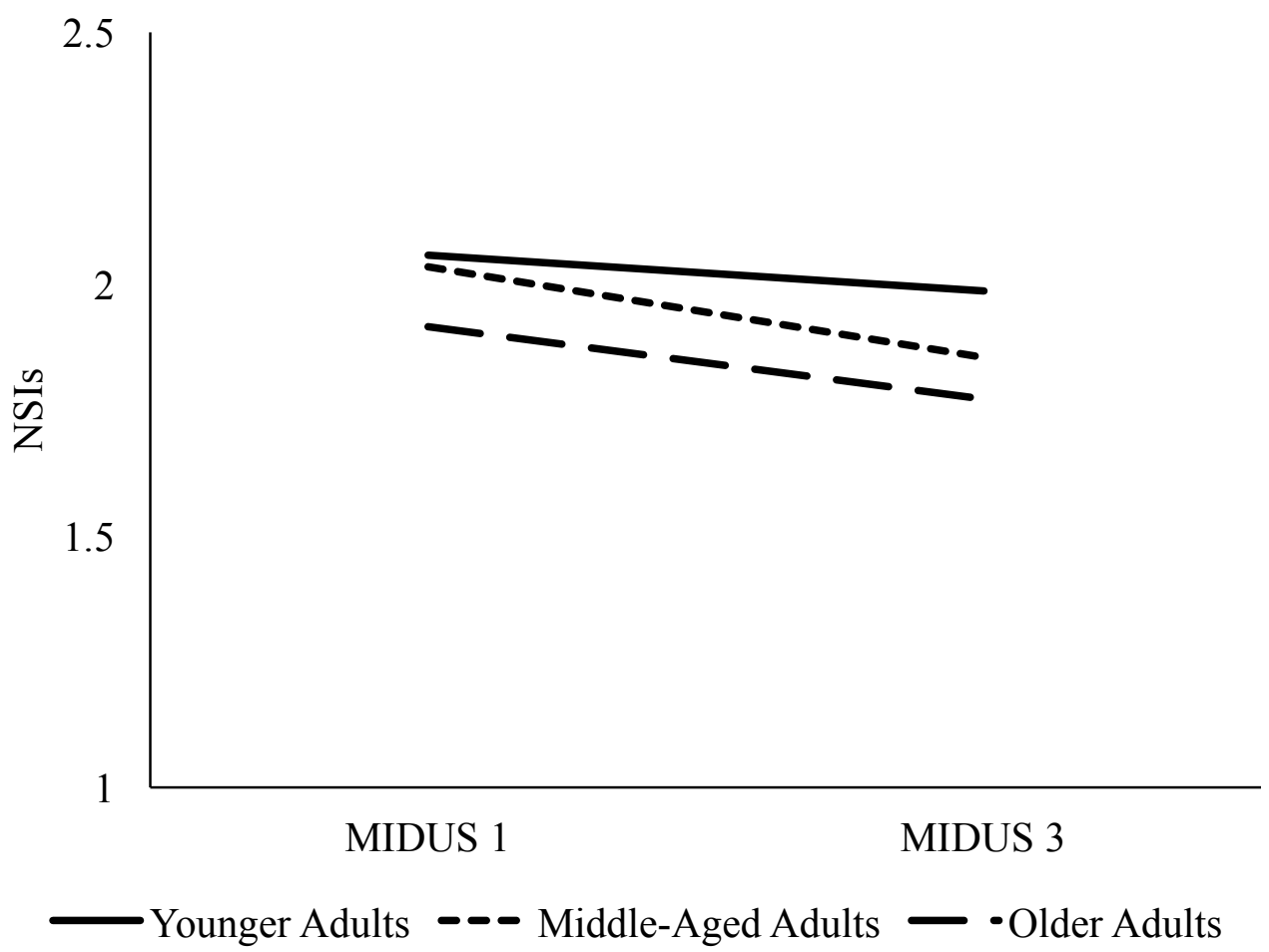

Figure 6. The trajectory of NSIs over 18 years for the younger adults (18 - 39 years old), middle-aged adults $(40-59$ years old $)$, and older adults (60+ years). The Likert-type scale of NSIs ranged from $1-4$, however, the scaling of the graph was modified because the average reported frequency of NSIs did not exceed 2.50. 


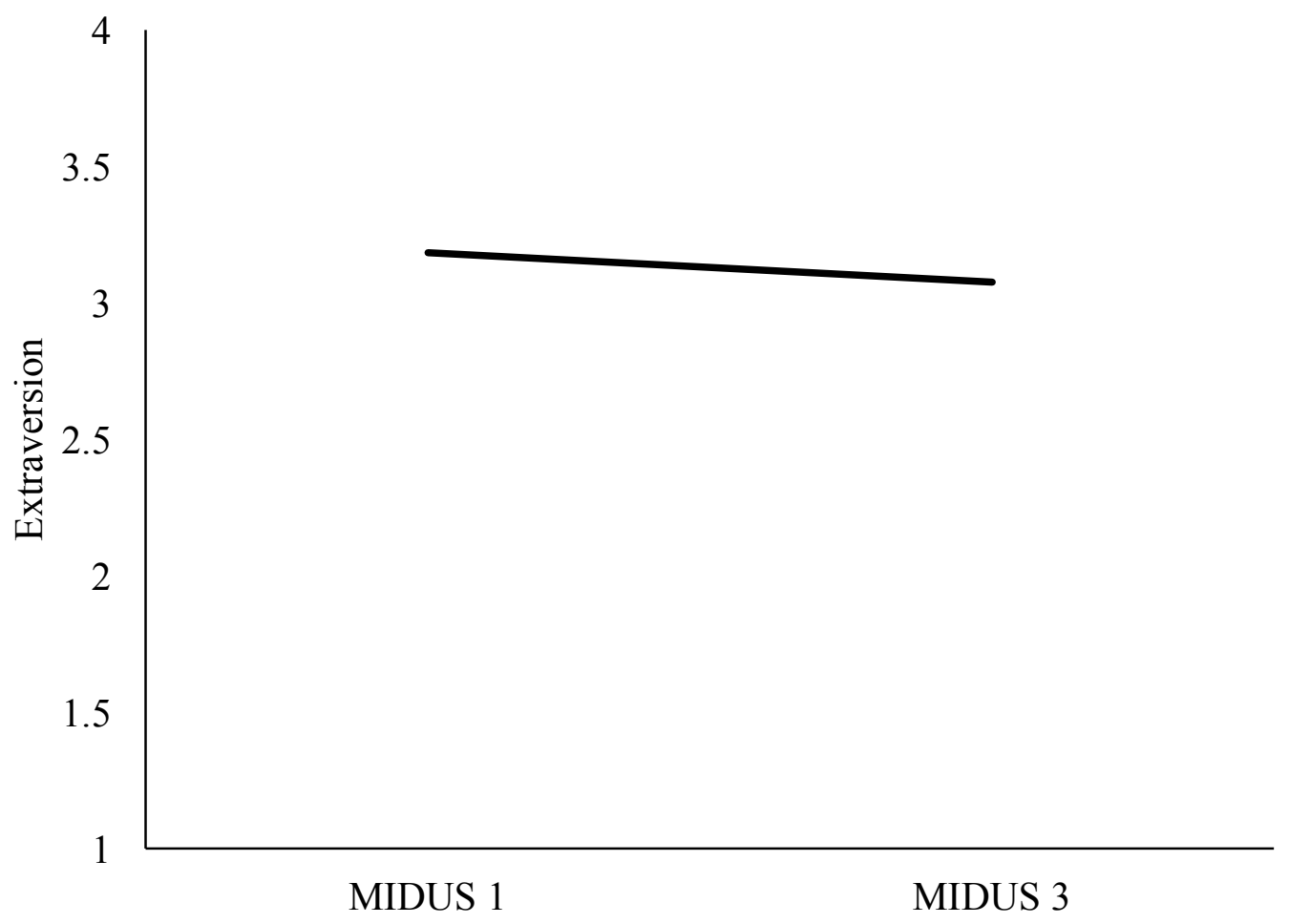

Figure 7. The trajectory of extraversion over 18 years for the entire sample. 


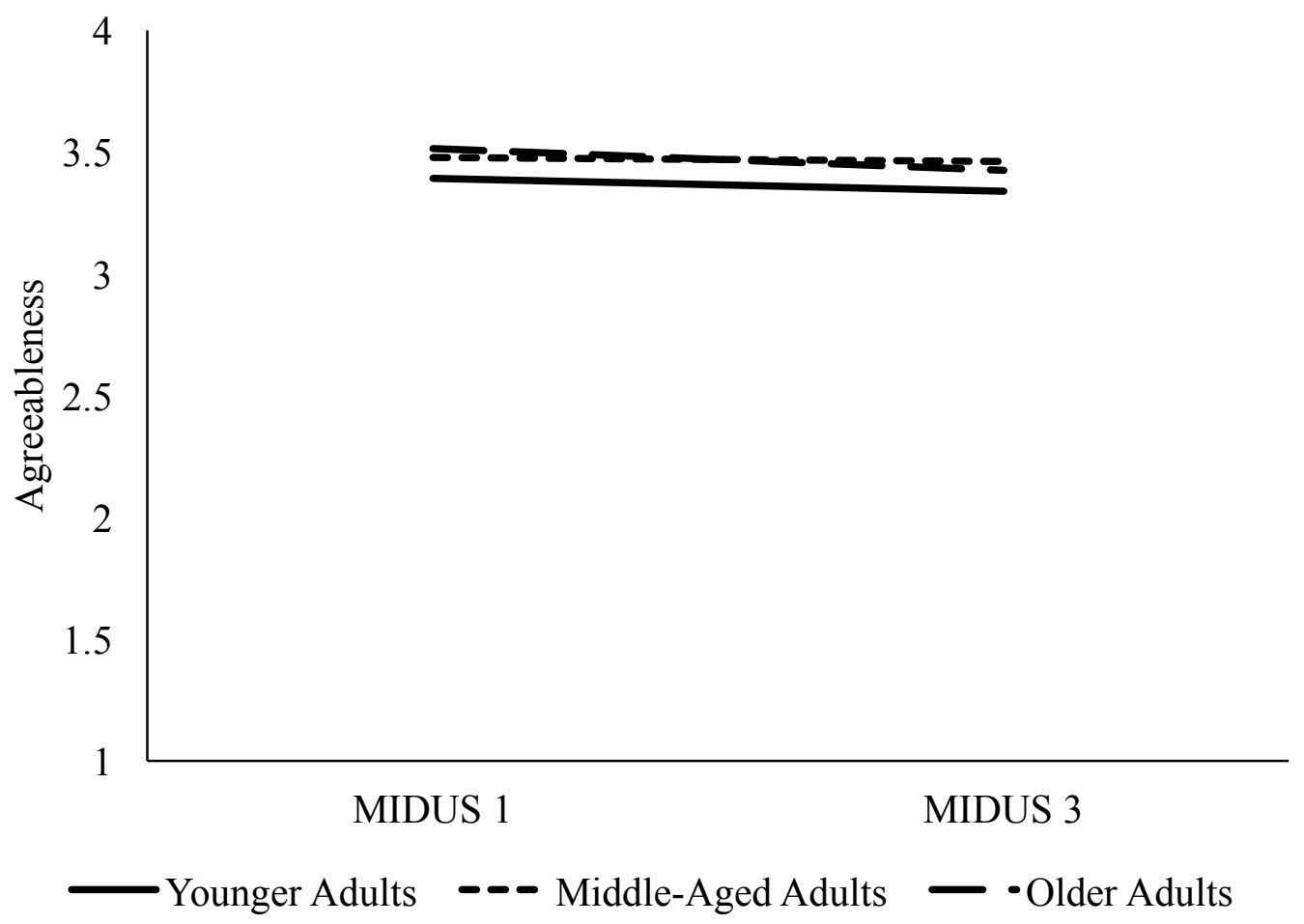

Figure 8 . The trajectory of agreeableness over 18 years for the younger adults $(18-39$ years old), middle-aged adults (40 - 59 years old), and older adults $(60+$ years $)$. 


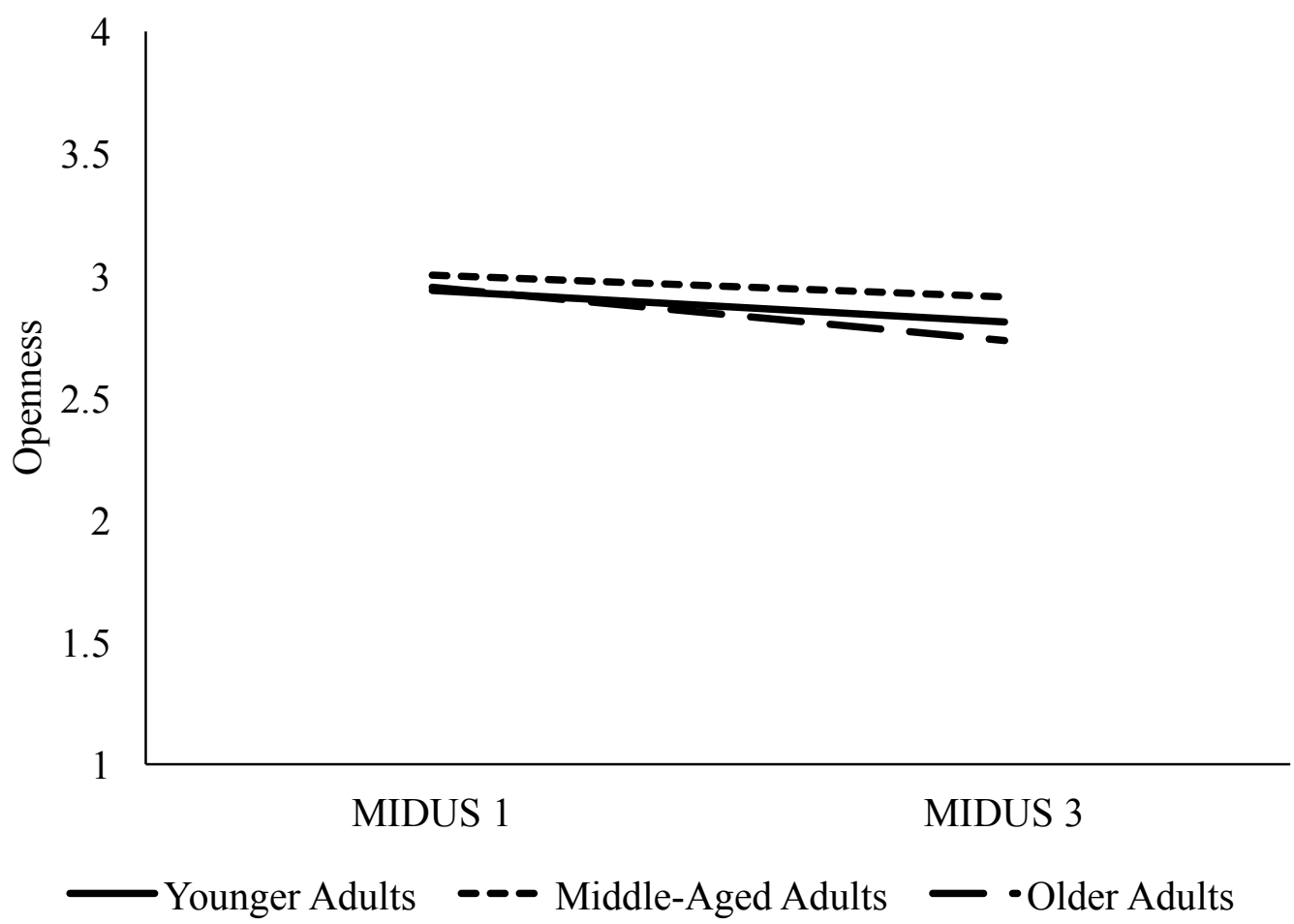

Figure 9. The trajectory of openness over 18 years for the younger adults $(18-39$ years old), middle-aged adults ( $40-59$ years old), and older adults (60+ years). 


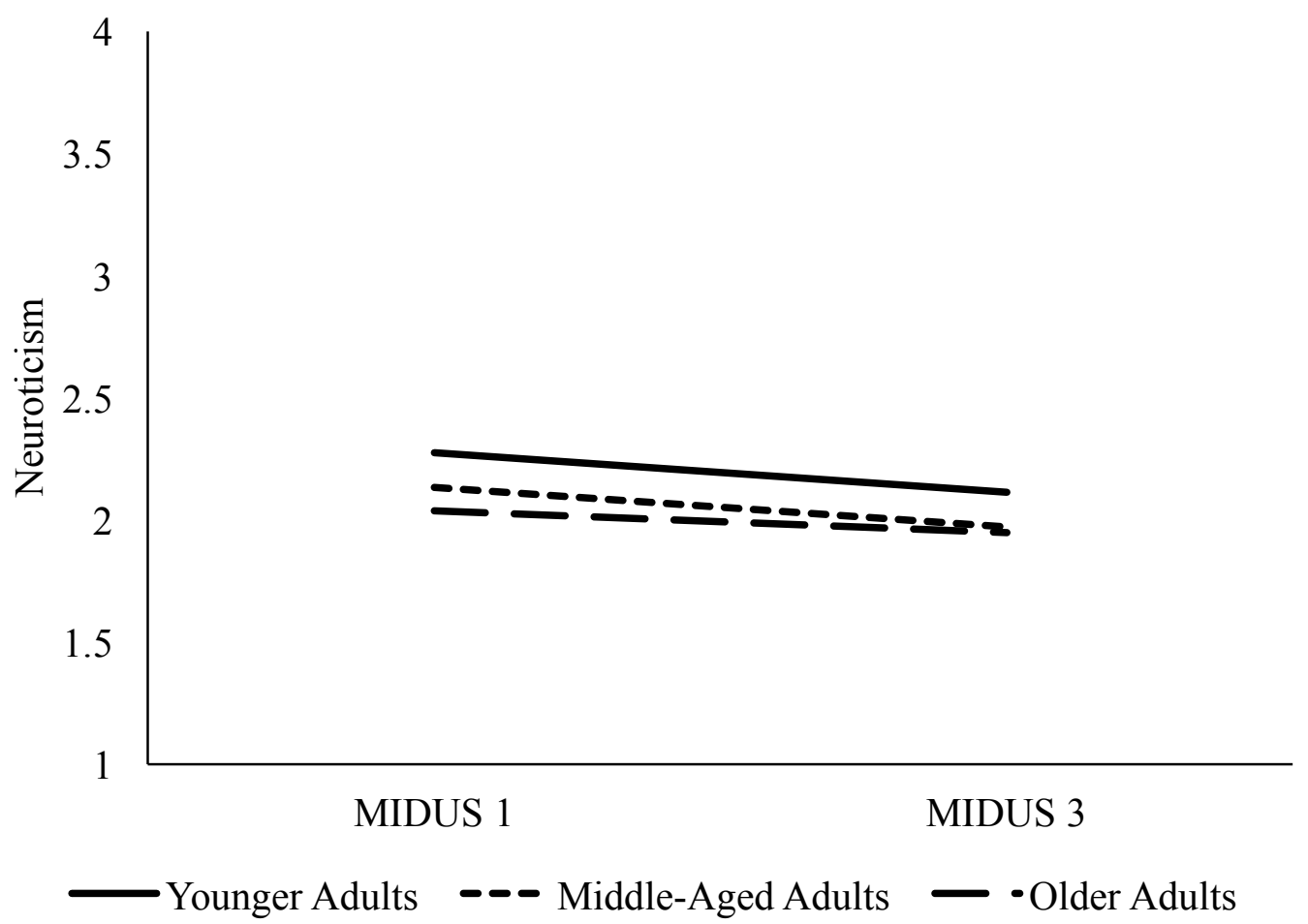

Figure 10. The trajectory of neuroticism over 18 years for the younger adults (18 - 39 years old), middle-aged adults ( $40-59$ years old), and older adults (60+ years). 


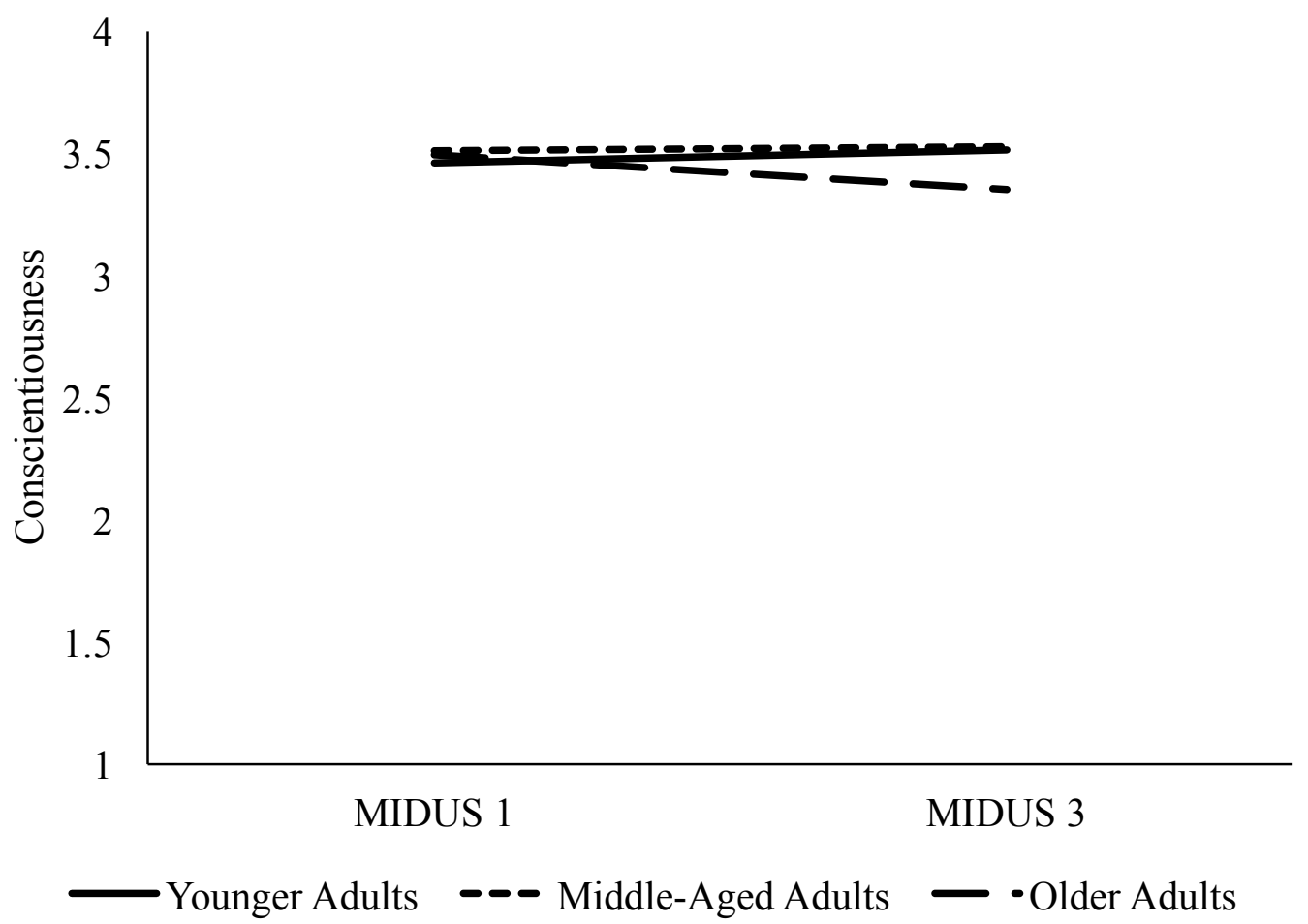

Figure 11. The trajectory of conscientiousness over 18 years for the younger adults $(18-39$ years old), middle-aged adults ( $40-59$ years old), and older adults (60+ years). 


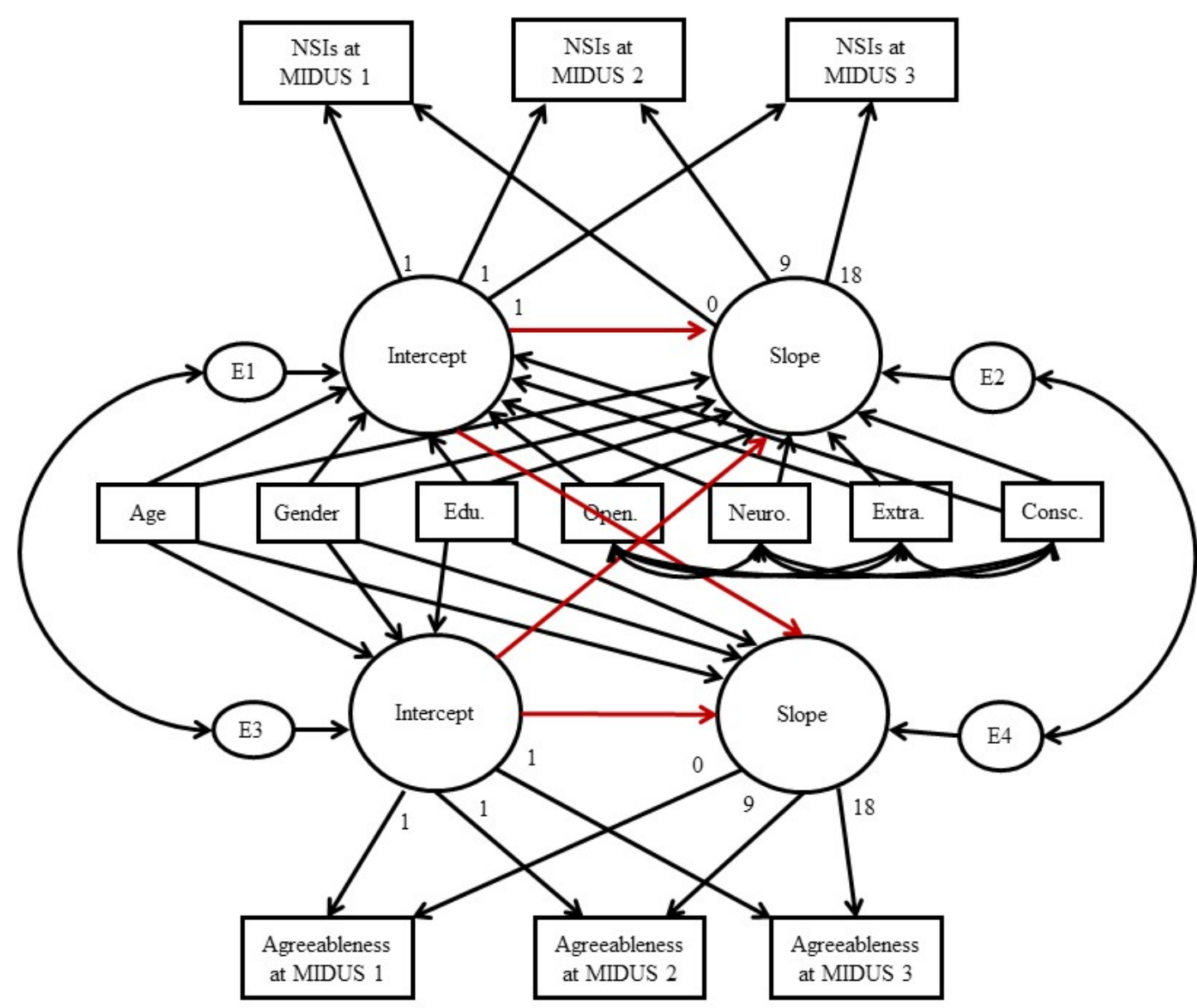

Figure 12. A modified cross-domain latent growth curve. The originally proposed cross-domain latent growth curves would not converge when the parameters of slope predicting slope were included in the model, even after multiple attempts to modify the models. 


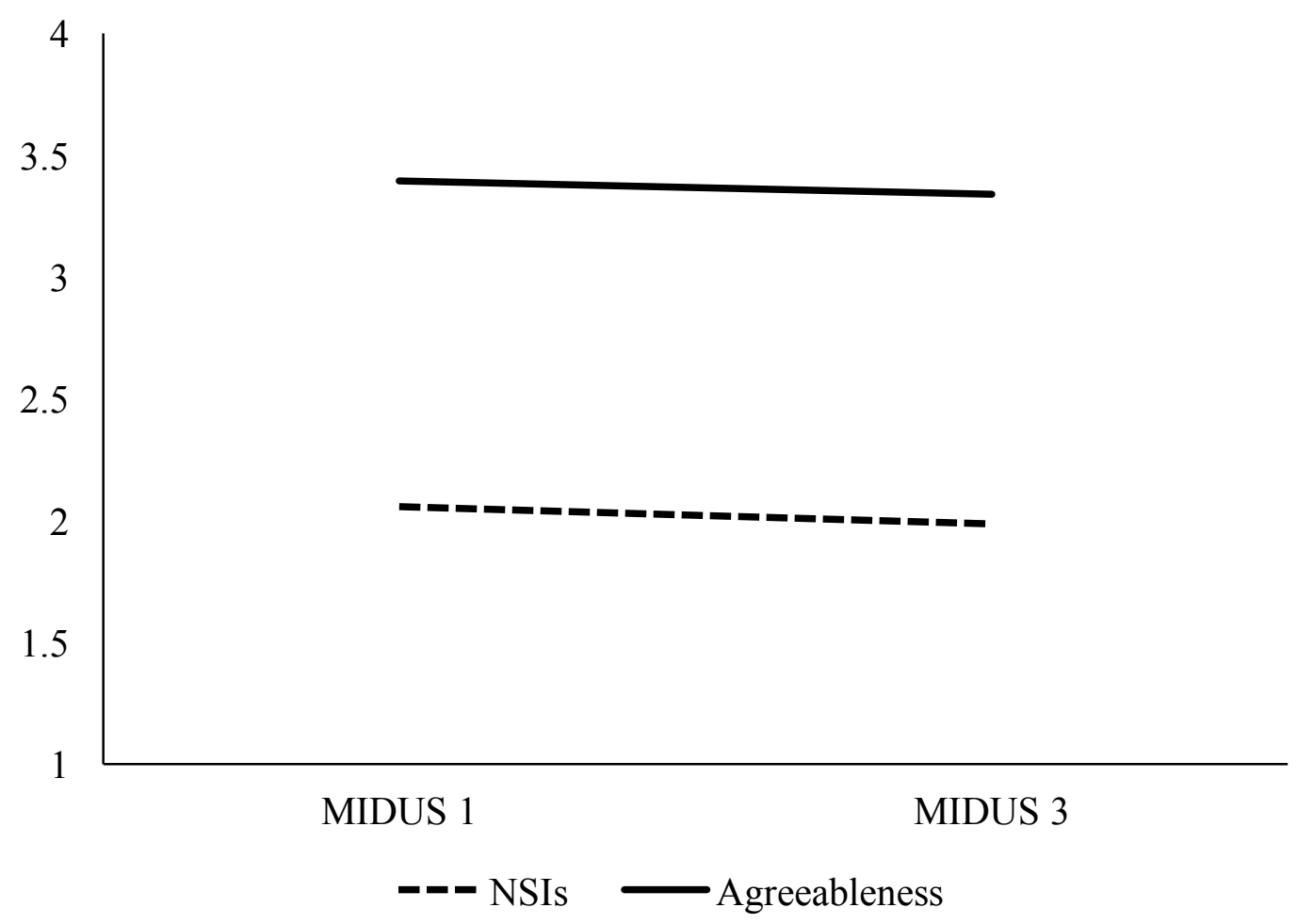

Figure 13. The trajectories of NSIs and agreeableness over 18 years for the younger adults (1839 years old). 


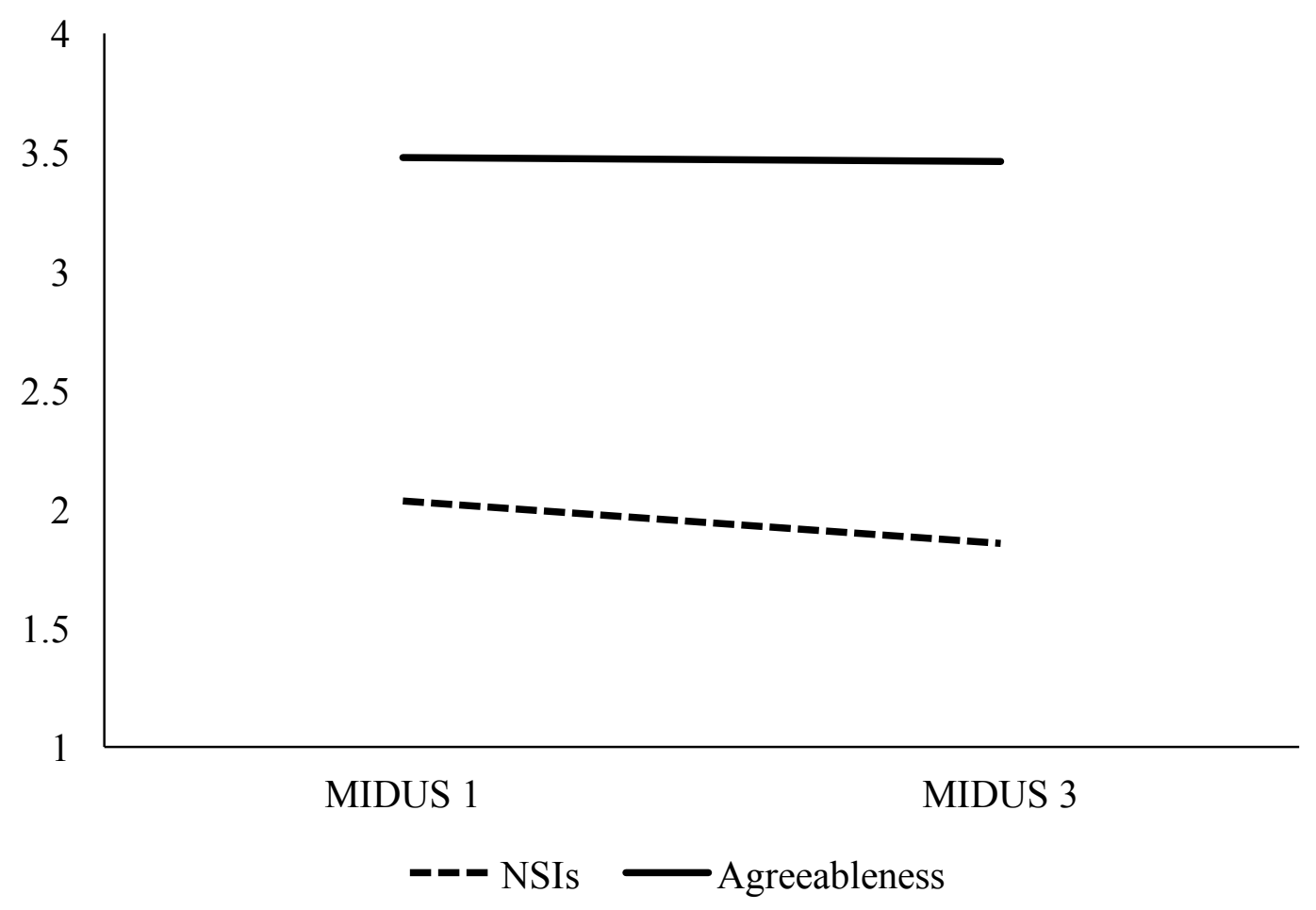

Figure 14. The trajectories of NSIs and agreeableness over 18 years for the middle-aged adults (40 - 59 years old). 


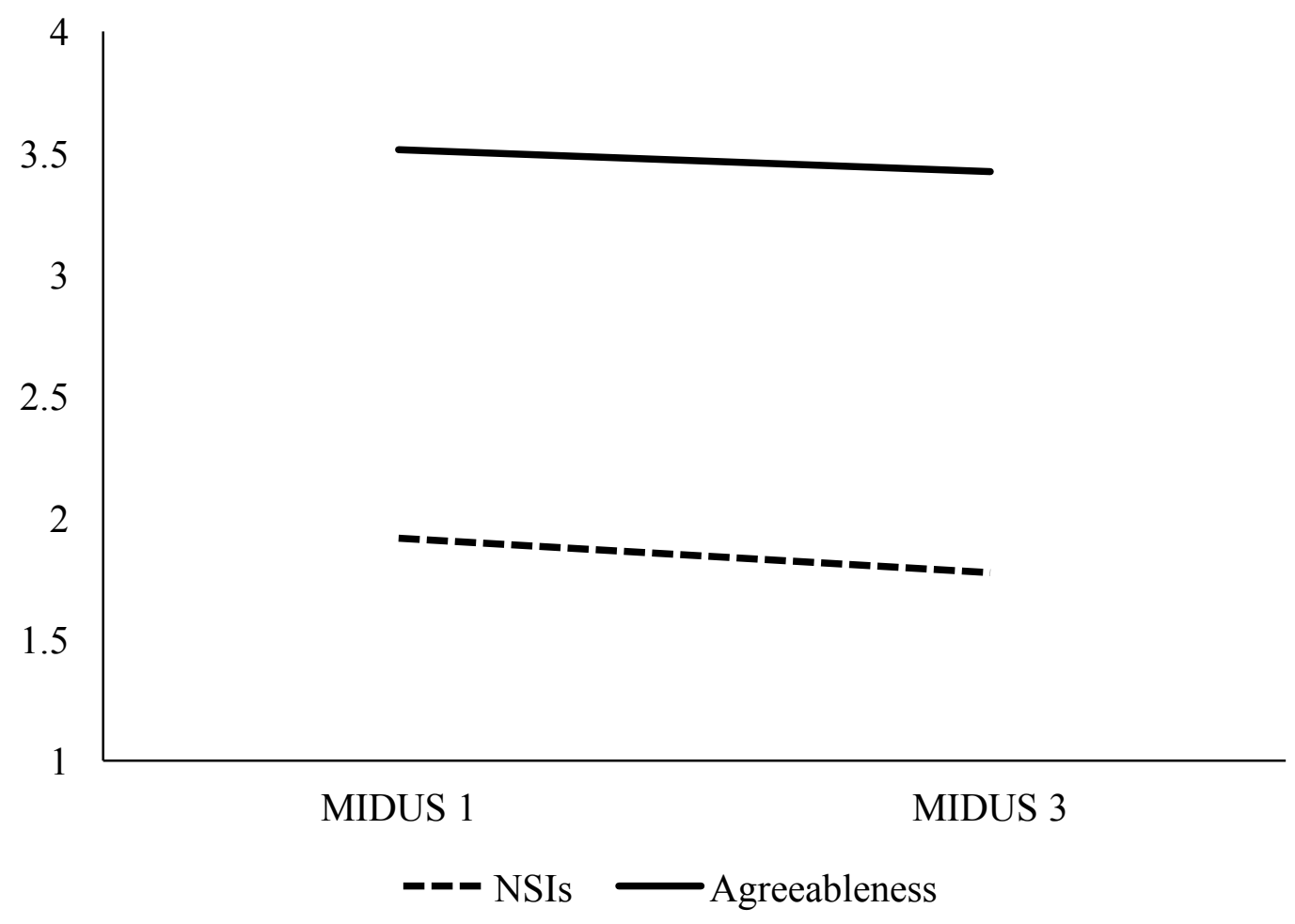

Figure 15. The trajectories of NSIs and agreeableness over 18 years for the older adults $(60+$ years old). 


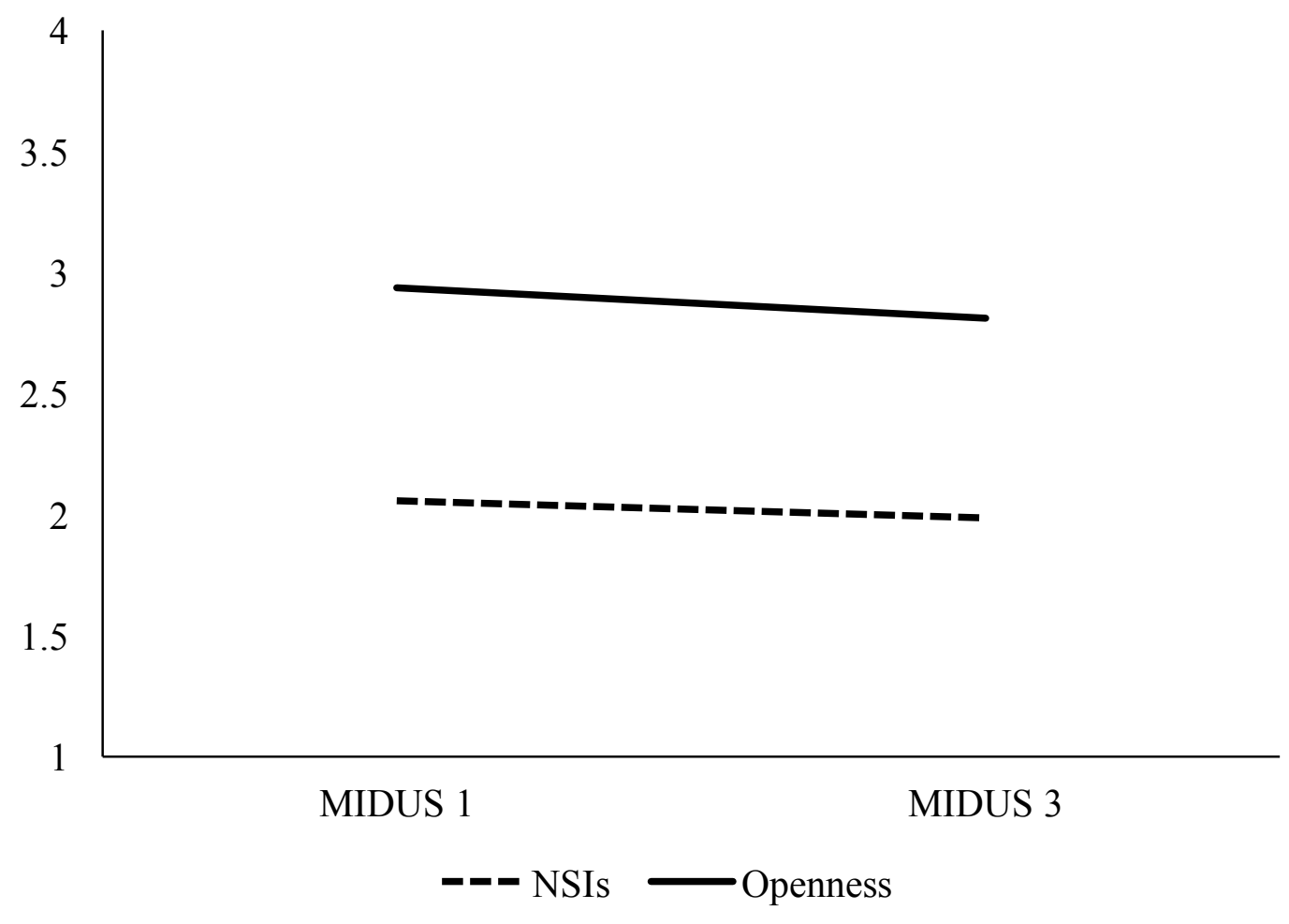

Figure 16. The trajectories of NSIs and openness over 18 years for the younger adults (18-39 years old). 


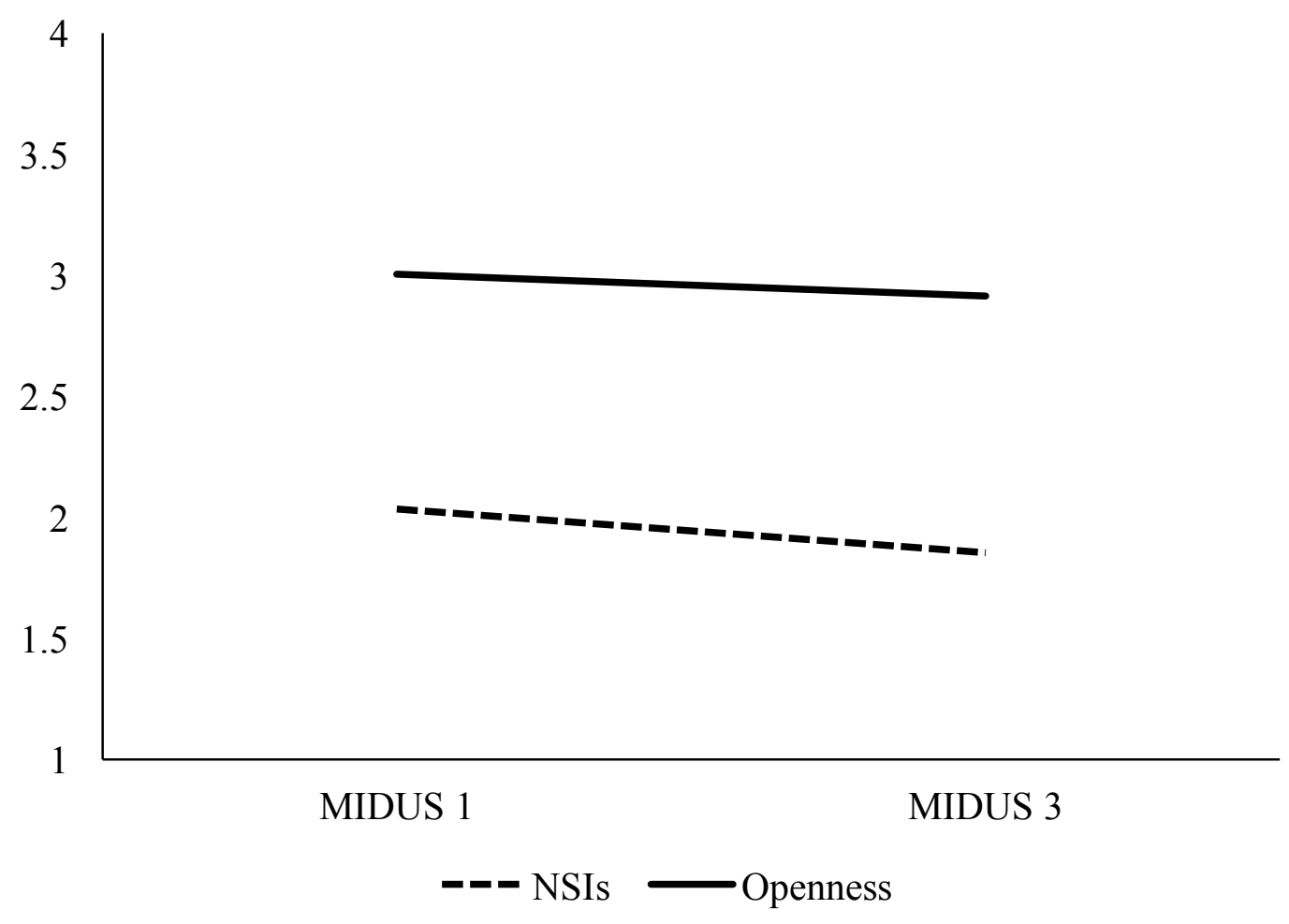

Figure 17. The trajectories of NSIs and openness over 18 years for the middle-aged adults (4059 years old). 


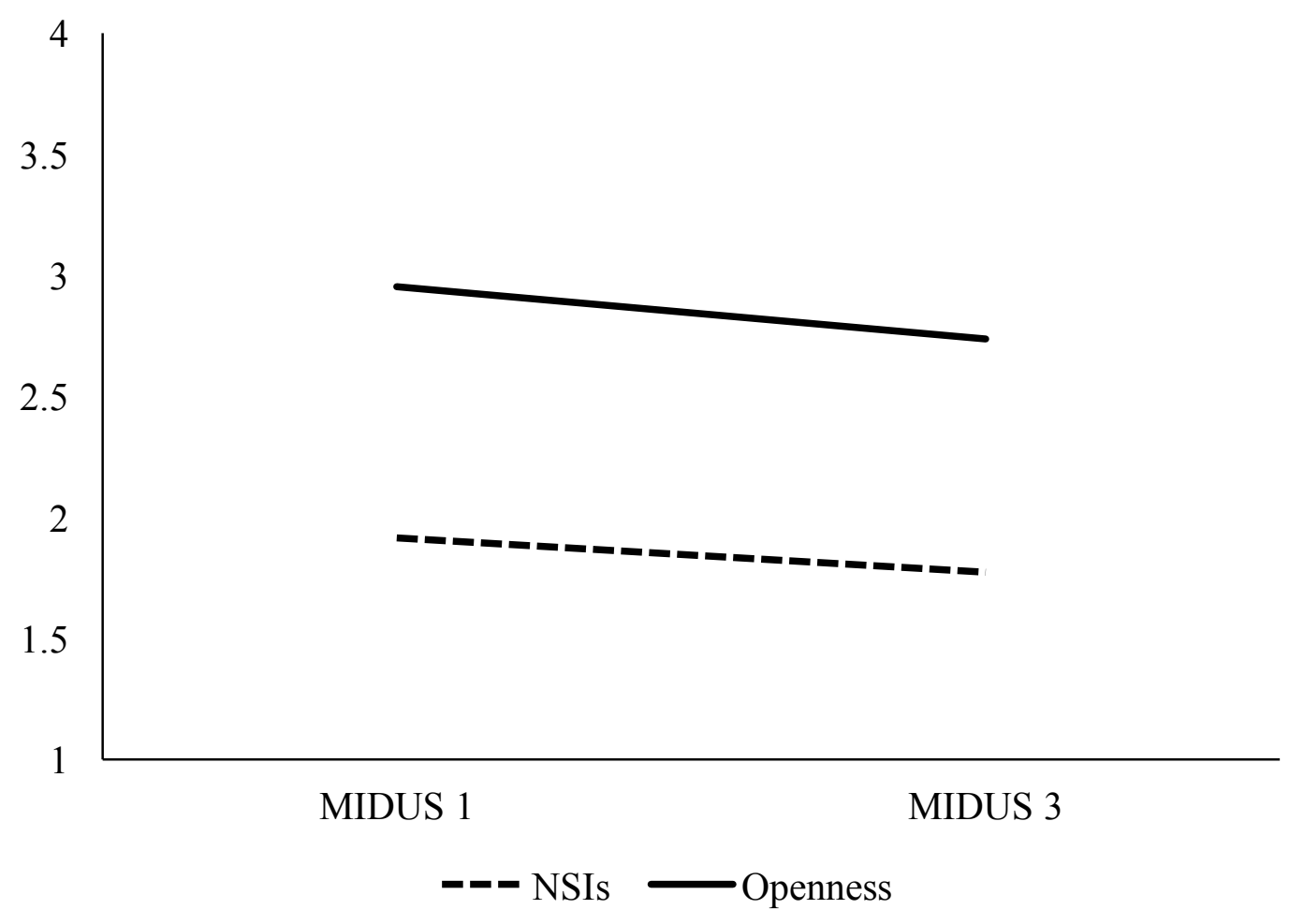

Figure 18. The trajectories of NSIs and openness over 18 years for the older adults $(60+$ years old). 


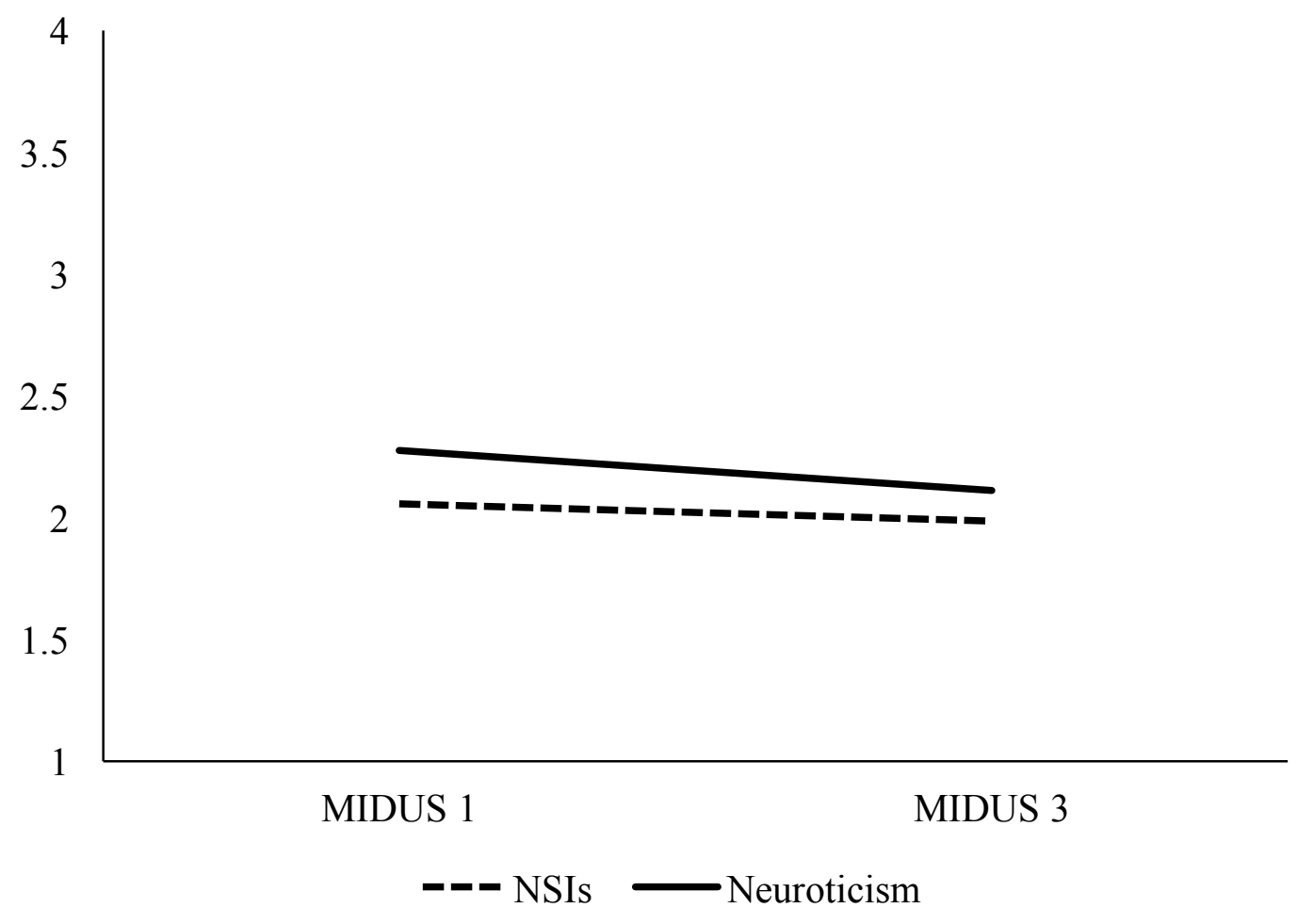

Figure 19. The trajectories of NSIs and neuroticism over 18 years for the younger adults $(18-39$ years old). 


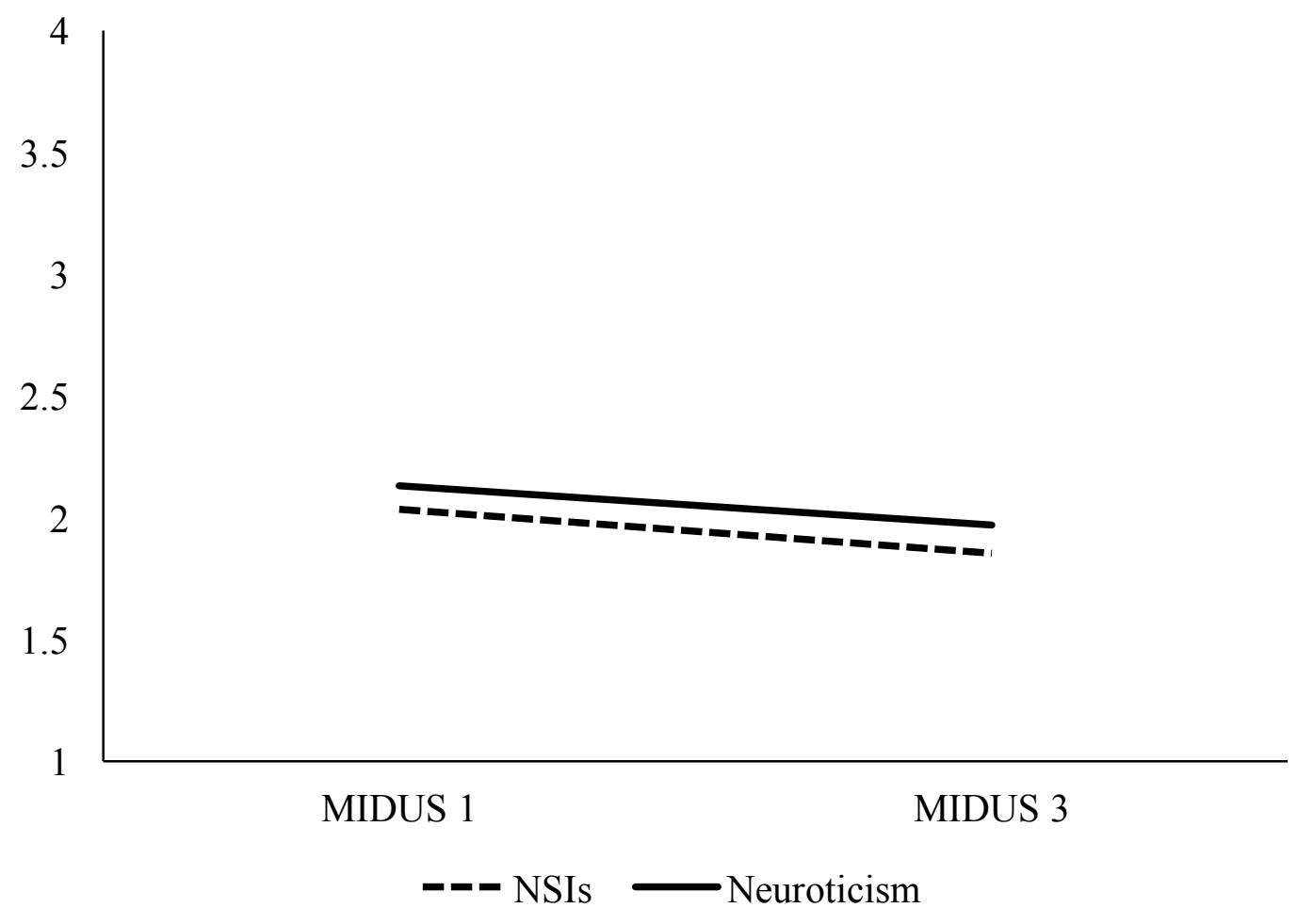

Figure 20. The trajectories of NSIs and neuroticism over 18 years for the middle-aged adults (40 -59 years old). 


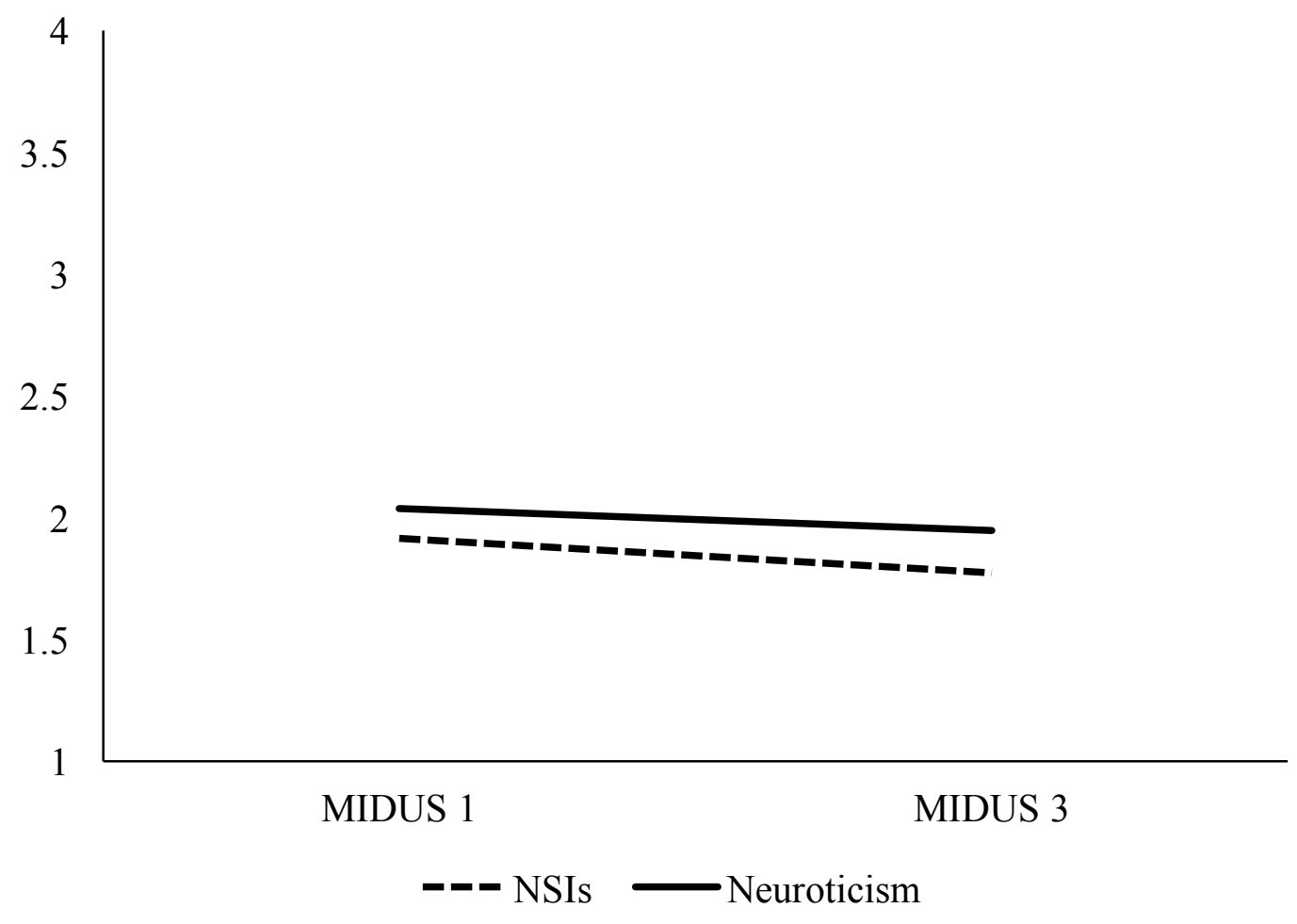

Figure 21. The trajectories of NSIs and neuroticism over 18 years for the older adults (60+ years old). 


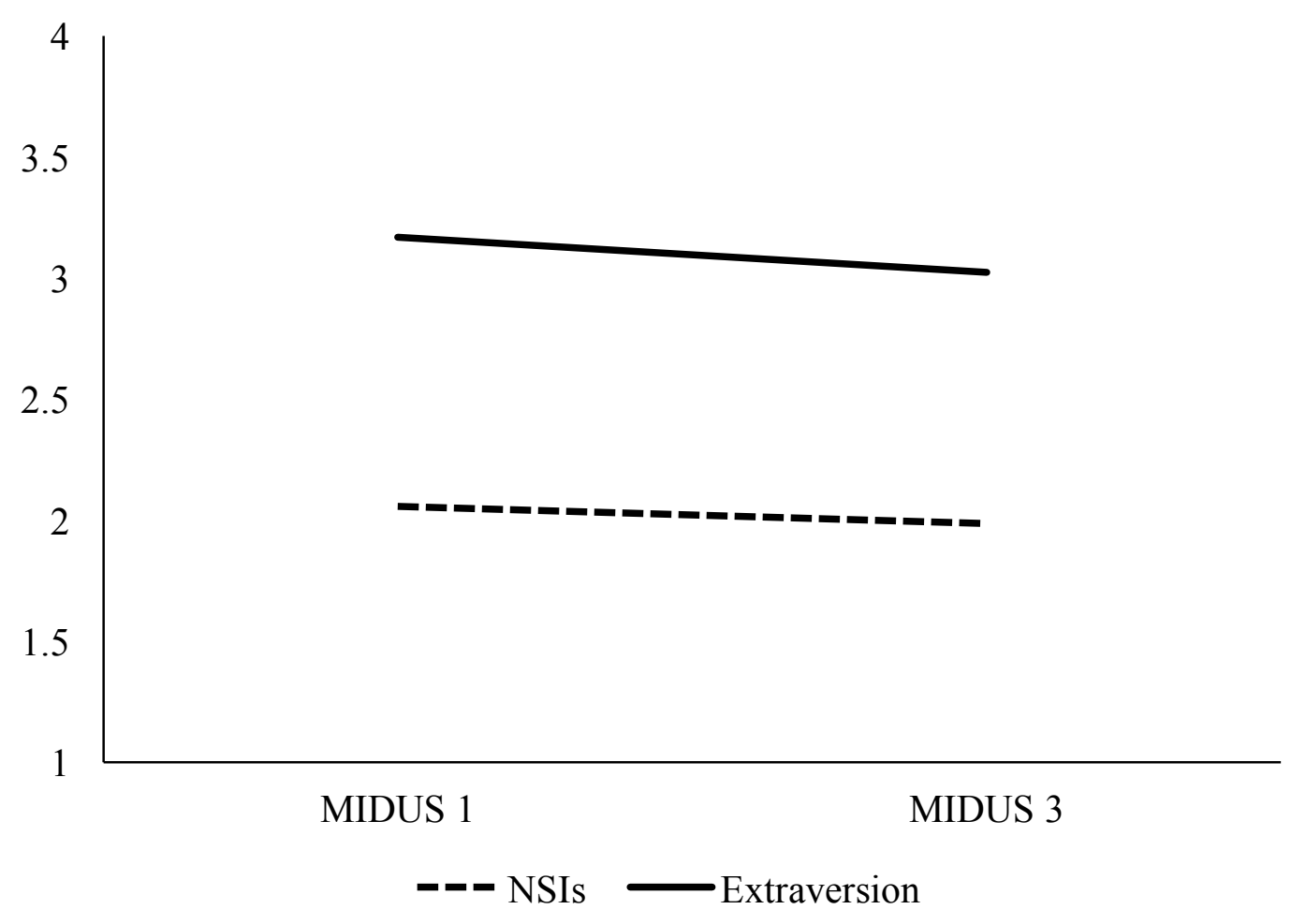

Figure 22. The trajectories of NSIs and extraversion over 18 years for the younger adults (1839 years old). 


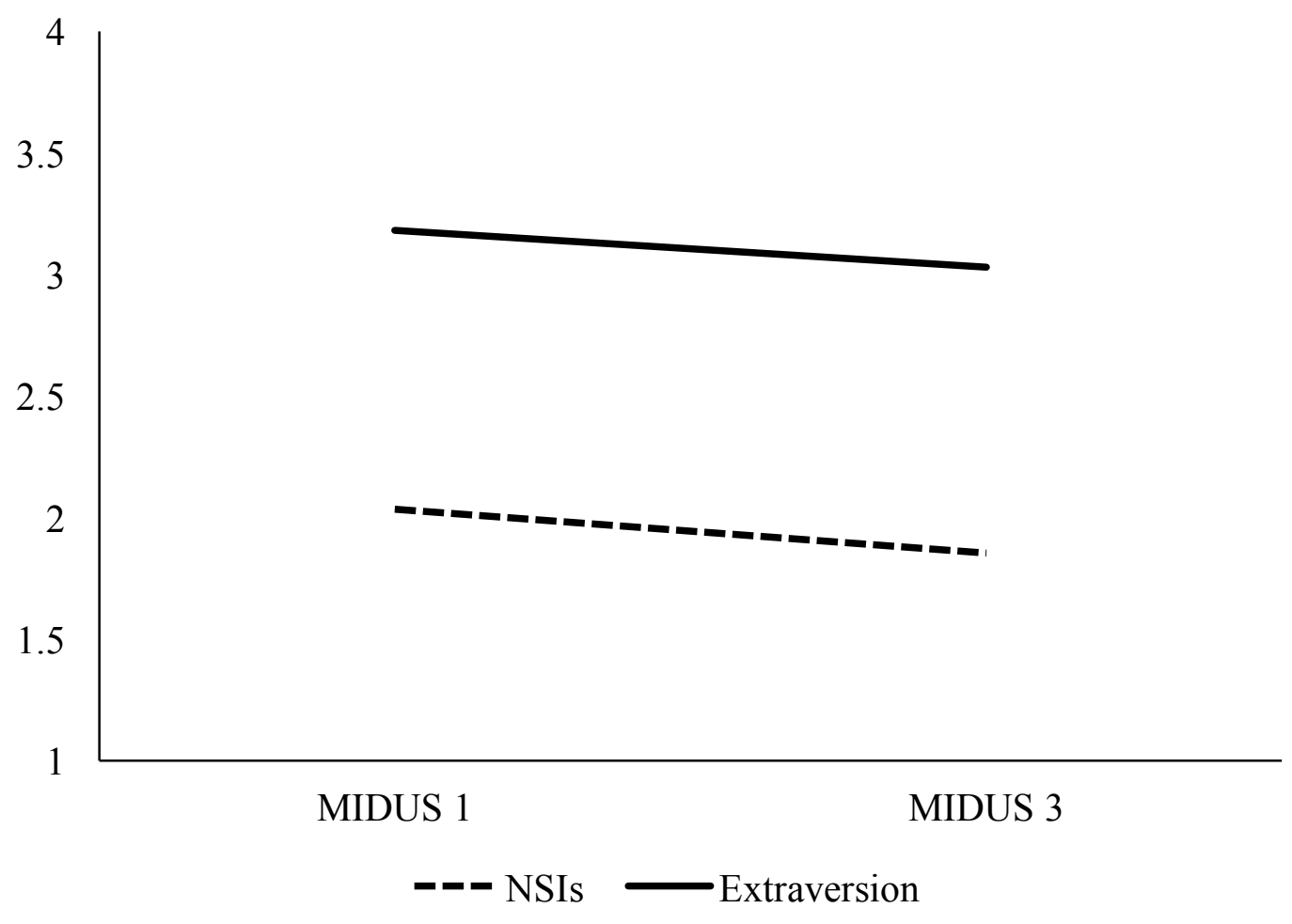

Figure 23. The trajectories of NSIs and extraversion over 18 years for the middle-aged adults (40 -59 years old). 


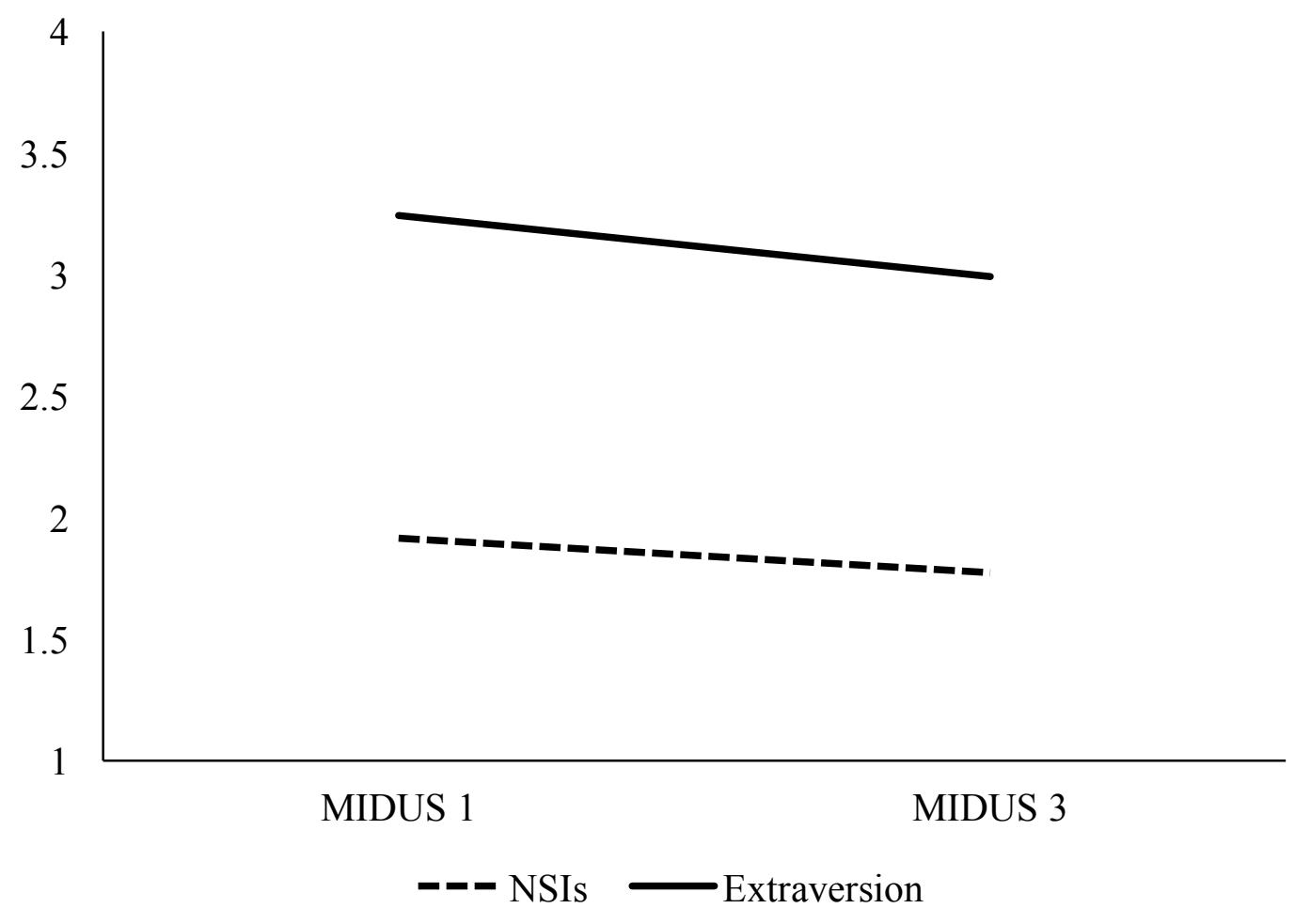

Figure 24. The trajectories of NSIs and extraversion over 18 years for the older adults $(60+$ years old). 


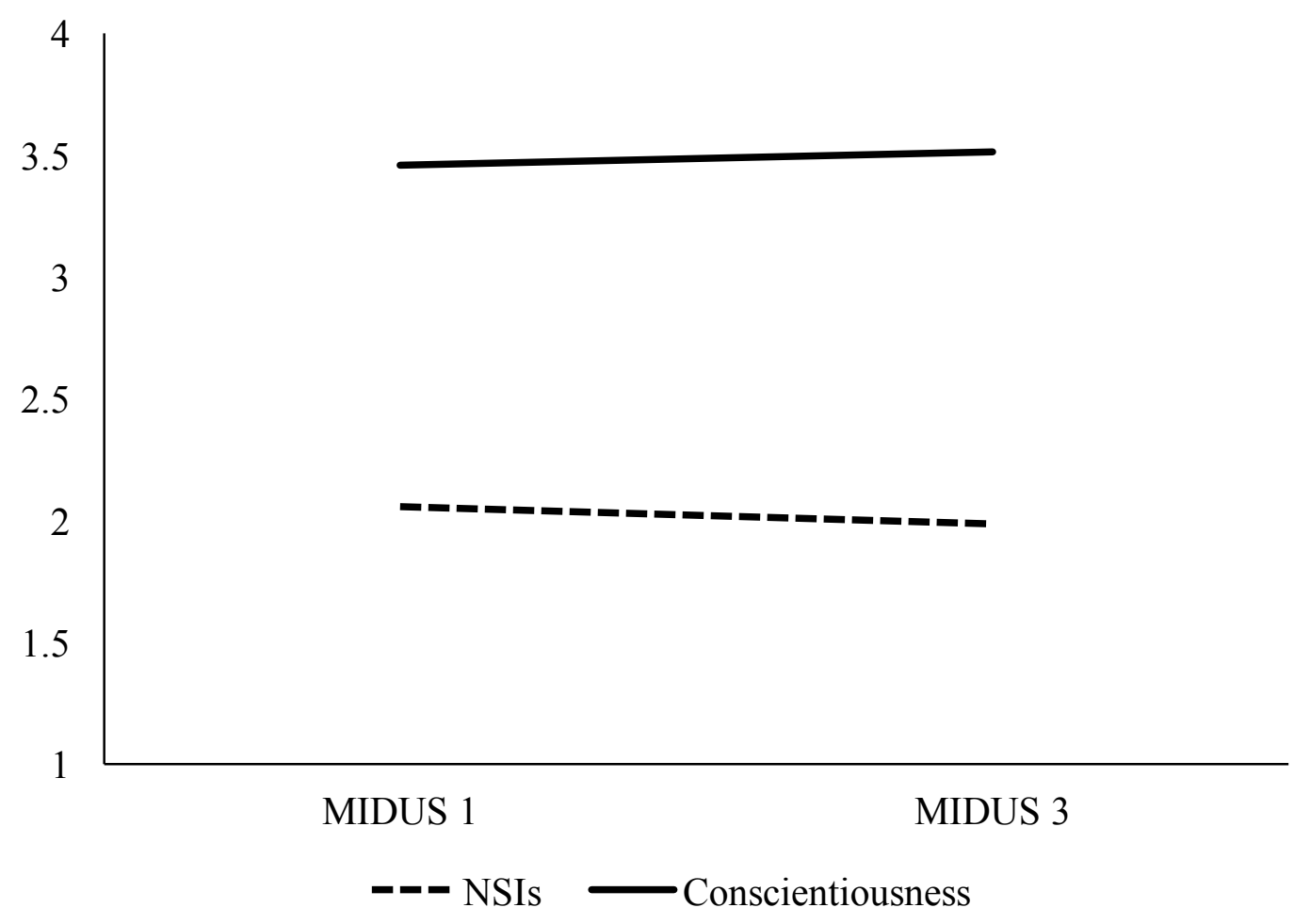

Figure 25. The trajectories of NSIs and conscientiousness over 18 years for the younger adults $(18-39$ years old). 


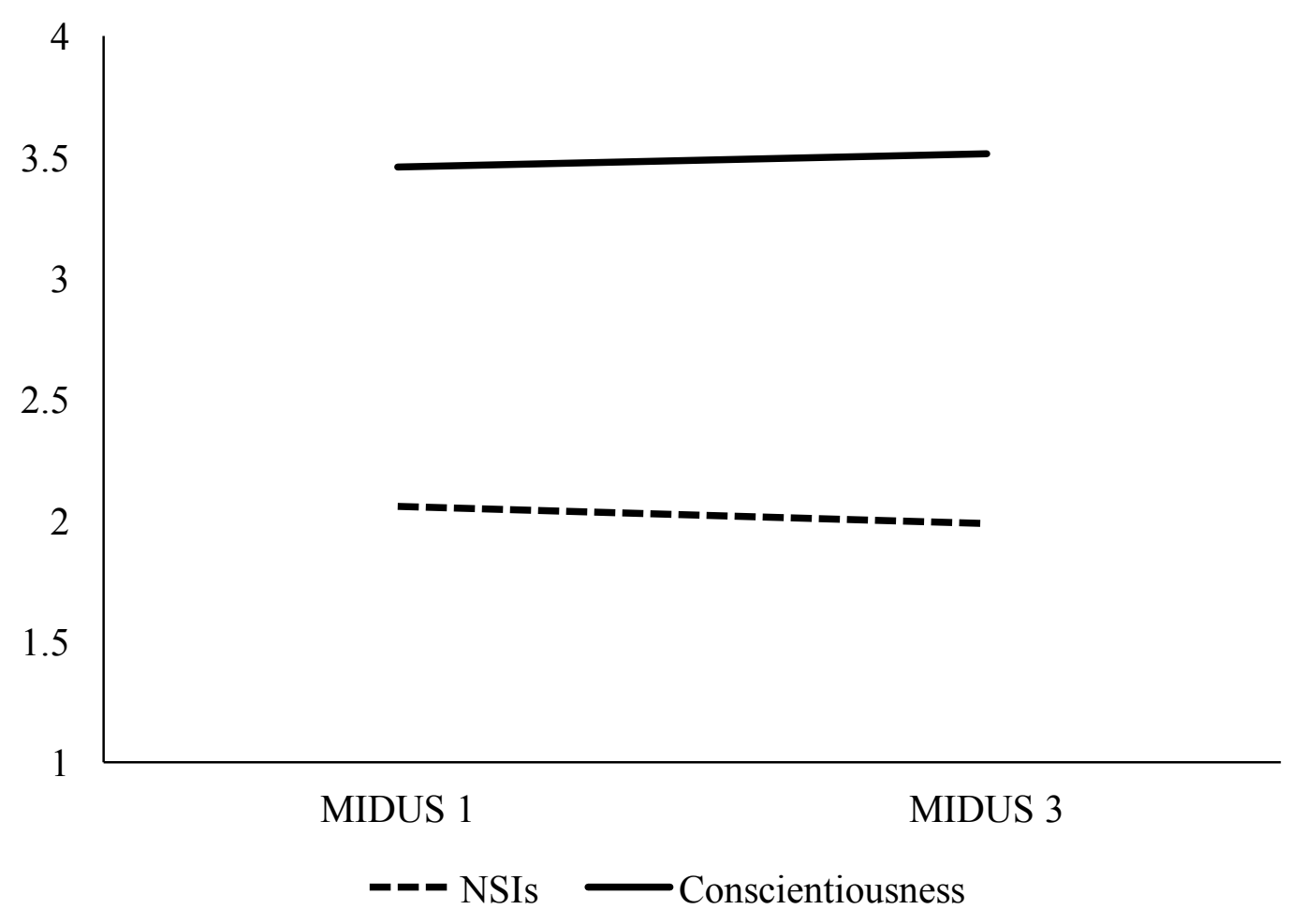

Figure 26. The trajectories of NSIs and conscientiousness over 18 years for the middle-aged adults $(40-59$ years old). 


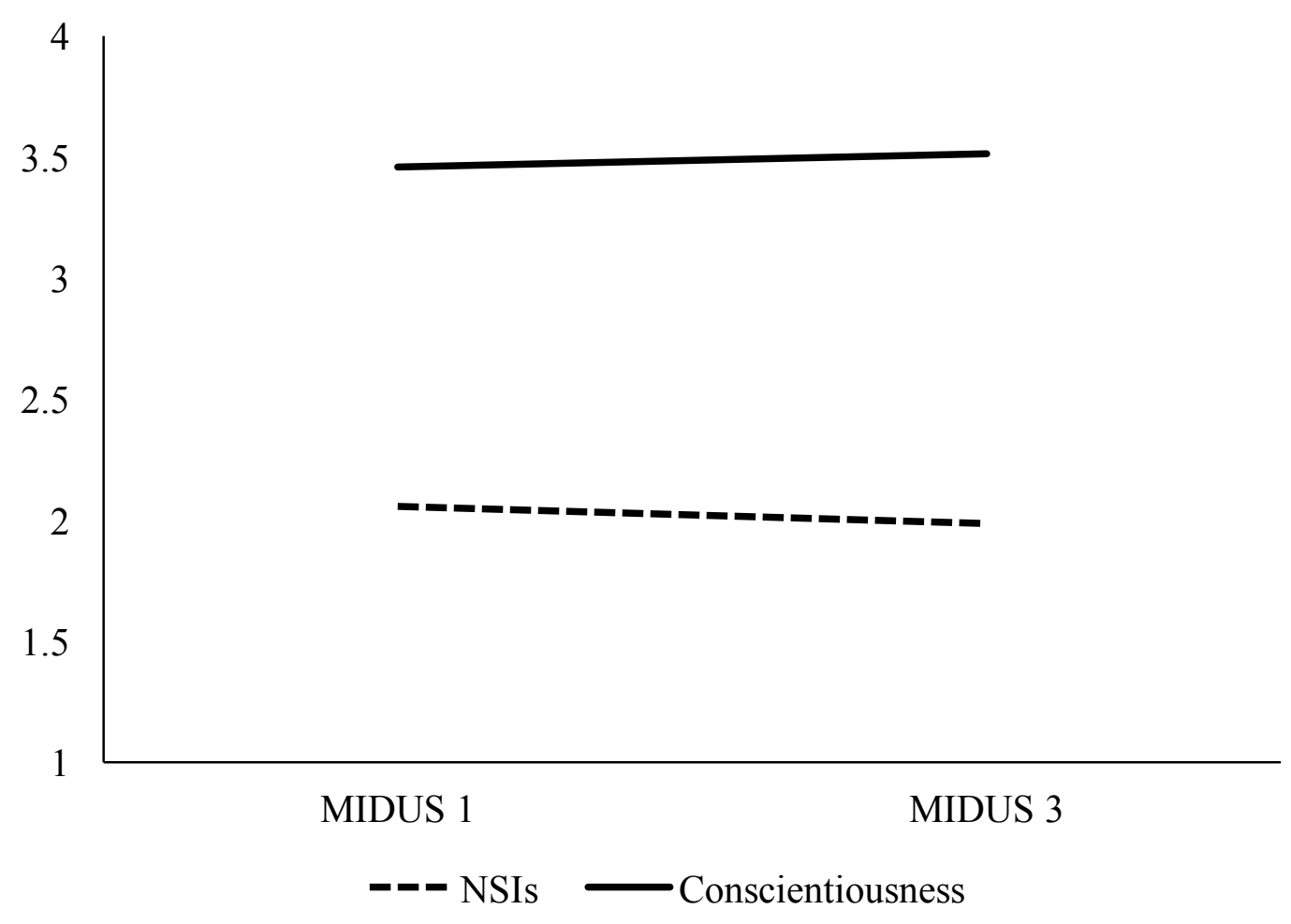

Figure 27. The trajectories of NSIs and conscientiousness over 18 years for the older adults $(60+$ years old). 
Appendix A

Completion Rates, Participant Characteristics for the General MIDUS Sample, and Attrition Analyses

Table A1

Completion Rates and Participant Characteristics for MIDUS 1

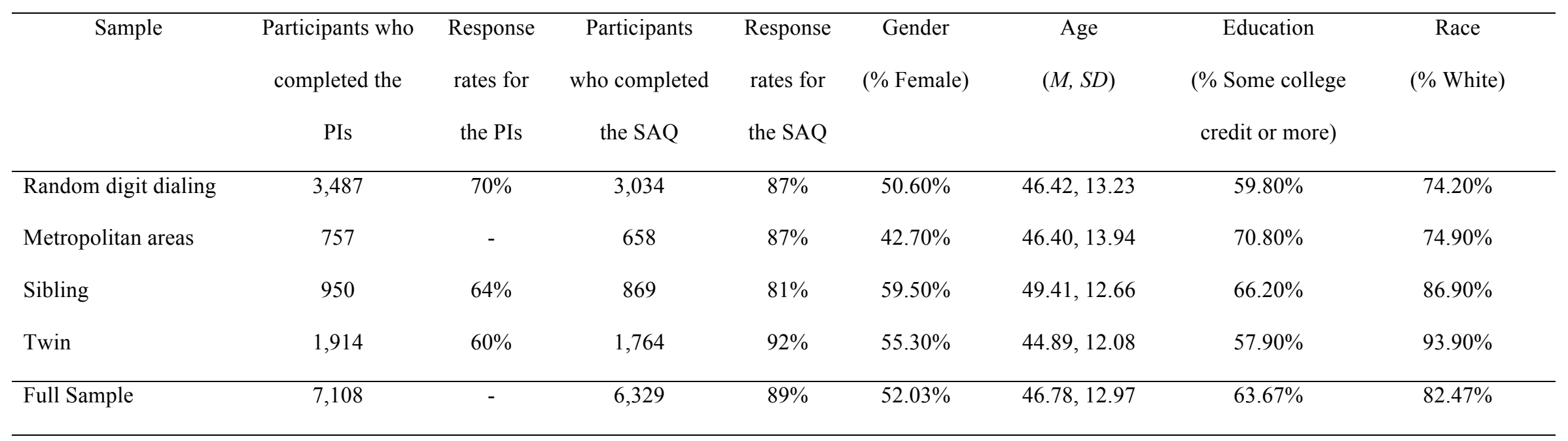

Note. $\mathrm{PI}=$ Phone interviews. $\mathrm{SAQ}=$ Self-administered questionnaires. The response rates for the oversampled metropolitan areas were not provided in the MIDUS 1 technical report. 
Table A2

Completion Rates and Participant Characteristics for MIDUS 2

\begin{tabular}{|c|c|c|c|c|c|c|c|c|}
\hline \multirow[t]{4}{*}{ Sample } & Participants & Retention & Participants & Response & Gender & Age & Education & Race \\
\hline & who & when & who & rates for & (\% Female $)$ & $(M, S D)$ & (\% Some college & (\% White) \\
\hline & completed the & adjusting for & completed the & the SAQ & & & credit or more) & \\
\hline & PIs & mortality & SAQ & & & & & \\
\hline Random digit dialing & 2,257 & $71 \%$ & 1,805 & $80 \%$ & $54.70 \%$ & $56.85,12.62$ & $65.60 \%$ & $89.40 \%$ \\
\hline Metropolitan areas & 489 & $71 \%$ & 386 & $79 \%$ & $45.30 \%$ & $57.31,13.02$ & $76.60 \%$ & $89.40 \%$ \\
\hline Sibling & 733 & $83 \%$ & 637 & $87 \%$ & $58.10 \%$ & $57.21,12.36$ & $69.80 \%$ & $95.00 \%$ \\
\hline Twin & 1,484 & $82 \%$ & 1,204 & $81 \%$ & $58.30 \%$ & $54.45,11.64$ & $63.90 \%$ & $93.60 \%$ \\
\hline Full Sample & 4,963 & $75 \%$ & 4,032 & $81 \%$ & $54.10 \%$ & $56.45,12.41$ & 68.97 & $91.85 \%$ \\
\hline
\end{tabular}

Note. $\mathrm{PI}=$ Phone interviews. $\mathrm{SAQ}=$ Self-administered questionnaires. Some reasons as to why participants did not complete the telephone interview consisted of refusal, being unable to participate for health or other related reasons, phone numbers that were no longer working, and death. 
Table A3

Completion Rates and Participant Characteristics for MIDUS 3

\begin{tabular}{|c|c|c|c|c|c|c|c|c|}
\hline \multirow[t]{4}{*}{ Sample } & Participants & Retention Rate & Participants & Response rates for the & Gender & Age & Education & Race \\
\hline & who & when adjusting & who & SAQ when adjusting for & $(\%$ Female $)$ & $(M, S D)$ & $(\%$ Some & (\% White) \\
\hline & completed the & for ineligible & completed & ineligible participants & & & college credit & \\
\hline & PIs & participants & the SAQ & & & & or more) & \\
\hline Random digit dialing & 1,730 & $74.40 \%$ & 1,427 & $82.80 \%$ & $53.30 \%$ & $64.72,11.37$ & $69.90 \%$ & $88.40 \%$ \\
\hline Metropolitan areas & - & - & - & - & $46.80 \%$ & $66.03,11.69$ & $77.80 \%$ & $87.60 \%$ \\
\hline Sibling & 544 & $84.10 \%$ & 473 & $87.10 \%$ & $58.80 \%$ & $65.68,11.40$ & $74.20 \%$ & $91.80 \%$ \\
\hline Twin & 1,020 & $77.90 \%$ & 832 & $81.80 \%$ & $58.60 \%$ & $63.17,10.50$ & $66.70 \%$ & $89.80 \%$ \\
\hline Full Sample & 3,294 & $76.90 \%$ & 2,732 & $83.20 \%$ & $54.38 \%$ & $64.90,11.24$ & $72.15 \%$ & $89.40 \%$ \\
\hline
\end{tabular}

Note. $\mathrm{PI}=$ Phone interviews. $\mathrm{SAQ}=$ Self-administered questionnaires. The response rates for the oversampled metropolitan areas were not provided in the MIDUS 3 technical report. Instead, they were included in the random digit dialing sample. Ineligible participants was defined as participants who died, were unable to participate for health or other related reasons, or participants who were no longer residing in the United States. 
Table A4

Attrition Analyses Comparing all Excluded Participants to Final Sample

\begin{tabular}{lccc}
\hline & $\begin{array}{c}\text { Excluded participants } \\
(n=5,519)\end{array}$ & $\begin{array}{c}\text { Remaining sample } \\
(n=1,530)\end{array}$ & Difference statistics \\
& $M, S D$ or $\%$ & $M, S D$ or $\%$ & \\
\hline Age & $46.48,13.61$ & $46.03,10.50$ & $t(3,097.98)=1.40$ \\
Gender & $53.00 \%$ Female & $49.40 \%$ Female & $\chi^{2}(\mathbf{1})=\mathbf{4 . 0 5}$ \\
Education & $6.59,2.48$ & $7.43,2.40$ & $\boldsymbol{t}(\mathbf{7 , 0 9 3})=\mathbf{- 1 1 . 9 2}$ \\
Marital Status & $57.70 \%$ Married & $94.70 \%$ Married & $\chi^{2}(\mathbf{1})=\mathbf{7 2 8 . 4 2}$ \\
\hline
\end{tabular}

Note. Significant statistics $(p<.05)$ are bolded. MIDUS 1 data were used for these attrition analyses. 
Table A5

Attrition Analyses Separated by Exclusion Criteria

Participants who did not complete MIDUS 1 SAQs

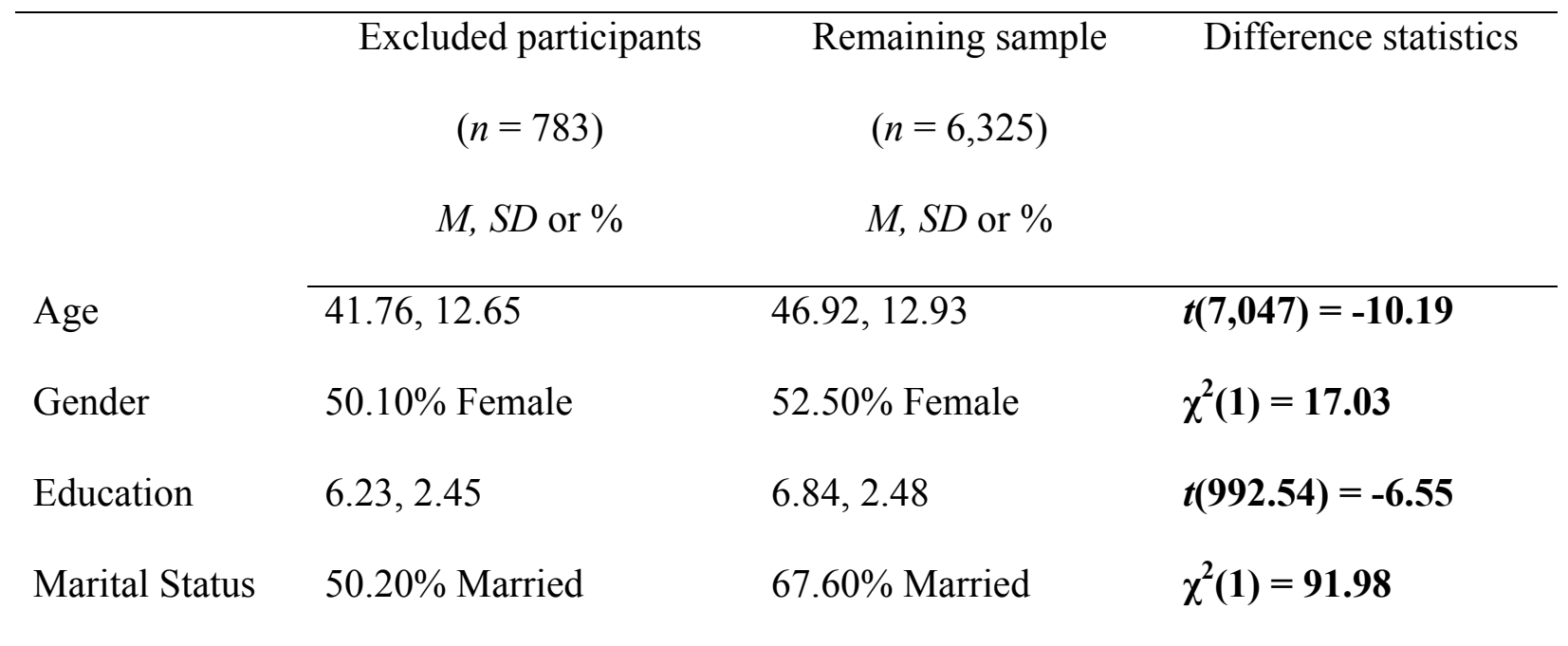

Participants who were ineligible to complete MIDUS 2

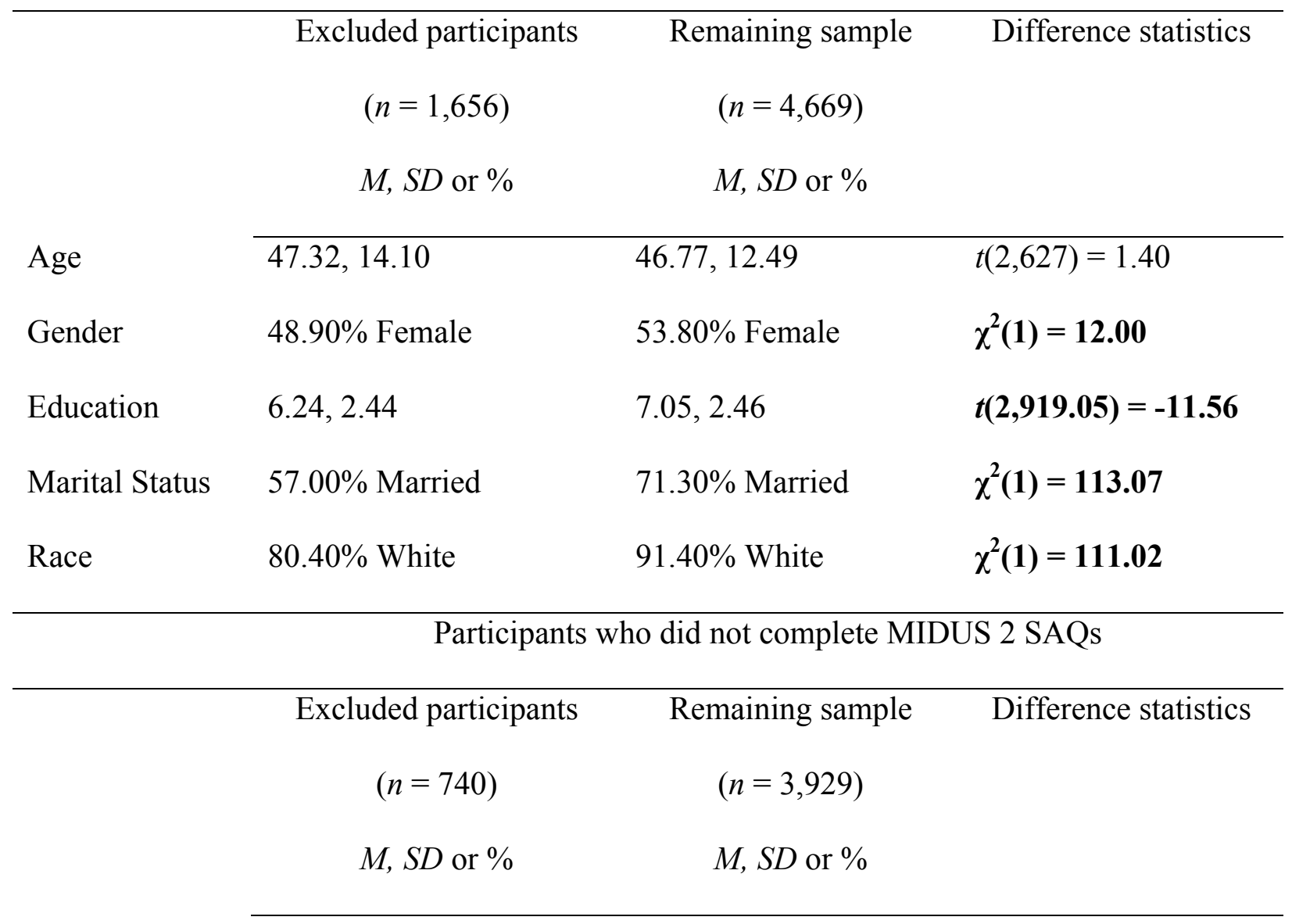




\begin{tabular}{|c|c|c|c|}
\hline Age & $52.61,12.27$ & $56.33,12.38$ & $t(4,667)=-7.50$ \\
\hline Gender & $44.9 \%$ Female & $55.50 \%$ Female & $\chi^{2}(1)=28.25$ \\
\hline Education & $6.92,2.37$ & $7.27,2.54$ & $t(1,084.21)=-3.62$ \\
\hline Marital Status & $70.10 \%$ Married & $71.20 \%$ Married & $\chi^{2}(1)=0.43$ \\
\hline Race & $85.80 \%$ White & $92.50 \%$ White & $\chi^{2}(1)=19.67$ \\
\hline
\end{tabular}

\section{Participants who were ineligible to complete MIDUS 3}

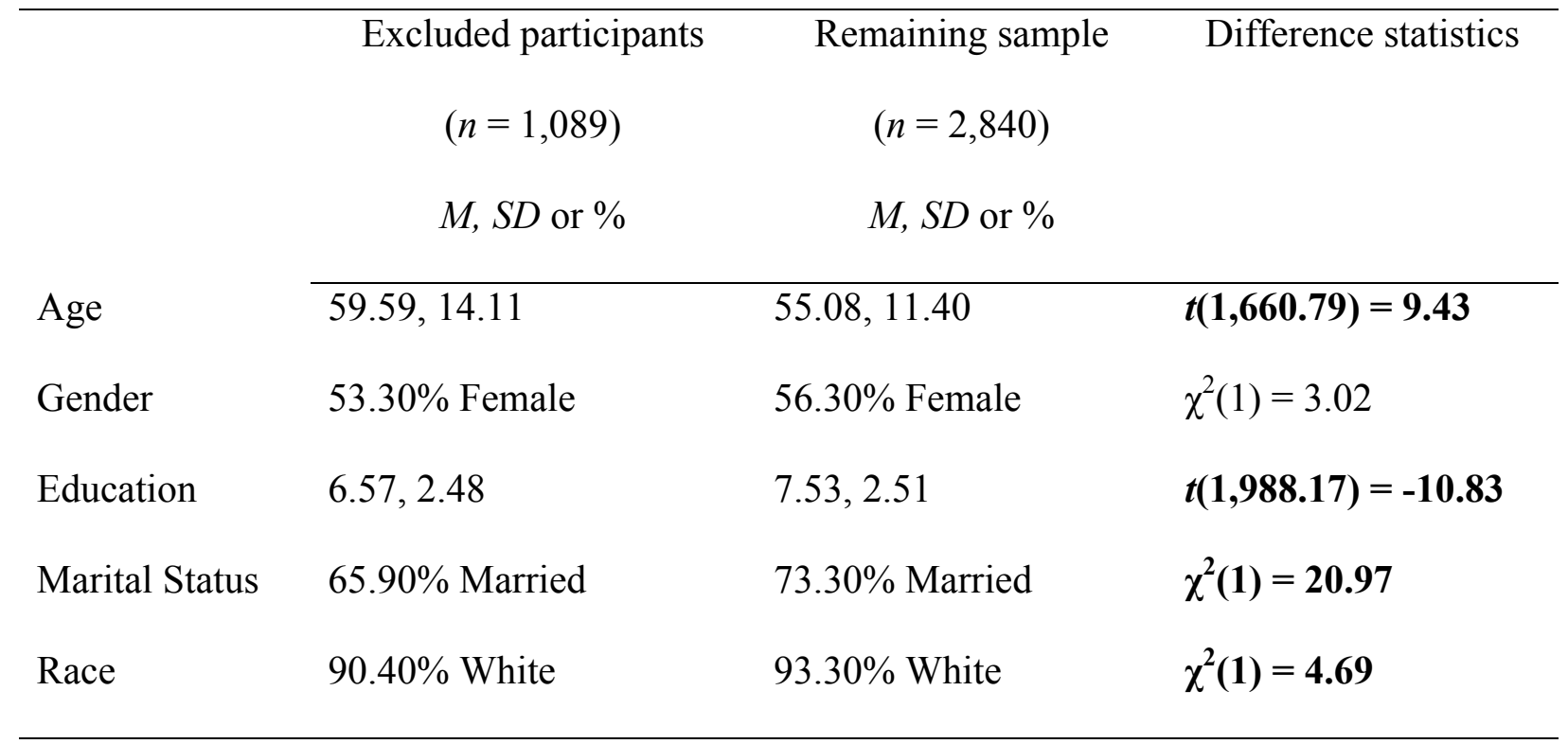

Participants who did not complete MIDUS 3 SAQs

Excluded participants $\quad$ Remaining sample Difference statistics

$\begin{array}{cr}(n=330) & (n=2,510) \\ M, S D \text { or } \% & M, S D \text { or } \%\end{array}$

\begin{tabular}{llll}
\cline { 2 - 3 } Age & $60.31,12.07$ & $64.69,11.22$ & $\boldsymbol{t}(\mathbf{2 , 8 3 8})=\mathbf{- 6 . 5 9}$ \\
Gender & $59.30 \%$ Female & $56.00 \%$ Female & $\chi^{2}(1)=1.30$ \\
Education & $7.64,2.54$ & $7.54,2.52$ & $t(2,828)=0.64$ \\
Marital Status & $69.90 \%$ Married & $67.00 \%$ Married & $\chi^{2}(1)=1.62$ \\
Race & $92.10 \%$ White & $93.40 \%$ White & $\chi^{2}(1)=1.61$
\end{tabular}


Participants who did not have a partner across all three MIDUS waves

\begin{tabular}{|c|c|c|c|}
\hline & $\begin{array}{l}\text { Excluded participants } \\
\qquad \begin{array}{c}(n=980) \\
M, S D \text { or } \%\end{array}\end{array}$ & $\begin{array}{l}\text { Remaining sample } \\
\qquad \begin{array}{c}(n=1,530) \\
M, S D \text { or } \%\end{array}\end{array}$ & Difference statistics \\
\hline Age & $47.72,12.31$ & $46.03,10.50$ & $t(1,847.89)=3.54$ \\
\hline Gender & $66.20 \%$ Female & $49.40 \%$ Female & $\chi^{2}(1)=68.52$ \\
\hline Education & $7.23,2.53$ & $7.43,2.40$ & $t(2,504)=-1.95$ \\
\hline Marital Status & 39.80\% Married & $94.70 \%$ Married & $\chi^{2}(1)=919.58$ \\
\hline Race & $90.60 \%$ White & $95.30 \%$ White & $\chi^{2}(1)=14.30$ \\
\hline
\end{tabular}

Note. Significant statistics $(p<.05)$ are bolded. SAQ $=$ Self-administered questionnaires. The variables that were in the telephone interview included age, gender, marital status, and education. The following sections were analyzed using MIDUS 1 data: participants who did not complete MIDUS 1 SAQs and participants who were ineligible to complete MIDUS 2. The following sections were analyzed using MIDUS 2 data: participants who did not complete MIDUS 2 SAQs, participants who were ineligible to complete MIDUS 3. The following section was analyzed using MIDUS 3 data: participants who did not complete MIDUS 3 SAQs and participants who did not have a partner across all three MIDUS waves. 
Table A6

Missing Data across all MIDUS Waves

\begin{tabular}{lcccccc}
\hline & \multicolumn{2}{c}{ MIDUS 1 } & \multicolumn{2}{c}{ MIDUS 2 } & \multicolumn{2}{c}{ MIDUS 3 } \\
\hline & Missing & Missing & Missing & Missing & Missing & Missing \\
& Count & Percent & Count & Percent & Count & Percent \\
\hline Age & 0 & $0.00 \%$ & 0 & $0.00 \%$ & 0 & $0.00 \%$ \\
Gender & 0 & $0.00 \%$ & 0 & $0.00 \%$ & 0 & $0.00 \%$ \\
Education & 1 & $0.10 \%$ & 2 & $0.10 \%$ & 2 & $0.10 \%$ \\
NSIs & 0 & $0.00 \%$ & 0 & $0.00 \%$ & 0 & $0.00 \%$ \\
Agreeableness & 2 & $0.10 \%$ & 5 & $0.30 \%$ & 5 & $0.30 \%$ \\
Openness & 3 & $0.20 \%$ & 12 & $0.80 \%$ & 7 & $0.50 \%$ \\
Neuroticism & 4 & $0.30 \%$ & 7 & $0.50 \%$ & 5 & $0.30 \%$ \\
Extraversion & 2 & $0.10 \%$ & 5 & $0.30 \%$ & 6 & $0.40 \%$ \\
Conscientiousness & 2 & $0.10 \%$ & 5 & $0.30 \%$ & 5 & $0.30 \%$ \\
\hline
\end{tabular}


Table A7

Attrition Analyses Comparing Non-Multivariate Outliers and Multivariate Outliers for MIDUS 1 Variables

\begin{tabular}{|c|c|c|c|}
\hline & Non-Multivariate & Multivariate & Difference \\
\hline & Outliers & Outliers & Statistic \\
\hline & $(n=1,403)$ & $(n=127)$ & \\
\hline & $M, S D$ or $\%$ & $M, S D$ or $\%$ & \\
\hline Age & $46.35,10.37$ & $42.43,11.31$ & $t(1,528)=4.05$ \\
\hline Gender & $49.10 \%$ Female & $52.80 \%$ Female & $\chi^{2}(1)=0.62$ \\
\hline Education & $7.47,2.40$ & $6.99,2.44$ & $t(1,527)=2.12$ \\
\hline Marital Status & $96.80 \%$ Married & $71.70 \%$ Married & $\chi^{2}(1)=146.79$ \\
\hline Race & $96.70 \%$ White & $80.30 \%$ White & $\chi^{2}(1)=82.42$ \\
\hline NSIs & $2.02,0.39$ & $2.08,0.41$ & $t(1,528)=-1.61$ \\
\hline Agreeableness & $3.45,0.48$ & $3.46,0.50$ & $t(1,526)=-0.25$ \\
\hline Openness & $2.97,0.50$ & $3.11,0.51$ & $t(1,525)=-2.91$ \\
\hline Neuroticism & $2.18,0.63$ & $2.28,0.68$ & $t(1,524)=-1.64$ \\
\hline Extraversion & $3.18,0.55$ & $3.23,0.56$ & $t(1,526)=-0.93$ \\
\hline Conscientiousness & $3.48,0.41$ & $3.42,0.45$ & $t(145.40)=1.48$ \\
\hline
\end{tabular}

Note. Significant statistics $(p<.05)$ are bolded. 
Table A 8

Attrition Analyses Comparing Non-Multivariate Outliers and Multivariate Outliers for MIDUS 2 Variables

\begin{tabular}{|c|c|c|c|}
\hline & Non-Multivariate & Multivariate & Difference \\
\hline & Outliers & Outliers & Statistic \\
\hline & $(n=1,403)$ & $(n=127)$ & \\
\hline & $M, S D$ or $\%$ & $M, S D$ or $\%$ & \\
\hline Age & $55.22,10.32$ & $51.43,11.29$ & $t(1,528)=3.93$ \\
\hline Gender & $49.10 \%$ Female & $52.80 \%$ Female & $\chi^{2}(1)=0.62$ \\
\hline Education & $7.56,2.47$ & $7.80,2.72$ & $t(145.57)=-0.97$ \\
\hline Marital Status & $99.40 \%$ Married & $65.40 \%$ Married & $\chi^{2}(1)=421.11$ \\
\hline Race & 96.50\% White & 71.70\% White & $\chi^{2}(1)=137.10$ \\
\hline NSIs & $1.94,0.40$ & $2.09,0.46$ & $t(143.76)=-3.50$ \\
\hline Agreeableness & $3.43,0.48$ & $3.41,0.53$ & $t(1,523)=0.48$ \\
\hline Openness & $2.89,0.51$ & $2.96,0.63$ & $t(141.34)=-1.21$ \\
\hline Neuroticism & $2.01,0.60$ & $2.17,0.70$ & $t(143.37)=-2.56$ \\
\hline Extraversion & $3.10,0.55$ & $3.09,0.60$ & $t(1,523)=0.24$ \\
\hline Conscientiousness & $3.52,0.40$ & $3.45,0.49$ & $t(141.75)=1.73$ \\
\hline
\end{tabular}

Note. Significant statistics $(p<.05)$ are bolded. 
Table A9

Attrition Analyses Comparing Non-Multivariate Outliers and Multivariate Outliers for MIDUS 3 Variables

\begin{tabular}{|c|c|c|c|}
\hline & Non-Multivariate & Multivariate & Difference \\
\hline & Outliers & Outliers & Statistic \\
\hline & $(n=1,403)$ & $(n=127)$ & \\
\hline & $M, S D$ or $\%$ & $M, S D$ or $\%$ & \\
\hline Age & $64.33,10.33$ & $60.53,11.29$ & $t(1,528)=3.94$ \\
\hline Gender & $49.10 \%$ Female & $52.80 \%$ Female & $\chi^{2}(1)=0.62$ \\
\hline Education & $7.62,2.46$ & $7.46,2.66$ & $t(1,526)=0.71$ \\
\hline Marital Status & 99.80\% Married & $63.80 \%$ Married & $\chi^{2}(1)=487.04$ \\
\hline Race & $92.20 \%$ White & 78.00\% White & $\chi^{2}(1)=32.85$ \\
\hline NSIs & $1.86,0.43$ & $2.02,0.50$ & $t(144.12)=-3.86$ \\
\hline Agreeableness & $3.43,0.48$ & $3.36,0.52$ & $t(1,523)=1.39$ \\
\hline Openness & $2.86,0.53$ & $2.95,0.58$ & $t(1,521)=-1.89$ \\
\hline Neuroticism & $2.02,0.60$ & $2.22,0.69$ & $t(1,523)=-3.53$ \\
\hline Extraversion & $3.08,0.57$ & $3.08,0.53$ & $t(1,522)=-0.05$ \\
\hline Conscientiousness & $3.50,0.43$ & $3.39,0.53$ & $t(141.37)=2.15$ \\
\hline
\end{tabular}

Note. Significant statistics $(p<.05)$ are bolded. 


\section{Appendix B}

Study Measures

\section{Demographics}

1. Respondent's age

2. Gender of respondent

1. Female

2. Male

3. What is the highest grade of school or year of college you completed?

1. No school/Some grade school

2. Eighth grade/Junior high school

3. Some high school

4. G.E.D

5. Graduated from high school

6. One to two years of college, no degree yet

7. Three or more years of college, no degree yet

8. Graduated two-year college, vocational school, or associates degree

9. Graduated four- or five-year college or Bachelor's degree

10. Some graduate school

11. Master's degree

12. Professional degree (Ph.D., Ed.D., M.D., D.D.S., L.L.B., L.L.D., J.D., or other professional degree). 
4. Are you married, separated, divorced, widowed, or never married?

1. Married

2. Separated

3. Divorced

4. Widowed

5. Never married

5. What race do you consider yourself to be?

1. White

2. Black and/or African American

3. Native American or Aleutian Islander/Eskimo

4. Asian or Pacific Islander

5. Other

6. Multiracial 
Table B1

The Indicators of Strain Scale

\section{Never Rarely Sometimes Often}

Not including your spouse or partner, how often do

members of your family...

1. Make too many demands on you?

12

3

4

2. Criticize you?

12

3

4

3. Let you down when you are counting on them?

12

3

4

4. Get on your nerves?

12

3

4

How often does your spouse or partner...

1. Make too many demands on you?

$1 \quad 2$

2

3

4

2. Criticize you?

12

3

4

3. Let you down when you are counting on them?

12

3

4

4. Get on your nerves?

12

3

4

5. Make you feel tense?

12

$1 \quad 2$

3

4

6. Argue with you?

How much do your friends...

1. Make too many demands on you?

2. Criticize you?

3. Let you down when you are counting on them?

4. Get on your nerves?

1

1

12

12

2

2

2

2

Note. Modified measure from Schuster, Kessler, \& Aseltine (1990). The Indicators of Strain Scale was utilized at all waves of collection. 
Table B2

Midlife Development Inventory Personality Scale

Not at all A little Some A lot

Please indicate how well each of the following describes you.

\begin{tabular}{|c|c|c|c|c|}
\hline 1. Outgoing & 1 & 2 & 3 & 4 \\
\hline 2. Helpful & 1 & 2 & 3 & 4 \\
\hline 3. Moody & 1 & 2 & 3 & 4 \\
\hline 4. Organized & 1 & 2 & 3 & 4 \\
\hline 5. Friendly & 1 & 2 & 3 & 4 \\
\hline 6. Warm & 1 & 2 & 3 & 4 \\
\hline 7. Worrying & 1 & 2 & 3 & 4 \\
\hline 8. Responsible & 1 & 2 & 3 & 4 \\
\hline 9. Lively & 1 & 2 & 3 & 4 \\
\hline 10. Caring & 1 & 2 & 3 & 4 \\
\hline 11. Nervous & 1 & 2 & 3 & 4 \\
\hline 12. Creative & 1 & 2 & 3 & 4 \\
\hline 13. Hardworking & 1 & 2 & 3 & 4 \\
\hline 14. Imaginative & 1 & 2 & 3 & 4 \\
\hline 15. Softhearted & 1 & 2 & 3 & 4 \\
\hline 16. Calm & 1 & 2 & 3 & 4 \\
\hline 17. Intelligent & 1 & 2 & 3 & 4 \\
\hline 18. Curious & 1 & 2 & 3 & 4 \\
\hline
\end{tabular}




\begin{tabular}{lllll} 
19. Active & 1 & 2 & 3 & 4 \\
20. Careless & 1 & 2 & 3 & 4 \\
21. Broad-minded & 1 & 2 & 3 & 4 \\
22. Sympathetic & 1 & 2 & 3 & 4 \\
23. Talkative & 1 & 2 & 3 & 4 \\
24. Sophisticated & 1 & 2 & 3 & 4 \\
25. Adventurous & 1 & 2 & 3 & 4 \\
\hline
\end{tabular}

Note. Measure from Lachman and Weaver (1997). The Midlife Development Inventory

Personality Scale was utilized at all waves of collection. 


\section{Appendix C}

Descriptive Statistics and Bivariate Correlations for Source of NSIs

\section{Table C1}

Descriptive Statistics and Bivariate Correlations for MIDUS 1

\begin{tabular}{|c|c|c|c|c|c|c|c|c|c|c|c|c|c|}
\hline & $M, S D$, or $\%$ & $\alpha$ & 1 & 2 & 3 & 4 & 5 & 6 & 7 & 8 & 9 & 10 & 11 \\
\hline 1. Age & $46.03,10.50$ & - & - & & & & & & & & & & \\
\hline 2. Gender $(0=$ Female $)$ & $49.40 \%$ Female & - & .11 & - & & & & & & & & & \\
\hline 3. Education & $7.43,2.40$ & - & -.05 & .13 & - & & & & & & & & \\
\hline 4. NSIs & $2.02,0.39$ & .81 & -.13 & -.08 & .01 & - & & & & & & & \\
\hline 5. NSIs from Family & $2.07,0.56$ & .77 & -.12 & -.14 & -.04 & .78 & - & & & & & & \\
\hline 6. NSIs from Partner & $2.11,0.58$ & .78 & -.05 & -.05 & .05 & .72 & .30 & - & & & & & \\
\hline 7. NSIs from Friends & $1.88,0.46$ & .76 & -.12 & .01 & .00 & .72 & .45 & .26 & - & & & & \\
\hline 8. Agreeableness & $3.45,0.49$ & .80 & .08 & -.26 & -.09 & -.08 & -.03 & -.08 & -.06 & - & & & \\
\hline 9. Openness & $2.98,0.50$ & .76 & .00 & .09 & .15 & -.02 & -.02 & -.03 & .00 & .32 & - & & \\
\hline 10. Neuroticism & $2.19,0.65$ & .74 & -.14 & -.14 & -.14 & .30 & .27 & .21 & .20 & -.05 & -.17 & - & \\
\hline 11. Extraversion & $3.19,0.55$ & .77 & .03 & -.53 & -.08 & -.09 & -.06 & -.10 & -.03 & .50 & .47 & -.13 & - \\
\hline 12. Conscientiousness & $3.48,0.41$ & .54 & .03 & -.14 & .06 & -.13 & -.08 & -.10 & -.11 & .25 & .24 & -.17 & .20 \\
\hline
\end{tabular}

Note. Significant statistics $(p<.05)$ are bolded. The NSIs variable is the average score across all sources of NSIs. 
Table C2

Descriptive Statistics and Bivariate Correlations for MIDUS 2

\begin{tabular}{|c|c|c|c|c|c|c|c|c|c|c|c|c|c|}
\hline & $M, S D$, or $\%$ & $\alpha$ & 1 & 2 & 3 & 4 & 5 & 6 & 7 & 8 & 9 & 10 & 11 \\
\hline 3. Education & $7.58,2.49$ & - & -.11 & .12 & - & & & & & & & & \\
\hline 5. NSIs from Family & $2.00,0.56$ & .78 & -.21 & -.12 & -.03 & .80 & - & & & & & & \\
\hline 6. NSIs from Partner & $2.07,0.58$ & .78 & -.11 & -.06 & .02 & .75 & .34 & - & & & & & \\
\hline 7. NSIs from Friends & $1.79,0.47$ & .78 & -.11 & .01 & .00 & .76 & .50 & .33 & - & & & & \\
\hline 10. Neuroticism & $2.02,0.61$ & .74 & -.17 & -.15 & -.13 & .29 & .25 & .21 & .21 & -.14 & -.23 & - & \\
\hline 11. Extraversion & $3.10,0.56$ & .75 & .07 & -.05 & -.07 & -.08 & -.05 & -.09 & -.03 & .47 & .49 & -.21 & - \\
\hline 12. Conscientiousness & $3.52,0.41$ & .55 & .00 & -.11 & .03 & -.17 & -.12 & -.12 & -.15 & .24 & .24 & -.18 & .22 \\
\hline
\end{tabular}

Note. Significant statistics $(p<.05)$ are bolded. The NSIs variable is the average score across all sources of NSIs. 
Table C3

Descriptive Statistics and Bivariate Correlations for MIDUS 3

\begin{tabular}{|c|c|c|c|c|c|c|c|c|c|c|c|c|c|}
\hline & $M, S D$, or $\%$ & $\alpha$ & 1 & 2 & 3 & 4 & 5 & 6 & 7 & 8 & 9 & 10 & 11 \\
\hline 1. Age & $64.02,10.47$ & - & - & & & & & & & & & & \\
\hline 2. Gender $(0=$ Female $)$ & $49.40 \%$ Female & - & .11 & - & & & & & & & & & \\
\hline 3. Education & $7.61,2.48$ & - & -.12 & .10 & - & & & & & & & & \\
\hline 4. NSIs & $1.88,0.44$ & .84 & -.19 & -.06 & .00 & - & & & & & & & \\
\hline 5. NSIs from Family & $1.92,0.59$ & .79 & -.24 & -.06 & -.01 & .81 & - & & & & & & \\
\hline 6. NSIs from Partner & $2.02,0.62$ & .79 & -.10 & -.10 & -.01 & .74 & .34 & - & & & & & \\
\hline 7. NSIs from Friends & $1.69,0.51$ & .81 & -.12 & .03 & .01 & .76 & .53 & .31 & - & & & & \\
\hline 8. Agreeableness & $3.42,0.49$ & .77 & .06 & -.30 & -.10 & -.10 & -.08 & -.05 & -.09 & - & & & \\
\hline 9. Openness & $2.87,0.53$ & .77 & -.01 & .04 & .17 & -.04 & -.03 & -.06 & .00 & .36 & - & & \\
\hline 10. Neuroticism & $2.04,0.61$ & .71 & -.12 & -.15 & -.14 & .31 & .25 & .25 & .20 & -.09 & -.19 & - & \\
\hline 11. Extraversion & $3.08,0.57$ & .74 & .01 & -.05 & -.06 & -.08 & -.04 & -.09 & -.05 & .47 & .52 & -.15 & - \\
\hline 12. Conscientiousness & $3.49,0.44$ & .55 & -.09 & -.10 & .08 & -.15 & -.10 & -.14 & -.11 & .25 & .28 & -.19 & .24 \\
\hline
\end{tabular}

Note. Significant statistics $(p<.05)$ are bolded. The NSIs variable is the average score across all sources of NSIs. 
Table C4

Descriptive Statistics and Bivariate Correlations for MIDUS 1 - Younger Adults

\begin{tabular}{|c|c|c|c|c|c|c|c|c|c|c|c|c|c|}
\hline & $M, S D$, or $\%$ & $\alpha$ & 1 & 2 & 3 & 4 & 5 & 6 & 7 & 8 & 9 & 10 & 11 \\
\hline 1. Age & $33.67,3.90$ & - & - & & & & & & & & & & \\
\hline 2. Gender $(0=$ Female $)$ & $55.40 \%$ Female & - & .00 & - & & & & & & & & & \\
\hline 3. Education & $7.54,2.29$ & - & -.04 & .02 & - & & & & & & & & \\
\hline 4. NSIs & $2.05,0.39$ & .78 & .01 & -.10 & -.07 & - & & & & & & & \\
\hline 5. NSIs from Family & $2.09,0.60$ & .79 & .01 & -.12 & -.15 & .77 & - & & & & & & \\
\hline 6. NSIs from Partner & $2.12,0.56$ & .79 & .01 & -.06 & .01 & .67 & .22 & - & & & & & \\
\hline 7. NSIs from Friends & $1.94,0.47$ & .75 & .01 & -.01 & -.02 & .69 & .37 & .19 & - & & & & \\
\hline 8. Agreeableness & $3.40,0.49$ & .79 & .03 & -.32 & -.01 & -.03 & -.02 & -.06 & .01 & - & & & \\
\hline 9. Openness & $2.95,0.49$ & .75 & -.09 & .13 & .13 & -.02 & -.05 & -.03 & .04 & .32 & - & & \\
\hline 10. Neuroticism & $2.29,0.67$ & .74 & -.06 & -.12 & -.18 & .32 & .30 & .18 & .21 & -.07 & -.11 & - & \\
\hline 11. Extraversion & $3.18,0.55$ & .77 & -.07 & -.07 & .00 & -.08 & -.06 & -.07 & -.03 & .43 & .44 & -.12 & - \\
\hline 12. Conscientiousness & $3.45,0.42$ & .52 & -.02 & -.26 & .03 & -.12 & -.05 & -.13 & -.08 & .24 & .07 & -.10 & .10 \\
\hline
\end{tabular}

Note. Significant statistics $(p<.05)$ are bolded. Younger adults' $(n=448)$ ages ranged from $25-39,91.10 \%$ were married, and 95.50\% identified as White/Caucasian. The NSIs variable is the average score across all sources of NSIs. 
Table C5

Descriptive Statistics and Bivariate Correlations for MIDUS 1 - Middle-Aged Adults

\begin{tabular}{|c|c|c|c|c|c|c|c|c|c|c|c|c|c|}
\hline & $M, S D$, or $\%$ & $\alpha$ & 1 & 2 & 3 & 4 & 5 & 6 & 7 & 8 & 9 & 10 & 11 \\
\hline 1. Age & $48.65,5.47$ & - & - & & & & & & & & & & \\
\hline 2. Gender $(0=$ Female $)$ & $48.20 \%$ Female & - & .08 & - & & & & & & & & & \\
\hline 3. Education & $7.42,2.41$ & - & -.04 & .19 & - & & & & & & & & \\
\hline 4. NSIs & $2.03,0.40$ & .82 & -.16 & -.09 & .03 & - & & & & & & & \\
\hline 5. NSIs from Family & $2.09,0.54$ & .76 & -.19 & -.17 & -.01 & .80 & - & & & & & & \\
\hline 6. NSIs from Partner & $2.11,0.59$ & .79 & -.09 & -.06 & .07 & .75 & .34 & - & & & & & \\
\hline 7. NSIs from Friends & $1.87,0.45$ & .76 & -.09 & .02 & .00 & .74 & .49 & .31 & - & & & & \\
\hline 8. Agreeableness & $3.47,0.47$ & .80 & .06 & -.25 & -.11 & -.11 & -.05 & -.11 & -.09 & - & & & \\
\hline 9. Openness & $3.00,0.50$ & .76 & .00 & .08 & .17 & -.04 & -.01 & -.06 & -.02 & .30 & - & & \\
\hline 10. Neuroticism & $2.16,0.64$ & .75 & -.15 & -.14 & -.13 & .30 & .28 & .22 & .19 & -.04 & -.21 & - & \\
\hline 11. Extraversion & $3.18,0.54$ & .76 & .07 & -.07 & -.11 & -.10 & -.06 & -.12 & -.04 & .51 & .46 & -.14 & - \\
\hline 12. Conscientiousness & $3.49,0.40$ & .55 & .02 & -.11 & .10 & -.14 & -.09 & -.11 & -.13 & .29 & .35 & -.19 & .28 \\
\hline
\end{tabular}

Note. Significant statistics $(p<.05)$ are bolded. Middle-aged adults' $(n=909)$ ages ranged from $40-59,95.70 \%$ were married, and 95.60\% identified as White/Caucasian. The NSIs variable is the average score across all sources of NSIs. 
Table C6

Descriptive Statistics and Bivariate Correlations for MIDUS 1 - Older Adults

\begin{tabular}{|c|c|c|c|c|c|c|c|c|c|c|c|c|c|}
\hline & $M, S D$, or $\%$ & $\alpha$ & 1 & 2 & 3 & 4 & 5 & 6 & 7 & 8 & 9 & 10 & 11 \\
\hline 1. Age & $64.28,3.71$ & - & - & & & & & & & & & & \\
\hline 2. Gender $(0=$ Female $)$ & $40.50 \%$ Female & - & .16 & - & & & & & & & & & \\
\hline 3. Education & $7.15,2.49$ & - & .02 & .19 & - & & & & & & & & \\
\hline 4. NSIs & $1.91,0.36$ & .80 & -.13 & .04 & .04 & - & & & & & & & \\
\hline 5. NSIs from Family & $1.91,0.47$ & .72 & -.12 & -.01 & .02 & .78 & - & & & & & & \\
\hline 6. NSIs from Partner & $2.06,0.55$ & .76 & -.09 & .00 & .03 & .73 & .30 & - & & & & & \\
\hline 7. NSIs from Friends & $1.76,0.44$ & .78 & -.07 & .12 & .04 & .74 & .49 & .26 & - & & & & \\
\hline 8. Agreeableness & $3.50,0.54$ & .85 & -.02 & -.23 & -.16 & -.06 & .01 & -.07 & -.09 & - & & & \\
\hline 9. Openness & $2.96,0.52$ & .77 & .07 & .07 & .16 & .04 & -.02 & .03 & .07 & .39 & - & & \\
\hline 10. Neuroticism & $2.07,0.61$ & .70 & .00 & -.15 & -.15 & .24 & .15 & .25 & .16 & -.02 & -.18 & - & \\
\hline 11. Extraversion & $3.23,0.58$ & .79 & .12 & .03 & -.15 & -.06 & -.05 & -.09 & -.02 & .63 & .59 & -.18 & - \\
\hline 12. Conscientiousness & $3.47,0.44$ & .53 & -.02 & -.04 & -.01 & -.13 & -.20 & -.01 & -.12 & .13 & .22 & -.24 & .16 \\
\hline
\end{tabular}

Note. Significant statistics $(p<.05)$ are bolded. Older adults' $(n=173)$ ages ranged from $60-74,98.80 \%$ were married, and 94.20\% identified as White/Caucasian. The NSIs variable is the average score across all sources of NSIs. 
Table C7

Descriptive Statistics and Bivariate Correlations for MIDUS 2 - Younger Adults

\begin{tabular}{|c|c|c|c|c|c|c|c|c|c|c|c|c|c|}
\hline & $M, S D$, or $\%$ & $\alpha$ & 1 & 2 & 3 & 4 & 5 & 6 & 7 & 8 & 9 & 10 & 11 \\
\hline 1. Age & $42.63,3.89$ & - & - & & & & & & & & & & \\
\hline 2. Gender $(0=$ Female $)$ & $55.40 \%$ Female & - & .01 & - & & & & & & & & & \\
\hline 3. Education & $7.87,2.41$ & - & -.08 & -.02 & - & & & & & & & & \\
\hline 4. NSIs & $2.02,0.42$ & .83 & -.06 & -.06 & -.06 & - & & & & & & & \\
\hline 5. NSIs from Family & $2.10,0.61$ & .81 & -.06 & -.14 & -.12 & .80 & - & & & & & & \\
\hline 6. NSIs from Partner & $2.12,0.60$ & .77 & -.06 & .03 & .00 & .73 & .30 & - & & & & & \\
\hline 7. NSIs from Friends & $1.83,0.46$ & .78 & -.01 & -.02 & -.02 & .74 & .48 & .30 & - & & & & \\
\hline 8. Agreeableness & $3.33,0.54$ & .83 & .08 & -.33 & -.01 & -.04 & -.03 & -.05 & .01 & - & & & \\
\hline 9. Openness & $2.83,0.56$ & .80 & -.04 & .12 & .15 & -.08 & -.03 & -.10 & -.04 & .27 & - & & \\
\hline 10. Neuroticism & $2.15,0.64$ & .75 & -.04 & -.12 & -.14 & .28 & .23 & .19 & .20 & -.15 & -.24 & - & \\
\hline 11. Extraversion & $3.05,0.58$ & .76 & -.01 & -.09 & .01 & -.02 & .01 & -.06 & .01 & .44 & .48 & -.19 & - \\
\hline 12. Conscientiousness & $3.50,0.42$ & .54 & .01 & -.17 & .01 & -.20 & -.16 & -.13 & -.18 & .20 & .09 & -.12 & .12 \\
\hline
\end{tabular}

Note. Significant statistics $(p<.05)$ are bolded. Younger adults' $(n=448)$ ages ranged from $34-49,94.40 \%$ were married, and $94.00 \%$ identified as White/Caucasian. The NSIs variable is the average score across all sources of NSIs. 
Table C8

Descriptive Statistics and Bivariate Correlations for MIDUS 2 - Middle-Aged Adults

\begin{tabular}{|c|c|c|c|c|c|c|c|c|c|c|c|c|c|}
\hline & $M, S D$, or $\%$ & $\alpha$ & 1 & 2 & 3 & 4 & 5 & 6 & 7 & 8 & 9 & 10 & 11 \\
\hline 1. Age & $57.50,5.48$ & - & - & & & & & & & & & & \\
\hline 2. Gender $(0=$ Female $)$ & $48.20 \%$ Female & - & .08 & - & & & & & & & & & \\
\hline 3. Education & $7.51,2.51$ & - & -.08 & .19 & - & & & & & & & & \\
\hline 4. NSIs & $1.95,0.41$ & .84 & -.20 & -.10 & .01 & - & & & & & & & \\
\hline 5. NSIs from Family & $1.98,0.54$ & .78 & -.20 & -.11 & .00 & .80 & - & & & & & & \\
\hline 6. NSIs from Partner & $2.05,0.58$ & .79 & -.14 & -.10 & .03 & .76 & .37 & - & & & & & \\
\hline 7. NSIs from Friends & $1.80,0.46$ & .78 & -.13 & .00 & -.01 & .76 & .50 & .34 & - & & & & \\
\hline 8. Agreeableness & $3.47,0.45$ & .76 & .06 & -.27 & -.18 & -.14 & -.09 & -.12 & -.11 & - & & & \\
\hline 9. Openness & $2.94,0.49$ & .74 & .02 & .06 & .16 & -.07 & -.05 & -.08 & -.03 & .27 & - & & \\
\hline 10. Neuroticism & $1.98,0.59$ & .73 & -.13 & -.13 & -.13 & .28 & .24 & .21 & .20 & -.14 & -.23 & - & \\
\hline 11. Extraversion & $3.13,0.54$ & .74 & .07 & -.05 & -.10 & -.11 & -.07 & -.12 & -.05 & .48 & .48 & -.22 & - \\
\hline 12. Conscientiousness & $3.54,0.39$ & .55 & .02 & -.07 & .07 & -.14 & -.08 & -.12 & -.11 & .24 & .33 & -.24 & .27 \\
\hline
\end{tabular}

Note. Significant statistics $(p<.05)$ are bolded. Middle-aged adults' $(n=909)$ ages ranged from $48-69,97.60 \%$ were married, and 94.60\% identified as White/Caucasian. The NSIs variable is the average score across all sources of NSIs. 
Table C9

Descriptive Statistics and Bivariate Correlations for MIDUS 2 - Older Adults

\begin{tabular}{|c|c|c|c|c|c|c|c|c|c|c|c|c|c|}
\hline & $M, S D$, or $\%$ & $\alpha$ & 1 & 2 & 3 & 4 & 5 & 6 & 7 & 8 & 9 & 10 & 11 \\
\hline 1. Age & $73.08,3.76$ & - & - & & & & & & & & & & \\
\hline 2. Gender $(0=$ Female $)$ & $40.50 \%$ Female & - & .16 & - & & & & & & & & & \\
\hline 3. Education & $7.19,2.56$ & - & .01 & .17 & - & & & & & & & & \\
\hline 4. NSIs & $1.84,0.38$ & .80 & -.13 & .05 & -.01 & - & & & & & & & \\
\hline 5. NSIs from Family & $1.79,0.45$ & .65 & -.14 & .00 & -.07 & .75 & - & & & & & & \\
\hline 6. NSIs from Partner & $2.01,0.56$ & .76 & -.12 & -.03 & .00 & .74 & .29 & - & & & & & \\
\hline 7. NSIs from Friends & $1.68,0.49$ & .80 & -.08 & .19 & .02 & .76 & .46 & .28 & - & & & & \\
\hline 8. Agreeableness & $3.49,0.50$ & .83 & -.07 & -.33 & -.19 & -.21 & -.14 & -.08 & -.29 & - & & & \\
\hline 9. Openness & $2.81,0.51$ & .75 & .03 & .00 & .16 & -.09 & -.09 & -.05 & -.06 & .37 & - & & \\
\hline 10. Neuroticism & $1.90,0.57$ & .72 & -.11 & -.21 & -.23 & .29 & .22 & .22 & .21 & .03 & -.14 & - & \\
\hline 11. Extraversion & $3.13,0.57$ & .76 & -.08 & .01 & -.09 & -.02 & -.03 & .00 & .01 & .48 & .56 & -.14 & - \\
\hline 12. Conscientiousness & $3.46,0.44$ & .59 & -.10 & -.12 & -.07 & -.25 & -.20 & -.08 & -.30 & .36 & .21 & -.08 & .24 \\
\hline
\end{tabular}

Note. Significant statistics $(p<.05)$ are bolded. Older adults' $(n=173)$ ages ranged from $68-83,97.10 \%$ were married, and 94.80\% identified as White/Caucasian. The NSIs variable is the average score across all sources of NSIs. 
Table C10

Descriptive Statistics and Bivariate Correlations for MIDUS 3 - Younger Adults

\begin{tabular}{|c|c|c|c|c|c|c|c|c|c|c|c|c|c|}
\hline & $M, S D$, or $\%$ & $\alpha$ & 1 & 2 & 3 & 4 & 5 & 6 & 7 & 8 & 9 & 10 & 11 \\
\hline 1. Age & $51.72,3.89$ & - & - & & & & & & & & & & \\
\hline 2. Gender $(0=$ Female $)$ & $55.40 \%$ Female & - & .02 & - & & & & & & & & & \\
\hline 3. Education & $7.93,2.37$ & - & -.07 & .00 & - & & & & & & & & \\
\hline 4. NSIs & $1.98,0.43$ & .82 & -.15 & -.01 & -.01 & - & & & & & & & \\
\hline 5. NSIs from Family & $2.08,0.61$ & .80 & -.10 & -.06 & -.04 & .78 & - & & & & & & \\
\hline 6. NSIs from Partner & $2.08,0.63$ & .79 & -.12 & .00 & -.01 & .71 & .27 & - & & & & & \\
\hline 7. NSIs from Friends & $1.78,0.50$ & .79 & -.12 & .05 & .04 & .72 & .45 & .24 & - & & & & \\
\hline 8. Agreeableness & $3.35,0.52$ & .79 & .11 & -.27 & -.03 & -.03 & -.01 & -.04 & -.02 & - & & & \\
\hline 9. Openness & $2.83,0.56$ & .80 & .04 & .11 & .16 & -.01 & -.02 & -.01 & .01 & .38 & - & & \\
\hline 10. Neuroticism & $2.14,0.64$ & .72 & -.12 & -.12 & -.15 & .26 & .24 & .21 & .11 & -.13 & -.18 & - & \\
\hline 11. Extraversion & $3.04,0.56$ & .73 & .00 & -.04 & -.03 & -.06 & .00 & -.10 & -.04 & .42 & .55 & -.13 & - \\
\hline 12. Conscientiousness & $3.51,0.46$ & .61 & .04 & -.16 & .03 & -.21 & -.16 & -.18 & -.11 & .24 & .16 & -.19 & .15 \\
\hline
\end{tabular}

Note. Significant statistics $(p<.05)$ are bolded. Younger adults' $(n=448)$ ages ranged from $42-58,94.40 \%$ were married, and 91.30\% identified as White/Caucasian. The NSIs variable is the average score across all sources of NSIs. 
Table C11

Descriptive Statistics and Bivariate Correlations for MIDUS 3 - Middle-Aged Adults

\begin{tabular}{|c|c|c|c|c|c|c|c|c|c|c|c|c|c|}
\hline & $M, S D$, or $\%$ & $\alpha$ & 1 & 2 & 3 & 4 & 5 & 6 & 7 & 8 & 9 & 10 & 11 \\
\hline 1. Age & $66.63,5.50$ & - & - & & & & & & & & & & \\
\hline 2. Gender $(0=$ Female $)$ & $48.20 \%$ Female & - & .08 & - & & & & & & & & & \\
\hline 3. Education & $7.54,2.48$ & - & -.07 & .15 & - & & & & & & & & \\
\hline 4. NSIs & $1.85,0.44$ & .85 & -.10 & -.08 & -.04 & - & & & & & & & \\
\hline 5. NSIs from Family & $1.87,0.58$ & .79 & -.15 & -.06 & -.03 & .81 & - & & & & & & \\
\hline 6. NSIs from Partner & $2.01,0.63$ & .81 & -.07 & -.15 & -.03 & .76 & .37 & - & & & & & \\
\hline 7. NSIs from Friends & $1.66,0.50$ & .82 & -.01 & .02 & -.04 & .77 & .54 & .33 & - & & & & \\
\hline 8. Agreeableness & $3.46,0.47$ & .76 & -.02 & -.33 & -.10 & -.11 & -.10 & -.05 & -.11 & - & & & \\
\hline 9. Openness & $2.91,0.51$ & .76 & -.03 & .03 & .18 & -.10 & -.07 & -.11 & -.04 & .33 & - & & \\
\hline 10. Neuroticism & $2.00,0.60$ & .71 & -.08 & -.14 & -.14 & .32 & .25 & .27 & .23 & -.09 & -.20 & - & \\
\hline 11. Extraversion & $3.12,0.55$ & .73 & .04 & -.04 & -.05 & -.12 & -.09 & -.10 & -.09 & .48 & .48 & -.18 & - \\
\hline 12. Conscientiousness & $3.51,0.41$ & .51 & -.07 & -.08 & .10 & -.16 & -.10 & -.15 & -.13 & .26 & .32 & -.21 & .25 \\
\hline
\end{tabular}

Note. Significant statistics $(p<.05)$ are bolded. Middle-aged adults' ages $(n=909)$ ranged from $57-78,98.00 \%$ were married, and 91.70\% identified as White/Caucasian. The NSIs variable is the average score across all sources of NSIs. 
Table C12

Descriptive Statistics and Bivariate Correlations for MIDUS 3 - Older Adults

\begin{tabular}{|c|c|c|c|c|c|c|c|c|c|c|c|c|c|}
\hline & $M, S D$, or $\%$ & $\alpha$ & 1 & 2 & 3 & 4 & 5 & 6 & 7 & 8 & 9 & 10 & 11 \\
\hline 1. Age & $82.16,3.79$ & - & - & & & & & & & & & & \\
\hline 2. Gender $(0=$ Female $)$ & $40.50 \%$ Female & - & .16 & - & & & & & & & & & \\
\hline 3. Education & $7.12,2.62$ & - & .00 & .18 & - & & & & & & & & \\
\hline 4. NSIs & $1.76,0.41$ & .82 & -.10 & .07 & .07 & - & & & & & & & \\
\hline 5. NSIs from Family & $1.72,0.50$ & .69 & -.16 & .10 & -.01 & .82 & - & & & & & & \\
\hline 6. NSIs from Partner & $1.93,0.56$ & .73 & -.04 & -.07 & .07 & .74 & .34 & - & & & & & \\
\hline 7. NSIs from Friends & $1.60,0.50$ & .46 & -.07 & .15 & .11 & .82 & .63 & .33 & - & & & & \\
\hline 8. Agreeableness & $3.40,0.48$ & .73 & -.04 & -.32 & -.19 & -.14 & -.11 & -.12 & -.10 & - & & & \\
\hline 9. Openness & $2.76,0.53$ & .77 & .00 & -.07 & .14 & .16 & .14 & .07 & .21 & .39 & - & & \\
\hline 10. Neuroticism & $1.98,0.57$ & .67 & -.04 & -.17 & -.21 & .25 & .21 & .22 & .17 & .07 & -.07 & - & \\
\hline 11. Extraversion & $2.99,0.63$ & .80 & -.03 & -.09 & -.14 & .07 & .09 & -.01 & .10 & .57 & .62 & -.03 & - \\
\hline 12. Conscientiousness & $3.34,0.49$ & .51 & -.04 & -.01 & .01 & -.09 & -.12 & -.04 & -.06 & .26 & .40 & -.17 & .34 \\
\hline
\end{tabular}

Note. Significant statistics $(p<.05)$ are bolded. Older adults' $(n=173)$ ages ranged from $73-92,96.50 \%$ were married, and 91.20\% identified as White/Caucasian. The NSIs variable is the average score across all sources of NSIs. 


\section{Appendix D}

Missing Data and Analyses Comparing Multivariate Outliers to Non-Multivariate Outliers Table D1

Missing Data across all MIDUS Waves

\begin{tabular}{lcccccc}
\hline & \multicolumn{2}{c}{ MIDUS 1 } & \multicolumn{2}{c}{ MIDUS 2 } & MIDUS 3 \\
& Missing & Missing & Missing & Missing & Missing & Missing \\
& Count & Percent & Count & Percent & Count & Percent \\
\hline Age & 0 & $0.00 \%$ & 0 & $0.00 \%$ & 0 & $0.00 \%$ \\
Gender & 0 & $0.00 \%$ & 0 & $0.00 \%$ & 0 & $0.00 \%$ \\
Education & 1 & $0.10 \%$ & 2 & $0.10 \%$ & 2 & $0.10 \%$ \\
NSIs & 0 & $0.00 \%$ & 0 & $0.00 \%$ & 0 & $0.00 \%$ \\
Agreeableness & 2 & $0.10 \%$ & 5 & $0.30 \%$ & 5 & $0.30 \%$ \\
Openness & 3 & $0.20 \%$ & 12 & $0.80 \%$ & 7 & $0.50 \%$ \\
Neuroticism & 4 & $0.30 \%$ & 7 & $0.50 \%$ & 5 & $0.30 \%$ \\
Extraversion & 2 & $0.10 \%$ & 5 & $0.30 \%$ & 6 & $0.40 \%$ \\
Conscientiousness & 2 & $0.10 \%$ & 5 & $0.30 \%$ & 5 & $0.30 \%$ \\
\hline
\end{tabular}


Table D2

Attrition Analyses Comparing Non-Multivariate Outliers and Multivariate Outliers for MIDUS 1 Variables

\begin{tabular}{|c|c|c|c|}
\hline & Non-Multivariate & Multivariate & Difference \\
\hline & Outliers & Outliers & Statistic \\
\hline & $(n=1,403)$ & $(n=127)$ & \\
\hline & $M, S D$ or $\%$ & $M, S D$ or $\%$ & \\
\hline Age & $46.35,10.37$ & $42.43,11.31$ & $t(1,528)=4.05$ \\
\hline Gender & $49.10 \%$ Female & $52.80 \%$ Female & $\chi^{2}(1)=0.62$ \\
\hline Education & $7.47,2.40$ & $6.99,2.44$ & $t(1,527)=2.12$ \\
\hline Marital Status & $96.80 \%$ Married & $71.70 \%$ Married & $\chi^{2}(1)=146.79$ \\
\hline Race & 96.70\% White & $80.30 \%$ White & $\chi^{2}(1)=82.42$ \\
\hline NSIs & $2.02,0.39$ & $2.08,0.41$ & $t(1,528)=-1.61$ \\
\hline Agreeableness & $3.45,0.48$ & $3.46,0.50$ & $t(1,526)=-0.25$ \\
\hline Openness & $2.97,0.50$ & $3.11,0.51$ & $t(1,525)=-2.91$ \\
\hline Neuroticism & $2.18,0.63$ & $2.28,0.68$ & $t(1,524)=-1.64$ \\
\hline Extraversion & $3.18,0.55$ & $3.23,0.56$ & $t(1,526)=-0.93$ \\
\hline Conscientiousness & $3.48,0.41$ & $3.42,0.45$ & $t(145.40)=1.48$ \\
\hline
\end{tabular}

Note. Significant statistics $(p<.05)$ are bolded. 
Table D3

Attrition Analyses Comparing Non-Multivariate Outliers and Multivariate Outliers for MIDUS 2 Variables

\begin{tabular}{|c|c|c|c|}
\hline & Non-Multivariate & Multivariate & Difference \\
\hline & Outliers & Outliers & Statistic \\
\hline & $(n=1,403)$ & $(n=127)$ & \\
\hline & $M, S D$ or $\%$ & $M, S D$ or $\%$ & \\
\hline Age & $55.22,10.32$ & $51.43,11.29$ & $t(1,528)=3.93$ \\
\hline Gender & $49.10 \%$ Female & $52.80 \%$ Female & $\chi^{2}(1)=0.62$ \\
\hline Education & $7.56,2.47$ & $7.80,2.72$ & $t(145.57)=-0.97$ \\
\hline Marital Status & $99.40 \%$ Married & $65.40 \%$ Married & $\chi^{2}(1)=421.11$ \\
\hline Race & 96.50\% White & 71.70\% White & $\chi^{2}(1)=137.10$ \\
\hline NSIs & $1.94,0.40$ & $2.09,0.46$ & $t(143.76)=-3.50$ \\
\hline Agreeableness & $3.43,0.48$ & $3.41,0.53$ & $t(1,523)=0.48$ \\
\hline Openness & $2.89,0.51$ & $2.96,0.63$ & $t(141.34)=-1.21$ \\
\hline Neuroticism & $2.01,0.60$ & $2.17,0.70$ & $t(143.37)=-2.56$ \\
\hline Extraversion & $3.10,0.55$ & $3.09,0.60$ & $t(1,523)=0.24$ \\
\hline Conscientiousness & $3.52,0.40$ & $3.45,0.49$ & $t(141.75)=1.73$ \\
\hline
\end{tabular}

Note. Significant statistics $(p<.05)$ are bolded. 
Table D4

Attrition Analyses Comparing Non-Multivariate Outliers and Multivariate Outliers for MIDUS 3 Variables

\begin{tabular}{|c|c|c|c|}
\hline & Non-Multivariate & Multivariate & Difference \\
\hline & Outliers & Outliers & Statistic \\
\hline & $(n=1,403)$ & $(n=127)$ & \\
\hline & $M, S D$ or $\%$ & $M, S D$ or $\%$ & \\
\hline Age & $64.33,10.33$ & $60.53,11.29$ & $t(1,528)=3.94$ \\
\hline Gender & $49.10 \%$ Female & $52.80 \%$ Female & $\chi^{2}(1)=0.62$ \\
\hline Education & $7.62,2.46$ & $7.46,2.66$ & $t(1,526)=0.71$ \\
\hline Marital Status & 99.80\% Married & $63.80 \%$ Married & $\chi^{2}(1)=487.04$ \\
\hline Race & $92.20 \%$ White & 78.00\% White & $\chi^{2}(1)=32.85$ \\
\hline NSIs & $1.86,0.43$ & $2.02,0.50$ & $t(144.12)=-3.86$ \\
\hline Agreeableness & $3.43,0.48$ & $3.36,0.52$ & $t(1,523)=1.39$ \\
\hline Openness & $2.86,0.53$ & $2.95,0.58$ & $t(1,521)=-1.89$ \\
\hline Neuroticism & $2.02,0.60$ & $2.22,0.69$ & $t(1,523)=-3.53$ \\
\hline Extraversion & $3.08,0.57$ & $3.08,0.53$ & $t(1,522)=-0.05$ \\
\hline Conscientiousness & $3.50,0.43$ & $3.39,0.53$ & $t(141.37)=2.15$ \\
\hline
\end{tabular}

Note. Significant statistics $(p<.05)$ are bolded. 


\section{Appendix E \\ Results that were Qualified by Age}

\section{Research Questions 1 and 2}

A univariate latent growth curve was modeled in AMOS to examine the rate of change in NSIs. The model fit the data well (see Table E1). NSIs significantly decreased over time (see Figure F1 in Appendix F). There was no significant association between participants' initial frequency of NSIs and their rate of change over time.

Age, gender, education, and the Big Five personality traits were added to the model to account for the significant variability surrounding the intercept and slope. The model fit was poor, which indicates that significant individual differences remained unaccounted for in the model (see Table E2). Being one standard deviation higher in openness or neuroticism was associated with more NSIs at MIDUS 1. Being older, male, or one standard deviation higher in agreeableness or conscientiousness was associated with fewer NSIs at MIDUS 1. Being older or one standard deviation higher in neuroticism was associated with a steeper decrease in NSIs over time.

\section{Research Questions 4 and 5}

Agreeableness. A univariate latent growth curve was modeled in AMOS to examine the rate of change in agreeableness. The model fit the data well (see Table E3). Agreeableness significantly decreased by 0.002 scale units every year (see Figure F2 in Appendix F). There was no significant association between participants' levels of agreeableness and their rate of change over time.

Age, gender, education, and NSIs were added to the model to account for the significant variability surrounding the intercept and slope. The model adequately fit the data (see Table E4). 
Being older was associated with higher levels of agreeableness at MIDUS 1, whereas being male or one standard deviation higher in education or NSIs was associated with lower levels of agreeableness. Being one standard deviation higher in NSIs was associated with an increase in agreeableness over time, which negated the overall decrease in NSIs.

Openness. A univariate latent growth curve was modeled in AMOS to examine the rate of change in openness. The model fit the data well (see Table E3). Openness significantly decreased by 0.006 scale units every year (see Figure F3 in Appendix F). There was no significant association between participants' levels of openness and their rate of change over time.

Age, gender, education, and NSIs were added to the model to account for the significant variability surrounding the intercept and slope. The model adequately fit the data (see Table E5). Being male or one standard deviation higher in education was associated with higher levels of openness at MIDUS 1. Being male was associated with a decrease in openness at a steeper rate.

Neuroticism. A univariate latent growth curve was modeled in AMOS to examine the rate of change in neuroticism. The model did not fit the data well (see Table E3). Neuroticism significantly decreased by 0.008 scale units every year (see Figure F4 in Appendix F). Participants who scored higher in neuroticism experienced a steeper decrease in neuroticism over time.

Age, gender, education, and NSIs were added to the model to account for the significant variability surrounding the intercept and slope. The model adequately fit the data (see Table E6). Being one standard deviation higher in NSIs was associated with higher levels of neuroticism at MIDUS 1, whereas being older, male, or one standard deviation higher in education was 
associated with lower levels of neuroticism. Being one standard deviation higher in NSIs was associated with a steeper decrease in neuroticism over time.

Conscientiousness. A univariate latent growth curve was modeled in AMOS to examine the rate of change in conscientiousness. The model fit the data well (see Table E3). Conscientiousness increased by 0.001 scale units every year, however, this change was not significant (see Figure F5 in Appendix F). There was no significant association between participants' initial levels of conscientiousness and their rate of change over time.

Age, gender, education, and NSIs were added to the model to account for the significant variability surrounding the intercept and slope. The model adequately fit the data (see Table E7). Being one standard deviation higher in education was associated with higher levels of conscientiousness at MIDUS 1, whereas being male or one standard deviation higher in NSIs was associated with lower levels of conscientiousness. Being older was associated with a less steep increase in conscientiousness over time, whereas being male was associated with a steeper increase in conscientiousness over time.

\section{Research Question 7}

Agreeableness. A cross-domain latent growth curve was modeled in AMOS to examine the association between NSIs and agreeableness. The model fit was poor, which indicates that significant individual differences remained unaccounted for in the model (see Table E8). Higher levels of agreeableness at MIDUS 1 was associated with a decrease in agreeableness at a steeper rate over time.

Openness. A cross-domain latent growth curve was modeled in AMOS to examine the association between NSIs and openness. The model fit was poor, which indicates that significant 
individual differences remained unaccounted for in the model (see Table E9). There were no significant associations between the latent growth curves of NSIs and openness.

Neuroticism. A cross-domain latent growth curve was modeled in AMOS to examine the association between NSIs and neuroticism. The model adequately fit the data (see Table E10). Higher levels of neuroticism at MIDUS 1 was associated with a decrease in neuroticism at a steeper rate over time. Higher levels of neuroticism at MIDUS 1 was also associated with a steeper decrease in NSIs over time.

Extraversion. A cross-domain latent growth curve was modeled in AMOS to examine the association between NSIs and extraversion. The model fit was poor, which indicates that significant individual differences remained unaccounted for in the model (see Table E11). There were no significant associations between the latent growth curves of NSIs and extraversion.

Conscientiousness. A cross-domain latent growth curve was modeled in AMOS to examine the association between NSIs and conscientiousness. The model fit was poor, which indicates that significant individual differences remained unaccounted for in the model (see Table E12). There were no significant associations between the latent growth curves of NSIs and conscientiousness. 
Table E1

Research Question 1 - The Latent Growth Curve of NSIs

\begin{tabular}{|c|c|c|c|c|c|c|c|}
\hline & Model Fit & & & Sl & & Cov & ance \\
\hline & & $b$ & S.E. & $b$ & S.E. & $b$ & S.E. \\
\hline NSIs & $\chi^{2}(3)=3.13$ & $2.027^{*}$ & 0.010 & $-0.008^{*}$ & 0.001 & 0.000 & 0.000 \\
\hline & $\mathrm{CMIN} / \mathrm{DF}=$ & & & & & & \\
\hline & 1.04 & & & & & & \\
\hline & $\mathrm{CFI}=1.000$ & & & & & & \\
\hline & RMSEA $=.00$ & & & & & & \\
\hline
\end{tabular}

Note. Significant statistics $(p<.05)$ are bolded. An asterisk indicates that there is significant variability surrounding the estimate. A blue value indicates that the estimate is significantly different than zero. 
Table E2

Research Question 2 - Demographic Variables and the Big Five Personality Traits Predicting the Latent Growth Curve of NSIS

NSIs Latent Growth Curve

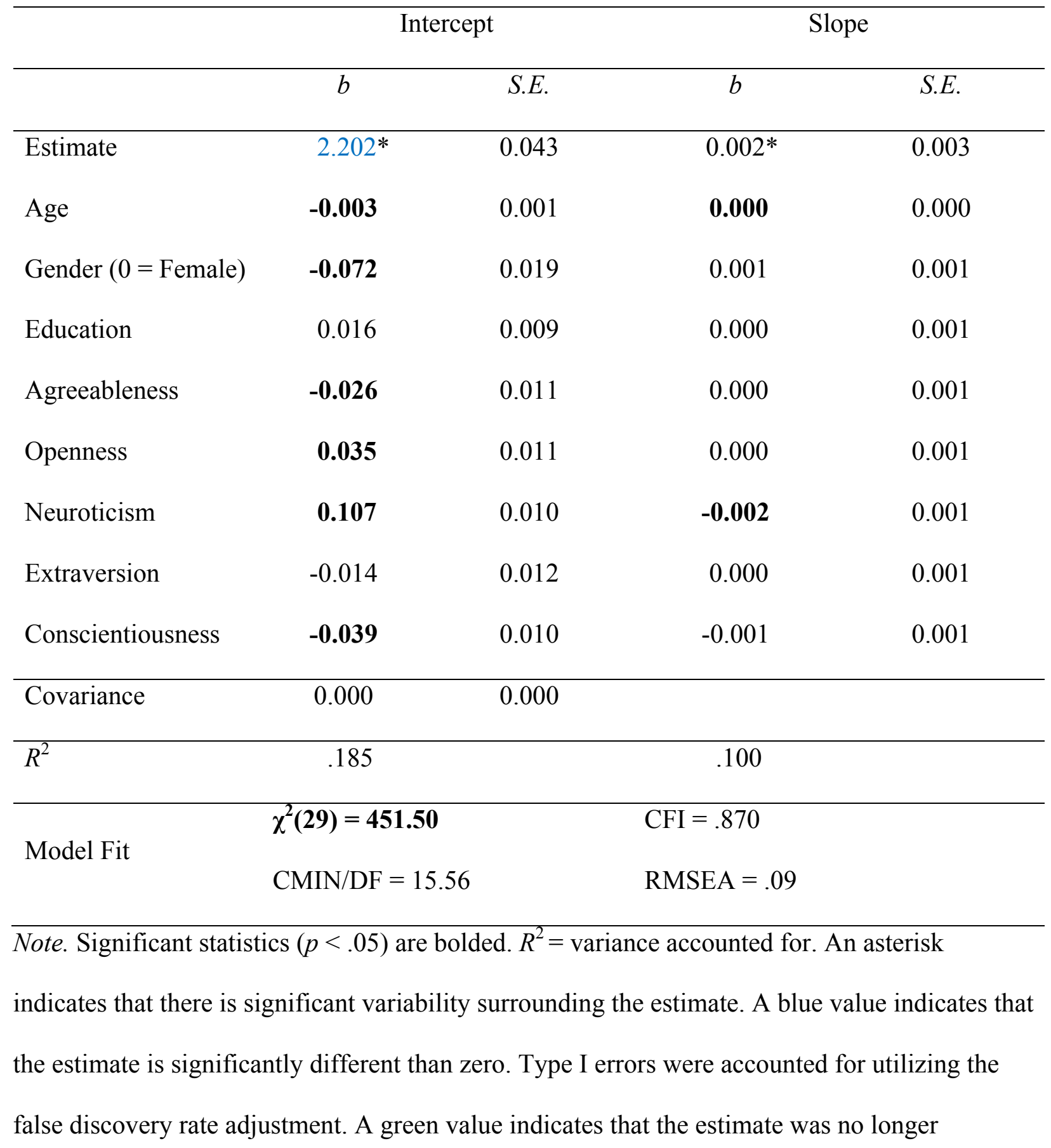


statistically significant after the false discovery rate adjustments. To aid in the interpretation of the estimates, education, agreeableness, openness, neuroticism, extraversion, and conscientiousness were standardized. As such, a unit increase or decrease in these variables represent a standard deviation increase or decrease, respectively. Age was not manipulated so that a unit increase or decrease represents an increase or decrease of a year, respectively. 


\section{Table E3}

Research Question 4 - The Latent Growth Curves of the Big Five Personality Traits

\begin{tabular}{|c|c|c|c|c|c|c|c|}
\hline & Model Fit & Inte & & Slc & & Cova & iance \\
\hline & & $b$ & S.E. & $b$ & S.E. & $b$ & S.E. \\
\hline Agreeableness & $\chi^{2}(3)=1.59$ & $3.456^{*}$ & 0.012 & $-0.002 *$ & 0.001 & -0.001 & 0.000 \\
\hline & $\mathrm{CMIN} / \mathrm{DF}=0.53$ & & & & & & \\
\hline & $\mathrm{CFI}=1.000$ & & & & & & \\
\hline & $\mathrm{RMSEA}=.00$ & & & & & & \\
\hline Openness & $\chi^{2}(3)=18.82$ & $2.977 *$ & 0.013 & $-0.006 *$ & 0.001 & 0.000 & 0.000 \\
\hline & $\mathrm{CMIN} / \mathrm{DF}=6.27$ & & & & & & \\
\hline & $\mathrm{CFI}=.993$ & & & & & & \\
\hline & $\mathrm{RMSEA}=.05$ & & & & & & \\
\hline Neuroticism & $\chi^{2}(3)=70.77$ & $2.162 *$ & 0.016 & $-0.008^{*}$ & 0.001 & -0.004 & 0.001 \\
\hline & $\mathrm{CMIN} / \mathrm{DF}=23.59$ & & & & & & \\
\hline & $\mathrm{CFI}=.965$ & & & & & & \\
\hline & $\mathrm{RMSEA}=.12$ & & & & & & \\
\hline Conscientiousness & $\chi^{2}(3)=31.60$ & $3.493 *$ & 0.010 & $0.001 *$ & 0.001 & 0.000 & 0.000 \\
\hline & $\mathrm{CMIN} / \mathrm{DF}=10.53$ & & & & & & \\
\hline & $\mathrm{CFI}=.982$ & & & & & & \\
\hline & RMSEA $=.07$ & & & & & & \\
\hline
\end{tabular}

Note. Significant statistics $(p<.05)$ are bolded. An asterisk indicates that there is significant variability surrounding the estimate. A blue value indicates that the estimate is significantly different than zero. 
Table E4

Research Question 5 - Demographics Variables and NSIs Predicting the Latent Growth Curve of Agreeableness

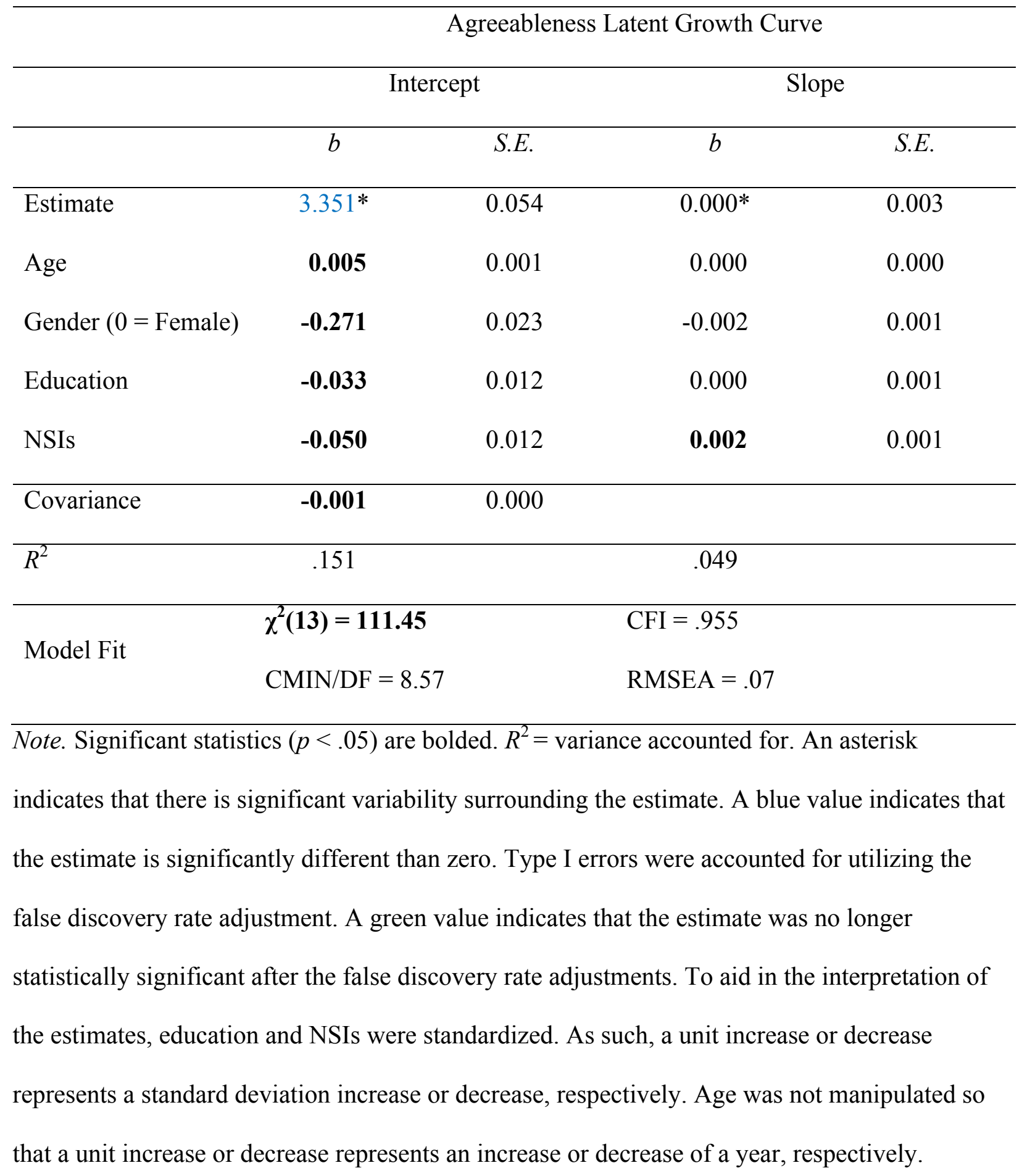


Table E5

Research Question 5 - Demographic Variables and NSIs Predicting the Latent Growth Curve of Openness

\section{Openness Latent Growth Curve}

\begin{tabular}{|c|c|c|c|c|}
\hline & \multicolumn{2}{|c|}{ Intercept } & \multicolumn{2}{|c|}{ Slope } \\
\hline & $b$ & S.E. & $b$ & S.E. \\
\hline Estimate & $2.909 *$ & 0.058 & $-0.003 *$ & 0.003 \\
\hline Age & 0.001 & 0.001 & 0.000 & 0.000 \\
\hline Gender $(0=$ Female $)$ & 0.072 & 0.025 & -0.003 & 0.001 \\
\hline Education & 0.073 & 0.013 & 0.000 & 0.001 \\
\hline NSIs & -0.016 & 0.013 & -0.001 & 0.001 \\
\hline Covariance & 0.000 & 0.000 & & \\
\hline$R^{2}$ & .037 & & .017 & \\
\hline \multirow{2}{*}{ Model Fit } & $\chi^{2}(13)=119.06$ & & $\mathrm{CFI}=.955$ & \\
\hline & $\mathrm{CMIN} / \mathrm{DF}=9.15$ & & $\mathrm{RMSEA}=.07$ & \\
\hline
\end{tabular}

Note. Significant statistics $(p<.05)$ are bolded. $R^{2}=$ variance accounted for. An asterisk

indicates that there is significant variability surrounding the estimate. A blue value indicates that the estimate is significantly different than zero. Type I errors were accounted for utilizing the false discovery rate adjustment. A green value indicates that the estimate was no longer statistically significant after the false discovery rate adjustments. To aid in the interpretation of the estimates, education and NSIs were standardized. As such, a unit increase or decrease represents a standard deviation increase or decrease, respectively. Age was not manipulated so that a unit increase or decrease represents an increase or decrease of a year, respectively. 
Table E6

Research Question 5 - Demographic Variables and NSIs Predicting the Latent Growth Curve of Neuroticism

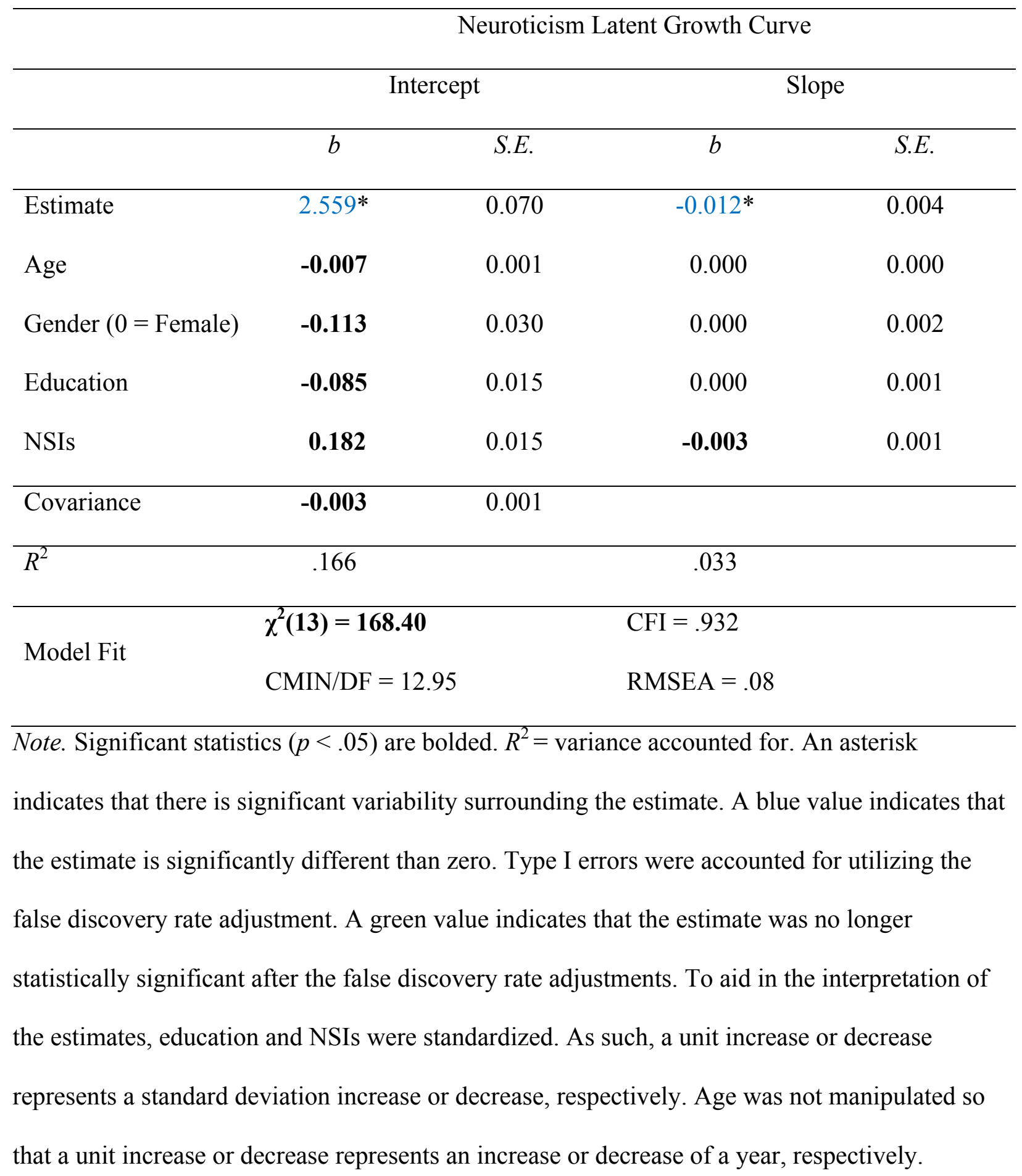


Table E7

Research Question 5 - Demographic Variables and NSIs Predicting the Latent Growth Curve of Conscientiousness

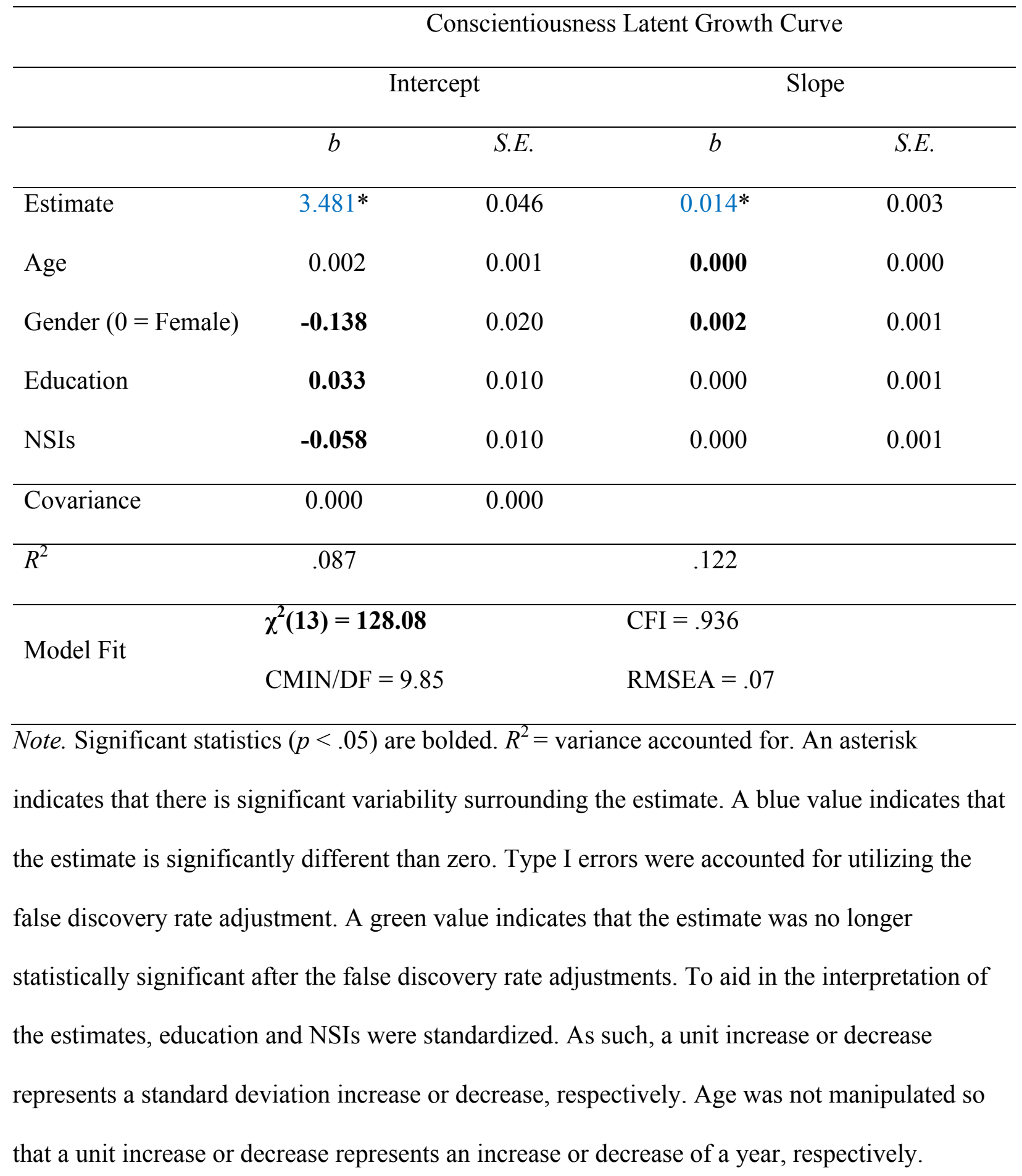


Table E8

Research Question 7 - Examination of the Association between the Latent Growth Curves of NSIs and Agreeableness After Accounting for the Demographic Variables and the Remaining Personality Traits

\begin{tabular}{|c|c|c|c|c|c|c|c|c|}
\hline & \multicolumn{4}{|c|}{ NSIs Latent Growth Curve } & \multicolumn{4}{|c|}{ Agreeableness Latent Growth Curve } \\
\hline & \multicolumn{2}{|c|}{ Intercept } & \multicolumn{2}{|c|}{ Slope } & \multicolumn{2}{|c|}{ Intercept } & \multicolumn{2}{|c|}{ Slope } \\
\hline & $b$ & S.E. & $b$ & S.E. & $b$ & S.E. & $b$ & S.E. \\
\hline Estimate & $2.209 *$ & 0.043 & $-0.003 *$ & 0.011 & $3.319 *$ & 0.054 & $0.008^{*}$ & 0.010 \\
\hline Age & -0.003 & 0.001 & 0.000 & 0.000 & 0.006 & 0.001 & 0.000 & 0.000 \\
\hline $\begin{array}{l}\text { Gender } \\
\qquad(0=\text { Female })\end{array}$ & -0.057 & 0.019 & 0.001 & 0.001 & -0.263 & 0.023 & -0.003 & 0.001 \\
\hline Education & 0.016 & 0.009 & -0.001 & 0.001 & -0.034 & 0.012 & 0.000 & 0.001 \\
\hline Openness & $\mathbf{0 . 0 3 7}$ & 0.011 & 0.000 & 0.001 & - & - & - & - \\
\hline Neuroticism & 0.105 & 0.010 & -0.003 & 0.001 & - & - & - & - \\
\hline Extraversion & -0.011 & 0.011 & 0.000 & 0.001 & - & - & - & - \\
\hline Conscientiousness & -0.037 & 0.010 & 0.000 & 0.001 & - & - & - & - \\
\hline NSIs Intercept & - & - & 0.003 & 0.003 & - & - & 0.004 & 0.002 \\
\hline
\end{tabular}




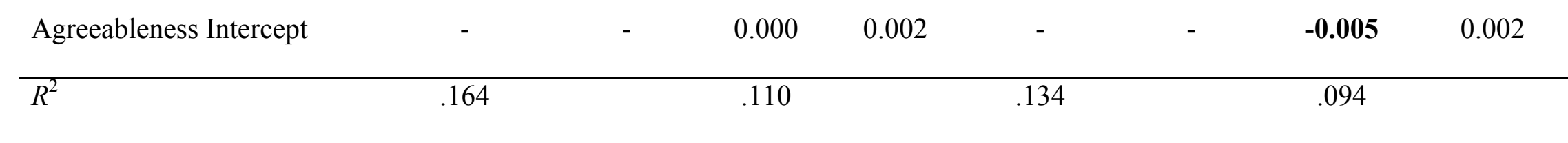

\begin{tabular}{ll}
\hline Model Fit & $\chi^{\mathbf{2}} \mathbf{4 9 )}=\mathbf{8 6 7 . 7 9}$ \\
$\mathrm{CMIN} / \mathrm{DF}=17.71$ \\
$\mathrm{CFI}=.844$ \\
$\mathrm{RMSEA}=.10$
\end{tabular}

Note. Significant statistics $(p<.05)$ are bolded. $R^{2}=$ variance accounted for. An asterisk indicates that there is significant variability surrounding the estimate. A blue value indicates that the estimate is significantly different than zero. Type I errors were accounted for utilizing the false discovery rate adjustment. A green value indicates that the estimate was no longer statistically significant after the false discovery rate adjustments. To aid in the interpretation of the estimates, education, openness, neuroticism, extraversion, and conscientiousness were standardized. As such, a unit increase or decrease in these variables represent a standard deviation increase or decrease, respectively. Age was not manipulated so that a unit increase or decrease represents an increase or decrease of a year, respectively. 
Table E9

Research Question 7 - Examination of the Association between the Latent Growth Curves of NSIs and Openness After Accounting for the Demographic Variables and the Remaining Personality Traits

\begin{tabular}{|c|c|c|c|c|c|c|c|c|}
\hline & \multicolumn{4}{|c|}{ NSIs Latent Growth Curve } & \multicolumn{4}{|c|}{ Openness Latent Growth Curve } \\
\hline & \multicolumn{2}{|c|}{ Intercept } & \multicolumn{2}{|c|}{ Slope } & \multicolumn{2}{|c|}{ Intercept } & \multicolumn{2}{|c|}{ Slope } \\
\hline & $b$ & S.E. & $b$ & S.E. & $b$ & S.E. & $b$ & S.E. \\
\hline Estimate & $2.204 *$ & 0.043 & $-0.011^{*}$ & 0.009 & $2.899 *$ & 0.058 & $0.012 *$ & 0.009 \\
\hline Age & -0.003 & 0.001 & 0.000 & 0.000 & 0.001 & 0.001 & 0.000 & 0.000 \\
\hline Gender & -0.059 & 0.019 & 0.001 & 0.001 & 0.075 & 0.025 & -0.003 & 0.001 \\
\hline \multicolumn{9}{|l|}{$(0=$ Female $)$} \\
\hline Education & 0.022 & 0.009 & -0.001 & 0.001 & 0.072 & 0.013 & 0.001 & 0.001 \\
\hline Agreeableness & -0.018 & 0.011 & 0.000 & 0.001 & - & - & - & - \\
\hline Neuroticism & 0.104 & 0.010 & -0.002 & 0.001 & - & - & - & - \\
\hline Extraversion & 0.007 & 0.011 & 0.000 & 0.001 & - & - & - & - \\
\hline Conscientiousness & -0.031 & 0.010 & -0.001 & 0.001 & - & - & - & - \\
\hline NSIs Intercept & - & - & 0.002 & 0.003 & - & - & -0.003 & 0.002 \\
\hline
\end{tabular}




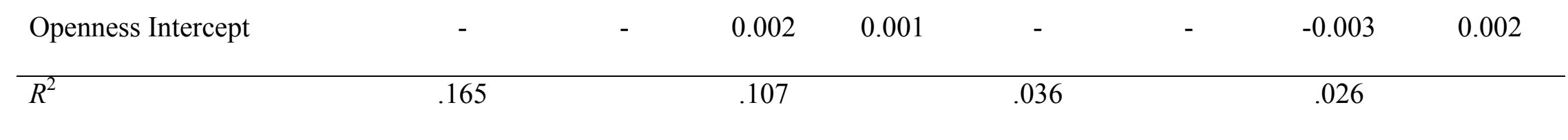

\begin{tabular}{ll}
\hline Model Fit & $\chi^{\mathbf{2}(49)=935.13}$ \\
& $\mathrm{CMIN} / \mathrm{DF}=19.08$ \\
$\mathrm{CFI}=.839$ \\
$\mathrm{RMSEA}=.10$
\end{tabular}

Note. Significant statistics $(p<.05)$ are bolded. $R^{2}=$ variance accounted for. An asterisk indicates that there is significant variability surrounding the estimate. A blue value indicates that the estimate is significantly different than zero. Type I errors were accounted for utilizing the false discovery rate adjustment. A green value indicates that the estimate was no longer statistically significant after the false discovery rate adjustments. To aid in the interpretation of the estimates, education, agreeableness, neuroticism, extraversion, and conscientiousness were standardized. As such, a unit increase or decrease in these variables represent a standard deviation increase or decrease, respectively. Age was not manipulated so that a unit increase or decrease represents an increase or decrease of a year, respectively. 
Table E10

Research Question 7 - Examination of the Bidirectional Association between the Latent Growth Curves of NSIs and Neuroticism After Accounting for the Demographic Variables and the Remaining Personality Traits

\begin{tabular}{|c|c|c|c|c|c|c|c|c|}
\hline & \multicolumn{4}{|c|}{ NSIs Latent Growth Curve } & \multicolumn{4}{|c|}{ Neuroticism Latent Growth Curve } \\
\hline & \multicolumn{2}{|c|}{ Intercept } & \multicolumn{2}{|c|}{ Slope } & \multicolumn{2}{|c|}{ Intercept } & \multicolumn{2}{|c|}{ Slope } \\
\hline & $b$ & S.E. & $b$ & S.E. & $b$ & S.E. & $b$ & S.E. \\
\hline Estimate & $2.282^{*}$ & 0.045 & $0.007^{*}$ & 0.007 & $2.673^{*}$ & 0.074 & $0.024 *$ & 0.007 \\
\hline Age & -0.005 & 0.001 & 0.000 & 0.000 & -0.010 & 0.002 & 0.000 & 0.000 \\
\hline $\begin{array}{l}\text { Gender } \\
\qquad(0=\text { Female })\end{array}$ & -0.093 & 0.020 & 0.001 & 0.001 & -0.141 & 0.032 & -0.002 & 0.001 \\
\hline Education & 0.001 & 0.010 & -0.001 & 0.001 & -0.083 & 0.016 & -0.001 & 0.001 \\
\hline Agreeableness & -0.025 & 0.011 & 0.000 & 0.001 & - & - & - & - \\
\hline Openness & 0.035 & 0.011 & 0.000 & 0.001 & - & - & - & - \\
\hline Extraversion & -0.011 & 0.012 & 0.000 & 0.001 & - & - & - & - \\
\hline Conscientiousness & -0.036 & 0.010 & 0.000 & 0.001 & - & - & - & - \\
\hline NSIs Intercept & - & - & 0.005 & 0.004 & - & - & -0.003 & 0.004 \\
\hline
\end{tabular}




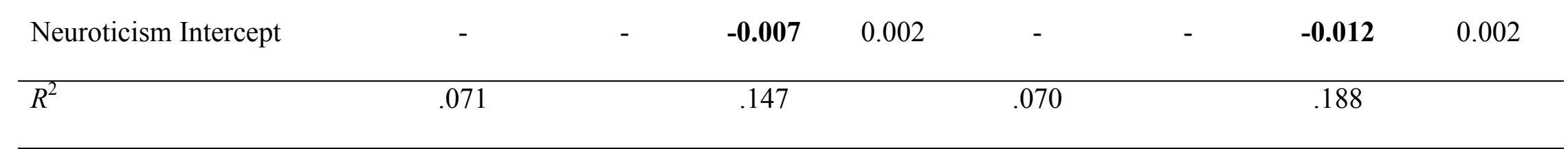

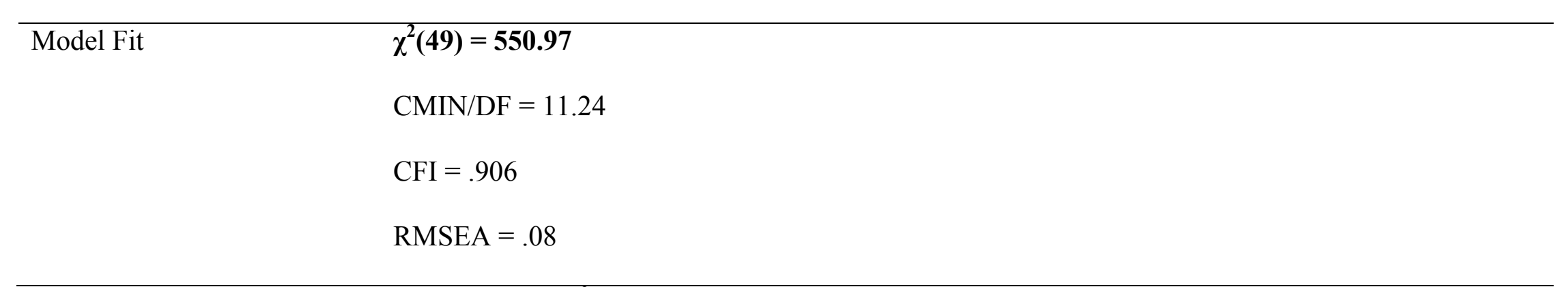

Note. Significant statistics $(p<.05)$ are bolded. $R^{2}=$ variance accounted for. An asterisk indicates that there is significant variability surrounding the estimate. A blue value indicates that the estimate is significantly different than zero. Type I errors were accounted for utilizing the false discovery rate adjustment. A green value indicates that the estimate was no longer statistically significant after the false discovery rate adjustments. To aid in the interpretation of the estimates, education, agreeableness, openness, extraversion, and conscientiousness were standardized. As such, a unit increase or decrease in these variables represent a standard deviation increase or decrease, respectively. Age was not manipulated so that a unit increase or decrease represents an increase or decrease of a year, respectively. 
Table E11

Research Question 7 - Examination of the Bidirectional Association between the Latent Growth Curves of NSIs and Extraversion After Accounting for the Demographic Variables and the Remaining Personality Traits

\begin{tabular}{|c|c|c|c|c|c|c|c|c|}
\hline & \multicolumn{4}{|c|}{ NSIs Latent Growth Curve } & \multicolumn{4}{|c|}{ Extraversion Latent Growth Curve } \\
\hline & \multicolumn{2}{|c|}{ Intercept } & \multicolumn{2}{|c|}{ Slope } & \multicolumn{2}{|c|}{ Intercept } & \multicolumn{2}{|c|}{ Slope } \\
\hline & $b$ & S.E. & $b$ & S.E. & $b$ & S.E. & $b$ & S.E. \\
\hline Estimate & $2.207 *$ & 0.043 & $-0.009 *$ & 0.010 & $3.088^{*}$ & 0.064 & $0.003 *$ & 0.009 \\
\hline Age & -0.003 & 0.001 & 0.000 & 0.000 & 0.003 & 0.001 & 0.000 & 0.000 \\
\hline $\begin{array}{l}\text { Gender } \\
\qquad(0=\text { Female })\end{array}$ & -0.068 & 0.019 & 0.001 & 0.001 & -0.054 & 0.028 & 0.000 & 0.001 \\
\hline Education & 0.016 & 0.009 & 0.000 & 0.001 & -0.045 & 0.014 & 0.000 & 0.001 \\
\hline Agreeableness & -0.019 & 0.010 & 0.000 & 0.001 & - & - & - & - \\
\hline Openness & 0.043 & 0.010 & 0.000 & 0.001 & - & - & - & - \\
\hline Neuroticism & 0.105 & 0.010 & -0.002 & 0.001 & - & - & - & - \\
\hline Conscientiousness & -0.038 & 0.010 & 0.000 & 0.001 & - & - & - & - \\
\hline NSIs Intercept & - & - & 0.003 & 0.003 & - & - & 0.000 & 0.002 \\
\hline
\end{tabular}




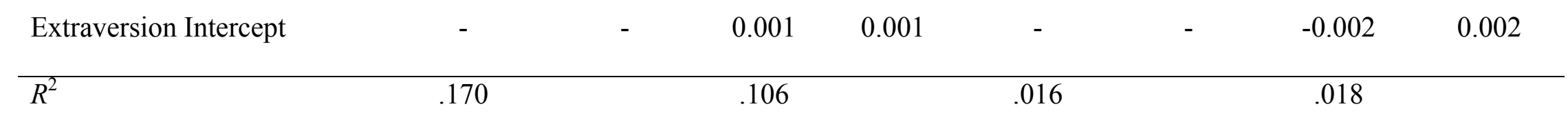

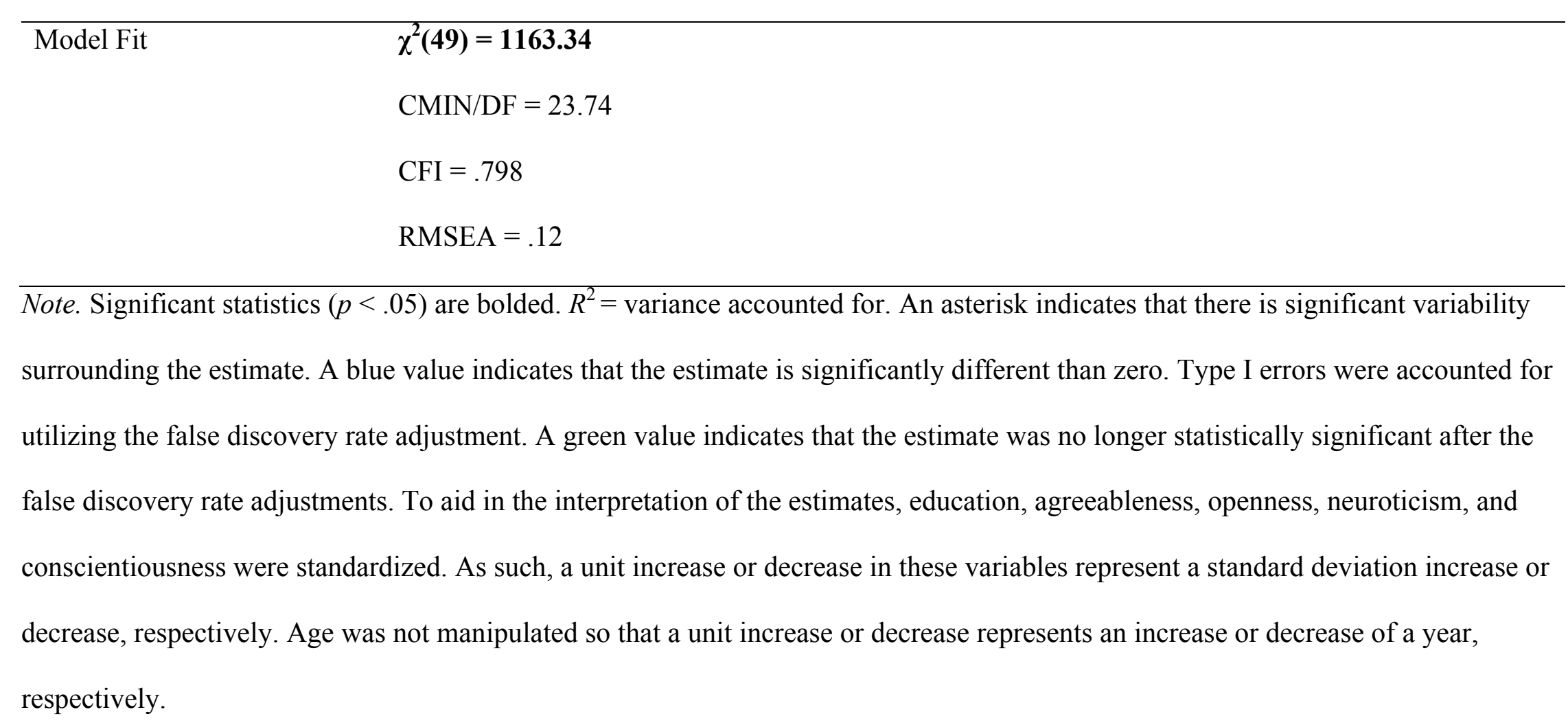


Table E12

Research Question 7 - Examination of the Bidirectional Association between the Latent Growth Curves of NSIs and

Conscientiousness After Accounting for the Demographic Variables and the Remaining Personality Traits

\begin{tabular}{|c|c|c|c|c|c|c|c|c|}
\hline & \multicolumn{4}{|c|}{ NSIs Latent Growth Curve } & \multicolumn{4}{|c|}{ Conscientiousness Latent Growth Curve } \\
\hline & \multicolumn{2}{|c|}{ Intercept } & \multicolumn{2}{|c|}{ Slope } & \multicolumn{2}{|c|}{ Intercept } & \multicolumn{2}{|c|}{ Slope } \\
\hline & $b$ & S.E. & $b$ & S.E. & $b$ & S.E. & $b$ & S.E. \\
\hline Estimate & $2.209 *$ & 0.043 & $-0.005^{*}$ & 0.013 & $3.445^{*}$ & 0.047 & $0.014 *$ & 0.003 \\
\hline Age & -0.003 & 0.001 & 0.000 & 0.000 & 0.002 & 0.001 & 0.000 & 0.000 \\
\hline $\begin{array}{l}\text { Gender } \\
\qquad(0=\text { Female })\end{array}$ & -0.059 & 0.019 & 0.001 & 0.001 & -0.129 & 0.020 & 0.002 & 0.001 \\
\hline Education & 0.011 & 0.009 & -0.001 & 0.001 & 0.032 & 0.010 & 0.000 & 0.001 \\
\hline Agreeableness & -0.024 & 0.011 & 0.000 & 0.001 & - & - & - & - \\
\hline Openness & 0.038 & 0.011 & 0.000 & 0.001 & - & - & - & - \\
\hline Neuroticism & 0.102 & 0.009 & -0.003 & 0.001 & - & - & - & - \\
\hline Extraversion & -0.012 & 0.012 & 0.000 & 0.001 & - & - & - & - \\
\hline NSIs Intercept & - & - & 0.003 & 0.004 & - & - & - & - \\
\hline
\end{tabular}




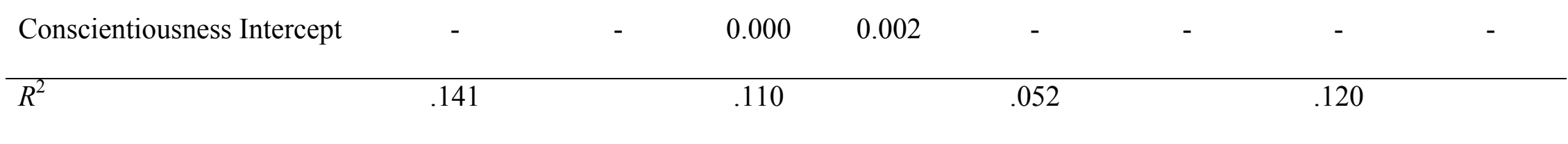

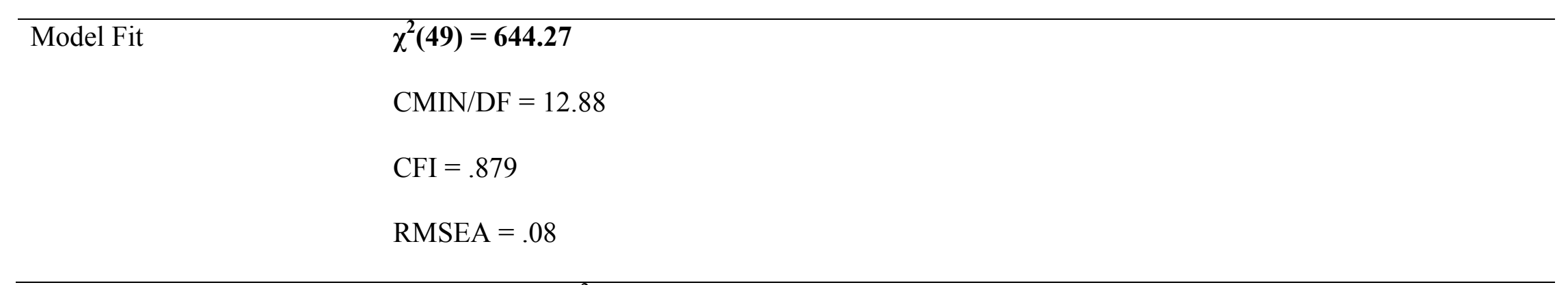

Note. Significant statistics $(p<.05)$ are bolded. $R^{2}=$ variance accounted for. An asterisk indicates that there is significant variability surrounding the estimate. A blue value indicates that the estimate is significantly different than zero. Type I errors were accounted for utilizing the false discovery rate adjustment. A green value indicates that the estimate was no longer statistically significant after the false discovery rate adjustments. To aid in the interpretation of the estimates, education, agreeableness, openness, neuroticism, and extraversion were standardized. As such, a unit increase or decrease in these variables represent a standard deviation increase or decrease, respectively. Age was not manipulated so that a unit increase or decrease represents an increase or decrease of a year, respectively. 


\section{Appendix F}

Figures for Results Qualified by Age

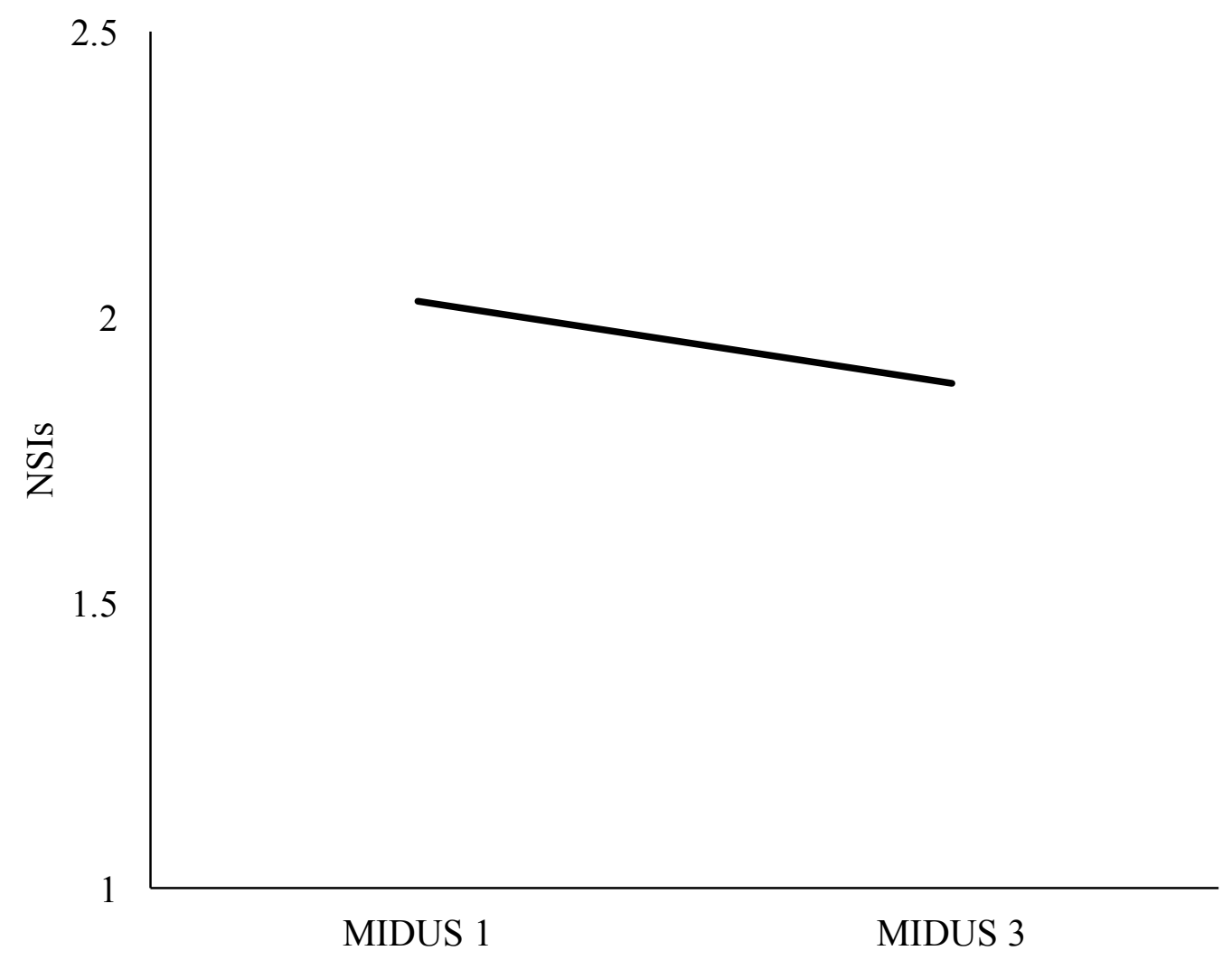

Figure F1. The trajectory of NSIs over 18 years for the entire sample. 


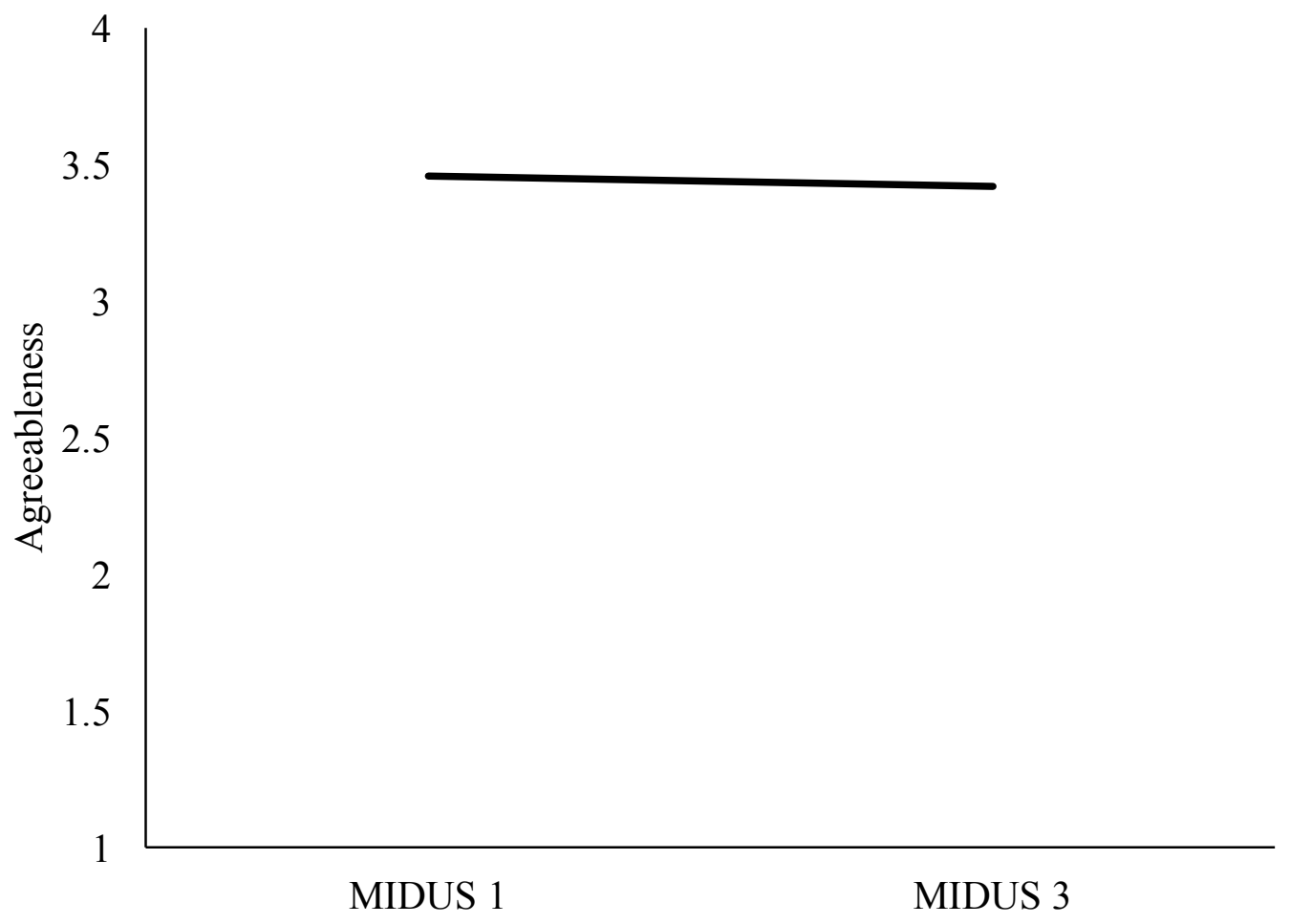

Figure F2. The trajectory of agreeableness over 18 years for the entire sample. 


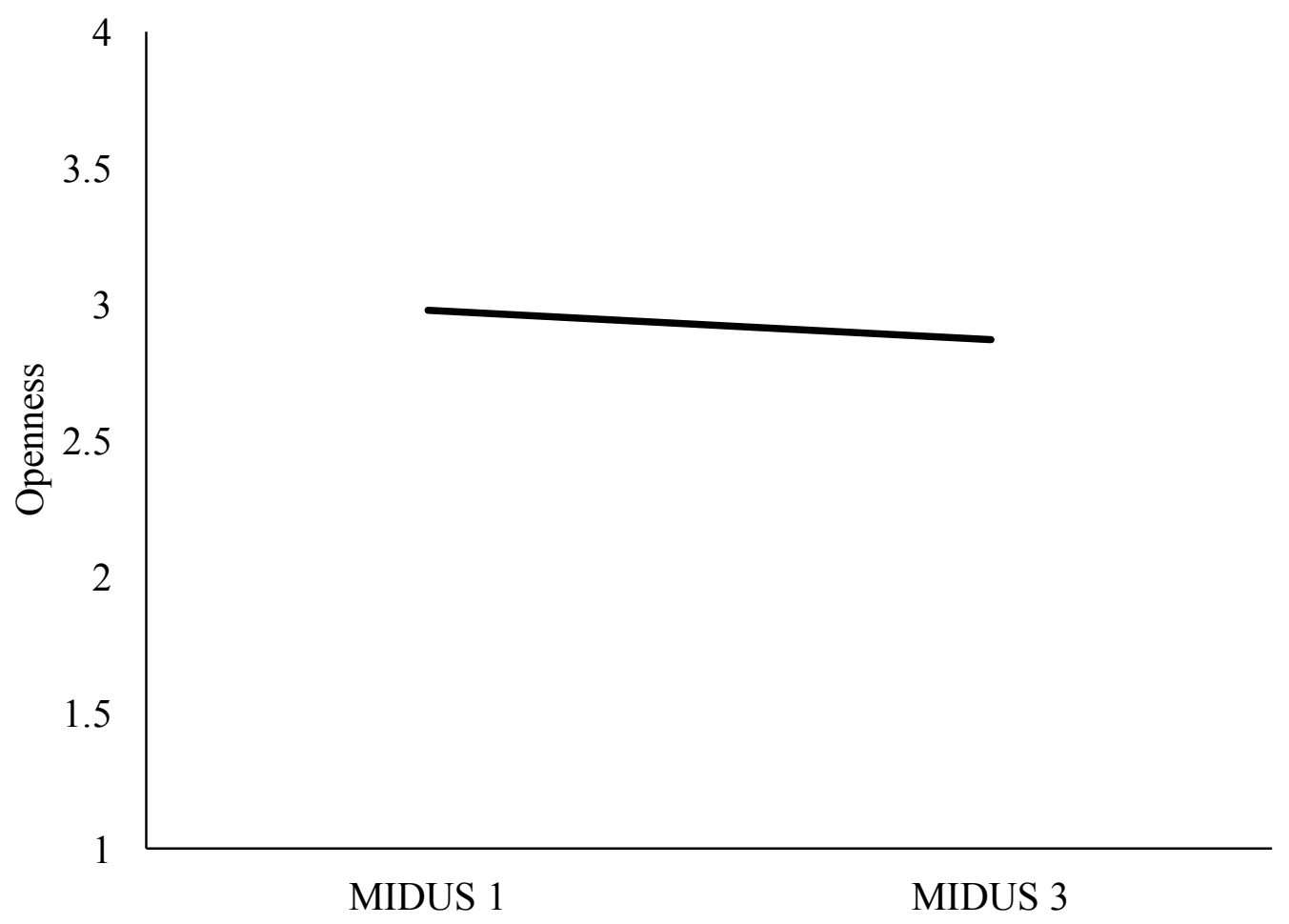

Figure F3. The trajectory of openness over 18 years for the entire sample. 


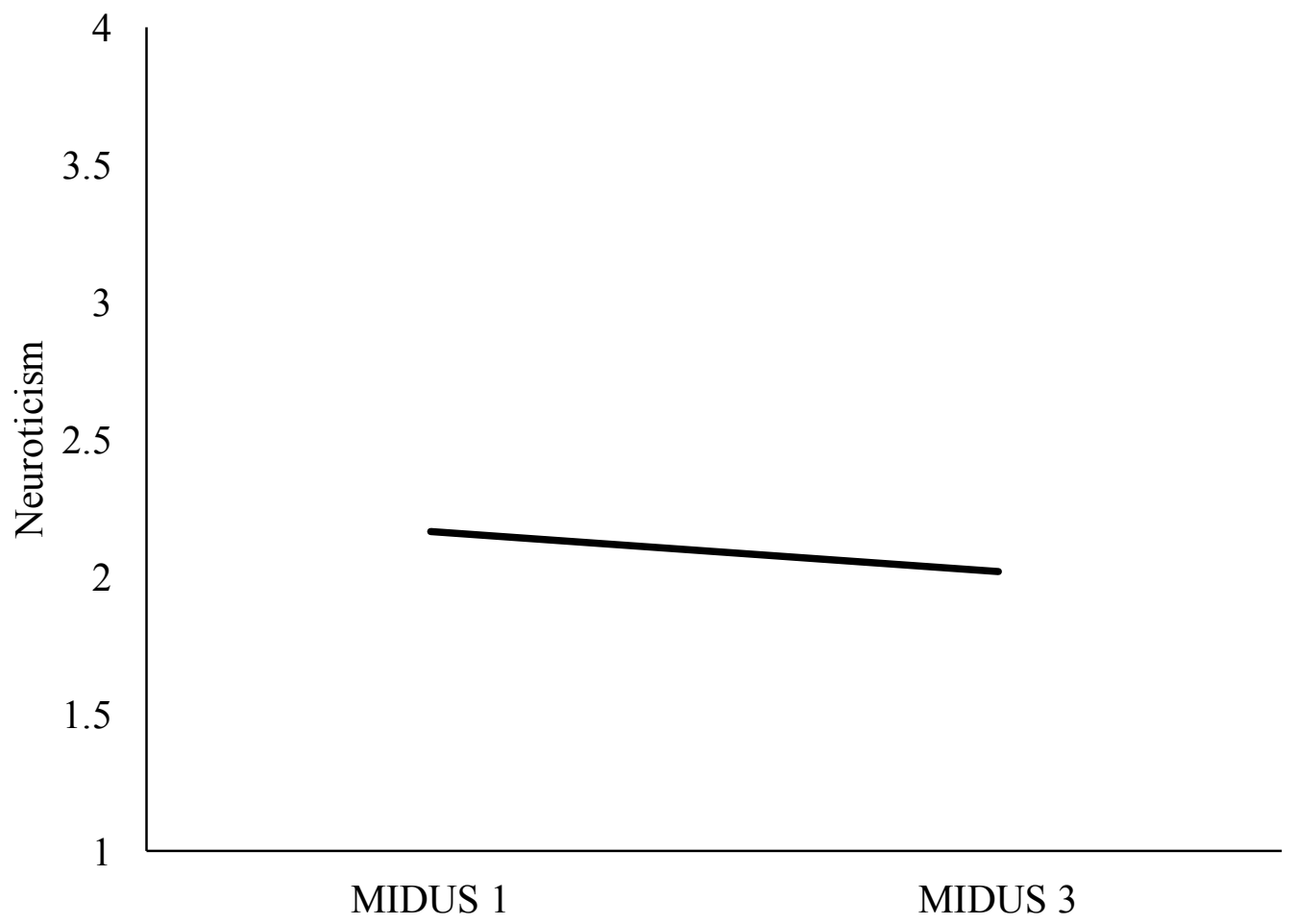

Figure F4. The trajectory of neuroticism over 18 years for the entire sample. 


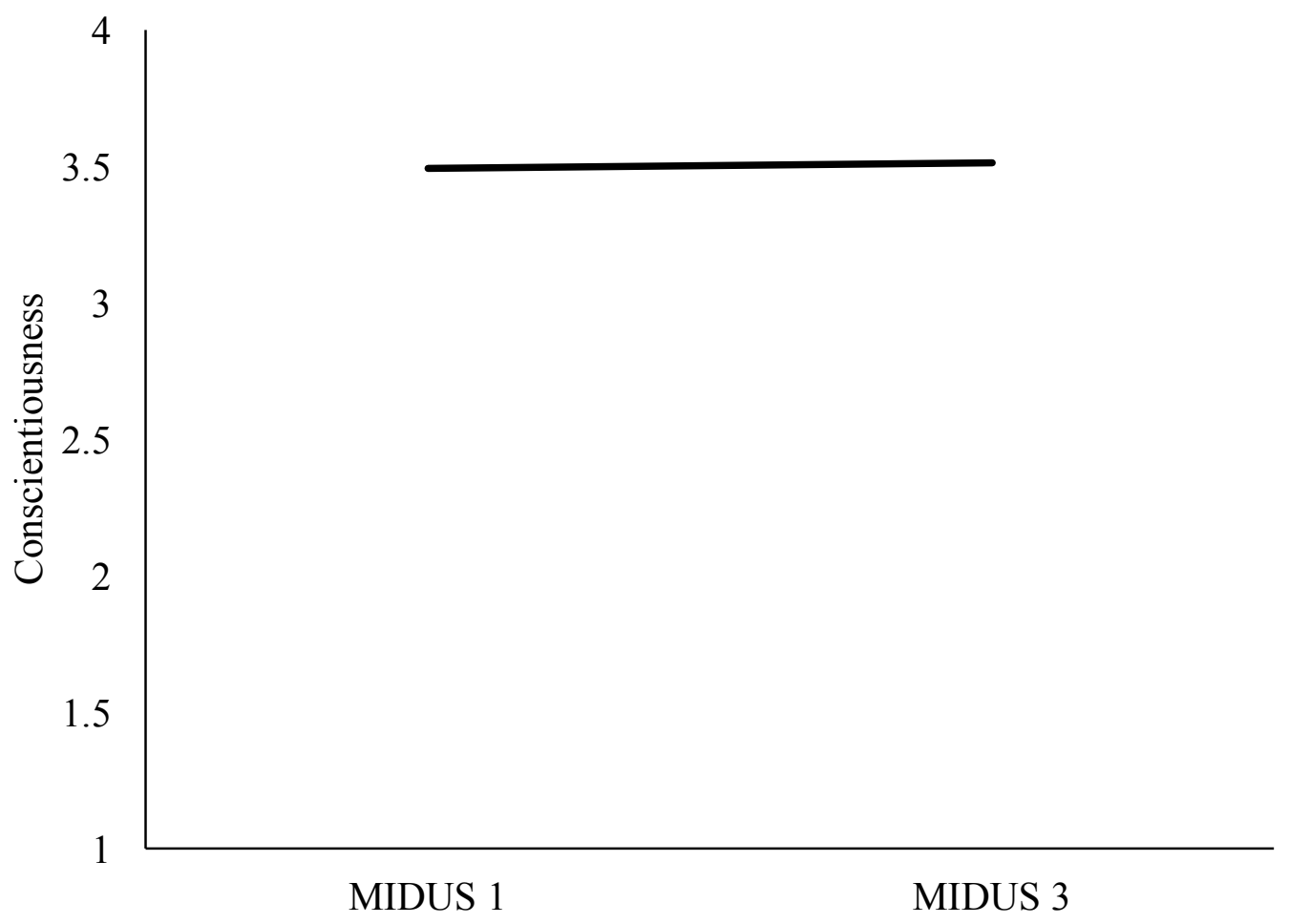

Figure F5. The trajectory of conscientiousness over 18 years for the entire sample. 
Appendix G

Summary of Results

Table G1

Summary Results of Research Questions 2 and 3 for NSIs

NSIs Latent Growth Curve

\begin{tabular}{|c|c|c|c|c|c|c|c|c|}
\hline & \multicolumn{2}{|c|}{ Entire Sample } & \multicolumn{2}{|c|}{ Younger Adults } & \multicolumn{2}{|c|}{ Middle-Aged Adults } & \multicolumn{2}{|c|}{ Older Adults } \\
\hline & Intercept & Slope & Intercept & Slope & Intercept & Slope & Intercept & Slope \\
\hline & & Decrease at a & & Decrease at a & & & & \\
\hline Age (Older) & - & steeper rate & & steeper rate & - & & & \\
\hline Gender (Males) & - & & - & & - & & + & \\
\hline Education & & & & & + & & & \\
\hline Agreeableness & - & & & & - & & & \\
\hline \multirow[t]{2}{*}{ Openness } & + & & & & + & & & \\
\hline & & Decrease at a & & Decrease at a & & & & \\
\hline Neuroticism & + & steeper rate & + & steeper rate & + & & + & \\
\hline Extraversion & & & & & & & & \\
\hline
\end{tabular}




\section{Conscientiousness}

Note. The summary of significant effects is presented above. A plus sign indicates that higher values of the variable are associated with a higher intercept. A minus sign indicates that higher values of the variable are associated with a lower intercept. 
Table G2

Summary Results of Research Questions 5 and 6 for Agreeableness

Agreeableness Latent Growth Curve

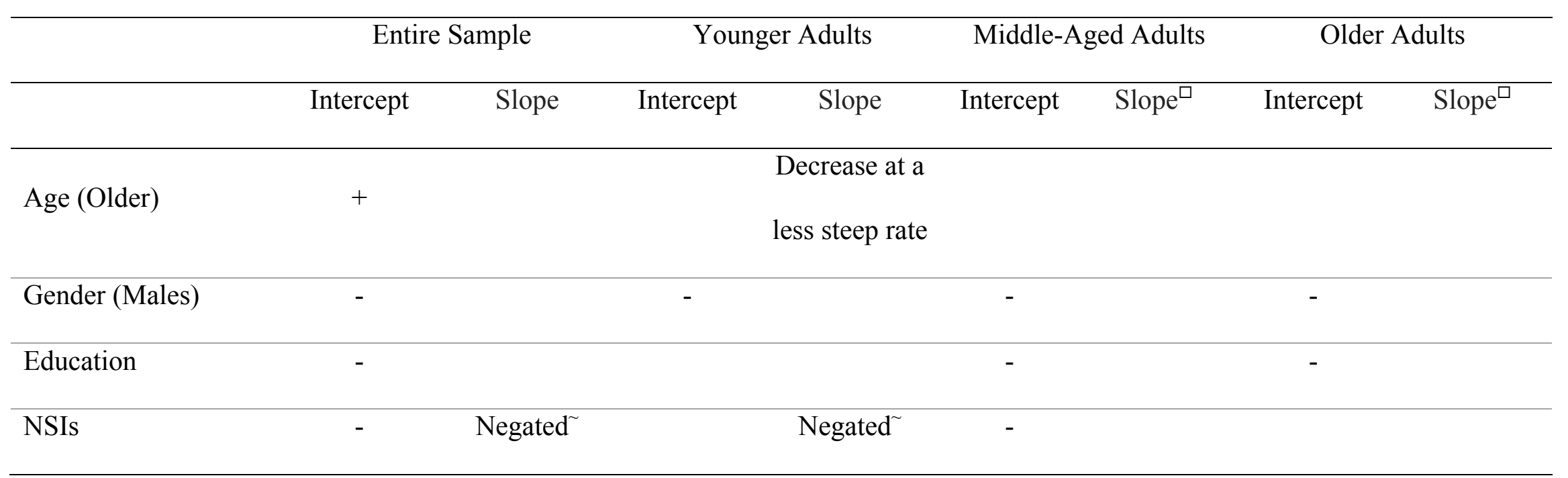

Note. The summary of significant effects is presented above. A plus sign indicates that higher values of the variable are associated with a higher intercept. A minus sign indicates that higher values of the variable are associated with a lower intercept. ${ }^{\square}$ indicates that

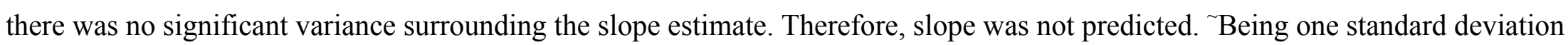
higher in NSIs was associated with an increase in agreeableness over time, which negated the overall decrease in agreeableness. 
Table G3

Summary Results of Research Questions 5 and 6 for Openness

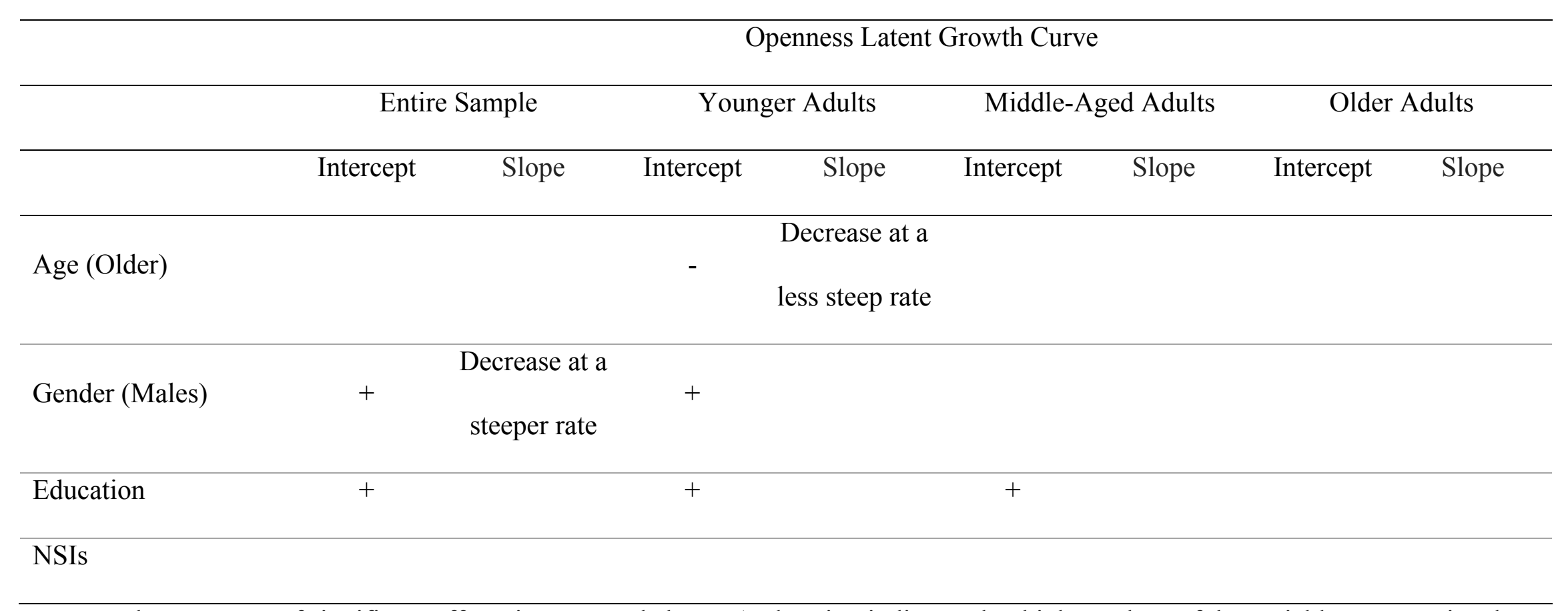

Note. The summary of significant effects is presented above. A plus sign indicates that higher values of the variable are associated with a higher intercept. A minus sign indicates that higher values of the variable are associated with a lower intercept. 
Table G4

Summary Results of Research Questions 5 and 6 for Neuroticism

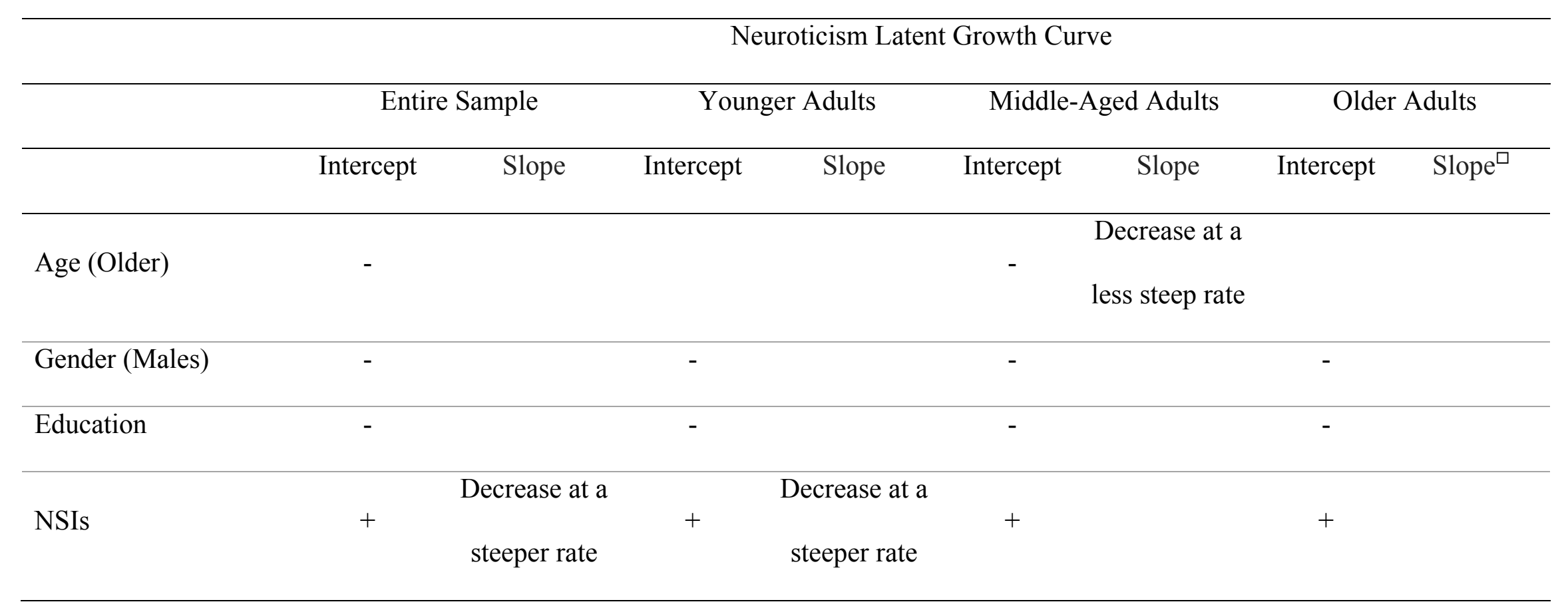

Note. The summary of significant effects is presented above. A plus sign indicates that higher values of the variable are associated

with a higher intercept. A minus sign indicates that higher values of the variable are associated with a lower intercept. ${ }^{\square}$ indicates that there was no significant variance surrounding the slope estimate. Therefore, slope was not predicted. 
Table G5

Summary Results of Research Questions 5 and 6 for Extraversion

Extraversion Latent Growth Curve

\begin{tabular}{ll}
\hline \multicolumn{3}{c}{ Entire Sample } & \\
\hline Intercept & Slope
\end{tabular}

Age (Older)

\section{Gender (Males)}

Education

NSIs

Note. The summary of significant effects is presented above. A plus sign indicates that higher values of the variable are associated with a higher intercept. A minus sign indicates that higher values of the variable are associated with a lower intercept. Age did not moderate the slope of extraversion. 
Table G6

Summary Results of Research Questions 5 and 6 for Conscientiousness

Conscientiousness Latent Growth Curve

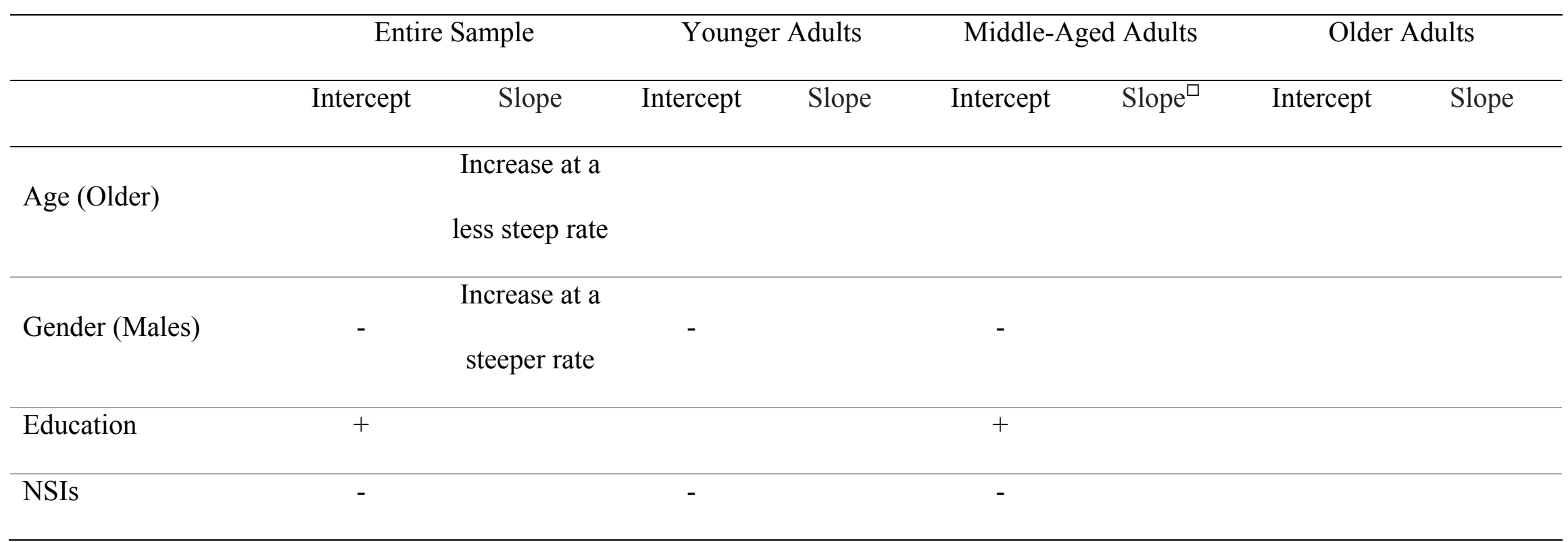

Note. The summary of significant effects is presented above. A plus sign indicates that higher values of the variable are associated

with a higher intercept. A minus sign indicates that higher values of the variable are associated with a lower intercept. ${ }^{\square}$ indicates that there was no significant variance surrounding the slope estimate. Therefore, slope was not predicted. 
Table G7

Summary Results of Research Questions 7 and 8 for NSIs and Agreeableness

\begin{tabular}{|c|c|c|c|c|c|c|c|c|}
\hline & \multicolumn{4}{|c|}{ NSIs Latent Growth Curve } & \multicolumn{4}{|c|}{ Agreeableness Latent Growth Curve } \\
\hline & \multicolumn{4}{|c|}{ NSIs Slope } & \multicolumn{4}{|c|}{ Agreeableness Slope } \\
\hline & Entire & Younger & Middle-Aged & Older & Entire & Younger & Middle- & Older \\
\hline & Sample & Adults & Adults & Adults & Sample & Adults & Aged Adults & Adults \\
\hline \multicolumn{9}{|l|}{ NSIs Intercept } \\
\hline Agreeableness & & & & & Decrease at a & & o & \\
\hline Intercept & & & & & steeper rate & & & \\
\hline
\end{tabular}

Note. The summary of significant effects is presented above. A plus sign indicates that higher values of the variable are associated

with a higher intercept. A minus sign indicates that higher values of the variable are associated with a lower intercept. ${ }^{\circ}$ indicates that the slope was not significant. Therefore, slope was not predicted. 
Table G8

Summary Results of Research Questions 7 and 8 for NSIs and Openness

\section{NSIs Latent Growth Curve}

Openness Latent Growth Curve

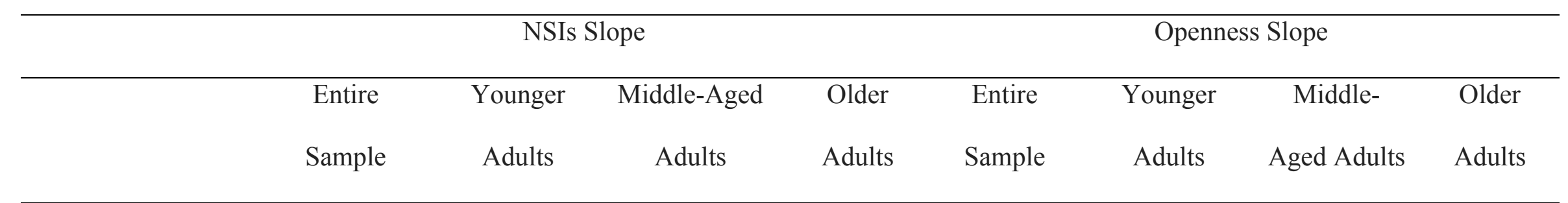

\section{NSIs Intercept}

Openness

Decrease at a

Decrease at a

Intercept

less steep rate

steeper rate

Note. The summary of significant effects is presented above. A plus sign indicates that higher values of the variable are associated

with a higher intercept. A minus sign indicates that higher values of the variable are associated with a lower intercept. 
Table G9

Summary Results of Research Questions 7 and 8 for NSIs and Neuroticism

\begin{tabular}{|c|c|c|c|c|c|c|c|c|}
\hline & \multicolumn{4}{|c|}{ NSIs Latent Growth Curve } & \multicolumn{4}{|c|}{ Neuroticism Latent Growth Curve } \\
\hline & \multicolumn{4}{|c|}{ NSIs Slope } & \multicolumn{4}{|c|}{ Neuroticism Slope } \\
\hline & Entire & Younger & Middle-Aged & Older & Entire & Younger & Middle-Aged & Older \\
\hline & Sample & Adults & Adults & Adults & Sample & Adults & Adults & Adults \\
\hline \multicolumn{9}{|c|}{ NSIs Intercept } \\
\hline Neuroticism & Decrease at a & Decrease at a & Decrease at a & & Decrease at a & Decrease at a & Decrease at a & \\
\hline Intercept & steeper rate & steeper rate & steeper rate & & steeper rate & steeper rate & steeper rate & \\
\hline
\end{tabular}

Note. The summary of significant effects is presented above. A plus sign indicates that higher values of the variable are associated

with a higher intercept. A minus sign indicates that higher values of the variable are associated with a lower intercept. 
Table G10

Summary Results of Research Questions 7 and 8 for NSIs and Extraversion

\begin{tabular}{cccccccc}
\hline & NSIs Latent Growth Curve & & Extraversion Latent Growth Curve \\
\hline & NSIs Slope & & & Extraversion Slope \\
\hline Entire & Younger & Middle-Aged & Older & Entire & Younger & Middle- & Older \\
Sample & Adults & Adults & Adults & Sample & Adults & Aged Adults & Adults \\
& & & & & & &
\end{tabular}

\section{NSIs Intercept}

\section{Extraversion}

Intercept

Note. The summary of significant effects is presented above. A plus sign indicates that higher values of the variable are associated with a higher intercept. A minus sign indicates that higher values of the variable are associated with a lower intercept. 
Table G11

Summary Results of Research Questions 7 and 8 for NSIs and Conscientiousness

\section{NSIs Latent Growth Curve}

$$
\text { NSIs Slope }
$$

Entire

Younger

Middle-Aged

Adults

Adults

Sample

\section{NSIs Intercept}

\section{Conscientiousness}

Intercept the slope was not significant. Therefore, slope was not predicted.
Conscientiousness Latent Growth Curve

Conscientiousness Slope

Entire

Younger

Middle-

Older

Adults

Sample

Adults

Aged Adults

Adults

\footnotetext{
Note. The summary of significant effects is presented above. A plus sign indicates that higher values of the variable are associated with a higher intercept. A minus sign indicates that higher values of the variable are associated with a lower intercept. ${ }^{\circ}$ indicates that
} 


\section{Appendix $\mathrm{H}$}

Measurement Equivalence

Table H1

Measurement Invariance for Agreeableness

$\chi^{2} \quad$ CFI $\quad \Delta \chi^{2} \quad \Delta$ CFI

Agreeableness for Entire Sample

Unconstrained Model

Weak Invariant Model

Strong Invariant Model

Strict Invariant Model

Agreeableness for Younger Adults

Unconstrained Model

Weak Invariant Model

Strong Invariant Model

Strict Invariant Model

$$
\begin{array}{llll}
\chi^{2}(\mathbf{7 2})=\mathbf{2 7 7 . 0 1} & .979 & & \\
\chi^{2}(\mathbf{8 2})=\mathbf{2 8 7 . 2 4} & .979 & \Delta \chi^{2}(10)=10.23 & .000 \\
\chi^{2}(\mathbf{9 2})=\mathbf{3 2 3 . 4 4} & .976 & \Delta \chi^{2}(\mathbf{1 0})=\mathbf{3 6 . 2 0} & .003 \\
\chi^{2}(\mathbf{1 0 2})=\mathbf{3 9 8 . 9 0} & .970 & \Delta \chi^{2}(\mathbf{1 0})=\mathbf{7 5 . 4 6} & .006
\end{array}
$$

Agreeableness for Middle-Aged Adults

Unconstrained Model

Weak Invariant Model

Strong Invariant Model

Strict Invariant Model $\chi^{2}(72)=134.66 \quad .979$

$\chi^{2}(\mathbf{8 2})=151.46 \quad .977 \quad \Delta \chi^{2}(10)=16.80 \quad .002$

$\chi^{2}(92)=181.35 \quad .971$

$\Delta \chi^{2}(\mathbf{1 0})=\mathbf{2 9 . 8 9} \quad .006$

$\chi^{2}(\mathbf{1 0 2})=\mathbf{2 0 4 . 5 7} .966$

Agreeableness for Older Adults

Unconstrained Model

Weak Invariant Model

$$
\chi^{2}(72)=188.65 \quad .978
$$$$
\chi^{2}(\mathbf{8 2})=\mathbf{2 0 4 . 6 2} \quad .977 \quad \Delta \chi^{2}(10)=15.97 \quad .001
$$$$
\chi^{2}(\mathbf{9 2})=\mathbf{2 2 6 . 4 2} \quad .975 \quad \Delta \chi^{2}(\mathbf{1 0})=\mathbf{2 1 . 8 0} \quad .002
$$

$\chi^{2}(\mathbf{1 0 2})=\mathbf{2 8 4 . 1 3} \quad .966$

$\Delta \chi^{2}(\mathbf{1 0})=\mathbf{5 7 . 7 1} \quad .009$ 
Strong Invariant Model

$$
\begin{array}{llll}
\chi^{2}(\mathbf{9 2})=\mathbf{1 5 1 . 3 5} & .953 & \Delta \chi^{2}(10)=16.16 & .005 \\
\chi^{2}(\mathbf{1 0 2})=\mathbf{2 0 6 . 5 3} & .917 & \Delta \chi^{2}(\mathbf{1 0})=\mathbf{5 5 . 1 8} & \mathbf{. 0 3 6}
\end{array}
$$

Strict Invariant Model

Note. Significant statistics $(p<.05$ or $\Delta \mathrm{CFI} \geq .01)$ are bolded. Both the chi-square and the CFI difference statistics were computed because the chi-square statistic is commonly used, however, it is sensitive to large sample sizes. The CFI is more robust to larger sample sizes (Little, 2013). Parameters were freely estimated in the unconstrained model. The factors loadings were constrained to be equal across time in the weak invariant model. The factors loadings and the intercepts were constrained to be equal across time in the strong invariant model. The factors loadings, the intercepts, and the measurement errors were constrained to be equal across time in the strict invariant model. The first listed difference test compares the unconstrained model to the weak invariant model. The second listed difference test compares the weak and strong invariant models. The third listed difference test compares the strong and strict invariant models.

In order to use latent variables, the assumption of strong invariance must be satisfied (Little, 2013). To determine whether this assumption was satisfied, the chi-square and the CFI difference tests comparing the unconstrained model to the weak invariant model and the weak to the strong invariant model must not be significant. Overall, agreeableness did satisfy the assumption of strong invariance. However, to keep models consistent and comparable across results, the decision to use composite scores instead of latent variables for agreeableness was made. 
Table H2

Measurement Invariance for Openness

$\chi^{2} \quad$ CFI $\quad \Delta \chi^{2} \quad \Delta$ CFI

Openness for Entire Sample

Unconstrained Model

Weak Invariant Model

Strong Invariant Model

Strict Invariant Model

Openness for Younger Adults

Unconstrained Model

Weak Invariant Model

Strong Invariant Model

Strict Invariant Model

Openness for Middle-Aged Adults

Unconstrained Model

Weak Invariant Model

Strong Invariant Model

Strict Invariant Model

Openness for Older Adults

Unconstrained Model

Weak Invariant Model

Strong Invariant Model

Strict Invariant Model

$$
\begin{array}{llll}
\chi^{2}(\mathbf{1 6 5})=\mathbf{1 3 2 5 . 2 0} & .923 & & \\
\chi^{2}(\mathbf{1 7 9})=\mathbf{1 3 4 0 . 4 3} & .923 & \Delta \chi^{2}(14)=15.23 & .000 \\
\chi^{2}(\mathbf{1 9 3})=\mathbf{1 5 3 0 . 6 9} & .911 & \Delta \chi^{2}(\mathbf{1 4})=\mathbf{1 9 0 . 2 6} & \mathbf{. 0 1 2} \\
\chi^{2}(\mathbf{2 0 7})=\mathbf{1 5 7 1 . 8 6} & .909 & \Delta \chi^{2}(\mathbf{1 4})=\mathbf{4 1 . 1 7} & .002
\end{array}
$$

.012


Note. Significant statistics $(p<.05$ or $\Delta \mathrm{CFI} \geq .01)$ are bolded. Both the chi-square and the CFI difference statistics were computed because the chi-square statistic is commonly used, however, it is sensitive to large sample sizes. The CFI is more robust to larger sample sizes (Little, 2013). Parameters were freely estimated in the unconstrained model. The factors loadings were constrained to be equal across time in the weak invariant model. The factors loadings and the intercepts were constrained to be equal across time in the strong invariant model. The factors loadings, the intercepts, and the measurement errors were constrained to be equal across time in the strict invariant model. The first listed difference test compares the unconstrained model to the weak invariant model. The second listed difference test compares the weak and strong invariant models. The third listed difference test compares the strong and strict invariant models.

In order to use latent variables, the assumption of strong invariance must be satisfied (Little, 2013). To determine whether this assumption was satisfied, the chi-square and the CFI difference tests comparing the unconstrained model to the weak invariant model and the weak to the strong invariant model must not be significant. Openness for middle-aged adults did satisfy the assumption of strong invariance. However, to keep models consistent and comparable across results, the decision to use composite scores instead of latent variables for openness was made. The critical ratios were examined to determine which indicators were invariant. Results indicated that the majority of problematic indicators were the factor loadings, intercepts, and measurement errors between MIDUS 1 and MIDUS 3 and MIDUS 2 and MIDUS 3. This suggests that the conceptual meaning of the MIDI (Lachman \& Weaver, 1997) is significantly different in older age. 
Table H3

Measurement Invariance for Neuroticism

$\chi^{2} \quad$ CFI $\quad \Delta \chi^{2} \quad \Delta$ CFI

Neuroticism for Entire Sample

Unconstrained Model

$\chi^{2}(\mathbf{3 9})=\mathbf{7 2 . 7 6} .996$

Weak Invariant Model

$\chi^{2}(\mathbf{4 7})=\mathbf{1 1 1 . 0 3} .992$

$\Delta \chi^{2}(\mathbf{8})=\mathbf{3 8 . 2 7} \quad .004$

Strong Invariant Model

$\chi^{2}(\mathbf{5 5})=\mathbf{3 7 5 . 6 3} \quad .958$

$\Delta \chi^{2}(8)=262.59 \quad .034$

Strict Invariant Model

$\chi^{2}(63)=399.32 \quad .956$

$\Delta \chi^{2}(8)=\mathbf{2 3 . 6 9} \quad .002$

Neuroticism for Younger Adults

Unconstrained Model

Weak Invariant Model

Strong Invariant Model

Strict Invariant Model

Neuroticism for Middle-Aged Adults

Unconstrained Model

Weak Invariant Model

Strong Invariant Model

Strict Invariant Model

$$
\begin{aligned}
& \chi^{2}(39)=64.49 \quad .989 \\
& \chi^{2}(\mathbf{4 7})=79.70 \quad .986 \quad \Delta \chi^{2}(8)=15.21 \quad .003 \\
& \chi^{2}(55)=174.70 \quad .947 \quad \Delta \chi^{2}(8)=95.00 \quad .039 \\
& \chi^{2}(63)=183.09 \quad .947 \quad \Delta \chi^{2}(8)=8.39 \quad .000
\end{aligned}
$$

.039

Neuroticism for Older Adults

$$
\begin{array}{llll}
\chi^{\mathbf{2}(39)}=\mathbf{5 6 . 2 5} & .996 & & \\
\chi^{\mathbf{2}(47)}=\mathbf{8 2 . 1 9} & .992 & \Delta \chi^{\mathbf{2}(8)}=\mathbf{2 5 . 9 4} & .004 \\
\chi^{\mathbf{2}(55)}=\mathbf{2 5 7 . 4 6} & .956 & \Delta \chi^{\mathbf{2}(8)}=\mathbf{1 7 5 . 2 7} & .036 \\
\chi^{\mathbf{2}(63)}=\mathbf{2 7 9 . 0 2} & .953 & \Delta \chi^{\mathbf{2}(8)}=\mathbf{2 1 . 5 6} & .003
\end{array}
$$

Unconstrained Model

Weak Invariant Model

Strong Invariant Model

Strict Invariant Model $\chi^{2}(39)=35.27 \quad 1.000$

$\chi^{2}(47)=43.89$

1.000

$\Delta \chi^{2}(8)=8.62$

.000

$\chi^{2}(55)=68.30 \quad .980$

$\Delta \chi^{2}(8)=24.41 \quad .020$

$\chi^{2}(63)=76.08 \quad .980$

$\Delta \chi^{2}(8)=7.78 \quad .000$ 
Note. Significant statistics $(p<.05$ or $\Delta \mathrm{CFI} \geq .01)$ are bolded. Both the chi-square and the CFI difference statistics were computed because the chi-square statistic is commonly used, however, it is sensitive to large sample sizes. The CFI is more robust to larger sample sizes (Little, 2013). Parameters were freely estimated in the unconstrained model. The factors loadings were constrained to be equal across time in the weak invariant model. The factors loadings and the intercepts were constrained to be equal across time in the strong invariant model. The factors loadings, the intercepts, and the measurement errors were constrained to be equal across time in the strict invariant model. The first listed difference test compares the unconstrained model to the weak invariant model. The second listed difference test compares the weak and strong invariant models. The third listed difference test compares the strong and strict invariant models.

In order to use latent variables, the assumption of strong invariance must be satisfied (Little, 2013). To determine whether this assumption was satisfied, the chi-square and the CFI difference tests comparing the unconstrained model to the weak invariant model and the weak to the strong invariant model must not be significant. Neuroticism for the entire sample and for the three age groups did not satisfy the assumption of strong invariance, therefore, the decision to use composite scores instead of latent variables for neuroticism was made. The critical ratios were examined to determine which indicators were invariant. Results indicated that the majority of problematic indicators were the factor loadings, intercepts, and measurement errors between MIDUS 1 and MIDUS 3 and MIDUS 2 and MIDUS 3. This suggests that the conceptual meaning of the MIDI (Lachman \& Weaver, 1997) is significantly different in older age. 
Table H4

Measurement Invariance for Extraversion

$\chi^{2} \quad$ CFI $\quad \Delta \chi^{2} \quad \Delta$ CFI

Extraversion for Entire Sample

Unconstrained Model

$\chi^{2}(72)=\mathbf{4 3 9 . 5 4} \quad .966$

Weak Invariant Model

$\chi^{2}(\mathbf{8 2})=\mathbf{4 5 1 . 9 7} .966$

$\Delta \chi^{2}(10)=12.43 \quad .000$

Strong Invariant Model

$\chi^{2}(92)=666.09$

.947

$\Delta \chi^{2}(10)=214.12 \quad .021$

Strict Invariant Model

$\chi^{2}(\mathbf{1 0 2})=763.23 \quad .939$

$\Delta \chi^{2}(\mathbf{1 0})=97.14 \quad .008$

Extraversion for Younger Adults

Unconstrained Model

$\chi^{2}(72)=174.10 \quad .967$

Weak Invariant Model

$\chi^{2}(\mathbf{8 2})=\mathbf{1 9 2 . 0 1} \quad .964$

$\Delta \chi^{2}(10)=17.91$

.003

Strong Invariant Model

$\chi^{2}(92)=272.75 \quad .941$

$\Delta \chi^{2}(10)=80.74$

.023

Strict Invariant Model

$\chi^{2}(\mathbf{1 0 2})=\mathbf{3 1 2 . 1 3} \quad .932$

$\Delta \chi^{2}(10)=39.38$

Extraversion for Middle-Aged Adults

Unconstrained Model

$\chi^{2}(72)=310.14 \quad .962$

Weak Invariant Model

$\chi^{2}(82)=327.06 \quad .961$

$\Delta \chi^{2}(10)=16.92$

.001

Strong Invariant Model

$\chi^{2}(92)=430.94 \quad .947$

$\Delta \chi^{2}(10)=103.88 \quad .014$

Strict Invariant Model

$\chi^{2}(\mathbf{1 0 2})=\mathbf{4 8 7 . 1 4} \quad .939$

$\Delta \chi^{2}(10)=56.20$

.008

Extraversion for Older Adults

Unconstrained Model

$\chi^{2}(72)=\mathbf{1 1 9 . 0 4} \quad .967$

Weak Invariant Model

$$
\chi^{2}(\mathbf{8 2})=\mathbf{1 1 3 . 6 5} \quad .963
$$

$\Delta \chi^{2}(10)=5.39$

.004

Strong Invariant Model

$\chi^{2}(\mathbf{9 2})=\mathbf{2 1 2 . 8 7} \quad .914$

$\Delta \chi^{2}(10)=99.22$

.049

Strict Invariant Model

$\chi^{2}(\mathbf{1 0 2})=\mathbf{2 2 5 . 0 5} \quad .913$

$\Delta \chi^{2}(10)=12.18$

.001 
Note. Significant statistics $(p<.05$ or $\Delta \mathrm{CFI} \geq .01)$ are bolded. Both the chi-square and the CFI difference statistics were computed because the chi-square statistic is commonly used, however, it is sensitive to large sample sizes. The CFI is more robust to larger sample sizes (Little, 2013). Parameters were freely estimated in the unconstrained model. The factors loadings were constrained to be equal across time in the weak invariant model. The factors loadings and the intercepts were constrained to be equal across time in the strong invariant model. The factors loadings, the intercepts, and the measurement errors were constrained to be equal across time in the strict invariant model. The first listed difference test compares the unconstrained model to the weak invariant model. The second listed difference test compares the weak and strong invariant models. The third listed difference test compares the strong and strict invariant models.

In order to use latent variables, the assumption of strong invariance must be satisfied (Little, 2013). To determine whether this assumption was satisfied, the chi-square and the CFI difference tests comparing the unconstrained model to the weak invariant model and the weak to the strong invariant model must not be significant. Extraversion for the entire sample and for the three age groups did not satisfy the assumption of strong invariance, therefore, the decision to use composite scores instead of latent variables for extraversion was made. The critical ratios were examined to determine which indicators were invariant. Results indicated that the majority of problematic indicators were the factor loadings, intercepts, and measurement errors between MIDUS 1 and MIDUS 3 and MIDUS 2 and MIDUS 3. This suggests that the conceptual meaning of the MIDI (Lachman \& Weaver, 1997) is significantly different in older age. 
Table H5

Measurement Invariance for Conscientiousness

$\chi^{2} \quad$ CFI $\quad \Delta \chi^{2} \quad \Delta$ CFI

Conscientiousness for Entire Sample

Unconstrained Model

Weak Invariant Model

Strong Invariant Model

Strict Invariant Model

Conscientiousness for Younger Adults

Unconstrained Model

Weak Invariant Model

Strong Invariant Model

Strict Invariant Model

Conscientiousness for Middle-Aged Adults

Unconstrained Model

Weak Invariant Model

Strong Invariant Model

Strict Invariant Model

Conscientiousness for Older Adults

Unconstrained Model

Weak Invariant Model

Strong Invariant Model

Strict Invariant Model

$$
\begin{aligned}
& \chi^{2}(39)=128.51 \quad .982 \\
& \chi^{2}(\mathbf{4 7})=\mathbf{1 7 6 . 8 5} \quad .974 \quad \Delta \chi^{2}(8)=\mathbf{4 8 . 3 4} \quad .008 \\
& \chi^{2}(55)=323.80 \quad .945 \quad \Delta \chi^{2}(8)=146.95 \quad .029 \\
& \chi^{2}(63)=469.53 \quad .917 \quad \Delta \chi^{2}(8)=145.73 \quad .028
\end{aligned}
$$


Note. Significant statistics $(p<.05$ or $\Delta \mathrm{CFI} \geq .01)$ are bolded. Both the chi-square and the CFI difference statistics were computed because the chi-square statistic is commonly used, however, it is sensitive to large sample sizes. The CFI is more robust to larger sample sizes (Little, 2013). Parameters were freely estimated in the unconstrained model. The factors loadings were constrained to be equal across time in the weak invariant model. The factors loadings and the intercepts were constrained to be equal across time in the strong invariant model. The factors loadings, the intercepts, and the measurement errors were constrained to be equal across time in the strict invariant model. The first listed difference test compares the unconstrained model to the weak invariant model. The second listed difference test compares the weak and strong invariant models. The third listed difference test compares the strong and strict invariant models.

In order to use latent variables, the assumption of strong invariance must be satisfied (Little, 2013). To determine whether this assumption was satisfied, the chi-square and the CFI difference tests comparing the unconstrained model to the weak invariant model and the weak to the strong invariant model must not be significant. Conscientiousness for the entire sample and for the three age groups did not satisfy the assumption of strong invariance, therefore, the decision to use composite scores instead of latent variables for conscientiousness was made. The critical ratios were examined to determine which indicators were invariant. Results indicated that the majority of problematic indicators were the factor loadings, intercepts, and measurement errors between MIDUS 1 and MIDUS 3 and MIDUS 2 and MIDUS 3. This suggests that the conceptual meaning of the MIDI (Lachman \& Weaver, 1997) is significantly different in older age. 
Table H6

Measurement Invariance for NSIs

$\chi^{2} \quad$ CFI $\quad \Delta \chi^{2} \quad \Delta$ CFI

NSIs for Entire Sample

Unconstrained Model

$\chi^{2}(\mathbf{5 5 5})=\mathbf{7 0 2 7 . 8 5} \quad .723$

Weak Invariant Model

$\chi^{2}(579)=7073.97 \quad .722$

$\Delta \chi^{2}(\mathbf{2 4})=\mathbf{4 6 . 1 2} \quad .001$

Strong Invariant Model

$\chi^{2}(603)=7368.65 \quad .710$

$\Delta \chi^{2}(24)=294.68 \quad .012$

Strict Invariant Model

$\chi^{2}(627)=\mathbf{7 4 4 1 . 4 0} \quad .708$

$\Delta \chi^{2}(\mathbf{2 4})=\mathbf{7 2 . 7 5} \quad .002$

NSIs for Younger Adults

Unconstrained Model

$\chi^{2}(\mathbf{5 5 5})=\mathbf{2 8 8 1 . 6 3} \quad .659$

Weak Invariant Model

$\chi^{2}(\mathbf{5 7 9})=\mathbf{2 8 9 8 . 3 4} \quad .660$

$\Delta \chi^{2}(24)=16.71 \quad .001$

Strong Invariant Model

$\chi^{2}(603)=3014.84 \quad .647$

$\Delta \chi^{2}(24)=11.65 \quad .013$

Strict Invariant Model

$\chi^{2}(627)=3058.60 \quad .644$

$\Delta \chi^{2}(\mathbf{2 4 )}=\mathbf{4 3 . 7 6} \quad .003$

NSIs for Middle-Aged Adults

Unconstrained Model

Weak Invariant Model

Strong Invariant Model

Strict Invariant Model

NSIs for Older Adults

Unconstrained Model

Weak Invariant Model

Strong Invariant Model

Strict Invariant Model $\chi^{2}(555)=4207.68 \quad .739$

$\chi^{2}(579)=4246.76 \quad .738$

$\Delta \chi^{2}(\mathbf{2 4})=\mathbf{3 9 . 0 8} \quad .001$

$\chi^{2}(603)=4492.17 \quad .723$

$\Delta \chi^{2}(24)=245.41 \quad .015$

$\chi^{2}(627)=4540.79 \quad .721$

$\Delta \chi^{2}(\mathbf{2 4})=\mathbf{4 8 . 6 2} \quad .002$

$\chi^{2}(\mathbf{5 5 5})=1239.77 \quad .714$

$\chi^{2}(579)=1261.48 \quad .715$

$\Delta \chi^{2}(24)=21.71 \quad .001$

$\chi^{2}(603)=1311.46 \quad .704$

$\Delta \chi^{2}(24)=49.98 \quad .011$

$\chi^{2}(627)=1349.50 \quad .699$

$\Delta \chi^{2}(\mathbf{2 4})=\mathbf{3 8 . 0 4} \quad .005$ 
Note. Significant statistics $(p<.05$ or $\Delta \mathrm{CFI} \geq .01)$ are bolded. Both the chi-square and the CFI difference statistics were computed because the chi-square statistic is commonly used, however, it is sensitive to large sample sizes. The CFI is more robust to larger sample sizes (Little, 2013). Parameters were freely estimated in the unconstrained model. The factors loadings were constrained to be equal across time in the weak invariant model. The factors loadings and the intercepts were constrained to be equal across time in the strong invariant model. The factors loadings, the intercepts, and the measurement errors were constrained to be equal across time in the strict invariant model. The first listed difference test compares the unconstrained model to the weak invariant model. The second listed difference test compares the weak and strong invariant models. The third listed difference test compares the strong and strict invariant models.

In order to use latent variables, the assumption of strong invariance must be satisfied (Little, 2013). To determine whether this assumption was satisfied, the chi-square and the CFI difference tests comparing the unconstrained model to the weak invariant model and the weak to the strong invariant model must not be significant. NSIs for the entire sample and for the three age groups did not satisfy the assumption of strong invariance, therefore, the decision to use composite scores instead of latent variables for NSIs was made. The critical ratios were examined to determine which indicators were invariant. Results indicated that the majority of problematic indicators were the factor loadings, intercepts, and measurement errors between MIDUS 1 and MIDUS 3 and MIDUS 2 and MIDUS 3. This suggests that the conceptual meaning of the Indicators of Stain Scale (Schuster et al., 1990) is significantly different in older age. 
Table H7

Measurement Invariance for NSIs from Family

$\chi^{2} \quad$ CFI $\quad \Delta \chi^{2} \quad \Delta$ CFI

NSIs from Family for Entire Sample

Unconstrained Model

Weak Invariant Model

Strong Invariant Model

Strict Invariant Model

NSIs from Family for Younger Adults

Unconstrained Model

Weak Invariant Model

Strong Invariant Model

Strict Invariant Model

$$
\begin{aligned}
& \chi^{2}(39)=73.83 \quad .995
\end{aligned}
$$

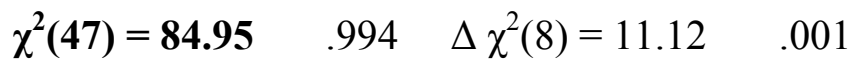

$$
\begin{aligned}
& \chi^{2}(55)=182.52 \quad .980 \quad \Delta \chi^{2}(8)=97.57 \quad .014 \\
& \chi^{2}(63)=197.44 \quad .979 \quad \Delta \chi^{2}(8)=14.92 \quad .001
\end{aligned}
$$

$\chi^{2}(39)=62.91 \quad .989$

$\chi^{2}(47)=66.64 \quad .991 \quad \Delta \chi^{2}(8)=3.73 \quad .002$

$\chi^{2}(55)=71.87 \quad .992 \quad \Delta \chi^{2}(8)=5.23 \quad .001$

$\chi^{2}(63)=74.51 \quad .995 \quad \Delta \chi^{2}(8)=2.64 \quad .003$

NSIs from Family for Middle-Aged Adults

Unconstrained Model

$\chi^{2}(39)=42.50 \quad .999$

Weak Invariant Model

$\chi^{2}(47)=56.48 \quad .997$

$\Delta \chi^{2}(8)=13.98 \quad .002$

Strong Invariant Model

$\chi^{2}(\mathbf{5 5})=\mathbf{1 7 1 . 3 2} .967$

$\Delta \chi^{2}(8)=114.84 \quad .030$

Strict Invariant Model

$\chi^{2}(63)=188.76 \quad .965$

$\Delta \chi^{2}(8)=17.44 \quad .002$

NSIs from Family for Older Adults

Unconstrained Model

$\chi^{2}(39)=43.62 \quad .990$

Weak Invariant Model

$\chi^{2}(47)=52.76 \quad .988$

$\Delta \chi^{2}(8)=9.14 \quad .002$

Strong Invariant Model

$\chi^{2}(55)=81.26 \quad .943$

$\Delta \chi^{2}(8)=28.50 \quad .045$

Strict Invariant Model

$\chi^{2}(63)=90.60 \quad .940$

$\Delta \chi^{2}(8)=9.34$

.003 
Note. Significant statistics $(p<.05$ or $\Delta \mathrm{CFI} \geq .01)$ are bolded. Both the chi-square and the CFI difference statistics were computed because the chi-square statistic is commonly used, however, it is sensitive to large sample sizes. The CFI is more robust to larger sample sizes (Little, 2013). Parameters are freely estimated in the unconstrained model. The factors loadings are constrained to be equal across time in the weak invariant model. The factors loadings and the intercepts are constrained to be equal across time in the strong invariant model. The factors loadings, the intercepts, and the measurement errors are constrained to be equal across time in the strict invariant model. The first listed difference test compares the unconstrained model to the weak invariant model. The second listed difference test compares the weak and strong invariant models. The third listed difference test compares the strong and strict invariant models.

In order to use latent variables, the assumption of strong invariance must be satisfied (Little, 2013). To determine whether this assumption was satisfied, the chi-square and the CFI difference tests comparing the unconstrained model to the weak invariant model and the weak to the strong invariant model must not be significant. NSIs from family for younger adults did satisfy the assumption of strong invariance. However, to keep models consistent and comparable across results, the decision to use composite scores instead of latent variables was made. The critical ratios were examined to determine which indicators were invariant. Results indicated that the majority of problematic indicators were the factor loadings, intercepts, and measurement errors between MIDUS 1 and MIDUS 3 and MIDUS 2 and MIDUS 3. This suggests that the conceptual meaning of the Indicators of Stain Scale (Schuster et al., 1990) is significantly different in older age. 
Table H8

Measurement Invariance for NSIs from Partner

$\chi^{2} \quad$ CFI $\quad \Delta \chi^{2} \quad \Delta$ CFI

NSIs from Partner for Entire Sample

Unconstrained Model

Weak Invariant Model

Strong Invariant Model

Strict Invariant Model

NSIs from Partner for Younger Adults

Unconstrained Model

Weak Invariant Model

Strong Invariant Model

Strict Invariant Model

NSIs from Partner for Middle-Aged Adults

Unconstrained Model

Weak Invariant Model

Strong Invariant Model

Strict Invariant Model

NSIs from Partner for Older Adults

Unconstrained Model

Weak Invariant Model

Strong Invariant Model

Strict Invariant Model

$$
\begin{array}{lllll}
\chi^{\mathbf{2}}(\mathbf{3 9})=\mathbf{7 8 . 4 7} & .995 & & \\
\chi^{\mathbf{2}(47)}=\mathbf{9 9 . 4 4} & .993 & \Delta \chi^{\mathbf{2}(8)}=\mathbf{2 0 . 9 7} & .003 \\
\chi^{\mathbf{2}(55)}=\mathbf{1 6 8 . 5 5} & .985 & \Delta \chi^{\mathbf{2}(8)}=\mathbf{6 9 . 1 1} & .008 \\
\chi^{\mathbf{2}(63)}=\mathbf{2 0 0 . 0 8} & .982 & \Delta \chi^{\mathbf{2}(8)}=\mathbf{3 1 . 5 3} & .003
\end{array}
$$

\begin{tabular}{|c|c|c|}
\hline$\chi^{2}(47)=60.28$ & .993 & $\Delta \chi^{2}(8)=10.04$ \\
\hline${ }^{2}(55)=93.53$ & .981 & $\Delta \chi^{2}(8)=33.25$ \\
\hline$(63)=108.80$ & .977 & $\Delta \chi^{2}(8)=15.27$ \\
\hline
\end{tabular}

$$
\begin{aligned}
& \chi^{2}(39)=50.24 \quad .994 \\
& \chi^{2}(47)=60.28 \quad .993 \quad \Delta \chi^{2}(8)=10.04 \quad .001 \\
& \chi^{2}(55)=93.53 \quad .981 \quad \Delta \chi^{2}(8)=33.25 \quad .012 \\
& \chi^{2}(63)=108.80 \quad .977 \quad \Delta \chi^{2}(8)=15.27 \quad .004
\end{aligned}
$$

$$
\begin{aligned}
& \chi^{2}(39)=61.32 \quad .995 \\
& \chi^{2}(47)=77.13 \quad .994 \quad \Delta \chi^{2}(8)=\mathbf{1 5 . 8 1} \quad .001 \\
& \chi^{2}(\mathbf{5 5})=\mathbf{1 1 6 . 8 3} \quad .987 \quad \Delta \chi^{2}(\mathbf{8})=\mathbf{3 9 . 7 0} \quad .007 \\
& \chi^{2}(63)=134.75 \quad .985 \quad \Delta \chi^{2}(8)=17.92 \quad .002
\end{aligned}
$$

$$
\chi^{2}(39)=75.79 \quad .955
$$$$
\chi^{2}(47)=87.36 \quad .951 \quad \Delta \chi^{2}(8)=11.57 \quad .004
$$$$
\chi^{2}(55)=112.76 \quad .929 \quad \Delta \chi^{2}(8)=25.40 \quad .022
$$$$
\chi^{2}(63)=120.50 \quad .930 \quad \Delta \chi^{2}(8)=7.74 \quad .001
$$ 
Note. Significant statistics $(p<.05$ or $\Delta \mathrm{CFI} \geq .01)$ are bolded. Both the chi-square and the CFI difference statistics were computed because the chi-square statistic is commonly used, however, it is sensitive to large sample sizes. The CFI is more robust to larger sample sizes (Little, 2013). Parameters are freely estimated in the unconstrained model. The factors loadings are constrained to be equal across time in the weak invariant model. The factors loadings and the intercepts are constrained to be equal across time in the strong invariant model. The factors loadings, the intercepts, and the measurement errors are constrained to be equal across time in the strict invariant model. The first listed difference test compares the unconstrained model to the weak invariant model. The second listed difference test compares the weak and strong invariant models. The third listed difference test compares the strong and strict invariant models.

In order to use latent variables, the assumption of strong invariance must be satisfied (Little, 2013). To determine whether this assumption was satisfied, the chi-square and the CFI difference tests comparing the unconstrained model to the weak invariant model and the weak to the strong invariant model must not be significant. NSIs from partner for the entire sample and middle-aged adults did satisfy the assumption of strong invariance. However, to keep models consistent and comparable across results, the decision to use composite scores instead of latent variables was made. The critical ratios were examined to determine which indicators were invariant. Results indicated that the majority of problematic indicators were the factor loadings, intercepts, and measurement errors between MIDUS 1 and MIDUS 3 and MIDUS 2 and MIDUS 3. This suggests that the conceptual meaning of the Indicators of Stain Scale (Schuster et al., 1990) is significantly different in older age. 
Table H9

Measurement Invariance for NSIs from Friends

$\chi^{2} \quad$ CFI $\quad \Delta \chi^{2} \quad \Delta$ CFI

NSIs from Friends for Entire Sample

Unconstrained Model

Weak Invariant Model

Strong Invariant Model

Strict Invariant Model

NSIs from Friends for Younger Adults

Unconstrained Model

Weak Invariant Model

Strong Invariant Model

Strict Invariant Model

NSIs from Friends for Middle-Aged Adults

Unconstrained Model

Weak Invariant Model

Strong Invariant Model

Strict Invariant Model

NSIs from Friends for Older Adults

Unconstrained Model
Weak Invariant Model

Strong Invariant Model

Strict Invariant Model $\chi^{2}(39)=54.32 \quad .998$

$\chi^{2}(47)=86.76 \quad .994$

$\Delta \chi^{2}(8)=32.44 \quad .004$

$\chi^{2}(55)=298.89$

$\Delta \chi^{2}(8)=212.13 \quad .033$

$\chi^{\mathbf{2}}(\mathbf{6 3})=\mathbf{3 1 7 . 9 6} .959$

$\Delta \chi^{2}(8)=19.07 \quad .002$ $\chi^{2}(39)=36.50 \quad 1.000$

$\chi^{2}(47)=51.95 \quad .997$

$\Delta \chi^{2}(8)=15.45$

.003

$\chi^{2}(55)=110.95 \quad .968$

$\Delta \chi^{2}(8)=59.00$

.029

$\chi^{2}(63)=121.41 \quad .967$

$\Delta \chi^{2}(8)=10.46$

.001 $\chi^{2}(39)=42.04 \quad .999$

$\chi^{2}(47)=62.38 \quad .996$

$\Delta \chi^{2}(8)=20.34 \quad .003$

$\chi^{2}(55)=224.99 \quad .955$

$\Delta \chi^{2}(8)=162.61$

.041

$\chi^{2}(63)=239.44 \quad .953$

$\Delta \chi^{2}(8)=14.45$

.002 
Note. Significant statistics $(p<.05$ or $\Delta \mathrm{CFI} \geq .01)$ are bolded. Both the chi-square and the CFI difference statistics were computed because the chi-square statistic is commonly used, however, it is sensitive to large sample sizes. The CFI is more robust to larger sample sizes (Little, 2013). Parameters are freely estimated in the unconstrained model. The factors loadings are constrained to be equal across time in the weak invariant model. The factors loadings and the intercepts are constrained to be equal across time in the strong invariant model. The factors loadings, the intercepts, and the measurement errors are constrained to be equal across time in the strict invariant model. The first listed difference test compares the unconstrained model to the weak invariant model. The second listed difference test compares the weak and strong invariant models. The third listed difference test compares the strong and strict invariant models.

In order to use latent variables, the assumption of strong invariance must be satisfied (Little, 2013). To determine whether this assumption was satisfied, the chi-square and the CFI difference tests comparing the unconstrained model to the weak invariant model and the weak to the strong invariant model must not be significant. NSIs from friends for the entire sample and the three age groups did not satisfy the assumption of strong invariance, therefore, the decision to use composite scores instead of latent variables for NSIs from friends was made. The critical ratios were examined to determine which indicators were invariant. Results indicated that the majority of problematic indicators were the factor loadings, intercepts, and measurement errors between MIDUS 1 and MIDUS 3 and MIDUS 2 and MIDUS 3. This suggests that the conceptual meaning of the Indicators of Stain Scale (Schuster et al., 1990) is significantly different in older age. 


\section{Appendix I}

Results for NSIs from Family

Table I1

Summary Results of Research Questions 2 and 3 for NSIs from Family

Latent Growth Curve of NSIs from Family

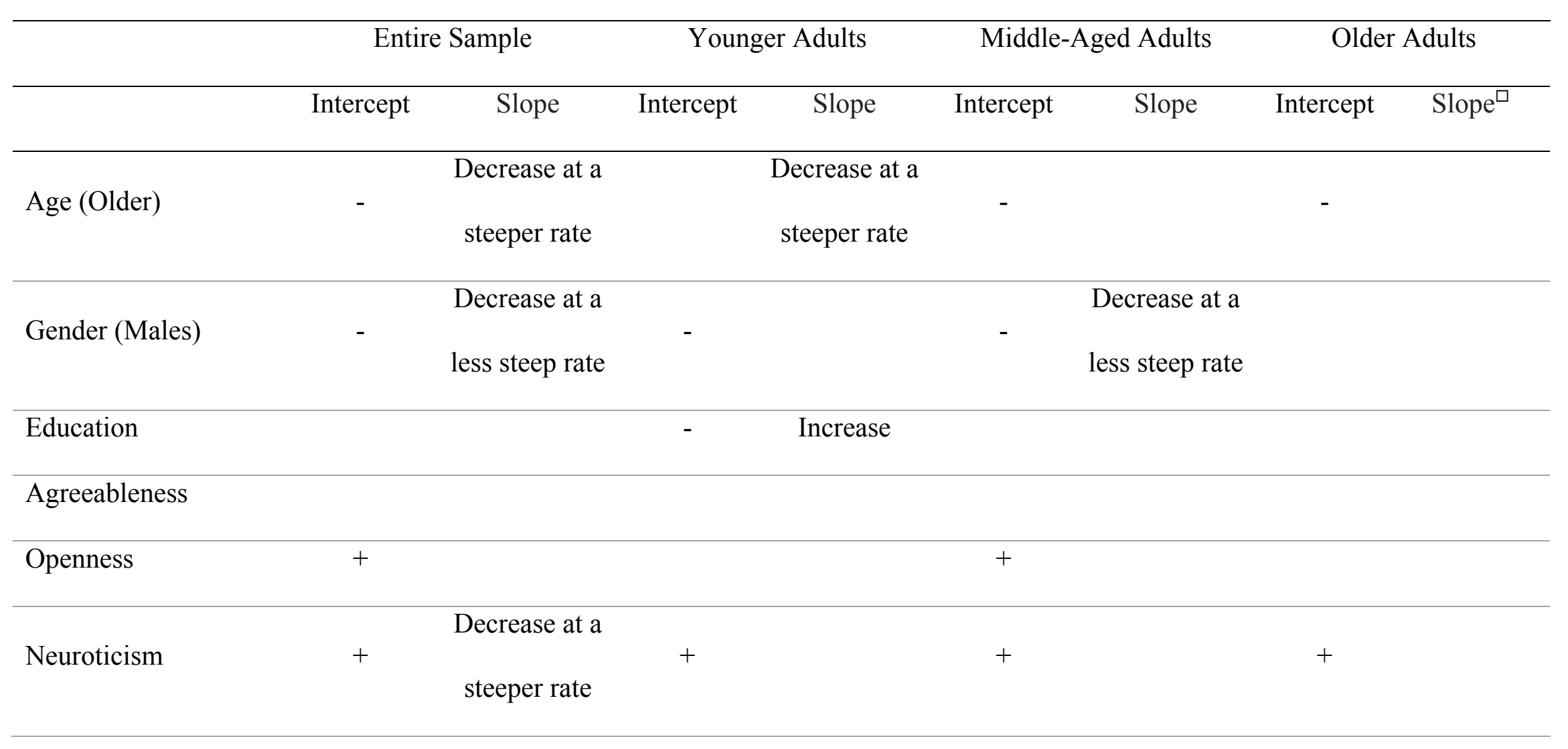




\section{Extraversion}

Conscientiousness

Note. The summary of significant effects is presented above. A plus sign indicates that higher values of the variable are associated

with a higher intercept. A minus sign indicates that higher values of the variable are associated with a lower intercept. ${ }^{\square}$ indicates that there was no significant variance surrounding the slope estimate. Therefore, slope was not predicted. 
Table I2

Summary Results of Research Questions 5 and 6 for Agreeableness

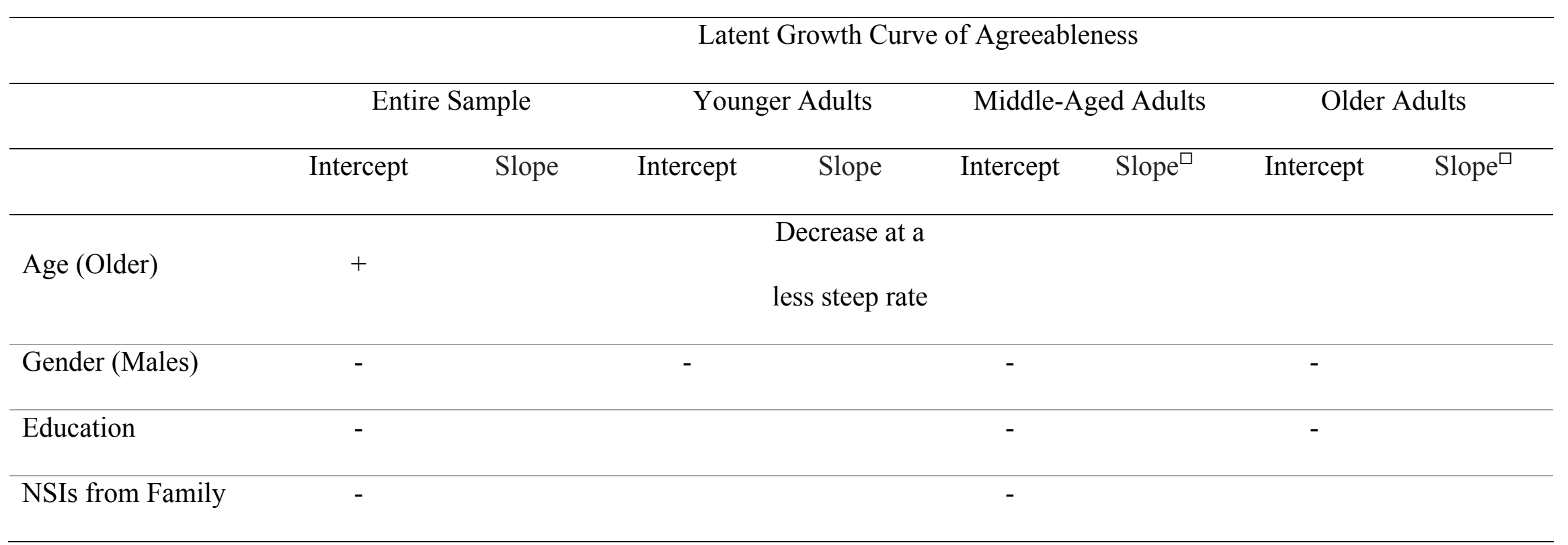

Note. The summary of significant effects is presented above. A plus sign indicates that higher values of the variable are associated with a higher intercept. A minus sign indicates that higher values of the variable are associated with a lower intercept. ${ }^{\square}$ indicates that there was no significant variance surrounding the slope estimate. Therefore, slope was not predicted. 
Table I3

Summary Results of Research Questions 5 and 6 for Openness

Latent Growth Curve of Openness

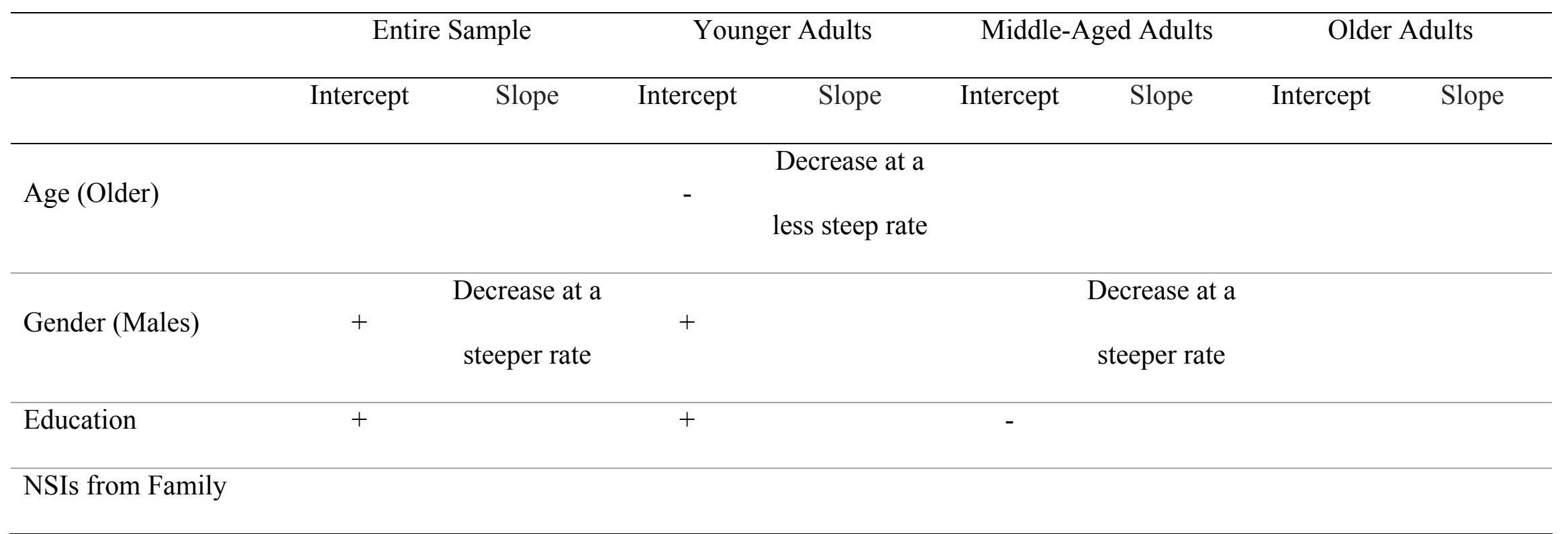

Note. The summary of significant effects is presented above. A plus sign indicates that higher values of the variable are associated

with a higher intercept. A minus sign indicates that higher values of the variable are associated with a lower intercept. 
Table I4

Summary Results of Research Questions 5 and 6 for Neuroticism

\section{Latent Growth Curve of Neuroticism}

\begin{tabular}{|c|c|c|c|c|c|c|c|c|}
\hline & \multicolumn{2}{|c|}{ Entire Sample } & \multicolumn{2}{|c|}{ Younger Adults } & \multicolumn{2}{|c|}{ Middle-Aged Adults } & \multicolumn{2}{|c|}{ Older Adults } \\
\hline & Intercept & Slope & Intercept & Slope & Intercept & Slope & Intercept & Slope $^{\square}$ \\
\hline Age (Older) & - & & & & - & $\begin{array}{l}\text { crease at } \\
\text { steep ra }\end{array}$ & & \\
\hline Gender (Males) & - & & - & & - & & - & \\
\hline Education & - & & - & & - & & - & \\
\hline NSIs from Family & + & crease & + & & + & & & \\
\hline
\end{tabular}

Note. The summary of significant effects is presented above. A plus sign indicates that higher values of the variable are associated

with a higher intercept. A minus sign indicates that higher values of the variable are associated with a lower intercept. ${ }^{\square}$ indicates that there was no significant variance surrounding the slope estimate. Therefore, slope was not predicted. 
Table I5

Summary Results of Research Questions 5 and 6 for Extraversion

Latent Growth Curve of Extraversion

\begin{tabular}{lll}
\hline \multicolumn{3}{c}{ Entire Sample } \\
\\
\hline Intercept & Slope
\end{tabular}

Age (Older)

Gender (Males)

Education

NSIs from Family

Note. The summary of significant effects is presented above. A plus sign indicates that higher values of the variable are associated with a higher intercept. A minus sign indicates that higher values of the variable are associated with a lower intercept. Age did not moderate the slope of extraversion. 
Table I6

Summary Results of Research Questions 5 and 6 for Conscientiousness

Latent Growth Curve of Conscientiousness

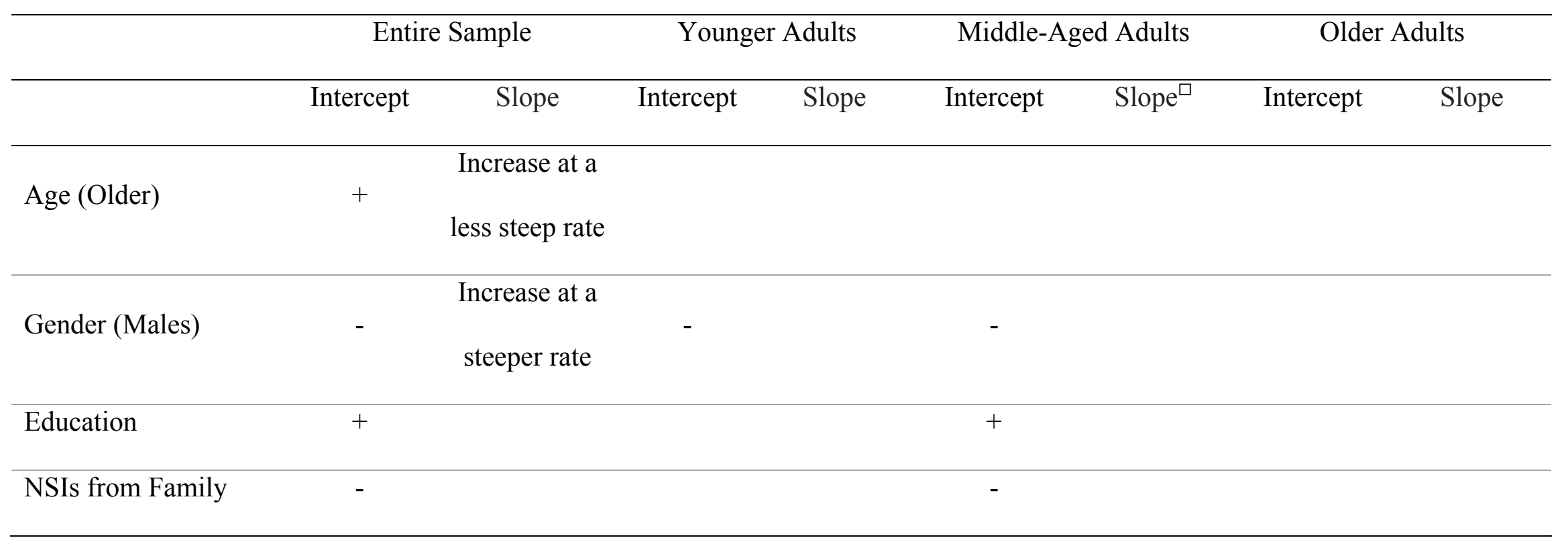

Note. The summary of significant effects is presented above. A plus sign indicates that higher values of the variable are associated

with a higher intercept. A minus sign indicates that higher values of the variable are associated with a lower intercept. ${ }^{\square}$ indicates that there was no significant variance surrounding the slope estimate. Therefore, slope was not predicted. 
Table I7

Summary Results of Research Questions 7 and 8 for NSIs from Family and Agreeableness

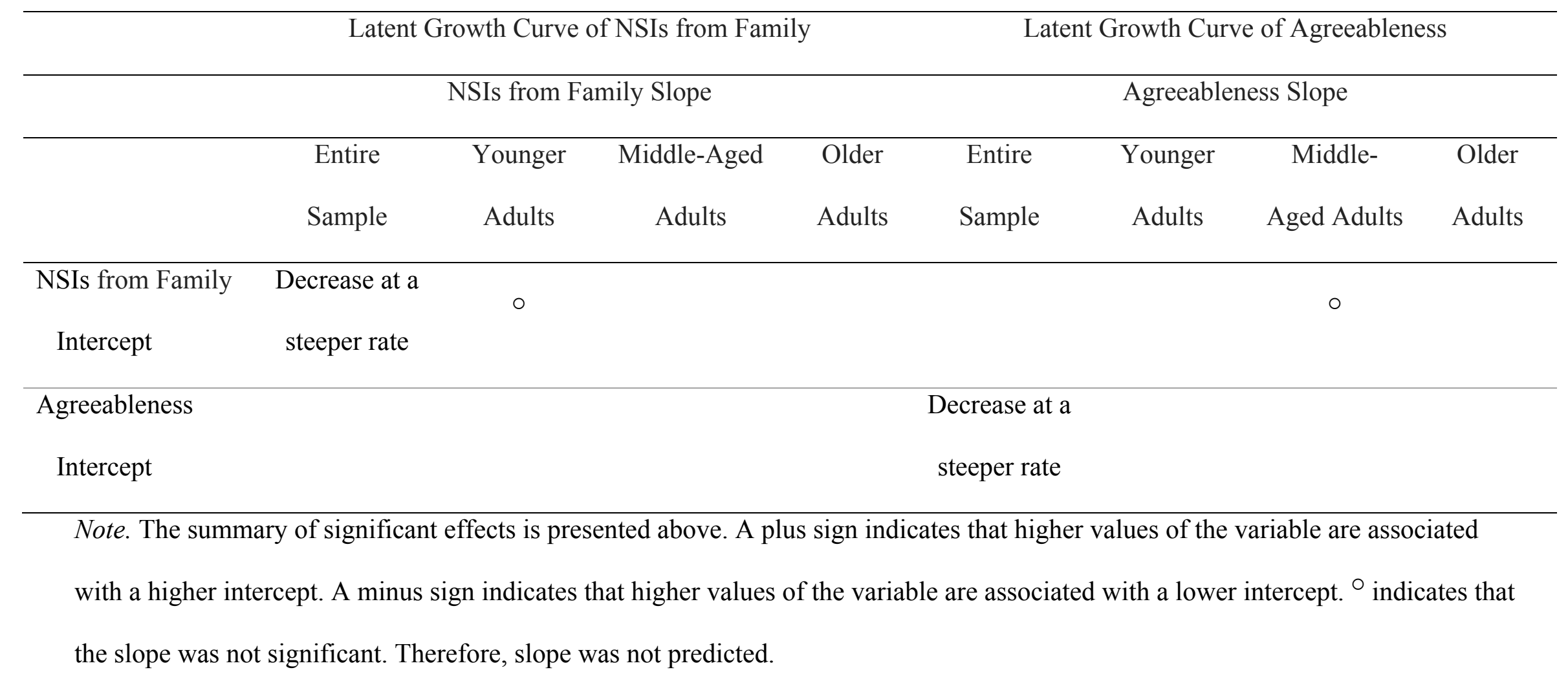


Table I8

Summary Results of Research Questions 7 and 8 for NSIs from Family and Openness

\section{Latent Growth Curve of NSIs from Family}

\begin{tabular}{cccccccccc}
\hline & \multicolumn{3}{c}{ NSIs from Family Slope } & & & Openness Slope \\
\hline Entire & Younger & Middle-Aged & Older & Entire & Younger & Middle- & Older \\
Sample & Adults & Adults & Adults & Sample & Adults & Aged Adults & Adults \\
& & & & & &
\end{tabular}

Intercept steeper rate

\section{Openness}

Intercept

Note. The summary of significant effects is presented above. A plus sign indicates that higher values of the variable are associated
Decrease at a

steeper rate

\footnotetext{
with a higher intercept. A minus sign indicates that higher values of the variable are associated with a lower intercept. ${ }^{\circ}$ indicates that the slope was not significant. Therefore, slope was not predicted.
} 
Table I9

Summary Results of Research Questions 7 and 8 for NSIs from Family and Neuroticism

\begin{tabular}{|c|c|c|c|c|c|c|c|c|}
\hline & \multicolumn{4}{|c|}{ Latent Growth Curve of NSIs from Family } & \multicolumn{4}{|c|}{ Latent Growth Curve of Neuroticism } \\
\hline & \multicolumn{4}{|c|}{ NSIs from Family Slope } & \multicolumn{4}{|c|}{ Neuroticism Slope } \\
\hline & Entire & Younger & Middle-Aged & Older & Entire & Younger & Middle- & Older \\
\hline & Sample & Adults & Adults & Adults & Sample & Adults & Aged Adults & Adults \\
\hline NSIs from Family & Decrease at a & O & & & & & & \\
\hline Intercept & steeper rate & & & & & & & \\
\hline Neuroticism & & & & & Decrease at a & & Decrease at a & \\
\hline Intercept & & & & & steeper rate & & steeper rate & \\
\hline
\end{tabular}

Note. The summary of significant effects is presented above. A plus sign indicates that higher values of the variable are associated with a higher intercept. A minus sign indicates that higher values of the variable are associated with a lower intercept. ${ }^{\circ}$ indicates that the slope was not significant. Therefore, slope was not predicted. 
Table I10

Summary Results of Research Questions 7 and 8 for NSIs from Family and Extraversion

\begin{tabular}{|c|c|c|c|c|c|c|c|c|}
\hline & \multicolumn{4}{|c|}{ Latent Growth Curve of NSIs from Family } & \multicolumn{4}{|c|}{ Latent Growth Curve of Extraversion } \\
\hline & \multicolumn{4}{|c|}{ NSIs from Family Slope } & \multicolumn{4}{|c|}{ Extraversion Slope } \\
\hline & Entire & Younger & Middle-Aged & Older & Entire & Younger & Middle- & Older \\
\hline & Sample & Adults & Adults & Adults & Sample & Adults & Aged Adults & Adults \\
\hline NSIs from Family & Decrease at a & 0 & & & & & & \\
\hline Intercept & steeper rate & & & & & & & \\
\hline
\end{tabular}

\section{Extraversion}

\section{Intercept}

Note. The summary of significant effects is presented above. A plus sign indicates that higher values of the variable are associated with a higher intercept. A minus sign indicates that higher values of the variable are associated with a lower intercept. ${ }^{\circ}$ indicates that the slope was not significant. Therefore, slope was not predicted. 
Table I11

Summary Results of Research Questions 7 and 8 for NSIs from Family and Conscientiousness

Latent Growth Curve of NSIs from Family NSIs from Family Slope

\begin{tabular}{|c|c|c|c|c|c|c|c|}
\hline Entire & Younger & Middle-Aged & Older & Entire & Younger & Middle- & Older \\
\hline Sample & Adults & Adults & Adults & Sample & Adults & Aged Adults & Adults \\
\hline
\end{tabular}

Latent Growth Curve of Conscientiousness

\section{Conscientiousness Slope}

NSIs from Family Decrease at a

Intercept steeper rate

Conscientiousness

○

Intercept

Note. The summary of significant effects is presented above. A plus sign indicates that higher values of the variable are associated with a higher intercept. A minus sign indicates that higher values of the variable are associated with a lower intercept. ${ }^{\circ}$ indicates that the slope was not significant. Therefore, slope was not predicted. 
Table I12

Missing Data for NSIs from Family across all MIDUS Waves

\begin{tabular}{lcc}
\hline & Missing Count & Missing Percent \\
\hline NSIs from Family - MIDUS 1 & 2 & $0.10 \%$ \\
NSIs from Family - MIDUS 2 & 4 & $0.30 \%$ \\
NSIs from Family - MIDUS 3 & 8 & $0.50 \%$ \\
\hline
\end{tabular}


Table I13

Attrition Analyses Comparing Non-Multivariate Outliers and Multivariate Outliers for NSIs from Family across all MIDUS Waves

\begin{tabular}{lccc}
\hline & Non-Multivariate & Multivariate & Difference Statistic \\
& Outliers & Outliers & \\
& $(n=1,403)$ & $(n=127)$ & \\
& $M, S D$ or $\%$ & $M, S D$ or $\%$ & \\
\hline NSIs from Family - MIDUS 1 & $2.06,0.55$ & $2.17,0.58$ & $\boldsymbol{t}(\mathbf{1 , 5 2 6})=\mathbf{- 2 . 0 9}$ \\
NSIs from Family - MIDUS 2 & $1.98,0.54$ & $2.18,0.66$ & $\boldsymbol{t}(\mathbf{1 4 0 . 8 0})=\mathbf{- 3 . 1 8}$ \\
NSIs from Family - MIDUS 3 & $1.90,0.58$ & $2.09,0.65$ & $\boldsymbol{t}(\mathbf{1 , 5 2 0})=\mathbf{- 3 . 4 5}$
\end{tabular}

Note. Significant statistics $(p<.05)$ are bolded. 
Table I14

Research Question 1 - The Latent Growth Curve of NSIs from Family

\begin{tabular}{|c|c|c|c|c|c|c|c|}
\hline & \multirow[t]{2}{*}{ Model Fit } & \multicolumn{2}{|c|}{ Intercept } & \multicolumn{2}{|c|}{ Slope } & \multicolumn{2}{|c|}{ Covariance } \\
\hline & & $b$ & S.E. & $b$ & S.E. & $b$ & S.E. \\
\hline \multirow[t]{4}{*}{ NSIs from Family } & $\chi^{2}(3)=0.75$ & $2.077 *$ & 0.014 & $-0.009 *$ & 0.001 & -0.002 & 0.001 \\
\hline & $\mathrm{CMIN} / \mathrm{DF}=0.25$ & & & & & & \\
\hline & $\mathrm{CFI}=1.000$ & & & & & & \\
\hline & $\mathrm{RMSEA}=.00$ & & & & & & \\
\hline
\end{tabular}

Note. Significant statistics $(p<.05)$ are bolded. An asterisk indicates that there is significant variability surrounding the estimate. A blue value indicates that the estimate is significantly different than zero. 
Table I15

Research Question 2 - Demographic Variables and the Big Five Personality Traits Predicting the Latent Growth Curve of NSIs from Family

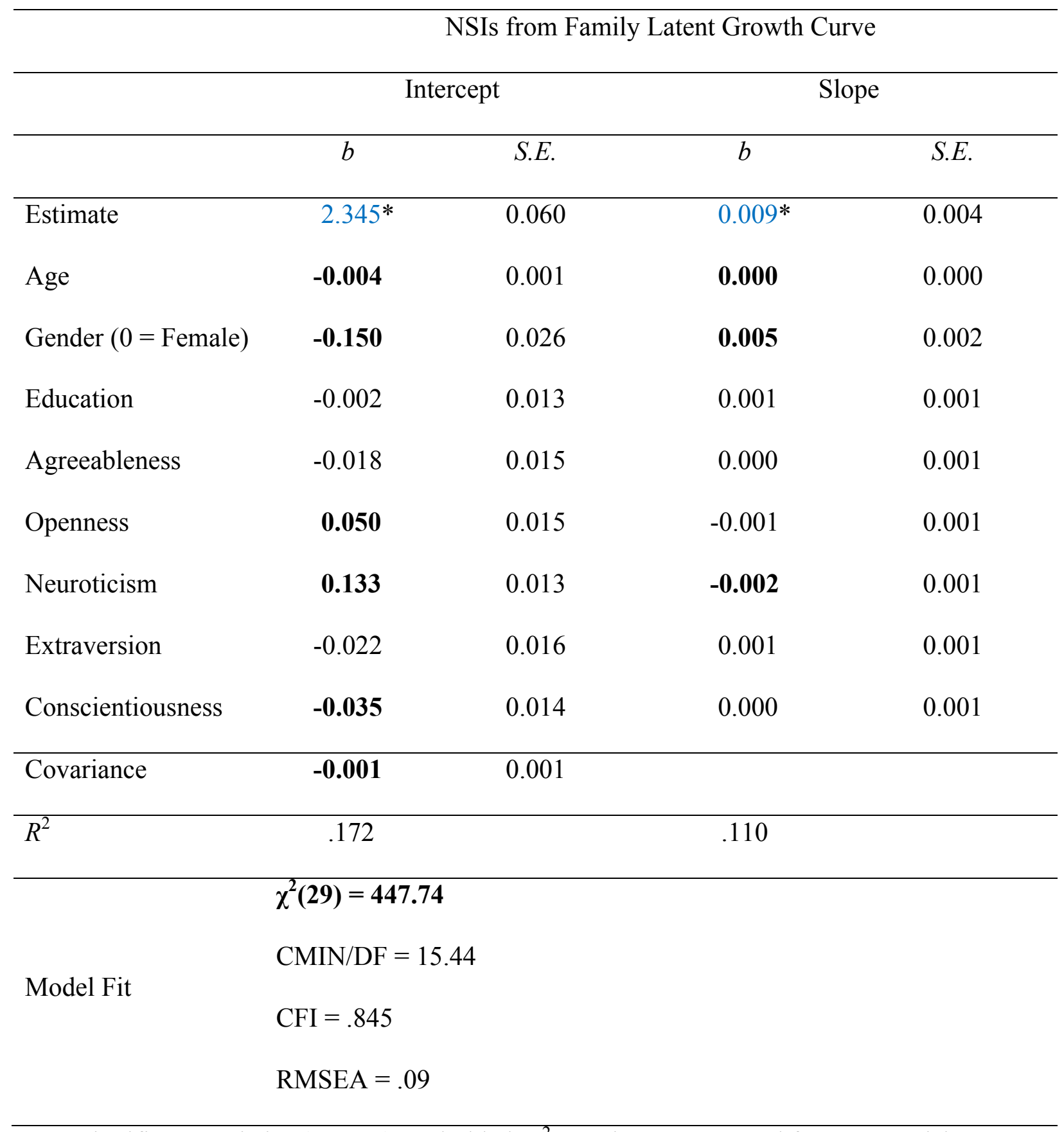

Note. Significant statistics $(p<.05)$ are bolded. $R^{2}=$ variance accounted for. An asterisk indicates that there is significant variability surrounding the estimate. A blue value indicates that 
the estimate is significantly different than zero. Type I errors were accounted for utilizing the false discovery rate adjustment. A green value indicates that the estimate was no longer statistically significant after the false discovery rate adjustments. To aid in the interpretation of the estimates, education, agreeableness, openness, neuroticism, extraversion, and conscientiousness were standardized. As such, a unit increase or decrease in these variables represent a standard deviation increase or decrease, respectively. Age was not manipulated so that a unit increase or decrease represents an increase or decrease of a year, respectively. 
Table I16

The Unconstrained Model Compared to the Constrained Model for the Multigroup Latent Growth Curve of NSIs from Family

\begin{tabular}{lcccc}
\hline & $\chi^{2}$ & CFI & $\Delta \chi^{2}$ & $\Delta$ CFI \\
\hline NSIs from Family & & & & \\
Unconstrained Model & $\chi^{2}(\mathbf{1 1})=\mathbf{8 . 8 9}$ & 1.000 & & \\
Constrained Model & $\chi^{2}(\mathbf{1 7})=\mathbf{9 7 . 1 3}$ & .912 & $\chi^{2}(\mathbf{6})=\mathbf{8 8 . 2 4}$ & $\mathbf{. 0 8 8}$ \\
\hline
\end{tabular}

Note. Significant statistics $(p<.05)$ are bolded. The unconstrained model is where the parameters were freely estimated across the three age groups. The constrained model is where the parameters were constrained to be equal across the three age groups. A change of .01 or greater for the CFI difference statistic was used to determine significance (Little, 2013). Both the chi-square and the CFI difference statistics were computed because the chi-square statistic is commonly used, however, it is sensitive to large sample sizes. The CFI is more robust to larger sample sizes (Little, 2013) 
Table I17

Research Question 3 - Multigroup Analyses for the Latent Growth Curve of NSIs from Family

\begin{tabular}{|c|c|c|c|c|c|c|c|c|}
\hline & \multirow[t]{2}{*}{ Model Fit } & & \multicolumn{2}{|c|}{ Intercept } & \multicolumn{2}{|c|}{ Slope } & \multicolumn{2}{|c|}{ Covariance } \\
\hline & & & $b$ & S.E. & $b$ & S.E. & $b$ & S.E. \\
\hline \multirow[t]{4}{*}{ NSIs from Family } & $\chi^{2}(11)=8.89$ & Younger Adults & $2.103 * b$ & 0.028 & $-0.001 * a, b$ & 0.002 & -0.003 & 0.001 \\
\hline & $\mathrm{CMIN} / \mathrm{DF}=0.80$ & Middle-Aged Adults & $2.097 *^{\mathrm{c}}$ & 0.017 & $-0.012 *$ & 0.001 & -0.002 & 0.001 \\
\hline & $\mathrm{CFI}=1.000$ & Older Adults & $1.906 *$ & 0.034 & -0.011 & 0.002 & 0.000 & 0.001 \\
\hline & $\mathrm{RMSEA}=.00$ & & & & & & & \\
\hline
\end{tabular}

Note. Significant statistics $(p<.05)$ are bolded. An asterisk indicates that there is significant variability surrounding the estimate. A blue value indicates that the estimate is significantly different than zero. ${ }^{a}$ indicates that the estimate is significantly different between younger and middle-aged adults. ${ }^{b}$ indicates that the estimate is significantly different between younger and older adults. ${ }^{c}$ indicates that the estimate is significantly different between middle-aged and older adults 
Table I18

Research Question 3 - Demographic Variables and the Big Five Personality Traits Predicting the Latent Growth Curve of NSIs from Family for Younger Adults

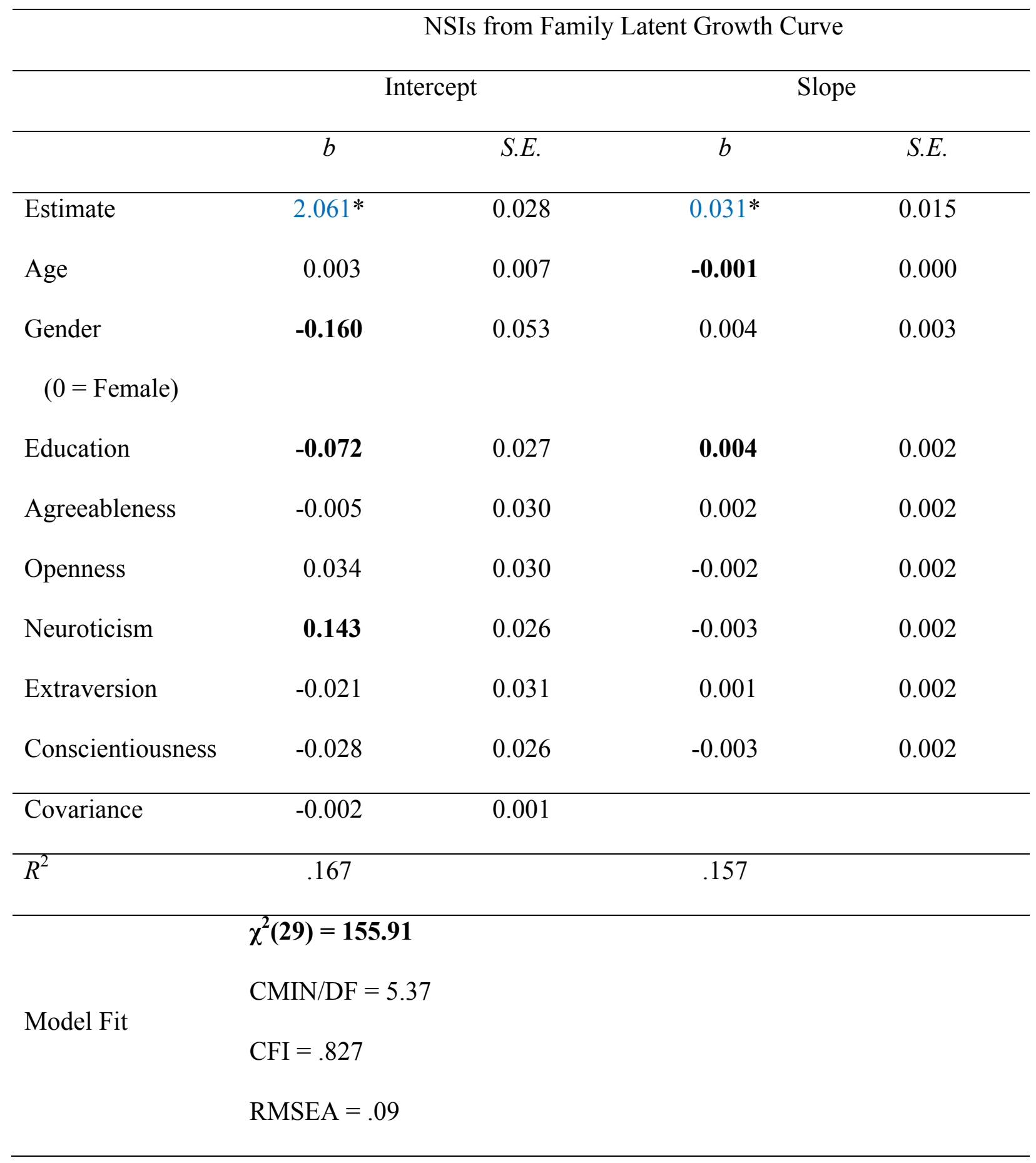


Note. Significant statistics $(p<.05)$ are bolded. $R^{2}=$ variance accounted for. An asterisk indicates that there is significant variability surrounding the estimate. A blue value indicates that the estimate is significantly different than zero. Type I errors were accounted for utilizing the false discovery rate adjustment. A green value indicates that the estimate was no longer statistically significant after the false discovery rate adjustments. To aid in the interpretation of the estimates, education, agreeableness, openness, neuroticism, extraversion, and conscientiousness were standardized. As such, a unit increase or decrease in these variables represent a standard deviation increase or decrease, respectively. Age was not manipulated so that a unit increase or decrease represents an increase or decrease of a year, respectively. 
Table I19

Research Question 3 - Demographic Variables and the Big Five Personality Traits Predicting the Latent Growth Curve of NSIs from Family for Middle-Aged Adults

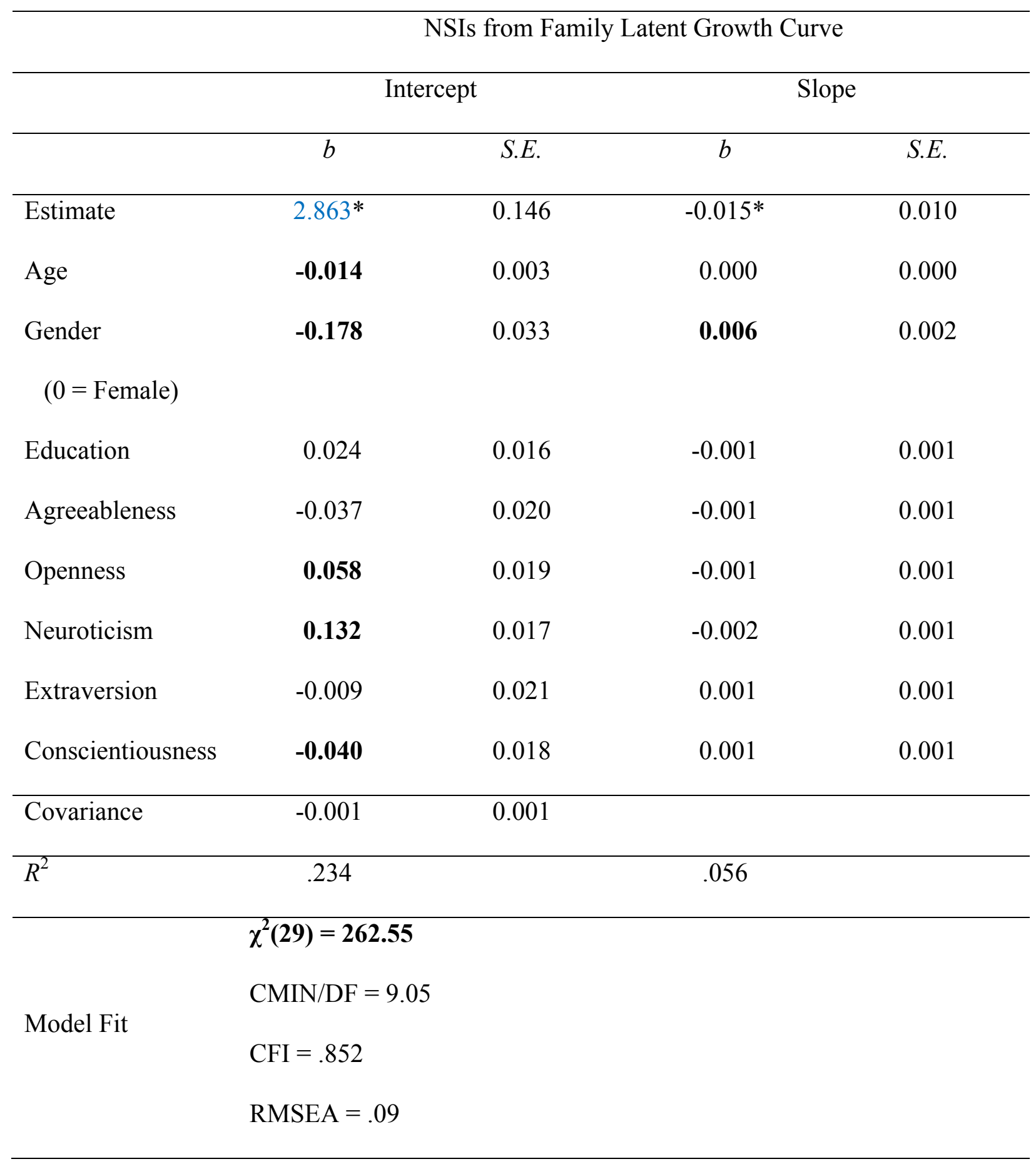


Note. Significant statistics $(p<.05)$ are bolded. $R^{2}=$ variance accounted for. An asterisk indicates that there is significant variability surrounding the estimate. A blue value indicates that the estimate is significantly different than zero. Type I errors were accounted for utilizing the false discovery rate adjustment. A green value indicates that the estimate was no longer statistically significant after the false discovery rate adjustments. To aid in the interpretation of the estimates, education, agreeableness, openness, neuroticism, extraversion, and conscientiousness were standardized. As such, a unit increase or decrease in these variables represent a standard deviation increase or decrease, respectively. Age was not manipulated so that a unit increase or decrease represents an increase or decrease of a year, respectively. 
Table I20

Research Question 3 - Demographic Variables and the Big Five Personality Traits Predicting the Latent Growth Curve of NSIs from Family for Older Adults

\begin{tabular}{|c|c|c|c|c|}
\hline & \multicolumn{4}{|c|}{ NSIs from Family Latent Growth Curve } \\
\hline & \multicolumn{2}{|c|}{ Intercept } & \multicolumn{2}{|c|}{ Slope } \\
\hline & $b$ & S.E. & $b$ & S.E. \\
\hline Estimate & $3.236^{*}$ & 0.467 & $-0.011^{*}$ & 0.002 \\
\hline Age & -0.021 & 0.007 & - & - \\
\hline $\begin{array}{l}\text { Gender } \\
\qquad(0=\text { Female })\end{array}$ & 0.056 & 0.054 & - & - \\
\hline Education & 0.006 & 0.026 & - & - \\
\hline Agreeableness & 0.003 & 0.032 & - & - \\
\hline Openness & 0.013 & 0.032 & - & - \\
\hline Neuroticism & 0.067 & 0.030 & - & - \\
\hline Extraversion & 0.009 & 0.038 & - & - \\
\hline Conscientiousness & -0.067 & 0.026 & - & - \\
\hline Covariance & -0.002 & 0.001 & & \\
\hline$R^{2}$ & .153 & & .000 & \\
\hline Model Fit & $\begin{array}{l}\chi^{2}(\mathbf{2 9})=\mathbf{7 0 . 8 8} \\
\text { CMIN/DF }=1.91 \\
\text { CFI }=.884 \\
\text { RMSEA }=.07\end{array}$ & & & \\
\hline
\end{tabular}


Note. Significant statistics $(p<.05)$ are bolded. $R^{2}=$ variance accounted for. An asterisk indicates that there is significant variability surrounding the estimate. A blue value indicates that the estimate is significantly different than zero. Type I errors were accounted for utilizing the false discovery rate adjustment. A green value indicates that the estimate was no longer statistically significant after the false discovery rate adjustments. To aid in the interpretation of the estimates, education, agreeableness, openness, neuroticism, extraversion, and conscientiousness were standardized. As such, a unit increase or decrease in these variables represent a standard deviation increase or decrease, respectively. Age was not manipulated so that a unit increase or decrease represents an increase or decrease of a year, respectively. 
Table I21

Research Question 5 - Demographic Variables and NSIs from Family Predicting the Latent Growth Curve of Agreeableness

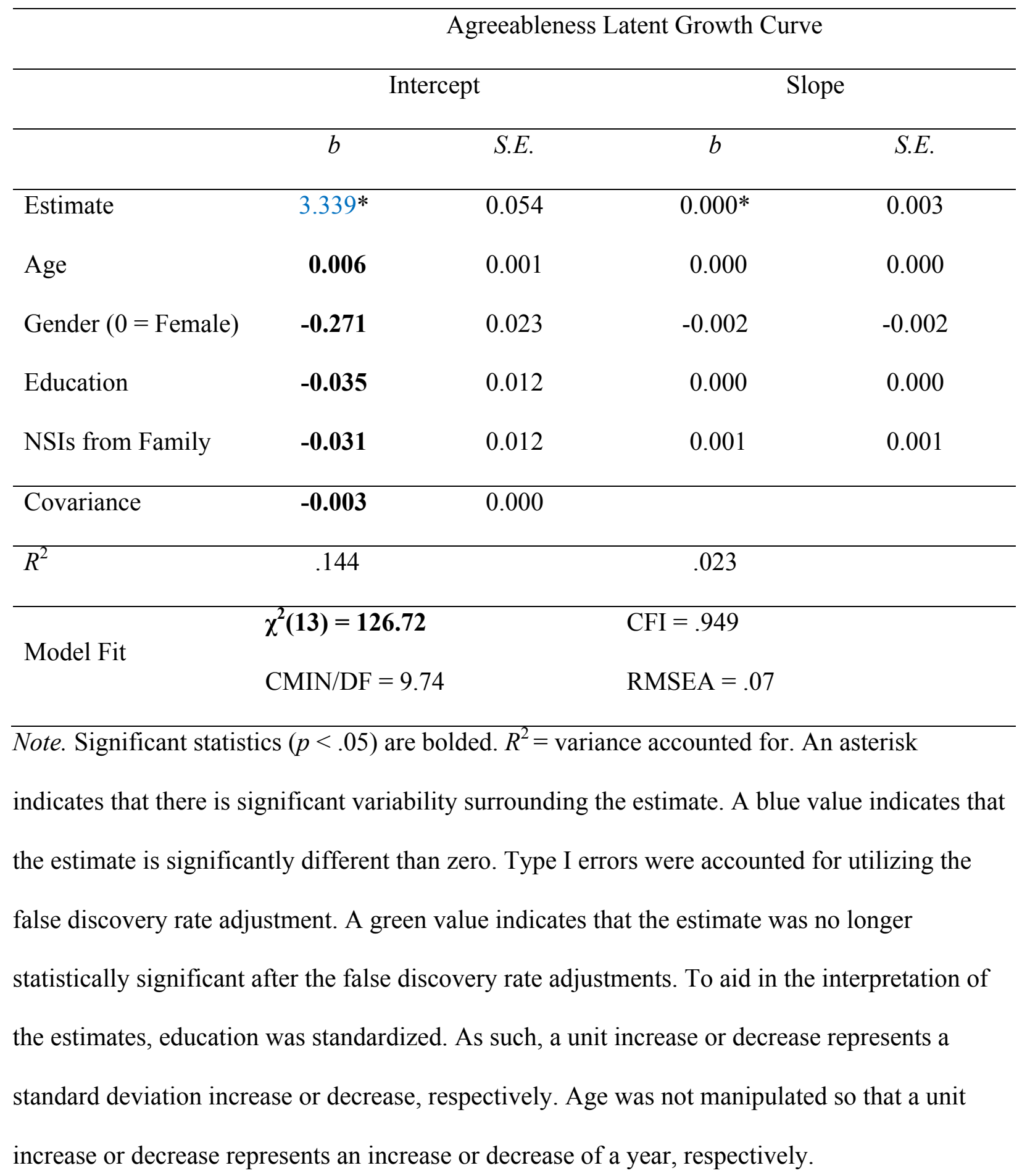


Table I22

Research Question 5 - Demographic Variables and NSIs from Family Predicting the Latent Growth Curve of Openness

\section{Openness Latent Growth Curve}

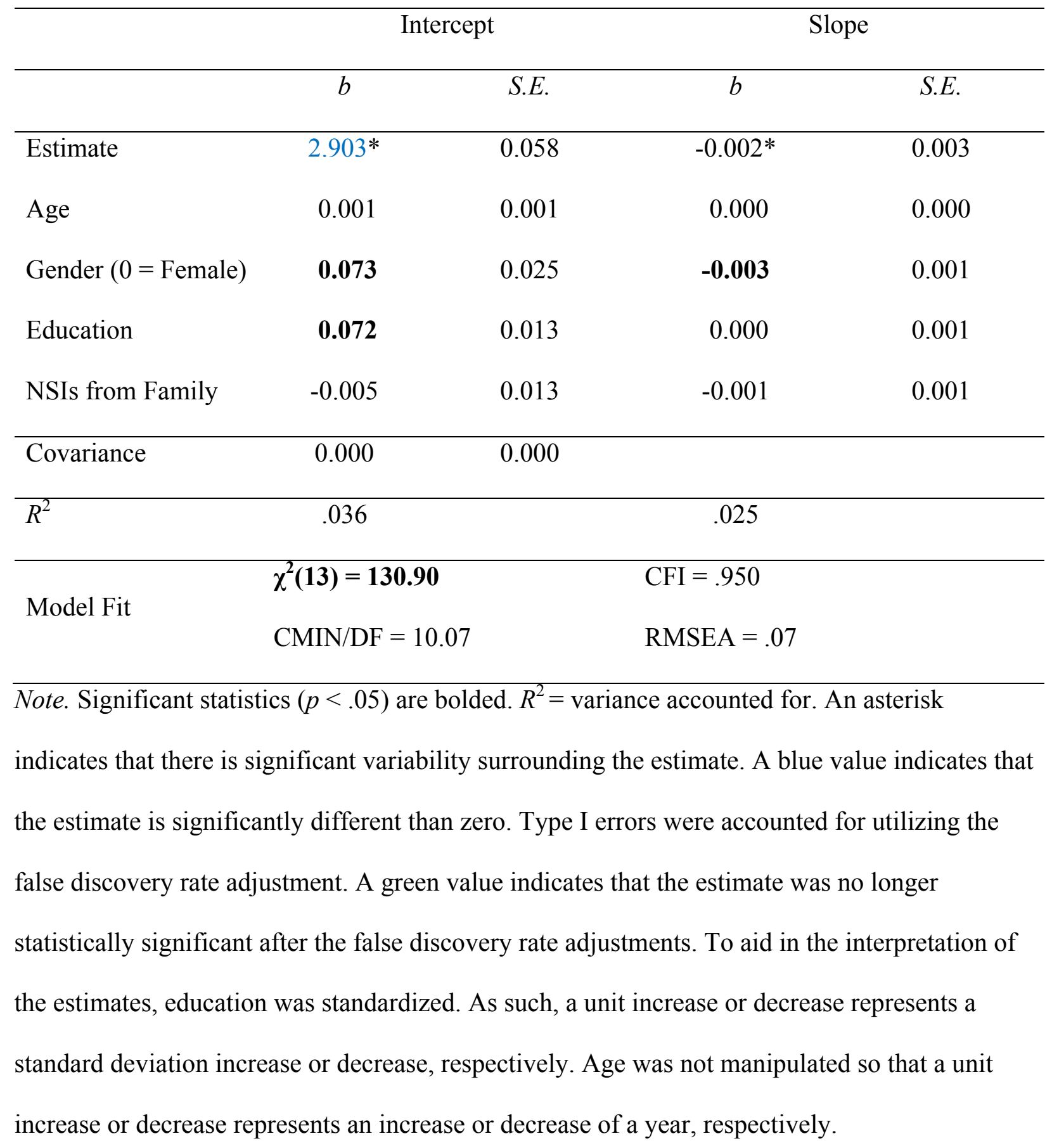


Table I23

Research Question 5 - Demographic Variables and NSIs from Family Predicting the Latent Growth Curve of Neuroticism

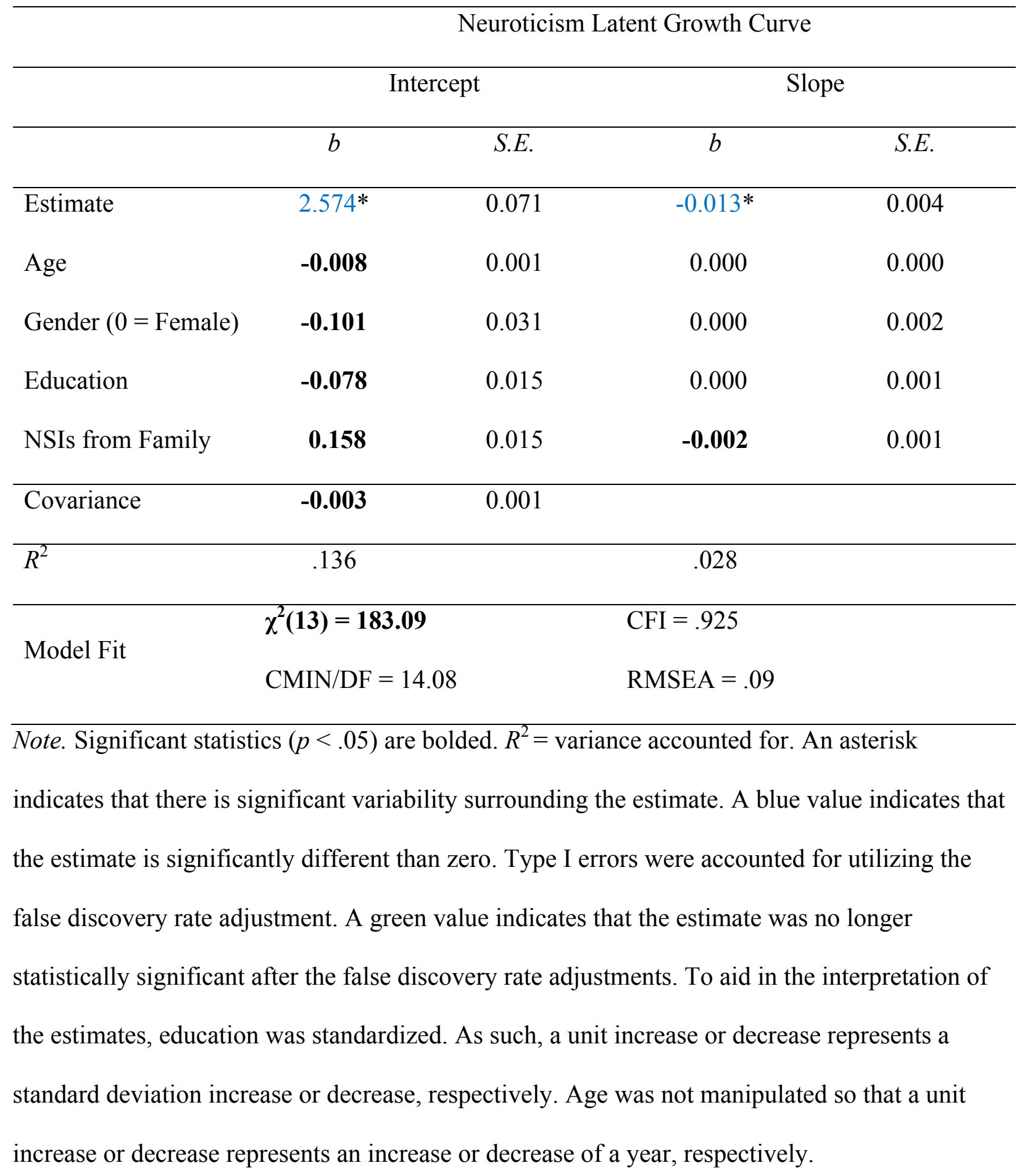


Table I24

Research Question 5 - Demographic Variables and NSIs from Family Predicting the Latent Growth Curve of Extraversion

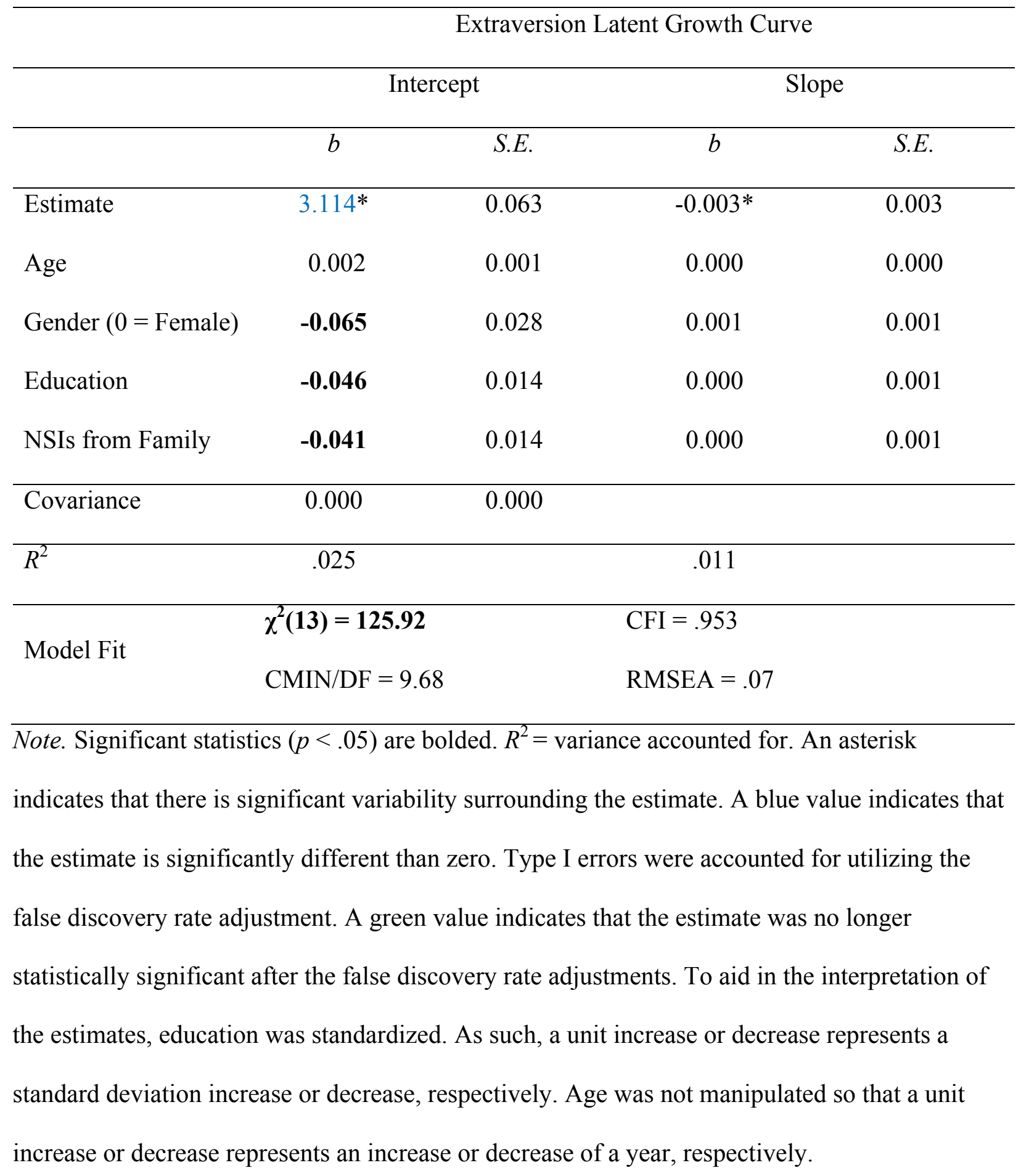


Table I25

Research Question 5 - Demographic Variables and NSIs from Family Predicting the Latent Growth Curve of Conscientiousness

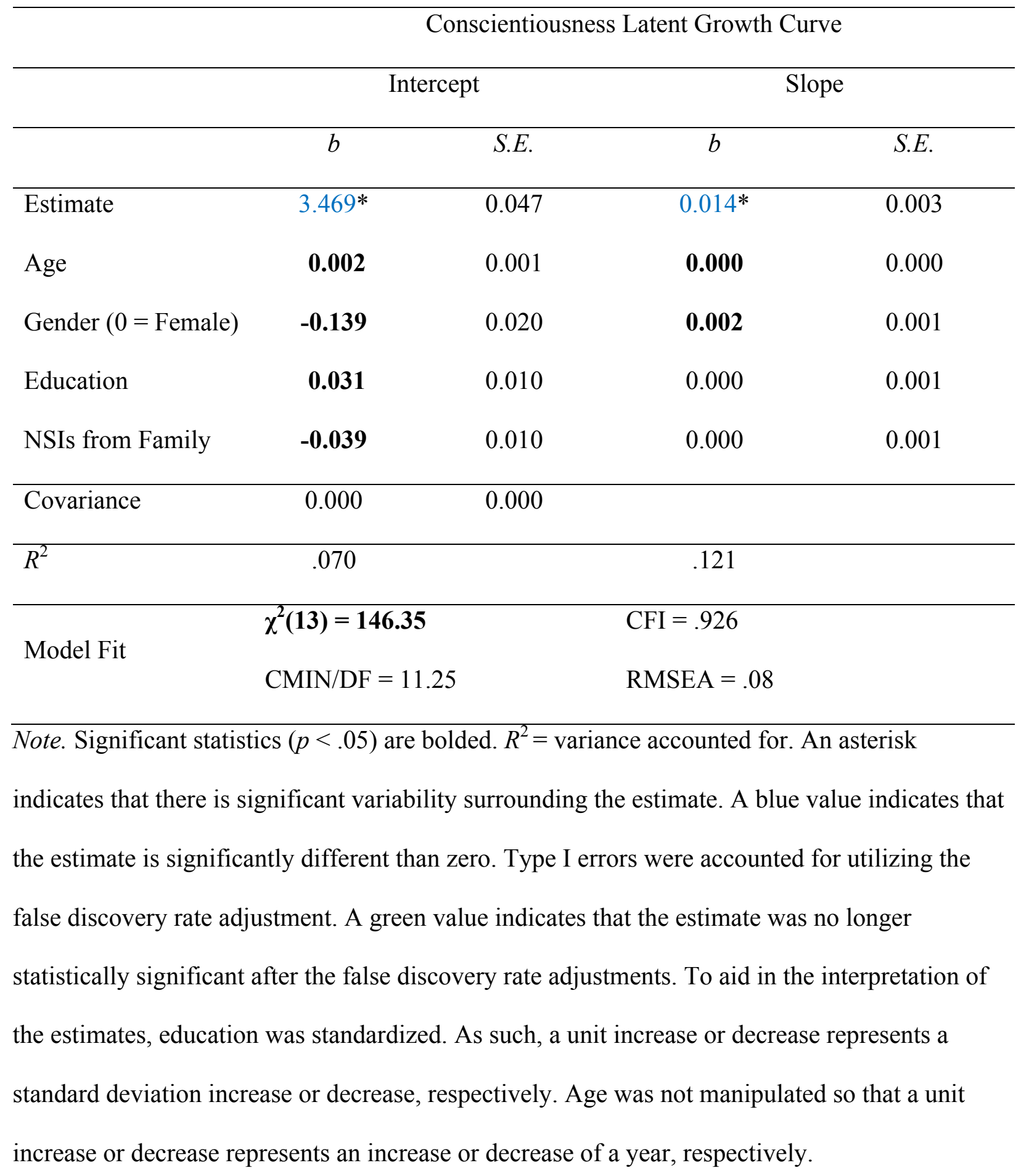


Table I26

Research Question 6 - Demographic Variables and NSIs from Family Predicting the Latent Growth Curve of Agreeableness for Younger Adults

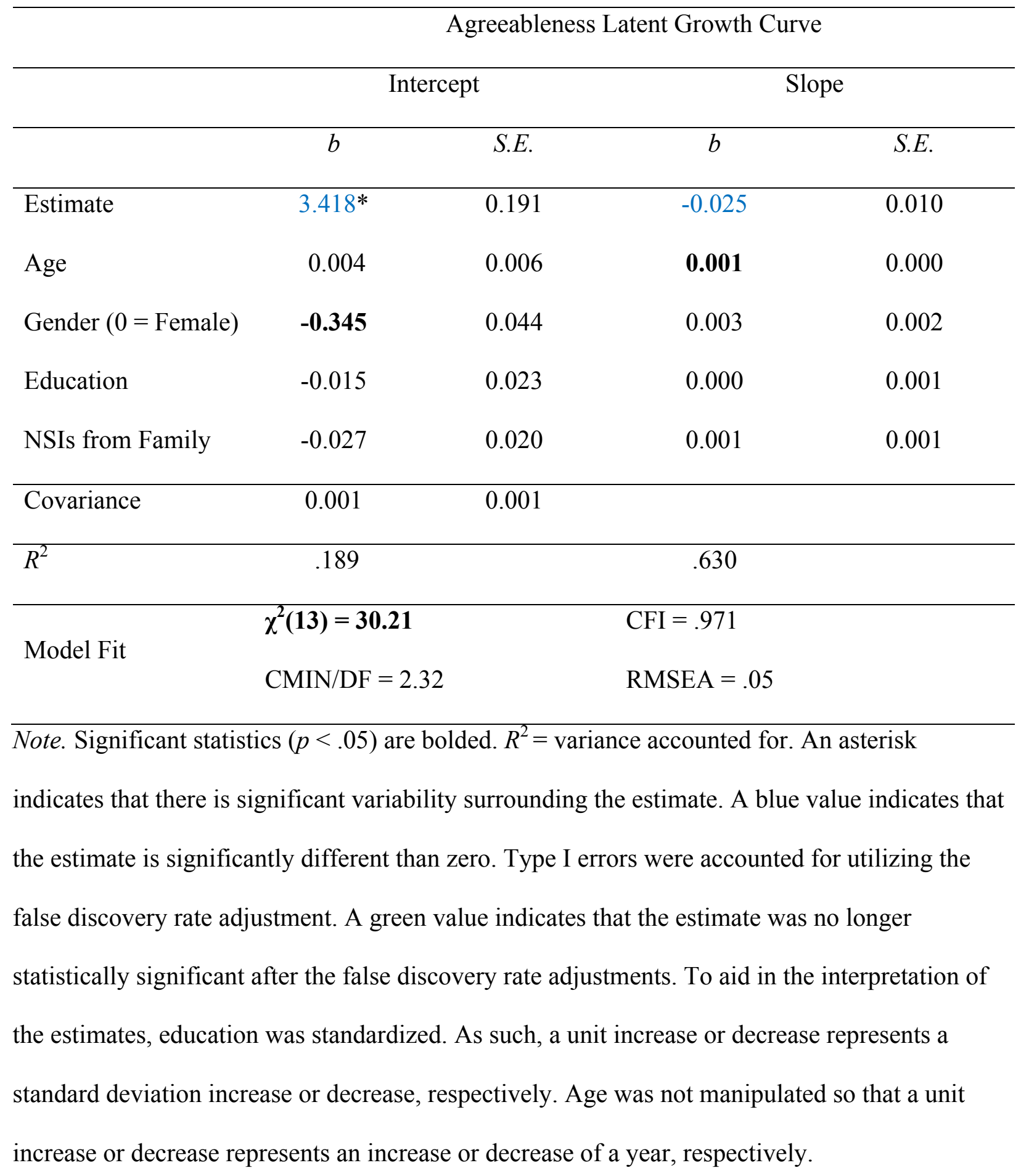


Table I27

Research Question 6 - Demographic Variables and NSIs from Family Predicting the Latent Growth Curve of Agreeableness for Middle-Aged Adults

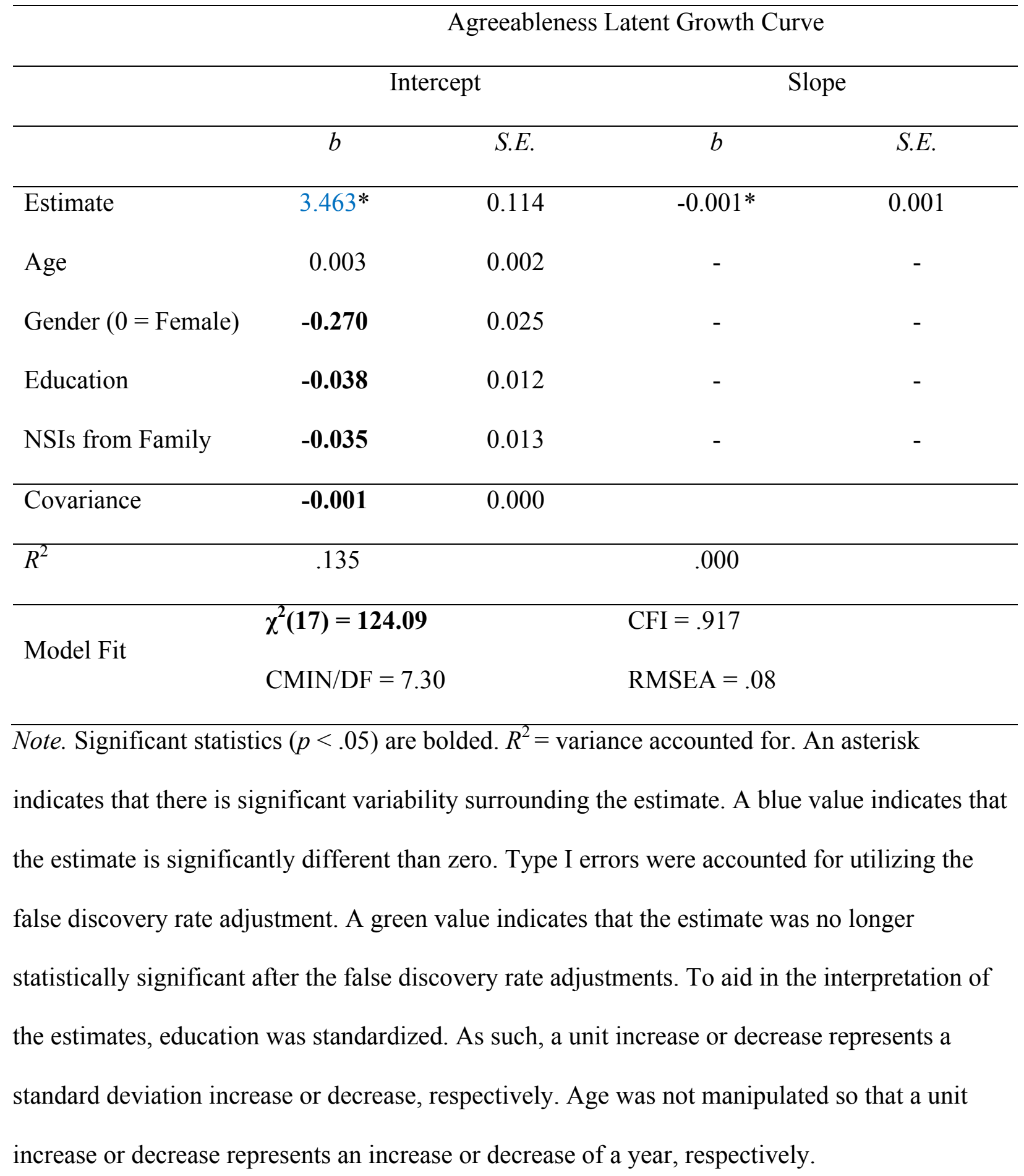


Table I28

Research Question 6 - Demographic Variables and NSIs from Family Predicting the Latent Growth Curve of Agreeableness for Older Adults

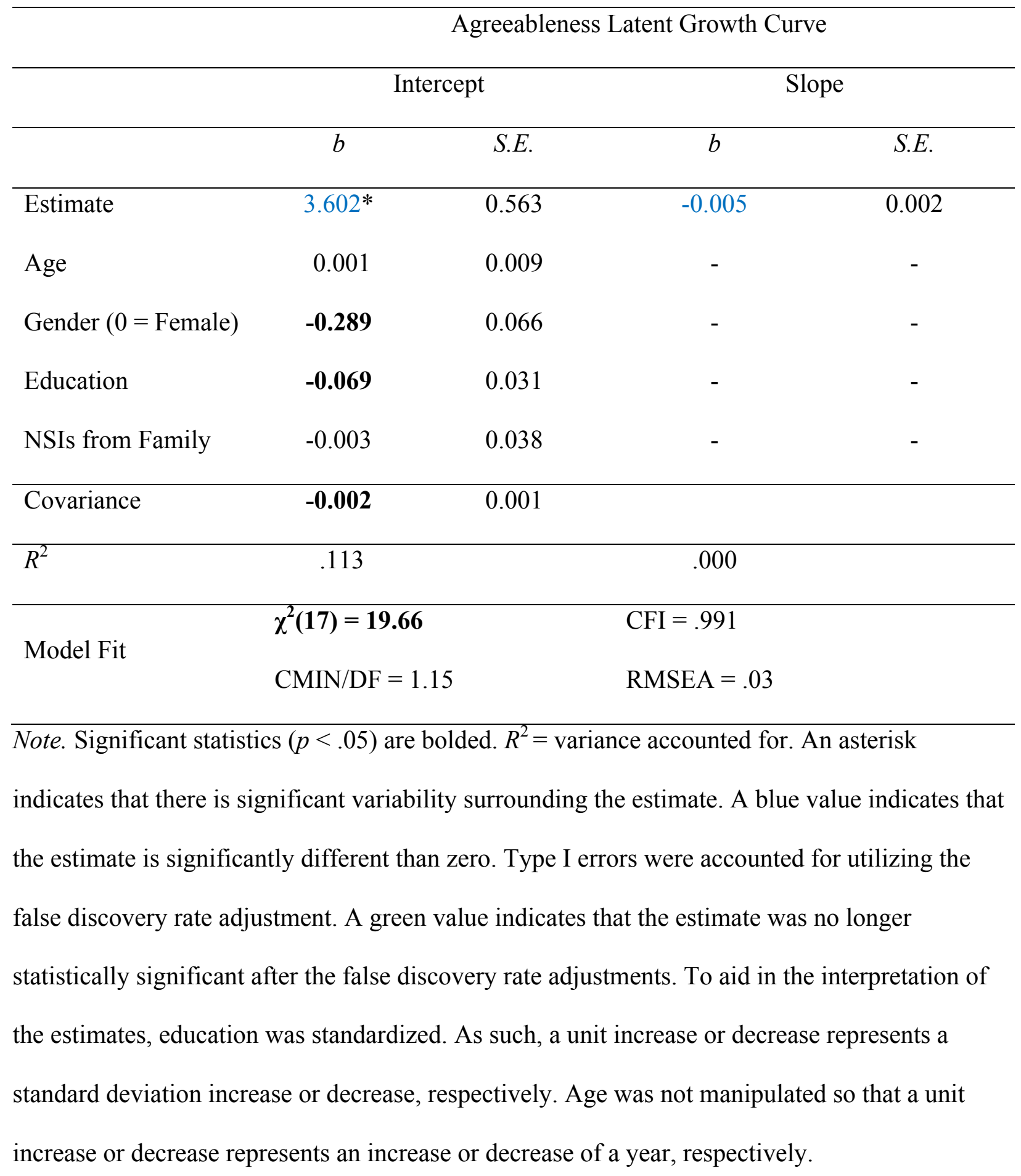


Table I29

Research Question 6 - Demographic Variables and NSIs from Family Predicting the Latent Growth Curve of Openness for Younger Adults

\section{Openness Latent Growth Curve}

\begin{tabular}{|c|c|c|c|c|}
\hline & \multicolumn{2}{|c|}{ Intercept } & \multicolumn{2}{|c|}{ Slope } \\
\hline & $b$ & S.E. & $b$ & S.E. \\
\hline Estimate & $3.273 *$ & 0.201 & $-0.038 *$ & 0.010 \\
\hline Age & -0.012 & 0.006 & 0.001 & 0.000 \\
\hline Gender $(0=$ Female $)$ & 0.127 & 0.046 & 0.000 & 0.002 \\
\hline Education & 0.071 & 0.024 & 0.001 & 0.001 \\
\hline NSIs from Family & -0.006 & 0.021 & -0.001 & 0.001 \\
\hline Covariance & 0.001 & 0.001 & & \\
\hline$R^{2}$ & .060 & & .121 & \\
\hline \multirow{2}{*}{ Model Fit } & $\chi^{2}(13)=44.28$ & & $\mathrm{CFI}=.956$ & \\
\hline & $\mathrm{CMIN} / \mathrm{DF}=3.40$ & & $\mathrm{RMSEA}=.07$ & \\
\hline
\end{tabular}

Note. Significant statistics $(p<.05)$ are bolded. $R^{2}=$ variance accounted for. An asterisk

indicates that there is significant variability surrounding the estimate. A blue value indicates that the estimate is significantly different than zero. Type I errors were accounted for utilizing the false discovery rate adjustment. A green value indicates that the estimate was no longer statistically significant after the false discovery rate adjustments. To aid in the interpretation of the estimates, education was standardized. As such, a unit increase or decrease represents a standard deviation increase or decrease, respectively. Age was not manipulated so that a unit increase or decrease represents an increase or decrease of a year, respectively. 
Table I30

Research Question 6 - Demographic Variables and NSIs from Family Predicting the Latent Growth Curve of Openness for Middle-Aged Adults

\section{Openness Latent Growth Curve}

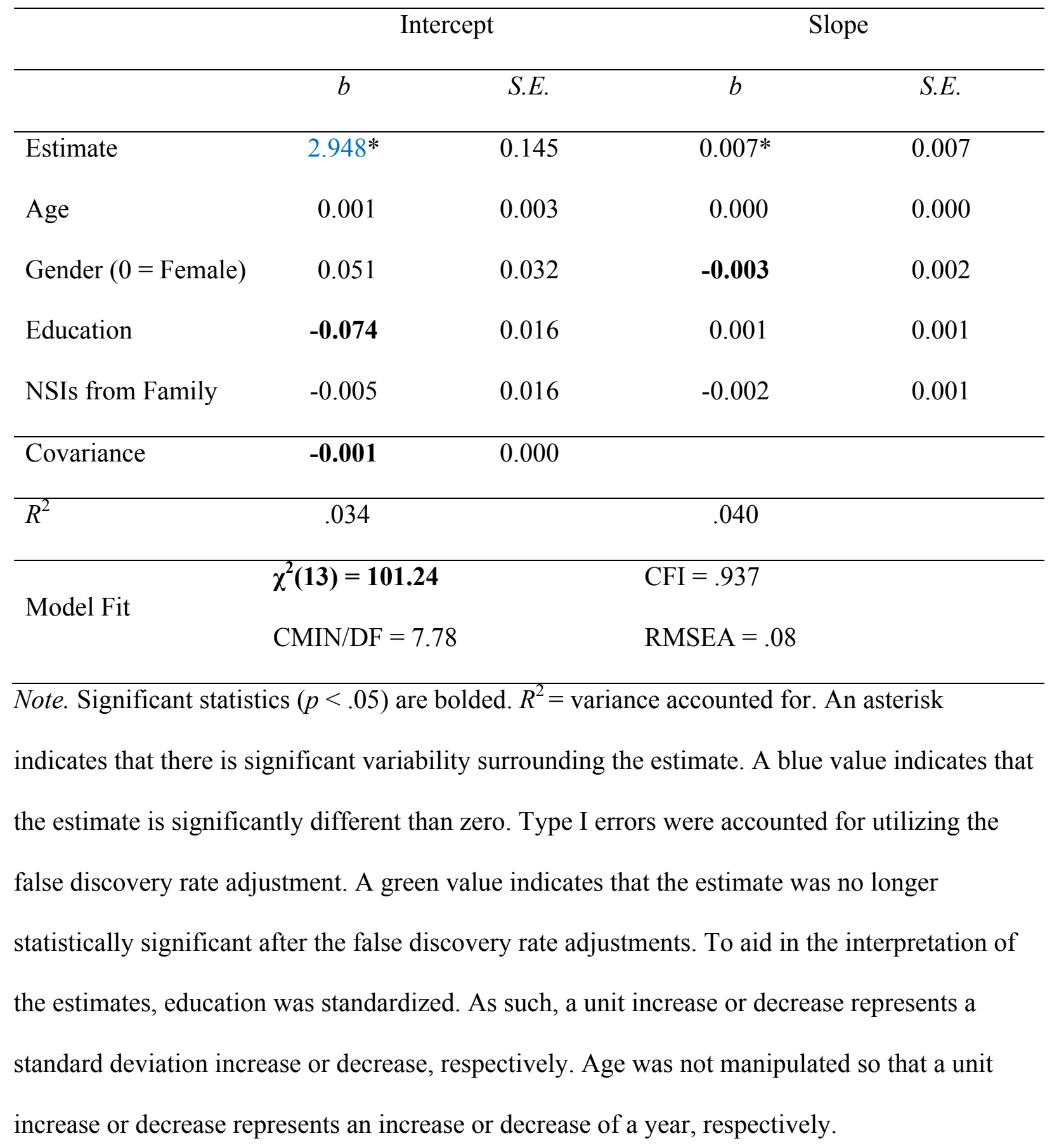


Table I31

Research Question 6 - Demographic Variables and NSIs from Family Predicting the Latent Growth Curve of Openness for Older Adults

\section{Openness Latent Growth Curve}

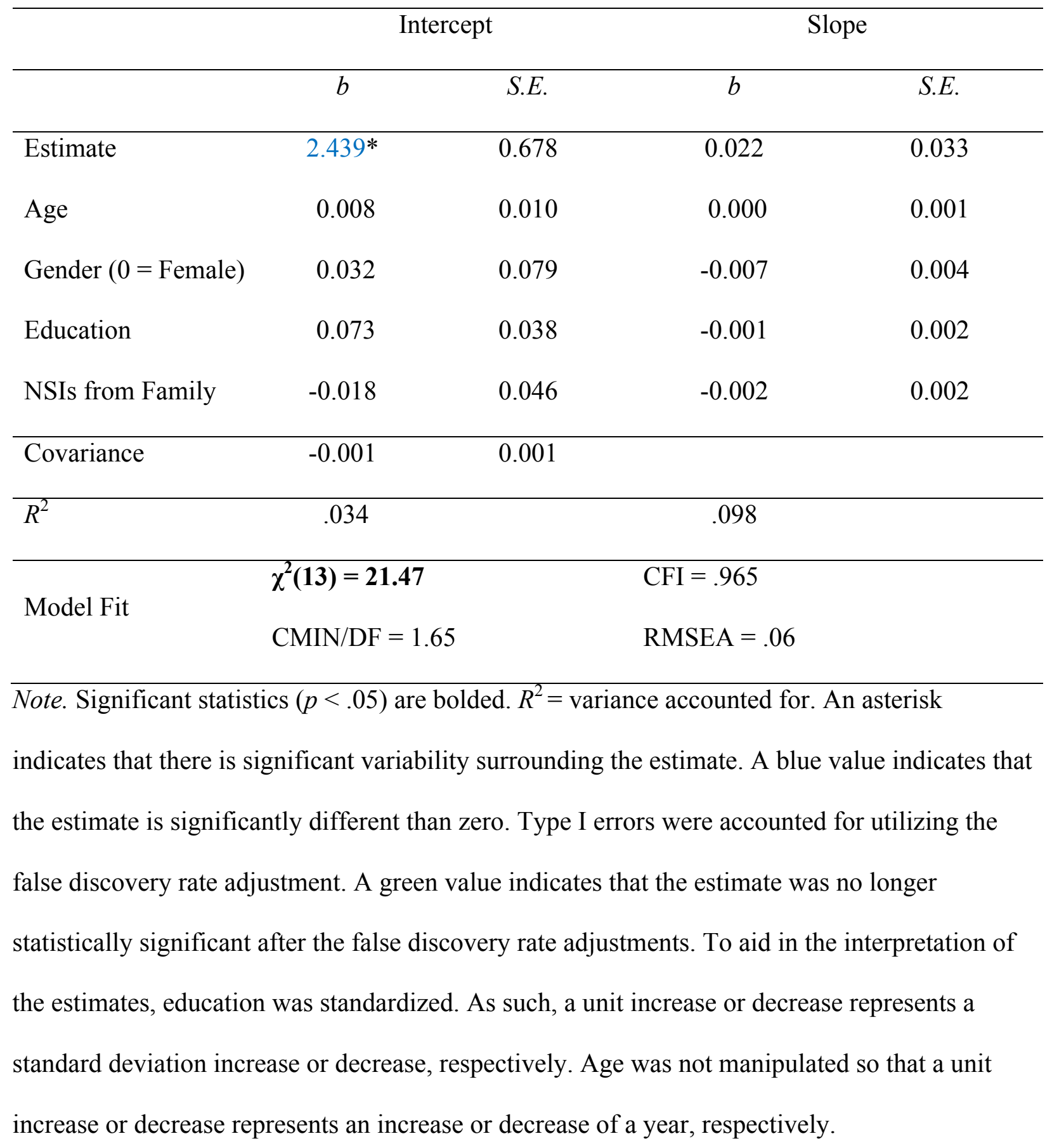


Table I32

Research Question 6 - Demographic Variables and NSIs from Family Predicting the Latent Growth Curve of Neuroticism for Younger Adults

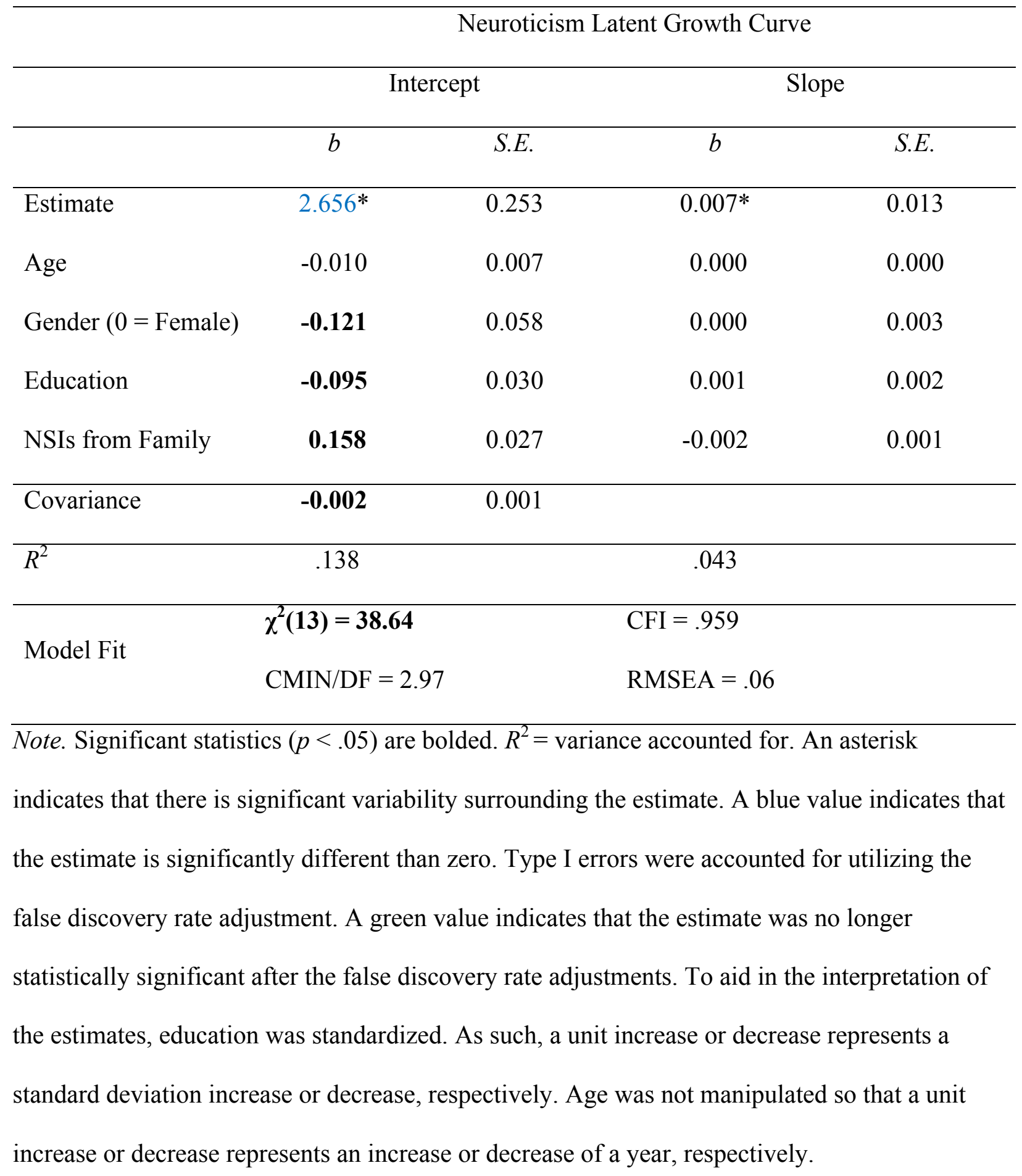


Table I33

Research Question 6 - Demographic Variables and NSIs from Family Predicting the Latent Growth Curve of Neuroticism for Middle-Aged Adults

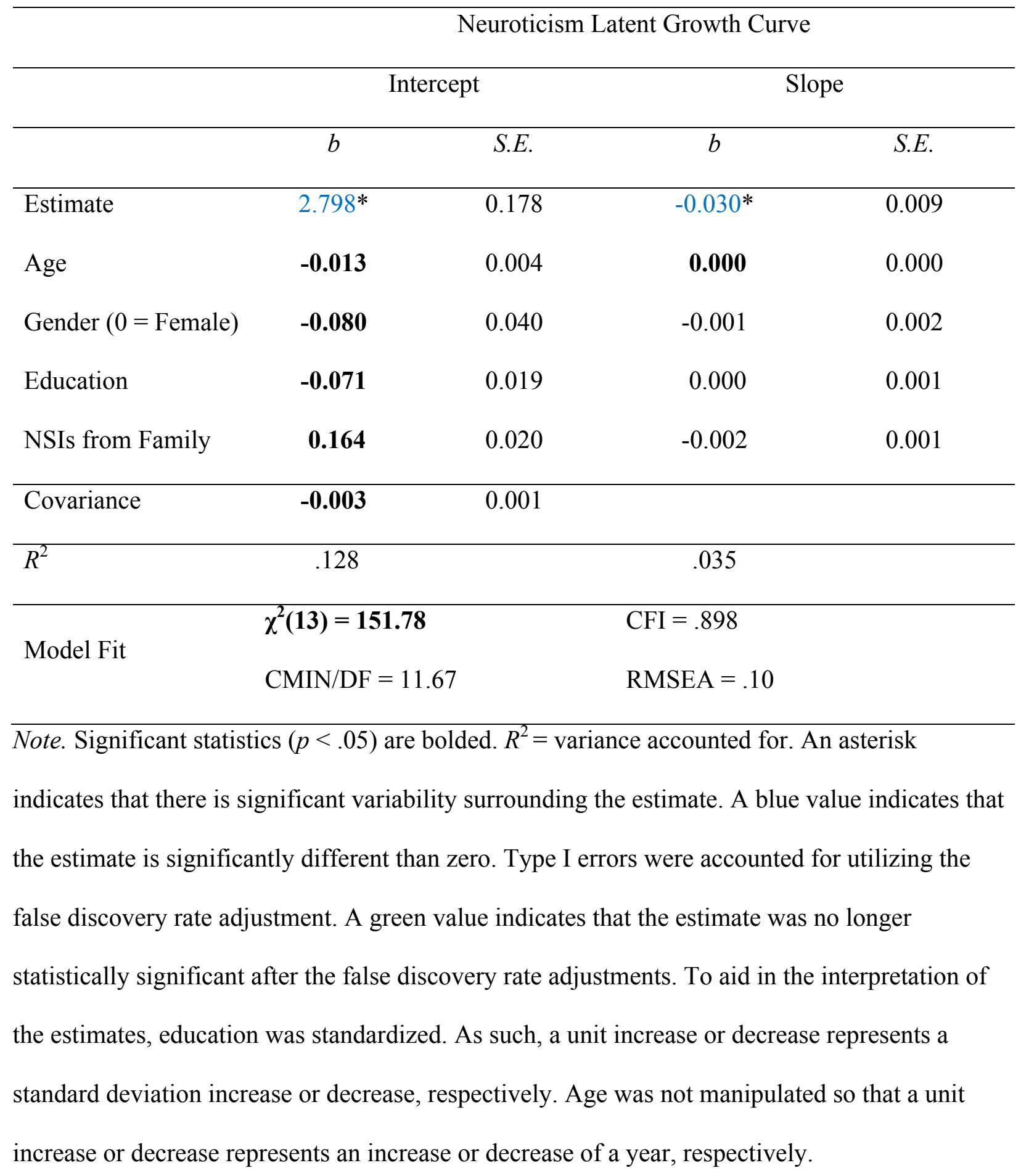


Table I34

Research Question 6 - Demographic Variables and NSIs from Family Predicting the Latent Growth Curve of Neuroticism for Older Adults

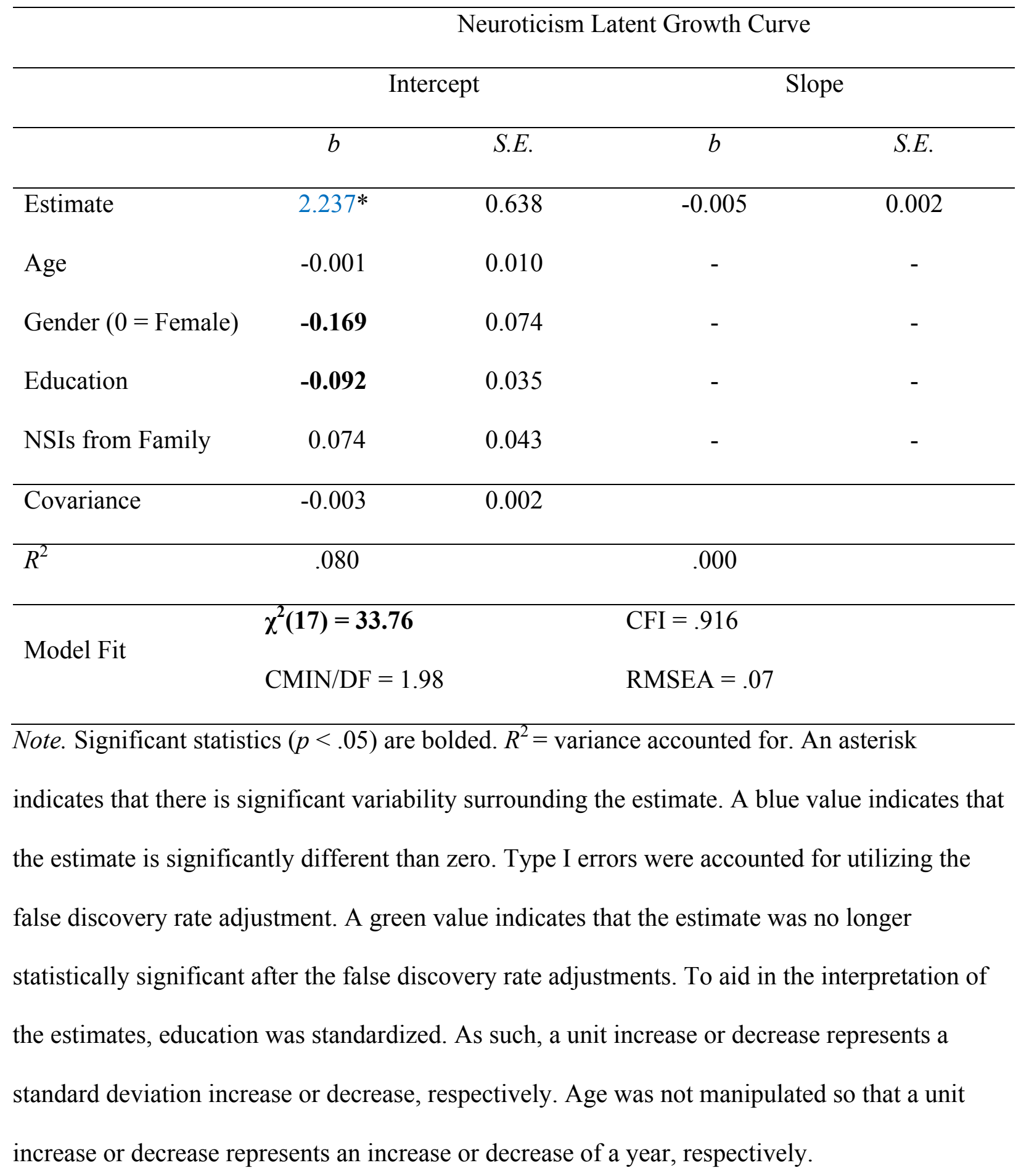


Table I35

Research Question 6 - Demographic Variables and NSIs from Family Predicting the Latent Growth Curve of Conscientiousness for Younger Adults

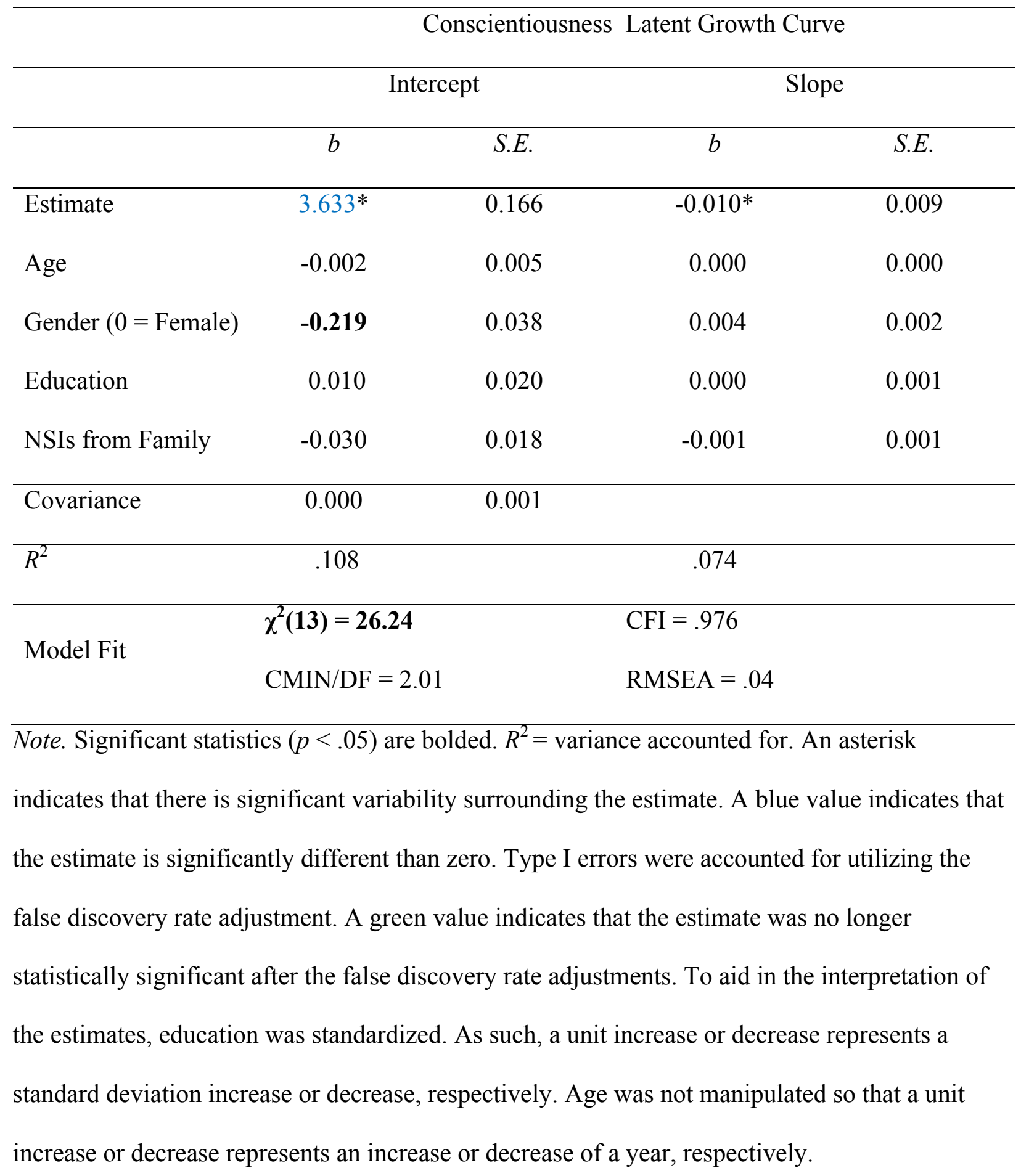


Table I36

Research Question 6 - Demographic Variables and NSIs from Family Predicting the Latent Growth Curve of Conscientiousness for Middle-Aged Adults

\begin{tabular}{|c|c|c|c|c|}
\hline & \multicolumn{4}{|c|}{ Conscientiousness Latent Growth Curve } \\
\hline & \multicolumn{2}{|c|}{ Intercept } & \multicolumn{2}{|c|}{ Slope } \\
\hline & $b$ & S.E. & $b$ & S.E. \\
\hline Estimate & $3.620^{*}$ & 0.102 & $0.001^{*}$ & 0.001 \\
\hline Age & -0.001 & 0.002 & - & - \\
\hline Gender $(0=$ Female $)$ & -0.102 & 0.023 & - & - \\
\hline Education & 0.044 & 0.011 & - & - \\
\hline NSIs from Family & -0.041 & 0.012 & - & - \\
\hline Covariance & 0.000 & 0.000 & & \\
\hline$R^{2}$ & .061 & & .000 & \\
\hline \multirow{2}{*}{ Model Fit } & $\chi^{2}(17)=126.53$ & & $\mathrm{CFI}=.897$ & \\
\hline & $\mathrm{CMIN} / \mathrm{DF}=7.44$ & & $\mathrm{RMSEA}=.08$ & \\
\hline
\end{tabular}

Note. Significant statistics $(p<.05)$ are bolded. $R^{2}=$ variance accounted for. An asterisk indicates that there is significant variability surrounding the estimate. A blue value indicates that the estimate is significantly different than zero. Type I errors were accounted for utilizing the false discovery rate adjustment. A green value indicates that the estimate was no longer statistically significant after the false discovery rate adjustments. To aid in the interpretation of the estimates, education was standardized. As such, a unit increase or decrease represents a standard deviation increase or decrease, respectively. Age was not manipulated so that a unit increase or decrease represents an increase or decrease of a year, respectively. 
Table I37

Research Question 6 - Demographic Variables and NSIs from Family Predicting the Latent Growth Curve of Conscientiousness for Older Adults

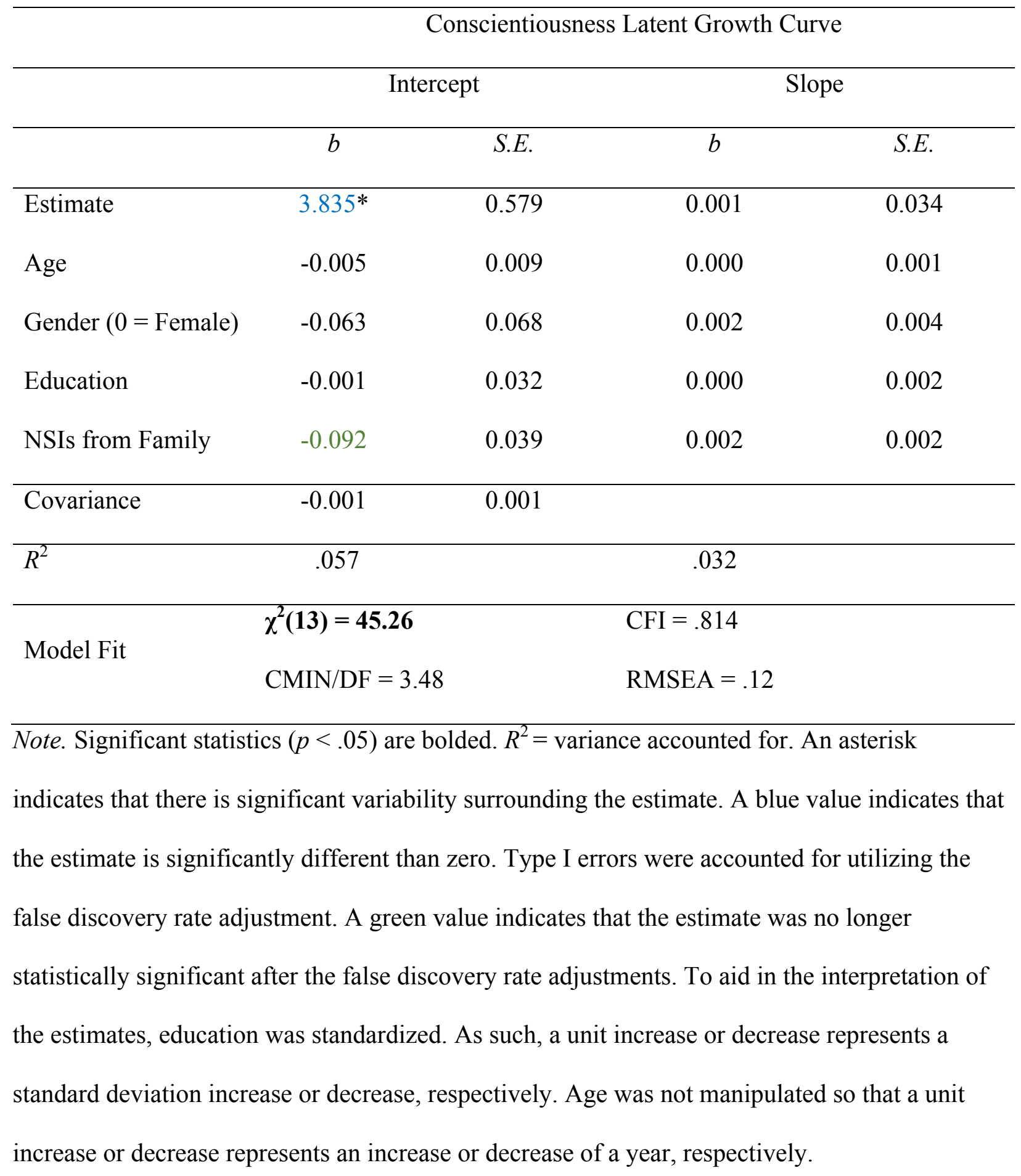


Table I38

Preliminary Models that Examine Significant Parameters that can be accounted for with the Cross-Domain Latent Growth Curves

\begin{tabular}{|c|c|c|c|c|c|c|c|}
\hline & \multirow[t]{2}{*}{ Model Fit } & \multicolumn{2}{|c|}{ Intercept } & \multicolumn{2}{|c|}{ Slope } & \multicolumn{2}{|c|}{ Covariance } \\
\hline & & $b$ & S.E. & $b$ & S.E. & $b$ & S.E. \\
\hline NSIs from Family and & $\chi^{2}(14)=29.87$ & $2.077^{*}$ & 0.014 & $-0.009 *$ & 0.001 & -0.002 & 0.001 \\
\hline \multirow[t]{3}{*}{ Agreeableness } & $\mathrm{CMIN} / \mathrm{DF}=2.13$ & $3.456^{*}$ & 0.012 & $-0.002 *$ & 0.001 & -0.001 & 0.000 \\
\hline & $\mathrm{CFI}=.994$ & & & & & & \\
\hline & RMSEA $=.02$ & & & & & & \\
\hline NSIs from Family and & $\chi^{2}(14)=30.49$ & $2.077 *$ & 0.014 & $-0.009 *$ & 0.001 & -0.002 & 0.001 \\
\hline \multirow[t]{3}{*}{ Openness } & $\mathrm{CMIN} / \mathrm{DF}=2.17$ & $2.977 *$ & 0.013 & $-0.006^{*}$ & 0.001 & 0.000 & 0.000 \\
\hline & $\mathrm{CFI}=.995$ & & & & & & \\
\hline & RMSEA $=.02$ & & & & & & \\
\hline NSIs from Family and & $\chi^{2}(14)=98.44$ & $2.077 *$ & 0.014 & $-0.009 *$ & 0.001 & -0.002 & 0.001 \\
\hline \multirow[t]{3}{*}{ Neuroticism } & $\mathrm{CMIN} / \mathrm{DF}=7.03$ & $2.163^{*}$ & 0.016 & $-0.008 *$ & 0.001 & -0.003 & 0.001 \\
\hline & $\mathrm{CFI}=.973$ & & & & & & \\
\hline & RMSEA $=.06$ & & & & & & \\
\hline
\end{tabular}




\begin{tabular}{|c|c|c|c|c|c|c|c|}
\hline NSIs from Family and & $\chi^{2}(14)=17.59$ & $2.077 *$ & 0.014 & $-0.009 *$ & 0.001 & -0.002 & 0.001 \\
\hline \multirow[t]{3}{*}{ Extraversion } & $\mathrm{CMIN} / \mathrm{DF}=1.25$ & $3.183 *$ & 0.014 & $-0.006^{*}$ & 0.001 & 0.000 & 0.000 \\
\hline & $\mathrm{CFI}=.999$ & & & & & & \\
\hline & $\mathrm{RMSEA}=.01$ & & & & & & \\
\hline NSIs from Family and & $\chi^{2}(14)=48.32$ & $2.077 *$ & 0.014 & $-0.009 *$ & 0.001 & -0.002 & 0.001 \\
\hline \multirow[t]{3}{*}{ Conscientiousness } & $\mathrm{CMIN} / \mathrm{DF}=3.45$ & $3.493^{*}$ & 0.010 & $0.001 *$ & 0.001 & 0.000 & 0.000 \\
\hline & $\mathrm{CFI}=.987$ & & & & & & \\
\hline & RMSEA $=.04$ & & & & & & \\
\hline
\end{tabular}

Note. Significant statistics $(p<.05)$ are bolded. An asterisk indicates that there is significant variability surrounding the estimate. A blue value indicates that the estimate is significantly different than zero. 
Table I39

Research Question 7 - Examination of the Association between the Latent Growth Curves of NSIs from Family and Agreeableness After Accounting for the Demographic Variables and Remaining Personality Traits

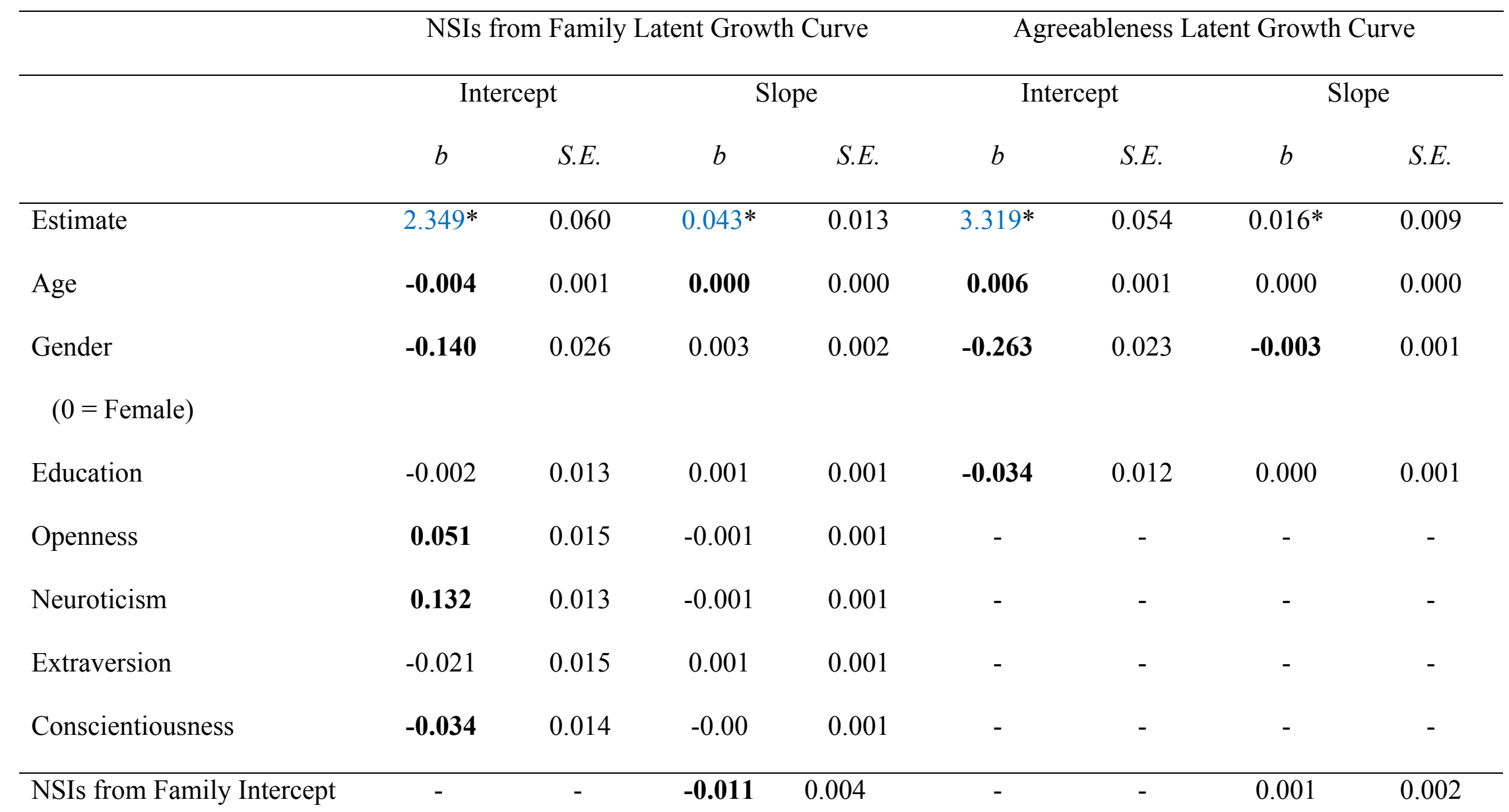




\begin{tabular}{|c|c|c|c|c|c|c|}
\hline Agreeableness Intercept & - & -0.003 & 0.002 & - & -0.005 & 0.002 \\
\hline$R^{2}$ & .163 & .166 & & .135 & .075 & \\
\hline
\end{tabular}

\begin{tabular}{ll}
\hline Model Fit & $\chi^{\mathbf{2}} \mathbf{4 9 )}=\mathbf{8 6 5 . 4 0}$ \\
$\mathrm{CMIN} / \mathrm{DF}=17.66$ \\
$\mathrm{CFI}=.827$ \\
$\mathrm{RMSEA}=.10$
\end{tabular}

Note. Significant statistics $(p<.05)$ are bolded. $R^{2}=$ variance accounted for. An asterisk indicates that there is significant variability surrounding the estimate. A blue value indicates that the estimate is significantly different than zero. Type I errors were accounted for utilizing the false discovery rate adjustment. A green value indicates that the estimate was no longer statistically significant after the false discovery rate adjustments. To aid in the interpretation of the estimates, education, agreeableness, neuroticism, openness, extraversion, and conscientiousness were standardized. As such, a unit increase or decrease in these variables represent a standard deviation increase or decrease, respectively. Age was not manipulated so that a unit increase or decrease represents an increase or decrease of a year, respectively. 
Table I40

Research Question 7 - Examination of the Bidirectional Association between the Latent Growth Curves of NSIs from Family and Openness After Accounting for the Demographic Variables and Remaining Personality Traits

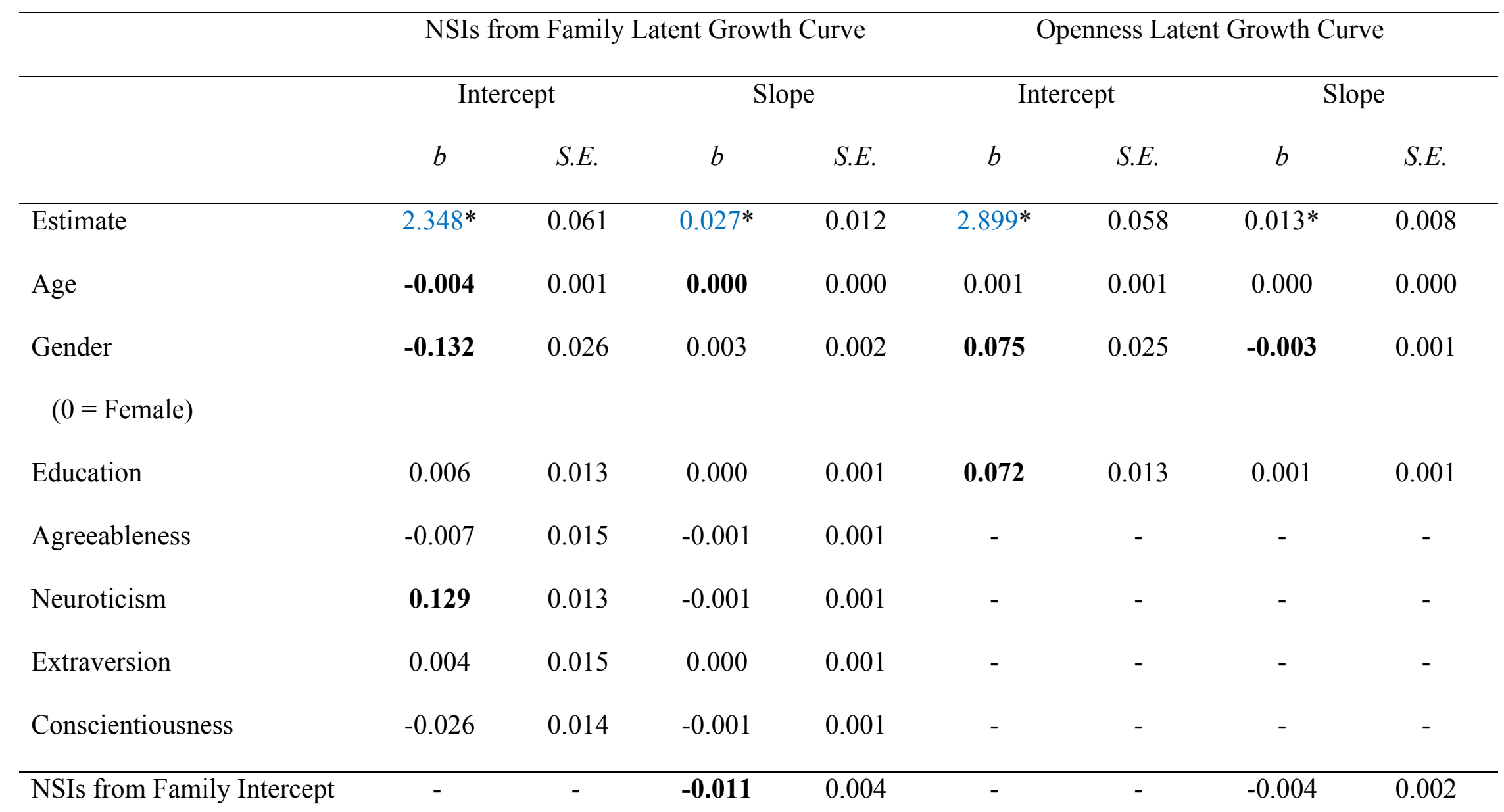




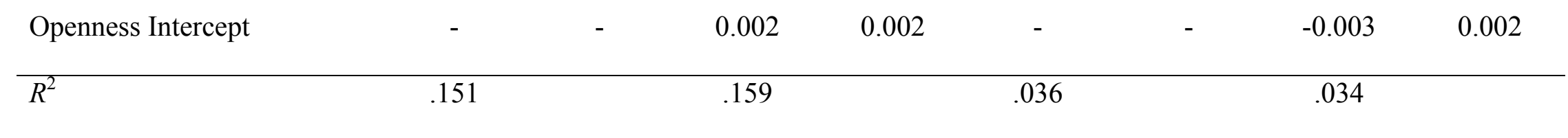

\begin{tabular}{ll}
\hline Model Fit & $\chi^{\mathbf{2}(49)=924.37}$ \\
& $\mathrm{CMIN} / \mathrm{DF}=18.86$ \\
$\mathrm{CFI}=.823$ \\
$\mathrm{RMSEA}=.10$
\end{tabular}

Note. Significant statistics $(p<.05)$ are bolded. $R^{2}=$ variance accounted for. An asterisk indicates that there is significant variability surrounding the estimate. A blue value indicates that the estimate is significantly different than zero. Type I errors were accounted for utilizing the false discovery rate adjustment. A green value indicates that the estimate was no longer statistically significant after the false discovery rate adjustments. To aid in the interpretation of the estimates, education, agreeableness, neuroticism, openness, extraversion, and conscientiousness were standardized. As such, a unit increase or decrease in these variables represent a standard deviation increase or decrease, respectively. Age was not manipulated so that a unit increase or decrease represents an increase or decrease of a year, respectively. 
Table I41

Research Question 7 - Examination of the Bidirectional Association between the Latent Growth Curves of NSIs from Family and Neuroticism After Accounting for the Demographic Variables and Remaining Personality Traits

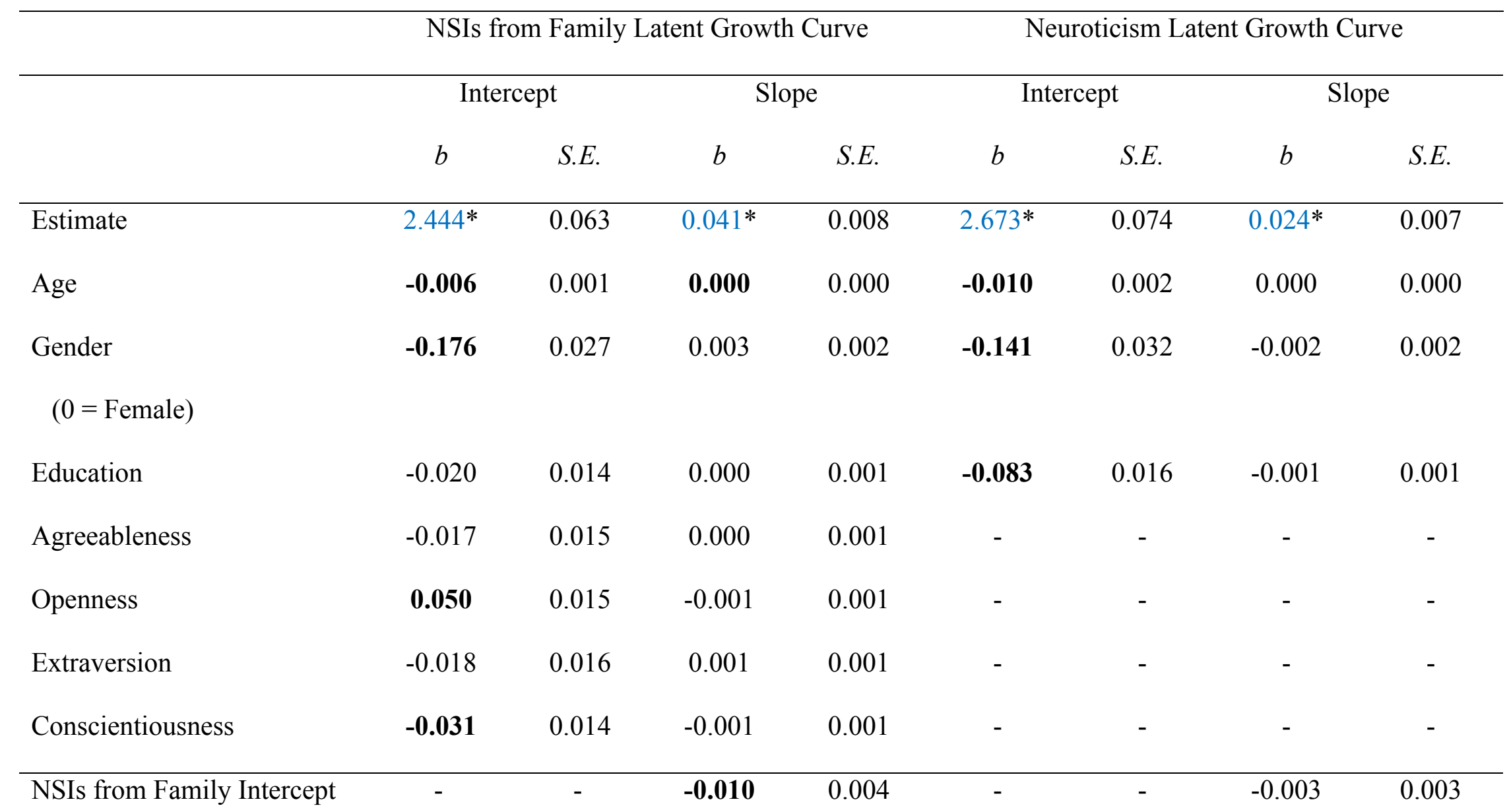




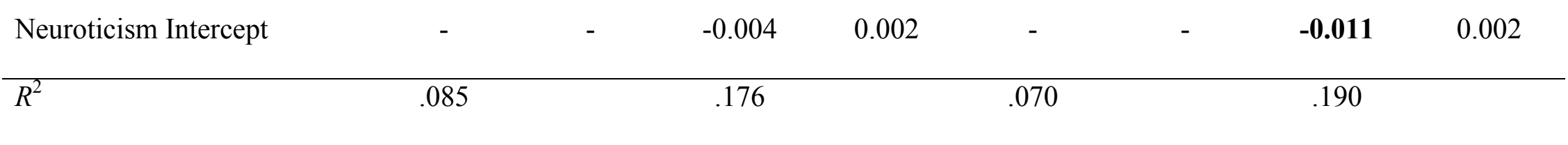

\begin{tabular}{ll}
\hline Model Fit & $\chi^{\mathbf{2}} \mathbf{4 9 )}=\mathbf{5 3 2 . 8 1}$ \\
$\mathrm{CMIN} / \mathrm{DF}=10.87$ \\
$\mathrm{CFI}=.898$ \\
$\mathrm{RMSEA}=.08$
\end{tabular}

Note. Significant statistics $(p<.05)$ are bolded. $R^{2}=$ variance accounted for. An asterisk indicates that there is significant variability surrounding the estimate. A blue value indicates that the estimate is significantly different than zero. Type I errors were accounted for utilizing the false discovery rate adjustment. A green value indicates that the estimate was no longer statistically significant after the false discovery rate adjustments. To aid in the interpretation of the estimates, education, agreeableness, neuroticism, openness, extraversion, and conscientiousness were standardized. As such, a unit increase or decrease in these variables represent a standard deviation increase or decrease, respectively. Age was not manipulated so that a unit increase or decrease represents an increase or decrease of a year, respectively. 
Table I42

Research Question 7 - Examination of the Bidirectional Association between the Latent Growth Curves of NSIs from Family and Extraversion After Accounting for the Demographic Variables and Remaining Personality Traits

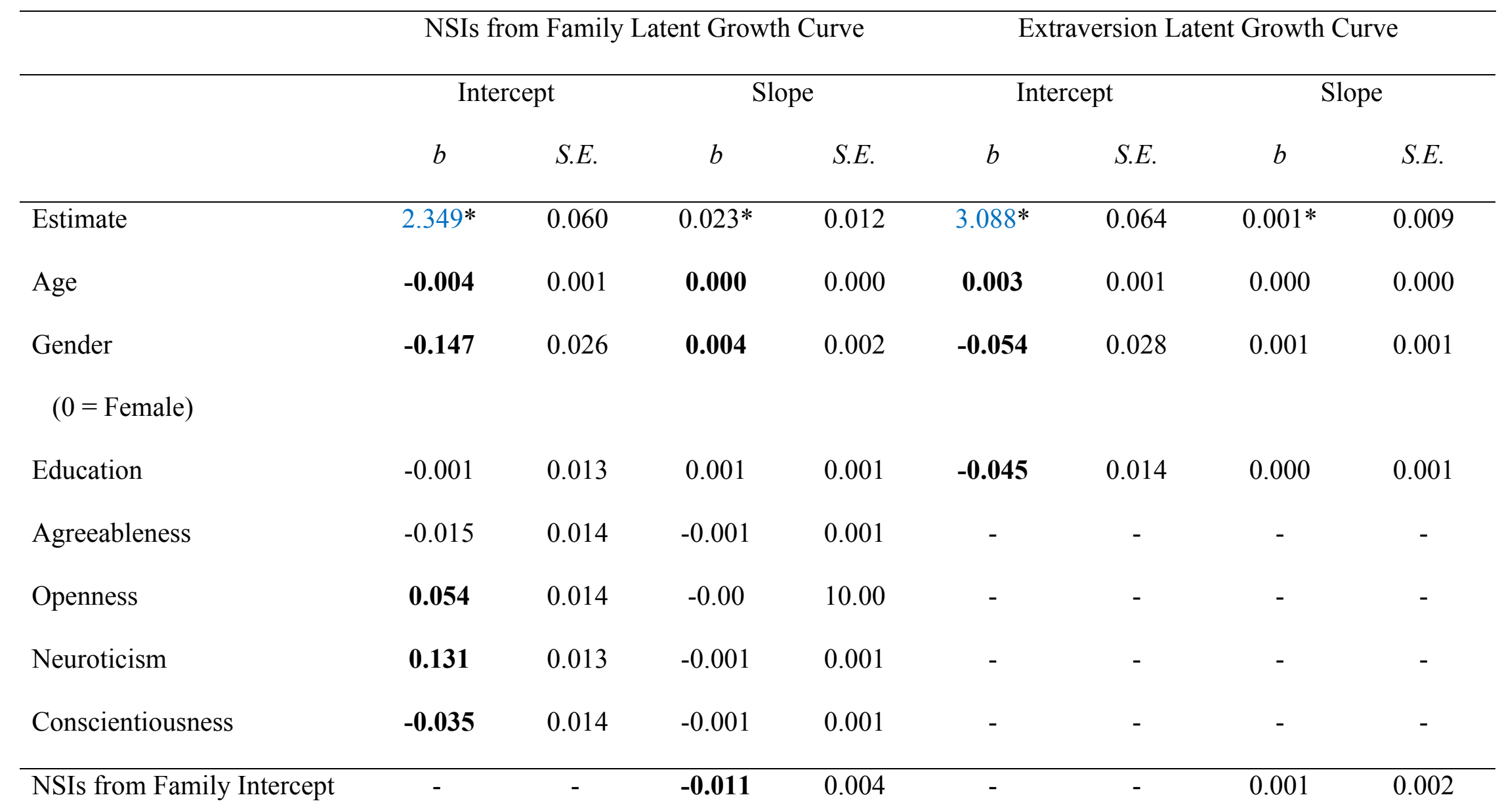




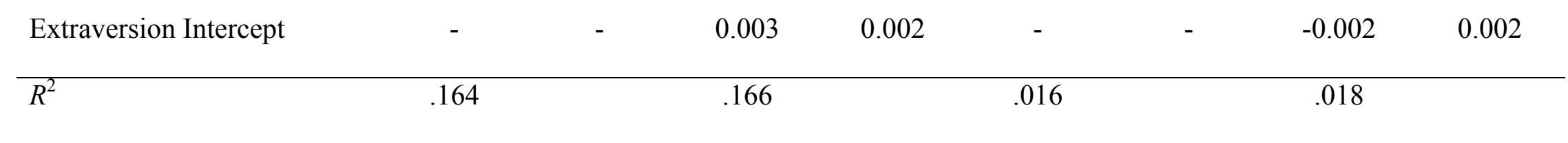

\begin{tabular}{ll}
\hline Model Fit & $\chi^{2}(\mathbf{4 9})=\mathbf{1 1 5 6 . 9 3}$ \\
$\mathrm{CMIN} / \mathrm{DF}=23.61$ \\
$\mathrm{CFI}=.777$ \\
$\mathrm{RMSEA}=.12$
\end{tabular}

Note. Significant statistics $(p<.05)$ are bolded. $R^{2}=$ variance accounted for. An asterisk indicates that there is significant variability surrounding the estimate. A blue value indicates that the estimate is significantly different than zero. Type I errors were accounted for utilizing the false discovery rate adjustment. A green value indicates that the estimate was no longer statistically significant after the false discovery rate adjustments. To aid in the interpretation of the estimates, education, agreeableness, neuroticism, openness, extraversion, and conscientiousness were standardized. As such, a unit increase or decrease in these variables represent a standard deviation increase or decrease, respectively. Age was not manipulated so that a unit increase or decrease represents an increase or decrease of a year, respectively. 
Table I43

Research Question 7 - Examination of the Bidirectional Association between the Latent Growth Curves of NSIs from Family and Conscientiousness After Accounting for the Demographic Variables and Remaining Personality Traits

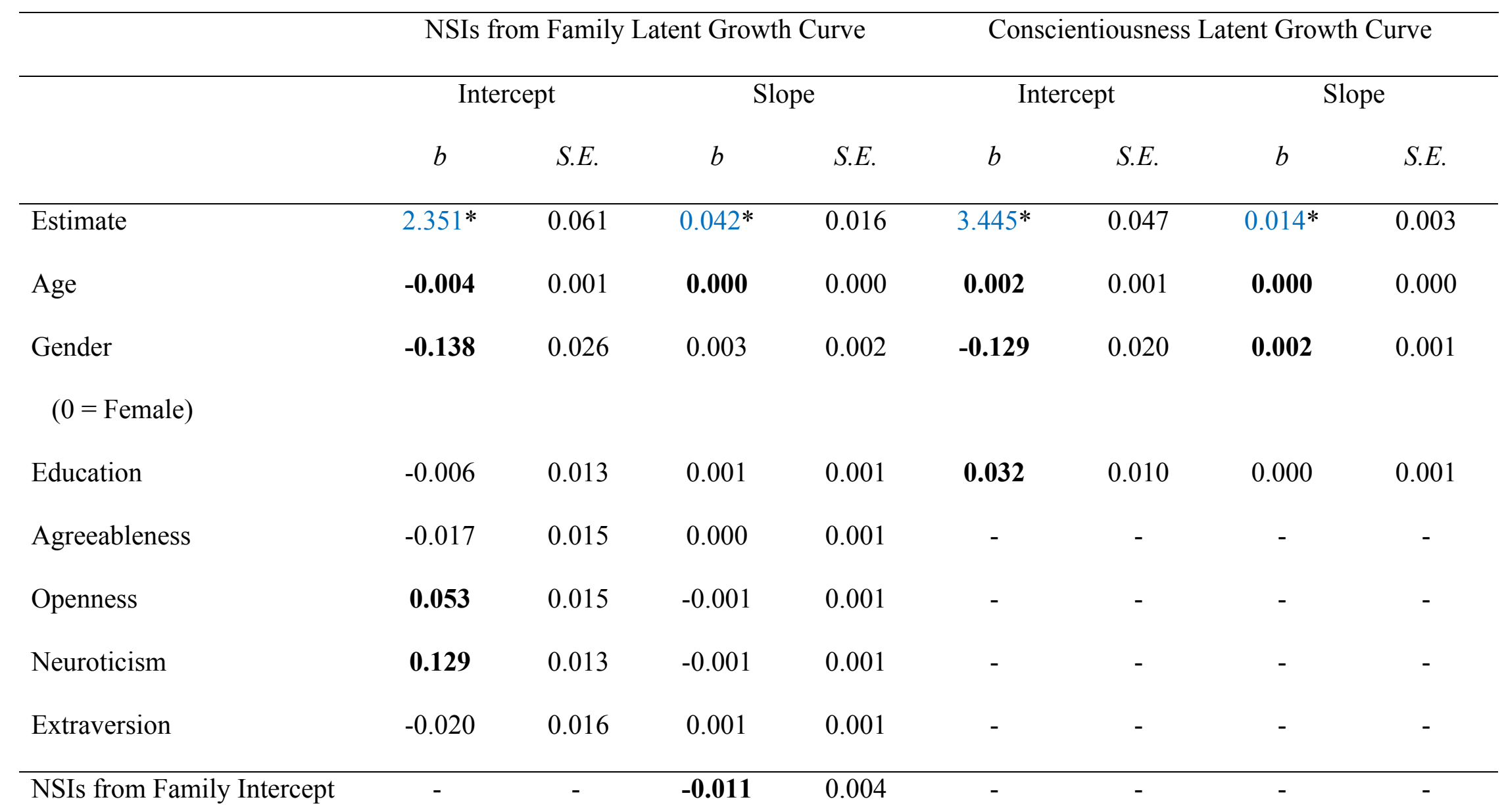




\begin{tabular}{|c|c|c|c|c|c|c|}
\hline Conscientiousness Intercept & - & -0.002 & 0.003 & - & - & - \\
\hline$R^{2}$ & .147 & .164 & & .053 & .120 & \\
\hline
\end{tabular}

\begin{tabular}{|c|c|}
\hline Model Fit & $\chi^{2}(50)=642.58$ \\
\hline & $\mathrm{CMIN} / \mathrm{DF}=12.85$ \\
\hline & $\mathrm{CFI}=.864$ \\
\hline & RMSEA $=.08$ \\
\hline
\end{tabular}

Note. Significant statistics $(p<.05)$ are bolded. $R^{2}=$ variance accounted for. An asterisk indicates that there is significant variability surrounding the estimate. A blue value indicates that the estimate is significantly different than zero. Type I errors were accounted for utilizing the false discovery rate adjustment. A green value indicates that the estimate was no longer statistically significant after the false discovery rate adjustments. To aid in the interpretation of the estimates, education, agreeableness, neuroticism, openness, extraversion, and conscientiousness were standardized. As such, a unit increase or decrease in these variables represent a standard deviation increase or decrease, respectively. Age was not manipulated so that a unit increase or decrease represents an increase or decrease of a year, respectively. 
Table I44

The Unconstrained Models Compared to the Constrained Models for the Multigroup Cross-Domain Latent Growth Curves

$\chi^{2}$

\section{CFI}

NSIs from Family and Agreeableness

Unconstrained Model

Constrained Model

NSIs from Family and Openness

Unconstrained Model
Constrained Model

NSIs from Family and Neuroticism

Unconstrained Model
Constrained Model

NSIs from Family and Extraversion

Unconstrained Model
Constrained Model

.968

$$
\begin{aligned}
& \chi^{2}(46)=84.83 \\
& \chi^{2}(58)=197.29
\end{aligned}
$$$$
\chi^{2}(46)=85.75
$$$$
\chi^{2}(58)=196.67
$$

.956$$
\chi^{2}(12)=110.92
$$

$$
\chi^{2}(12)=96.61
$$


NSIs from Family and Conscientiousness

$\begin{array}{llll}\text { Unconstrained Model } & \chi^{\mathbf{2}}(\mathbf{4 6})=\mathbf{9 1 . 9 3} & .982 & \\ \text { Constrained Model } & \chi^{2}(\mathbf{5 8})=\mathbf{2 2 0 . 5 6} & .937 & \chi^{2}(\mathbf{1 2})=\mathbf{1 2 8 . 6 3}\end{array}$

Note. Significant statistics $(p<.05)$ are bolded. The unconstrained model is where the parameters were freely estimated across the three age groups. The constrained model is where the parameters were constrained to be equal across the three age groups. A change of .01 or greater for the CFI difference statistic was used to determine significance (Little, 2013). Both the chi-square and the CFI difference statistics were computed because the chi-square statistic is commonly used, however, it is sensitive to large sample sizes. The CFI is more robust to larger sample sizes (Little, 2013). 
Table I45

Research Question 8 - Multigroup Cross-Domain Latent Growth Curves

\begin{tabular}{|c|c|c|c|c|c|c|c|c|c|c|c|c|c|c|}
\hline & & & \multicolumn{6}{|c|}{ NSIs from Family } & \multicolumn{6}{|c|}{ Personality Trait } \\
\hline & \multicolumn{2}{|l|}{ Model Fit } & \multicolumn{2}{|c|}{ Intercept } & \multicolumn{2}{|c|}{ Slope } & \multicolumn{2}{|c|}{ Covariance } & \multicolumn{2}{|c|}{ Intercept } & \multicolumn{2}{|c|}{ Slope } & \multicolumn{2}{|c|}{ Covariance } \\
\hline NSIs and & $\chi^{2}(46)=84.83$ & Younger Adults & $2.103 *^{b}$ & 0.028 & $-0.001 * a, b$ & 0.002 & -0.003 & 0.001 & $3.392 * \mathrm{a}, \mathrm{b}$ & 0.023 & $-0.003 *$ & 0.001 & -0.001 & 0.001 \\
\hline \multirow[t]{3}{*}{ Agree } & $\mathrm{CMIN} / \mathrm{DF}=1.84$ & Middle-Aged Adults & $2.097 *^{\mathrm{c}}$ & 0.017 & $-0.012 *$ & 0.001 & -0.002 & 0.001 & $3.477 *$ & 0.015 & $-0.001^{\mathrm{c}}$ & 0.001 & -0.001 & 0.000 \\
\hline & $\mathrm{CFI}=.986$ & Older Adults & $1.906^{*}$ & 0.034 & -0.011 & 0.002 & 0.000 & 0.001 & $3.513 *$ & 0.040 & -0.005 & 0.002 & -0.002 & 0.001 \\
\hline & $\mathrm{RMSEA}=.02$ & & & & & & & & & & & & & \\
\hline NSIs and & $\chi^{2}(46)=85.75$ & Younger Adults & $2.103 *^{\mathrm{b}}$ & 0.028 & $-0.001 * a, b$ & 0.002 & -0.003 & 0.001 & $2.937 *^{\mathrm{a}}$ & 0.024 & $-0.007 * \mathrm{a}$ & 0.001 & 0.000 & 0.001 \\
\hline \multirow[t]{2}{*}{ Open } & $\mathrm{CMIN} / \mathrm{DF}=1.86$ & Middle-Aged Adults & $2.097 *^{\mathrm{c}}$ & 0.017 & $-0.012 *$ & 0.001 & -0.002 & 0.001 & $3.002 *$ & 0.016 & $-0.005 * \mathrm{c}$ & 0.001 & -0.001 & 0.000 \\
\hline & RMSEA $=.02$ & & & & & & & & & & & & & \\
\hline NSIs and & $\chi^{2}(46)=152.13$ & Younger Adults & $2.103 * \mathrm{~b}$ & 0.028 & $-0.001 * a, b$ & 0.002 & -0.003 & 0.001 & $2.274^{* a, b}$ & 0.031 & $-0.009 *$ & 0.002 & -0.003 & 0.001 \\
\hline \multirow[t]{2}{*}{ Neuro } & $\mathrm{CMIN} / \mathrm{DF}=3.30$ & Middle-Aged Adults & $2.097 * \mathrm{c}$ & 0.017 & $-0.012 *$ & 0.001 & -0.002 & 0.001 & $2.131 *$ & 0.021 & $-0.009 *$ & 0.001 & -0.003 & 0.001 \\
\hline & $\mathrm{CFI}=.965$ & Older Adults & $1.906^{*}$ & 0.034 & -0.011 & 0.002 & 0.000 & 0.001 & $2.037 *$ & 0.045 & -0.005 & 0.002 & -0.002 & 0.002 \\
\hline
\end{tabular}




\begin{tabular}{|c|c|c|c|c|c|c|c|c|c|c|c|c|c|c|}
\hline NSIs and & $\chi^{2}(46)=49.30$ & Younger Adults & $2.103 * \mathrm{~b}$ & 0.028 & $-0.001 * a, b$ & 0.002 & -0.003 & 0.001 & $3.167 * \mathrm{a}, \mathrm{b}$ & 0.026 & $-0.008 *$ & 0.001 & -0.001 & 0.001 \\
\hline \multirow[t]{3}{*}{ Extra } & $\mathrm{CMIN} / \mathrm{DF}=1.07$ & Middle-Aged Adults & $2.097 *^{\mathrm{c}}$ & 0.017 & $-0.012 *$ & 0.001 & -0.002 & 0.001 & $3.180^{*}$ & 0.018 & $-0.004^{* c}$ & 0.001 & 0.000 & 0.001 \\
\hline & $\mathrm{CFI}=.999$ & Older Adults & $1.906^{*}$ & 0.034 & -0.011 & 0.002 & 0.000 & 0.001 & $3.243 *$ & 0.043 & -0.014 & 0.002 & 0.001 & 0.001 \\
\hline & $\mathrm{RMSEA}=.00$ & & & & & & & & & & & & & \\
\hline NSIs and & $\chi^{2}(46)=91.93$ & Younger Adults & $2.103 * b$ & 0.028 & $-0.001 * \mathrm{a}, \mathrm{b}$ & 0.002 & -0.003 & 0.001 & $3.459 *^{\mathrm{a}}$ & 0.020 & $0.003^{\mathrm{a}, \mathrm{b}}$ & 0.001 & 0.000 & 0.000 \\
\hline \multirow[t]{2}{*}{ Consc } & $\mathrm{CMIN} / \mathrm{DF}=1.99$ & Middle-Aged Adults & $2.097 *^{\mathrm{c}}$ & 0.017 & $-0.012 *$ & 0.001 & -0.002 & 0.001 & $3.510 *$ & 0.013 & $0.001^{\mathrm{c}}$ & 0.001 & 0.000 & 0.000 \\
\hline & $\mathrm{CFI}=.982$ & Older Adults & $1.906^{*}$ & 0.034 & -0.011 & 0.002 & 0.000 & 0.001 & $3.495^{*}$ & 0.034 & $-0.008 *$ & 0.002 & -0.002 & 0.001 \\
\hline
\end{tabular}

Note. Significant statistics $(p<.05)$ are bolded. Agree $=$ agreeableness. Open $=$ openness. Neuro $=$ neuroticism. Extra $=$ extraversion.

Consc $=$ conscientiousness. An asterisk indicates that there is significant variability surrounding the estimate. A blue value indicates that the estimate is significantly different than zero. ${ }^{a}$ indicates that the estimate is significantly different between younger and middleaged adults. ${ }^{b}$ indicates that the estimate is significantly different between younger and older adults. ${ }^{c}$ indicates that the estimate is significantly different between middle-aged and older adults. 
Table I46

Research Question 8 - Examination of the Bidirectional Association between the Latent Growth Curves of NSIs from Family and Agreeableness After Accounting for the Demographic Variables and Remaining Personality Traits for Younger Adults

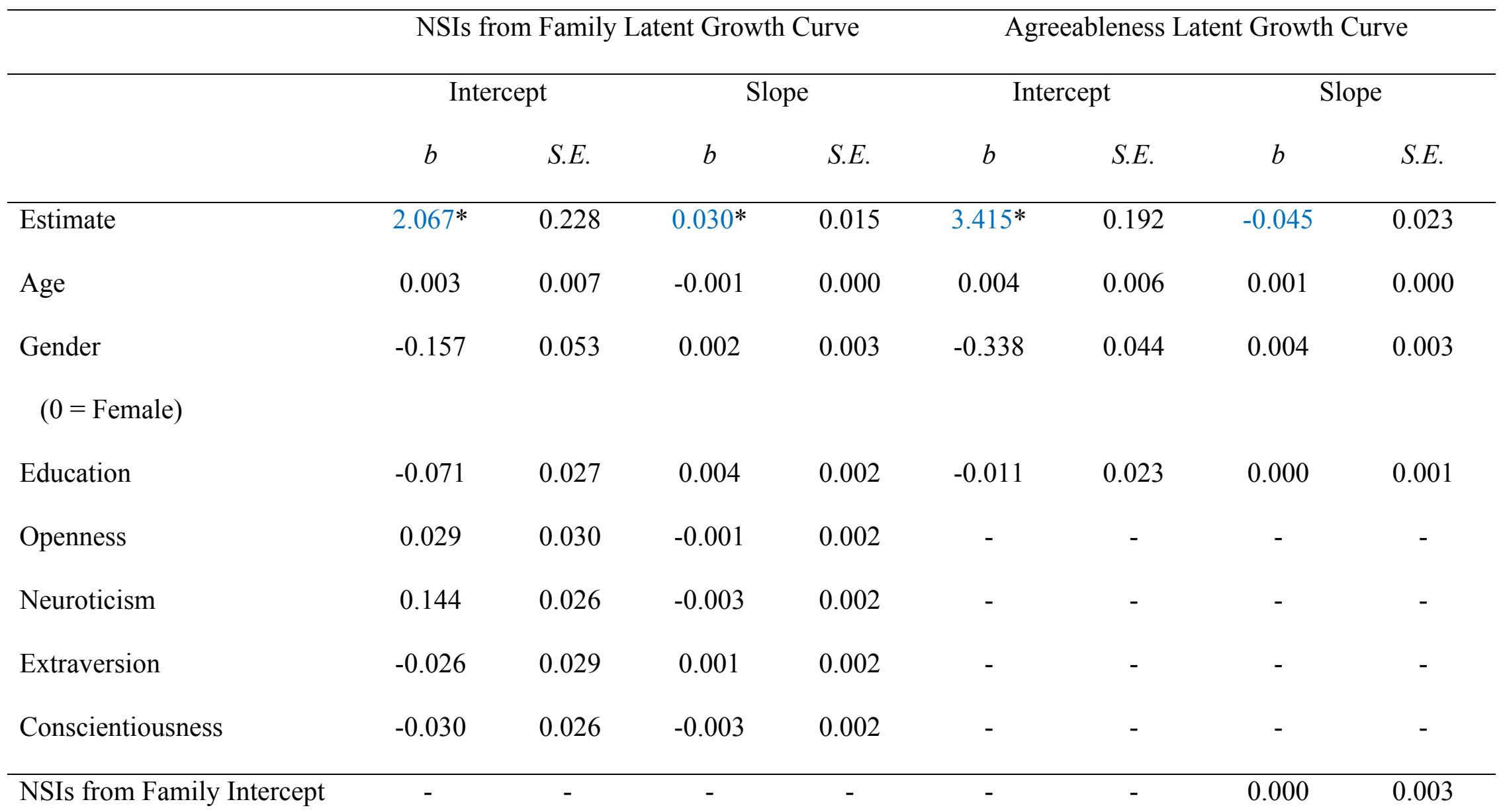




\begin{tabular}{|c|c|c|c|c|c|}
\hline Agreeableness Intercept & - & - & - & 0.006 & 0.006 \\
\hline$R^{2}$ & .168 & .134 & .178 & .786 & \\
\hline
\end{tabular}

$\chi^{2} \mathbf{( 5 0 )}=\mathbf{2 7 2 . 3 1}$
Model Fit
$\mathrm{CFI}=.828$
$\mathrm{RMSEA}=.10$

Note. Significant statistics $(p<.05)$ are bolded. $R^{2}=$ variance accounted for. An asterisk indicates that there is significant variability surrounding the estimate. A blue value indicates that the estimate is significantly different than zero. Type I errors were accounted for utilizing the false discovery rate adjustment. A green value indicates that the estimate was no longer statistically significant after the false discovery rate adjustments. To aid in the interpretation of the estimates, education, agreeableness, neuroticism, openness, extraversion, and conscientiousness were standardized. As such, a unit increase or decrease in these variables represent a standard deviation increase or decrease, respectively. Age was not manipulated so that a unit increase or decrease represents an increase or decrease of a year, respectively. 
Table I47

Research Question 8 - Examination of the Bidirectional Association between the Latent Growth Curves of NSIs from Family and Agreeableness After Accounting for the Demographic Variables and Remaining Personality Traits for Middle-Aged Adults

\begin{tabular}{|c|c|c|c|c|c|c|c|c|}
\hline & \multicolumn{4}{|c|}{ NSIs from Family Latent Growth Curve } & \multicolumn{4}{|c|}{ Agreeableness Latent Growth Curve } \\
\hline & \multicolumn{2}{|c|}{ Intercept } & \multicolumn{2}{|c|}{ Slope } & \multicolumn{2}{|c|}{ Intercept } & \multicolumn{2}{|c|}{ Slope } \\
\hline & $b$ & S.E. & $b$ & S.E. & $b$ & S.E. & $b$ & S.E. \\
\hline Estimate & $2.879 *$ & 0.147 & $0.021 *$ & 0.025 & $3.379 *$ & 0.114 & -0.001 & 0.001 \\
\hline Age & -0.014 & 0.003 & 0.000 & 0.000 & 0.005 & 0.002 & - & - \\
\hline $\begin{array}{l}\text { Gender } \\
\qquad(0=\text { Female })\end{array}$ & -0.160 & 0.033 & 0.004 & 0.003 & -0.255 & 0.025 & - & - \\
\hline Education & 0.026 & 0.016 & 0.000 & 0.001 & $-\mathbf{0 . 0 3 9}$ & 0.013 & - & - \\
\hline Openness & 0.057 & 0.019 & 0.000 & 0.001 & - & - & - & - \\
\hline Neuroticism & 0.131 & 0.017 & -0.001 & 0.001 & - & - & - & - \\
\hline Extraversion & -0.009 & 0.019 & 0.000 & 0.001 & - & - & - & - \\
\hline Conscientiousness & -0.039 & 0.018 & 0.001 & 0.001 & - & - & - & - \\
\hline NSIs from Family Intercept & - & - & -0.008 & 0.006 & - & - & - & - \\
\hline
\end{tabular}




\begin{tabular}{|c|c|c|c|c|c|}
\hline Agreeableness Intercept & - & -0.004 & 0.004 & - & - \\
\hline$R^{2}$ & .218 & .088 & & .135 & .000 \\
\hline
\end{tabular}

\begin{tabular}{|c|c|}
\hline Model Fit & $\chi^{2}(54)=567.38$ \\
\hline & $\mathrm{CMIN} / \mathrm{DF}=10.50$ \\
\hline & $\mathrm{CFI}=.813$ \\
\hline & RMSEA $=.10$ \\
\hline
\end{tabular}

Note. Significant statistics $(p<.05)$ are bolded. $R^{2}=$ variance accounted for. An asterisk indicates that there is significant variability surrounding the estimate. A blue value indicates that the estimate is significantly different than zero. Type I errors were accounted for utilizing the false discovery rate adjustment. A green value indicates that the estimate was no longer statistically significant after the false discovery rate adjustments. To aid in the interpretation of the estimates, education, agreeableness, neuroticism, openness, extraversion, and conscientiousness were standardized. As such, a unit increase or decrease in these variables represent a standard deviation increase or decrease, respectively. Age was not manipulated so that a unit increase or decrease represents an increase or decrease of a year, respectively. 
Table I48

Research Question 8 - Examination of the Bidirectional Association between the Latent Growth Curves of NSIs from Family and Agreeableness After Accounting for the Demographic Variables and Remaining Personality Traits for Older Adults

\begin{tabular}{|c|c|c|c|c|c|c|c|c|}
\hline & \multicolumn{4}{|c|}{ NSIs from Family Latent Growth Curve } & \multicolumn{4}{|c|}{ Agreeableness Latent Growth Curve } \\
\hline & \multicolumn{2}{|c|}{ Intercept } & \multicolumn{2}{|c|}{ Slope } & \multicolumn{2}{|c|}{ Intercept } & \multicolumn{2}{|c|}{ Slope } \\
\hline & $b$ & S.E. & $b$ & S.E. & $b$ & S.E. & $b$ & S.E. \\
\hline Estimate & $3.304 *$ & 0.519 & 0.025 & 0.027 & $3.651^{*}$ & 0.618 & 0.024 & 0.020 \\
\hline Age & -0.022 & 0.008 & - & - & 0.000 & 0.010 & - & - \\
\hline $\begin{array}{l}\text { Gender } \\
\qquad(0=\text { Female })\end{array}$ & 0.033 & 0.060 & - & - & -0.292 & 0.073 & - & - \\
\hline Education & 0.009 & 0.028 & - & - & -0.073 & 0.034 & - & - \\
\hline Openness & 0.017 & 0.035 & - & - & - & - & - & - \\
\hline Neuroticism & 0.076 & 0.033 & - & - & - & - & - & - \\
\hline Extraversion & 0.020 & 0.035 & - & - & - & - & - & - \\
\hline Conscientiousness & -0.072 & 0.029 & - & - & - & - & - & - \\
\hline NSIs from Family Intercept & - & - & -0.011 & .010 & - & - & -0.002 & 0.006 \\
\hline
\end{tabular}




\begin{tabular}{|c|c|c|c|c|c|c|}
\hline Agreeableness Intercept & - & -0.004 & 0.005 & - & -0.007 & 0.004 \\
\hline$R^{2}$ & .183 & .079 & & .125 & 1.431 & \\
\hline
\end{tabular}

\begin{tabular}{ll}
\hline Model Fit & $\left.\chi^{\mathbf{2}} \mathbf{5 9}\right)=\mathbf{1 7 3 . 1 9}$ \\
$\mathrm{CMIN} / \mathrm{DF}=2.93$ \\
$\mathrm{CFI}=.802$ \\
$\mathrm{RMSEA}=.10$
\end{tabular}

Note. Significant statistics $(p<.05)$ are bolded. $R^{2}=$ variance accounted for. An asterisk indicates that there is significant variability surrounding the estimate. A blue value indicates that the estimate is significantly different than zero. Type I errors were accounted for utilizing the false discovery rate adjustment. A green value indicates that the estimate was no longer statistically significant after the false discovery rate adjustments. To aid in the interpretation of the estimates, education, agreeableness, neuroticism, openness, extraversion, and conscientiousness were standardized. As such, a unit increase or decrease in these variables represent a standard deviation increase or decrease, respectively. Age was not manipulated so that a unit increase or decrease represents an increase or decrease of a year, respectively. 
Table I49

Research Question 8 - Examination of the Bidirectional Association between the Latent Growth Curves of NSIs from Family and Openness After Accounting for the Demographic Variables and Remaining Personality Traits for Younger Adults

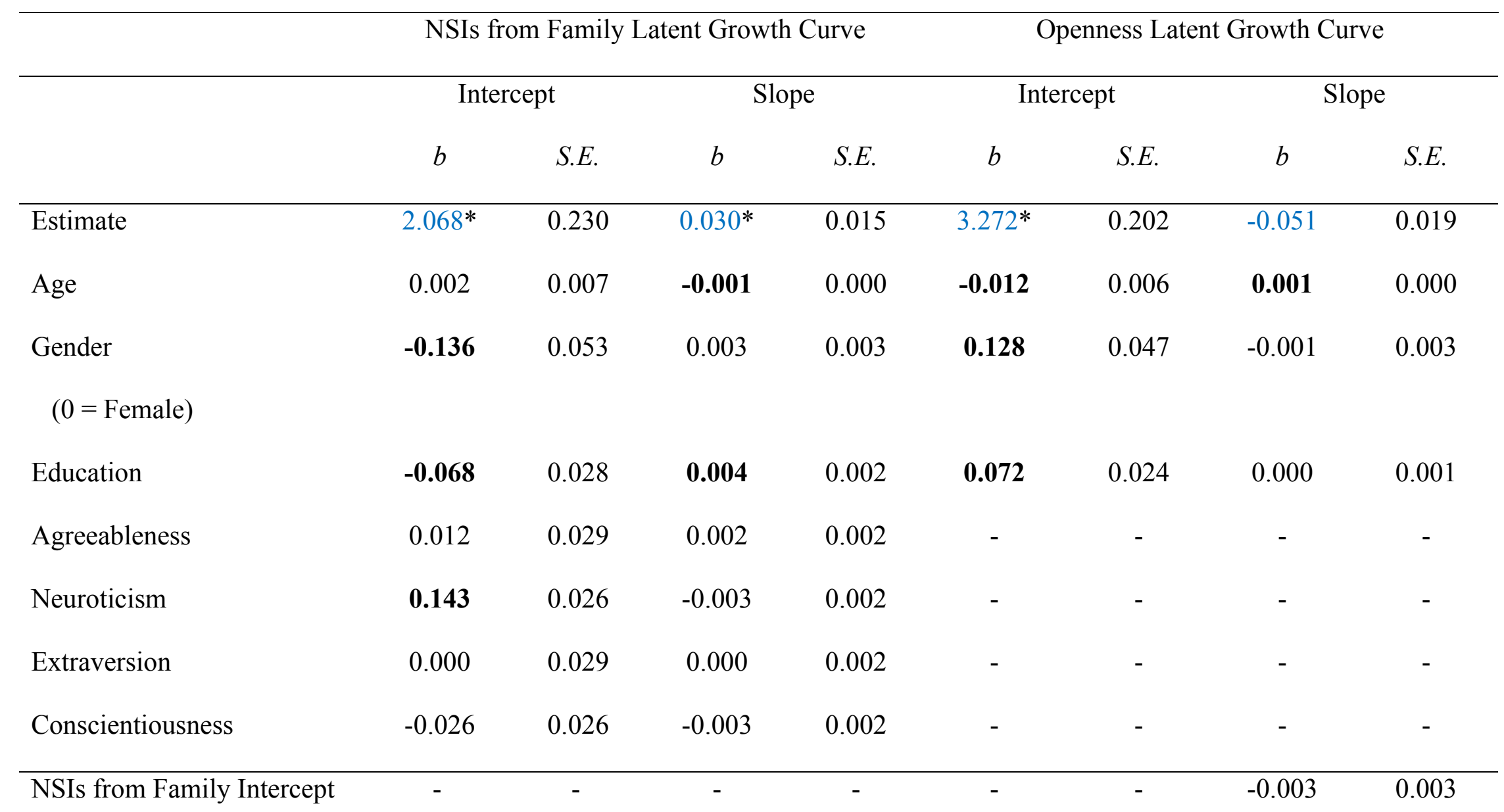




\begin{tabular}{|c|c|c|c|c|c|}
\hline Openness Intercept & - & - & - & 0.006 & 0.004 \\
\hline$R^{2}$ & .149 & .136 & .060 & .174 & \\
\hline
\end{tabular}

\begin{tabular}{ll}
\hline Model Fit & $\chi^{2} \mathbf{( 5 0 )}=\mathbf{2 9 6 . 3 6}$ \\
& $\mathrm{CMIN} / \mathrm{DF}=5.927$ \\
$\mathrm{CFI}=.828$ \\
$\mathrm{RMSEA}=.10$
\end{tabular}

Note. Significant statistics $(p<.05)$ are bolded. $R^{2}=$ variance accounted for. An asterisk indicates that there is significant variability surrounding the estimate. A blue value indicates that the estimate is significantly different than zero. Type I errors were accounted for utilizing the false discovery rate adjustment. A green value indicates that the estimate was no longer statistically significant after the false discovery rate adjustments. To aid in the interpretation of the estimates, education, agreeableness, neuroticism, openness, extraversion, and conscientiousness were standardized. As such, a unit increase or decrease in these variables represent a standard deviation increase or decrease, respectively. Age was not manipulated so that a unit increase or decrease represents an increase or decrease of a year, respectively. 
Table I50

Research Question 8 - Examination of the Bidirectional Association between the Latent Growth Curves of NSIs from Family and Openness After Accounting for the Demographic Variables and Remaining Personality Traits for Middle-Aged Adults

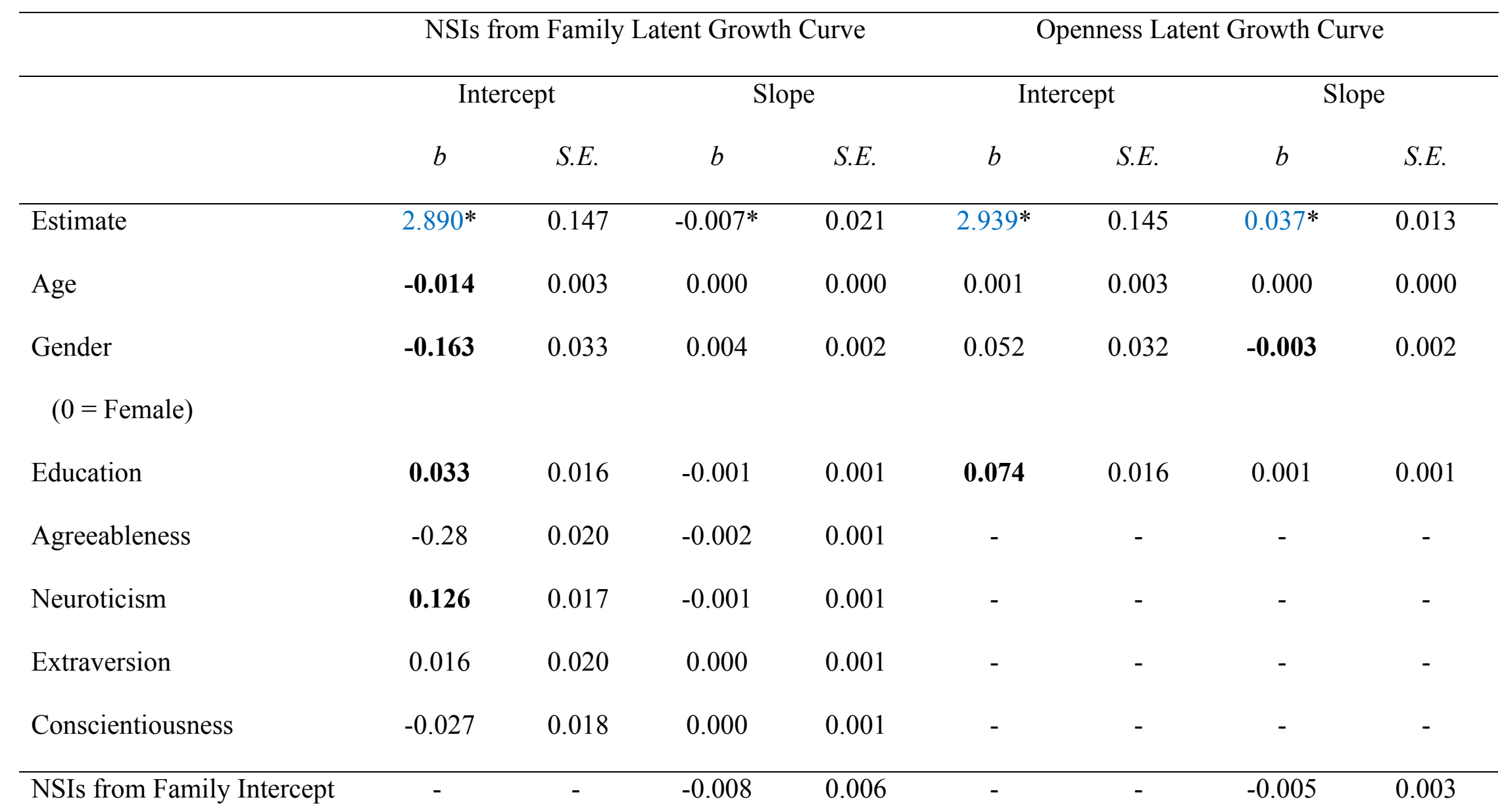




\begin{tabular}{|c|c|c|c|c|c|c|}
\hline Openness Intercept & - & 0.005 & 0.003 & - & -0.006 & 0.002 \\
\hline$R^{2}$ & .215 & .097 & & .034 & .075 & \\
\hline
\end{tabular}

\begin{tabular}{l}
\hline Model Fit \\
CMIN $=\mathbf{5 7 5 . 6 7 9}$ \\
$\mathrm{CFI}=.818$ \\
$\mathrm{RMSEA}=.10$ \\
\hline
\end{tabular}

Note. Significant statistics $(p<.05)$ are bolded. $R^{2}=$ variance accounted for. An asterisk indicates that there is significant variability surrounding the estimate. A blue value indicates that the estimate is significantly different than zero. Type I errors were accounted for utilizing the false discovery rate adjustment. A green value indicates that the estimate was no longer statistically significant after the false discovery rate adjustments. To aid in the interpretation of the estimates, education, agreeableness, neuroticism, openness, extraversion, and conscientiousness were standardized. As such, a unit increase or decrease in these variables represent a standard deviation increase or decrease, respectively. Age was not manipulated so that a unit increase or decrease represents an increase or decrease of a year, respectively. 
Table I51

Research Question 8 - Examination of the Bidirectional Association between the Latent Growth Curves of NSIs from Family and Openness After Accounting for the Demographic Variables and Remaining Personality Traits for Older Adults

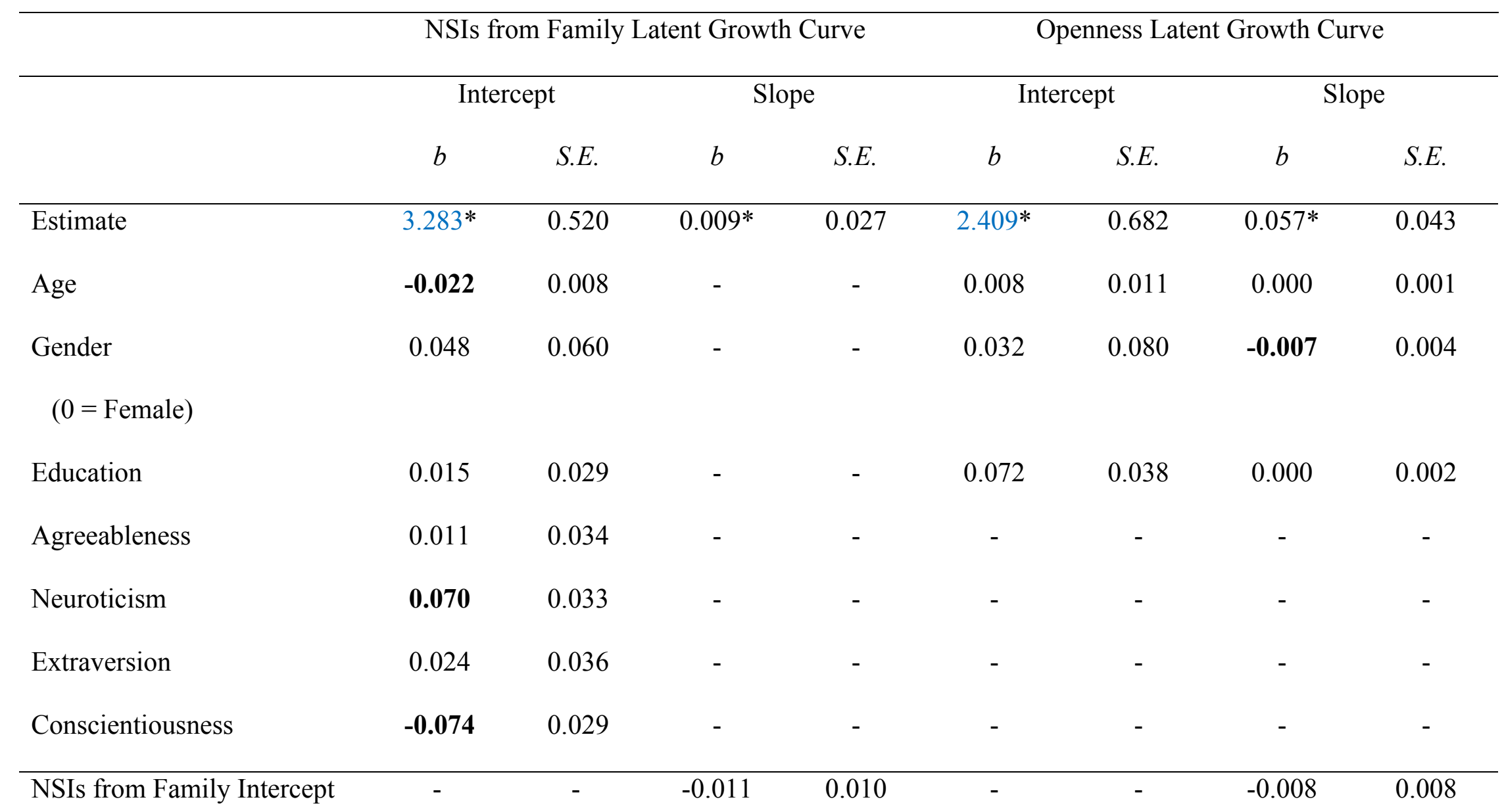




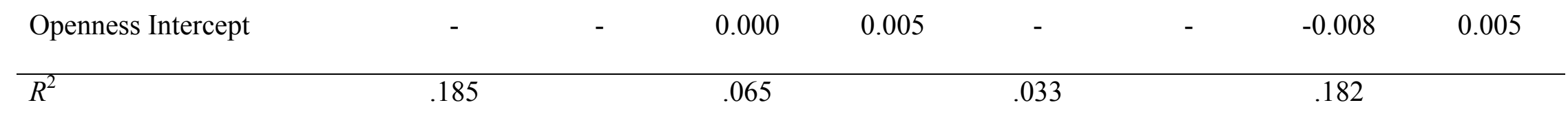

Model Fit $\chi^{2}(\mathbf{5 6})=\mathbf{1 7 6 . 5 2}$

$$
\mathrm{CMIN} / \mathrm{DF}=3.15
$$

$$
\mathrm{CFI}=.781
$$

$$
\mathrm{RMSEA}=.11
$$

Note. Significant statistics $(p<.05)$ are bolded. $R^{2}=$ variance accounted for. An asterisk indicates that there is significant variability surrounding the estimate. A blue value indicates that the estimate is significantly different than zero. Type I errors were accounted for utilizing the false discovery rate adjustment. A green value indicates that the estimate was no longer statistically significant after the false discovery rate adjustments. To aid in the interpretation of the estimates, education, agreeableness, neuroticism, openness, extraversion, and conscientiousness were standardized. As such, a unit increase or decrease in these variables represent a standard deviation increase or decrease, respectively. Age was not manipulated so that a unit increase or decrease represents an increase or decrease of a year, respectively. 
Table I52

Research Question 8 - Examination of the Bidirectional Association between the Latent Growth Curves of NSIs from Family and Neuroticism After Accounting for the Demographic Variables and Remaining Personality Traits for Younger Adults

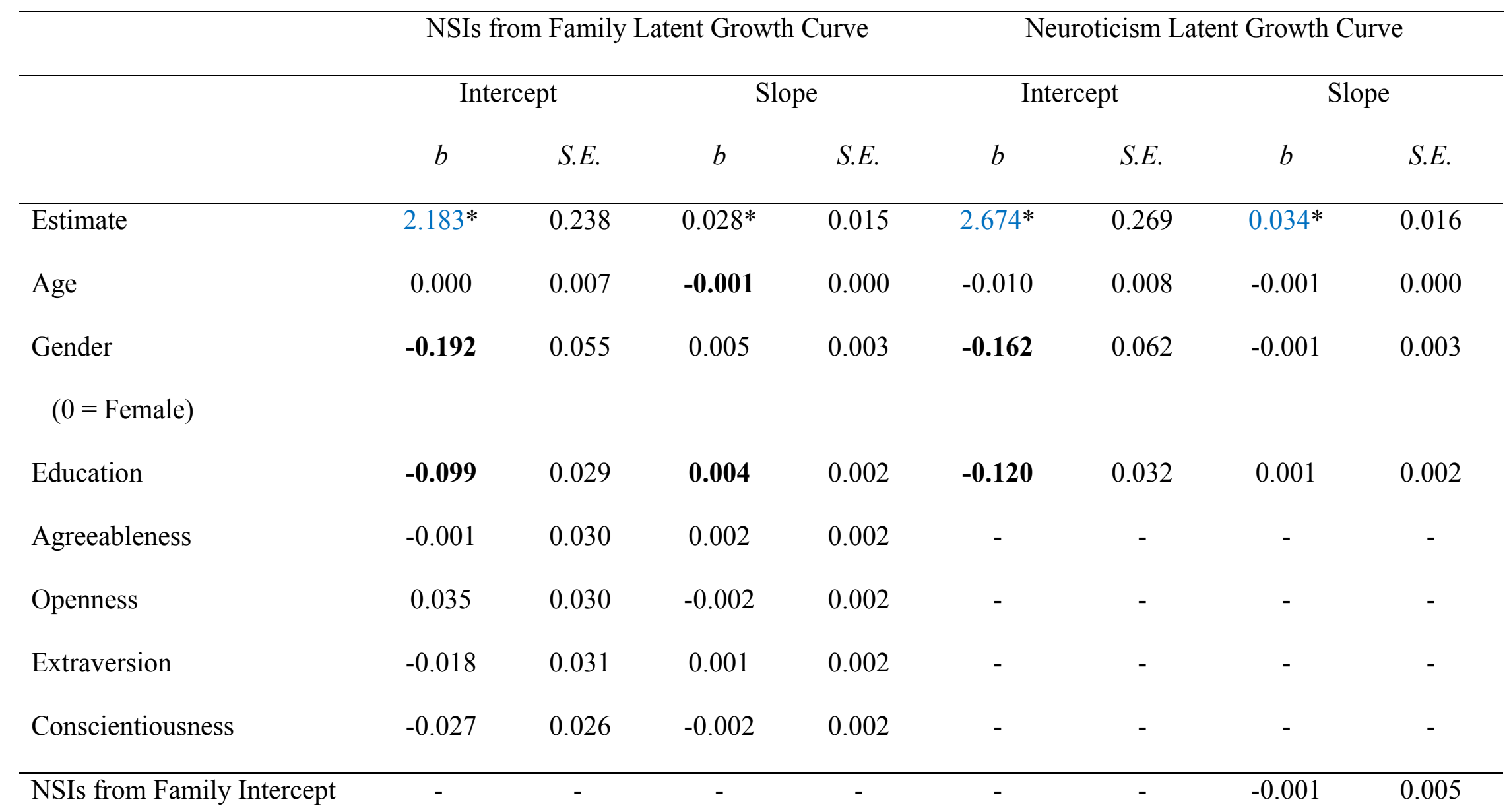




\begin{tabular}{|c|c|c|c|c|c|}
\hline Neuroticism Intercept & - & - & - & -0.009 & 0.004 \\
\hline$R^{2}$ & .089 & .149 & .063 & .139 & \\
\hline
\end{tabular}

$\chi^{\mathbf{2}(\mathbf{5 0})=\mathbf{1 8 3 . 2 5}}$
$\mathrm{CMIN} / \mathrm{DF}=3.66$
$\mathrm{CFI}=.898$
$\mathrm{RMSEA}=.07$

Note. Significant statistics $(p<.05)$ are bolded. $R^{2}=$ variance accounted for. An asterisk indicates that there is significant variability surrounding the estimate. A blue value indicates that the estimate is significantly different than zero. Type I errors were accounted for utilizing the false discovery rate adjustment. A green value indicates that the estimate was no longer statistically significant after the false discovery rate adjustments. To aid in the interpretation of the estimates, education, agreeableness, neuroticism, openness, extraversion, and conscientiousness were standardized. As such, a unit increase or decrease in these variables represent a standard deviation increase or decrease, respectively. Age was not manipulated so that a unit increase or decrease represents an increase or decrease of a year, respectively. 
Table I53

Research Question 8 - Examination of the Bidirectional Association between the Latent Growth Curves of NSIs from Family and Neuroticism After Accounting for the Demographic Variables and Remaining Personality Traits for Middle-Aged Adults

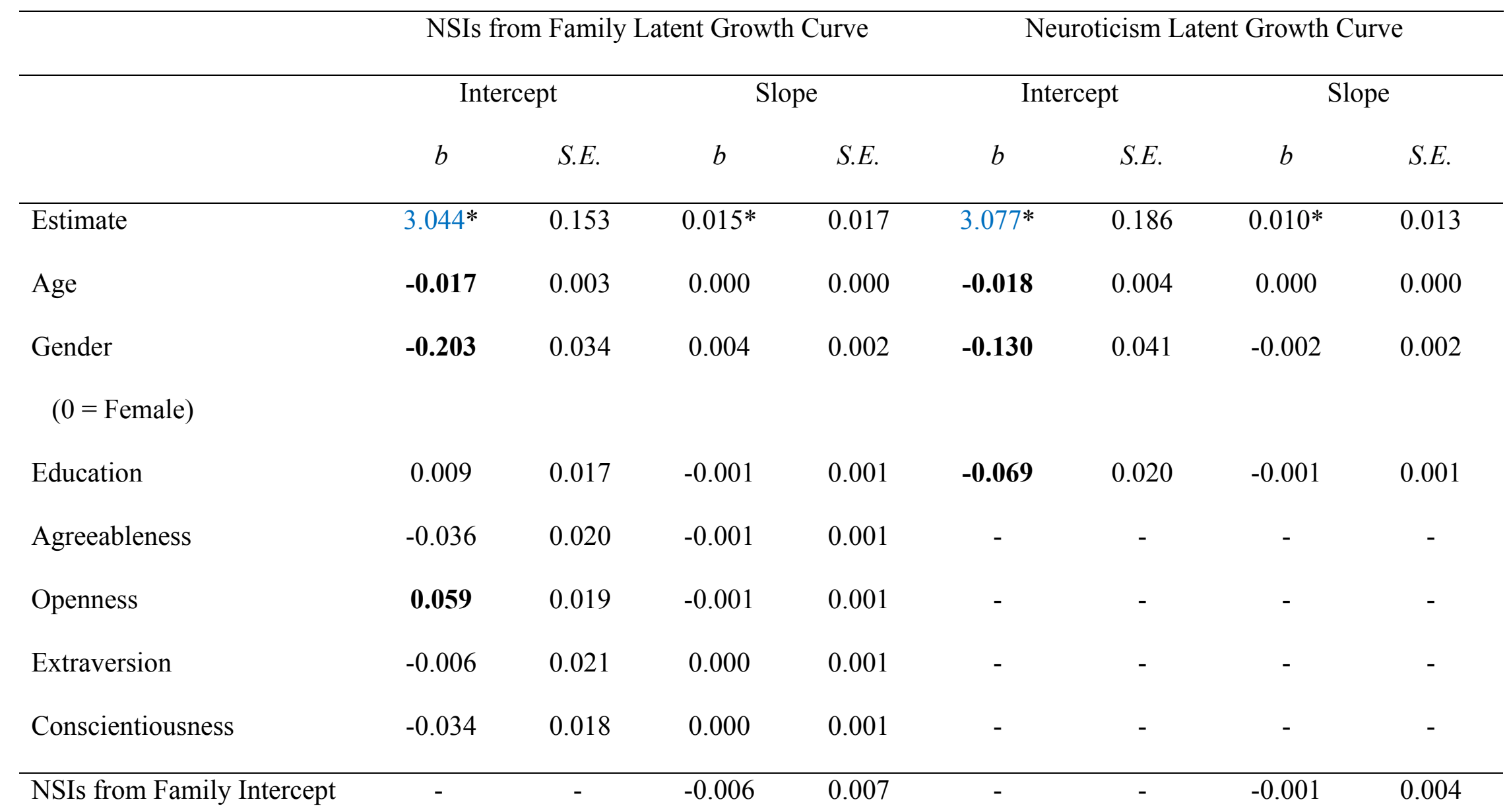




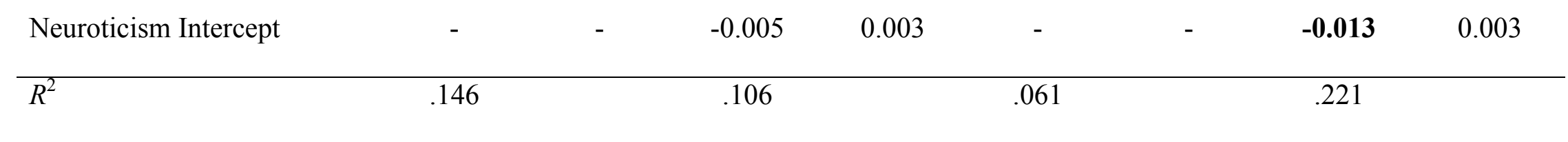

\begin{tabular}{ll}
\hline Model Fit & $\chi^{\mathbf{2}} \mathbf{4 9 )}=\mathbf{3 4 4 . 0 4}$ \\
$\mathrm{CMIN} / \mathrm{DF}=7.02$ \\
$\mathrm{CFI}=.894$ \\
$\mathrm{RMSEA}=.08$
\end{tabular}

Note. Significant statistics $(p<.05)$ are bolded. $R^{2}=$ variance accounted for. An asterisk indicates that there is significant variability surrounding the estimate. A blue value indicates that the estimate is significantly different than zero. Type I errors were accounted for utilizing the false discovery rate adjustment. A green value indicates that the estimate was no longer statistically significant after the false discovery rate adjustments. To aid in the interpretation of the estimates, education, agreeableness, neuroticism, openness, extraversion, and conscientiousness were standardized. As such, a unit increase or decrease in these variables represent a standard deviation increase or decrease, respectively. Age was not manipulated so that a unit increase or decrease represents an increase or decrease of a year, respectively. 
Table I54

Research Question 8 - Examination of the Bidirectional Association between the Latent Growth Curves of NSIs from Family and Neuroticism After Accounting for the Demographic Variables and Remaining Personality Traits for Older Adults

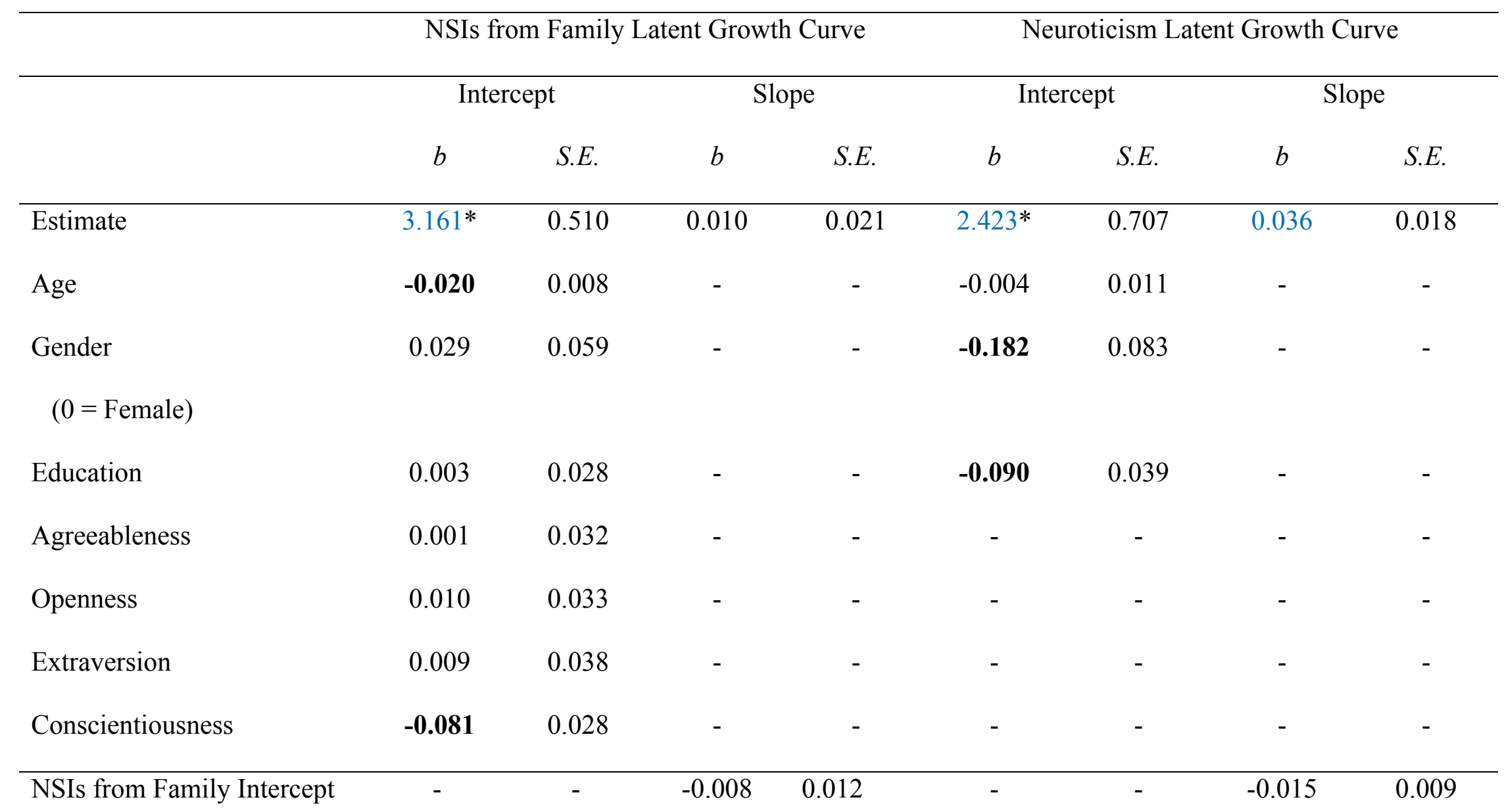




\begin{tabular}{|c|c|c|c|c|c|c|}
\hline Neuroticism Intercept & - & -0.002 & 0.005 & - & -0.006 & 0.007 \\
\hline$R^{2}$ & .128 & .053 & & .071 & .549 & \\
\hline
\end{tabular}

$\chi^{\mathbf{2}(\mathbf{5 9})=\mathbf{1 1 1 . 2 6}}$
$\mathrm{CMIN} / \mathrm{DF}=1.88$
$\mathrm{CFI}=.893$
$\mathrm{RMSEA}=.07$

Note. Significant statistics $(p<.05)$ are bolded. $R^{2}=$ variance accounted for. An asterisk indicates that there is significant variability surrounding the estimate. A blue value indicates that the estimate is significantly different than zero. Type I errors were accounted for utilizing the false discovery rate adjustment. A green value indicates that the estimate was no longer statistically significant after the false discovery rate adjustments. To aid in the interpretation of the estimates, education, agreeableness, neuroticism, openness, extraversion, and conscientiousness were standardized. As such, a unit increase or decrease in these variables represent a standard deviation increase or decrease, respectively. Age was not manipulated so that a unit increase or decrease represents an increase or decrease of a year, respectively. 
Table I55

Research Question 8 - Examination of the Bidirectional Association between the Latent Growth Curves of NSIs from Family and Extraversion After Accounting for the Demographic Variables and Remaining Personality Traits for Younger Adults

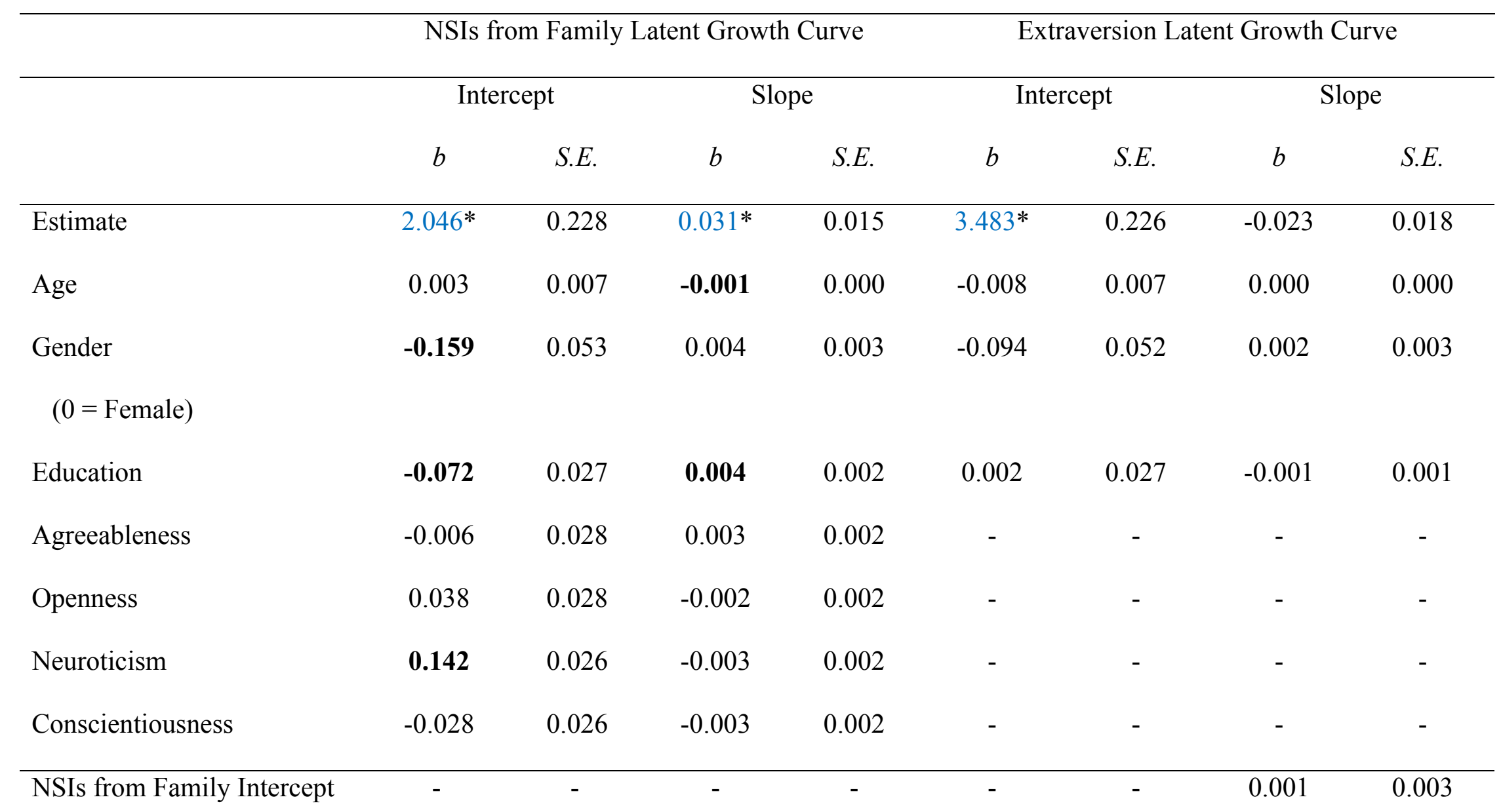




\begin{tabular}{|c|c|c|c|c|c|}
\hline Extraversion Intercept & - & - & - & -0.001 & 0.004 \\
\hline$R^{2}$ & .164 & .162 & .014 & .056 & \\
\hline
\end{tabular}

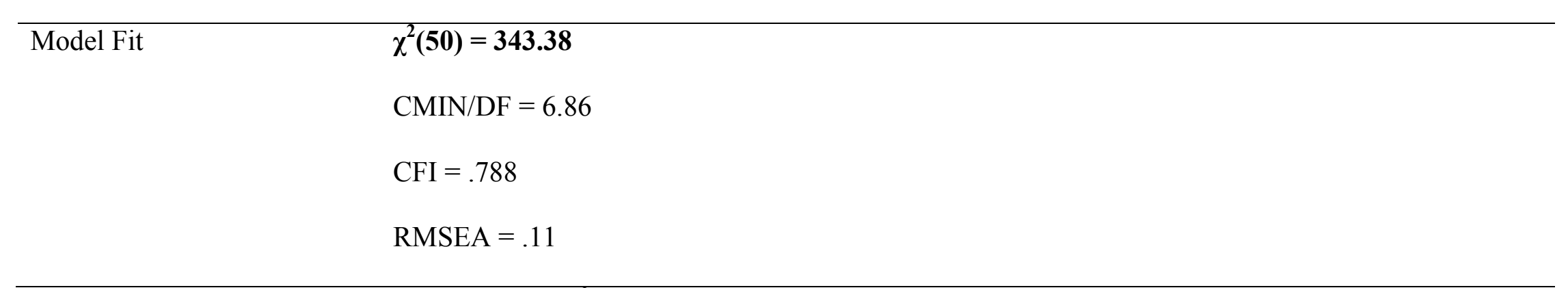

Note. Significant statistics $(p<.05)$ are bolded. $R^{2}=$ variance accounted for. An asterisk indicates that there is significant variability surrounding the estimate. A blue value indicates that the estimate is significantly different than zero. Type I errors were accounted for utilizing the false discovery rate adjustment. A green value indicates that the estimate was no longer statistically significant after the false discovery rate adjustments. To aid in the interpretation of the estimates, education, agreeableness, neuroticism, openness, extraversion, and conscientiousness were standardized. As such, a unit increase or decrease in these variables represent a standard deviation increase or decrease, respectively. Age was not manipulated so that a unit increase or decrease represents an increase or decrease of a year, respectively. 
Table I56

Research Question 8 - Examination of the Bidirectional Association between the Latent Growth Curves of NSIs from Family and Extraversion After Accounting for the Demographic Variables and Remaining Personality Traits for Middle-Aged Adults

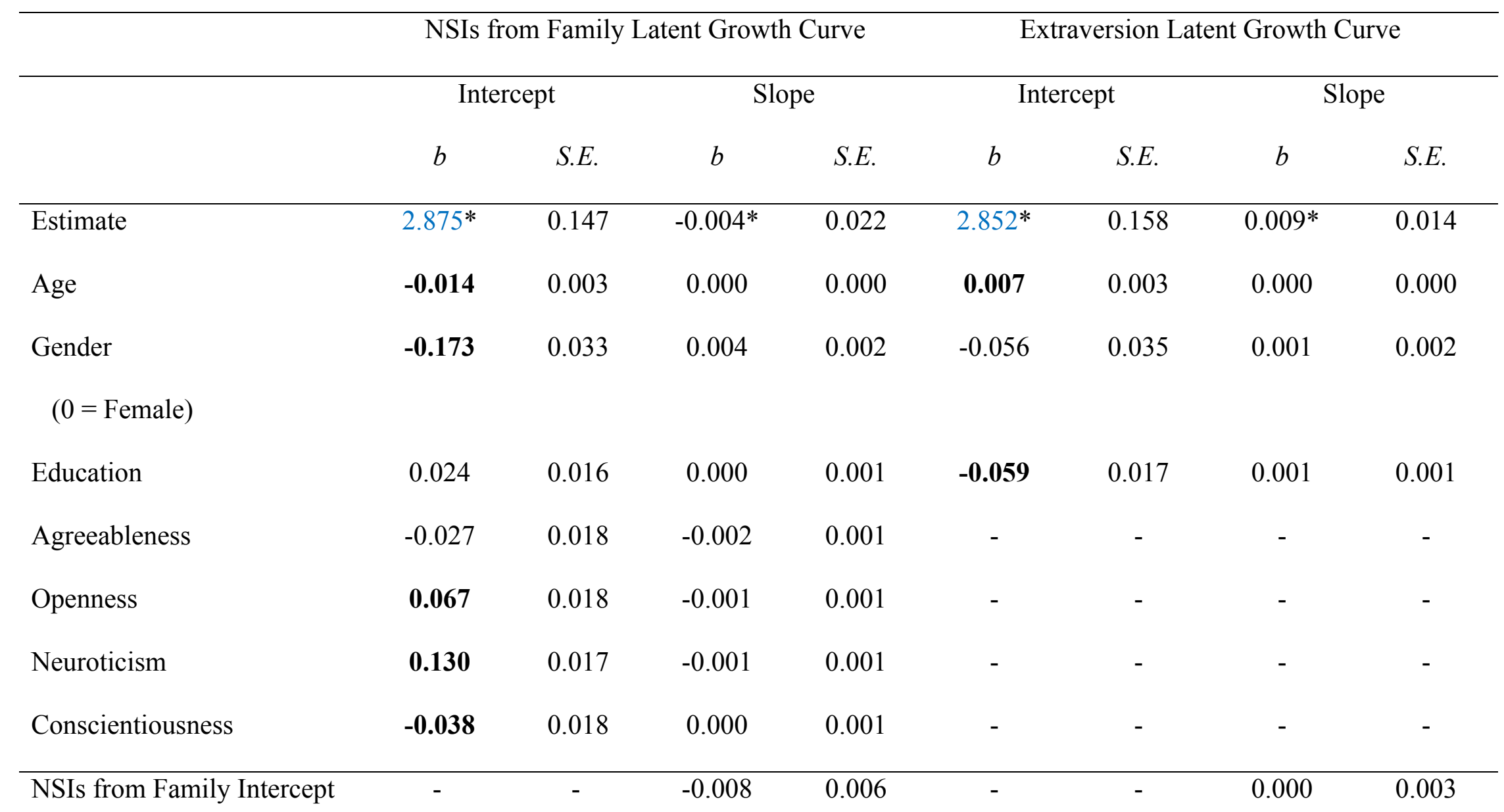




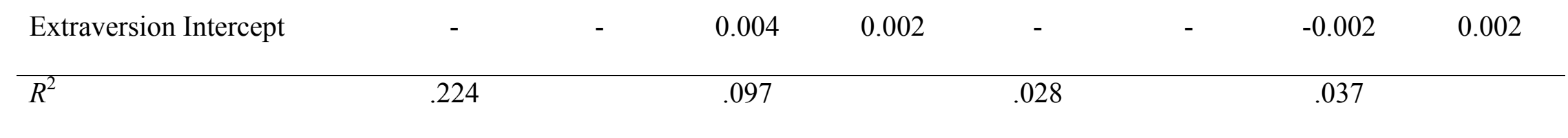

\begin{tabular}{ll}
\hline Model Fit & $\chi^{\mathbf{2}(49)=682.73}$ \\
& $\mathrm{CMIN} / \mathrm{DF}=13.93$ \\
$\mathrm{CFI}=.783$ \\
$\mathrm{RMSEA}=.11$
\end{tabular}

Note. Significant statistics $(p<.05)$ are bolded. $R^{2}=$ variance accounted for. An asterisk indicates that there is significant variability surrounding the estimate. A blue value indicates that the estimate is significantly different than zero. Type I errors were accounted for utilizing the false discovery rate adjustment. A green value indicates that the estimate was no longer statistically significant after the false discovery rate adjustments. To aid in the interpretation of the estimates, education, agreeableness, neuroticism, openness, extraversion, and conscientiousness were standardized. As such, a unit increase or decrease in these variables represent a standard deviation increase or decrease, respectively. Age was not manipulated so that a unit increase or decrease represents an increase or decrease of a year, respectively. 
Table I57

Research Question 8 - Examination of the Bidirectional Association between the Latent Growth Curves of NSIs from Family and Extraversion After Accounting for the Demographic Variables and Remaining Personality Traits for Older Adults

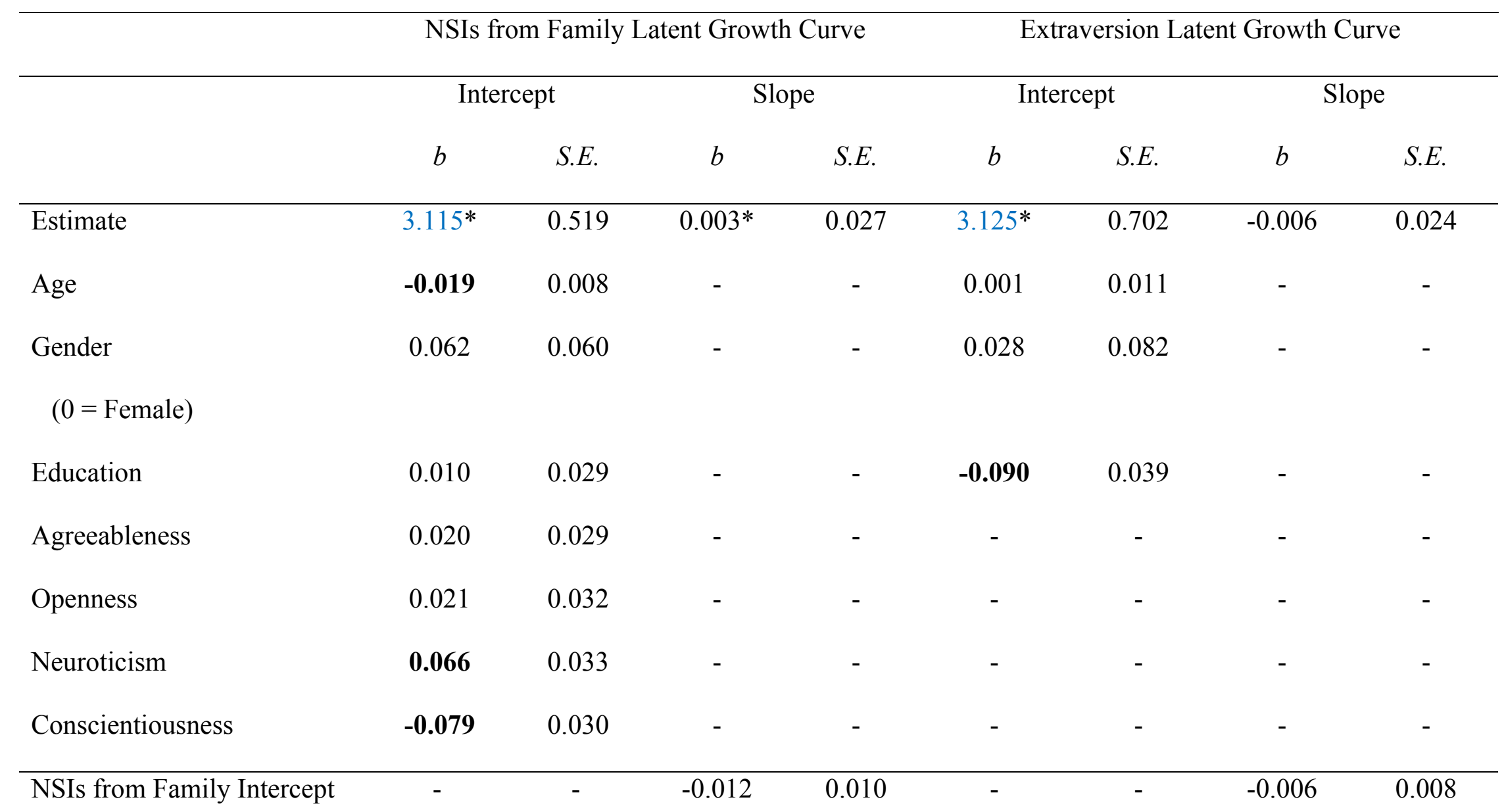




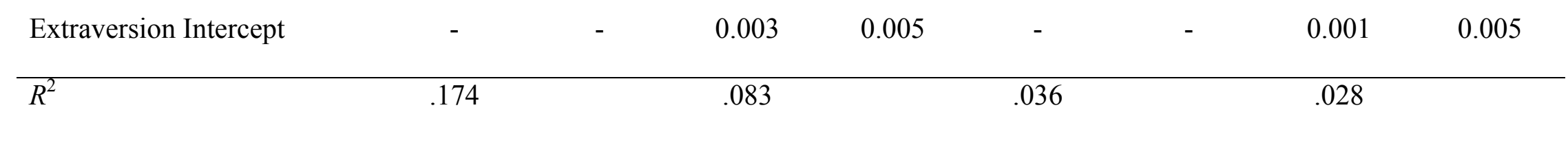

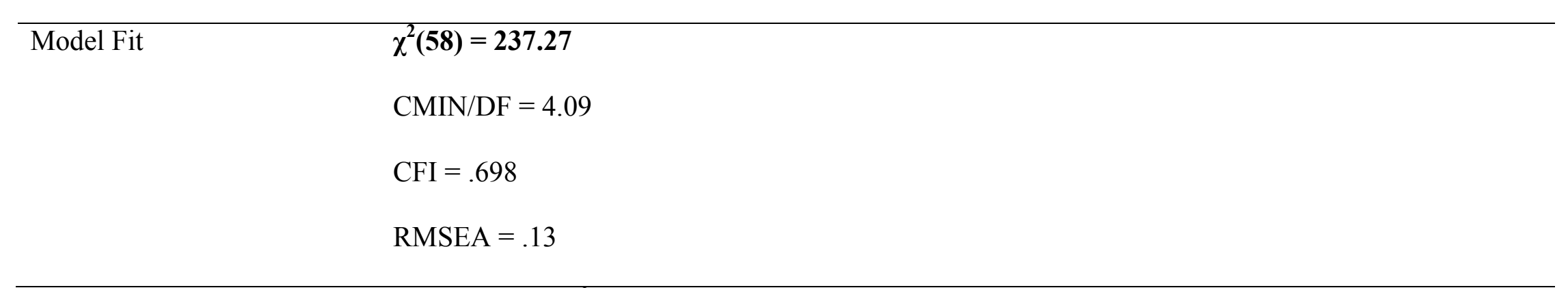

Note. Significant statistics $(p<.05)$ are bolded. $R^{2}=$ variance accounted for. An asterisk indicates that there is significant variability surrounding the estimate. A blue value indicates that the estimate is significantly different than zero. Type I errors were accounted for utilizing the false discovery rate adjustment. A green value indicates that the estimate was no longer statistically significant after the false discovery rate adjustments. To aid in the interpretation of the estimates, education, agreeableness, neuroticism, openness, extraversion, and conscientiousness were standardized. As such, a unit increase or decrease in these variables represent a standard deviation increase or decrease, respectively. Age was not manipulated so that a unit increase or decrease represents an increase or decrease of a year, respectively. 
Table I58

Research Question 8 - Examination of the Bidirectional Association between the Latent Growth Curves of NSIs from Family and Conscientiousness After Accounting for the Demographic Variables and Remaining Personality Traits for Younger Adults

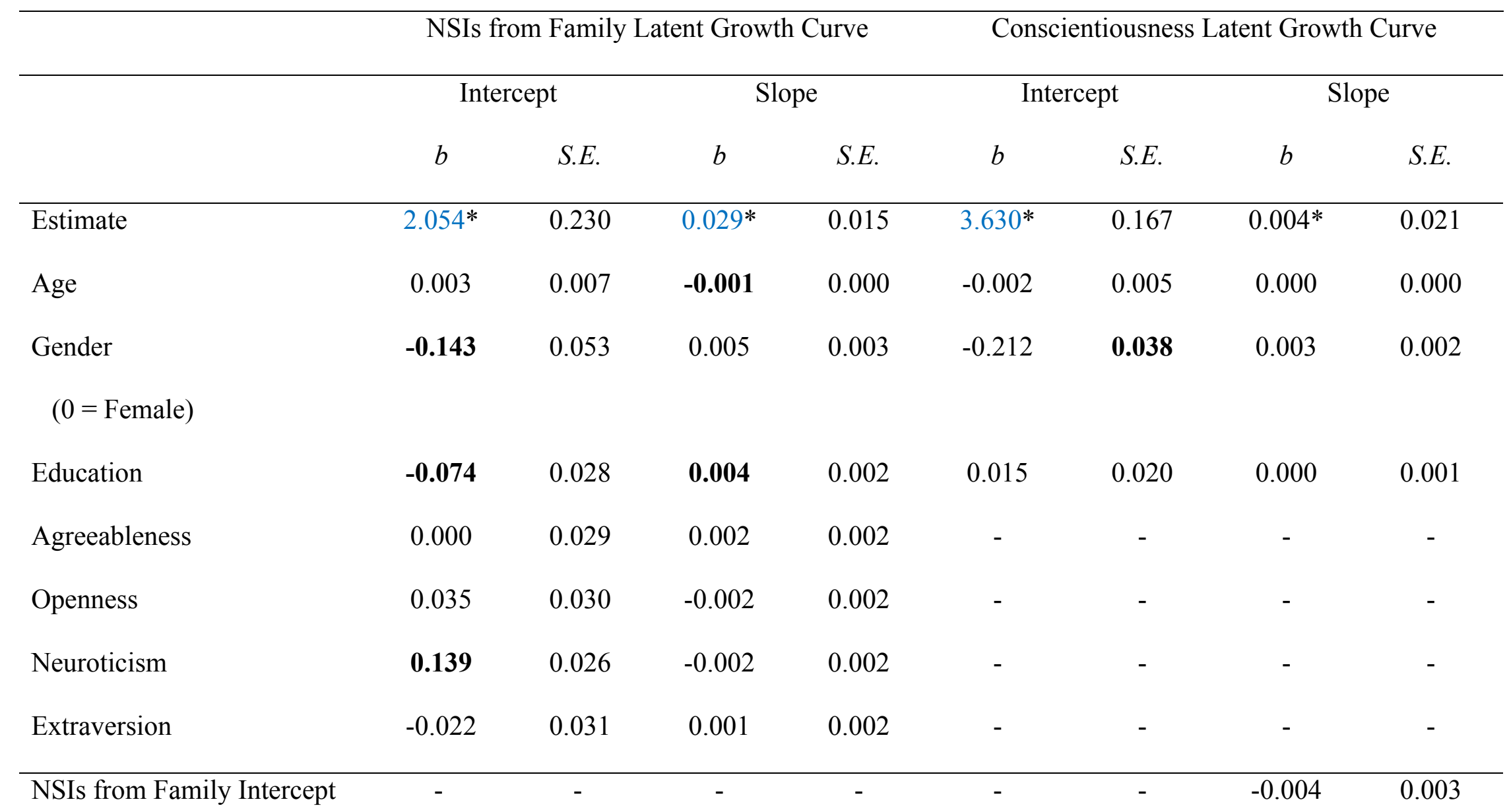




\begin{tabular}{|c|c|c|c|c|c|}
\hline Conscientiousness Intercept & - & - & - & -0.002 & 0.005 \\
\hline$R^{2}$ & .143 & .146 & .093 & 084 & \\
\hline
\end{tabular}

\begin{tabular}{ll}
\hline Model Fit & $\chi^{\mathbf{2}(\mathbf{5 0})=\mathbf{1 7 4 . 6 3}}$ \\
$\mathrm{CMIN} / \mathrm{DF}=3.49$ \\
$\mathrm{CFI}=.900$ \\
$\mathrm{RMSEA}=.07$
\end{tabular}

Note. Significant statistics $(p<.05)$ are bolded. $R^{2}=$ variance accounted for. An asterisk indicates that there is significant variability surrounding the estimate. A blue value indicates that the estimate is significantly different than zero. Type I errors were accounted for utilizing the false discovery rate adjustment. A green value indicates that the estimate was no longer statistically significant after the false discovery rate adjustments. To aid in the interpretation of the estimates, education, agreeableness, neuroticism, openness, extraversion, and conscientiousness were standardized. As such, a unit increase or decrease in these variables represent a standard deviation increase or decrease, respectively. Age was not manipulated so that a unit increase or decrease represents an increase or decrease of a year, respectively. 
Table I59

Research Question 8 - Examination of the Bidirectional Association between the Latent Growth Curves of NSIs from Family and Conscientiousness After Accounting for the Demographic Variables and Remaining Personality Traits for Middle-Aged Adults

\begin{tabular}{|c|c|c|c|c|c|c|c|c|}
\hline & \multicolumn{4}{|c|}{ NSIs from Family Latent Growth Curve } & \multicolumn{4}{|c|}{ Conscientiousness Latent Growth Curve } \\
\hline & \multicolumn{2}{|c|}{ Intercept } & \multicolumn{2}{|c|}{ Slope } & \multicolumn{2}{|c|}{ Intercept } & \multicolumn{2}{|c|}{ Slope } \\
\hline & $b$ & S.E. & $b$ & S.E. & $b$ & S.E. & $b$ & S.E. \\
\hline Estimate & $2.872^{*}$ & 0.147 & $0.001 *$ & 0.027 & $3.550 *$ & 0.102 & $0.001 *$ & 0.001 \\
\hline Age & -0.014 & 0.003 & 0.000 & 0.000 & 0.000 & 0.002 & - & - \\
\hline $\begin{array}{l}\text { Gender } \\
\qquad(0=\text { Female })\end{array}$ & -0.168 & 0.033 & 0.005 & 0.002 & -0.089 & 0.023 & - & - \\
\hline Education & 0.019 & 0.016 & -0.001 & 0.001 & 0.043 & 0.011 & - & - \\
\hline Agreeableness & -0.038 & 0.020 & -0.001 & 0.001 & - & - & - & - \\
\hline Openness & 0.060 & 0.019 & 0.000 & 0.001 & - & - & - & - \\
\hline Neuroticism & 0.129 & 0.017 & -0.001 & 0.001 & - & - & - & - \\
\hline Extraversion & -0.007 & 0.021 & 0.000 & 0.001 & - & - & - & - \\
\hline NSIs from Family Intercept & - & - & -0.008 & 0.006 & - & - & - & - \\
\hline
\end{tabular}




\begin{tabular}{lllllll} 
Conscientiousness Intercept & - & - & 0.002 & 0.004 & - & - \\
\hline$R^{2}$ & .208 & .083 & .039 & .000
\end{tabular}

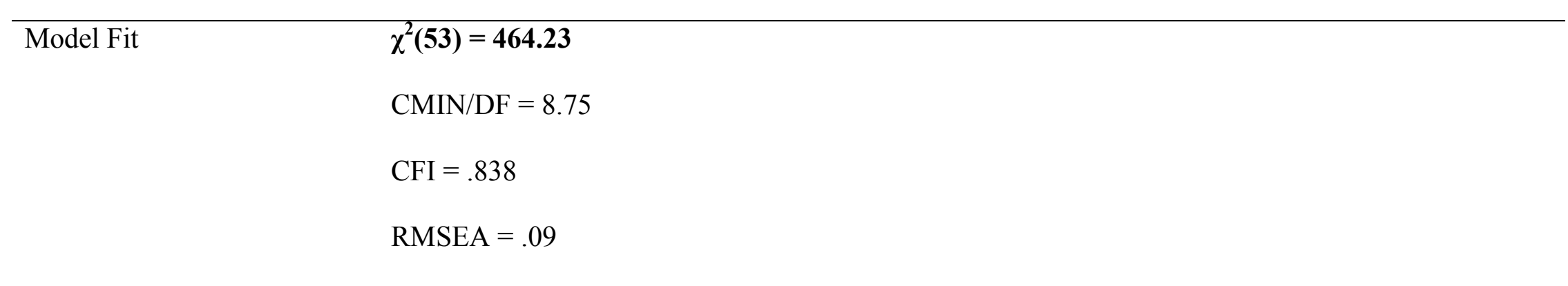

Note. Significant statistics $(p<.05)$ are bolded. $R^{2}=$ variance accounted for. An asterisk indicates that there is significant variability surrounding the estimate. A blue value indicates that the estimate is significantly different than zero. Type I errors were accounted for utilizing the false discovery rate adjustment. A green value indicates that the estimate was no longer statistically significant after the false discovery rate adjustments. To aid in the interpretation of the estimates, education, agreeableness, neuroticism, openness, extraversion, and conscientiousness were standardized. As such, a unit increase or decrease in these variables represent a standard deviation increase or decrease, respectively. Age was not manipulated so that a unit increase or decrease represents an increase or decrease of a year, respectively. 
Table I60

Research Question 8 - Examination of the Bidirectional Association between the Latent Growth Curves of NSIs from Family and Conscientiousness After Accounting for the Demographic Variables and Remaining Personality Traits for Older Adults

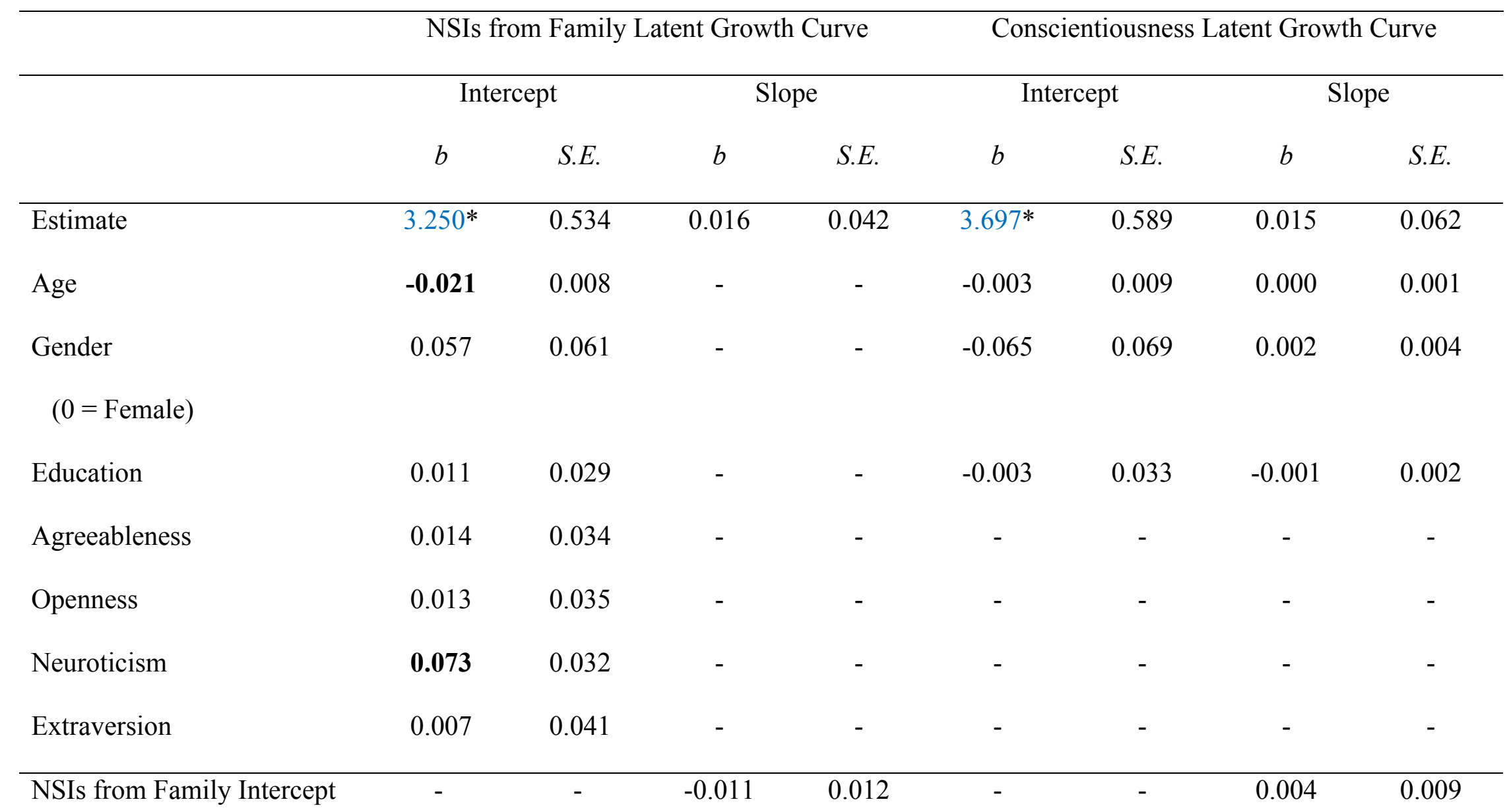




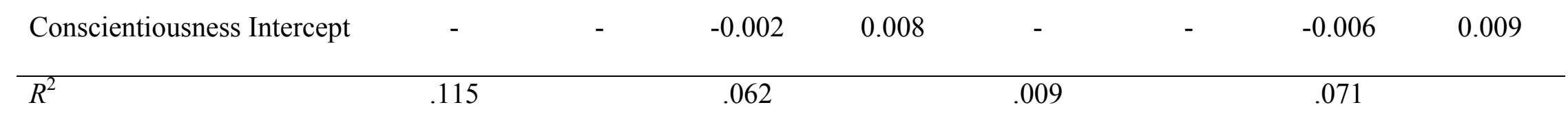

\begin{tabular}{ll}
\hline Model Fit & $\chi^{\mathbf{2}(\mathbf{5 6})=\mathbf{1 3 7 . 8 2}}$ \\
$\mathrm{CMIN} / \mathrm{DF}=2.46$ \\
$\mathrm{CFI}=.826$ \\
$\mathrm{RMSEA}=.09$
\end{tabular}

Note. Significant statistics $(p<.05)$ are bolded. $R^{2}=$ variance accounted for. An asterisk indicates that there is significant variability surrounding the estimate. A blue value indicates that the estimate is significantly different than zero. Type I errors were accounted for utilizing the false discovery rate adjustment. A green value indicates that the estimate was no longer statistically significant after the false discovery rate adjustments. To aid in the interpretation of the estimates, education, agreeableness, neuroticism, openness, extraversion, and conscientiousness were standardized. As such, a unit increase or decrease in these variables represent a standard deviation increase or decrease, respectively. Age was not manipulated so that a unit increase or decrease represents an increase or decrease of a year, respectively. 


\section{Appendix J}

Results for NSIs from Partner

Table J1

Summary Results of Research Questions 2 and 3 for NSIs from Partner

NSIs from Partner Latent Growth Curve

Entire Sample

\begin{tabular}{lcc}
\hline & Intercept & Slope \\
\hline Age (Older) & & Decrease at a steeper rate \\
\hline Gender (Males) & - & \\
\hline Education & + & Decrease at a steeper rate \\
\hline Agreeableness & & \\
\hline
\end{tabular}

\section{Openness}

Neuroticism $\quad+\quad$ Decrease at a steeper rate

\section{Extraversion}

\section{Conscientiousness}

Note. The summary of significant effects is presented above. A plus sign indicates that higher values of the variable are associated with a higher intercept. A minus sign indicates that higher values of the variable are associated with a lower intercept. Age did not moderate the slope of NSIs from partner. 
Table J2

Summary Results of Research Questions 5 and 6 for Agreeableness

Agreeableness Latent Growth Curve

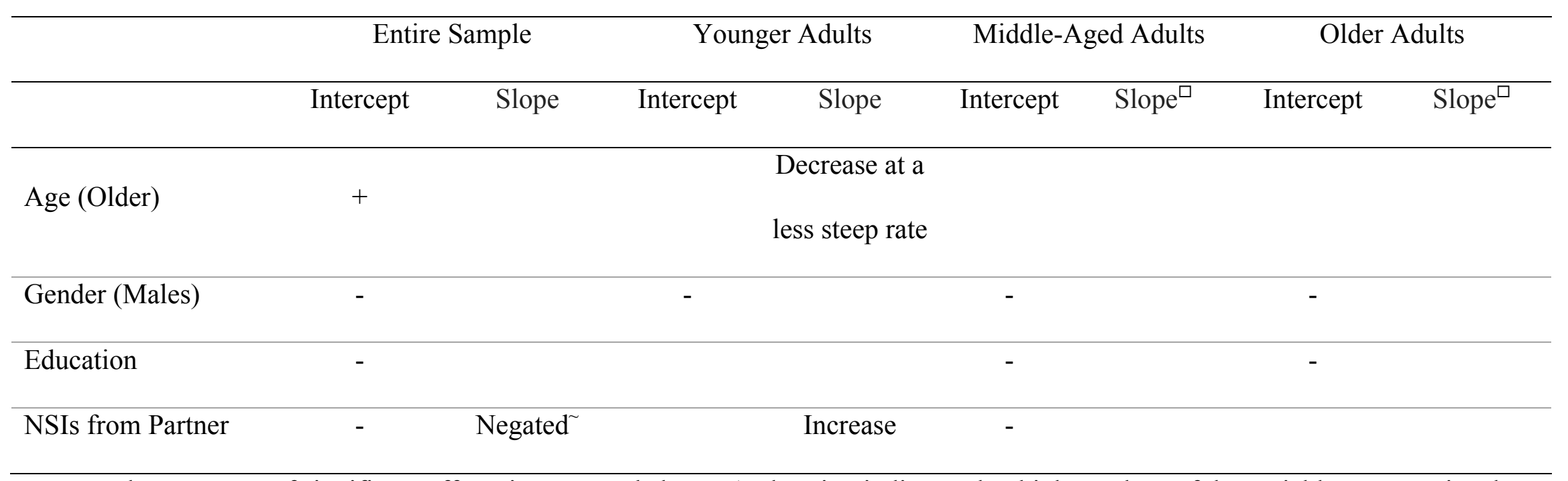

Note. The summary of significant effects is presented above. A plus sign indicates that higher values of the variable are associated with a higher intercept. A minus sign indicates that higher values of the variable are associated with a lower intercept. ${ }^{\square}$ indicates that there was no significant variance surrounding the slope estimate. Therefore, slope was not predicted. Being one standard deviation higher in NSIs was associated with an increase in agreeableness over time, which negated the overall decrease in agreeableness. 
Table J3

Summary Results of Research Questions 5 and 6 for Openness

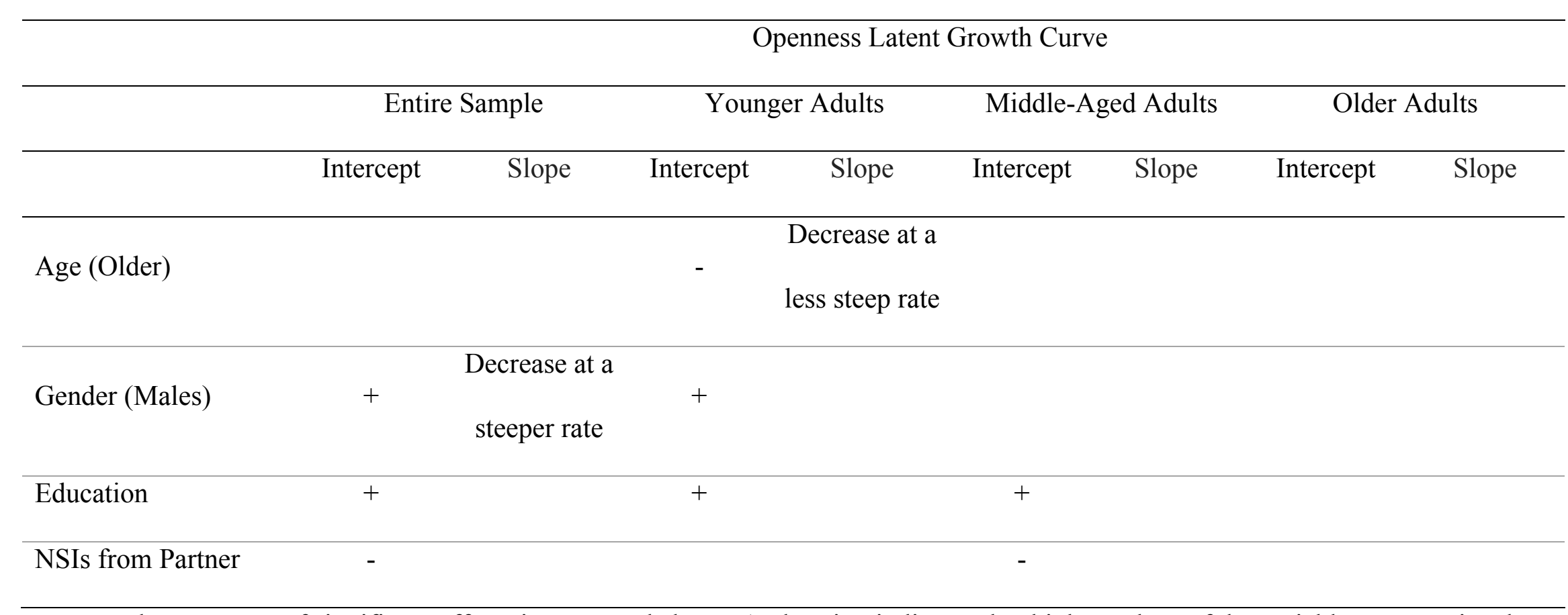

Note. The summary of significant effects is presented above. A plus sign indicates that higher values of the variable are associated

with a higher intercept. A minus sign indicates that higher values of the variable are associated with a lower intercept. 
Table J4

Summary Results of Research Questions 5 and 6 for Neuroticism

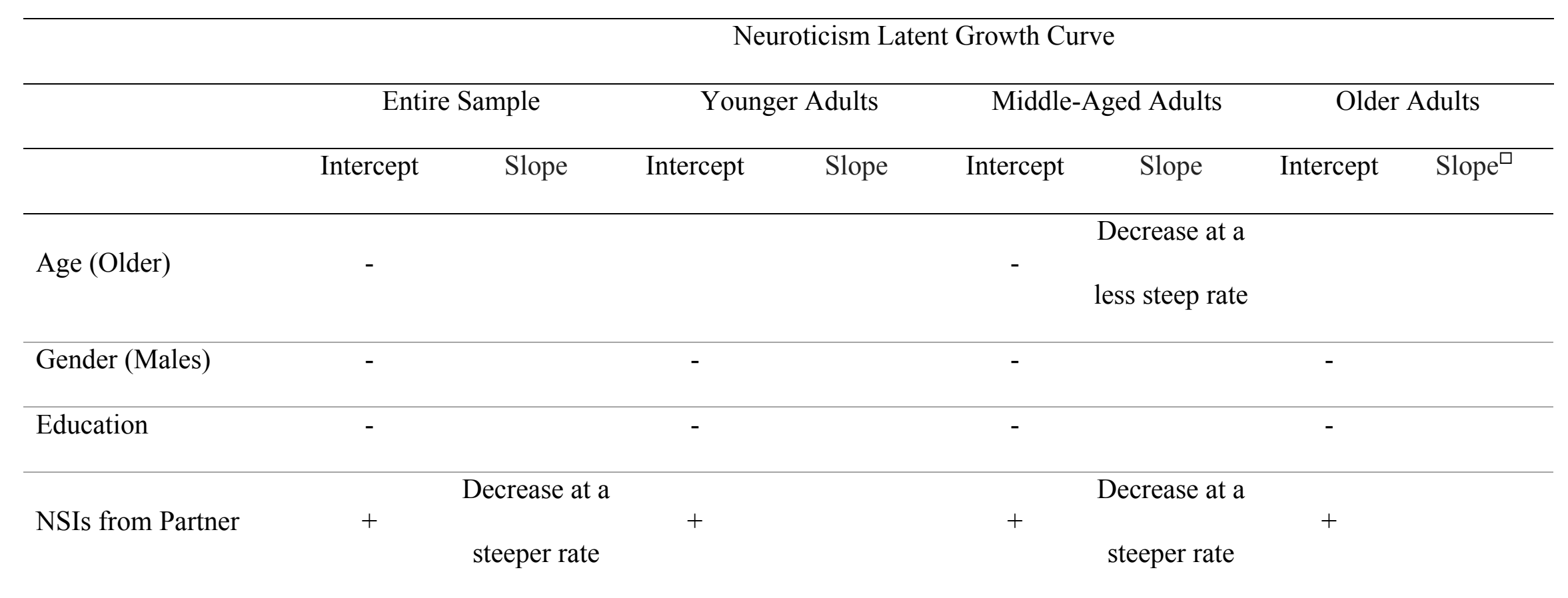

Note. The summary of significant effects is presented above. A plus sign indicates that higher values of the variable are associated

with a higher intercept. A minus sign indicates that higher values of the variable are associated with a lower intercept. ${ }^{\square}$ indicates that there was no significant variance surrounding the slope estimate. Therefore, slope was not predicted. 
Table J5

Summary Results of Research Questions 5 and 6 for Extraversion

Extraversion Latent Growth Curve

\begin{tabular}{ll}
\hline \multicolumn{3}{c}{ Entire Sample } & \\
\hline Intercept & Slope
\end{tabular}

Age (Older)

\section{Gender (Males)}

Education

\section{NSIs from Partner}

Note. The summary of significant effects is presented above. A plus sign indicates that higher values of the variable are associated with a higher intercept. A minus sign indicates that higher values of the variable are associated with a lower intercept. Age did not moderate the slope of extraversion. 
Table J6

Summary Results of Research Questions 5 and 6 for Conscientiousness

Conscientiousness Latent Growth Curve

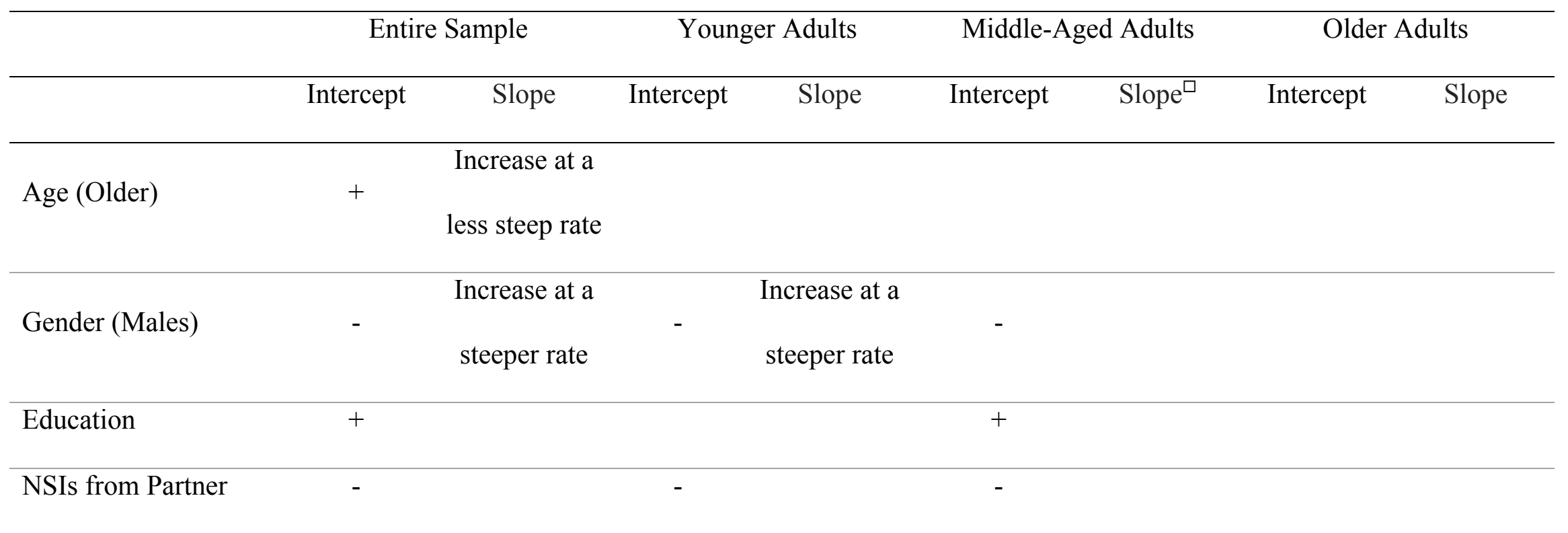

Note. The summary of significant effects is presented above. A plus sign indicates that higher values of the variable are associated

with a higher intercept. A minus sign indicates that higher values of the variable are associated with a lower intercept. ${ }^{\square}$ indicates that there was no significant variance surrounding the slope estimate. Therefore, slope was not predicted. 
Table J7

Summary Results of Research Questions 7 and 8 for NSIs from Partner and Agreeableness

NSIs from Partner Latent Growth Curve

NSIs from Partner Slope

Entire

Sample
Agreeableness Latent Growth Curve

Agreeableness Slope

\begin{tabular}{lll}
\hline & Entire & Entire Sample \\
Sample &
\end{tabular}

\section{NSIs from Partner}

Intercept

\section{Agreeableness}

Intercept

Decrease at a steeper rate

Note. The summary of significant effects is presented above. A plus sign indicates that higher values of the variable are associated with a higher intercept. A minus sign indicates that higher values of the variable are associated with a lower intercept. Age did not moderate the cross-domain latent growth curve between NSIs from partner and agreeableness. 
Table J8

Summary Results of Research Questions 7 and 8 for NSIs from Partner and Openness

NSIs from Partner Latent Growth Curve

NSIs from Partner Slope

Entire

Sample
Openness Latent Growth Curve

Openness Slope

\begin{tabular}{ccc}
\hline & Entire & Sntire Sample \\
Sample & Sam
\end{tabular}

\section{NSIs from Partner}

Intercept

\section{Openness}

Intercept

Decrease at a less steep rate

Note. The summary of significant effects is presented above. A plus sign indicates that higher values of the variable are associated with a higher intercept. A minus sign indicates that higher values of the variable are associated with a lower intercept. Age did not moderate the cross-domain latent growth curve between NSIs from partner and openness. 
Table J9

Summary Results of Research Questions 7 and 8 for NSIs from Partner and Neuroticism

\section{NSIs from Partner Latent Growth Curve}

Neuroticism Latent Growth Curve

Neuroticism Slope

\section{Entire}

Entire Sample

Sample

\section{NSIs from Partner}

Intercept

\section{Neuroticism}

Intercept

Note. The summary of significant effects is presented above. A plus sign indicates that higher values of the variable are associated with a higher intercept. A minus sign indicates that higher values of the variable are associated with a lower intercept. Age did not moderate the cross-domain latent growth curve between NSIs from partner and neuroticism. 
Table J10

Summary Results of Research Questions 7 and 8 for NSIs from Partner and Extraversion

NSIs from Partner Latent Growth Curve

NSIs from Partner Slope

Entire

Sample

\section{NSIs from Partner}

Intercept

\section{Extraversion}

\section{Intercept}

Note. The summary of significant effects is presented above. A plus sign indicates that higher values of the variable are associated with a higher intercept. A minus sign indicates that higher values of the variable are associated with a lower intercept. Age did not moderate the cross-domain latent growth curve between NSIs from partner and extraversion. 
Table J11

Summary Results of Research Questions 7 and 8 for NSIs from Partner and Conscientiousness

\section{NSIs Latent Growth Curve}

$$
\text { NSIs Slope }
$$

Entire

Younger

Middle-Aged

Older

Adults

Adults

Sample

Adults

○

\section{NSIs Intercept}

Conscientiousness

Intercept

Note. The summary of significant effects is presented above. A plus sign indicates that higher values of the variable are associated

with a higher intercept. A minus sign indicates that higher values of the variable are associated with a lower intercept. ${ }^{\circ}$ indicates that the slope was not significant. Therefore, slope was not predicted.
Conscientiousness Latent Growth Curve

Conscientiousness Slope

○

$\begin{array}{llcc}\text { Entire } & \text { Younger } & \text { Middle- } & \text { Older } \\ \text { Sample } & \text { Adults } & \text { Aged Adults } & \text { Adults }\end{array}$


Table J12

Missing Data for NSIs from Partner across all MIDUS Waves

\begin{tabular}{lcc}
\hline & Missing Count & Missing Percent \\
\hline NSIs from Partner - MIDUS 1 & 0 & $0.00 \%$ \\
NSIs from Partner - MIDUS 2 & 0 & $0.00 \%$ \\
NSIs from Partner - MIDUS 3 & 0 & $0.00 \%$ \\
\hline
\end{tabular}


Table J13

Attrition Analyses Comparing Non-Multivariate Outliers and Multivariate Outliers for NSIs from Partner across all MIDUS Waves

\begin{tabular}{lccc}
\hline & Non-Multivariate & Multivariate & Difference Statistic \\
& Outliers & Outliers & \\
& $(n=1,403)$ & $(n=127)$ & \\
& $M, S D$ or $\%$ & $M, S D$ or $\%$ & \\
\hline NSIs from Partner - MIDUS 1 & $2.11,0.57$ & $2.15,0.68$ & $t(142.47)=-0.69$ \\
NSIs from Partner - MIDUS 2 & $2.05,0.57$ & $2.24,0.69$ & $\boldsymbol{t}(\mathbf{1 4 2 . 2 7})=\mathbf{- 2 . 8 9}$ \\
NSIs from Partner - MIDUS 3 & $2.01,0.61$ & $2.15,0.75$ & $\boldsymbol{t ( 1 4 1 . 6 2 )}=\mathbf{- 1 . 9 9}$
\end{tabular}

Note. Significant statistics $(p<.05)$ are bolded. 
Table J14

Research Question 1 - The Latent Growth Curves of NSIs from Partner

\begin{tabular}{|c|c|c|c|c|c|c|c|}
\hline & \multirow[t]{2}{*}{ Model Fit } & \multicolumn{2}{|c|}{ Intercept } & \multicolumn{2}{|c|}{ Slope } & \multicolumn{2}{|c|}{ Covariance } \\
\hline & & $b$ & S.E. & $b$ & S.E. & $b$ & S.E. \\
\hline \multirow[t]{4}{*}{ NSIs from Partner } & $\chi^{2}(3)=7.68$ & $2.114^{*}$ & 0.014 & $-0.005^{*}$ & 0.001 & -0.001 & 0.001 \\
\hline & $\mathrm{CMIN} / \mathrm{DF}=2.56$ & & & & & & \\
\hline & $\mathrm{CFI}=.997$ & & & & & & \\
\hline & RMSEA $=.03$ & & & & & & \\
\hline
\end{tabular}

Note. Significant statistics $(p<.05)$ are bolded. An asterisk indicates that there is significant variability surrounding the estimate. A blue value indicates that the estimate . 
Table J15

Research Question 2 - Demographic Variables and the Big Five Personality Traits Predicting the Latent Growth Curve of NSIs from Partner

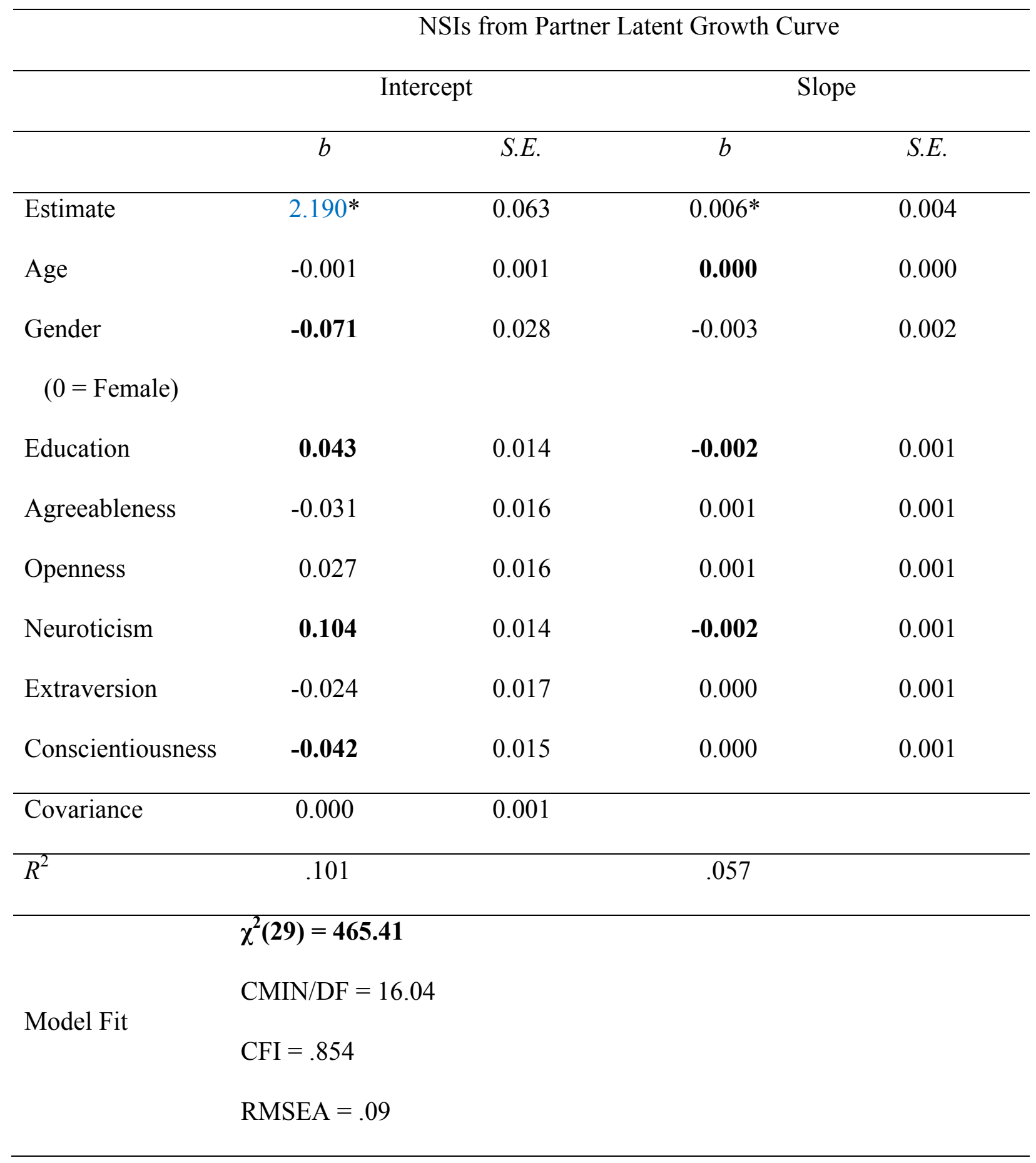


Note. Significant statistics $(p<.05)$ are bolded. $R^{2}=$ variance accounted for. An asterisk indicates that there is significant variability surrounding the estimate. A blue value indicates that the estimate is significantly different than zero. Type I errors were accounted for utilizing the false discovery rate adjustment. A green value indicates that the estimate was no longer statistically significant after the false discovery rate adjustments. To aid in the interpretation of the estimates, education, agreeableness, openness, neuroticism, extraversion, and conscientiousness were standardized. As such, a unit increase or decrease in these variables represent a standard deviation increase or decrease, respectively. Age was not manipulated so that a unit increase or decrease represents an increase or decrease of a year, respectively. 
Table J16

The Unconstrained Model Compared to the Constrained Model for the Multigroup Latent Growth Curve of NSIs from Partner

\begin{tabular}{lcccc}
\hline & $\chi^{2}$ & CFI & $\Delta \chi^{2}$ & $\Delta$ CFI \\
\hline NSIs from Partner & & & & \\
Unconstrained Model & $\chi^{\mathbf{2}(\mathbf{1 1})=\mathbf{3 8 . 6 1}}$ & .981 & & \\
Constrained Model & $\chi^{2}(\mathbf{1 7})=\mathbf{4 8 . 9 4}$ & .977 & $\chi^{2}(6)=10.33$ & .004 \\
\hline
\end{tabular}

Note. Significant statistics $(p<.05)$ are bolded. The unconstrained model is where the parameters were freely estimated across the three age groups. The constrained model is where the parameters were constrained to be equal across the three age groups. A change of .01 or greater for the CFI difference statistic was used to determine significance (Little, 2013). Both the chi-square and the CFI difference statistics were computed because the chi-square statistic is commonly used, however, it is sensitive to large sample sizes. The CFI is more robust to larger sample sizes (Little, 2013). 
Table J17

Research Question 5 - Demographic Variables and NSIs from Partner Predicting the Latent Growth Curve of Agreeableness

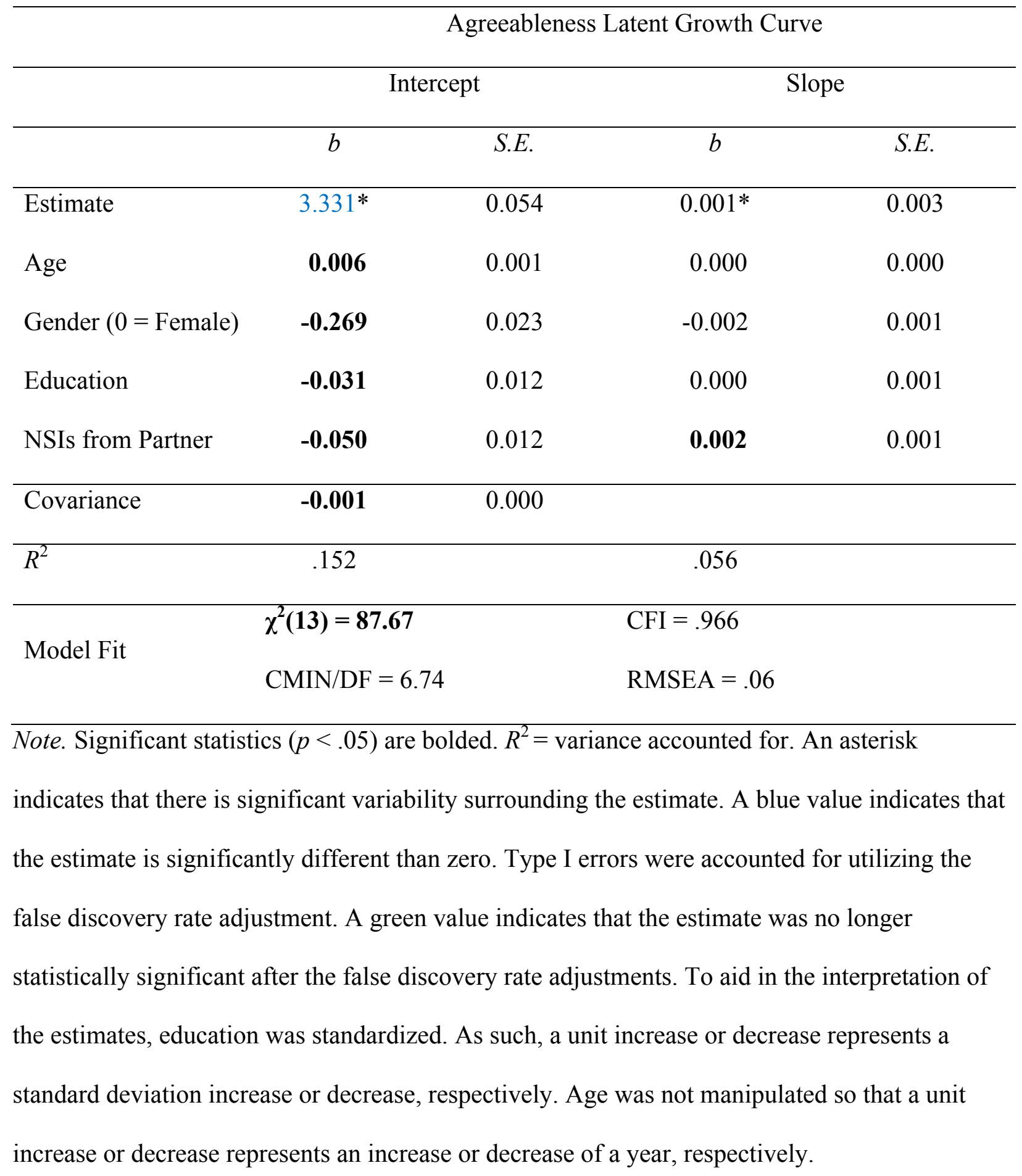


Table J18

Research Question 5 - Demographic Variables and from Partner Predicting the Latent Growth Curve of Openness

\section{Openness Latent Growth Curve}

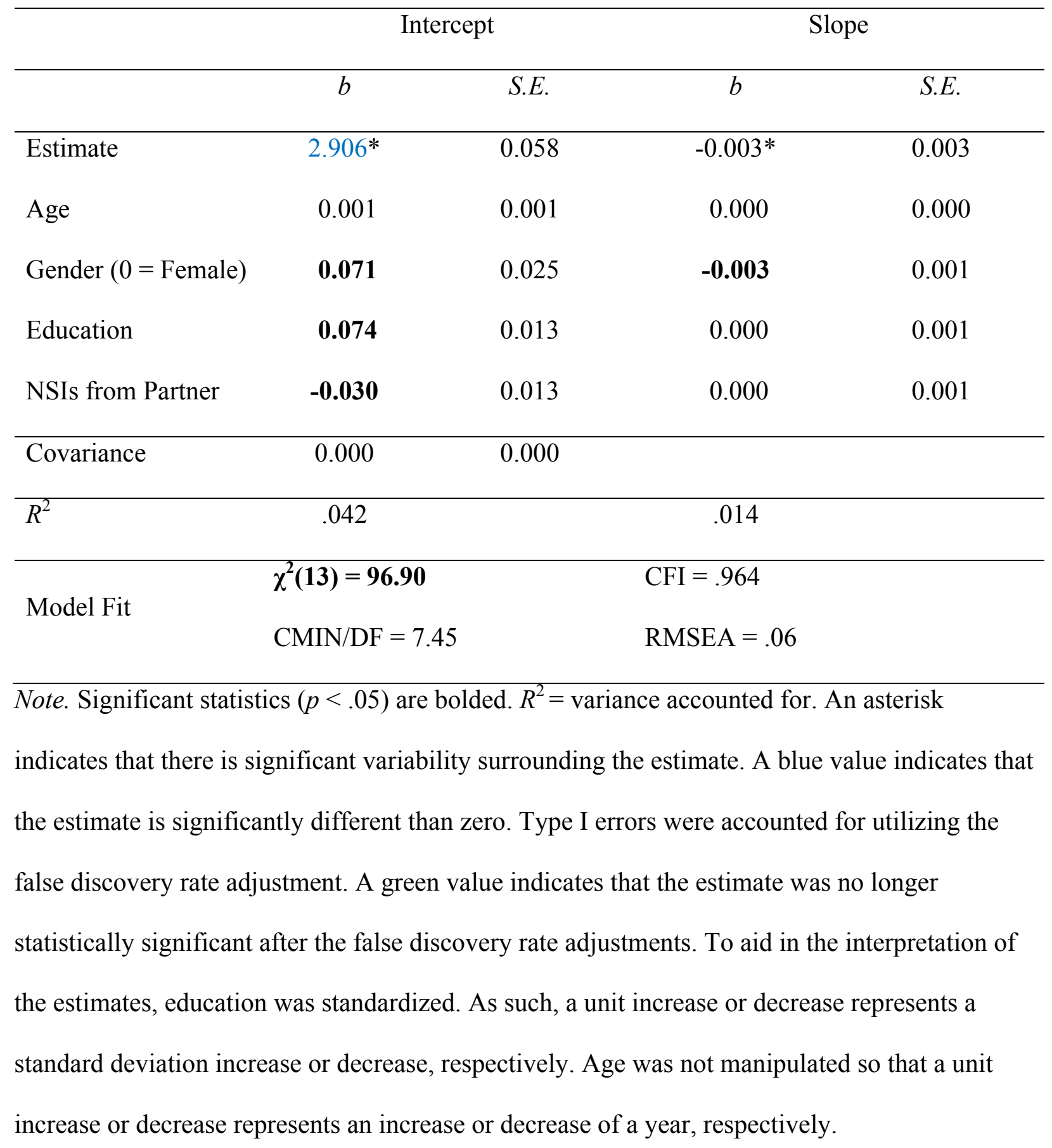


Table J19

Research Question 5 - Demographic Variables and NSIs from Partner Predicting the Latent Growth Curve of Neuroticism

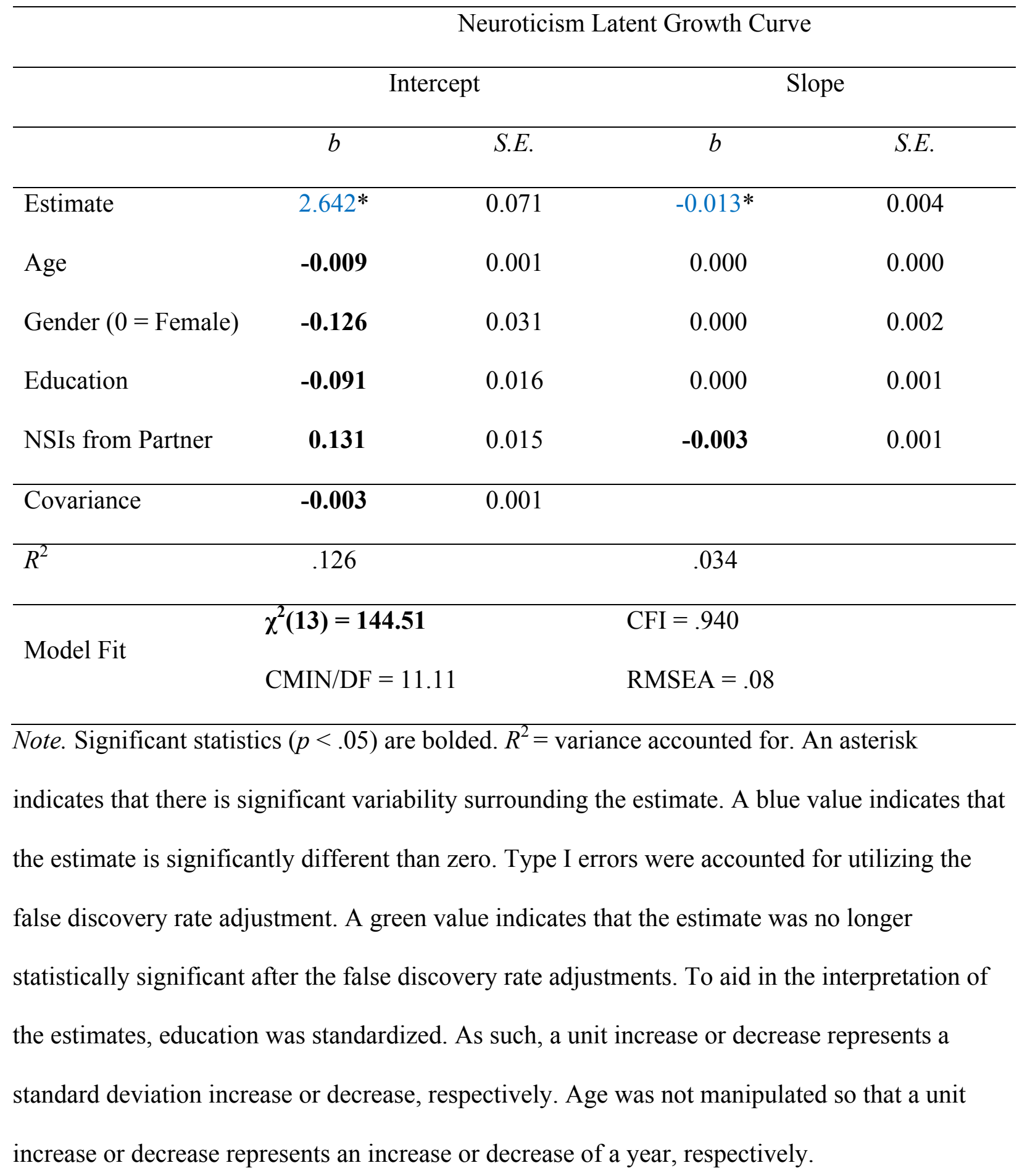


Table J20

Research Question 5 - Demographic Variables and NSIs from Partner Predicting the Latent Growth Curve of Extraversion

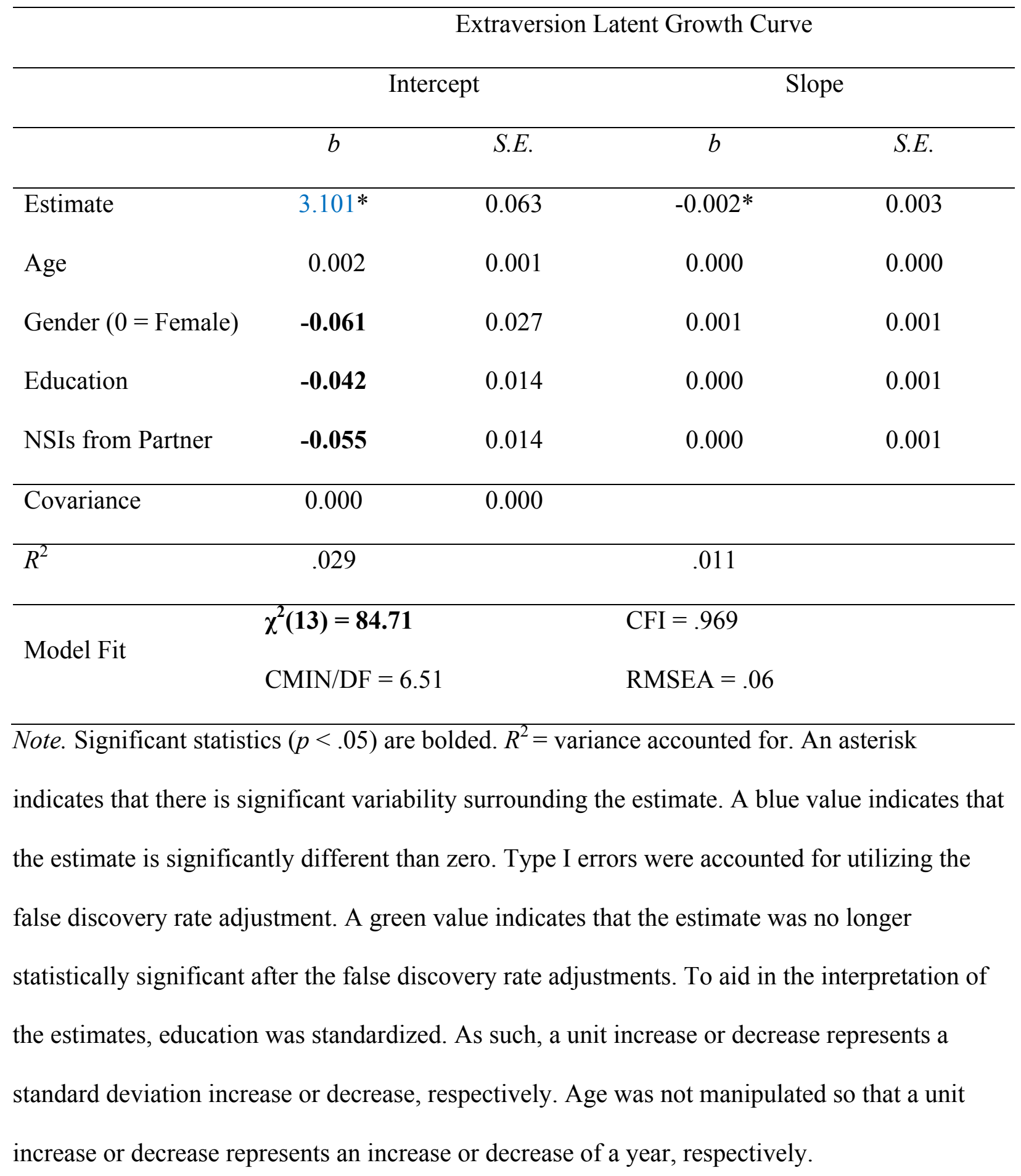


Table $\mathrm{J} 21$

Research Question 5 - Demographic Variables and NSIs from Partner Predicting the Latent Growth Curve of Conscientiousness

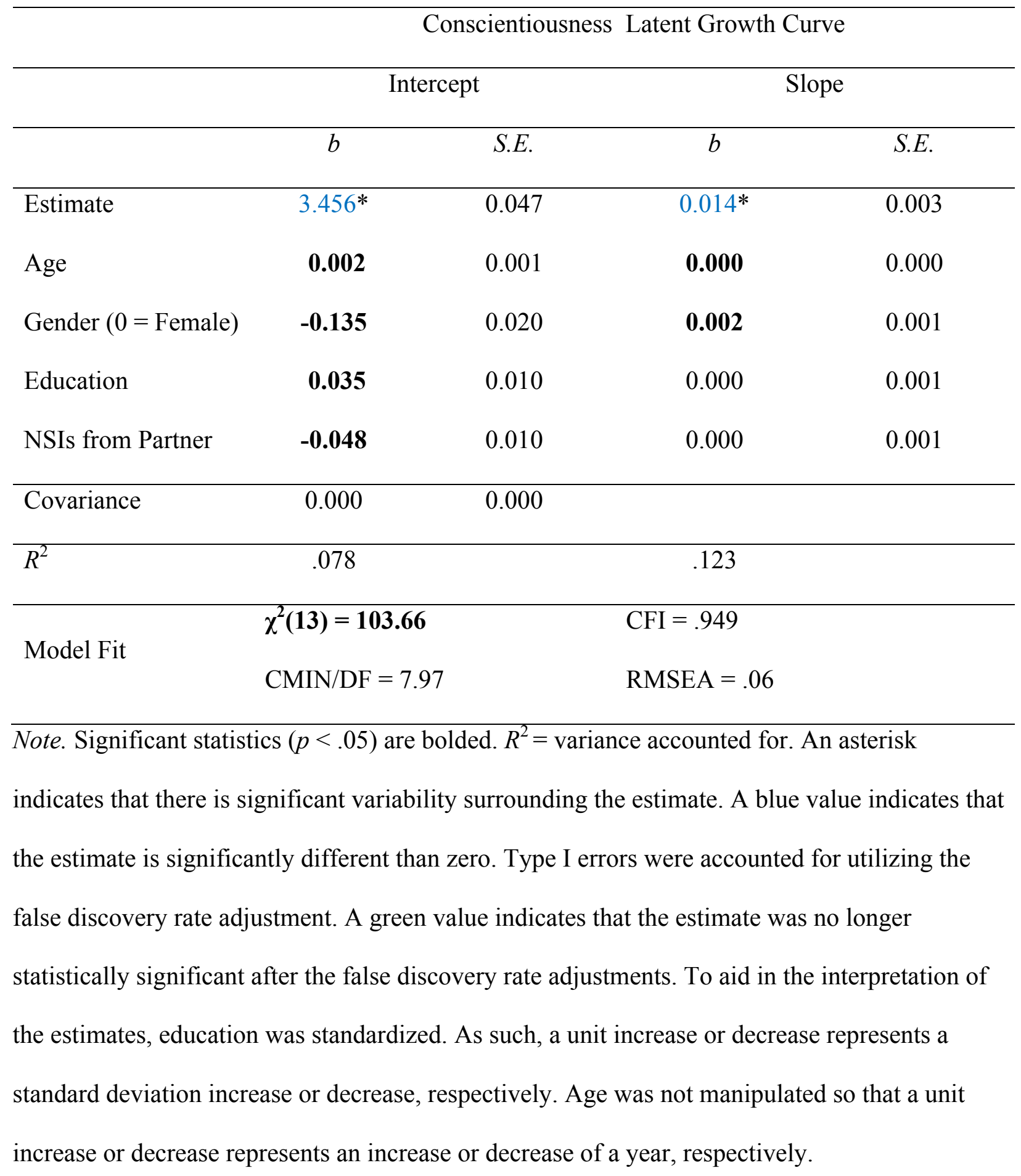


Table J22

Research Question 6 - Demographic Variables and NSIs from Partner Predicting the Latent Growth Curve of Agreeableness for Younger Adults

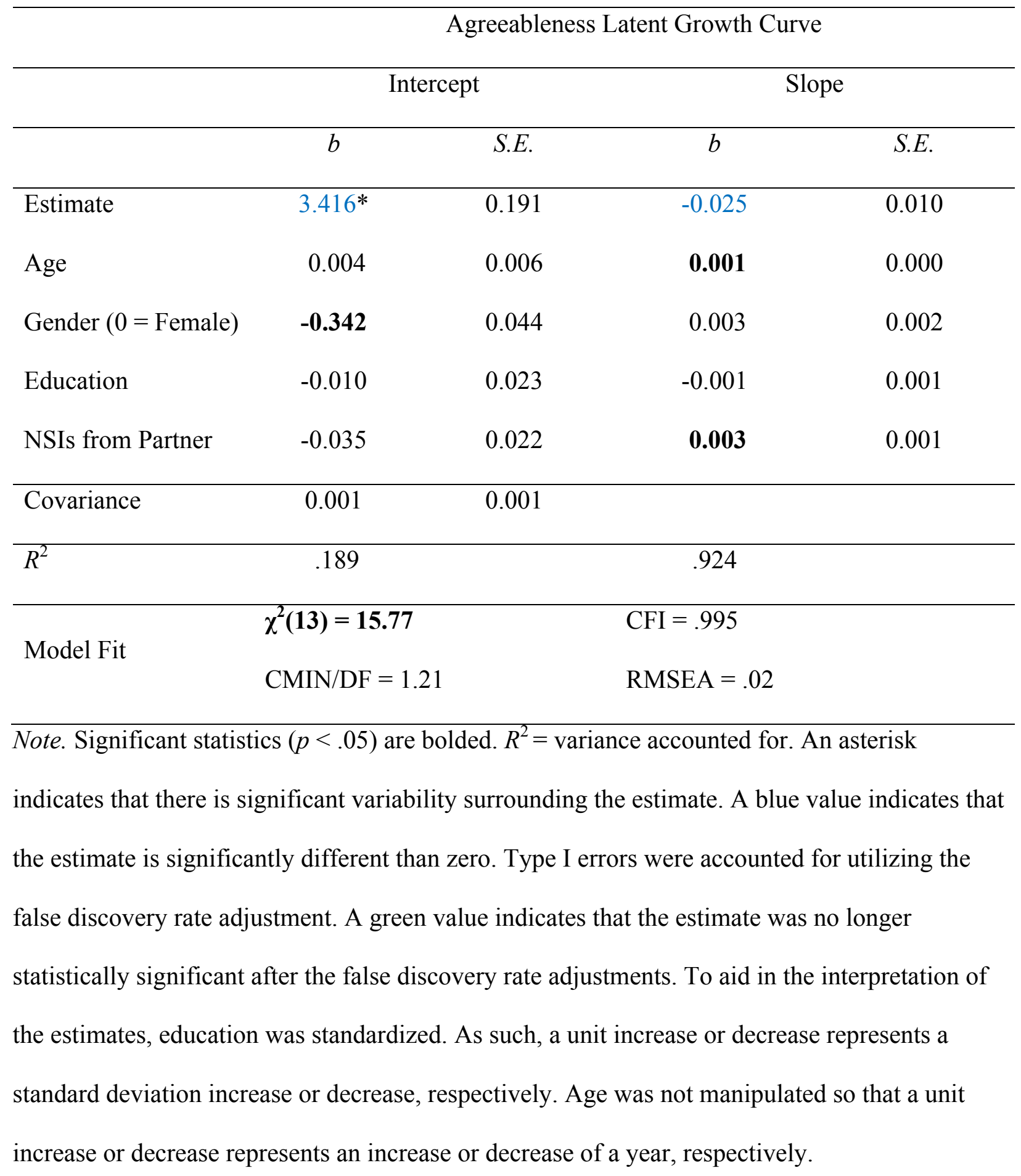


Table J23

Research Question 6 - Demographic Variables and NSIs from Partner Predicting the Latent Growth Curve of Agreeableness for Middle-Aged Adults

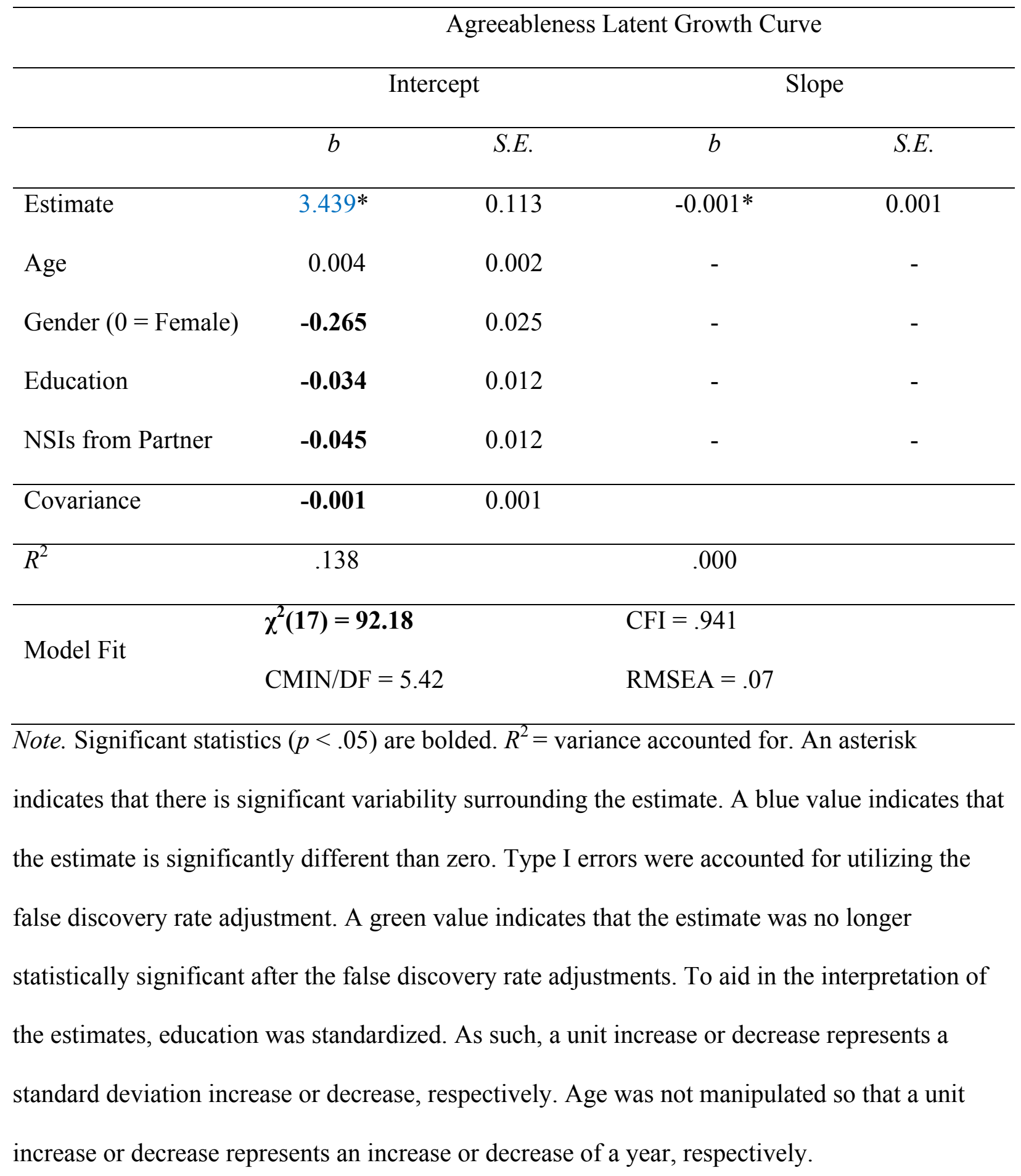


Table J24

Research Question 6 - Demographic Variables and NSIs from Partner Predicting the Latent Growth Curve of Agreeableness for Older Adults

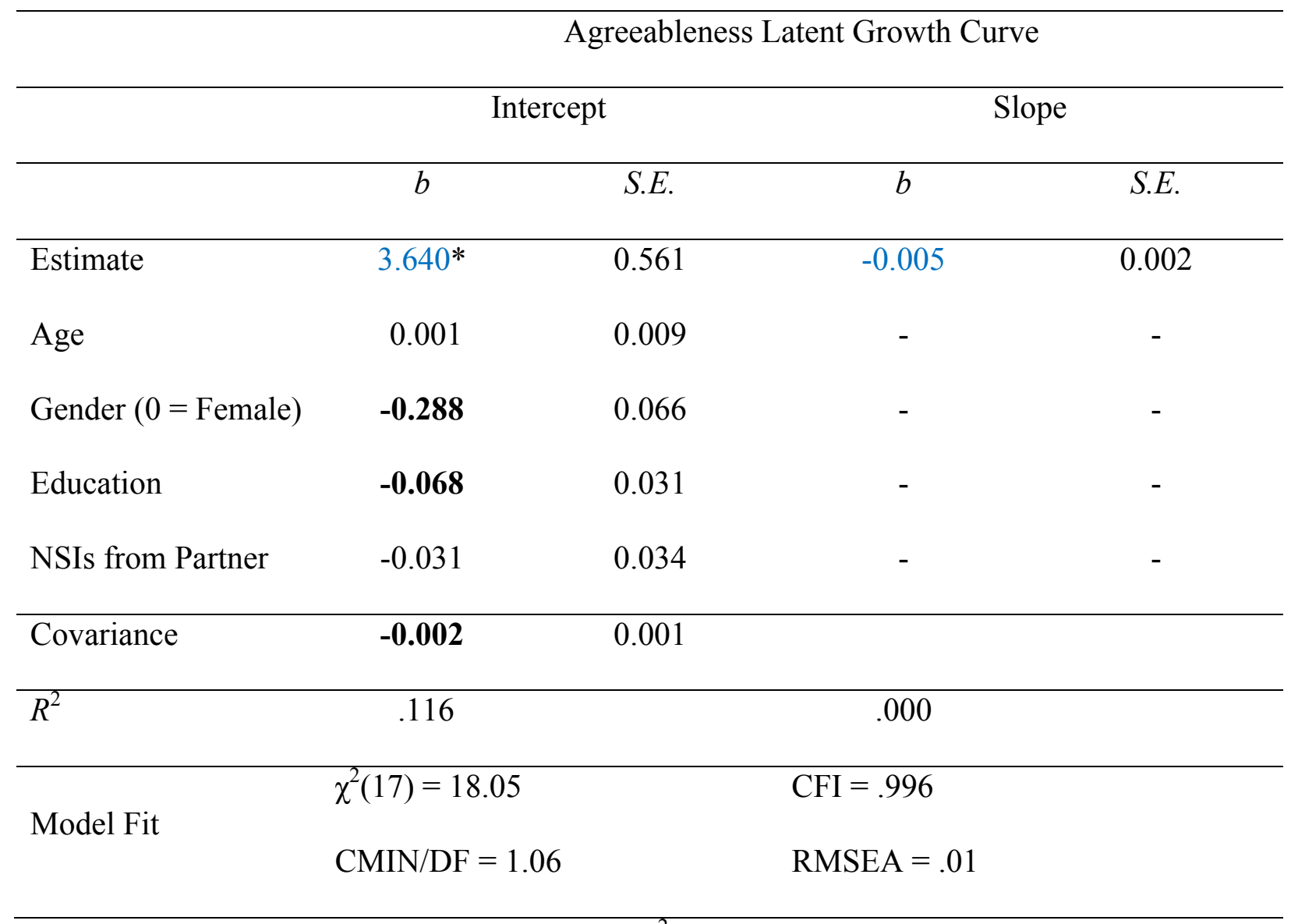

Note. Significant statistics $(p<.05)$ are bolded. $R^{2}=$ variance accounted for. An asterisk indicates that there is significant variability surrounding the estimate. A blue value indicates that the estimate is significantly different than zero. Type I errors were accounted for utilizing the false discovery rate adjustment. A green value indicates that the estimate was no longer statistically significant after the false discovery rate adjustments. To aid in the interpretation of the estimates, education was standardized. As such, a unit increase or decrease represents a standard deviation increase or decrease, respectively. Age was not manipulated so that a unit increase or decrease represents an increase or decrease of a year, respectively. 
Table $\mathbf{J} 25$

Research Question 6 - Demographic Variables and from Partner Predicting the Latent Growth Curve of Openness for Younger Adults

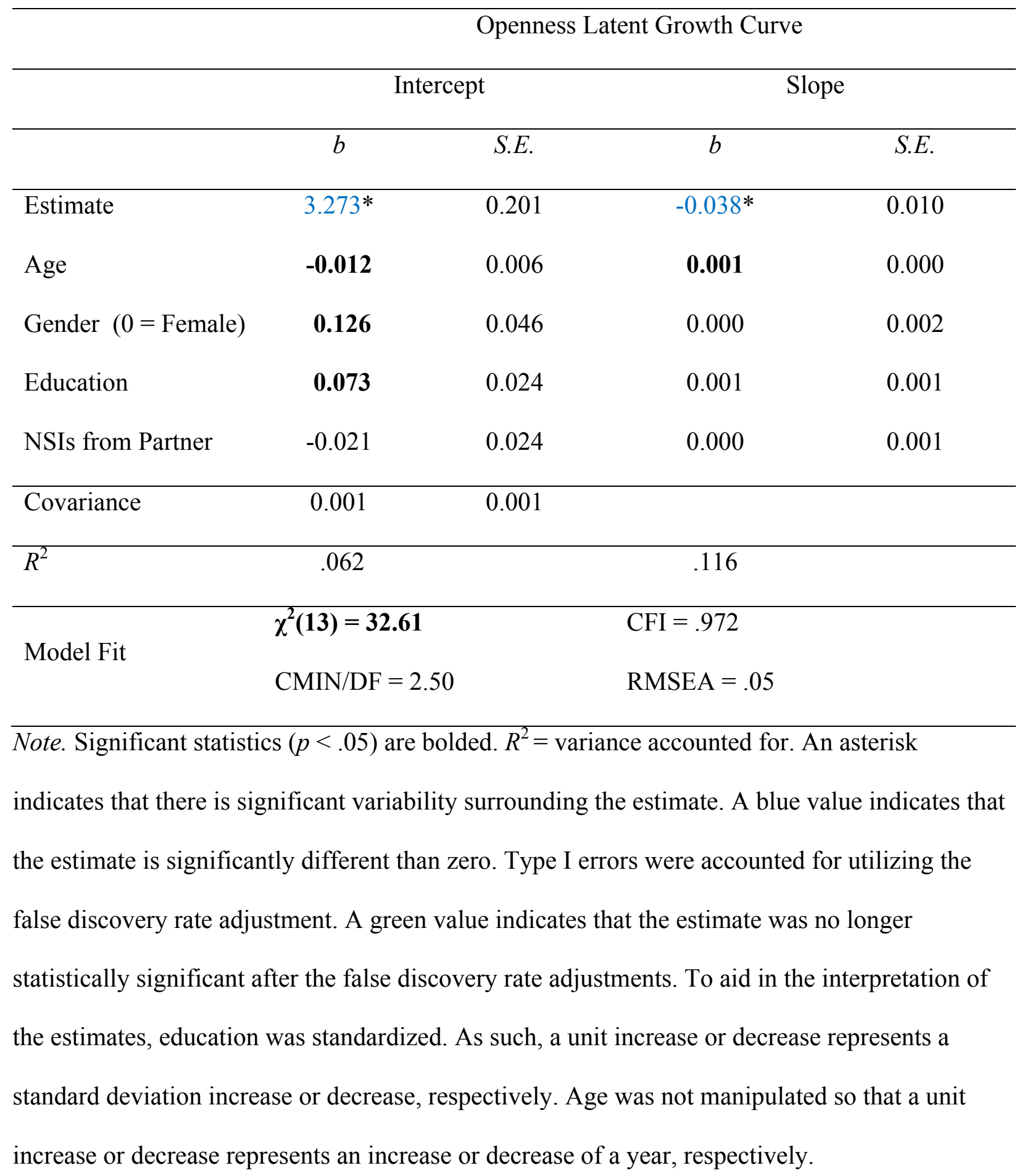


Table J26

Research Question 6 - Demographic Variables and from Partner Predicting the Latent Growth Curve of Openness for Middle-Aged Adults

\section{Openness Latent Growth Curve}

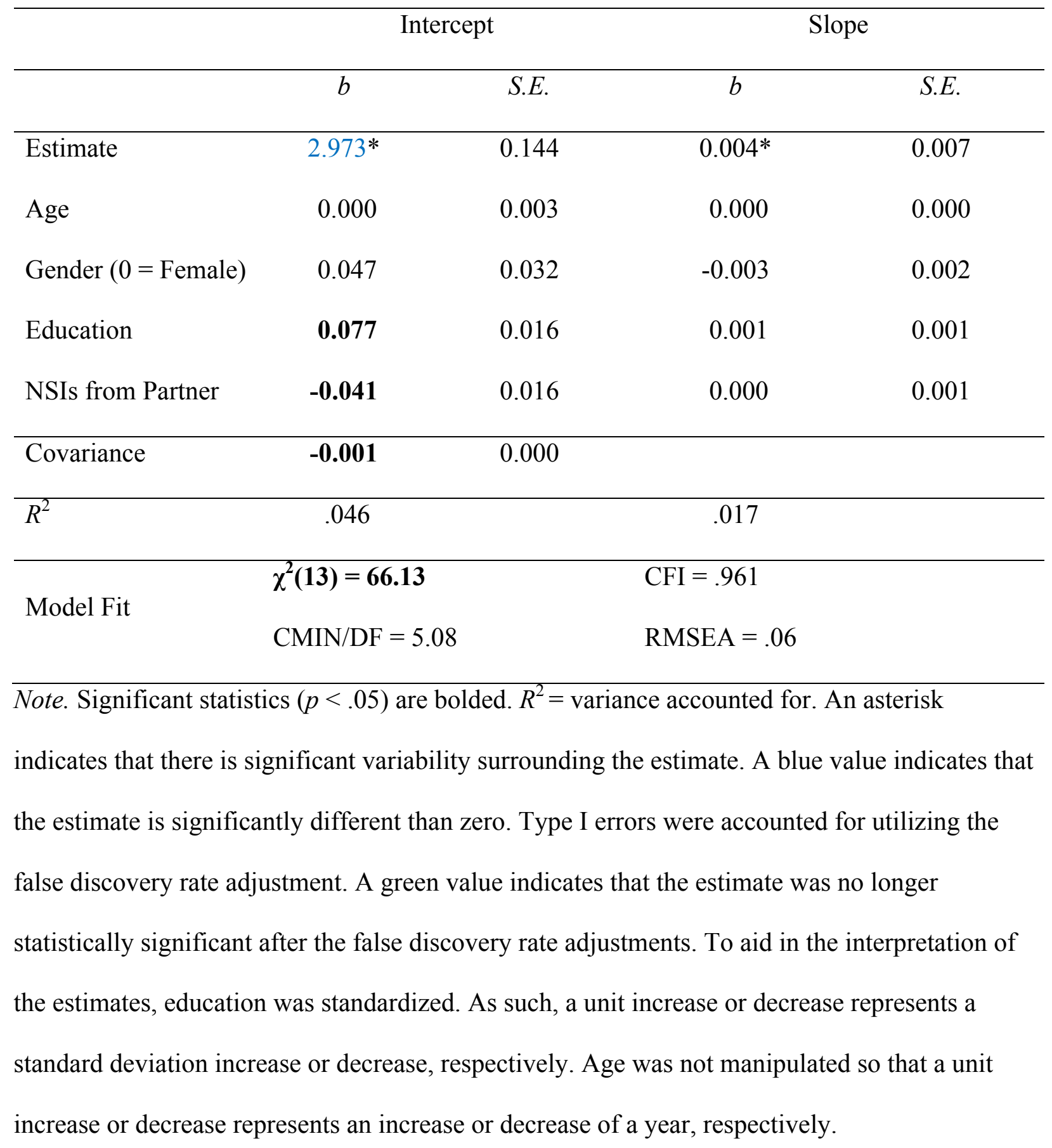


Table J27

Research Question 6 - Demographic Variables and from Partner Predicting the Latent Growth Curve of Openness for Older Adults

\section{Openness Latent Growth Curve}

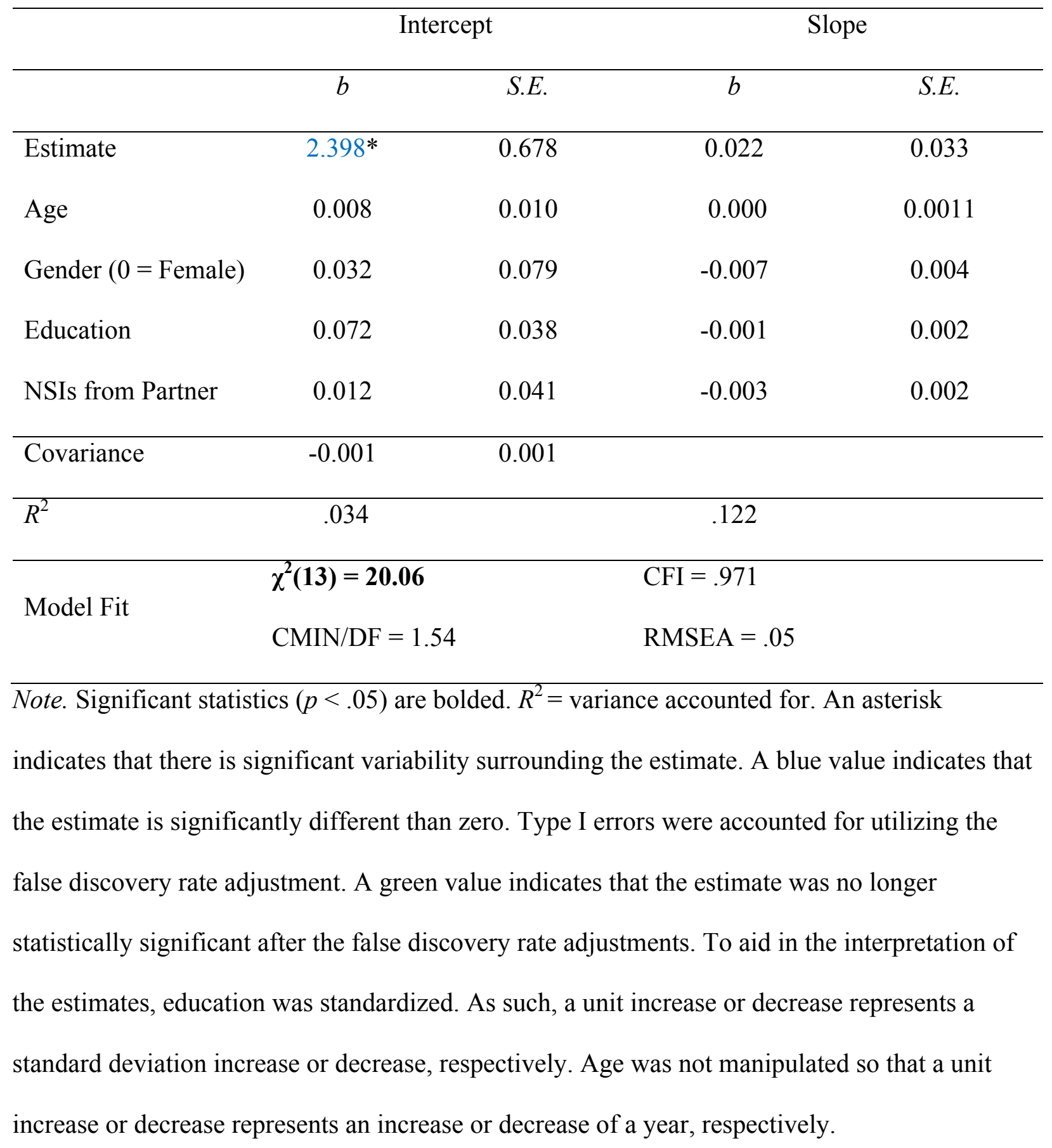


Table J28

Research Question 6 - Demographic Variables and NSIs from Partner Predicting the Latent Growth Curve of Neuroticism for Younger Adults

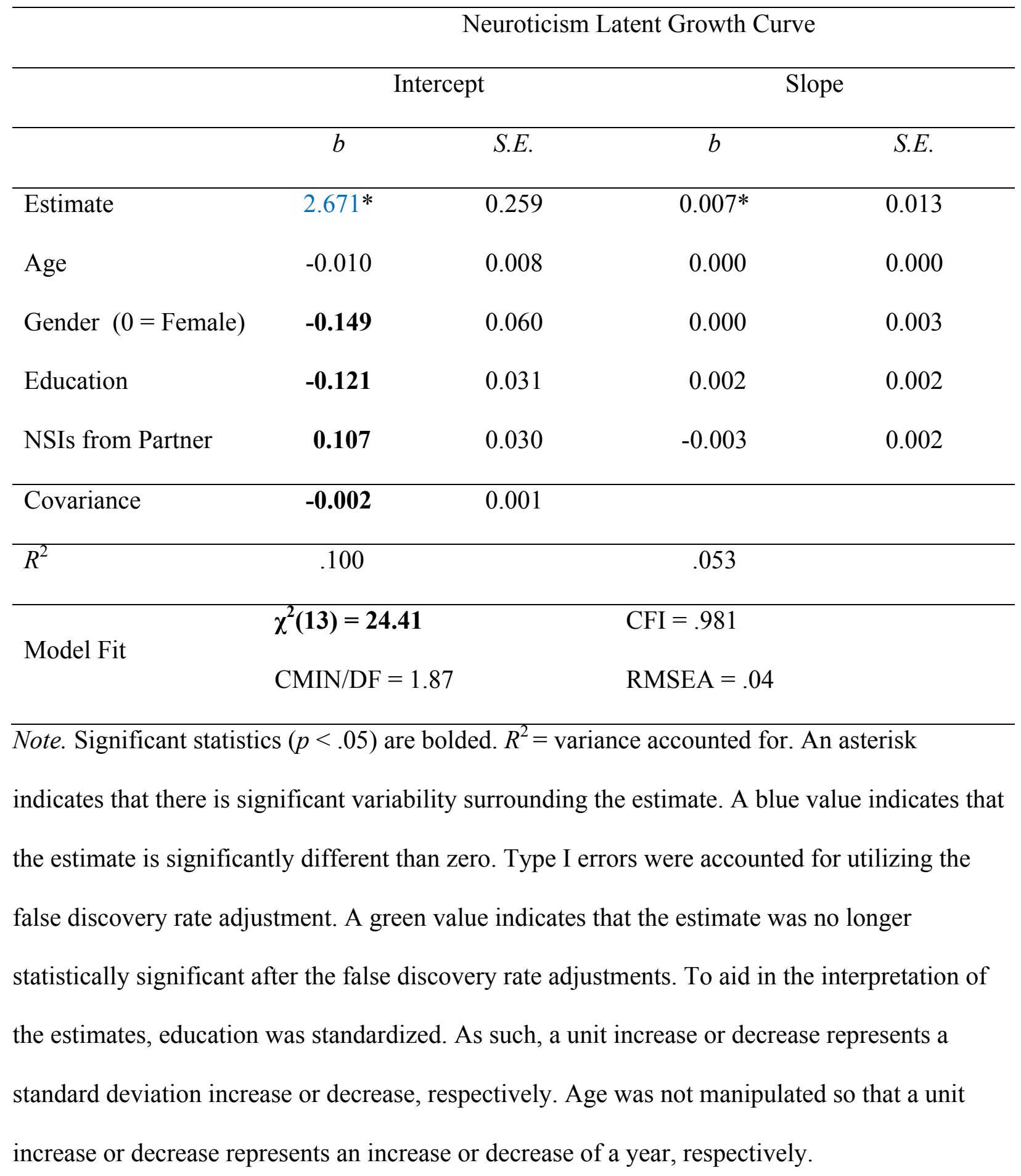


Table J29

Research Question 6 - Demographic Variables and NSIs from Partner Predicting the Latent Growth Curve of Neuroticism for Middle-Aged Adults

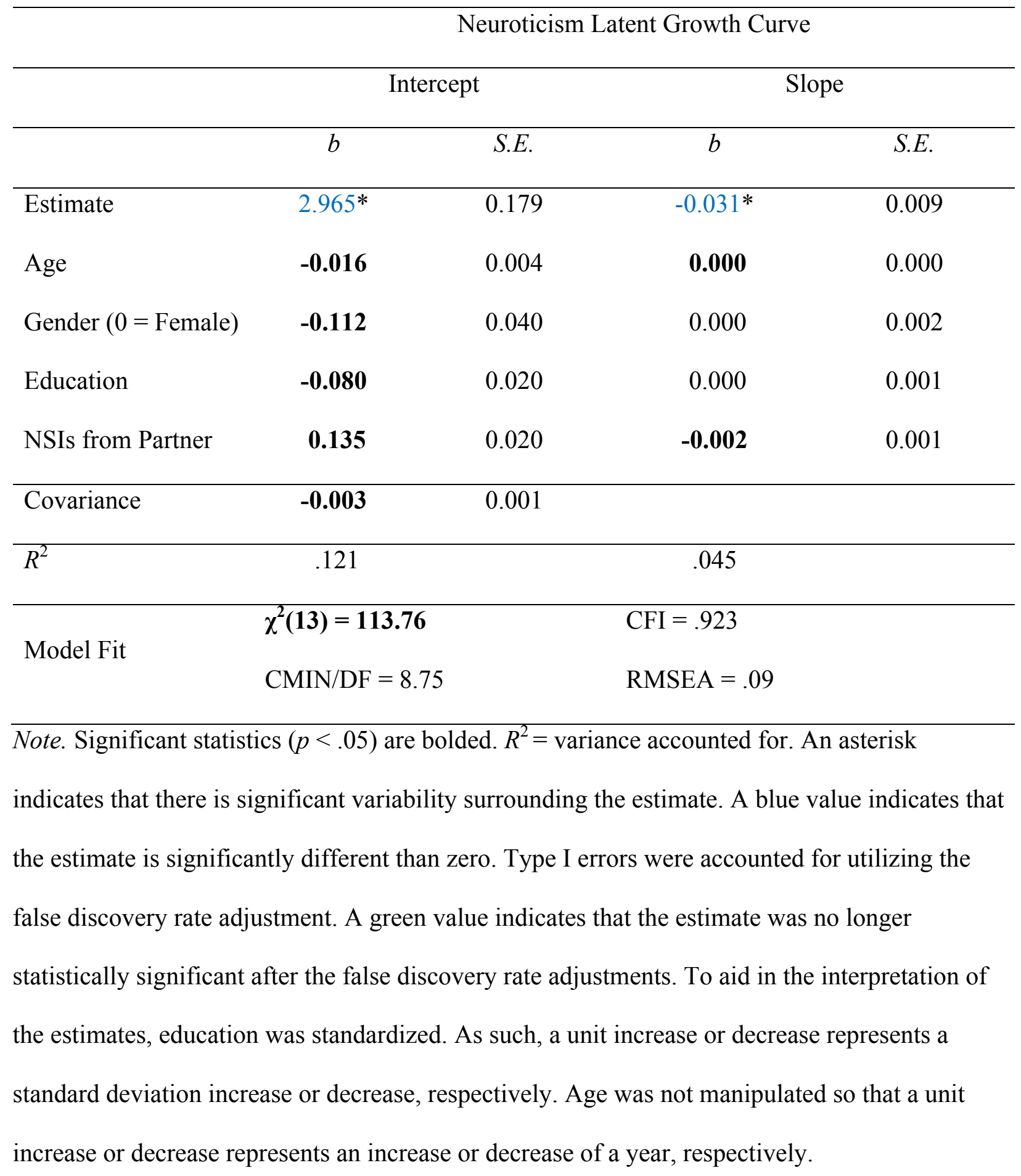


Table J30

Research Question 6 - Demographic Variables and NSIs from Partner Predicting the Latent Growth Curve of Neuroticism for Older Adults

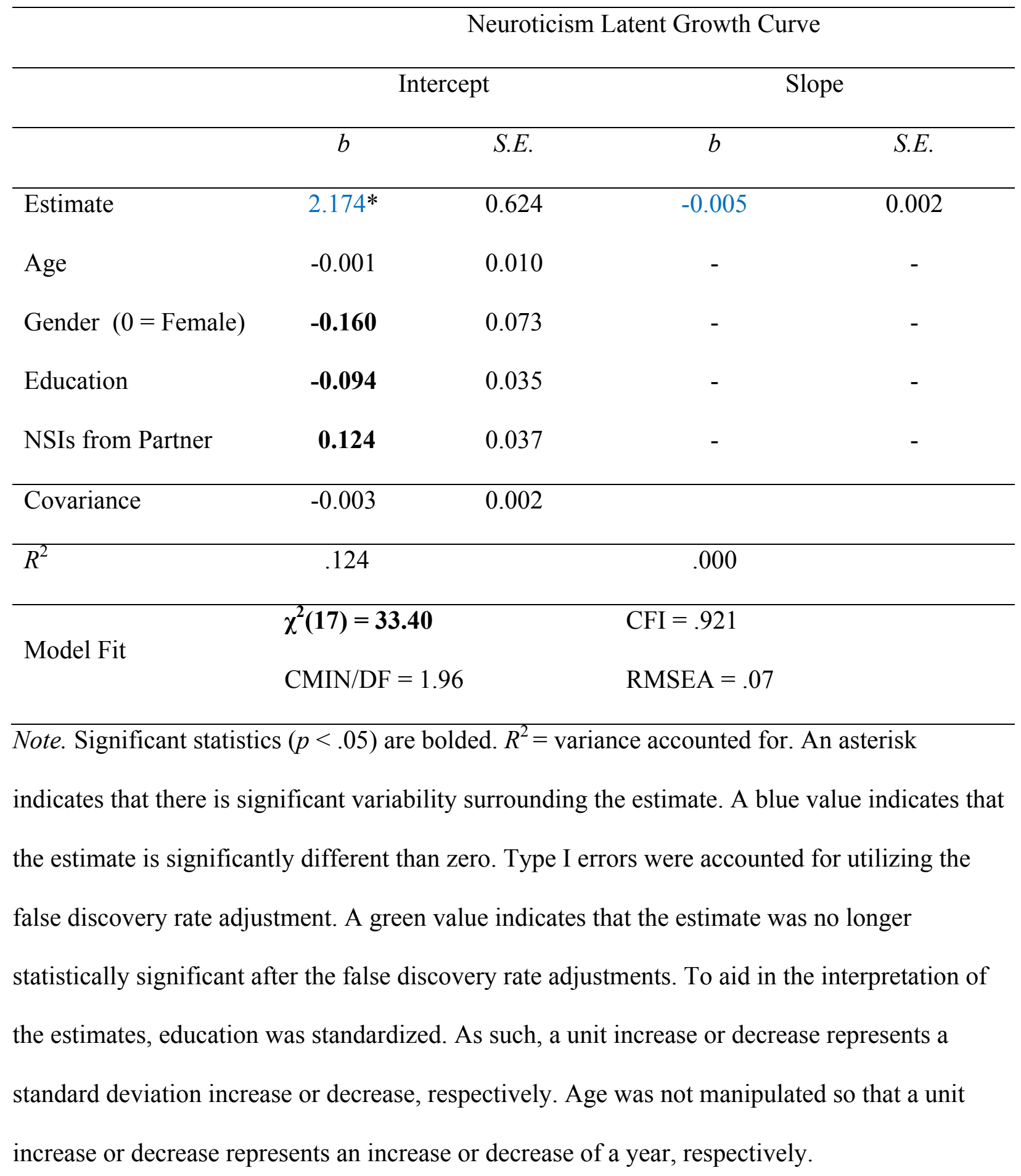


Table J31

Research Question 6 - Demographic Variables and NSIs from Partner Predicting the Latent Growth Curve of Conscientiousness for Younger Adults

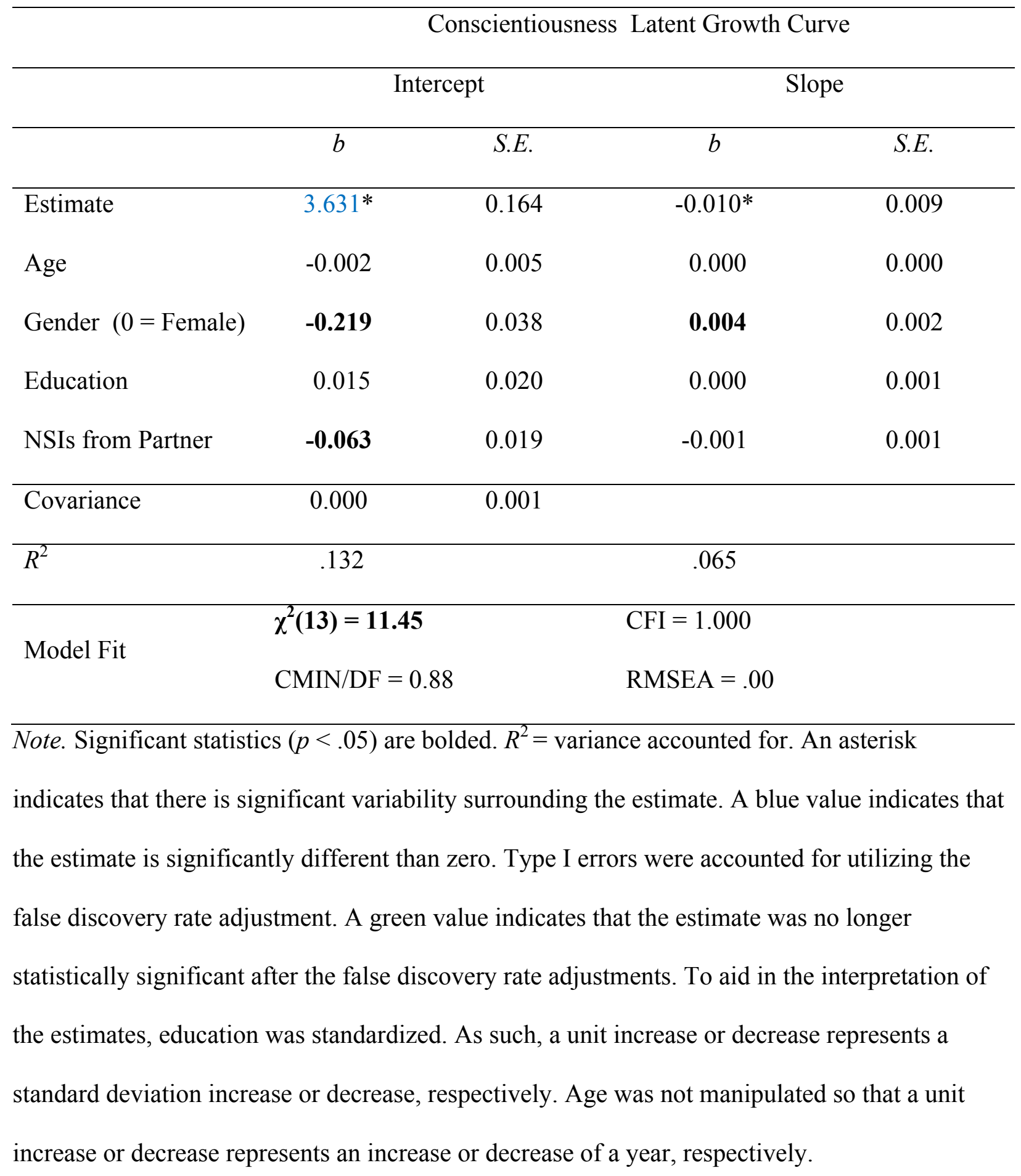


Table J32

Research Question 6 - Demographic Variables and NSIs from Partner Predicting the Latent Growth Curve of Conscientiousness for Middle-Aged Adults

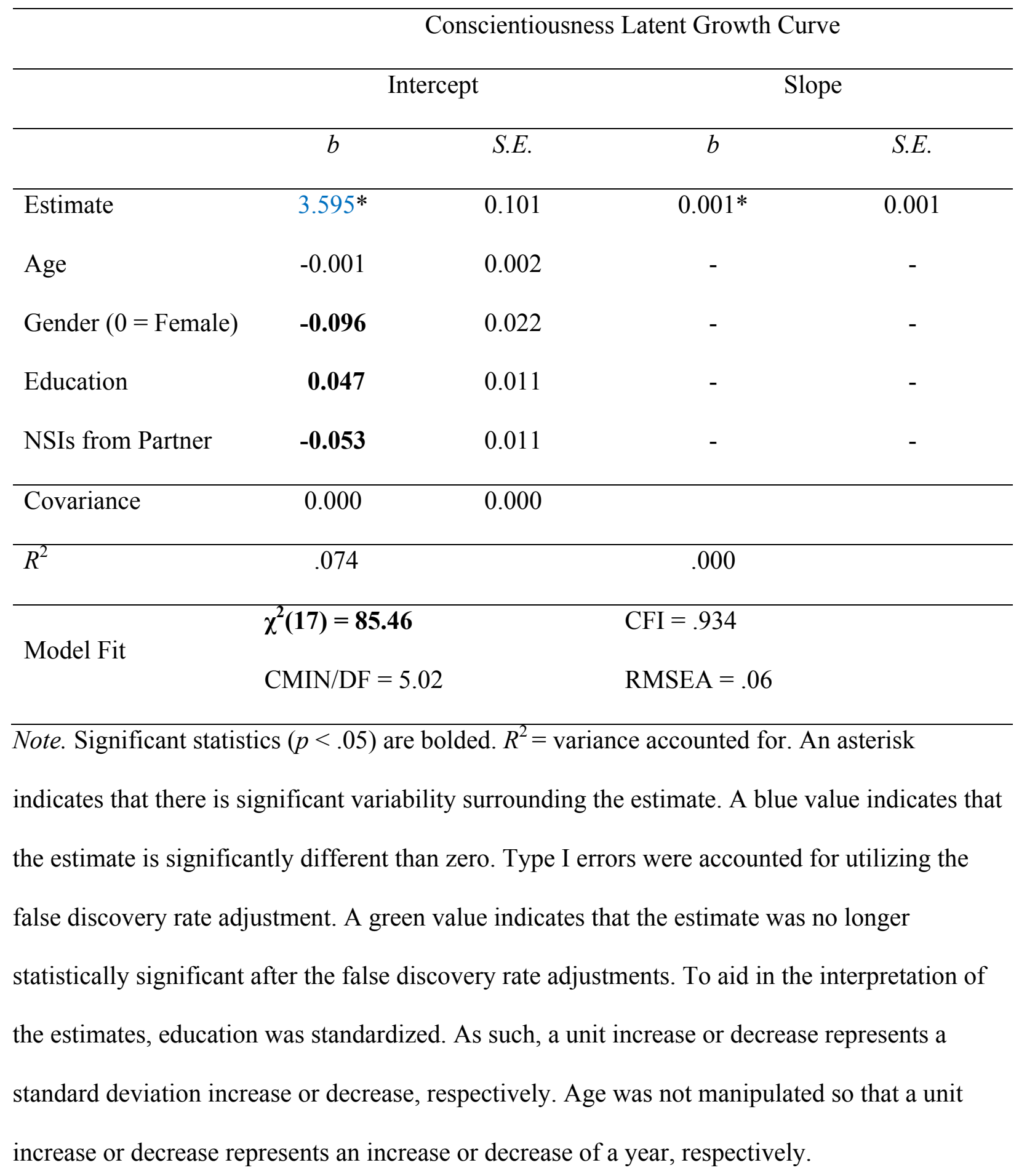


Table J33

Research Question 6 - Demographic Variables and NSIs from Partner Predicting the Latent Growth Curve of Conscientiousness for Older Adults

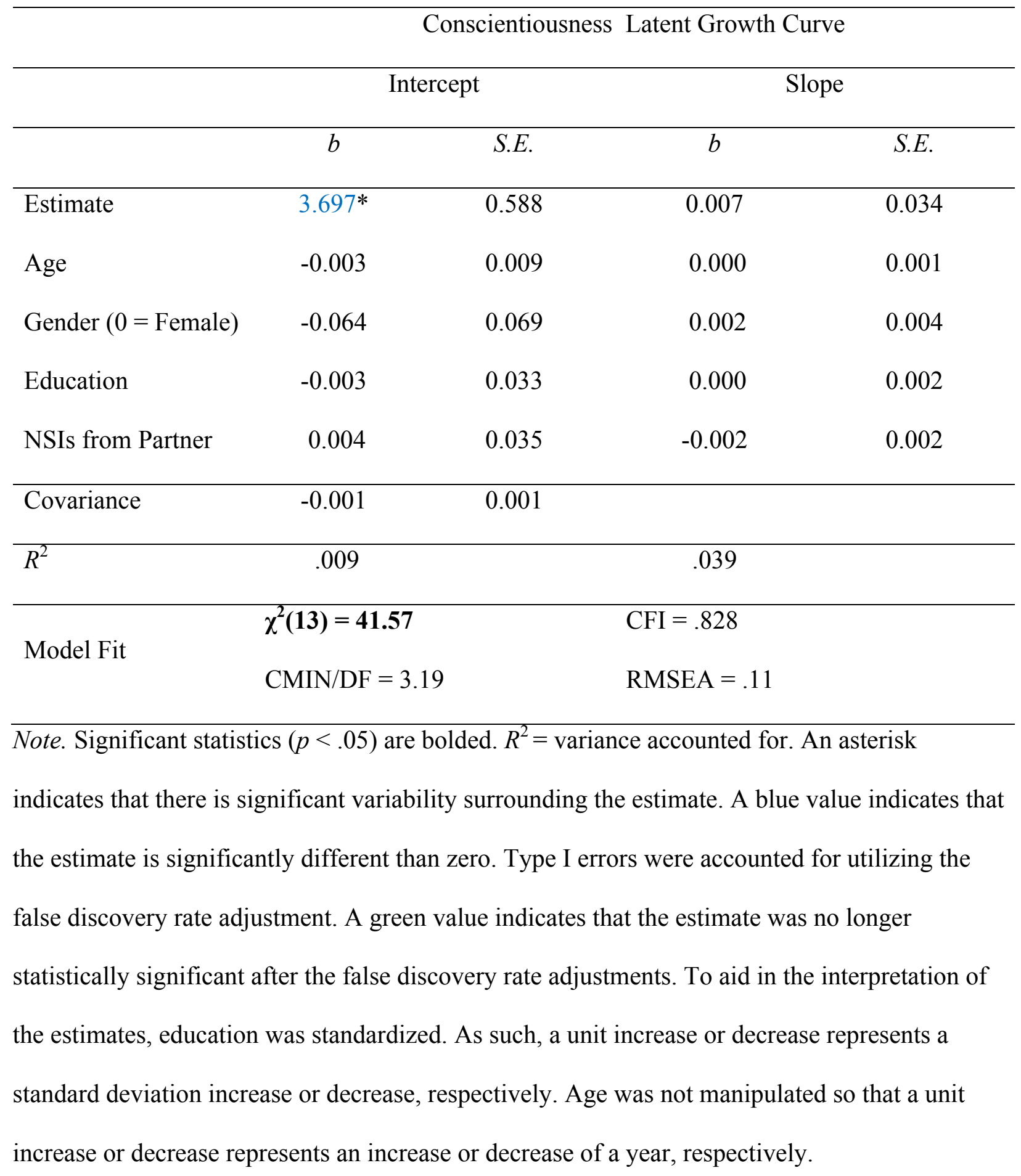


Table J34

Preliminary Models that Examine Significant Parameters that can be accounted for with the Cross-Domain Latent Growth Curves

\begin{tabular}{|c|c|c|c|c|c|c|c|}
\hline & \multirow[t]{2}{*}{ Model Fit } & \multicolumn{2}{|c|}{ Intercept } & \multicolumn{2}{|c|}{ Slope } & \multicolumn{2}{|c|}{ Covariance } \\
\hline & & $b$ & S.E. & $b$ & S.E. & $b$ & S.E. \\
\hline NSIs from Partner and & $\chi^{2}(14)=31.48$ & $2.114^{*}$ & 0.014 & $-0.005^{*}$ & 0.001 & -0.001 & 0.001 \\
\hline \multirow[t]{3}{*}{ Agreeableness } & $\mathrm{CMIN} / \mathrm{DF}=2.24$ & $3.456^{*}$ & 0.012 & $-0.002 *$ & 0.001 & -0.001 & 0.000 \\
\hline & $\mathrm{CFI}=.995$ & & & & & & \\
\hline & RMSEA $=.02$ & & & & & & \\
\hline NSIs from Partner and & $\chi^{2}(14)=39.76$ & $2.114^{*}$ & 0.014 & $-0.005^{*}$ & 0.001 & -0.001 & 0.001 \\
\hline \multirow[t]{3}{*}{ Openness } & $\mathrm{CMIN} / \mathrm{DF}=2.84$ & $2.977 *$ & 0.013 & $-0.006 *$ & 0.001 & 0.000 & 0.000 \\
\hline & $\mathrm{CFI}=.993$ & & & & & & \\
\hline & RMSEA $=.03$ & & & & & & \\
\hline NSIs from Partner and & $\chi^{2}(14)=128.97$ & $2.114^{*}$ & 0.014 & $-0.005^{*}$ & 0.001 & -0.001 & 0.001 \\
\hline \multirow[t]{3}{*}{ Neuroticism } & $\mathrm{CMIN} / \mathrm{DF}=9.21$ & $2.163^{*}$ & 0.016 & $-0.008 *$ & 0.001 & -0.003 & 0.001 \\
\hline & $\mathrm{CFI}=.967$ & & & & & & \\
\hline & $\mathrm{RMSEA}=.07$ & & & & & & \\
\hline
\end{tabular}




\begin{tabular}{|c|c|c|c|c|c|c|c|}
\hline NSIs from Partner and & $\chi^{2}(14)=28.91$ & $2.114^{*}$ & 0.014 & $-0.005^{*}$ & 0.001 & -0.001 & 0.001 \\
\hline \multirow[t]{3}{*}{ Extraversion } & $\mathrm{CMIN} / \mathrm{DF}=2.06$ & $3.183^{*}$ & 0.014 & $-0.006^{*}$ & 0.001 & 0.000 & 0.000 \\
\hline & $\mathrm{CFI}=.996$ & & & & & & \\
\hline & $\mathrm{RMSEA}=.01$ & & & & & & \\
\hline NSIs from Partner and & $\chi^{2}(14)=45.09$ & $2.114^{*}$ & 0.014 & $-0.005^{*}$ & 0.001 & -0.001 & 0.001 \\
\hline \multirow[t]{3}{*}{ Conscientiousness } & $\mathrm{CMIN} / \mathrm{DF}=3.22$ & $3.493 *$ & 0.010 & $0.001 *$ & 0.001 & 0.000 & 0.000 \\
\hline & $\mathrm{CFI}=.990$ & & & & & & \\
\hline & RMSEA $=.03$ & & & & & & \\
\hline
\end{tabular}

Note. Significant statistics $(p<.05)$ are bolded. An asterisk indicates that there is significant variability surrounding the estimate. A blue value indicates that the estimate is significantly different than zero. 
Table J35

Research Question 7 - Examination of the Bidirectional Association between the Latent Growth Curves of NSIs from Partner and Agreeableness After Accounting for the Demographic Variables and Remaining Personality Traits

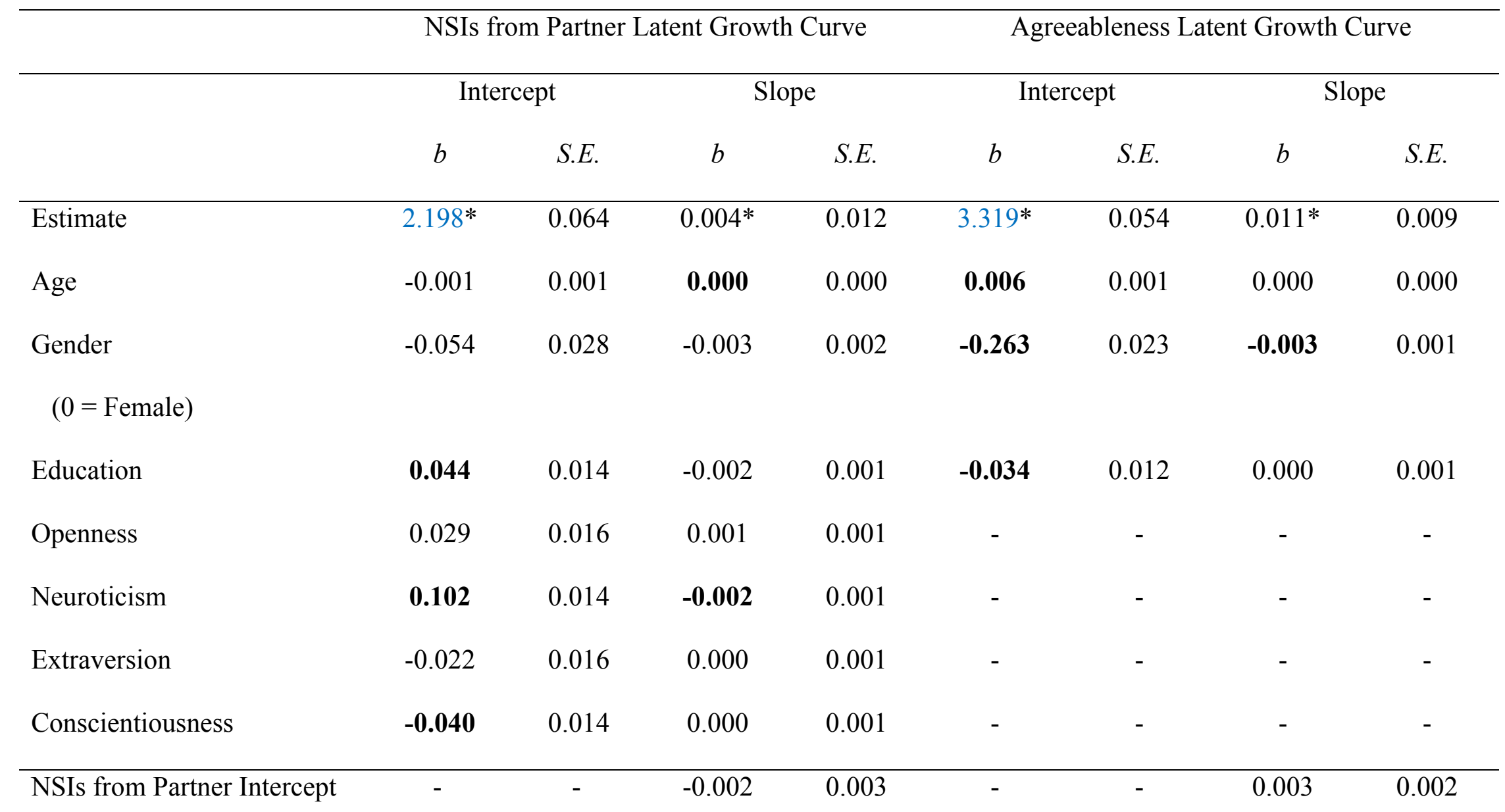




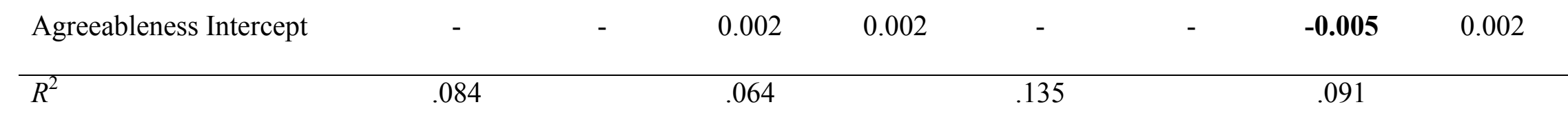

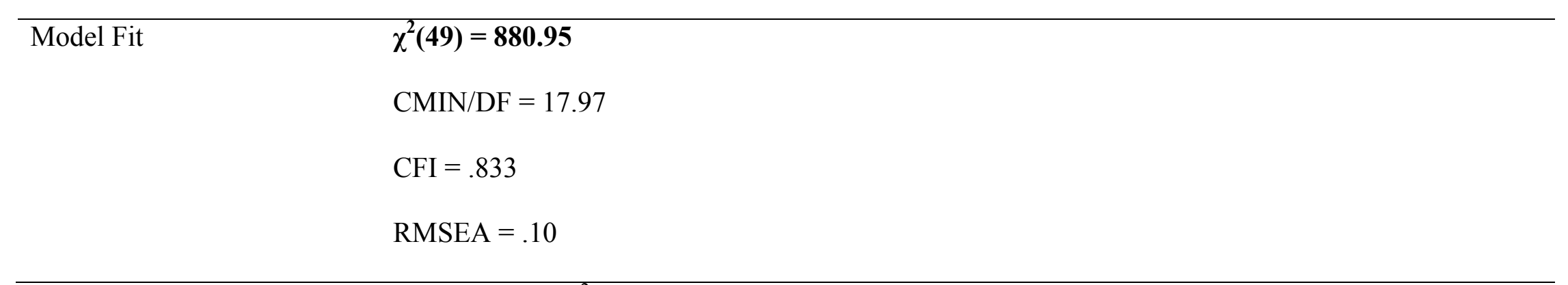

Note. Significant statistics $(p<.05)$ are bolded. $R^{2}=$ variance accounted for. An asterisk indicates that there is significant variability surrounding the estimate. A blue value indicates that the estimate is significantly different than zero. Type I errors were accounted for utilizing the false discovery rate adjustment. A green value indicates that the estimate was no longer statistically significant after the false discovery rate adjustments. To aid in the interpretation of the estimates, education, agreeableness, openness, neuroticism, extraversion, and conscientiousness were standardized. As such, a unit increase or decrease in these variables represent a standard deviation increase or decrease, respectively. Age was not manipulated so that a unit increase or decrease represents an increase or decrease of a year, respectively. 
Table J36

Research Question 7 - Examination of the Bidirectional Association between the Latent Growth Curves of NSIs from Partner and Openness After Accounting for the Demographic Variables and Remaining Personality Traits

\begin{tabular}{|c|c|c|c|c|c|c|c|c|}
\hline & \multicolumn{4}{|c|}{ NSIs from Partner Latent Growth Curve } & \multicolumn{4}{|c|}{ Openness Latent Growth Curve } \\
\hline & \multicolumn{2}{|c|}{ Intercept } & \multicolumn{2}{|c|}{ Slope } & \multicolumn{2}{|c|}{ Intercept } & \multicolumn{2}{|c|}{ Slope } \\
\hline & $b$ & S.E. & $b$ & S.E. & $b$ & S.E. & $b$ & S.E. \\
\hline Estimate & $2.194 *$ & 0.064 & $-0.002 *$ & 0.011 & $2.899 *$ & 0.058 & $0.008^{*}$ & 0.008 \\
\hline Age & -0.001 & 0.001 & 0.000 & 0.000 & 0.001 & 0.001 & 0.000 & 0.000 \\
\hline $\begin{array}{l}\text { Gender } \\
\qquad(0=\text { Female })\end{array}$ & -0.059 & 0.028 & -0.004 & 0.002 & 0.074 & 0.025 & -0.002 & 0.001 \\
\hline Education & 0.048 & 0.014 & -0.002 & 0.001 & 0.072 & 0.013 & 0.001 & 0.001 \\
\hline Agreeableness & -0.023 & 0.016 & 0.000 & 0.001 & - & - & - & - \\
\hline Neuroticism & 0.100 & 0.014 & -0.002 & 0.001 & - & - & - & - \\
\hline Extraversion & -0.002 & 0.016 & -0.001 & 0.001 & - & - & - & - \\
\hline Conscientiousness & -0.034 & 0.015 & -0.001 & 0.001 & - & - & - & - \\
\hline NSIs from Partner Intercept & - & - & -0.002 & 0.003 & - & - & -0.001 & 0.002 \\
\hline
\end{tabular}




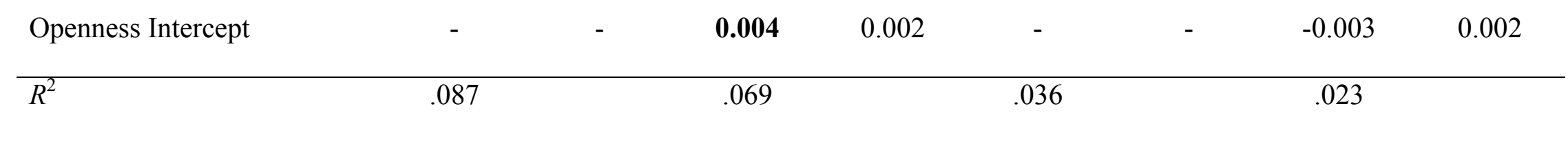

\begin{tabular}{ll}
\hline Model Fit & $\chi^{\mathbf{2}(49)=941.77}$ \\
& $\mathrm{CMIN} / \mathrm{DF}=19.22$ \\
$\mathrm{CFI}=.830$ \\
$\mathrm{RMSEA}=.10$
\end{tabular}

Note. Significant statistics $(p<.05)$ are bolded. $R^{2}=$ variance accounted for. An asterisk indicates that there is significant variability surrounding the estimate. A blue value indicates that the estimate is significantly different than zero. Type I errors were accounted for utilizing the false discovery rate adjustment. A green value indicates that the estimate was no longer statistically significant after the false discovery rate adjustments. To aid in the interpretation of the estimates, education, agreeableness, openness, neuroticism, extraversion, and conscientiousness were standardized. As such, a unit increase or decrease in these variables represent a standard deviation increase or decrease, respectively. Age was not manipulated so that a unit increase or decrease represents an increase or decrease of a year, respectively. 
Table J37

Research Question 7 - Examination of the Bidirectional Association between the Latent Growth Curves of NSIs from Partner and Neuroticism After Accounting for the Demographic Variables and Remaining Personality Traits

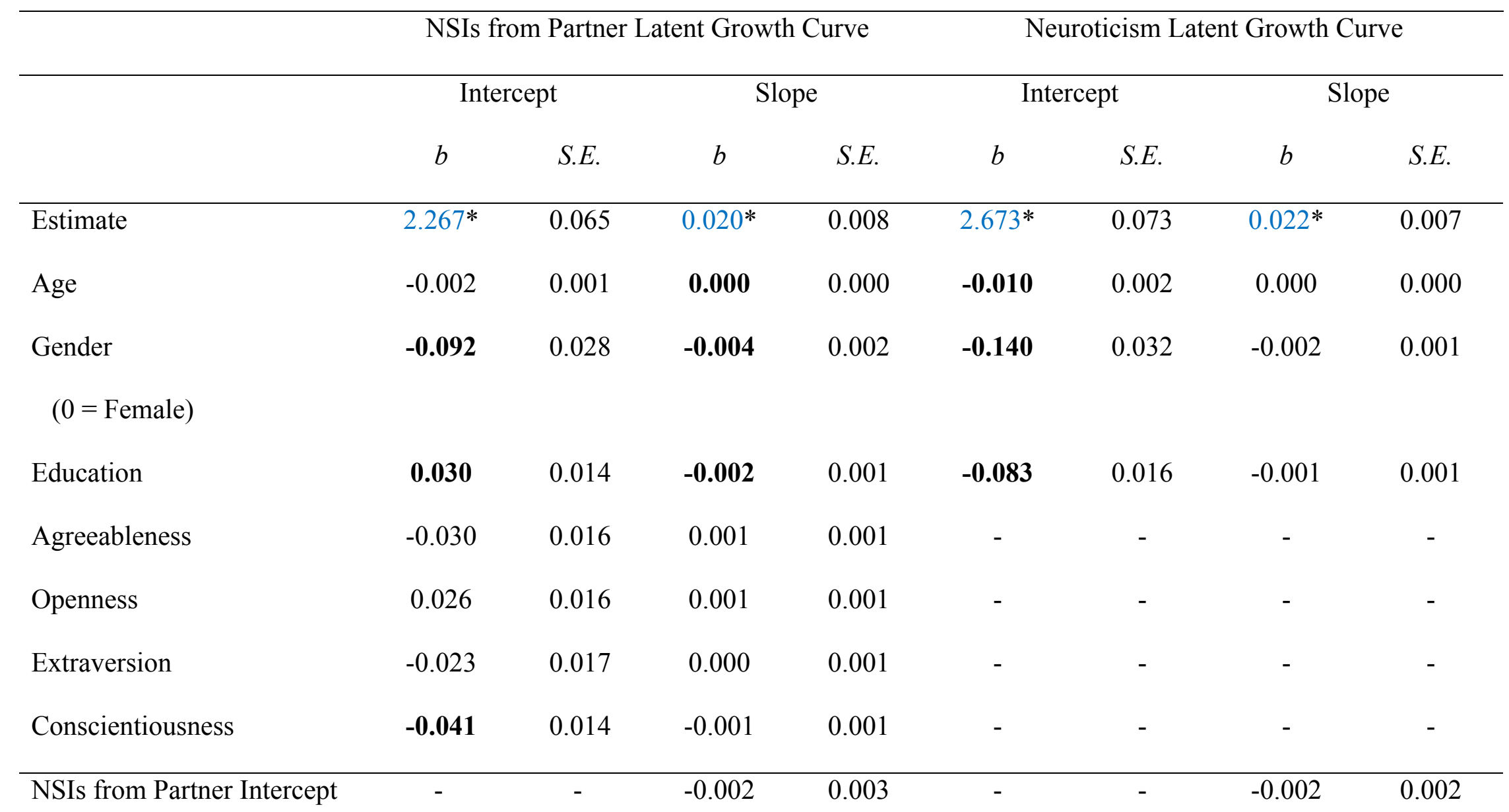




\begin{tabular}{|c|c|c|c|c|c|c|}
\hline Neuroticism Intercept & - & -0.005 & 0.002 & - & -0.012 & 0.002 \\
\hline$R^{2}$ & .039 & .055 & & .072 & .178 & \\
\hline
\end{tabular}

\begin{tabular}{ll}
\hline Model Fit & $\chi^{2} \mathbf{( 4 9 )}=\mathbf{5 7 0 . 6 8}$ \\
& $\mathrm{CMIN} / \mathrm{DF}=11.64$ \\
$\mathrm{CFI}=.897$ \\
$\mathrm{RMSEA}=.08$
\end{tabular}

Note. Significant statistics $(p<.05)$ are bolded. $R^{2}=$ variance accounted for. An asterisk indicates that there is significant variability surrounding the estimate. A blue value indicates that the estimate is significantly different than zero. Type I errors were accounted for utilizing the false discovery rate adjustment. A green value indicates that the estimate was no longer statistically significant after the false discovery rate adjustments. To aid in the interpretation of the estimates, education, agreeableness, openness, neuroticism, extraversion, and conscientiousness were standardized. As such, a unit increase or decrease in these variables represent a standard deviation increase or decrease, respectively. Age was not manipulated so that a unit increase or decrease represents an increase or decrease of a year, respectively. 
Table J38

Research Question 7 - Examination of the Bidirectional Association between the Latent Growth Curves of NSIs from Partner and Extraversion After Accounting for the Demographic Variables and Remaining Personality Traits

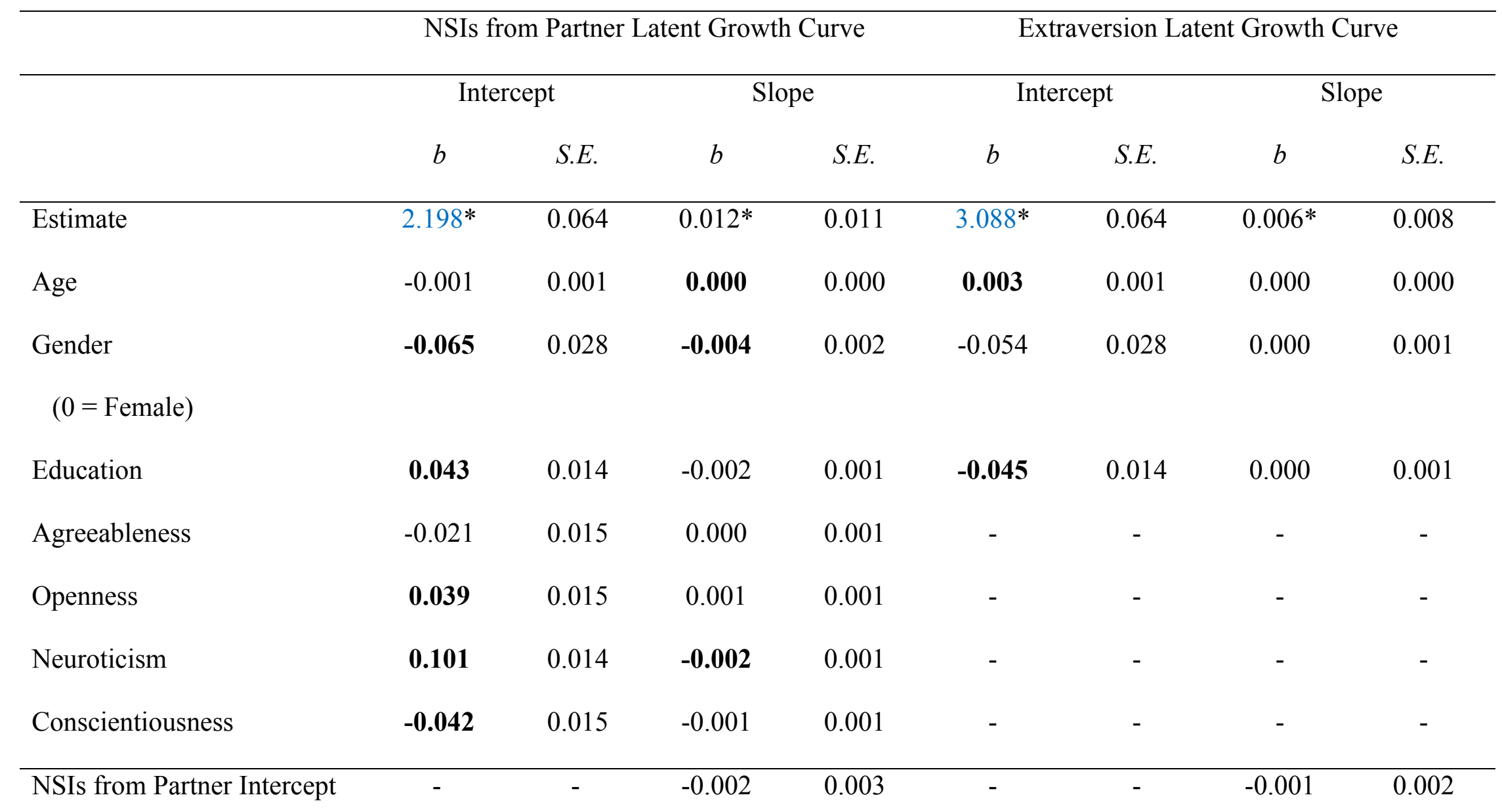




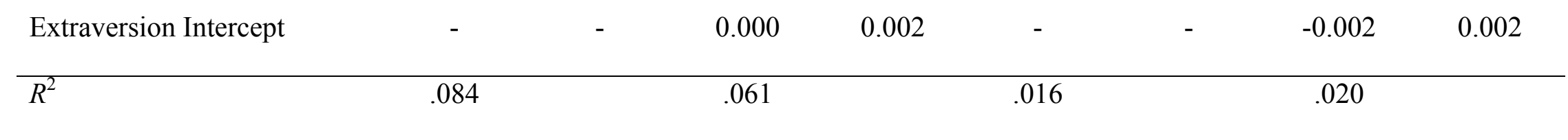

\begin{tabular}{ll}
\hline Model Fit & $\chi^{\mathbf{2}(49)=\mathbf{1 1 7 9 . 0 5}}$ \\
& $\mathrm{CMIN} / \mathrm{DF}=24.06$ \\
$\mathrm{CFI}=.785$ \\
$\mathrm{RMSEA}=.12$
\end{tabular}

Note. Significant statistics $(p<.05)$ are bolded. $R^{2}=$ variance accounted for. An asterisk indicates that there is significant variability surrounding the estimate. A blue value indicates that the estimate is significantly different than zero. Type I errors were accounted for utilizing the false discovery rate adjustment. A green value indicates that the estimate was no longer statistically significant after the false discovery rate adjustments. To aid in the interpretation of the estimates, education, agreeableness, openness, neuroticism, extraversion, and conscientiousness were standardized. As such, a unit increase or decrease in these variables represent a standard deviation increase or decrease, respectively. Age was not manipulated so that a unit increase or decrease represents an increase or decrease of a year, respectively. 
Table J39

Research Question 7 - Examination of the Bidirectional Association between the Latent Growth Curves of NSIs from Partner and Conscientiousness After Accounting for the Demographic Variables and Remaining Personality Traits

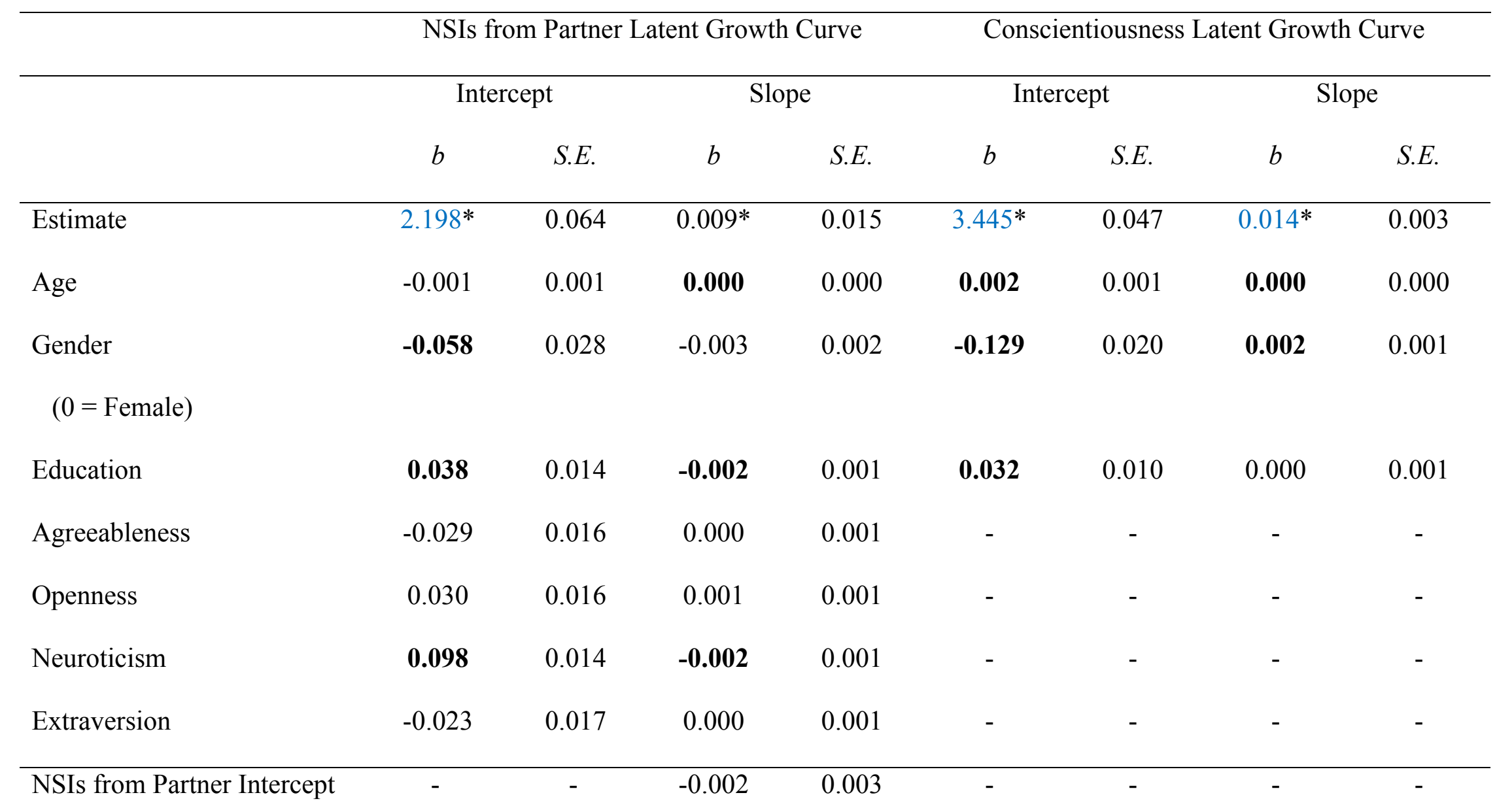




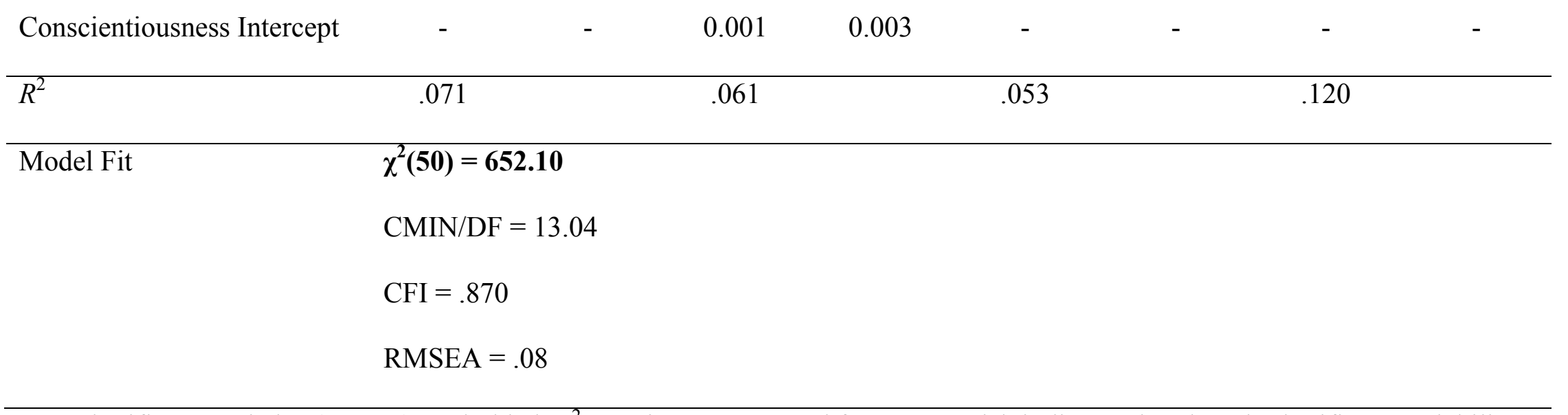

Note. Significant statistics $(p<.05)$ are bolded. $R^{2}=$ variance accounted for. An asterisk indicates that there is significant variability surrounding the estimate. A blue value indicates that the estimate is significantly different than zero. Type I errors were accounted for utilizing the false discovery rate adjustment. A green value indicates that the estimate was no longer statistically significant after the false discovery rate adjustments. To aid in the interpretation of the estimates, education, agreeableness, openness, neuroticism, extraversion, and conscientiousness were standardized. As such, a unit increase or decrease in these variables represent a standard deviation increase or decrease, respectively. Age was not manipulated so that a unit increase or decrease represents an increase or decrease of a year, respectively. 
Table J40

The Unconstrained Models Compared to the Constrained Models for the Multigroup Cross-Domain Latent Growth Curves

$\chi^{2}$

CFI

.982

Unconstrained Model

Constrained Model

NSIs from Partner and Openness

Unconstrained Model
Constrained Model

$$
\begin{aligned}
& \chi^{2}(46)=102.39 \\
& \chi^{2}(58)=138.28
\end{aligned}
$$

.975

$$
\chi^{2}(12)=36.09
$$

.984

.978

$$
\chi^{2}(12)=35.89
$$

NSIs from Partner and Neuroticism

Unconstrained Model
Constrained Model

$$
\begin{aligned}
& \chi^{2}(46)=191.46 \\
& \chi^{2}(58)=224.36
\end{aligned}
$$

$$
\chi^{2}(12)=32.90
$$

NSIs from Partner and Extraversion

Unconstrained Model
Constrained Model

$$
\begin{aligned}
& \chi^{2}(46)=81.70 \\
& \chi^{2}(58)=118.49
\end{aligned}
$$


NSIs from Partner and Conscientiousness

\begin{tabular}{|c|c|c|c|c|}
\hline Unconstrained Model & $\chi^{2}(46)=110.68$ & .979 & & \\
\hline Constrained Model & $\chi^{2}(58)=157.44$ & .968 & $\chi^{2}(12)=46.76$ & .011 \\
\hline
\end{tabular}

$\overline{\text { Note. Significant statistics }(p<.05) \text { are bolded. The unconstrained model is where the parameters were freely estimated across the }}$ three age groups. The constrained model is where the parameters were constrained to be equal across the three age groups. A change of .01 or greater for the CFI difference statistic was used to determine significance (Little, 2013). Both the chi-square and the CFI difference statistics were computed because the chi-square statistic is commonly used, however, it is sensitive to large sample sizes. The CFI is more robust to larger sample sizes (Little, 2013). 
Table J41

Research Question 8 - Multigroup Cross-Domain Latent Growth Curves

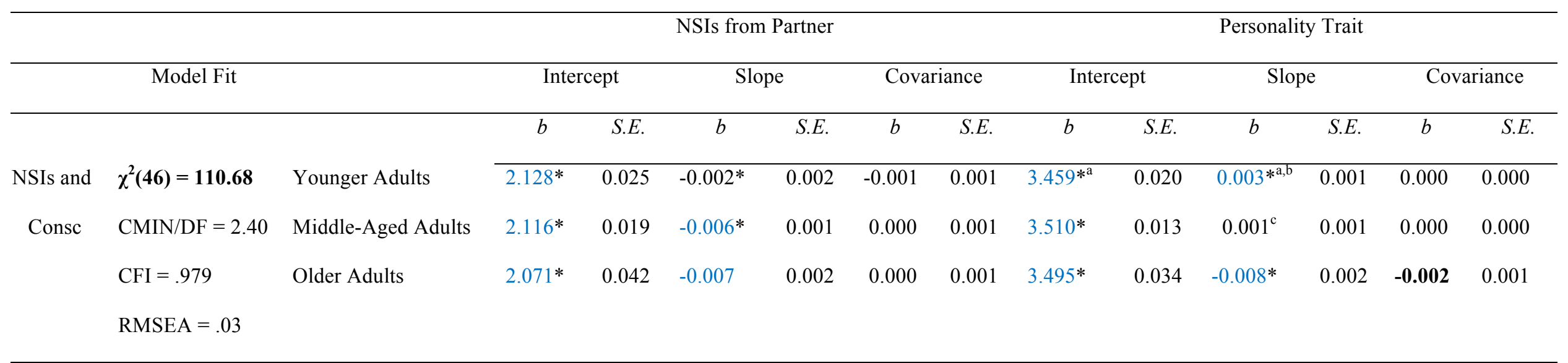

Note. Significant statistics $(p<.05)$ are bolded. Consc $=$ conscientiousness. An asterisk indicates that there is significant variability surrounding the estimate. A blue value indicates that the estimate is significantly different than zero. ${ }^{a}$ indicates that the estimate is significantly different between younger and middle-aged adults. ${ }^{b}$ indicates that the estimate is significantly different between younger and older adults. ${ }^{\mathrm{c}}$ indicates that the estimate is significantly different between middle-aged and older adults. 
Table J42

Research Question 8 - Examination of the Bidirectional Association between the Latent Growth Curves of NSIs from Partner and Conscientiousness After Accounting for the Demographic Variables and Remaining Personality Traits for Younger Adults

\begin{tabular}{|c|c|c|c|c|c|c|c|c|}
\hline & \multicolumn{4}{|c|}{ NSIs from Partner Latent Growth Curve } & \multicolumn{4}{|c|}{ Conscientiousness Latent Growth Curve } \\
\hline & \multicolumn{2}{|c|}{ Intercept } & \multicolumn{2}{|c|}{ Slope } & \multicolumn{2}{|c|}{ Intercept } & \multicolumn{2}{|c|}{ Slope } \\
\hline & $b$ & S.E. & $b$ & S.E. & $b$ & S.E. & $b$ & S.E. \\
\hline Estimate & $2.099 *$ & 0.217 & 0.027 & 0.015 & $3.596^{*}$ & 0.166 & $0.000 *$ & 0.024 \\
\hline Age & 0.001 & 0.006 & -0.001 & 0.000 & -0.002 & 0.005 & 0.000 & 0.000 \\
\hline Gender $(0=$ Female $)$ & -0.045 & 0.050 & 0.003 & 0.004 & -0.214 & 0.038 & 0.004 & 0.002 \\
\hline Education & 0.018 & 0.026 & -0.001 & 0.002 & 0.016 & 0.020 & 0.000 & 0.001 \\
\hline Agreeableness & -0.019 & 0.027 & 0.001 & 0.002 & - & - & - & - \\
\hline Openness & 0.006 & 0.028 & 0.000 & 0.002 & - & - & - & - \\
\hline Neuroticism & 0.073 & 0.024 & -0.005 & 0.002 & - & - & - & - \\
\hline Extraversion & -0.013 & 0.029 & -0.002 & 0.002 & - & - & - & - \\
\hline NSIs from Partner Intercept & - & - & - & - & - & - & -0.002 & 0.005 \\
\hline Conscientiousness Intercept & - & - & - & - & - & - & -0.003 & 0.004 \\
\hline
\end{tabular}




$$
\begin{aligned}
& \begin{array}{llllll}
R^{2} & .055 & .184 & .109 & .073
\end{array} \\
& \text { Model Fit } \chi^{2}(\mathbf{6 9})=237.46 \\
& \mathrm{CMIN} / \mathrm{DF}=3.44 \\
& \mathrm{CFI}=.859 \\
& \text { RMSEA }=.07
\end{aligned}
$$


Table J43

Research Question 8 - Examination of the Bidirectional Association between the Latent Growth Curves of NSIs from Partner and Conscientiousness After Accounting for the Demographic Variables and Remaining Personality Traits for Middle-Aged Adults

\begin{tabular}{|c|c|c|c|c|c|c|c|c|}
\hline & \multicolumn{4}{|c|}{ NSIs from Partner Latent Growth Curve } & \multicolumn{4}{|c|}{ Conscientiousness Latent Growth Curve } \\
\hline & \multicolumn{2}{|c|}{ Intercept } & \multicolumn{2}{|c|}{ Slope } & \multicolumn{2}{|c|}{ Intercept } & \multicolumn{2}{|c|}{ Slope } \\
\hline & $b$ & S.E. & $b$ & S.E. & $b$ & S.E. & $b$ & S.E. \\
\hline Estimate & $2.522 *$ & 0.164 & $-0.003^{*}$ & 0.021 & $3.543^{*}$ & 0.102 & $0.001 *$ & 0.001 \\
\hline Age & -0.007 & 0.003 & 0.000 & 0.000 & 0.000 & 0.002 & - & - \\
\hline Gender $(0=$ Female $)$ & -0.077 & 0.036 & -0.005 & 0.002 & -0.089 & 0.023 & - & - \\
\hline Education & 0.052 & 0.018 & -0.003 & 0.001 & 0.042 & 0.011 & - & - \\
\hline Agreeableness & -0.039 & 0.022 & 0.000 & 0.001 & - & - & - & - \\
\hline Openness & 0.032 & 0.021 & 0.001 & 0.001 & - & - & - & - \\
\hline Neuroticism & 0.103 & 0.019 & -0.001 & 0.001 & - & - & - & - \\
\hline Extraversion & -0.021 & 0.023 & 0.001 & 0.001 & - & - & - & - \\
\hline NSIs from Partner Intercept & - & - & -0.004 & 0.004 & - & - & - & - \\
\hline Conscientiousness Intercept & - & - & 0.002 & 0.004 & - & - & - & - \\
\hline
\end{tabular}




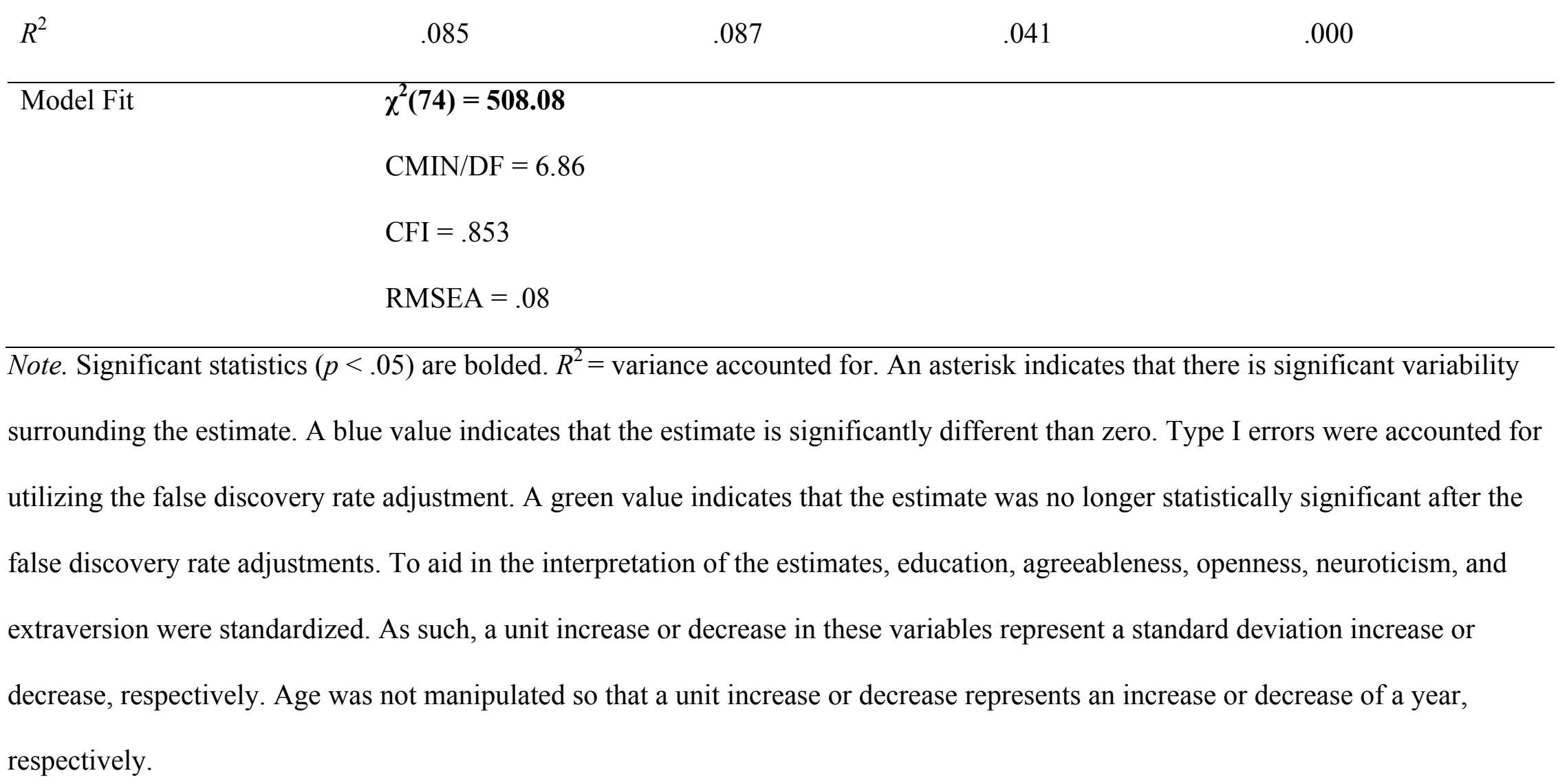


Table J44

Research Question 8 - Examination of the Bidirectional Association between the Latent Growth Curves of NSIs from Partner and Conscientiousness After Accounting for the Demographic Variables and Remaining Personality Traits for Older Adults

\begin{tabular}{|c|c|c|c|c|c|c|c|c|}
\hline & \multicolumn{4}{|c|}{ NSIs from Partner Latent Growth Curve } & \multicolumn{4}{|c|}{ Conscientiousness Latent Growth Curve } \\
\hline & \multicolumn{2}{|c|}{ Intercept } & \multicolumn{2}{|c|}{ Slope } & \multicolumn{2}{|c|}{ Intercept } & \multicolumn{2}{|c|}{ Slope } \\
\hline & $b$ & S.E. & $b$ & S.E. & $b$ & S.E. & $b$ & S.E. \\
\hline Estimate & $3.081^{*}$ & 0.660 & 0.015 & 0.027 & $3.766^{*}$ & 0.585 & $0.037 *$ & 0.047 \\
\hline Age & -0.015 & 0.010 & - & - & -0.003 & 0.009 & 0.000 & 0.001 \\
\hline Gender $(0=$ Female $)$ & -0.004 & 0.077 & - & - & -0.074 & 0.068 & 0.002 & 0.004 \\
\hline Education & 0.022 & 0.037 & - & - & -0.002 & 0.033 & 0.000 & 0.002 \\
\hline Agreeableness & -0.024 & 0.045 & - & - & - & - & - & - \\
\hline Openness & 0.080 & 0.045 & - & - & - & - & - & - \\
\hline Neuroticism & 0.140 & 0.042 & - & - & - & - & - & - \\
\hline Extraversion & -0.038 & 0.054 & - & - & - & - & - & - \\
\hline NSIs from Partner Intercept & - & - & -0.009 & 0.006 & - & - & -0.004 & 0.004 \\
\hline Conscientiousness Intercept & - & - & -0.001 & 0.007 & - & - & -0.006 & 0.008 \\
\hline
\end{tabular}




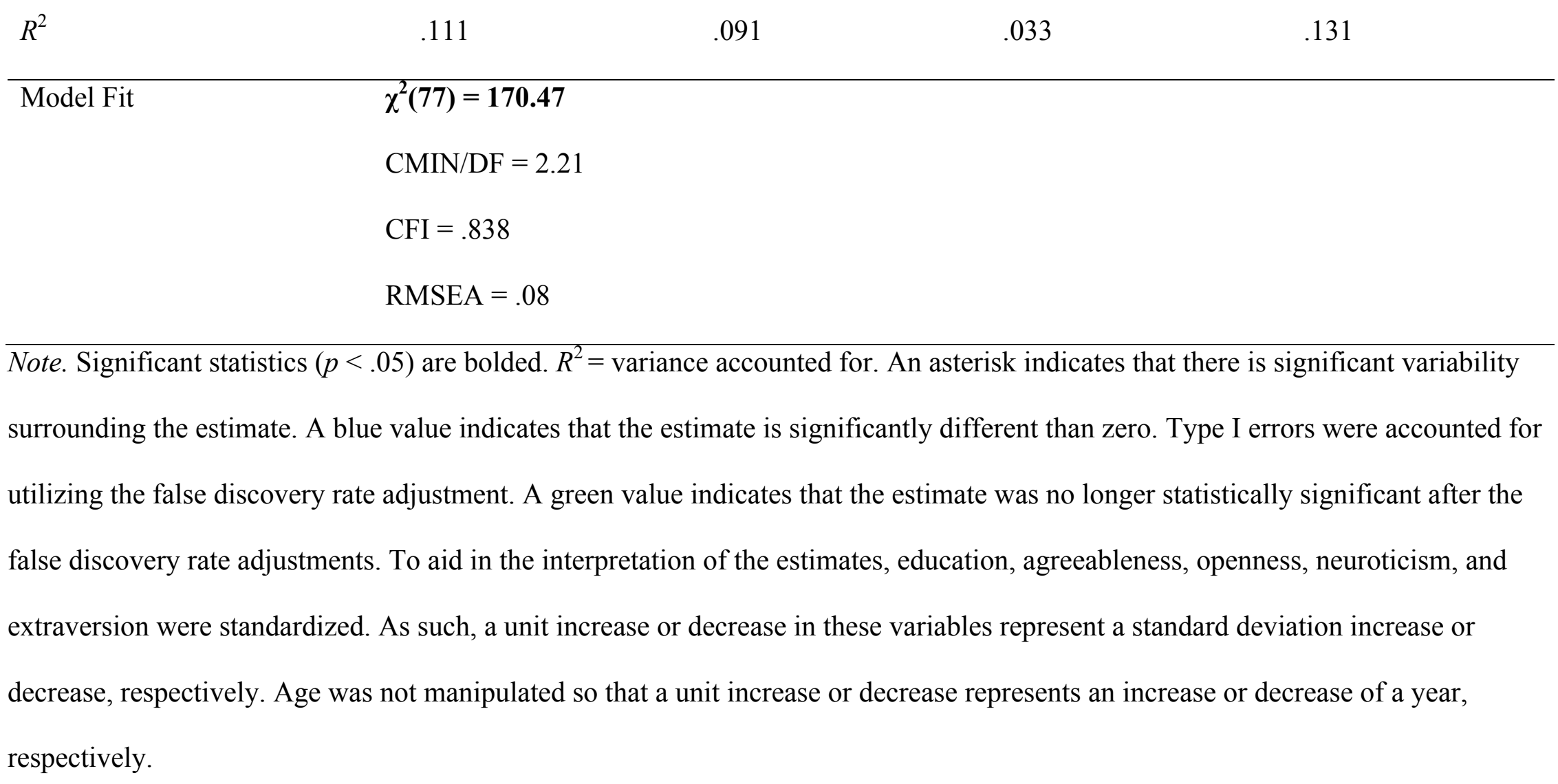


Appendix K

Results for NSIs from Friends

Table K1

Summary Results of Research Questions 2 and 3 for NSIs from Friends

\section{NSIs from Friends Latent Growth Curve}

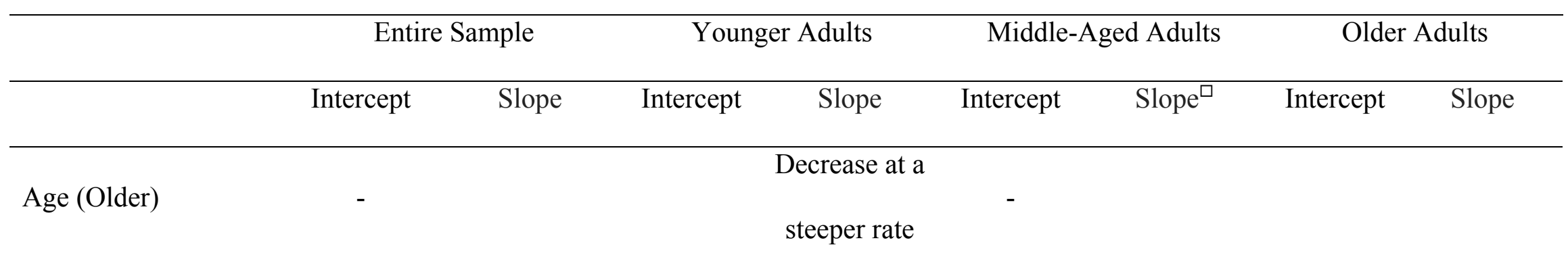

Gender (Males)

Education

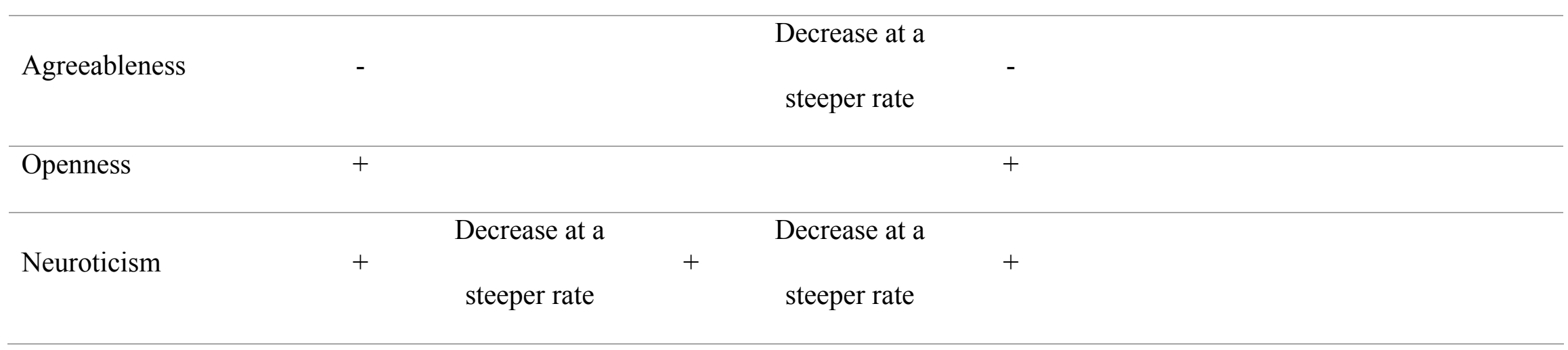




\section{Extraversion}

Conscientiousness

Note. The summary of significant effects is presented above. A plus sign indicates that higher values of the variable are associated

with a higher intercept. A minus sign indicates that higher values of the variable are associated with a lower intercept. ${ }^{\square}$ indicates that there was no significant variance surrounding the slope estimate. Therefore, slope was not predicted. 
Table K2

Summary Results of Research Questions 5 and 6 for Agreeableness

Agreeableness Latent Growth Curve

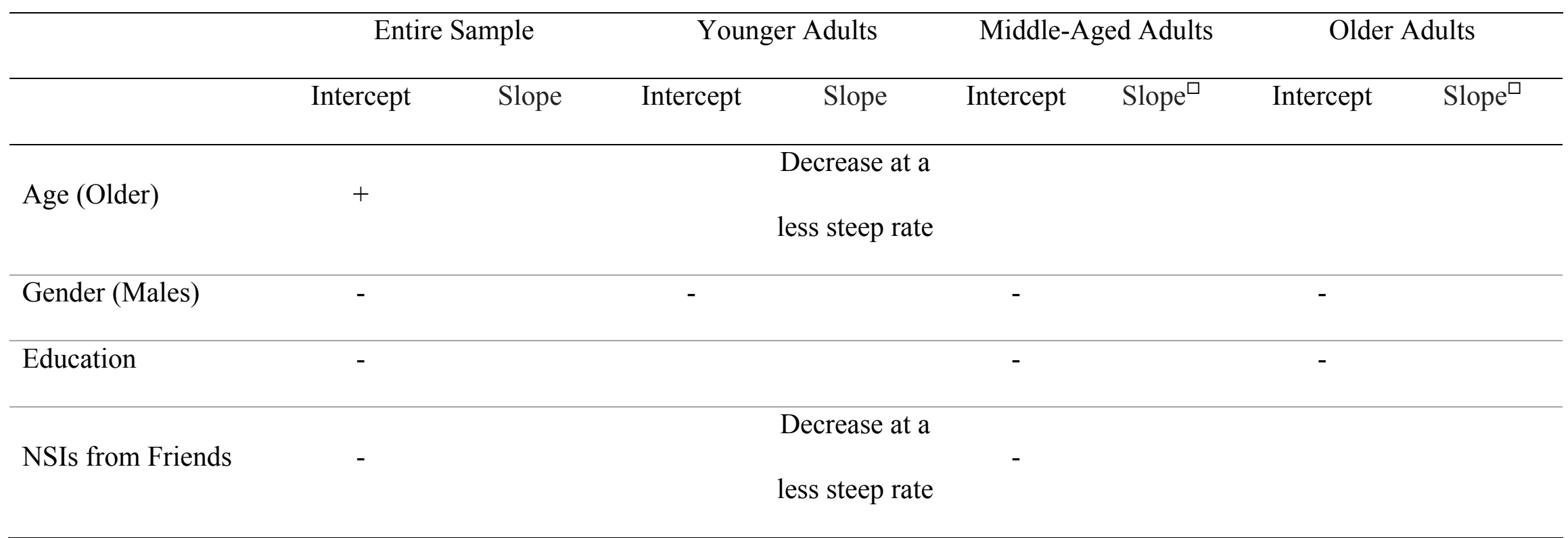

Note. The summary of significant effects is presented above. A plus sign indicates that higher values of the variable are associated

with a higher intercept. A minus sign indicates that higher values of the variable are associated with a lower intercept. ${ }^{\square}$ indicates that there was no significant variance surrounding the slope estimate. Therefore, slope was not predicted. 
Table K3

Summary Results of Research Questions 5 and 6 for Openness

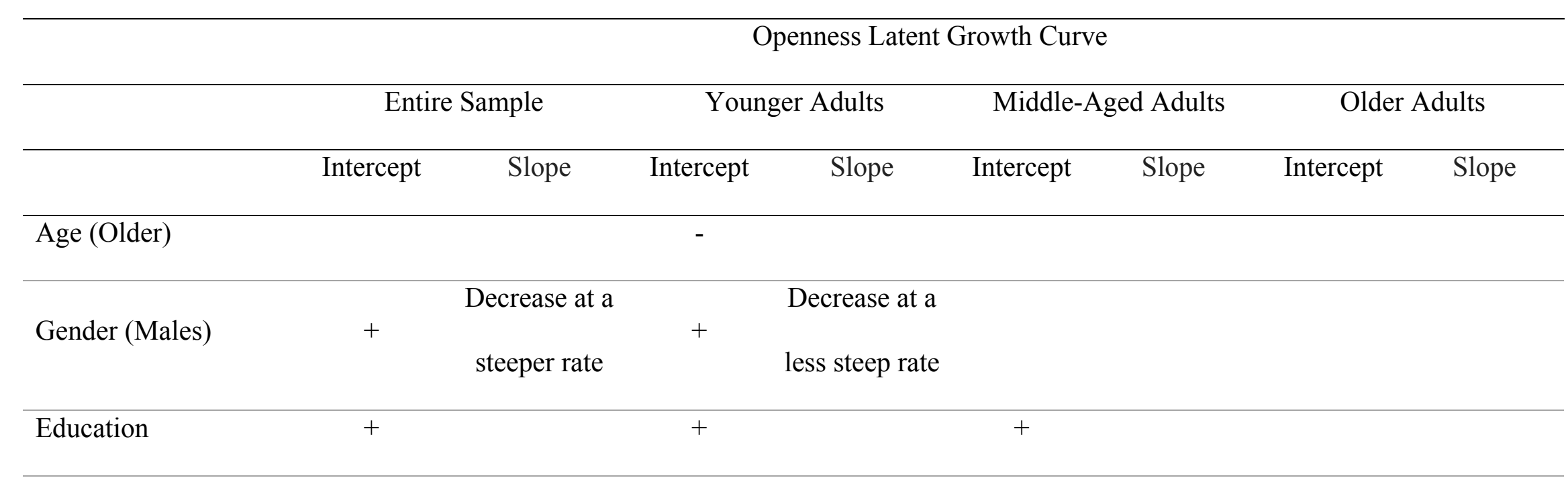

NSIs from Friends

Note. The summary of significant effects is presented above. A plus sign indicates that higher values of the variable are associated with a higher intercept. A minus sign indicates that higher values of the variable are associated with a lower intercept. 
Table K4

Summary Results of Research Questions 5 and 6 for Neuroticism

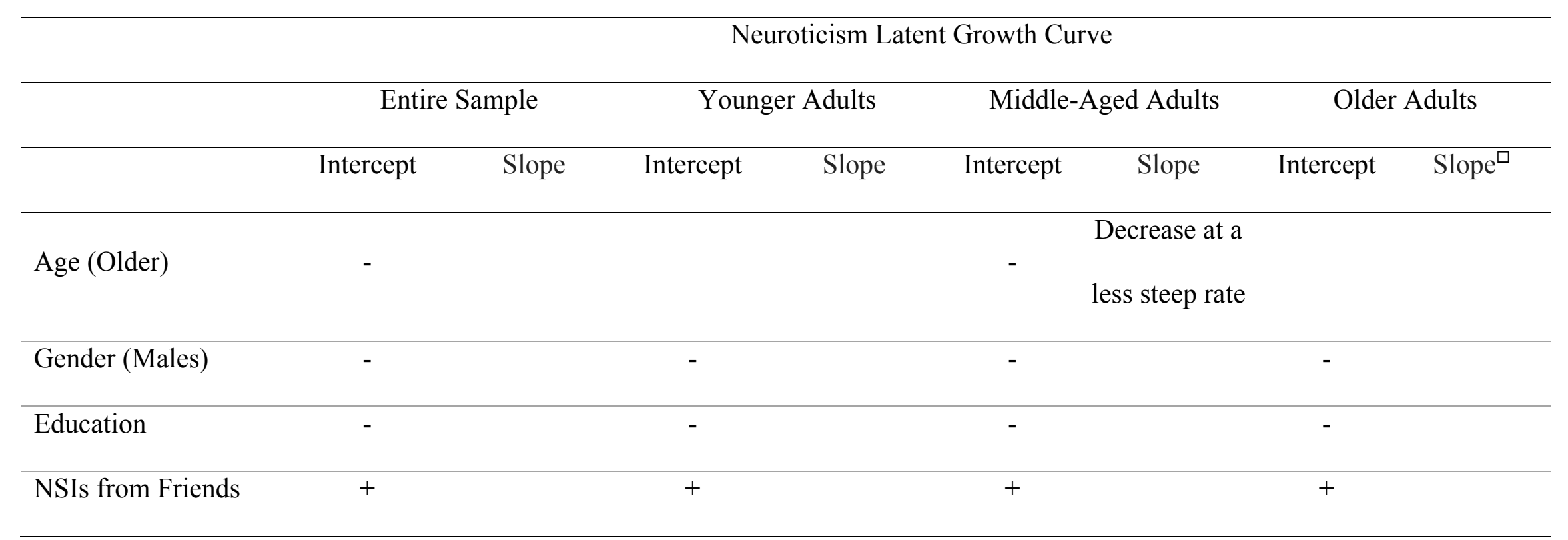

Note. The summary of significant effects is presented above. A plus sign indicates that higher values of the variable are associated with a higher intercept. A minus sign indicates that higher values of the variable are associated with a lower intercept. ${ }^{\square}$ indicates that there was no significant variance surrounding the slope estimate. Therefore, slope was not predicted. 
Table K5

Summary Results of Research Questions 5 and 6 for Extraversion

\begin{tabular}{cc}
\hline \multicolumn{3}{c}{ Extraversion Latent Growth Curve } \\
\hline \multicolumn{3}{c}{ Entire Sample } \\
\hline Intercept & Slope \\
\hline
\end{tabular}

Age (Older)

Gender (Males)

Education

NSIs from Friends

Note. The summary of significant effects is presented above. A plus sign indicates that higher values of the variable are associated with a higher intercept. A minus sign indicates that higher values of the variable are associated with a lower intercept. Age did not moderate the slope of extraversion. 
Table K6

Summary Results of Research Questions 5 and 6 for Conscientiousness

Conscientiousness Latent Growth Curve

\begin{tabular}{|c|c|c|c|c|c|c|c|}
\hline & \multicolumn{2}{|c|}{ Entire Sample } & \multicolumn{2}{|c|}{ Younger Adults } & \multicolumn{2}{|c|}{ Middle-Aged Adults } & Older Adults \\
\hline & Intercept & Slope & Intercept & Slope & Intercept & Slope $^{\square}$ & Intercept \\
\hline Age (Older) & + & $\begin{array}{l}\text { Increase at a } \\
\text { steeper rate }\end{array}$ & & & & & \\
\hline Gender (Males) & - & $\begin{array}{l}\text { Increase at a } \\
\text { steeper rate }\end{array}$ & - & $\begin{array}{l}\text { Increase at a } \\
\text { steeper rate }\end{array}$ & - & & \\
\hline Education & + & & & & + & & \\
\hline NSIs from Friends & - & & & & - & & \\
\hline
\end{tabular}

Note. The summary of significant effects is presented above. A plus sign indicates that higher values of the variable are associated

with a higher intercept. A minus sign indicates that higher values of the variable are associated with a lower intercept. ${ }^{\square}$ indicates that there was no significant variance surrounding the slope estimate. Therefore, slope was not predicted. 
Table K7

Summary Results of Research Questions 7 and 8 for NSIs from Friends and Agreeableness

\section{NSIs from Friends Latent Growth Curve}

Agreeableness Latent Growth Curve

\begin{tabular}{ccccccccc}
\hline & \multicolumn{4}{c}{ NSIs from Friends Slope } & & & Agreeableness Slope \\
\hline Entire & Younger & Middle-Aged & Older & Entire & Younger & Middle- & Older \\
& Sample & Adults & Adults & Adults & Sample & Adults & Aged Adults & Adults
\end{tabular}

NSIs from Friends

Intercept

Agreeableness

Decrease at a

Decrease at a

Intercept

steeper rate

steeper rate

Note. The summary of significant effects is presented above. A plus sign indicates that higher values of the variable are associated

with a higher intercept. A minus sign indicates that higher values of the variable are associated with a lower intercept. ${ }^{\circ}$ indicates that the slope was not significant. Therefore, slope was not predicted. 
Table K8

Summary Results of Research Questions 7 and 8 for NSIs from Friends and Openness

NSIs from Friends Latent Growth Curve

Openness Latent Growth Curve

\begin{tabular}{ccccccccc}
\hline & \multicolumn{3}{c}{ NSIs from Friends Slope } & & Openness Slope \\
\hline Entire & Younger & Middle-Aged & Older & Entire & Younger & Middle- & Older \\
& Sample & Adults & Adults & Adults & Sample & Adults & Aged Adults Adults \\
& & & & & &
\end{tabular}

NSIs from Friends

Intercept

Openness Decrease at a Decrease at a

Intercept less steep rate steeper rate

Note. The summary of significant effects is presented above. A plus sign indicates that higher values of the variable are associated with a higher intercept. A minus sign indicates that higher values of the variable are associated with a lower intercept. 
Table K9

Summary Results of Research Questions 7 and 8 for NSIs from Friends and Neuroticism

\begin{tabular}{|c|c|c|c|c|c|c|c|c|}
\hline & \multicolumn{4}{|c|}{ NSIs from Friends Latent Growth Curve } & \multicolumn{4}{|c|}{ Neuroticism Latent Growth Curve } \\
\hline & \multicolumn{4}{|c|}{ NSIs from Friends Slope } & \multicolumn{4}{|c|}{ Neuroticism Slope } \\
\hline & Entire & Younger & Middle-Aged & Older & Entire & Younger & Middle-Aged & Older \\
\hline & Sample & Adults & Adults & Adults & Sample & Adults & Adults & Adults \\
\hline NSIs from Friends & Decrease at a & & & & & & & \\
\hline Intercept & steeper rate & & & & & & & \\
\hline Neuroticism & & & & & Decrease at a & & & \\
\hline Intercept & & & & & steeper rate & & & \\
\hline
\end{tabular}

Note. The summary of significant effects is presented above. A plus sign indicates that higher values of the variable are associated with a higher intercept. A minus sign indicates that higher values of the variable are associated with a lower intercept. 
Table K10

Summary Results of Research Questions 7 and 8 for NSIs from Friends and Extraversion

\begin{tabular}{|c|c|c|c|c|c|c|c|c|}
\hline & \multicolumn{4}{|c|}{ NSIs from Friends Latent Growth Curve } & \multicolumn{4}{|c|}{ Extraversion Latent Growth Curve } \\
\hline & \multicolumn{4}{|c|}{ NSIs from Friends Slope } & \multicolumn{4}{|c|}{ Extraversion Slope } \\
\hline & Entire & Younger & Middle-Aged & Older & Entire & Younger & Middle- & Older \\
\hline & Sample & Adults & Adults & Adults & Sample & Adults & Aged Adults & Adults \\
\hline \multicolumn{9}{|l|}{ NSIs from Friends } \\
\hline Intercept & & & & & & & & \\
\hline
\end{tabular}

Extraversion

Intercept

Note. The summary of significant effects is presented above. A plus sign indicates that higher values of the variable are associated with a higher intercept. A minus sign indicates that higher values of the variable are associated with a lower intercept. 
Table K11

Summary Results of Research Questions 7 and 8 for NSIs from Friends and Conscientiousness

\section{NSIs from Friends Latent Growth Curve}

Conscientiousness Latent Growth Curve

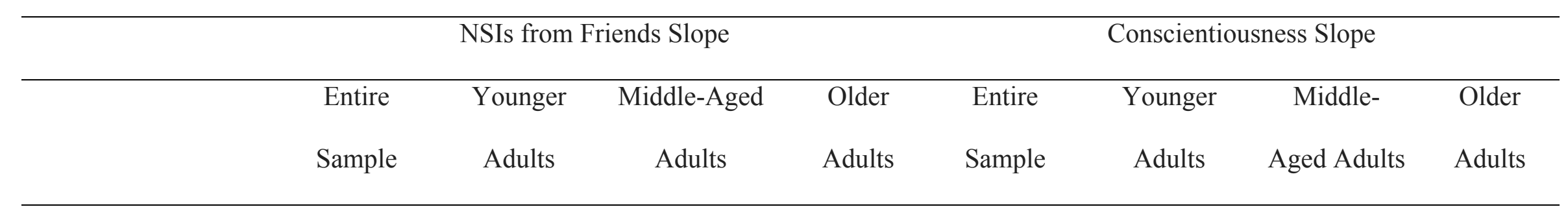

NSIs from Friends

Intercept

Conscientiousness

Intercept

Note. The summary of significant effects is presented above. A plus sign indicates that higher values of the variable are associated with a higher intercept. A minus sign indicates that higher values of the variable are associated with a lower intercept. ${ }^{\circ}$ indicates that the slope was not significant. Therefore, slope was not predicted. 
Table K12

Missing Data for NSIs from Friends across all MIDUS Waves

\begin{tabular}{lcc}
\hline & Missing Count & Missing Percent \\
\hline NSIs from Friends - MIDUS 1 & 4 & $0.40 \%$ \\
NSIs from Friends - MIDUS 2 & 9 & $0.60 \%$ \\
NSIs from Friends - MIDUS 3 & 14 & $0.90 \%$ \\
\hline
\end{tabular}


Table K13

Attrition Analyses Comparing Non-Multivariate Outliers and Multivariate Outliers for NSIs from Friends Across all MIDUS Waves

\begin{tabular}{llcl}
\hline & Non-Multivariate & Multivariate & Difference Statistic \\
& Outliers & Outliers & \\
& $(n=1,403)$ & $(n=127)$ & \\
& $M, S D$ or $\%$ & $M, S D$ or $\%$ & \\
\hline NSIs from Friends - MIDUS 1 & $1.98,0.54$ & $2.18,0.66$ & $\boldsymbol{t ( 1 4 0 . 8 0 )}=\mathbf{- 3 . 1 8}$ \\
NSIs from Friends - MIDUS 2 & $2.05,0.57$ & $2.24,0.69$ & $\boldsymbol{t ( 1 4 2 . 2 7 )}=\mathbf{- 2 . 8 9}$ \\
NSIs from Friends - MIDUS 3 & $1.68,0.49$ & $1.83,0.60$ & $\boldsymbol{t ( 1 4 2 . 3 6 )}=\mathbf{- 2 . 8 4}$
\end{tabular}

Note. Significant statistics $(p<.05)$ are bolded. 
Table K14

Research Question 1 - The Latent Growth Curves of NSIs from Friends

\begin{tabular}{|c|c|c|c|c|c|c|c|}
\hline & \multirow[t]{2}{*}{ Model Fit } & \multicolumn{2}{|c|}{ Intercept } & \multicolumn{2}{|c|}{ Slope } & \multicolumn{2}{|c|}{ Covariance } \\
\hline & & $b$ & S.E. & $b$ & S.E. & $b$ & S.E. \\
\hline \multirow[t]{4}{*}{ NSIs from Friends } & $\chi^{2}(3)=11.39$ & $1.888^{*}$ & 0.011 & $-0.011^{*}$ & 0.001 & 0.000 & 0.000 \\
\hline & $\mathrm{CMIN} / \mathrm{DF}=3.79$ & & & & & & \\
\hline & $\mathrm{CFI}=.988$ & & & & & & \\
\hline & $\mathrm{RMSEA}=.04$ & & & & & & \\
\hline
\end{tabular}

Note. Significant statistics $(p<.05)$ are bolded. An asterisk indicates that there is significant variability surrounding the estimate. A blue value indicates that the estimate is significantly different than zero. 
Table K15

Research Question 2 - Demographic Variables and the Big Five Personality Traits Predicting the Latent Growth Curve of NSIs from Friends

\begin{tabular}{|c|c|c|c|c|}
\hline & \multicolumn{4}{|c|}{ NSIs from Friends Latent Growth Curve } \\
\hline & \multicolumn{2}{|c|}{ Intercept } & \multicolumn{2}{|c|}{ Slope } \\
\hline & $b$ & S.E. & $b$ & S.E. \\
\hline Estimate & $2.067 *$ & 0.050 & $-0.009 *$ & 0.004 \\
\hline Age & -0.004 & 0.001 & 0.000 & 0.000 \\
\hline Gender & 0.009 & 0.022 & 0.000 & 0.002 \\
\hline \multicolumn{5}{|l|}{$(0=$ Female $)$} \\
\hline Education & 0.004 & 0.011 & 0.000 & 0.001 \\
\hline Agreeableness & -0.026 & 0.013 & -0.001 & 0.001 \\
\hline Openness & 0.027 & 0.013 & 0.000 & 0.001 \\
\hline Neuroticism & 0.085 & 0.011 & -0.002 & 0.001 \\
\hline Extraversion & 0.005 & 0.014 & 0.000 & 0.001 \\
\hline Conscientiousness & -0.039 & 0.012 & -0.001 & 0.001 \\
\hline Covariance & 0.000 & 0.000 & & \\
\hline$R^{2}$ & .131 & & .044 & \\
\hline \multicolumn{5}{|c|}{$\chi^{2}(29)=457.29$} \\
\hline \multirow{3}{*}{ Model Fit } & $\mathrm{CMIN} / \mathrm{DF}=15.76$ & & & \\
\hline & $\mathrm{CFI}=.816$ & & & \\
\hline & RMSEA $=.09$ & & & \\
\hline
\end{tabular}


Note. Significant statistics $(p<.05)$ are bolded. $R^{2}=$ variance accounted for. An asterisk indicates that there is significant variability surrounding the estimate. A blue value indicates that the estimate is significantly different than zero. Type I errors were accounted for utilizing the false discovery rate adjustment. A green value indicates that the estimate was no longer statistically significant after the false discovery rate adjustments. To aid in the interpretation of the estimates, education, agreeableness, openness, neuroticism, extraversion, and conscientiousness were standardized. As such, a unit increase or decrease in these variables represent a standard deviation increase or decrease, respectively. Age was not manipulated so that a unit increase or decrease represents an increase or decrease of a year, respectively. 
Table K16

The Unconstrained Model Compared to the Constrained Model for the Multigroup Latent Growth Curve of NSIs from Friends

\begin{tabular}{lcccc}
\hline & $\chi^{2}$ & CFI & $\Delta \chi^{2}$ & $\Delta$ CFI \\
\hline NSIs from Friends & & & & \\
Unconstrained Model & $\chi^{2}(\mathbf{1 1})=\mathbf{2 2 . 8 3}$ & .983 & & \\
Constrained Model & $\chi^{2}(\mathbf{1 7})=\mathbf{5 6 . 4 3}$ & .944 & $\chi^{2}(\mathbf{6})=\mathbf{3 3 . 6 0}$ & $\mathbf{. 0 3 9}$
\end{tabular}

Note. Significant statistics $(p<.05)$ are bolded. The unconstrained model is where the parameters were freely estimated across the three age groups. The constrained model is where the parameters were constrained to be equal across the three age groups. A change of .01 or greater for the CFI difference statistic was used to determine significance (Little, 2013). Both the chi-square and the CFI difference statistics were computed because the chi-square statistic is commonly used, however, it is sensitive to large sample sizes. The CFI is more robust to larger sample sizes (Little, 2013). 
Table K17

Research Question 3 - Multigroup Analyses for the Latent Growth Curve of NSIs from Friends

\begin{tabular}{|c|c|c|c|c|c|c|c|c|}
\hline & \multirow[t]{2}{*}{ Model Fit } & & \multicolumn{2}{|c|}{ Intercept } & \multicolumn{2}{|c|}{ Slope } & \multicolumn{2}{|c|}{ Covariance } \\
\hline & & & $b$ & S.E. & $b$ & S.E. & $b$ & S.E. \\
\hline \multirow[t]{4}{*}{ NSIs from Friends } & $\chi^{2}(11)=22.83$ & Younger Adults & $1.936 * b$ & 0.021 & $-0.009 *$ & 0.001 & -0.001 & 0.001 \\
\hline & $\mathrm{CMIN} / \mathrm{DF}=2.07$ & Middle-Aged Adults & $1.888 *^{\mathrm{c}}$ & 0.014 & -0.012 & 0.001 & 0.000 & 0.001 \\
\hline & $\mathrm{CFI}=.983$ & Older Adults & $1.763 *$ & 0.033 & $-0.009 *$ & 0.002 & -0.001 & 0.001 \\
\hline & $\mathrm{RMSEA}=.02$ & & & & & & & \\
\hline
\end{tabular}

Note. Significant statistics $(p<.05)$ are bolded. An asterisk indicates that there is significant variability surrounding the estimate. A blue value indicates that the estimate is significantly different than zero. ${ }^{a}$ indicates that the estimate is significantly different between younger and middle-aged adults. ${ }^{b}$ indicates that the estimate is significantly different between younger and older adults. ${ }^{c}$ indicates that the estimate is significantly different between middle-aged and older adults. 
Table K18

Research Question 3 -Demographic Variables and the Big Five Personality Traits Predicting the Latent Growth Curve of NSIs from Friends for Younger Adults

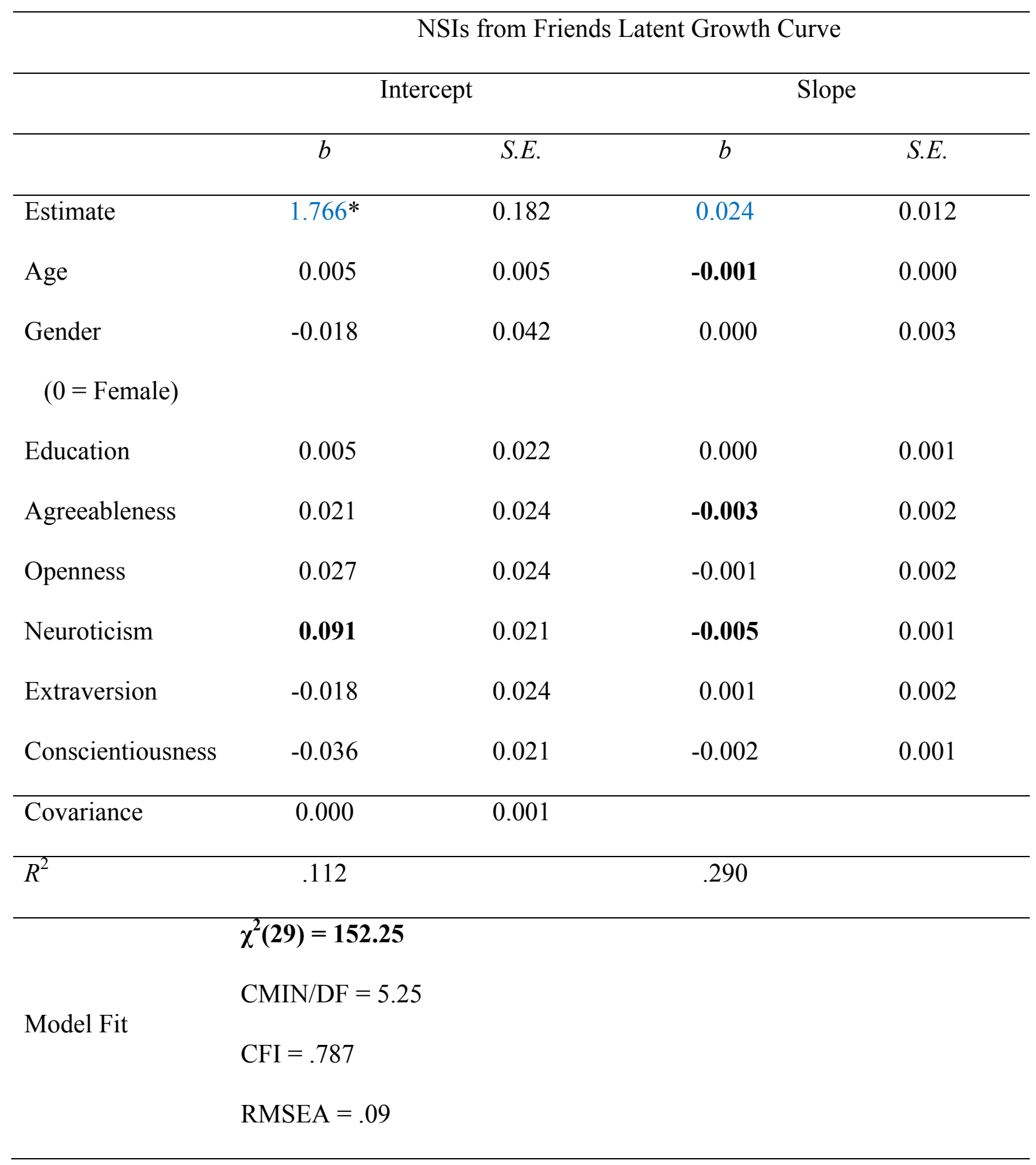


Note. Significant statistics $(p<.05)$ are bolded. $R^{2}=$ variance accounted for. An asterisk indicates that there is significant variability surrounding the estimate. A blue value indicates that the estimate is significantly different than zero. Type I errors were accounted for utilizing the false discovery rate adjustment. A green value indicates that the estimate was no longer statistically significant after the false discovery rate adjustments. To aid in the interpretation of the estimates, education, agreeableness, openness, neuroticism, extraversion, and conscientiousness were standardized. As such, a unit increase or decrease in these variables represent a standard deviation increase or decrease, respectively. Age was not manipulated so that a unit increase or decrease represents an increase or decrease of a year, respectively. 
Table K19

Research Question 3 - Demographic Variables and the Big Five Personality Traits Predicting the Latent Growth Curve of NSIs from Friends for Middle-Aged Adults

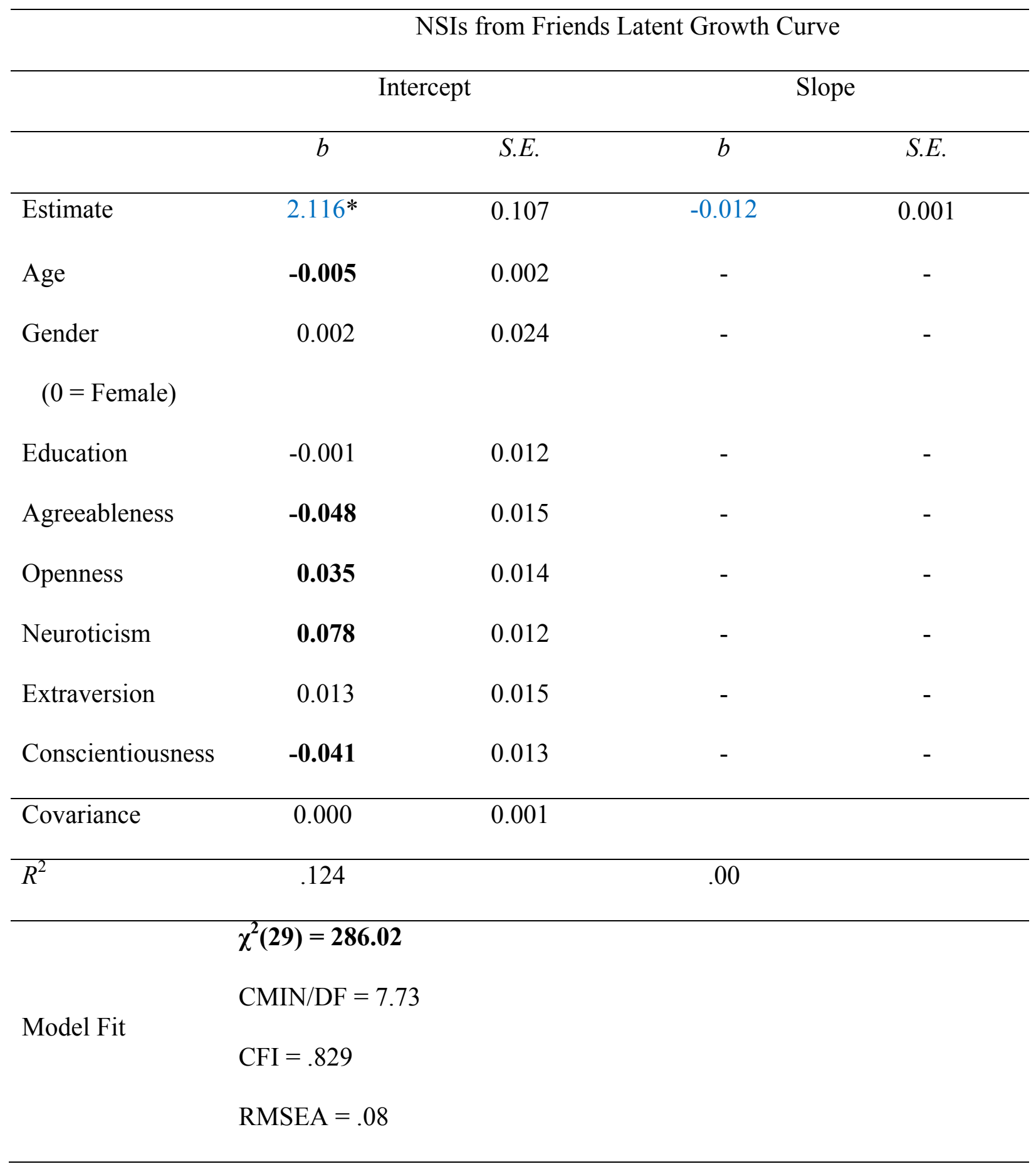


Note. Significant statistics $(p<.05)$ are bolded. $R^{2}=$ variance accounted for. An asterisk indicates that there is significant variability surrounding the estimate. A blue value indicates that the estimate is significantly different than zero. Type I errors were accounted for utilizing the false discovery rate adjustment. A green value indicates that the estimate was no longer statistically significant after the false discovery rate adjustments. To aid in the interpretation of the estimates, education, agreeableness, openness, neuroticism, extraversion, and conscientiousness were standardized. As such, a unit increase or decrease in these variables represent a standard deviation increase or decrease, respectively. Age was not manipulated so that a unit increase or decrease represents an increase or decrease of a year, respectively. 
Table K20

Research Question 3 - Demographic Variables and the Big Five Personality Traits Variables Predicting the Latent Growth Curve of NSIs from Friends for Older Adults

\begin{tabular}{|c|c|c|c|c|}
\hline & \multicolumn{4}{|c|}{ NSIs from Friends Latent Growth Curve } \\
\hline & \multicolumn{2}{|c|}{ Intercept } & \multicolumn{2}{|c|}{ Slope } \\
\hline & $b$ & S.E. & $b$ & S.E. \\
\hline Estimate & $2.494^{*}$ & 0.544 & 0.022 & 0.043 \\
\hline Age & -0.012 & 0.008 & -0.001 & 0.001 \\
\hline Gender & 0.123 & 0.064 & 0.003 & 0.005 \\
\hline \multicolumn{5}{|l|}{$(0=$ Female $)$} \\
\hline Education & -0.006 & 0.030 & 0.002 & 0.002 \\
\hline Agreeableness & -0.070 & 0.037 & 0.003 & 0.003 \\
\hline Openness & 0.030 & 0.038 & 0.000 & 0.003 \\
\hline Neuroticism & 0.081 & 0.035 & -0.002 & 0.003 \\
\hline Extraversion & 0.025 & 0.044 & 0.001 & 0.003 \\
\hline Conscientiousness & -0.057 & 0.031 & 0.000 & 0.002 \\
\hline Covariance & 0.000 & 0.001 & & \\
\hline$R^{2}$ & .289 & & .162 & \\
\hline \multirow{4}{*}{ Model Fit } & $\chi^{2}(29)=82.84$ & & & \\
\hline & $\mathrm{CMIN} / \mathrm{DF}=2.85$ & & & \\
\hline & $\mathrm{CFI}=.810$ & & & \\
\hline & $\mathrm{RMSEA}=.10$ & & & \\
\hline
\end{tabular}


Note. Significant statistics $(p<.05)$ are bolded. $R^{2}=$ variance accounted for. An asterisk indicates that there is significant variability surrounding the estimate. A blue value indicates that the estimate is significantly different than zero. Type I errors were accounted for utilizing the false discovery rate adjustment. A green value indicates that the estimate was no longer statistically significant after the false discovery rate adjustments. To aid in the interpretation of the estimates, education, agreeableness, openness, neuroticism, extraversion, and conscientiousness were standardized. As such, a unit increase or decrease in these variables represent a standard deviation increase or decrease, respectively. Age was not manipulated so that a unit increase or decrease represents an increase or decrease of a year, respectively. 
Table K21

Research Question 5 - Demographic Variables and NSIs from Friends Predicting the Latent Growth Curve of Agreeableness

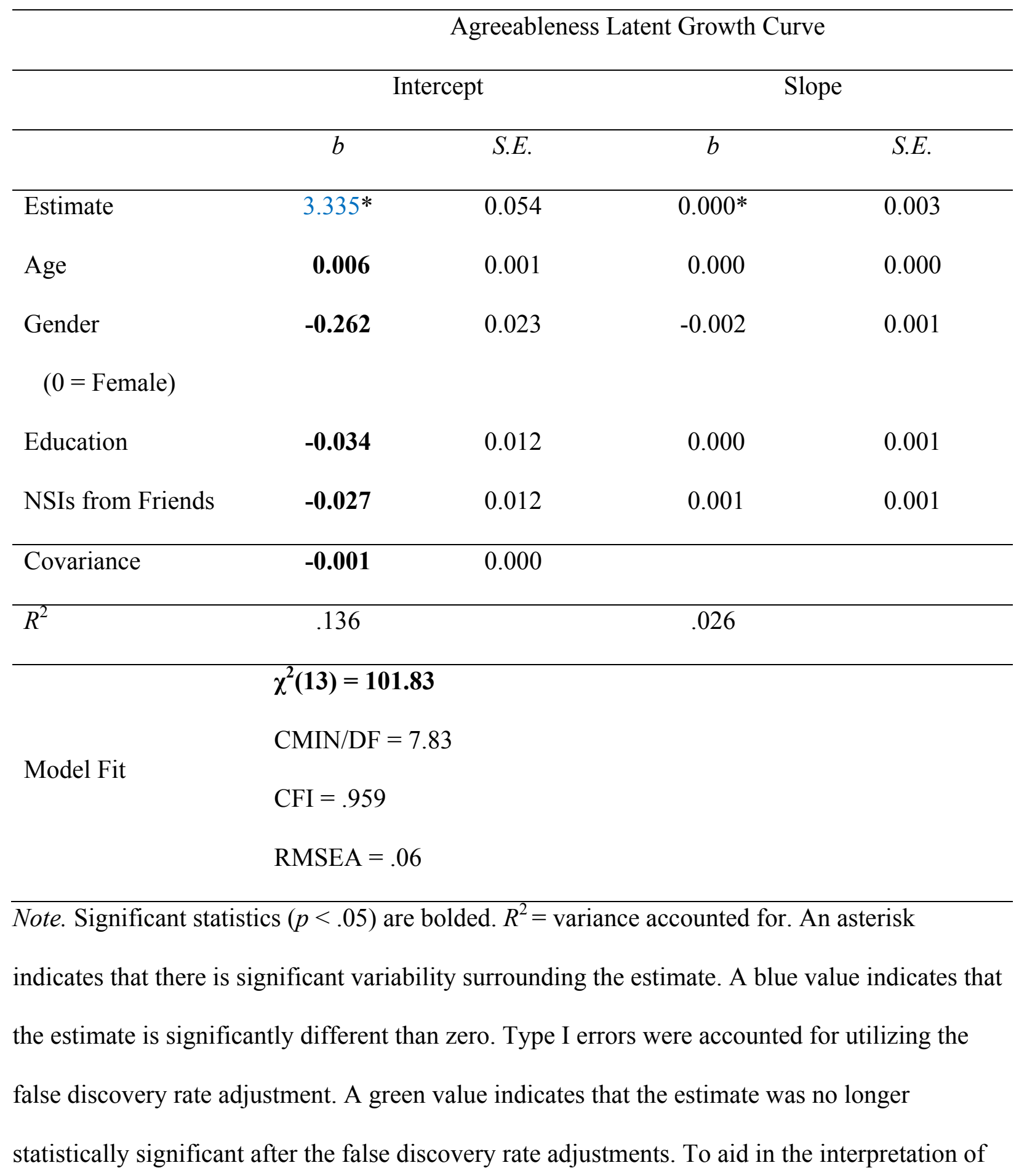


the estimates, education and NSIs from Friends were standardized. As such, a unit increase or decrease in these variables represent a standard deviation increase or decrease, respectively. Age was not manipulated so that a unit increase or decrease represents an increase or decrease of a year, respectively. 
Table K22

Research Question 5 - Demographic Variables and NSIs from Friends Predicting the Latent Growth Curve of Openness

\section{Openness Latent Growth Curve}

\begin{tabular}{|c|c|c|c|c|}
\hline & \multicolumn{2}{|c|}{ Intercept } & \multicolumn{2}{|c|}{ Slope } \\
\hline & $b$ & S.E. & $b$ & S.E. \\
\hline Estimate & $2.899 *$ & 0.058 & $-0.003 *$ & 0.003 \\
\hline Age & 0.001 & 0.001 & 0.000 & 0.000 \\
\hline Gender & 0.075 & 0.025 & -0.003 & 0.001 \\
\hline \multicolumn{5}{|l|}{$(0=$ Female $)$} \\
\hline Education & 0.072 & 0.013 & 0.000 & 0.001 \\
\hline NSIs from Friends & 0.000 & 0.013 & 0.000 & 0.001 \\
\hline Covariance & 0.000 & 0.000 & & \\
\hline$R^{2}$ & .036 & & .014 & \\
\hline \multicolumn{5}{|c|}{$\chi^{2}(13)=104.91$} \\
\hline \multirow{3}{*}{ Model Fit } & \multicolumn{4}{|c|}{$\mathrm{CMIN} / \mathrm{DF}=8.07$} \\
\hline & \multicolumn{4}{|c|}{$\mathrm{CFI}=.961$} \\
\hline & \multicolumn{4}{|c|}{ RMSEA $=.06$} \\
\hline
\end{tabular}

Note. Significant statistics $(p<.05)$ are bolded. $R^{2}=$ variance accounted for. An asterisk indicates that there is significant variability surrounding the estimate. A blue value indicates that the estimate is significantly different than zero. Type I errors were accounted for utilizing the false discovery rate adjustment. A green value indicates that the estimate was no longer statistically significant after the false discovery rate adjustments. To aid in the interpretation of 
the estimates, education and NSIs from Friends were standardized. As such, a unit increase or decrease in these variables represent a standard deviation increase or decrease, respectively. Age was not manipulated so that a unit increase or decrease represents an increase or decrease of a year, respectively. 
Table K23

Research Question 5 - Demographic Variables and NSIs from Friends Predicting the Latent Growth Curve of Neuroticism

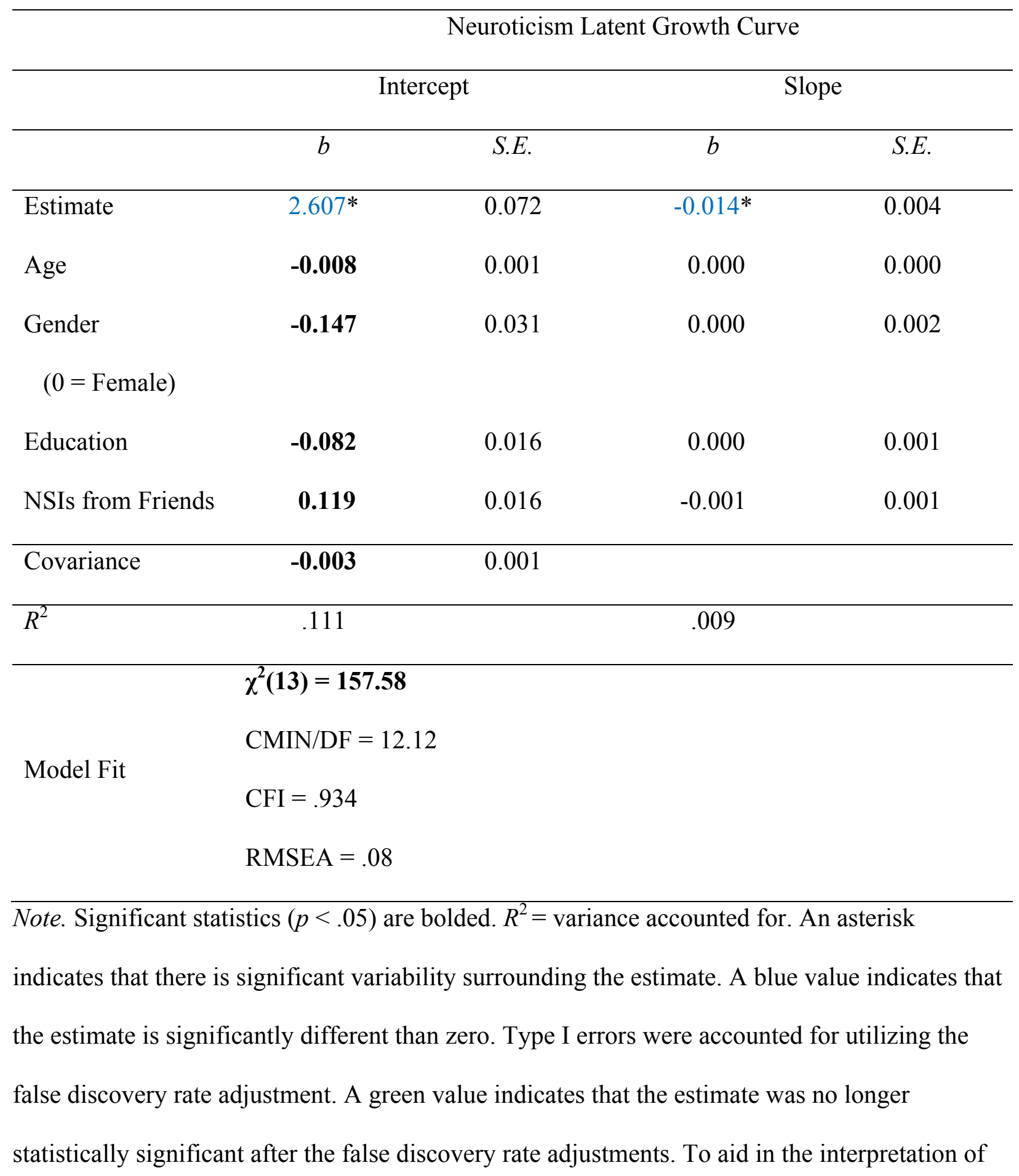


the estimates, education and NSIs from Friends were standardized. As such, a unit increase or decrease in these variables represent a standard deviation increase or decrease, respectively. Age was not manipulated so that a unit increase or decrease represents an increase or decrease of a year, respectively. 
Table K24

Research Question 5 - Demographic Variables and NSIs from Friends Predicting the Latent Growth Curve of Extraversion

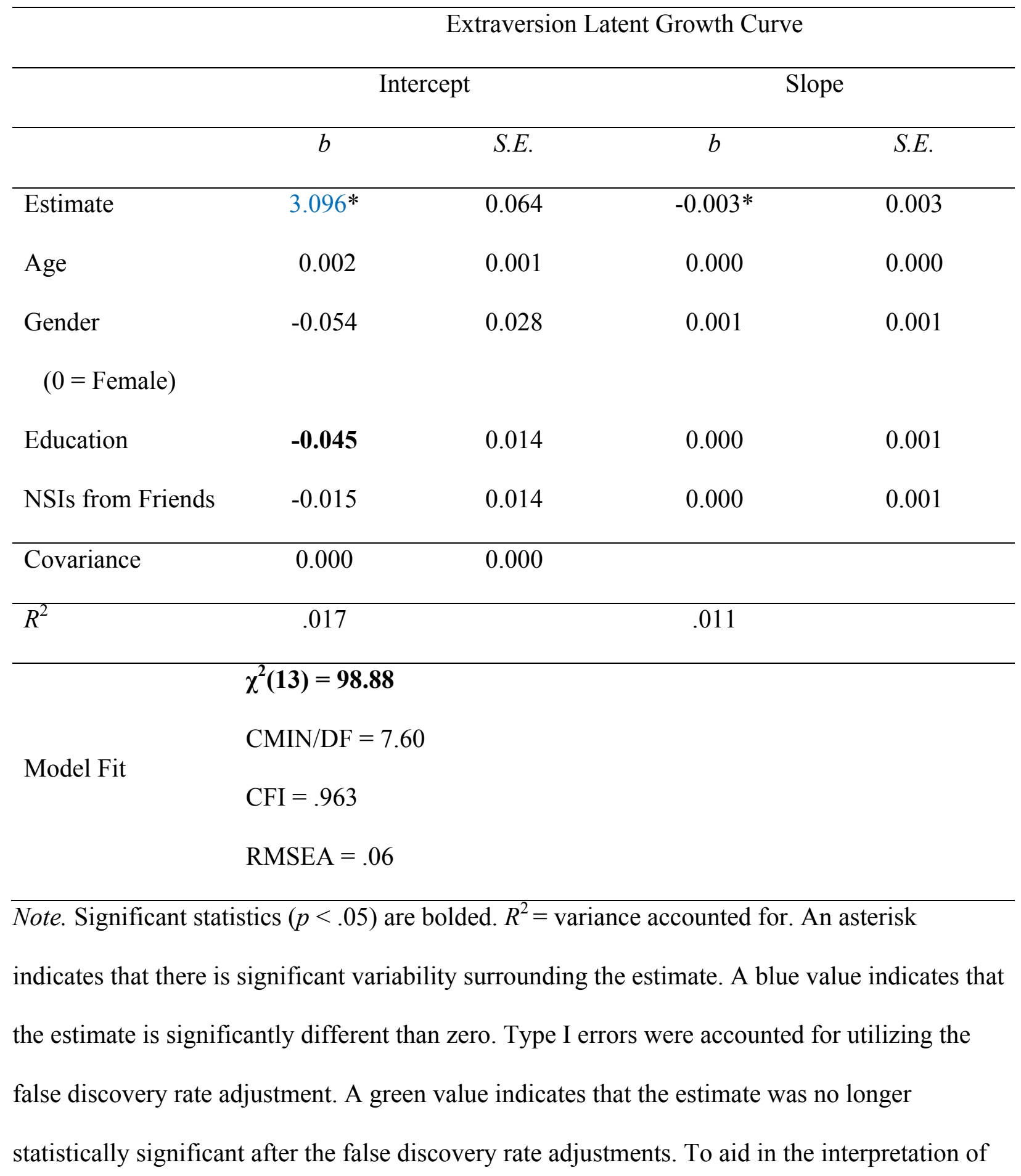


the estimates, education and NSIs from Friends were standardized. As such, a unit increase or decrease in these variables represent a standard deviation increase or decrease, respectively. Age was not manipulated so that a unit increase or decrease represents an increase or decrease of a year, respectively. 
Table K25

Research Question 5 - Demographic Variables and NSIs from Friends Predicting the Latent Growth Curve of Conscientiousness

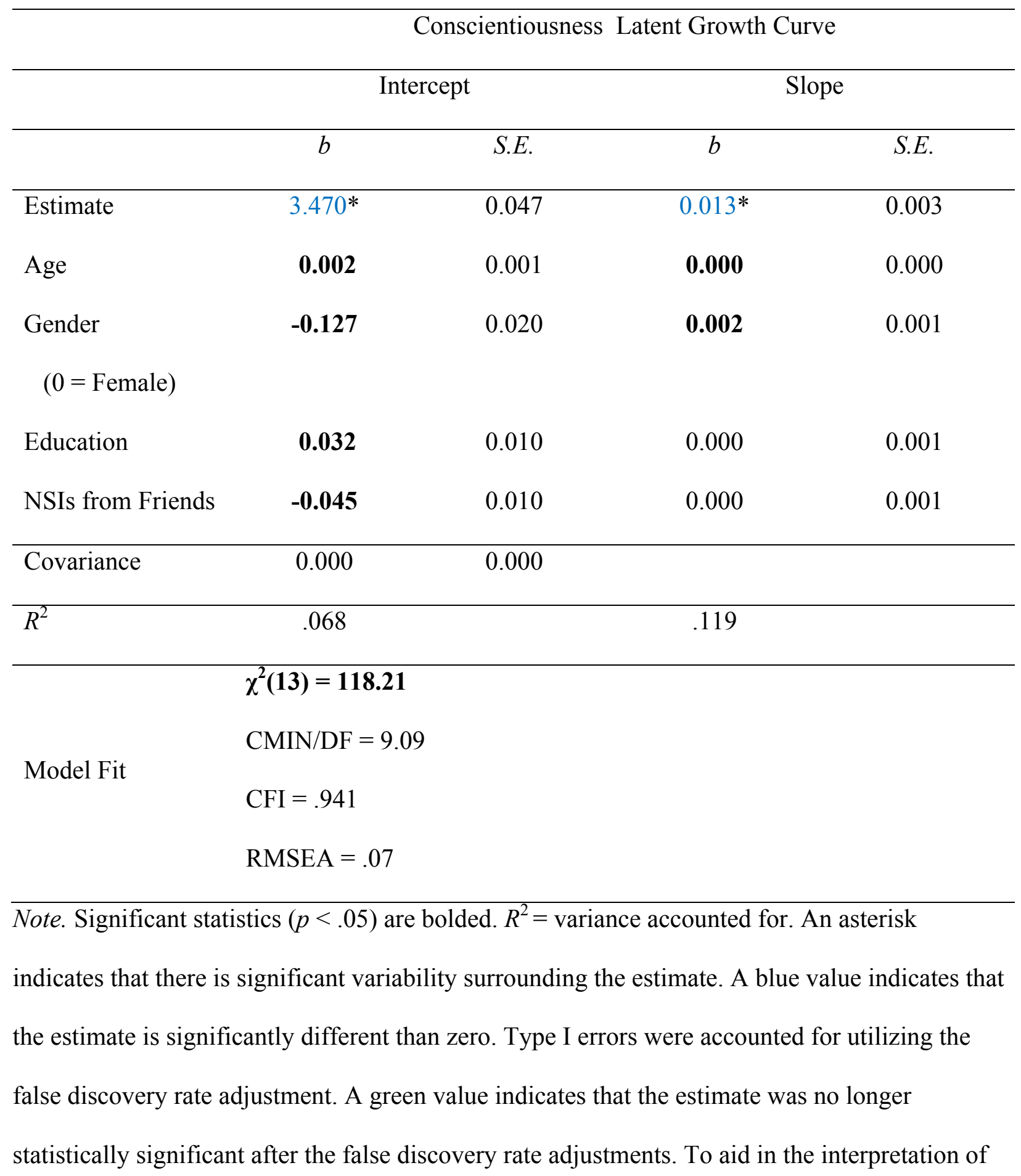


the estimates, education and NSIs from Friends were standardized. As such, a unit increase or decrease in these variables represent a standard deviation increase or decrease, respectively. Age was not manipulated so that a unit increase or decrease represents an increase or decrease of a year, respectively. 
Table K26

Research Question 6 - Demographic Variables and NSIs from Friends Predicting the Latent Growth Curve of Agreeableness for Younger Adults

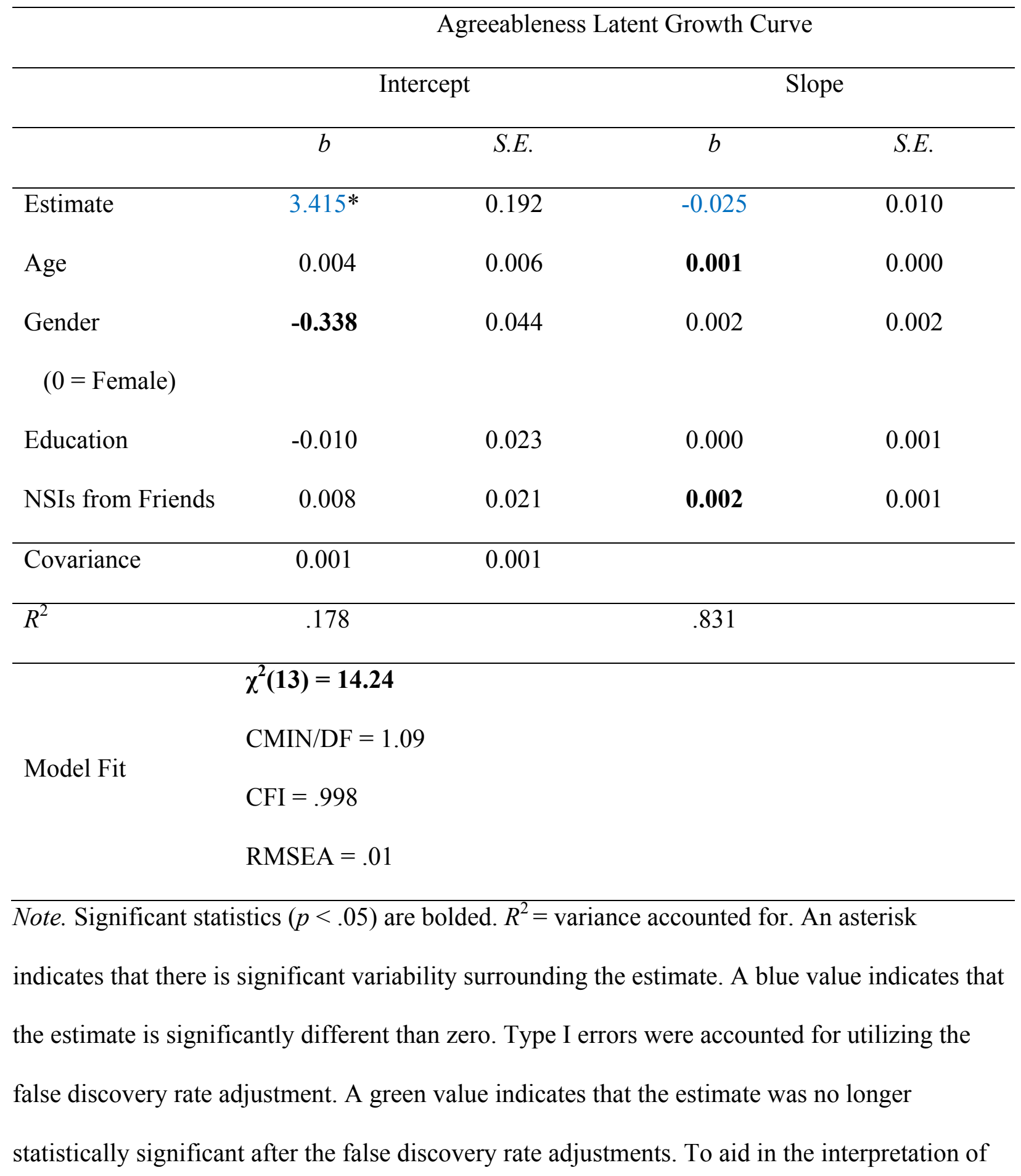


the estimates, education and NSIs from Friends were standardized. As such, a unit increase or decrease in these variables represent a standard deviation increase or decrease, respectively. Age was not manipulated so that a unit increase or decrease represents an increase or decrease of a year, respectively. 
Table K27

Research Question 6 - Demographic Variables and NSIs from Friends Predicting the Latent Growth Curve of Agreeableness for Middle-Aged Adults

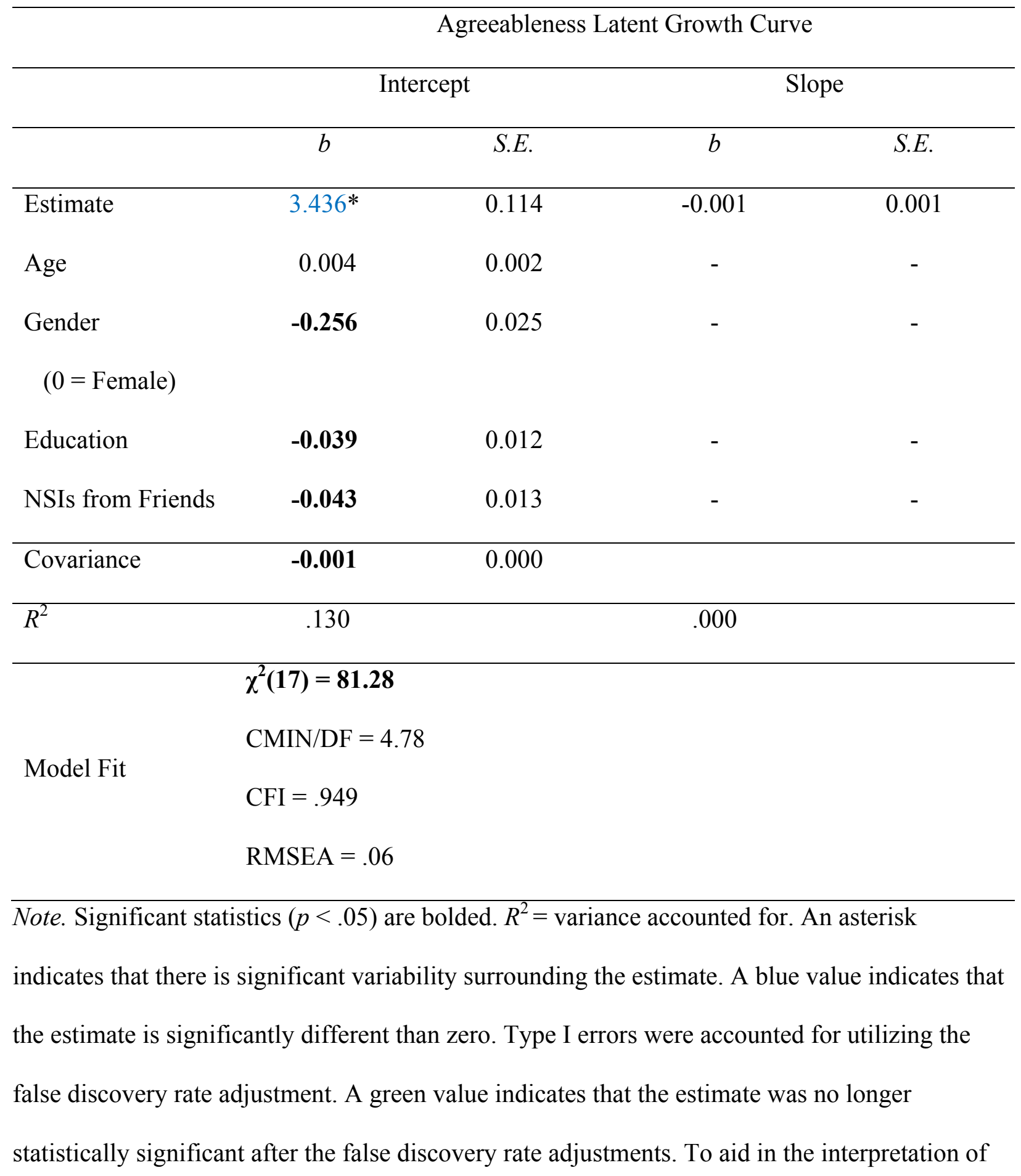


the estimates, education and NSIs from Friends were standardized. As such, a unit increase or decrease in these variables represent a standard deviation increase or decrease, respectively. Age was not manipulated so that a unit increase or decrease represents an increase or decrease of a year, respectively. 
Table K28

Research Question 6 - Demographic Variables and NSIs from Friends Predicting the Latent Growth Curve of Agreeableness for Older Adults

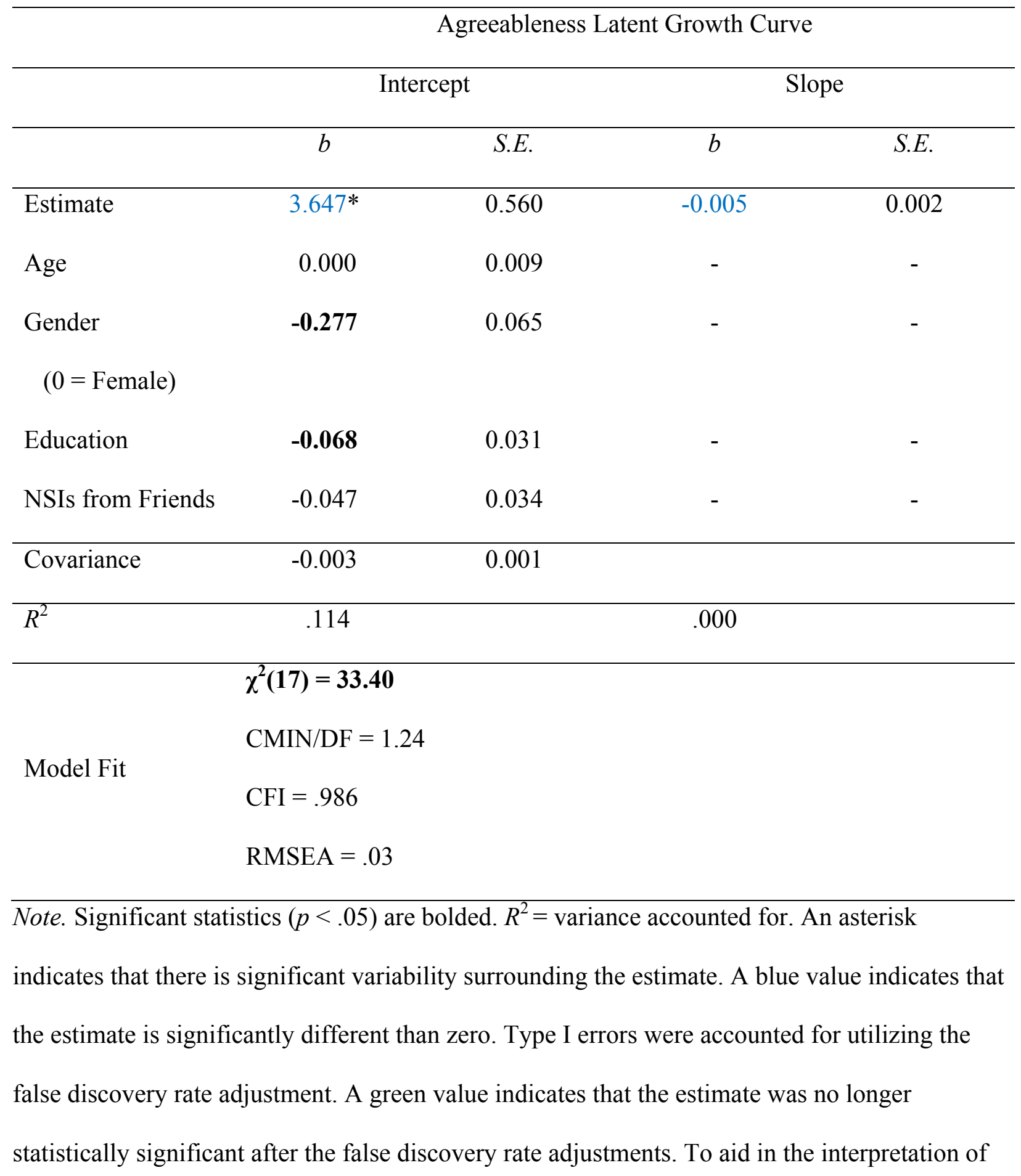


the estimates, education and NSIs from Friends were standardized. As such, a unit increase or decrease in these variables represent a standard deviation increase or decrease, respectively. Age was not manipulated so that a unit increase or decrease represents an increase or decrease of a year, respectively. 
Table K29

Research Question 6 - Demographic Variables and NSIs from Friends Predicting the Latent Growth Curve of Openness for Younger Adults

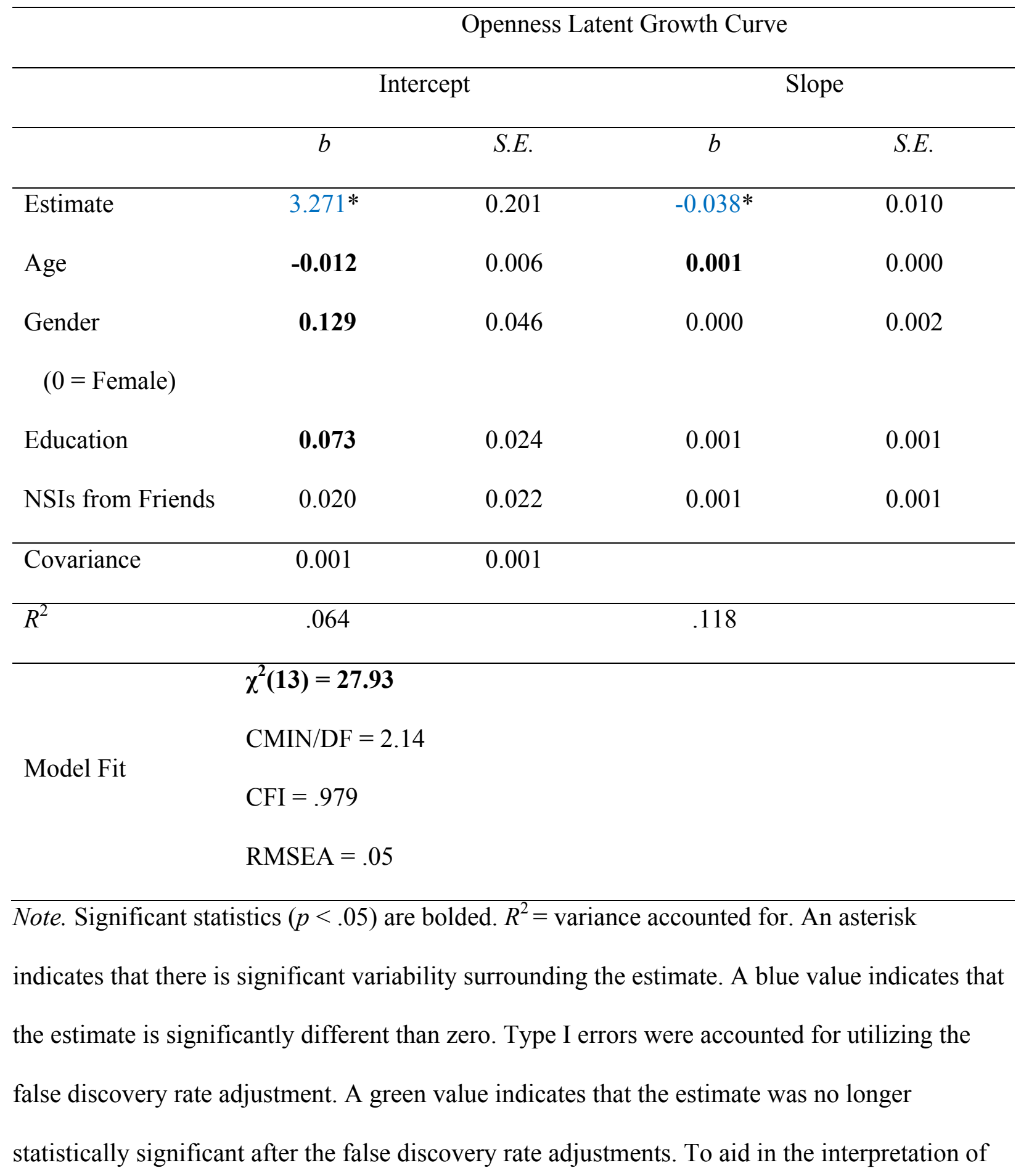


the estimates, education and NSIs from Friends were standardized. As such, a unit increase or decrease in these variables represent a standard deviation increase or decrease, respectively. Age was not manipulated so that a unit increase or decrease represents an increase or decrease of a year, respectively. 
Table K30

Research Question 6 - Demographic Variables and NSIs from Friends Predicting the Latent Growth Curve of Openness for Middle-Aged Adults

\section{Openness Latent Growth Curve}

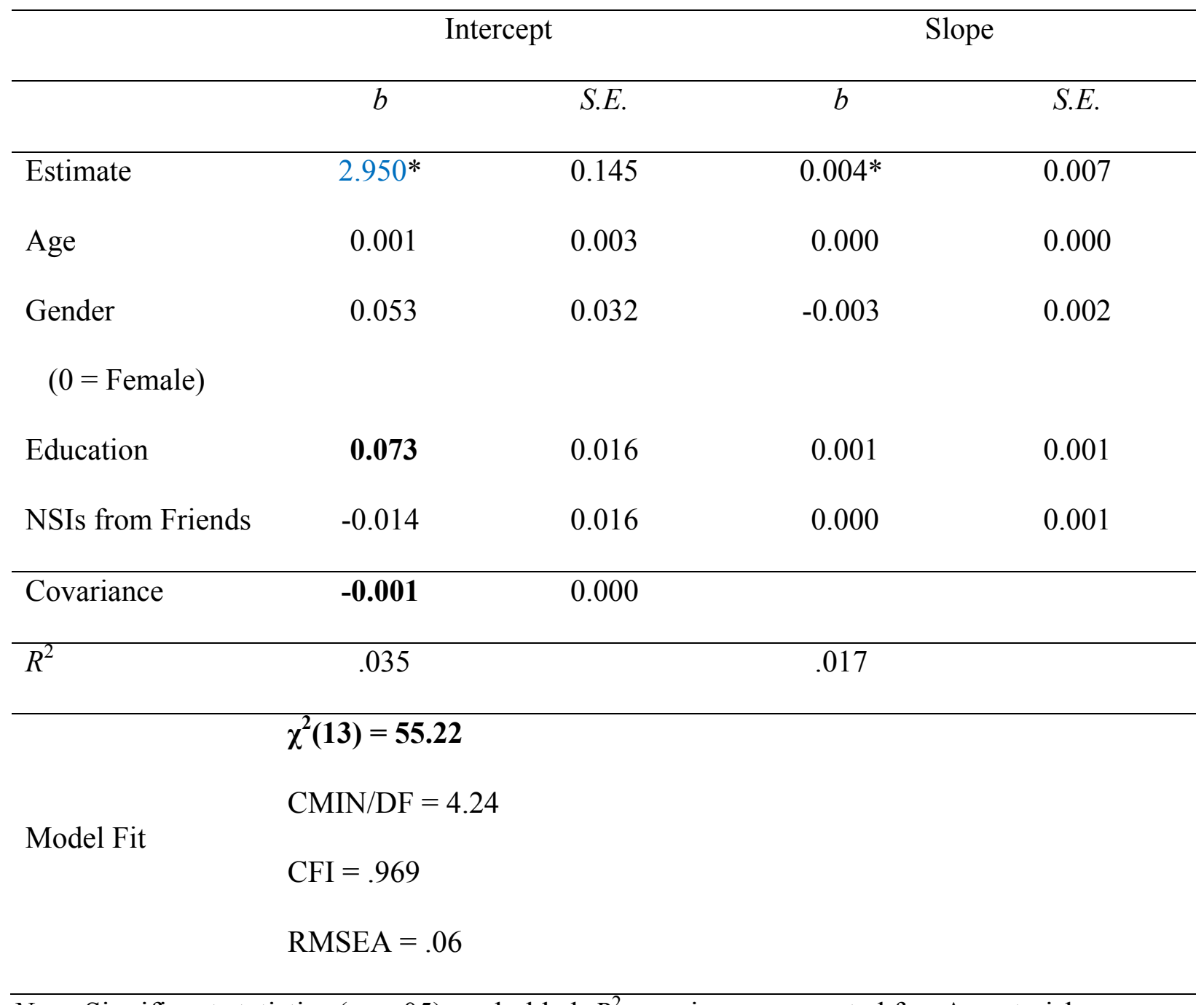

Note. Significant statistics $(p<.05)$ are bolded. $R^{2}=$ variance accounted for. An asterisk indicates that there is significant variability surrounding the estimate. A blue value indicates that the estimate is significantly different than zero. Type I errors were accounted for utilizing the false discovery rate adjustment. A green value indicates that the estimate was no longer statistically significant after the false discovery rate adjustments. To aid in the interpretation of 
the estimates, education and NSIs from Friends were standardized. As such, a unit increase or decrease in these variables represent a standard deviation increase or decrease, respectively. Age was not manipulated so that a unit increase or decrease represents an increase or decrease of a year, respectively. 
Table K31

Research Question 6 - Demographic Variables and NSIs from Friends Predicting the Latent Growth Curve of Openness for Older Adults

\section{Openness Latent Growth Curve}

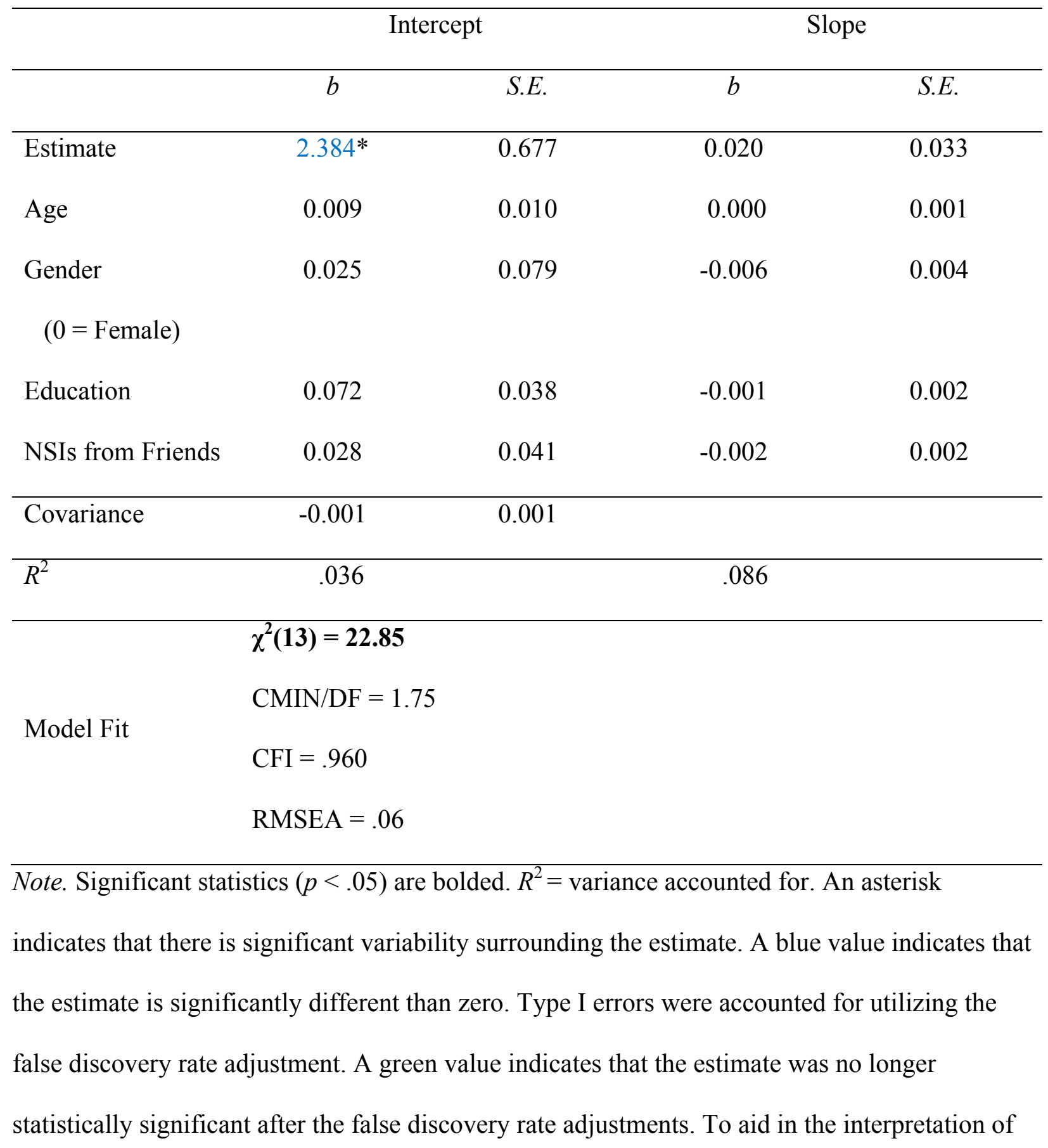


the estimates, education and NSIs from Friends were standardized. As such, a unit increase or decrease in these variables represent a standard deviation increase or decrease, respectively. Age was not manipulated so that a unit increase or decrease represents an increase or decrease of a year, respectively. 
Table K32

Research Question 6 - Demographic Variables and NSIs from Friends Predicting the Latent Growth Curve of Neuroticism for Younger Adults

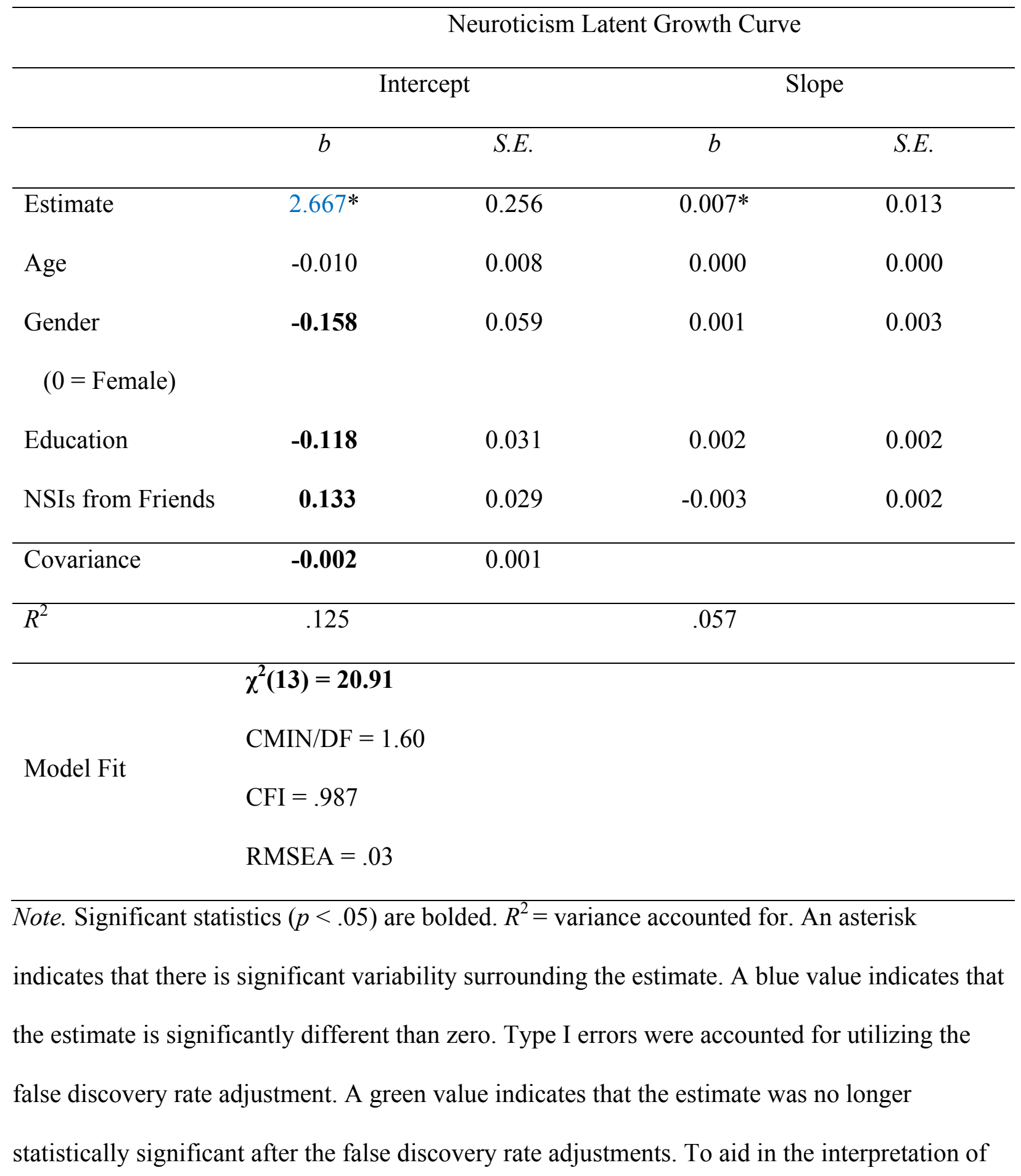


the estimates, education and NSIs from Friends were standardized. As such, a unit increase or decrease in these variables represent a standard deviation increase or decrease, respectively. Age was not manipulated so that a unit increase or decrease represents an increase or decrease of a year, respectively. 
Table K33

Research Question 6 - Demographic Variables and NSIs from Friends Predicting the Latent Growth Curve of Neuroticism for Middle-Aged Adults

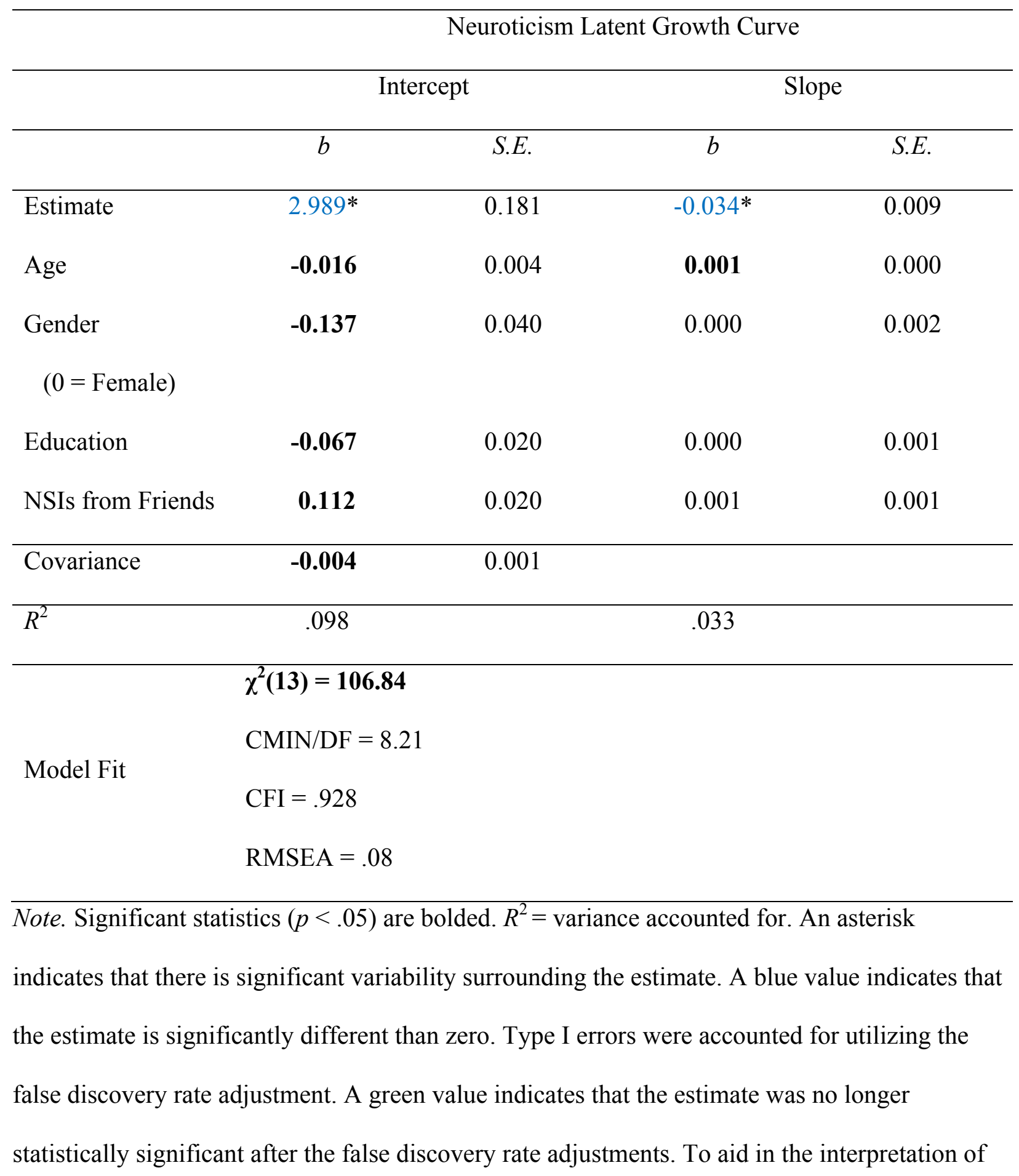


the estimates, education and NSIs from Friends were standardized. As such, a unit increase or decrease in these variables represent a standard deviation increase or decrease, respectively. Age was not manipulated so that a unit increase or decrease represents an increase or decrease of a year, respectively. 
Table K34

Research Question 6 - Demographic Variables and NSIs from Friends Predicting the Latent Growth Curve of Neuroticism for Older Adults

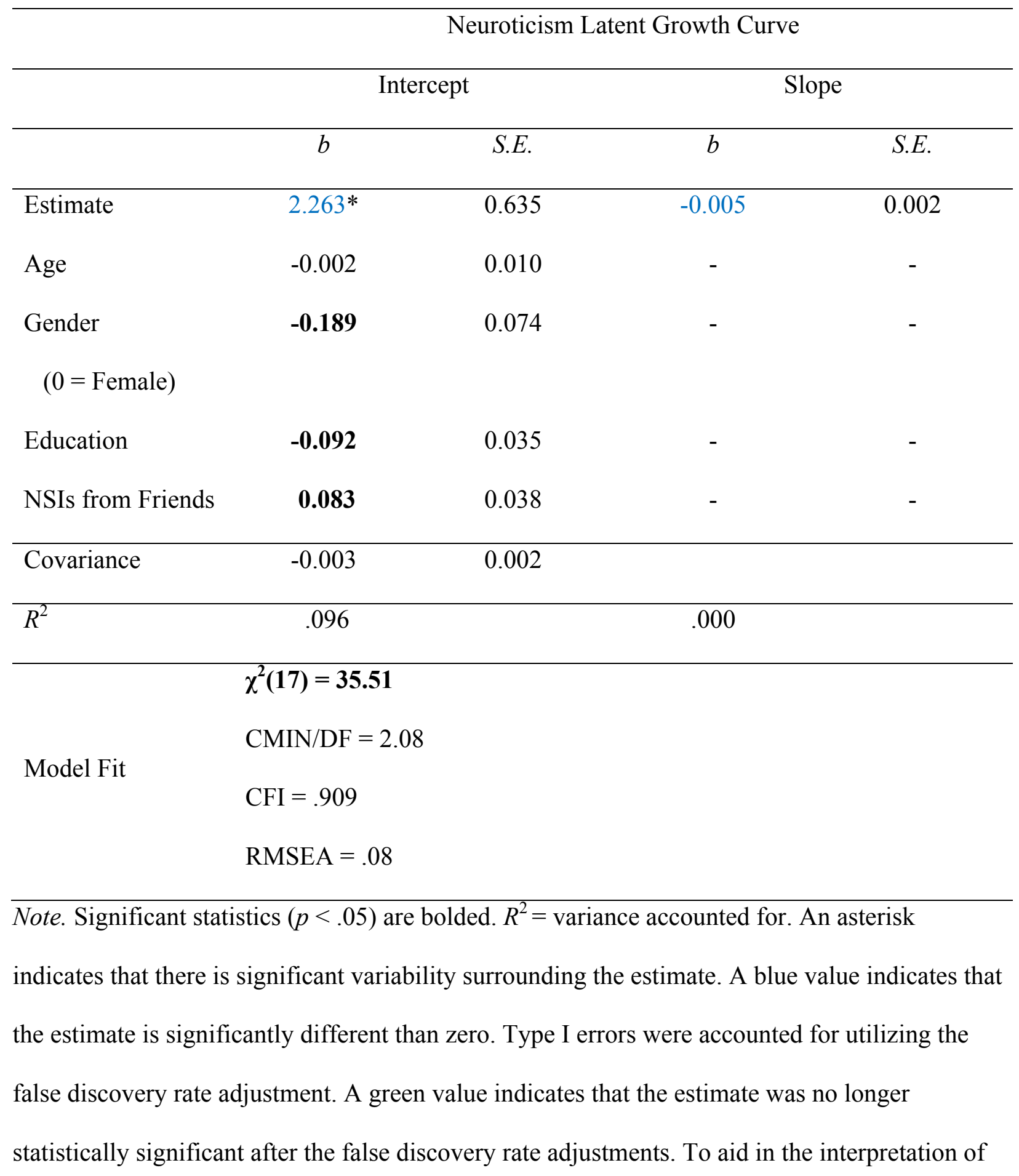


the estimates, education and NSIs from Friends were standardized. As such, a unit increase or decrease in these variables represent a standard deviation increase or decrease, respectively. Age was not manipulated so that a unit increase or decrease represents an increase or decrease of a year, respectively. 
Table K35

Research Question 6 - Demographic Variables and NSIs from Friends Predicting the Latent Growth Curve of Conscientiousness for Younger Adults

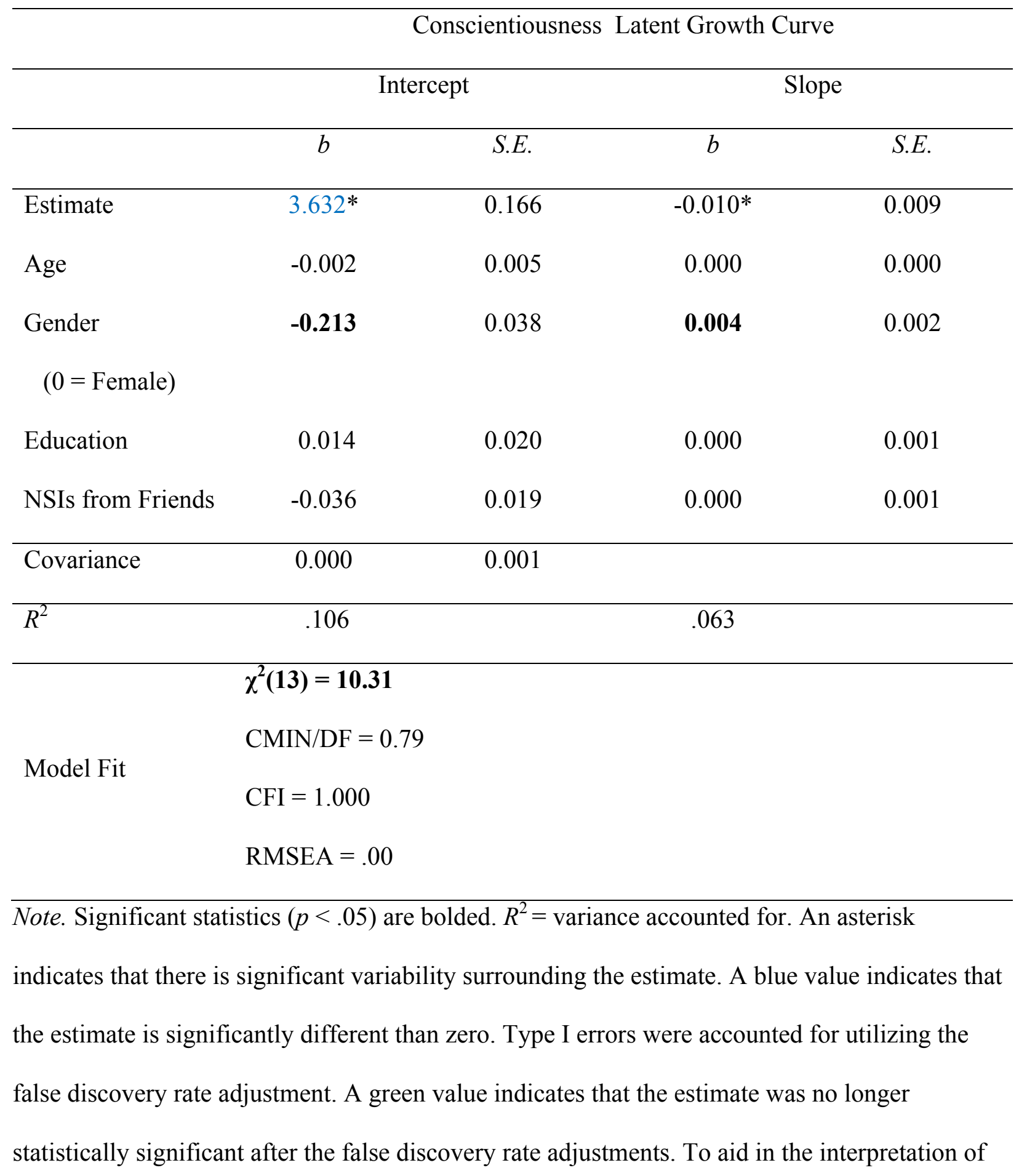


the estimates, education and NSIs from Friends were standardized. As such, a unit increase or decrease in these variables represent a standard deviation increase or decrease, respectively. Age was not manipulated so that a unit increase or decrease represents an increase or decrease of a year, respectively. 
Table K36

Research Question 6 - Demographic Variables and NSIs from Friends Predicting the Latent Growth Curve of Conscientiousness for Middle-Aged Adults

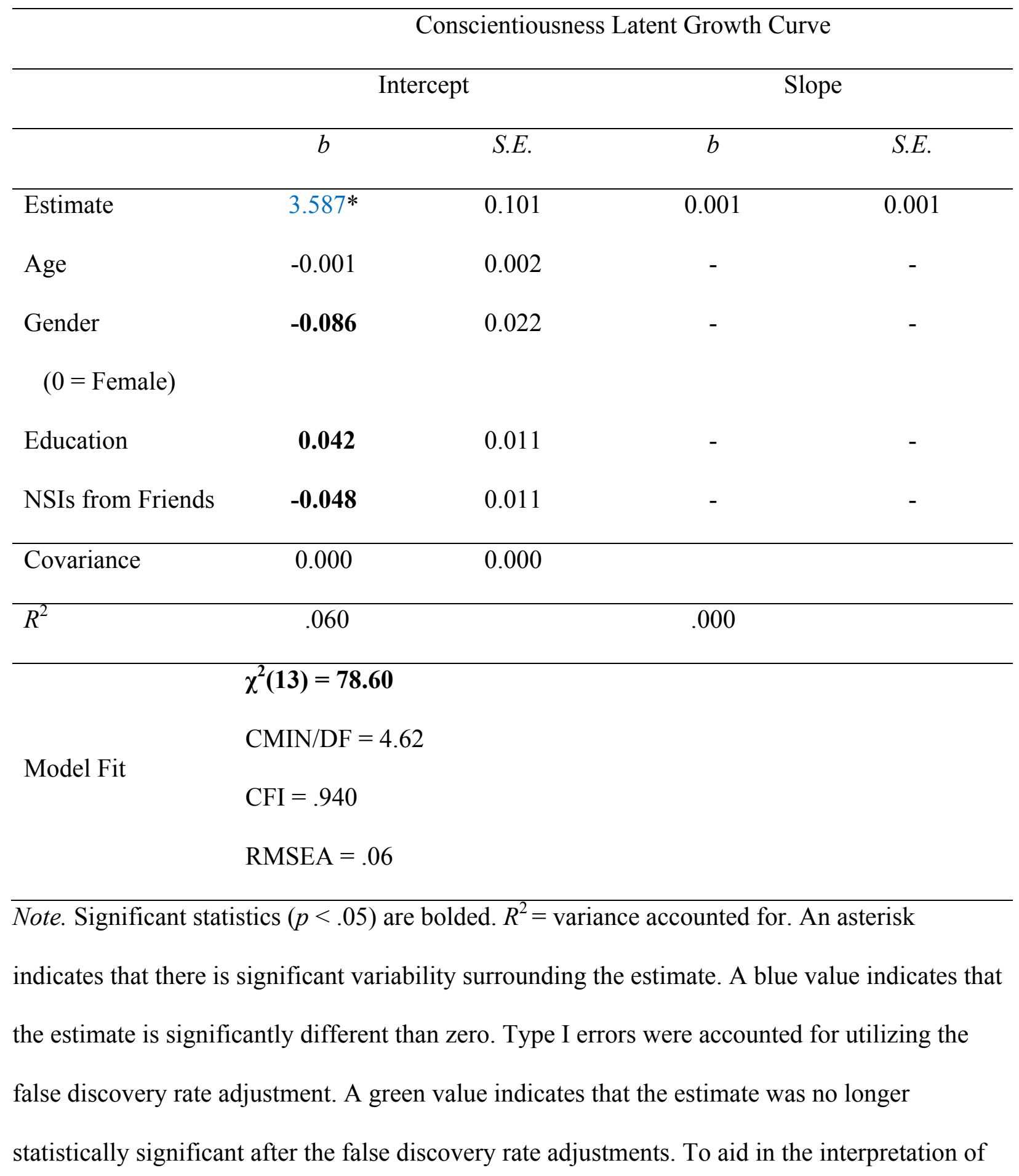


the estimates, education and NSIs from Friends were standardized. As such, a unit increase or decrease in these variables represent a standard deviation increase or decrease, respectively. Age was not manipulated so that a unit increase or decrease represents an increase or decrease of a year, respectively. 
Table K37

Research Question 6 - Demographic Variables and NSIs from Friends Predicting the Latent Growth Curve of Conscientiousness for Older Adults

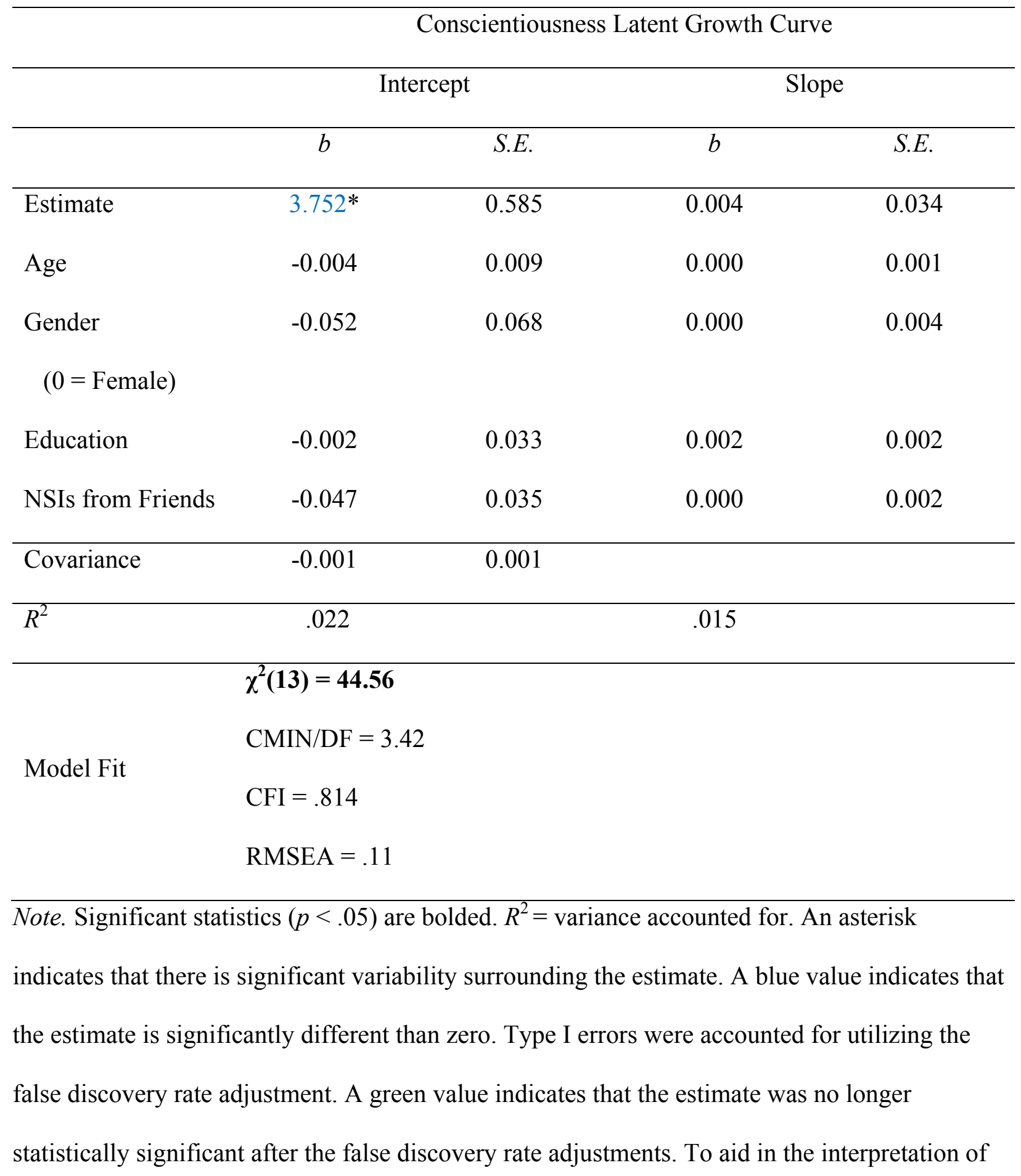


the estimates, education and NSIs from Friends were standardized. As such, a unit increase or decrease in these variables represent a standard deviation increase or decrease, respectively. Age was not manipulated so that a unit increase or decrease represents an increase or decrease of a year, respectively. 
Table K38

Preliminary Models that Examine Significant Parameters that can be accounted for with the Cross-Domain Latent Growth Curves

\begin{tabular}{|c|c|c|c|c|c|c|c|}
\hline & \multirow[t]{2}{*}{ Model Fit } & \multicolumn{2}{|c|}{ Intercept } & \multicolumn{2}{|c|}{ Slope } & \multicolumn{2}{|c|}{ Covariance } \\
\hline & & $b$ & S.E. & $b$ & S.E. & $b$ & S.E. \\
\hline NSIs from Friends and & $\chi^{2}(14)=25.54$ & $1.888 *$ & 0.011 & $-0.011 *$ & 0.001 & 0.000 & 0.000 \\
\hline \multirow[t]{3}{*}{ Agreeableness } & $\mathrm{CMIN} / \mathrm{DF}=1.82$ & $3.456^{*}$ & 0.012 & $-0.002 *$ & 0.001 & -0.001 & 0.000 \\
\hline & $\mathrm{CFI}=.996$ & & & & & & \\
\hline & $\mathrm{RMSEA}=.02$ & & & & & & \\
\hline NSIs from Friends and & $\chi^{2}(14)=50.64$ & $1.888 *$ & 0.011 & $-0.011 *$ & 0.001 & 0.000 & 0.000 \\
\hline \multirow[t]{3}{*}{ Openness } & $\mathrm{CMIN} / \mathrm{DF}=3.61$ & $2.977 *$ & 0.013 & $-0.006^{*}$ & 0.001 & 0.000 & 0.000 \\
\hline & $\mathrm{CFI}=.988$ & & & & & & \\
\hline & $\mathrm{RMSEA}=.04$ & & & & & & \\
\hline NSIs from Friends and & $\chi^{2}(14)=95.57$ & $1.888^{*}$ & 0.011 & $-0.011^{*}$ & 0.001 & 0.000 & 0.000 \\
\hline \multirow[t]{3}{*}{ Neuroticism } & $\mathrm{CMIN} / \mathrm{DF}=6.82$ & $2.163 *$ & 0.016 & $-0.008 *$ & 0.001 & -0.003 & 0.001 \\
\hline & $\mathrm{CFI}=.971$ & & & & & & \\
\hline & $\mathrm{RMSEA}=.06$ & & & & & & \\
\hline
\end{tabular}




\begin{tabular}{|c|c|c|c|c|c|c|c|}
\hline NSIs from Friends and & $\chi^{2}(14)=27.70$ & $1.888^{*}$ & 0.011 & $-0.011 *$ & 0.001 & 0.000 & 0.000 \\
\hline \multirow[t]{3}{*}{ Extraversion } & $\mathrm{CMIN} / \mathrm{DF}=1.97$ & $3.183 *$ & 0.014 & $-0.006^{*}$ & 0.001 & 0.000 & 0.000 \\
\hline & $\mathrm{CFI}=.995$ & & & & & & \\
\hline & RMSEA $=.02$ & & & & & & \\
\hline NSIs from Friends and & $\chi^{2}(14)=49.68$ & $1.888^{*}$ & 0.011 & $-0.011 *$ & 0.001 & 0.000 & 0.000 \\
\hline \multirow[t]{3}{*}{ Conscientiousness } & $\mathrm{CMIN} / \mathrm{DF}=3.54$ & $3.493 *$ & 0.010 & $0.001 *$ & 0.001 & 0.000 & 0.000 \\
\hline & $\mathrm{CFI}=.985$ & & & & & & \\
\hline & RMSEA $=.04$ & & & & & & \\
\hline
\end{tabular}

Note. Significant statistics $(p<.05)$ are bolded. An asterisk indicates that there is significant variability surrounding the estimate. A blue value indicates that the estimate is significantly different than zero. 
Table K39

Research Question 7 - Examination of the Bidirectional Association between the Latent Growth Curves of NSIs from Friend and Agreeableness After Accounting for the Demographic Variables and Remaining Personality Traits

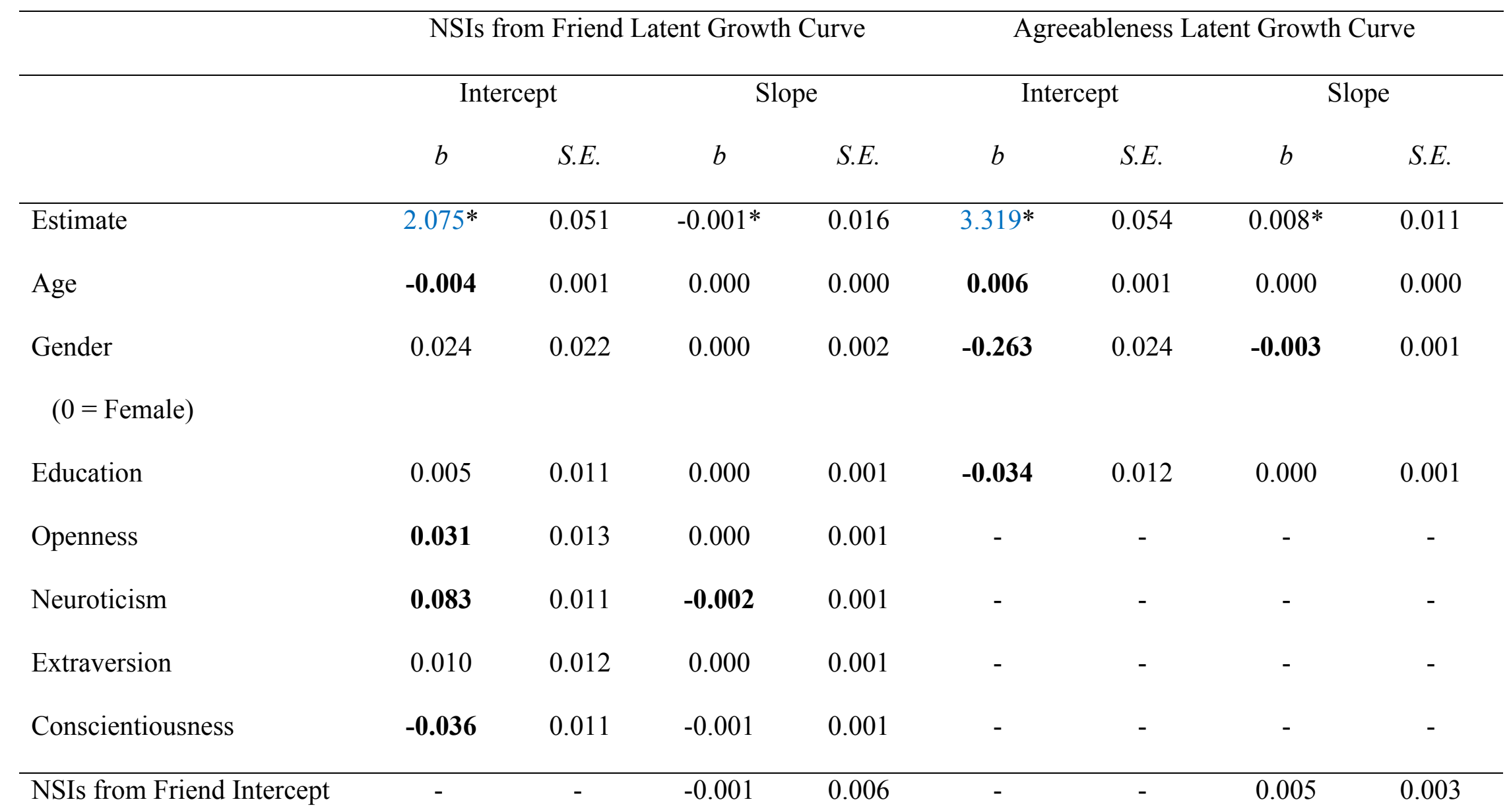




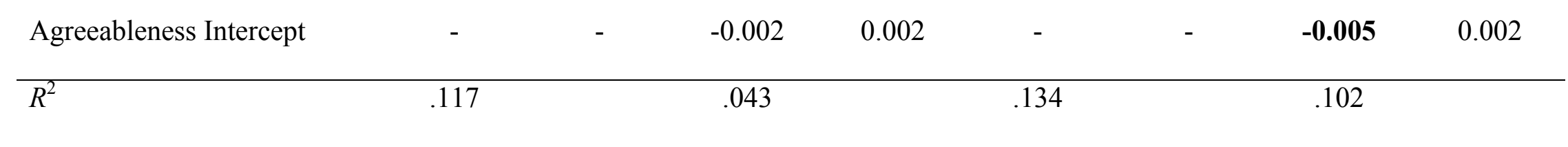

\begin{tabular}{ll}
\hline Model Fit & $\chi^{2}(\mathbf{4 9})=\mathbf{8 6 7 . 2 1}$ \\
$\mathrm{CMIN} / \mathrm{DF}=17.69$ \\
$\mathrm{CFI}=.811$ \\
$\mathrm{RMSEA}=.10$
\end{tabular}

Note. Significant statistics $(p<.05)$ are bolded. $R^{2}=$ variance accounted for. An asterisk indicates that there is significant variability surrounding the estimate. A blue value indicates that the estimate is significantly different than zero. Type I errors were accounted for utilizing the false discovery rate adjustment. A green value indicates that the estimate was no longer statistically significant after the false discovery rate adjustments. To aid in the interpretation of the estimates, education, openness, neuroticism, extraversion, and conscientiousness were standardized. As such, a unit increase or decrease in these variables represent a standard deviation increase or decrease, respectively. Age was not manipulated so that a unit increase or decrease represents an increase or decrease of a year, respectively. 
Table K40

Research Question 7 - Examination of the Bidirectional Association between the Latent Growth Curves of NSIs from Friend and Openness After Accounting for the Demographic Variables and Remaining Personality Traits

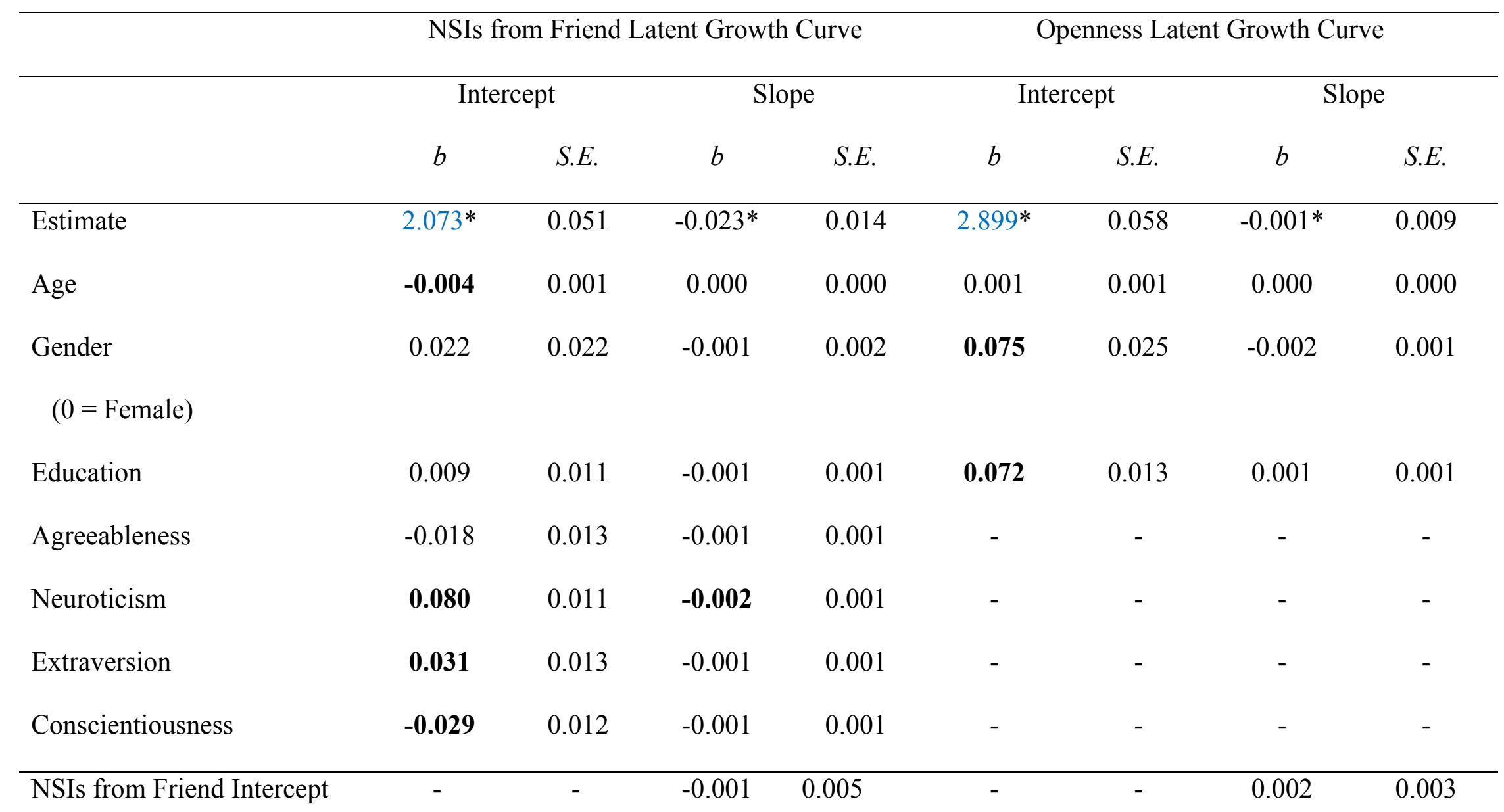




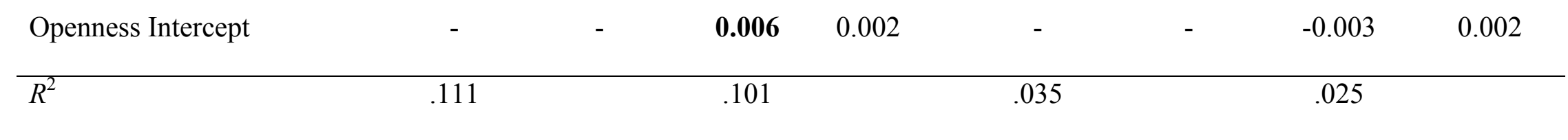

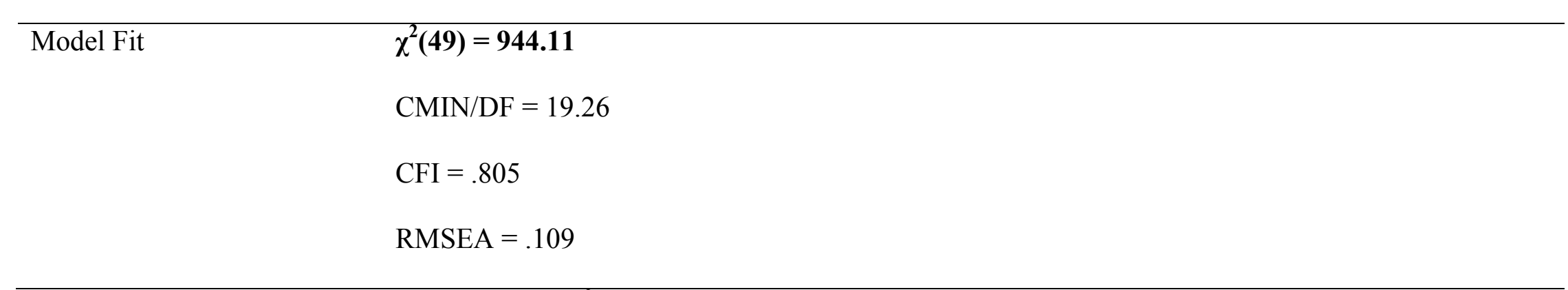

Note. Significant statistics $(p<.05)$ are bolded. $R^{2}=$ variance accounted for. An asterisk indicates that there is significant variability surrounding the estimate. A blue value indicates that the estimate is significantly different than zero. Type I errors were accounted for utilizing the false discovery rate adjustment. A green value indicates that the estimate was no longer statistically significant after the false discovery rate adjustments. To aid in the interpretation of the estimates, education, agreeableness, neuroticism, extraversion, and conscientiousness were standardized. As such, a unit increase or decrease in these variables represent a standard deviation increase or decrease, respectively. Age was not manipulated so that a unit increase or decrease represents an increase or decrease of a year, respectively. 
Table K41

Research Question 7 - Examination of the Bidirectional Association between the Latent Growth Curves of NSIs from Friend and Neuroticism After Accounting for the Demographic Variables and Remaining Personality Traits

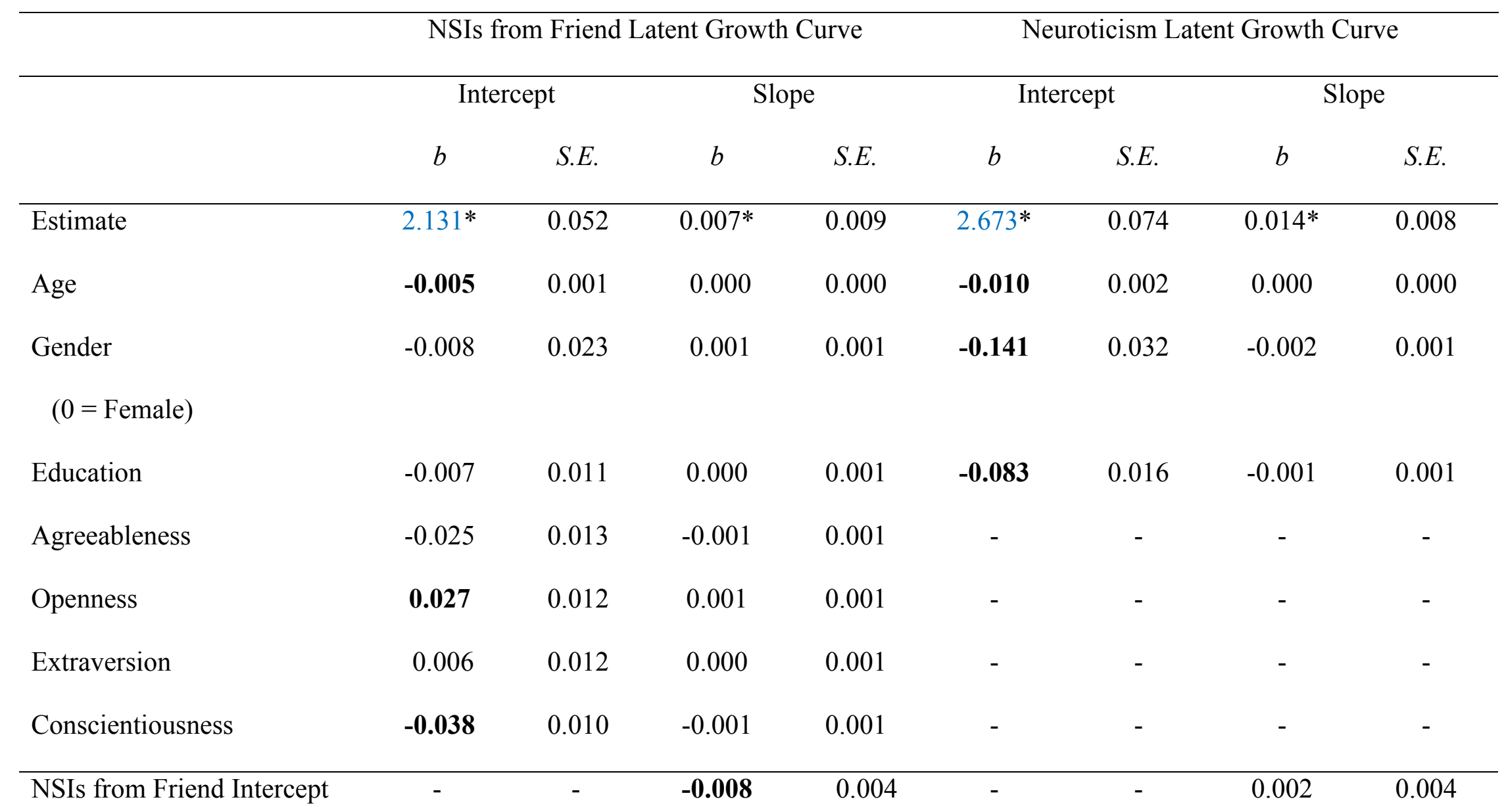




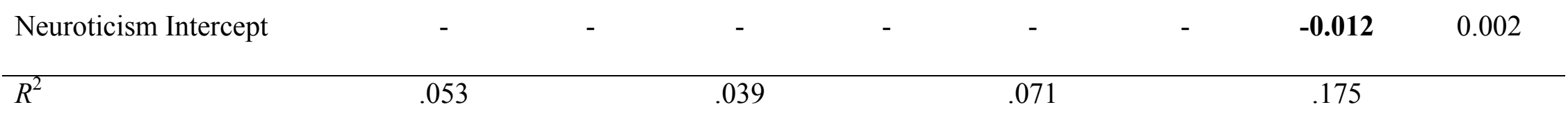

\begin{tabular}{ll}
\hline Model Fit & $\chi^{2} \mathbf{5 0}=\mathbf{5 4 5 . 0 8}$ \\
& $\mathrm{CMIN} / \mathrm{DF}=10.90$ \\
$\mathrm{CFI}=.886$ \\
$\mathrm{RMSEA}=.08$
\end{tabular}

Note. Significant statistics $(p<.05)$ are bolded. $R^{2}=$ variance accounted for. An asterisk indicates that there is significant variability surrounding the estimate. A blue value indicates that the estimate is significantly different than zero. Type I errors were accounted for utilizing the false discovery rate adjustment. A green value indicates that the estimate was no longer statistically significant after the false discovery rate adjustments. To aid in the interpretation of the estimates, education, agreeableness, openness, extraversion, and conscientiousness were standardized. As such, a unit increase or decrease in these variables represent a standard deviation increase or decrease, respectively. Age was not manipulated so that a unit increase or decrease represents an increase or decrease of a year, respectively. 
Table K42

Research Question 7 - Examination of the Bidirectional Association between the Latent Growth Curves of NSIs from Friend and Extraversion After Accounting for the Demographic Variables and Remaining Personality Traits

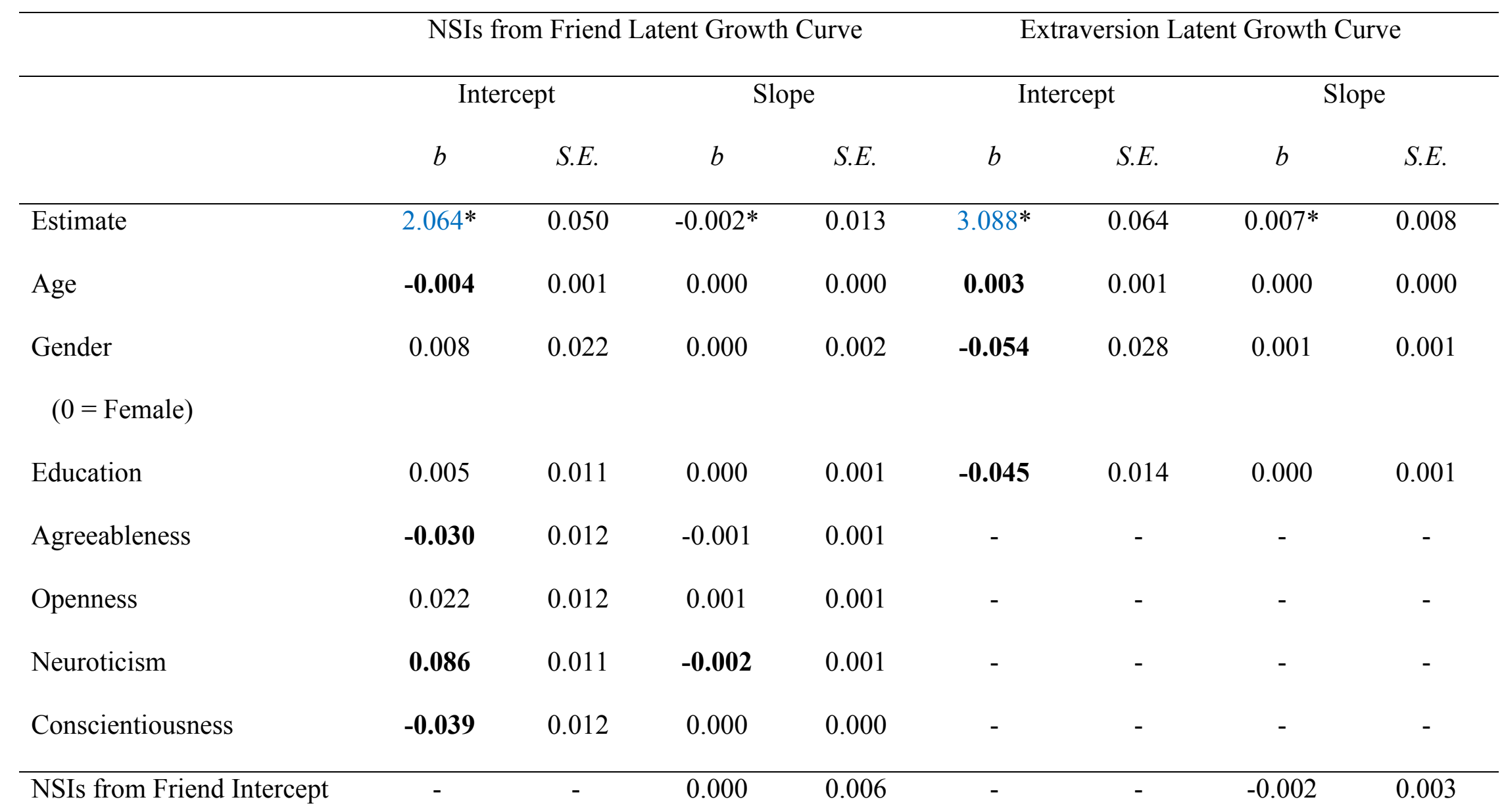




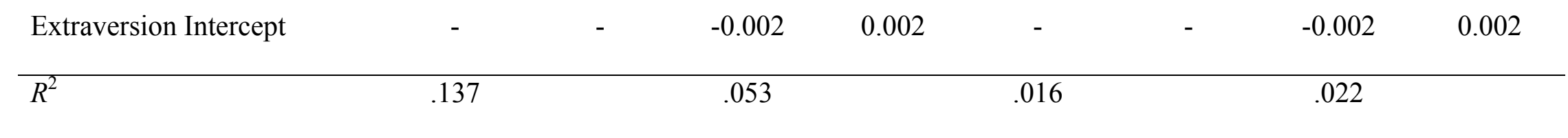

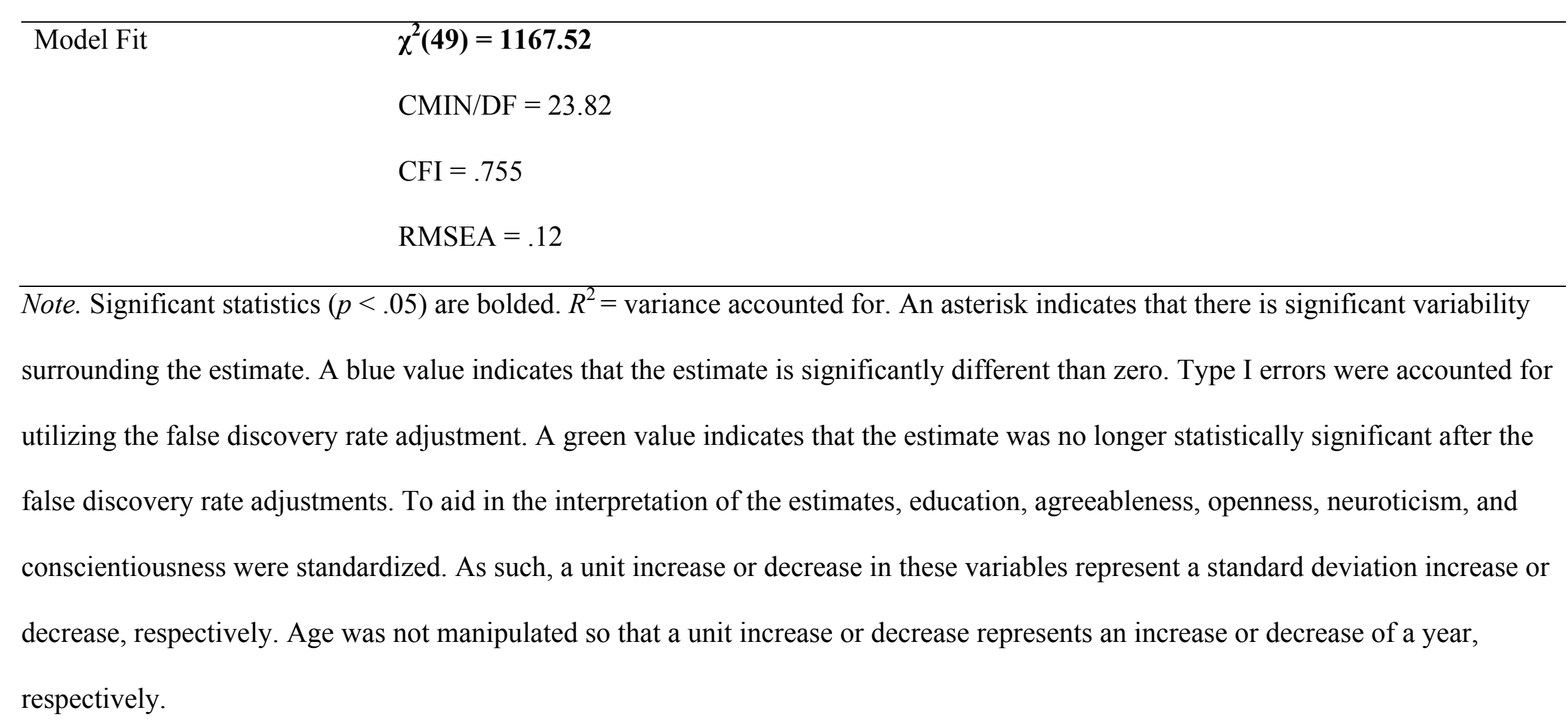


Table K43

Research Question 7 - Examination of the Bidirectional Association between the Latent Growth Curves of NSIs from Friend and Conscientiousness After Accounting for the Demographic Variables and Remaining Personality Traits

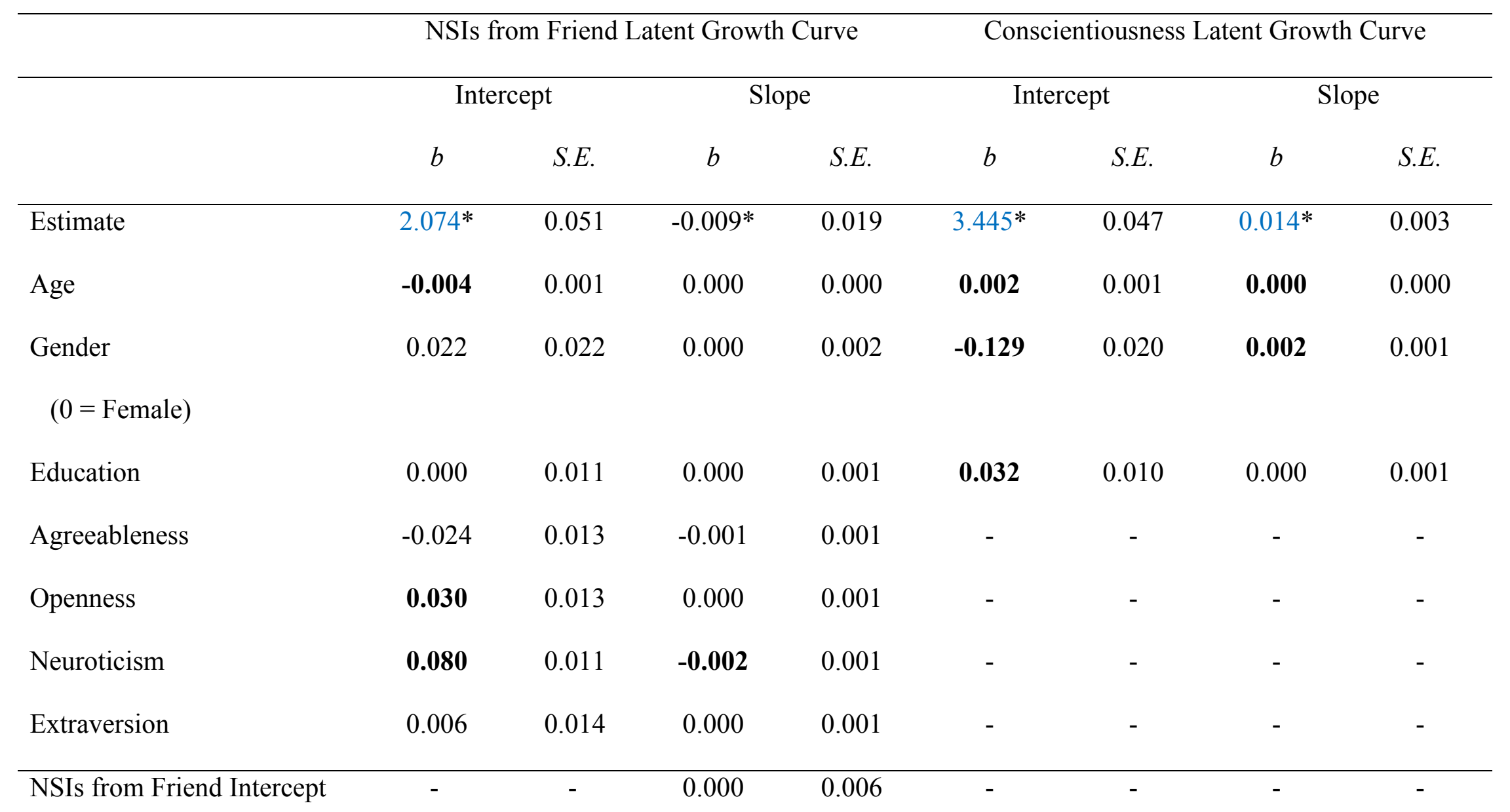




\begin{tabular}{lllllll} 
Conscientiousness Intercept & - & - & 0.000 & 0.003 & - & - \\
\hline$R^{2}$ & .097 & .044 & .053 & .120
\end{tabular}

$\chi^{\mathbf{2}} \mathbf{( 5 0 )}=\mathbf{6 4 4 . 8 1}$
Model Fit
CFI $=.851$
RMSEA $=.08$

Note. Significant statistics $(p<.05)$ are bolded. $R^{2}=$ variance accounted for. An asterisk indicates that there is significant variability surrounding the estimate. A blue value indicates that the estimate is significantly different than zero. Type I errors were accounted for utilizing the false discovery rate adjustment. A green value indicates that the estimate was no longer statistically significant after the false discovery rate adjustments. To aid in the interpretation of the estimates, education, agreeableness, openness, neuroticism, and extraversion were standardized. As such, a unit increase or decrease in these variables represent a standard deviation increase or decrease, respectively. Age was not manipulated so that a unit increase or decrease represents an increase or decrease of a year, respectively. 
Table K44

The Unconstrained Models Compared to the Constrained Models for the Multigroup Cross-Domain Latent Growth Curves

$\chi^{2}$

CFI

$\Delta \chi^{2}$ $\triangle \mathrm{CFI}$

NSIs from Friend and Agreeableness

Unconstrained Model

Constrained Model

NSIs from Friend and Openness

Unconstrained Model
Constrained Model

$$
\chi^{2}(46)=109.03
$$

.978

$\chi^{2}(58)=168.16$

.962

$$
\chi^{2}(12)=59.13
$$

.016

NSIs from Friend and Neuroticism

Unconstrained Model
Constrained Model

$$
\begin{aligned}
& \chi^{2}(46)=145.89 \\
& \chi^{2}(58)=195.96
\end{aligned}
$$

$$
\chi^{2}(12)=50.07
$$

NSIs from Friend and Extraversion

Unconstrained Model
Constrained Model

$$
\begin{aligned}
& \chi^{2}(46)=69.44 \\
& \chi^{2}(58)=128.51
\end{aligned}
$$

$$
\chi^{2}(12)=59.07
$$


NSIs from Friend and Conscientiousness

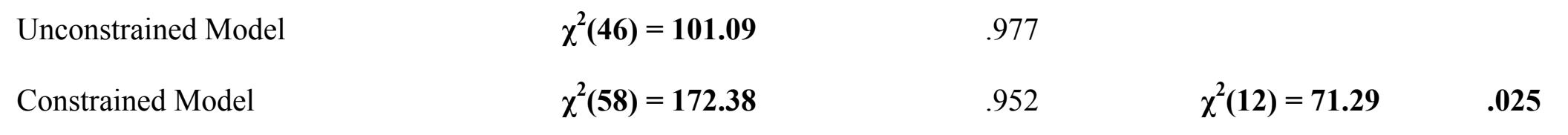

Note. Significant statistics $(p<.05)$ are bolded. The unconstrained model is where the parameters were freely estimated across the three age groups. The constrained model is where the parameters were constrained to be equal across the three age groups. A change of .01 or greater for the CFI difference statistic was used to determine significance (Little, 2013). Both the chi-square and the CFI difference statistics were computed because the chi-square statistic is commonly used, however, it is sensitive to large sample sizes. The CFI is more robust to larger sample sizes (Little, 2013). 
Table K45

Research Question 8 - Multigroup Cross-Domain Latent Growth Curves

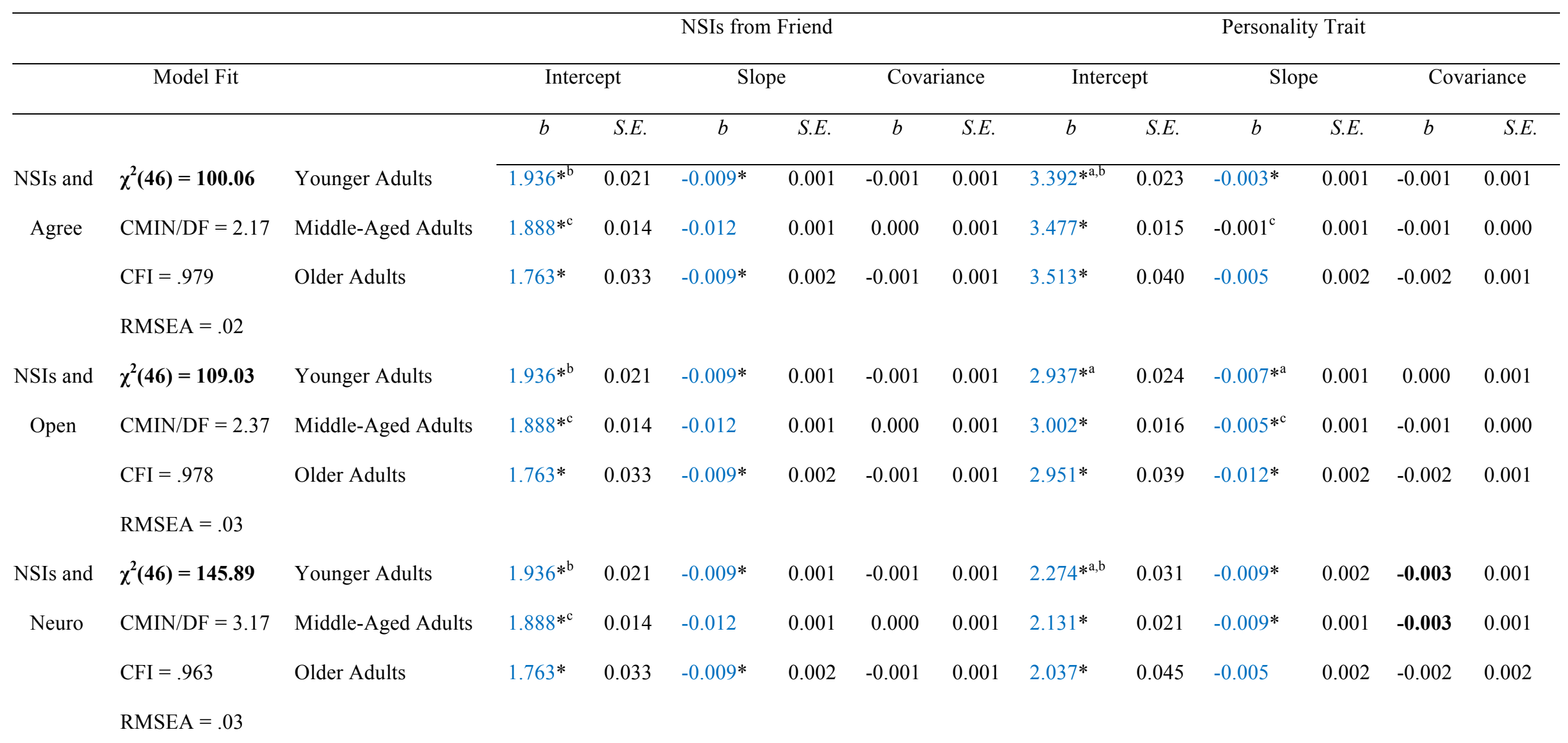




\begin{tabular}{|c|c|c|c|c|c|c|c|c|c|c|c|c|c|c|}
\hline NSIs and & $\chi^{2}(46)=69.44$ & Younger Adults & $1.936^{* b}$ & 0.021 & $-0.009 *$ & 0.001 & -0.001 & 0.001 & $3.167 *$ a,b & 0.026 & $-0.008 *$ & 0.001 & -0.001 & 0.001 \\
\hline \multirow[t]{3}{*}{ Extra } & $\mathrm{CMIN} / \mathrm{DF}=1.51$ & Middle-Aged Adults & $1.888 *^{\mathrm{c}}$ & 0.014 & -0.012 & 0.001 & 0.000 & 0.001 & $3.180 *$ & 0.018 & $-0.004 * \mathrm{c}$ & 0.001 & 0.000 & 0.001 \\
\hline & $\mathrm{CFI}=.992$ & Older Adults & $1.763^{*}$ & 0.033 & $-0.009 *$ & 0.002 & -0.001 & 0.001 & $3.243 *$ & 0.043 & -0.014 & 0.002 & 0.001 & 0.001 \\
\hline & $\mathrm{RMSEA}=.01$ & & & & & & & & & & & & & \\
\hline NSIs and & $\chi^{2}(46)=101.09$ & Younger Adults & $1.936^{* b}$ & 0.021 & $-0.009 *$ & 0.001 & -0.001 & 0.001 & $3.459 *^{\mathrm{a}}$ & 0.020 & $0.003 * a, b$ & 0.001 & 0.000 & 0.000 \\
\hline \multirow[t]{2}{*}{ Consc } & $\mathrm{CMIN} / \mathrm{DF}=2.19$ & Middle-Aged Adults & $1.888^{* \mathrm{c}}$ & 0.014 & -0.012 & 0.001 & 0.000 & 0.001 & $3.510 *$ & 0.013 & $0.001^{\mathrm{c}}$ & 0.001 & 0.000 & 0.000 \\
\hline & $\mathrm{CFI}=.977$ & Older Adults & $1.763 *$ & 0.033 & $-0.009 *$ & 0.002 & -0.001 & 0.001 & $3.495^{*}$ & 0.034 & $-0.008 *$ & 0.002 & -0.002 & 0.001 \\
\hline
\end{tabular}

Note. Significant statistics $(p<.05)$ are bolded. Agree $=$ agreeableness. Open $=$ openness. Neuro $=$ neuroticism. Extra $=$ extraversion.

Consc $=$ conscientiousness. An asterisk indicates that there is significant variability surrounding the estimate. A blue value indicates that the estimate is significantly different than zero. ${ }^{a}$ indicates that the estimate is significantly different between younger and middleaged adults. ${ }^{\mathrm{b}}$ indicates that the estimate is significantly different between younger and older adults. ${ }^{\mathrm{c}}$ indicates that the estimate is significantly different between middle-aged and older adults. 
Table K46

Research Question 8 - Examination of the Bidirectional Association between the Latent Growth Curves of NSIs from Friend and Agreeableness After Accounting for the Demographic Variables and Remaining Personality Traits for Younger Adults

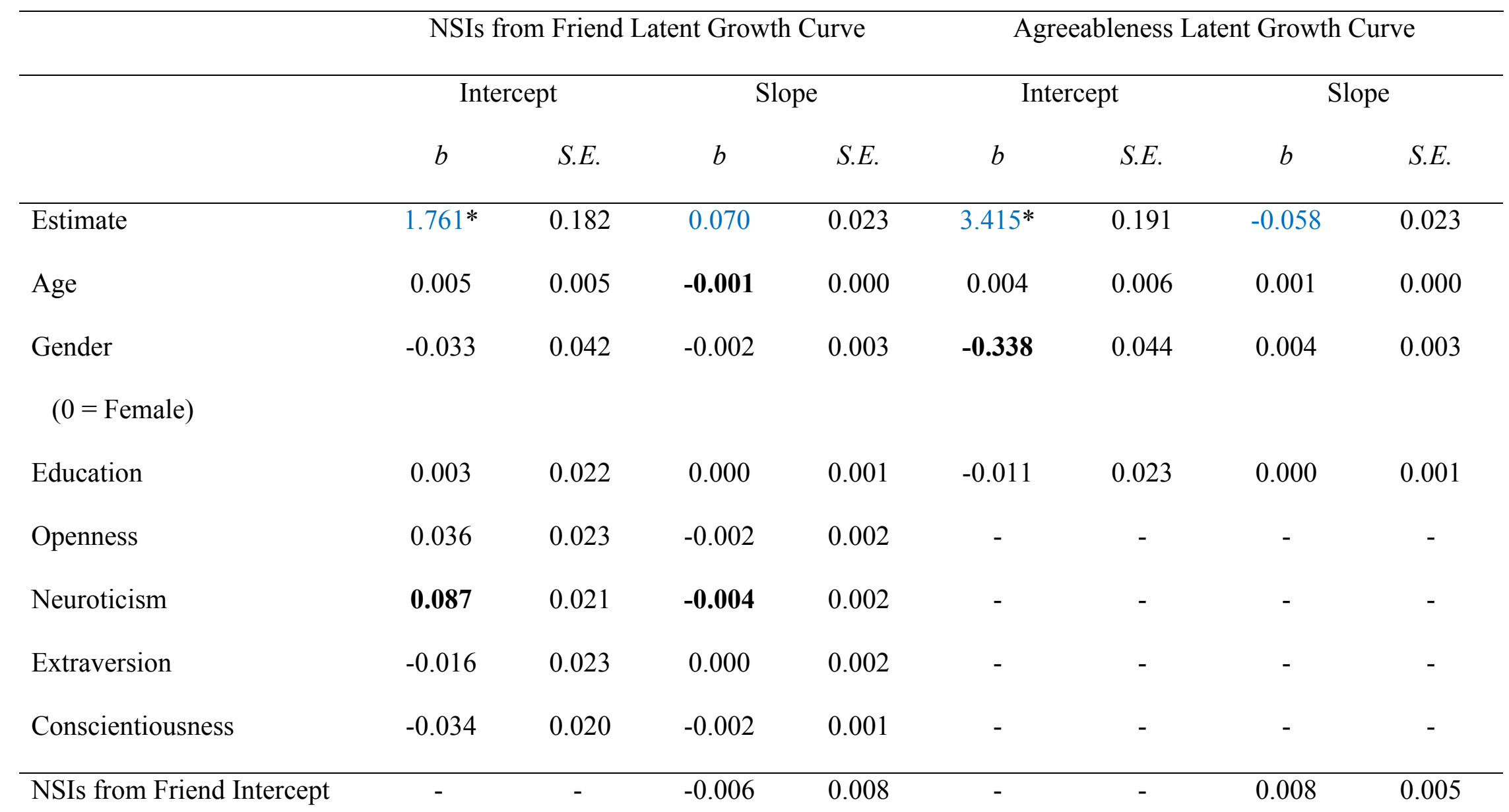




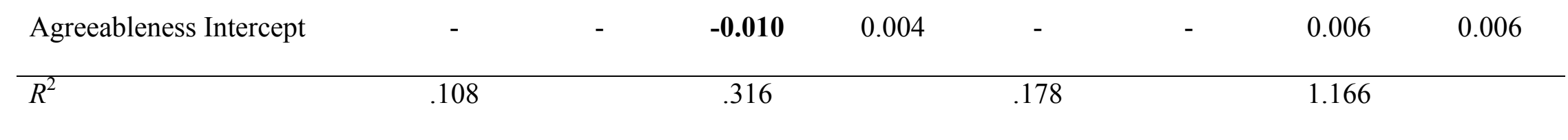

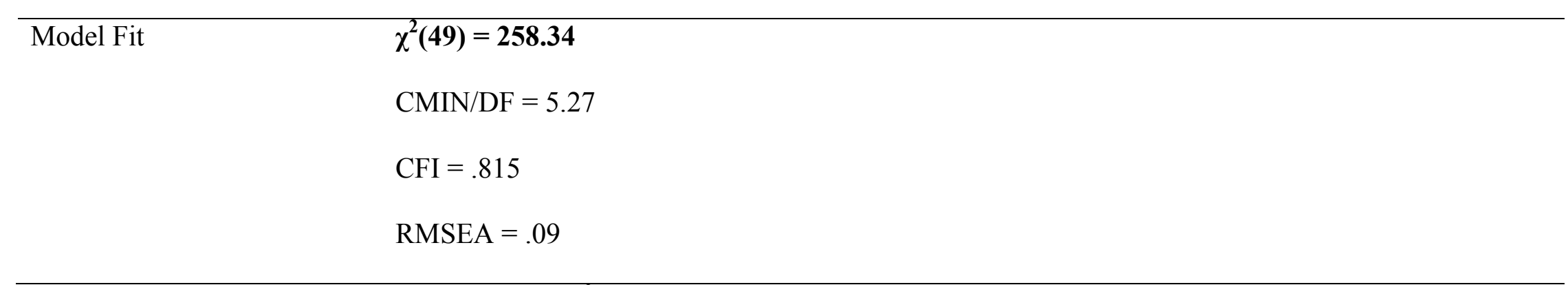

Note. Significant statistics $(p<.05)$ are bolded. $R^{2}=$ variance accounted for. An asterisk indicates that there is significant variability surrounding the estimate. A blue value indicates that the estimate is significantly different than zero. Type I errors were accounted for utilizing the false discovery rate adjustment. A green value indicates that the estimate was no longer statistically significant after the false discovery rate adjustments. To aid in the interpretation of the estimates, education, openness, neuroticism, extraversion, and conscientiousness were standardized. As such, a unit increase or decrease in these variables represent a standard deviation increase or decrease, respectively. Age was not manipulated so that a unit increase or decrease represents an increase or decrease of a year, respectively. 
Table K47

Research Question 8 - Examination of the Bidirectional Association between the Latent Growth Curves of NSIs from Friends and Agreeableness After Accounting for the Demographic Variables and Remaining Personality Traits for Middle-Aged Adults

\begin{tabular}{|c|c|c|c|c|c|c|c|c|}
\hline & \multicolumn{4}{|c|}{ NSIs from Friends Latent Growth Curve } & \multicolumn{4}{|c|}{ Agreeableness Latent Growth Curve } \\
\hline & \multicolumn{2}{|c|}{ Intercept } & \multicolumn{2}{|c|}{ Slope } & \multicolumn{2}{|c|}{ Intercept } & \multicolumn{2}{|c|}{ Slope } \\
\hline & $b$ & S.E. & $b$ & S.E. & $b$ & S.E. & $b$ & S.E. \\
\hline Estimate & $2.143^{*}$ & 0.109 & $-0.012 *$ & 0.017 & $3.399 *$ & 0.115 & $-0.001^{*}$ & 0.001 \\
\hline Age & -0.005 & 0.002 & - & - & 0.004 & 0.002 & - & - \\
\hline $\begin{array}{l}\text { Gender } \\
\qquad(0=\text { Female })\end{array}$ & 0.025 & 0.025 & - & - & -0.259 & 0.025 & - & - \\
\hline Education & 0.002 & 0.012 & - & - & -0.038 & 0.013 & - & - \\
\hline Openness & 0.034 & 0.014 & - & - & - & - & - & - \\
\hline Neuroticism & 0.077 & 0.013 & - & - & - & - & - & - \\
\hline Extraversion & 0.015 & 0.014 & - & - & - & - & - & - \\
\hline Conscientiousness & -0.039 & 0.013 & - & - & - & - & - & - \\
\hline NSIs from Friends Intercept & - & - & 0.000 & 0.006 & - & - & - & - \\
\hline
\end{tabular}




\begin{tabular}{lcccccc} 
Agreeableness Intercept & - & - & 0.000 & 0.003 & - & - \\
\hline$R^{2}$ & .094 & .000 & .121 & .000
\end{tabular}

\begin{tabular}{ll}
\hline Model Fit & $\chi^{\mathbf{2}(60)}=\mathbf{5 7 5 . 3 9}$ \\
& $\mathrm{CMIN} / \mathrm{DF}=9.59$ \\
$\mathrm{CFI}=.802$ \\
$\mathrm{RMSEA}=.09$
\end{tabular}

Note. Significant statistics $(p<.05)$ are bolded. $R^{2}=$ variance accounted for. An asterisk indicates that there is significant variability surrounding the estimate. A blue value indicates that the estimate is significantly different than zero. Type I errors were accounted for utilizing the false discovery rate adjustment. A green value indicates that the estimate was no longer statistically significant after the false discovery rate adjustments. To aid in the interpretation of the estimates, education, openness, neuroticism, extraversion, and conscientiousness were standardized. As such, a unit increase or decrease in these variables represent a standard deviation increase or decrease, respectively. Age was not manipulated so that a unit increase or decrease represents an increase or decrease of a year, respectively. 
Table K48

Research Question 8 - Examination of the Bidirectional Association between the Latent Growth Curves of NSIs from Friends and Agreeableness After Accounting for the Demographic Variables and Remaining Personality Traits for Older Adults

\begin{tabular}{|c|c|c|c|c|c|c|c|c|}
\hline & \multicolumn{4}{|c|}{ NSIs from Friends Latent Growth Curve } & \multicolumn{4}{|c|}{ Agreeableness Latent Growth Curve } \\
\hline & \multicolumn{2}{|c|}{ Intercept } & \multicolumn{2}{|c|}{ Slope } & \multicolumn{2}{|c|}{ Intercept } & \multicolumn{2}{|c|}{ Slope } \\
\hline & $b$ & S.E. & $b$ & S.E. & $b$ & S.E. & $b$ & S.E. \\
\hline Estimate & $2.483 *$ & 0.548 & -0.004 & 0.118 & $3.651^{*}$ & 0.622 & 0.040 & 0.028 \\
\hline Age & -0.013 & 0.008 & 0.000 & 0.001 & 0.000 & 0.010 & - & - \\
\hline $\begin{array}{l}\text { Gender } \\
\qquad(0=\text { Female })\end{array}$ & 0.166 & 0.064 & 0.001 & 0.007 & -0.287 & 0.073 & - & - \\
\hline Education & 0.004 & 0.030 & 0.002 & 0.003 & -0.074 & 0.035 & - & - \\
\hline Openness & 0.029 & 0.038 & 0.000 & 0.003 & - & - & - & - \\
\hline Neuroticism & 0.081 & 0.035 & -0.002 & 0.004 & - & - & - & - \\
\hline Extraversion & 0.023 & 0.037 & 0.002 & 0.003 & - & - & - & - \\
\hline Conscientiousness & -0.058 & 0.031 & 0.001 & 0.003 & - & - & - & - \\
\hline NSIs from Friends Intercept & - & - & 0.006 & 0.034 & - & - & -0.007 & 0.009 \\
\hline
\end{tabular}




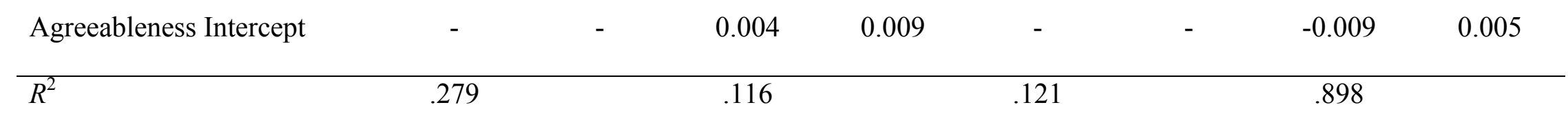

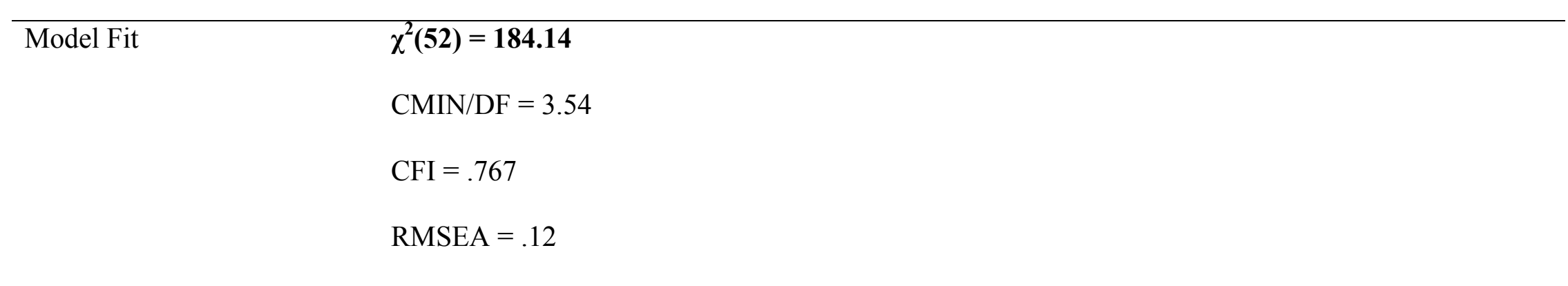

Note. Significant statistics $(p<.05)$ are bolded. $R^{2}=$ variance accounted for. An asterisk indicates that there is significant variability surrounding the estimate. A blue value indicates that the estimate is significantly different than zero. Type I errors were accounted for utilizing the false discovery rate adjustment. A green value indicates that the estimate was no longer statistically significant after the false discovery rate adjustments. To aid in the interpretation of the estimates, education, openness, neuroticism, extraversion, and conscientiousness were standardized. As such, a unit increase or decrease in these variables represent a standard deviation increase or decrease, respectively. Age was not manipulated so that a unit increase or decrease represents an increase or decrease of a year, respectively. 
Table K49

Research Question 8 - Examination of the Bidirectional Association between the Latent Growth Curves of NSIs from Friend and Openness After Accounting for the Demographic Variables and Remaining Personality Traits for Younger Adults

\begin{tabular}{|c|c|c|c|c|c|c|c|c|}
\hline & \multicolumn{4}{|c|}{ NSIs from Friend Latent Growth Curve } & \multicolumn{4}{|c|}{ Openness Latent Growth Curve } \\
\hline & \multicolumn{2}{|c|}{ Intercept } & \multicolumn{2}{|c|}{ Slope } & \multicolumn{2}{|c|}{ Intercept } & \multicolumn{2}{|c|}{ Slope } \\
\hline & $b$ & S.E. & $b$ & S.E. & $b$ & S.E. & $b$ & S.E. \\
\hline Estimate & $1.778 *$ & 0.182 & 0.037 & 0.022 & $3.272 *$ & 0.201 & -0.055 & 0.019 \\
\hline Age & 0.004 & 0.005 & -0.001 & 0.000 & -0.012 & 0.006 & 0.001 & 0.000 \\
\hline $\begin{array}{l}\text { Gender } \\
\qquad(0=\text { Female })\end{array}$ & -0.005 & 0.042 & -0.001 & 0.003 & 0.128 & 0.046 & -0.001 & 0.003 \\
\hline Education & 0.009 & 0.022 & 0.000 & 0.001 & 0.072 & 0.024 & 0.001 & 0.001 \\
\hline Agreeableness & 0.028 & 0.023 & -0.003 & 0.002 & - & - & - & - \\
\hline Neuroticism & 0.091 & 0.020 & -0.005 & 0.002 & - & - & - & - \\
\hline Extraversion & -0.010 & 0.023 & 0.000 & 0.002 & - & - & - & - \\
\hline Conscientiousness & -0.036 & 0.021 & -0.002 & 0.001 & - & - & - & - \\
\hline NSIs from Friend Intercept & - & - & -0.005 & 0.009 & - & - & -0.002 & 0.005 \\
\hline
\end{tabular}




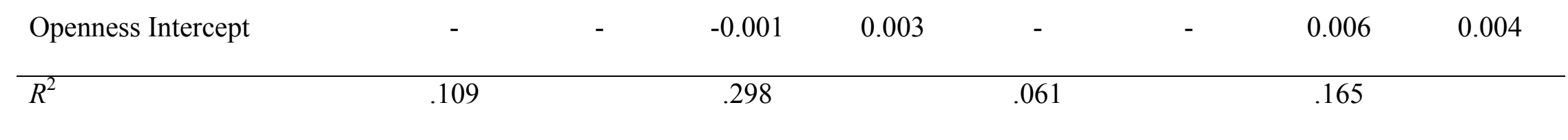

\begin{tabular}{ll}
\hline Model Fit & $\chi^{\mathbf{2}(49)=\mathbf{2 9 7 . 5 1}}$ \\
& $\mathrm{CMIN} / \mathrm{DF}=6.07$ \\
$\mathrm{CFI}=.806$ \\
$\mathrm{RMSEA}=.10$
\end{tabular}

Note. Significant statistics $(p<.05)$ are bolded. $R^{2}=$ variance accounted for. An asterisk indicates that there is significant variability surrounding the estimate. A blue value indicates that the estimate is significantly different than zero. Type I errors were accounted for utilizing the false discovery rate adjustment. A green value indicates that the estimate was no longer statistically significant after the false discovery rate adjustments. To aid in the interpretation of the estimates, education, agreeableness, neuroticism, extraversion, and conscientiousness were standardized. As such, a unit increase or decrease in these variables represent a standard deviation increase or decrease, respectively. Age was not manipulated so that a unit increase or decrease represents an increase or decrease of a year, respectively. 
Table K50

Research Question 8 - Examination of the Bidirectional Association between the Latent Growth Curves of NSIs from Friends and Openness After Accounting for the Demographic Variables and Remaining Personality Traits for Middle-Aged Adults

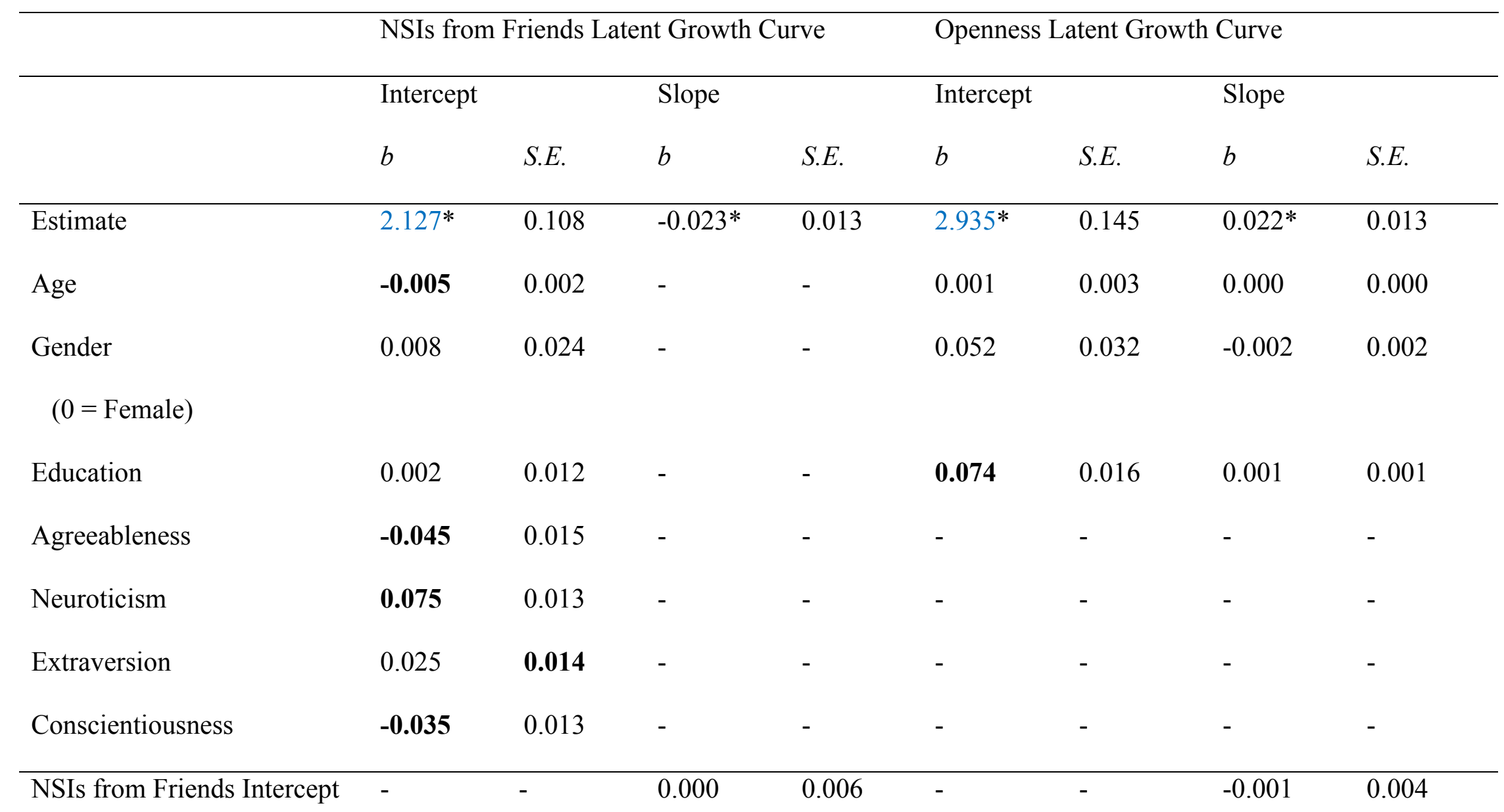




\begin{tabular}{|c|c|c|c|c|c|c|}
\hline Openness Intercept & - & 0.004 & 0.002 & - & -0.006 & 0.002 \\
\hline$R^{2}$ & 0.110 & 0.022 & & 0.034 & 0.052 & \\
\hline
\end{tabular}

\begin{tabular}{ll}
\hline Model Fit & $\left.\chi^{2} \mathbf{5 6}\right)=\mathbf{5 8 8 . 6 7}$ \\
& $\mathrm{CMIN} / \mathrm{DF}=10.51$ \\
$\mathrm{CFI}=.807$ \\
$\mathrm{RMSEA}=.10$
\end{tabular}

Note. Significant statistics $(p<.05)$ are bolded. $R^{2}=$ variance accounted for. An asterisk indicates that there is significant variability surrounding the estimate. A blue value indicates that the estimate is significantly different than zero. Type I errors were accounted for utilizing the false discovery rate adjustment. A green value indicates that the estimate was no longer statistically significant after the false discovery rate adjustments. To aid in the interpretation of the estimates, education, agreeableness, neuroticism, extraversion, and conscientiousness were standardized. As such, a unit increase or decrease in these variables represent a standard deviation increase or decrease, respectively. Age was not manipulated so that a unit increase or decrease represents an increase or decrease of a year, respectively. 
Table K51

Research Question 8 - Examination of the Bidirectional Association between the Latent Growth Curves of NSIs from Friends and Openness After Accounting for the Demographic Variables and Remaining Personality Traits for Older Adults

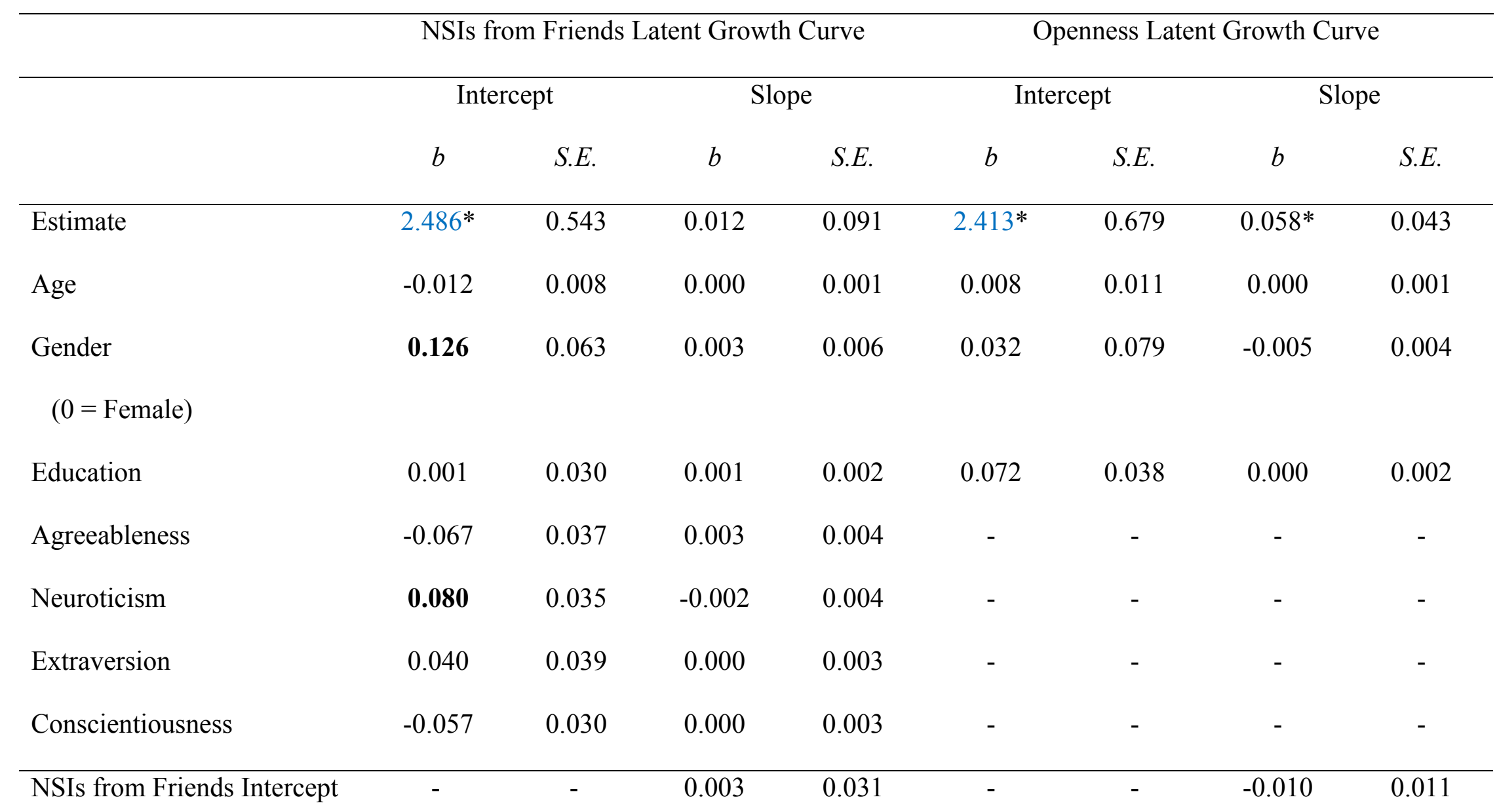




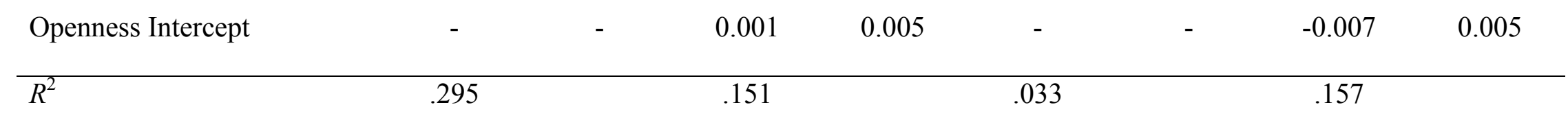

\begin{tabular}{ll}
\hline Model Fit & $\chi^{\mathbf{2}(49)}=\mathbf{1 8 2 . 1 7}$ \\
& $\mathrm{CMIN} / \mathrm{DF}=3.718$ \\
$\mathrm{CFI}=.751$ \\
$\mathrm{RMSEA}=.12$
\end{tabular}

Note. Significant statistics $(p<.05)$ are bolded. $R^{2}=$ variance accounted for. An asterisk indicates that there is significant variability surrounding the estimate. A blue value indicates that the estimate is significantly different than zero. Type I errors were accounted for utilizing the false discovery rate adjustment. A green value indicates that the estimate was no longer statistically significant after the false discovery rate adjustments. To aid in the interpretation of the estimates, education, agreeableness, neuroticism, extraversion, and conscientiousness were standardized. As such, a unit increase or decrease in these variables represent a standard deviation increase or decrease, respectively. Age was not manipulated so that a unit increase or decrease represents an increase or decrease of a year, respectively. 
Table K52

Research Question 8 - Examination of the Bidirectional Association between the Latent Growth Curves of NSIs from Friend and Neuroticism After Accounting for the Demographic Variables and Remaining Personality Traits for Younger Adults

\begin{tabular}{|c|c|c|c|c|c|c|c|c|}
\hline & \multicolumn{4}{|c|}{ NSIs from Friend Latent Growth Curve } & \multicolumn{4}{|c|}{ Neuroticism Latent Growth Curve } \\
\hline & \multicolumn{2}{|c|}{ Intercept } & \multicolumn{2}{|c|}{ Slope } & \multicolumn{2}{|c|}{ Intercept } & \multicolumn{2}{|c|}{ Slope } \\
\hline & $b$ & S.E. & $b$ & S.E. & $b$ & S.E. & $b$ & S.E. \\
\hline Estimate & $1.865^{*}$ & 0.193 & 0.037 & 0.024 & $3.381 *$ & 0.242 & -0.026 & 0.021 \\
\hline Age & 0.002 & 0.006 & -0.001 & 0.000 & -0.005 & 0.007 & 0.000 & 0.000 \\
\hline Gender $(0=$ Female $)$ & -0.021 & 0.044 & 0.001 & 0.003 & -0.122 & 0.055 & 0.002 & 0.003 \\
\hline Education & 0.026 & 0.023 & -0.001 & 0.002 & 0.011 & 0.029 & -0.001 & 0.001 \\
\hline Agreeableness & 0.020 & 0.024 & -0.002 & 0.002 & - & - & - & - \\
\hline Openness & 0.020 & 0.024 & 0.000 & 0.002 & - & - & - & - \\
\hline Extraversion & 0.085 & 0.021 & -0.004 & 0.002 & - & - & - & - \\
\hline Conscientiousness & -0.023 & 0.022 & -0.003 & 0.001 & - & - & - & - \\
\hline NSIs from Friend Intercept & - & - & -0.007 & 0.009 & - & - & 0.004 & 0.005 \\
\hline Neuroticism Intercept & - & - & -0.002 & 0.003 & - & - & 0.000 & 0.004 \\
\hline
\end{tabular}




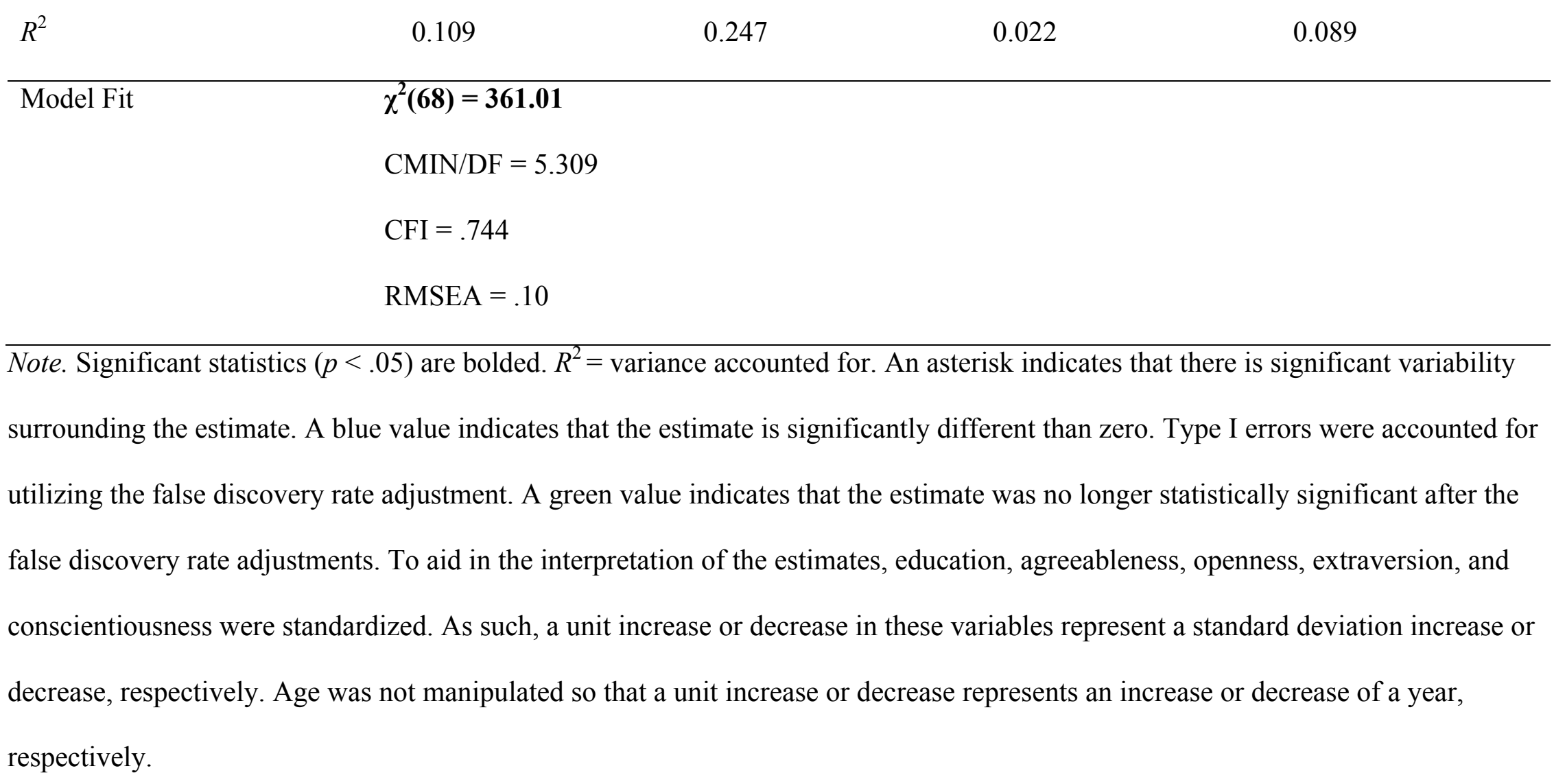


Table K53

Research Question 8 - Examination of the Bidirectional Association between the Latent Growth Curves of NSIs from Friends and Neuroticism After Accounting for the Demographic Variables and Remaining Personality Traits for Middle-Aged Adults

\begin{tabular}{|c|c|c|c|c|c|c|c|c|}
\hline & \multicolumn{4}{|c|}{ NSIs from Friends Latent Growth Curve } & \multicolumn{4}{|c|}{ Neuroticism Latent Growth Curve } \\
\hline & Intercept & & Slope & & Intercep & & Slope & \\
\hline & $b$ & S.E. & $b$ & S.E. & $b$ & S.E. & $b$ & S.E. \\
\hline Estimate & $2.300 *$ & 0.130 & $-0.051 *$ & 0.021 & $2.886^{*}$ & 0.149 & 0.021 & 0.013 \\
\hline Age & -0.008 & 0.003 & 0.000 & 0.000 & 0.002 & 0.003 & 0.000 & 0.000 \\
\hline Gender $(0=$ Female $)$ & 0.024 & 0.029 & -0.002 & 0.002 & 0.048 & 0.033 & -0.002 & 0.002 \\
\hline Education & 0.003 & 0.014 & -0.001 & 0.001 & 0.070 & 0.016 & 0.000 & 0.001 \\
\hline Agreeableness & -0.044 & 0.018 & 0.000 & 0.001 & - & - & - & - \\
\hline Openness & 0.084 & 0.015 & -0.001 & 0.001 & - & - & - & - \\
\hline Extraversion & 0.035 & 0.017 & -0.001 & 0.001 & - & - & - & - \\
\hline Conscientiousness & -0.034 & 0.016 & 0.000 & 0.001 & - & - & - & - \\
\hline NSIs from Friends Intercept & - & - & 0.003 & 0.008 & - & - & -0.005 & 0.002 \\
\hline Neuroticism Intercept & - & - & -0.001 & 0.004 & - & - & 0.005 & 0.002 \\
\hline
\end{tabular}




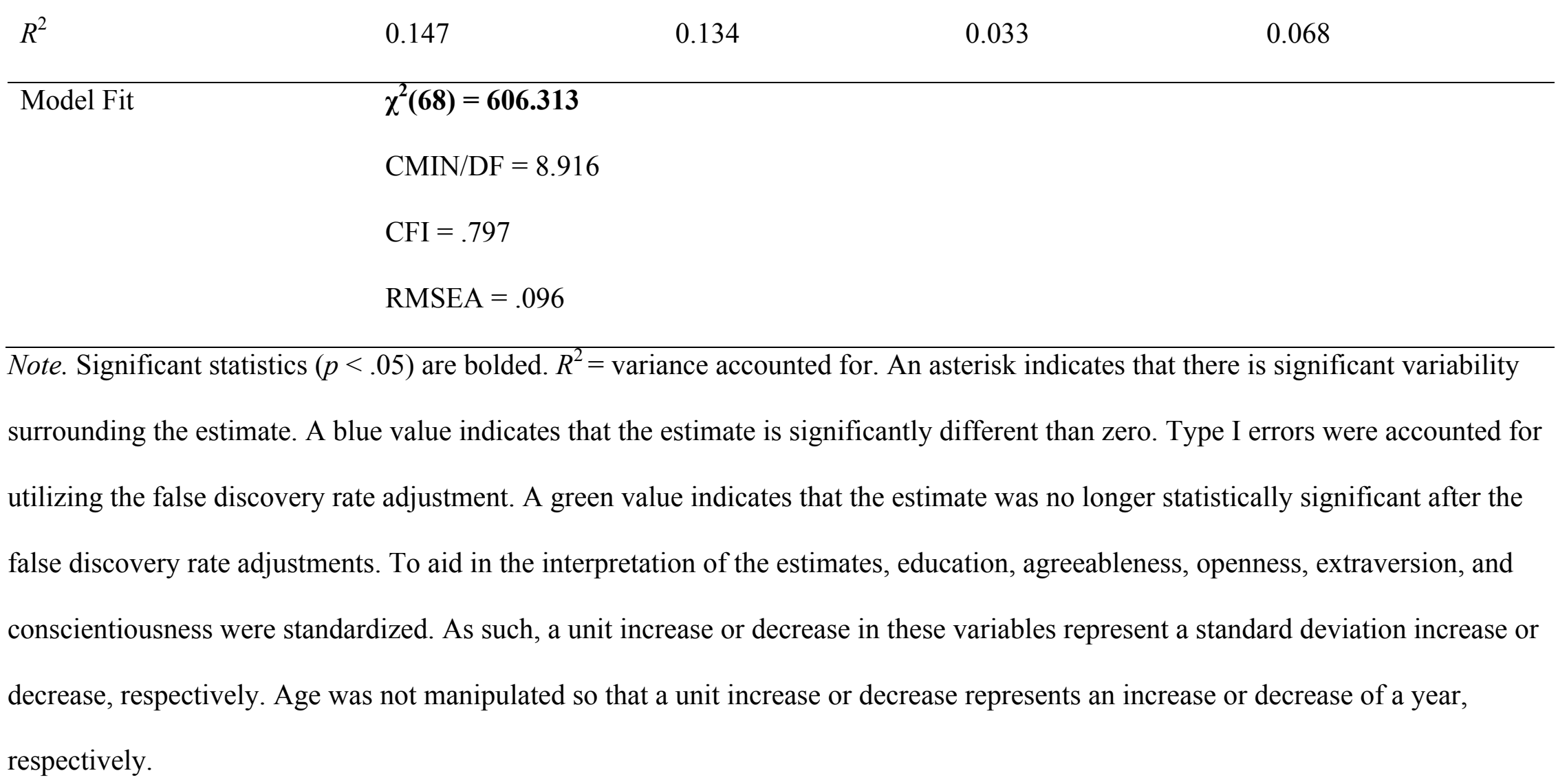


Table K54

Research Question 8 - Examination of the Bidirectional Association between the Latent Growth Curves of NSIs from Friends and Neuroticism After Accounting for the Demographic Variables and Remaining Personality Traits for Older Adults

\begin{tabular}{|c|c|c|c|c|c|c|c|c|}
\hline & \multicolumn{4}{|c|}{ NSIs from Friends Latent Growth Curve } & \multicolumn{4}{|c|}{ Neuroticism Latent Growth Curve } \\
\hline & \multicolumn{2}{|l|}{ Intercept } & \multicolumn{2}{|l|}{ Slope } & \multicolumn{2}{|l|}{ Intercept } & \multicolumn{2}{|l|}{ Slope } \\
\hline & $b$ & S.E. & $b$ & S.E. & $b$ & S.E. & $b$ & S.E. \\
\hline Estimate & $2.953^{*}$ & 0.471 & -0.011 & 0.028 & $2.254^{*}$ & 0.696 & 0.010 & 0.047 \\
\hline Age & 0.020 & 0.007 & - & - & 0.010 & 0.011 & 0.000 & 0.001 \\
\hline Gender $(0=$ Female $)$ & 0.179 & 0.056 & - & - & 0.076 & 0.081 & -0.008 & 0.004 \\
\hline Education & 0.018 & 0.025 & - & - & 0.061 & 0.038 & 0.000 & 0.002 \\
\hline Agreeableness & -0.050 & 0.030 & - & - & - & - & - & - \\
\hline Openness & 0.056 & 0.030 & - & - & - & - & - & - \\
\hline Extraversion & 0.057 & 0.032 & - & - & - & - & - & - \\
\hline Conscientiousness & -0.052 & 0.026 & - & - & - & - & - & - \\
\hline NSIs from Friends Intercept & - & - & 0.000 & 0.013 & - & - & -0.005 & 0.006 \\
\hline Neuroticism Intercept & - & - & 0.000 & 0.011 & - & - & 0.000 & 0.005 \\
\hline
\end{tabular}




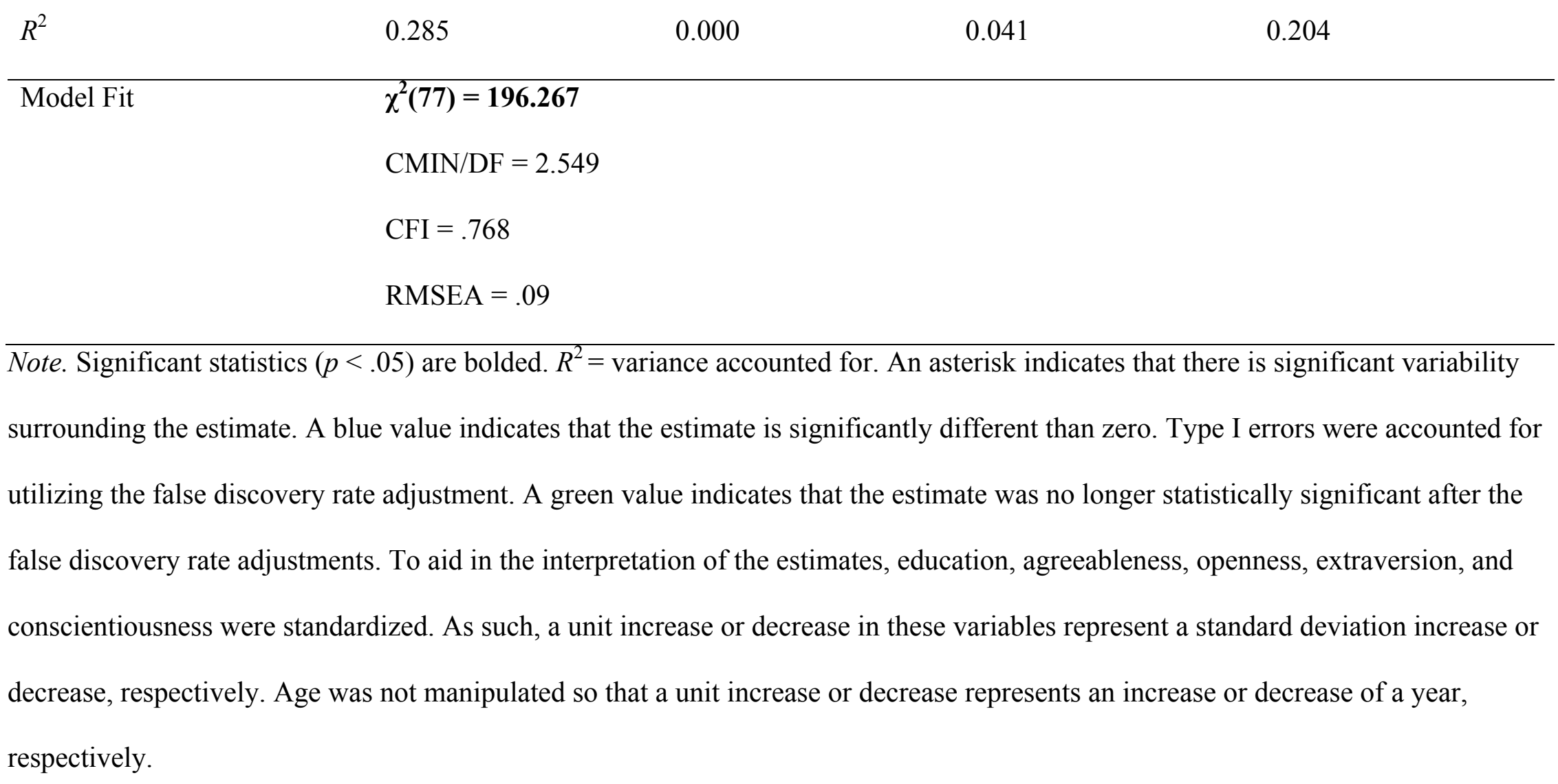


Table K55

Research Question 8 - Examination of the Bidirectional Association between the Latent Growth Curves of NSIs from Friend and Extraversion After Accounting for the Demographic Variables and Remaining Personality Traits for Younger Adults

\begin{tabular}{|c|c|c|c|c|c|c|c|c|}
\hline & \multicolumn{4}{|c|}{ NSIs from Friend Latent Growth Curve } & \multicolumn{4}{|c|}{ Extraversion Latent Growth Curve } \\
\hline & \multicolumn{2}{|l|}{ Intercept } & \multicolumn{2}{|l|}{ Slope } & \multicolumn{2}{|l|}{ Intercept } & \multicolumn{2}{|l|}{ Slope } \\
\hline & $b$ & S.E. & $b$ & S.E. & $b$ & S.E. & $b$ & S.E. \\
\hline Estimate & $1.755^{*}$ & 0.182 & 0.035 & 0.022 & $3.483 *$ & 0.226 & -0.029 & 0.019 \\
\hline Age & 0.005 & 0.005 & -0.001 & 0.000 & -0.008 & 0.007 & 0.000 & 0.000 \\
\hline Gender & -0.019 & 0.042 & 0.000 & 0.003 & -0.094 & 0.052 & 0.002 & 0.002 \\
\hline \multicolumn{9}{|l|}{$(0=$ Female $)$} \\
\hline Education & 0.005 & 0.022 & 0.000 & 0.001 & 0.002 & 0.027 & -0.001 & 0.001 \\
\hline Agreeableness & 0.015 & 0.022 & -0.003 & 0.001 & - & - & - & - \\
\hline Openness & 0.027 & 0.022 & -0.001 & 0.001 & - & - & - & - \\
\hline Neuroticism & 0.091 & 0.020 & -0.005 & 0.002 & - & - & - & - \\
\hline Conscientiousness & -0.036 & 0.21 & -0.002 & 0.001 & - & - & - & - \\
\hline NSIs from Friend Intercept & - & - & -0.005 & 0.008 & - & - & 0.005 & 0.005 \\
\hline
\end{tabular}




\begin{tabular}{|c|c|c|c|c|c|c|}
\hline Extraversion Intercept & - & 0.000 & 0.003 & - & -0.001 & 0.004 \\
\hline$R^{2}$ & .110 & .301 & & .014 & .080 & \\
\hline
\end{tabular}

\begin{tabular}{ll}
\hline Model Fit & $\chi^{\mathbf{2}(49)=341.89}$ \\
& $\mathrm{CMIN} / \mathrm{DF}=6.97$ \\
$\mathrm{CFI}=.761$ \\
$\mathrm{RMSEA}=.11$
\end{tabular}

Note. Significant statistics $(p<.05)$ are bolded. $R^{2}=$ variance accounted for. An asterisk indicates that there is significant variability surrounding the estimate. A blue value indicates that the estimate is significantly different than zero. Type I errors were accounted for utilizing the false discovery rate adjustment. A green value indicates that the estimate was no longer statistically significant after the false discovery rate adjustments. To aid in the interpretation of the estimates, education, agreeableness, openness, neuroticism, and conscientiousness were standardized. As such, a unit increase or decrease in these variables represent a standard deviation increase or decrease, respectively. Age was not manipulated so that a unit increase or decrease represents an increase or decrease of a year, respectively. 
Table K56

Research Question 8 - Examination of the Bidirectional Association between the Latent Growth Curves of NSIs from Friends and Extraversion After Accounting for the Demographic Variables and Remaining Personality Traits for Middle-Aged Adults

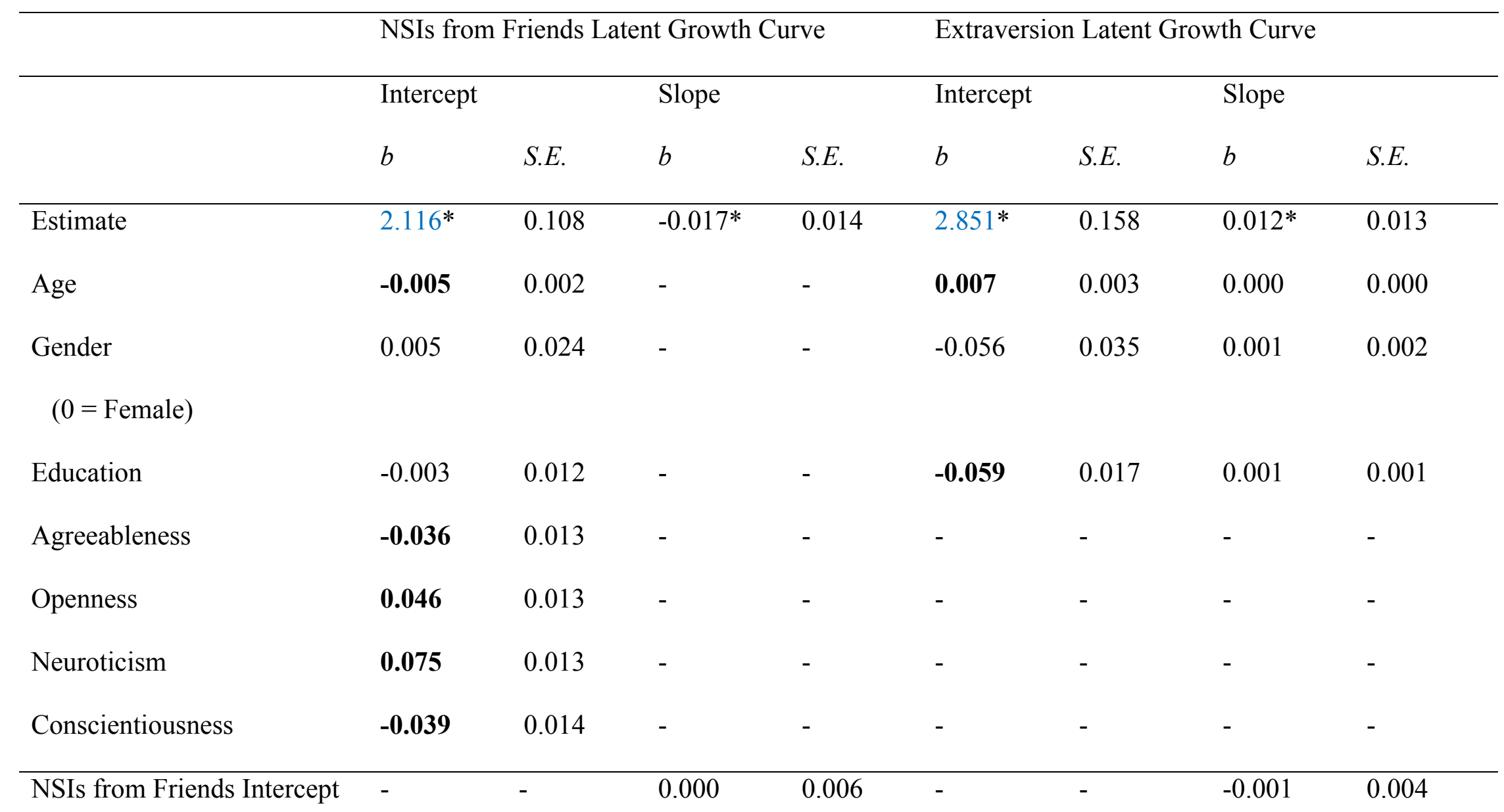




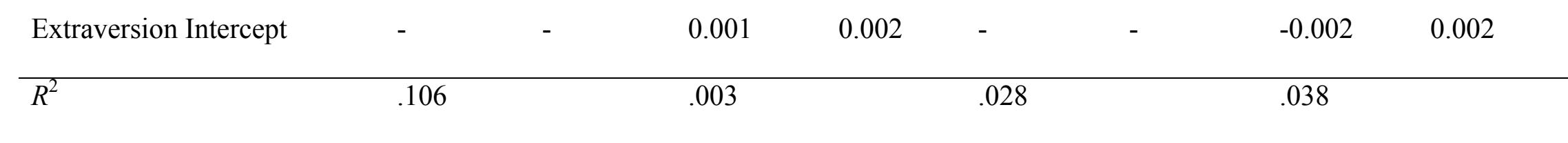

\begin{tabular}{ll}
\hline Model Fit & $\left.\chi^{2} \mathbf{5 6}\right)=\mathbf{7 0 7 . 8 0}$ \\
& $\mathrm{CMIN} / \mathrm{DF}=12.63$ \\
$\mathrm{CFI}=.767$ \\
$\mathrm{RMSEA}=.11$
\end{tabular}

Note. Significant statistics $(p<.05)$ are bolded. $R^{2}=$ variance accounted for. An asterisk indicates that there is significant variability surrounding the estimate. A blue value indicates that the estimate is significantly different than zero. Type I errors were accounted for utilizing the false discovery rate adjustment. A green value indicates that the estimate was no longer statistically significant after the false discovery rate adjustments. To aid in the interpretation of the estimates, education, agreeableness, openness, neuroticism, and conscientiousness were standardized. As such, a unit increase or decrease in these variables represent a standard deviation increase or decrease, respectively. Age was not manipulated so that a unit increase or decrease represents an increase or decrease of a year, respectively. 
Table K57

Research Question 8 - Examination of the Bidirectional Association between the Latent Growth Curves of NSIs from Friends and Extraversion After Accounting for the Demographic Variables and Remaining Personality Traits for Older Adults

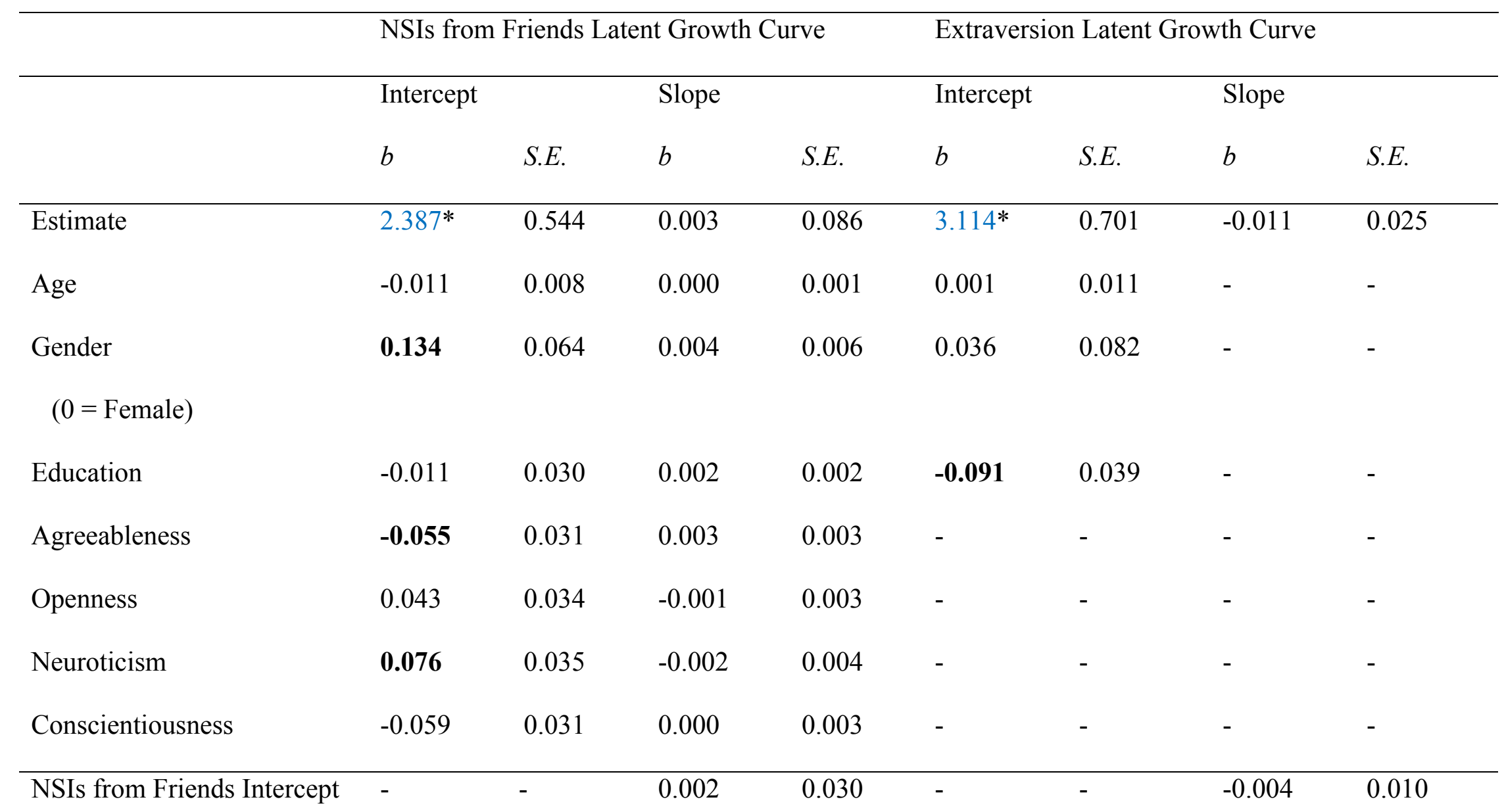




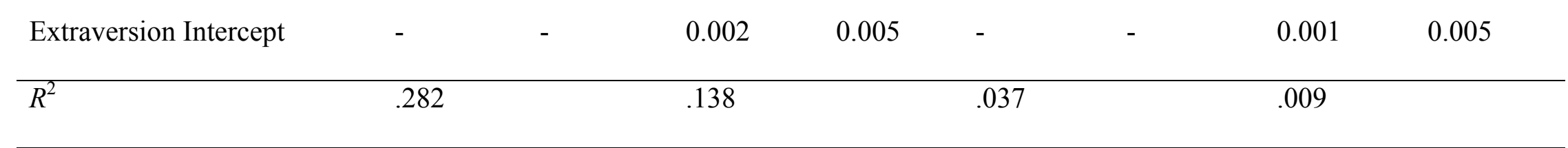

\begin{tabular}{ll}
\hline Model Fit & $\chi^{2} \mathbf{( 5 2 )}=\mathbf{2 5 6 . 0 2}$ \\
& $\mathrm{CMIN} / \mathrm{DF}=4.92$ \\
$\mathrm{CFI}=.654$ \\
$\mathrm{RMSEA}=.15$
\end{tabular}

Note. Significant statistics $(p<.05)$ are bolded. $R^{2}=$ variance accounted for. An asterisk indicates that there is significant variability surrounding the estimate. A blue value indicates that the estimate is significantly different than zero. Type I errors were accounted for utilizing the false discovery rate adjustment. A green value indicates that the estimate was no longer statistically significant after the false discovery rate adjustments. To aid in the interpretation of the estimates, education, agreeableness, openness, neuroticism, and conscientiousness were standardized. As such, a unit increase or decrease in these variables represent a standard deviation increase or decrease, respectively. Age was not manipulated so that a unit increase or decrease represents an increase or decrease of a year, respectively. 
Table K58

Research Question 8 - Examination of the Bidirectional Association between the Latent Growth Curves of NSIs from Friend and Conscientiousness After Accounting for the Demographic Variables and Remaining Personality Traits for Younger Adults

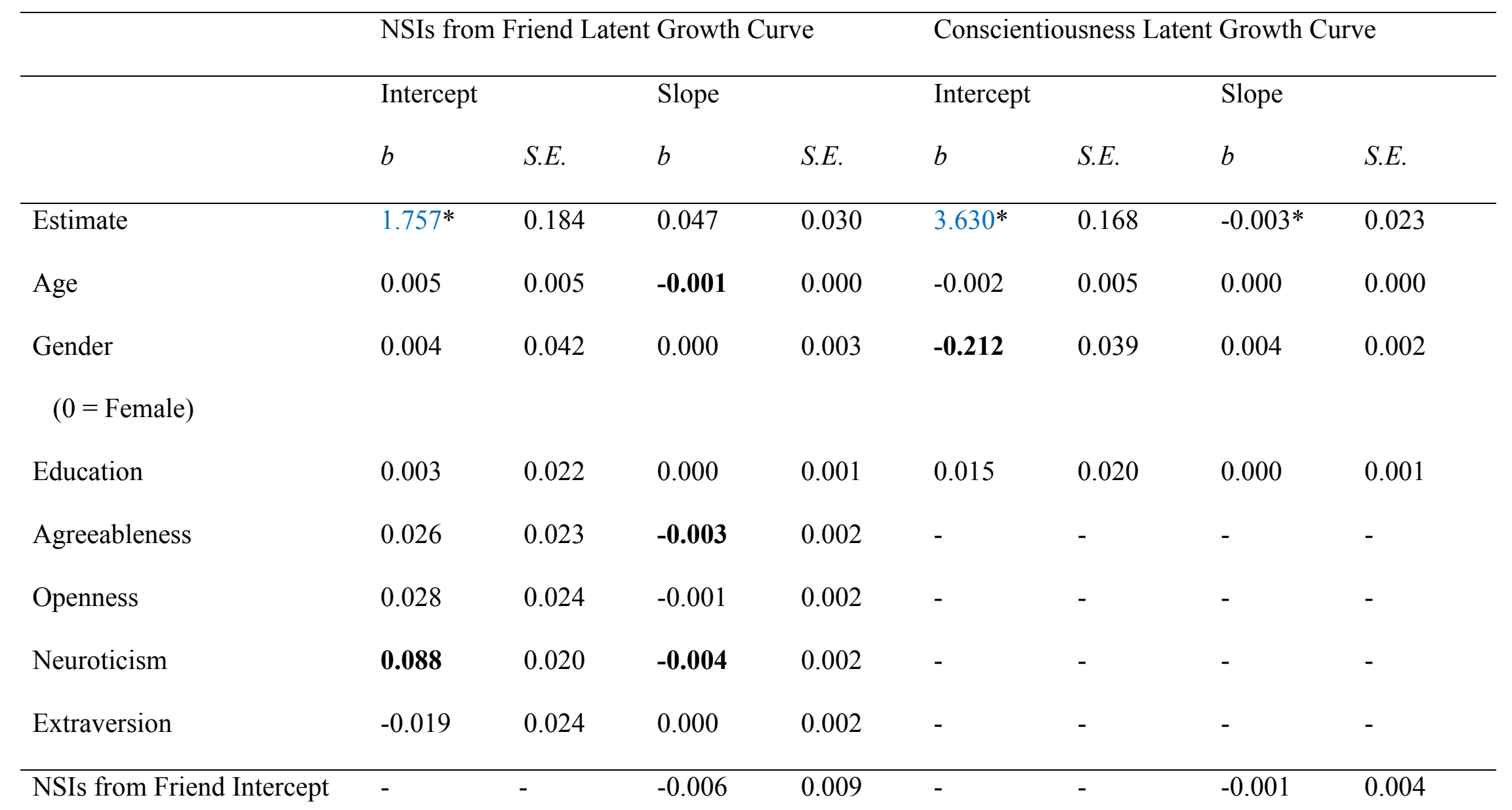




\begin{tabular}{|c|c|c|c|c|c|c|}
\hline Conscientiousness Intercept & - & -0.004 & 0.005 & - & -0.001 & 0.005 \\
\hline$R^{2}$ & .089 & .289 & & .092 & .063 & \\
\hline
\end{tabular}

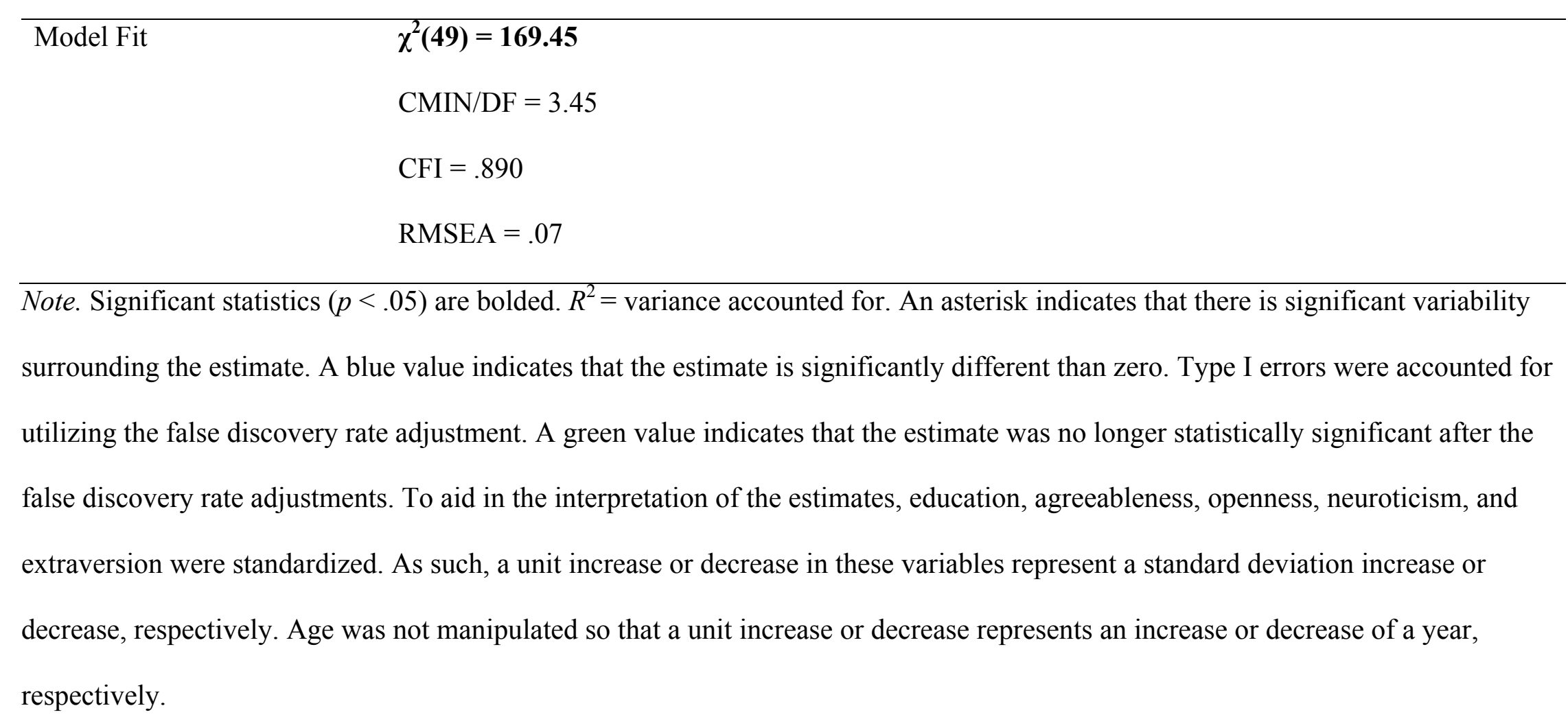


Table K59

Research Question 8 - Examination of the Bidirectional Association between the Latent Growth Curves of NSIs from Friends and Conscientiousness After Accounting for the Demographic Variables and Remaining Personality Traits for Middle-Aged Adults

\begin{tabular}{|c|c|c|c|c|c|c|c|c|}
\hline & \multicolumn{4}{|c|}{ NSIs from Friends Latent Growth Curve } & \multicolumn{4}{|c|}{ Conscientiousness Latent Growth Curve } \\
\hline & Intercept & & Slope & & Intercept & & Slope & \\
\hline & $b$ & S.E. & $b$ & S.E. & $b$ & S.E. & $b$ & S.E. \\
\hline Estimate & $2.130 *$ & 0.108 & $-0.024 *$ & 0.020 & $3.545^{*}$ & 0.102 & $0.001 *$ & 0.001 \\
\hline Age & -0.005 & 0.002 & - & - & 0.000 & 0.002 & - & - \\
\hline $\begin{array}{l}\text { Gender } \\
\qquad(0=\text { Female })\end{array}$ & 0.013 & 0.024 & - & - & -0.089 & 0.023 & - & - \\
\hline Education & -0.008 & 0.012 & - & - & 0.43 & 0.011 & - & - \\
\hline Agreeableness & -0.050 & 0.014 & - & - & - & - & - & - \\
\hline Openness & 0.037 & 0.014 & - & - & - & - & - & - \\
\hline Neuroticism & 0.073 & 0.013 & - & - & - & - & - & - \\
\hline Extraversion & 0.014 & 0.015 & - & - & - & - & - & - \\
\hline NSIs from Friends Intercept & - & - & 0.001 & 0.006 & - & - & - & - \\
\hline
\end{tabular}




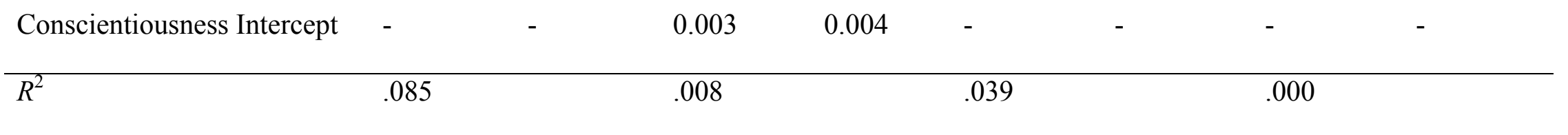

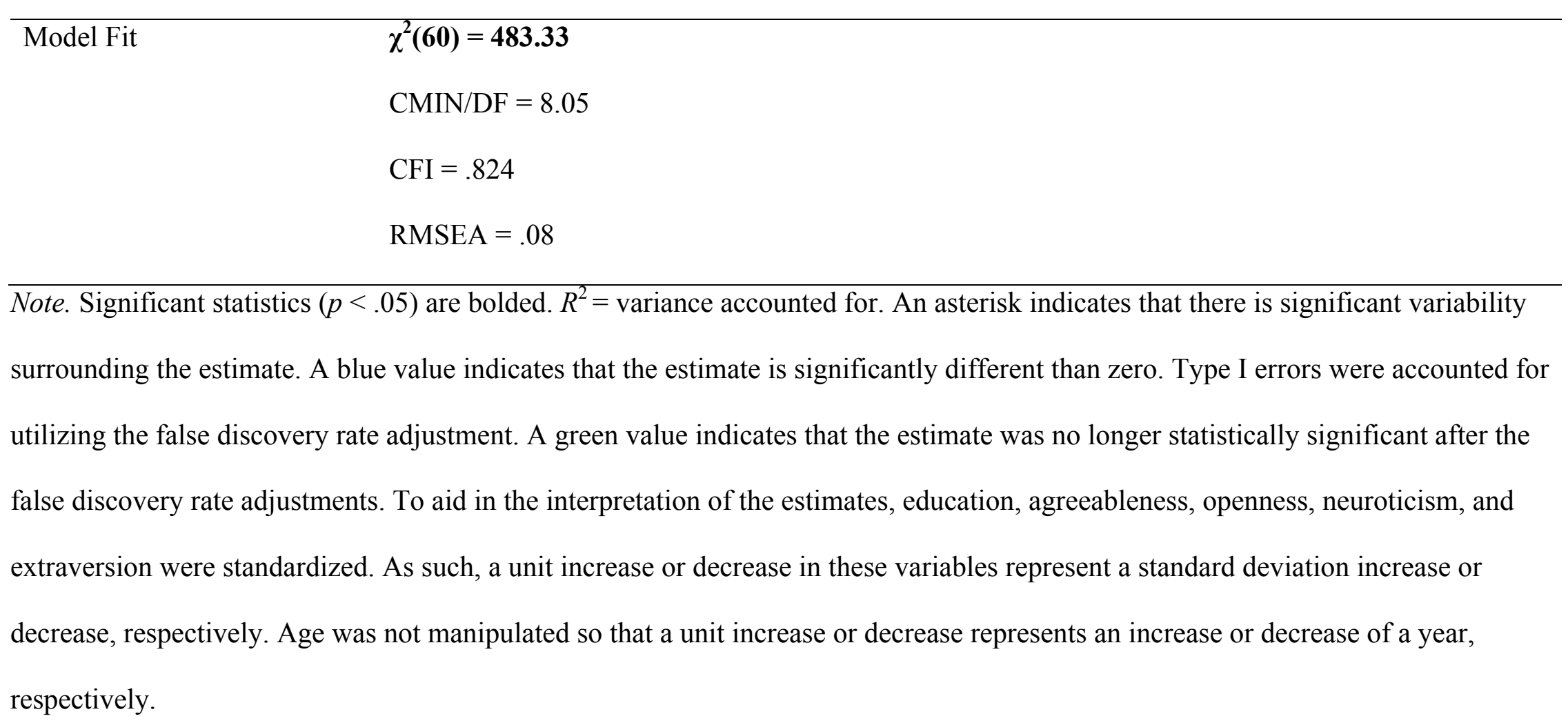


Table K60

Research Question 8 - Examination of the Bidirectional Association between the Latent Growth Curves of NSIs from Friends and Conscientiousness After Accounting for the Demographic Variables and Remaining Personality Traits for Older Adults

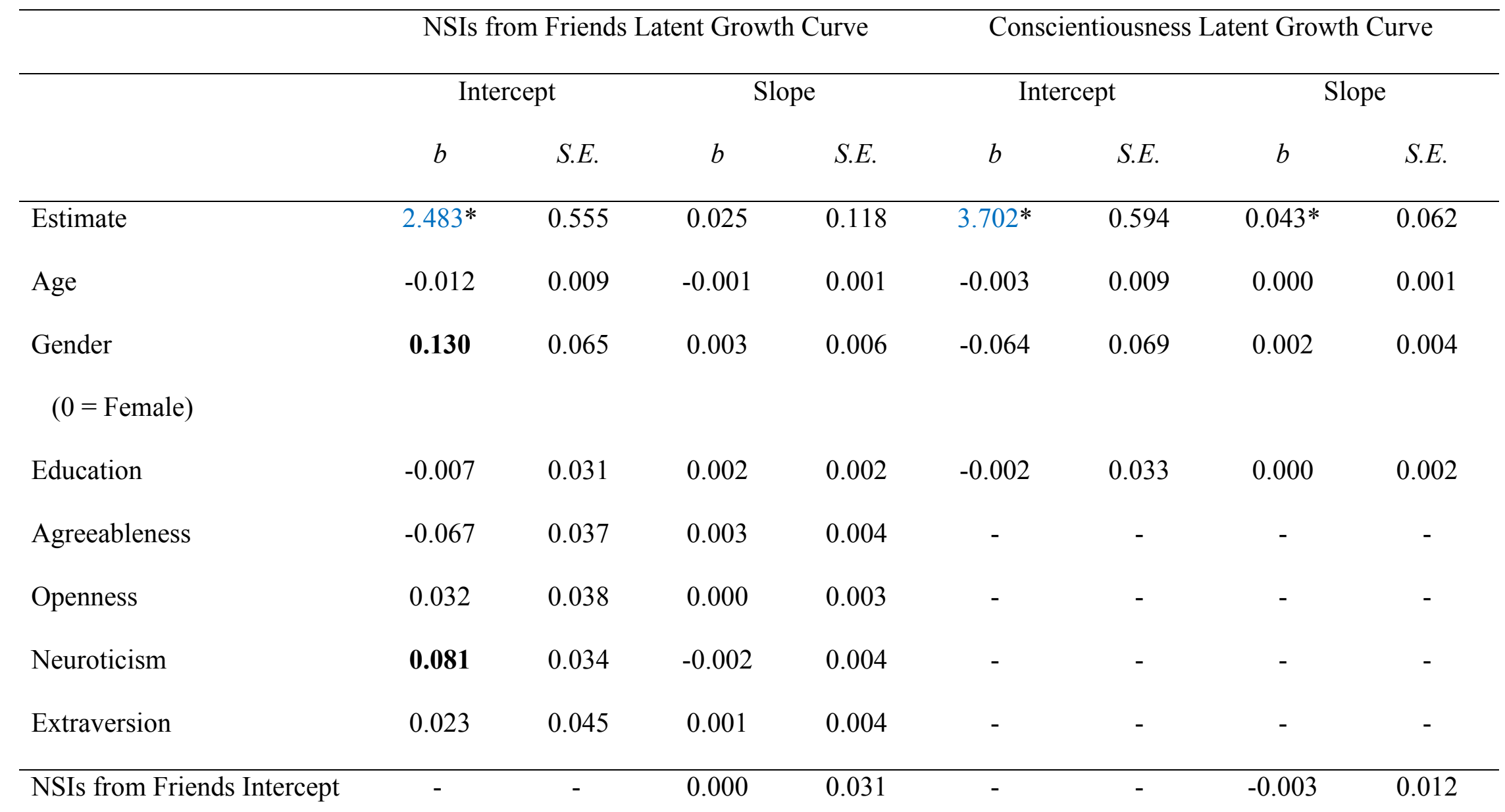




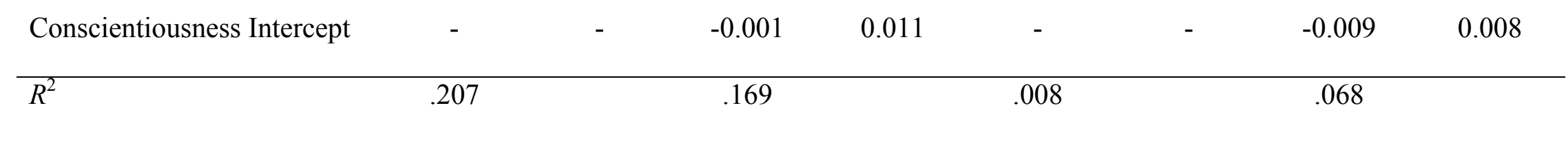

\begin{tabular}{l}
\hline Model Fit \\
CMIN $=\mathbf{1 5 0 . 2 9}$ \\
$\mathrm{CFI}=.781$ \\
$\mathrm{RMSEA}=.11$ \\
\hline
\end{tabular}

Note. Significant statistics $(p<.05)$ are bolded. $R^{2}=$ variance accounted for. An asterisk indicates that there is significant variability surrounding the estimate. A blue value indicates that the estimate is significantly different than zero. Type I errors were accounted for utilizing the false discovery rate adjustment. A green value indicates that the estimate was no longer statistically significant after the false discovery rate adjustments. To aid in the interpretation of the estimates, education, agreeableness, openness, neuroticism, and extraversion were standardized. As such, a unit increase or decrease in these variables represent a standard deviation increase or decrease, respectively. Age was not manipulated so that a unit increase or decrease represents an increase or decrease of a year, respectively. 